\title{
Magnetotelluric Data, Rainier Mesa/Shoshone Mountain, Nevada Test Site, Nevada
}

Open-File Report 2006-1215 


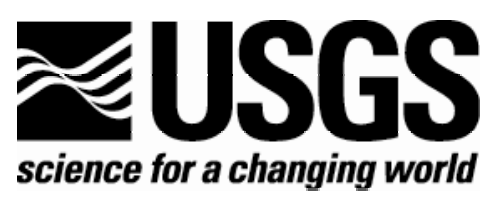

\section{Magnetotelluric Data, Rainier Mesa/Shoshone Mountain, Nevada Test Site, Nevada}

By Jackie M. Williams, Jay A. Sampson, Brian D. Rodriguez, and Theodore H. Asch

Open-File Report 2006-1215

U.S. DEPARTMENT OF THE INTERIOR

U.S. GEOLOGICAL SURVEY 


\section{U.S. Department of the Interior DIRK KEMPTHORNE, Secretary}

\section{U.S. Geological Survey Mark D. Myers, Director}

U.S. Geological Survey, Reston, Virginia 2006

For product and ordering information:

World Wide Web: http://www.usgs.gov/pubprod

Telephone: 1-888-ASK-USGS

For more information on the USGS - the Federal source for science about the Earth, its natural and living resources, natural hazards, and the environment:

World Wide Web: http://www.usgs.gov

Telephone: 1-888-ASK-USGS

Any use of trade, product, or firm names is for descriptive purposes only and does not imply endorsement by the U.S. Government.

Although this report is in the public domain, permission must be secured from the individual copyright owners to reproduce any copyrighted material contained within this report. 


\section{Contents}

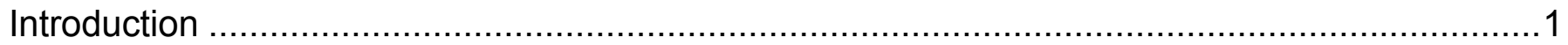

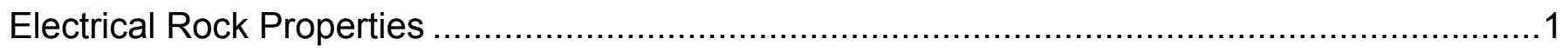

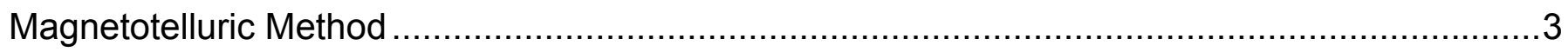

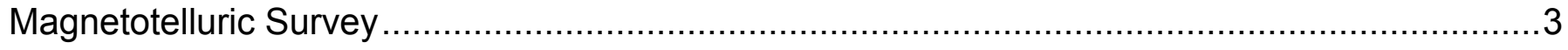

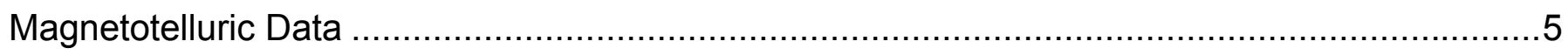

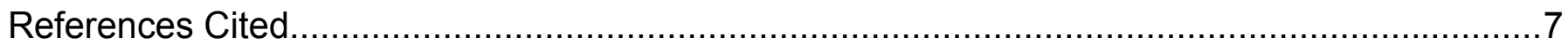

\section{Figure}

1. Index map showing magnetotelluric (MT) stations in the areas of Rainier Mesa and

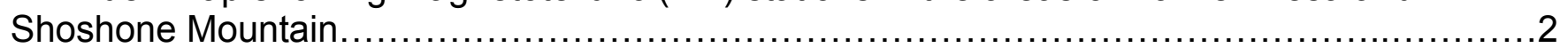

\section{Table}

1. Station Locations.

\section{Appendix}

Magnetotelluric Data Plots 


\section{Introduction}

The United States Department of Energy (DOE) and the National Nuclear Security Administration (NNSA) at their Nevada Site Office (NSO) are addressing ground-water contamination resulting from historical underground nuclear testing through the Environmental Management (EM) program and, in particular, the Underground Test Area (UGTA) project.

From 1951 to 1992,828 underground nuclear tests were conducted at the Nevada Test Site northwest of Las Vegas. Most of these tests were conducted hundreds of feet above the ground-water table; however, more than 200 of the tests were near or within the water table. This underground testing was limited to specific areas of the Nevada Test Site, including Pahute Mesa, Rainier Mesa/Shoshone Mountain, Frenchman Flat, and Yucca Flat.

One issue of concern is the nature of the somewhat poorly constrained pre-Tertiary geology, and its effects on ground-water flow. Ground-water modelers would like to know more about the hydrostratigraphy and geologic structure to support a hydrostratigraphic framework model that is under development for the Rainier Mesa/Shoshone Mountain Corrective Action Unit (Bechtel Nevada, 2006).

During 2005, the U.S. Geological Survey (USGS), in cooperation with the DOE and NNSA-NSO, collected and processed data from twenty-six magnetotelluric (MT) and audiomagnetotelluric (AMT) sites at the Nevada Test Site. The 2005 data stations were located on and near Rainier Mesa and Shoshone Mountain to assist in characterizing the pre-Tertiary geology in those areas. These new stations extend the area of the hydrogeologic study previously conducted in Yucca Flat. This work will help refine what is known about the character, thickness, and lateral extent of pre-Tertiary confining units. In particular, a major goal has been to define the upper clastic confining unit (UCCU - late Devonian to Mississippian-age siliciclastic rocks assigned to the Eleana Formation and Chainman Shale) from the Yucca Flat area and west towards Shoshone Mountain, to Buckboard Mesa in the south, and onto Rainier Mesa in the north. Subsequent interpretation will include a three-dimensional (3-D) character analysis and a two-dimensional (2-D) resistivity model. The purpose of this report is to release the MT sounding data for the twenty-six stations shown in figure 1. No interpretation of the data is included here.

\section{Electrical Rock Properties}

Electromagnetic geophysical methods detect variations in the electrical properties of rocks-in particular, electrical resistivity, or its inverse, electrical conductivity. Electrical resistivity can be correlated with geologic units on the surface and at depth using lithologic logs to provide a 3-D picture of subsurface geology. In the upper crust the resistivity of geologic units is largely dependent upon their fluid content, pore-volume porosity, interconnected fracture porosity, and conductive mineral content (Keller, 1989). Although there is not a one-to-one relation between lithology and resistivity, there are general correlations that can be made using typical resistivity values, even though values can be found at other localities that may fall outside of the ranges presented below (Palacky, 1987). Fluids within the pore spaces and fracture openings, especially if saline, can reduce resitivities in what would otherwise be a resistive rock matrix. Resistivity also can be lowered by the presence of electrically conductive clay minerals, graphitic carbon, and metallic mineralization. It is common, for example, for altered volcanic 


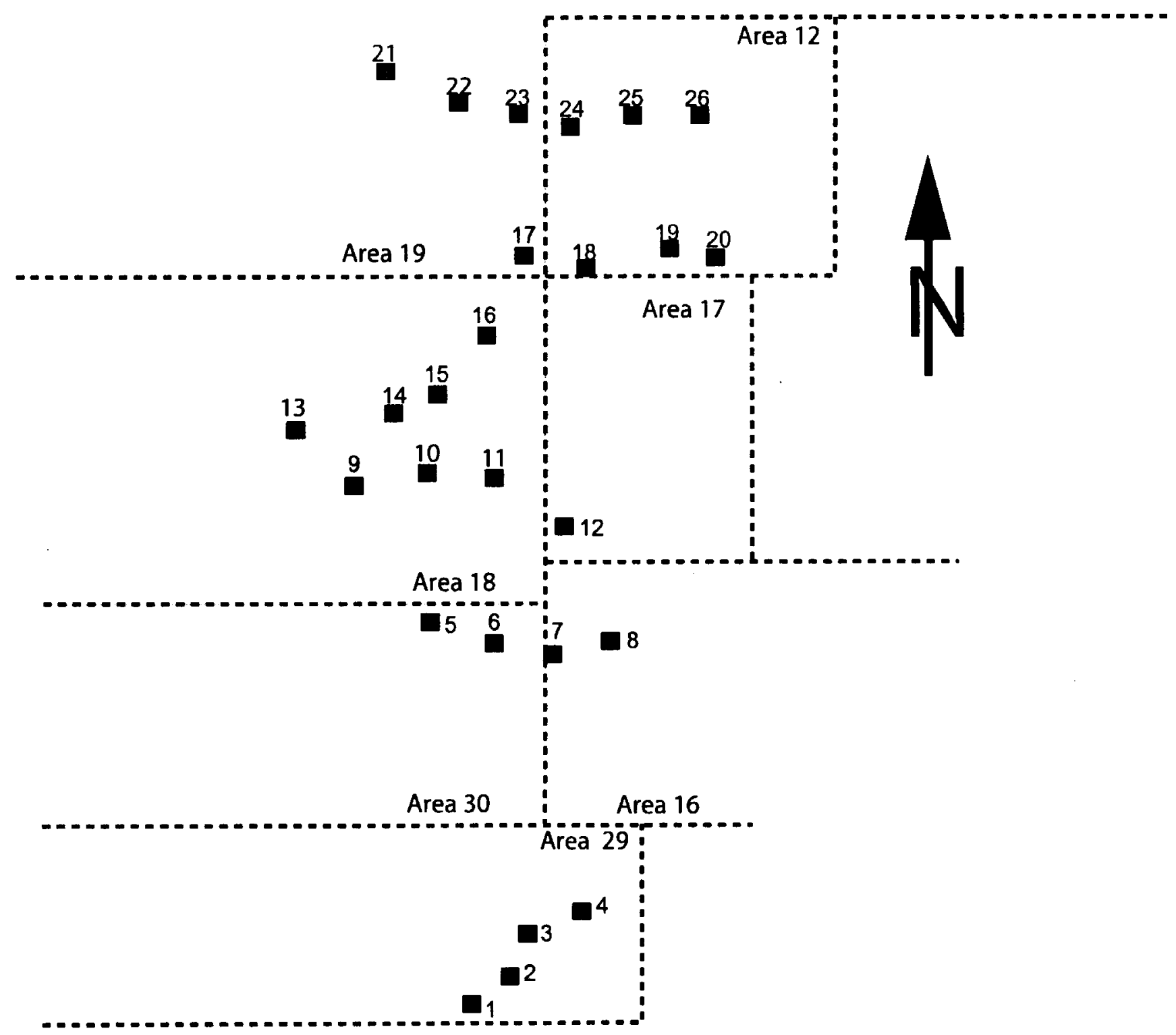

Figure 1. Index map showing magnetotelluric (MT) stations in the areas of Rainier Mesa and Shoshone Mountain. The 26 stations were collected in 2005. Dashed lines are Nevada Test Site areas. 
rocks to contain replacement minerals that have resistivities ten times lower than those of the surrounding rocks (Nelson and Anderson, 1992). Fine-grained sediments, such as clay-rich alluvium, marine shales, and other mudstones, are normally conductive from a few ohm-m to a few tens of ohm-m (Keller, 1987; Palacky, 1987). Metamorphic rocks (non-graphitic) and unaltered, unfractured igneous rocks are normally moderately to highly resistive (a few hundreds to thousands of ohm-m). Carbonate rocks can have similarly high resistivities depending on their fluid content, porosity, and impurities (Keller, 1987; Palacky, 1987). Fault zones may be moderately conductive (tens of ohm-m) when comprised of rocks fractured enough to have hosted fluid transport and consequent mineralogical alteration (Eberhart-Phillips and others, 1995). Higher subsurface temperatures cause higher ionic mobility that reduces rock resistivities (Keller, 1987; Palacky, 1987). Tables of electrical resistivities for a variety of rocks, minerals, and geological environments may be found in Keller (1987) and Palacky (1987).

\section{Magnetotelluric Method}

The MT method is a passive-surface electromagnetic geophysical technique that measures variations in the Earth's natural electromagnetic fields to investigate the electrical resistivity structure of the subsurface from depths of tens of meters to tens of kilometers (Vozoff, 1991). Worldwide lightning activity at frequencies of 10,000 to $1 \mathrm{Hertz}(\mathrm{Hz})$ and geomagnetic micro-pulsations at frequencies of 1 to $0.001 \mathrm{~Hz}$ provide the majority of natural signal used by the MT method. The natural electromagnetic wave propagates vertically into the Earth due to the large resistivity contrast between the air and the Earth, causing a vertical refraction of the electromagnetic wave transmitted into the Earth (Vozoff, 1972).

The natural fields are recorded in the xyz direction for the magnetic field and the xy direction for the electric field at the Earth's surface. The resulting time-series signals are used to derive tensor apparent-resistivities and phases by first converting them to complex cross-spectra using Fourier-transform techniques. Least squares, cross-spectral analysis (Bendat and Piersol, 1971) are used to solve for a tensor transfer function. Prior to conversion to apparent resistivity and phase, the tensor is normally rotated into principal directions that usually correspond to the direction of maximum and minimum apparent resistivity. For a two-dimensional (2-D) Earth, in which Earth's resistivity structure varies with depth and in one lateral direction, the MT fields can be decoupled into transverse-electric (TE) and transverse-magnetic (TM) modes; 2-D resistivity modeling is generally computed to fit both modes. When the geology satisfies the 2-D assumption, the MT data for the TE mode represents electric fields that are oriented parallel to geologic strike, and the data for the TM mode represents electric fields oriented perpendicular to strike. The MT method is well suited for studying complicated geological environments because the electric and magnetic fields are sensitive to vertical and horizontal variations in resistivity. The method is capable of establishing whether the electromagnetic fields are responding to subsurface rock bodies of effectively 1, 2, or 3 dimensions. An introduction to the MT method and references for a more advanced understanding are contained in Dobrin and Savit (1988) and Vozoff (1991).

\section{Magnetotelluric Survey}

In May of 2005, data were collected at 26 stations on and near Rainier Mesa and Shoshone Mountain. The station locations were chosen to constrain the geologic/hydrostratigraphic interpretation, for proximity to roads, and to avoid, where possible, 
electrical noise from power lines and vehicles. The low-frequency data $(0.0002$ to $200 \mathrm{~Hz}) \mathrm{were}$ collected with an Electromagnetic Instruments, Inc., (EMI) MT24/LF 24-bit system (EMI, 2002), and the high-frequency data (4 Hz to $23,000 \mathrm{~Hz}$ ) were collected with a portable EMI MT1 system (EMI, 1996). For the low-frequency data, horizontal electric fields were measured using three copper/copper sulfate porous-pot electrodes placed in an L-shaped array with dipole lengths of 30 meters $(\mathrm{m})$. Titanium electrodes were used in a similar array for the highfrequency data acquisition. The orthogonal, horizontal magnetic fields in the direction of the electric-field measurement array were sensed using EMI's high-magnetic-permeability, mumetal-cored induction coils. For the low-frequency data, two single-station recordings of the orthogonal, horizontal components of the electric and magnetic fields and the vertical magnetic field were acquired at Global Positioning System (GPS) referenced times and were used as remote references for each other as shown in Table 1. The high-frequency data were recorded as non-remote referenced single stations.

The following table lists the $26 \mathrm{MT}$ and AMT station locations as recorded using a GPS during field acquisition. Coordinates are referenced to the 1866 Clarke spheroid and North American 1983 Western United States datum. Longitude and latitude format is degrees, minutes, seconds. Universal Transverse Mercator (UTM) Zone 11 North units are in meters. Station elevation is given in meters (NAVD29) above sea level. The accuracy of the $x, y$, component is $\pm 5 \mathrm{~m}$. The accuracy of the $z$ component is $\pm 10 \mathrm{~m}$.

Table 1. Station Locations $[\mathrm{d}, \mathrm{m}, \mathrm{s}$ degrees, minutes,seconds; $m$, meters]

\begin{tabular}{|c|c|c|c|c|c|c|}
\hline Station & $\begin{array}{r}\text { Latitude } \\
(\mathrm{d}, \mathrm{m}, \mathrm{s})\end{array}$ & $\begin{array}{l}\text { Longitude } \\
\qquad(\mathrm{d}, \mathrm{m}, \mathrm{s})\end{array}$ & $\begin{array}{c}\text { Elevation } \\
\text { above sea } \\
\text { level } \\
\text { (m) }\end{array}$ & $\begin{array}{l}\text { Zone } 11 \\
\text { Northings } \\
\quad(\mathrm{m})\end{array}$ & $\begin{array}{c}\text { Zone } 11 \\
\text { Eastings } \\
\quad(\mathrm{m})\end{array}$ & $\begin{array}{c}\text { Remote } \\
\text { Reference } \\
\text { d to } \\
\text { Station }\end{array}$ \\
\hline 1 & $36,55^{\prime} 28.2$ & $116,16^{\prime} 20.1$ & 1,603 & 4086744 & 564819 & 5 \\
\hline 2 & $36,56^{\prime} 03.7$ & $116,15^{\prime} 14.9$ & 1,729 & 4087851 & 566420 & 6 \\
\hline 3 & $36,56 ' 48.6$ & $116,14 ' 52.9$ & 1,929 & 4089239 & 566954 & 7 \\
\hline 4 & $36,57^{\prime} 15.4$ & $116,13^{\prime} 30.8$ & 1,648 & 4090080 & 568979 & 8 \\
\hline 5 & $37,04^{\prime} 45.8$ & $116,19^{\prime} 17.8$ & 1,454 & 4100906 & 562963 & 1 \\
\hline 6 & $37,02^{\prime} 45.2$ & $116,15^{\prime} 42.8$ & 1,537 & 4100216 & 565636 & 2 \\
\hline 7 & $37,02^{\prime} 31.6$ & $116,14^{\prime} 01.8$ & 1,665 & 4099819 & 568133 & 3 \\
\hline 8 & $37,02^{\prime} 49.1$ & $116,12^{\prime} 36.8$ & 1,602 & 4100375 & 570229 & 4 \\
\hline 9 & $37,05^{\prime} 53.5$ & $116,19^{\prime} 15.2$ & 1,521 & 4105982 & 560348 & 12 \\
\hline 10 & $37,06^{\prime} 05.6$ & $116,17^{\prime} 42.4$ & 1,581 & 4106370 & 562632 & 21 \\
\hline 11 & $37,06^{\prime} 06.1$ & $116,15^{\prime} 48.0$ & 1,632 & 4106408 & 565460 & 17 \\
\hline 12 & $37,05^{\prime} 02.6$ & $116,13^{\prime} 42.5$ & 1,640 & 4104474 & 568573 & 9 \\
\hline 13 & $37,07^{\prime} 04.2$ & $116,20^{\prime} 38.3$ & 1,566 & 4108146 & 558283 & 22 \\
\hline 14 & $37,07^{\prime} 21.3$ & $116,18^{\prime} 28.3$ & 1,578 & 4108693 & 561479 & 20 \\
\hline 15 & $37,07^{\prime} 42.9$ & $116,16 ' 33.1$ & 1,686 & 4109380 & 564321 & 26 \\
\hline 16 & $37,08^{\prime} 53.4$ & $116,15^{\prime} 41.2$ & 1,733 & 4111563 & 565505 & 25 \\
\hline 17 & $37,10^{\prime} 33.3$ & $116,14^{\prime} 48.5$ & 1,828 & 4114650 & 566860 & 11 \\
\hline 18 & $37,09 ' 57.1$ & $116,13^{\prime} 35.8$ & 2,096 & 4113551 & 568664 & 24 \\
\hline 19 & $37,10^{\prime} 31.7$ & $116,11^{\prime} 00.7$ & 1,772 & 4114652 & 572478 & 23 \\
\hline 20 & $37,10^{\prime} 36.5$ & $116,10^{\prime} 12.5$ & 1,867 & 4114810 & 573666 & 14 \\
\hline 21 & $37,14^{\prime} 00.6$ & $116,18^{\prime} 23.2$ & 2,127 & 4121001 & 561521 & 10 \\
\hline
\end{tabular}




\begin{tabular}{lllllll}
$\mathbf{2 2}$ & $37,13^{\prime} 30.6$ & $116,16^{\prime} 30.0$ & 2,063 & 4120102 & 564318 & 13 \\
$\mathbf{2 3}$ & $37,13^{\prime} 05.9$ & $116,15^{\prime} 02.1$ & 2,097 & 4119357 & 566489 & 19 \\
$\mathbf{2 4}$ & $37,13^{\prime} 30.7$ & $116,13^{\prime} 19.1$ & 2,107 & 4120130 & 569030 & 18 \\
$\mathbf{2 5}$ & $37,13^{\prime} 36.7$ & $116,12^{\prime} 20.6$ & 2,059 & 4120333 & 570460 & 16 \\
$\mathbf{2 6}$ & $37,13^{\prime} 03.8$ & $116,10^{\prime} 37.8$ & 2,062 & 4119342 & 573002 & 15 \\
\hline
\end{tabular}

\section{Magnetotelluric Data}

The recorded time-series data were transformed to the frequency domain and processed to determine a 2-D apparent resistivity and phase tensor at each site. Rotation of the impedance tensor allows for decoupling into the TE and TM modes. The data provided here have not been rotated from the original north-south, east-west acquisition orientation. During the analysis and interpretation process, each station will be rotated to a fixed angle determined by the given nominal profile orientation. Low-frequency time-series data were edited, and cross-power files were created with Egbert's (1997) multiple-station magnetotelluric data-processing algorithms using remote references. Cross-power files were sorted to select optimal signal-to-noise timeseries data sets (see appendix 1).

The effects of near-surface resistivity anomalies can cause what are known as "static shifts" in the data (Sternberg and others, 1988). Cultural features also can affect the measured magnetotelluric responses. These include fences, pipelines, communication lines, railways, and other manmade conductors.

The figures in appendix 1 represent the field-processed MT data for each station, after the time-series data were converted to the frequency domain and the tensor-transfer function was developed.

For each station, nine separate plots are given:

1. Apparent Resistivity ( $\mathrm{x}$ and $\mathrm{y}$ symbols are $\mathrm{xy}$ and $\mathrm{yx}$ components)

2. Impedance Phase ( $\mathrm{x}$ and $\mathrm{y}$ symbols are $\mathrm{xy}$ and $\mathrm{yx}$ components)

3. Rotation Angle

4. Impedance Skew

5. Multiple Coherency ( $\mathrm{x}$ and $\mathrm{y}$ symbols are $\mathrm{xy}$ and $\mathrm{yx}$ components)

6. Impedance Polar Plots

7. Tipper Magnitude

8. Tipper Strike

9. $\mathrm{HzHx}$ (x symbol) and HzHy (o symbol) Coherency

Error bars (],[) on the Apparent Resistivity, Impedance Phase, Skew, Tipper Magnitude, and Tipper Strike plots represent probable errors within one standard deviation of the sample variance (Gamble and others, 1979).

Apparent resistivity is the approximate ratio of the electric-field strength to the magnetic-field strength at a given frequency. The impedance phase is proportional to the slope of the apparent resistivity curve on a log-log plot, but from baselines at \pm 45 degrees (Vozoff, 1991). A measure of the dimensionality for MT data is provided by the impedance skew of the impedance tensor (Vozoff, 1972). If the effective measured resistivity response to the geology beneath a MT station truly is one or two dimensional, then the skew will be zero. Instrumental 
and environmental sources of electrical noise can cause non-zero skew values. Skew values typically are small (about 0.1 ) for relatively low-noise recordings. Higher skews (above 0.2) are an indication of either the resistivity response to 3-D geology or higher levels of noise. Manmade electrical noise, such as power lines, power generators, and moving vehicles and trains, can have a negative effect on MT data quality. All of these local disturbances can produce incoherent noise that mainly affects frequencies above $1 \mathrm{~Hz}$. Other manmade electrical noise, such as direct-current electric trains and active cathodic protection of pipelines, produces coherent electromagnetic signals that mainly affect frequencies below $1 \mathrm{~Hz}$.

In the survey area, noise from a number of small power lines and small moving vehicles was negligible at distances greater than $0.4 \mathrm{~km}$ from the noise source. Power-line signal levels were measured at each site and typically were less than 20 percent of the maximum recordable signals. Noise from larger power lines, power generators, pipelines, and trains was negligible at distances greater than $5 \mathrm{~km}$. Local lightning, wind, and rainstorms also can degrade data quality. Burying the magnetic induction coils and the electric dipole wires minimized wind noise.

Predicted values of the electric field can be computed from the measured values of the magnetic field (Vozoff, 1991). The coherence of the predicted electric field with the measured electric field is a measure of the signal-to-noise ratio provided in the multiple coherency plots. Values are normalized between 0 and 1; values at 0.5 signify signal levels equal to noise levels. For this data set, coherencies generally were at an acceptable level, except at times in the frequency ranges of 0.01 to $5 \mathrm{~Hz}$ (often referred to as the "dead band").

The field-processed MT data include some scatter and poor signal-to-noise ratios. Spectral results were inspected visually for noisy data, and the best signal-to-noise field data were combined into the final plots.

The magnetotelluric impedance polar plots provide a measure of MT data dimensionality (Reddy and others, 1977). For 1-D resistivity structures, the principal impedance polar diagram (dashed line) is a circle. For 2-D or 3-D resistivity structures, the principal impedance polar diagram (dashed line) elongates either parallel or perpendicular to strike direction. Over resistors, the principal impedance polar diagram elongates perpendicular to strike direction, and over conductors, it elongates parallel to strike direction. For 2-D resistivity structures, the additional impedance polar diagram (solid line) attains the shape of a symmetric clover leaf. For 3-D resistivity structures, the additional impedance polar diagram (solid line) elongates in one direction, and its amplitude is comparable to that of the principal impedance polar diagram (dashed line).

The magnetotelluric "tipper" is calculated from the vertical component of the magnetic field. The tipper magnitude is a measure of the "tipping" of the magnetic field out of the horizontal plane (Vozoff, 1991). It will equal zero for the 1-D case. It typically increases to values between 0.1 to 0.5 and seldom approaches 1 , as it responds primarily to vertical and subvertical structures. The tipper magnitude of the stations discussed in this report ranged from 0.1 to 0.6 over the lower frequencies, indicating some vertical structure at depth. The tipper strike is used to help resolve the 90-degree ambiguity in the impedance rotation angle. The $\mathrm{HzHx}$ and HzHy coherency is a measure of the signal-to-noise ratio of the vertical magnetic field with respect to each of the orthogonal, horizontal magnetic-field directions. Values are normalized between 0 and 1 ; values at 0.5 signify signal levels equal to noise levels. These three-component magnetic-field coherencies provide a check on the signal-to-noise ratio of the measured values in the tipper magnitude and tipper strike plots. 


\section{References Cited}

Bechtel Navada, 2006, A hydrostratigraphic model and alternatives for the ground-water flow and contaiminant transport model of Corrective Action Unit 97: Yucca Flat-Climax Mine, Lincoln and Nye counties, Nevada: Report DOE/NV/11718-1119, in press.

Bendat, J.S., and Piersol, A.G., 1971, Random data-analysis and measurement procedures: New York, Wiley Interscience, 407p.

Dobrin, M.D., and Savit, C.H., 1988, Introduction to geophysical prospecting (4th ed.): New York: McGraw-Hill, 867 p.

Eberhart-Phillips, Donna, Stanley, W.D., Rodriguez, B.D., and Lutter, W.J., 1995, Surface seismic and electrical methods to detect fluids related to faulting: Journal of Geophysical Research, v. 100, no. B7, p. 12,919-12,936.

Egbert, G.D., 1997, Robust multiple station magnetotelluric data processing: Geophysics Journal International, 130, p. $475-496$.

EMI, Inc., 1996, MT-1 magnetotelluric system operation manual, version 3.2: Richmond, Calif., ElectroMagnetic Instruments, Inc., 220 p.

EMI,Inc., 2002, MT24/LF system operation and maintenance manual, version 1.0: Richmond, Calif., ElectroMagnetic Instruments, Inc., 72 p.

Gamble, T.D., Goubau, W.M., and Clarke, J., 1979, Error analysis for remote reference magnetotellurics: Geophysics, v. 44, no. 5, p. 959-968.

Keller, G.V., 1987, Rock and mineral properties, in Nabighian, M.N., ed., Electromagnetic methods in applied geophysics theory: Tulsa, Okla., Society of Exploration Geophysicists, v. 1, p. 13-51.

Keller, G.V., 1989, Electrical properties, in Carmichael, R.S., ed., Practical handbook of physical properties of rocks and minerals: Boca Raton, Fla., CRC Press, p. 359-427.

Nelson, P.H., and Anderson, L.A., 1992, Physical properties of ash flow tuff from Yucca Mountain, Nevada: Journal of Geophysical Research, v. 97, no. B5, p. 6,823-6,841.

Palacky, G.J., 1987, Resistivity characteristics of geologic targets, in Nabighian, M.N., ed., Electromagnetic methods in applied geophysics theory: Tulsa, Okla., Society of Exploration Geophysicists, v. 1, p. 53-129. 
Reddy, I.K., Rankin, David, and Phillips, R.J., 1977, Three-dimensional modelling in magnetotelluric and magnetic variational sounding: Geophysics Journal of the Royal Astronomical Society, v. 51, p. 313-325.

Sternberg, B.K., Washburne, J.C., and Pellerin, Louise, 1988, Correction for the static shift in magnetotellurics using transient electromagnetic soundings: Geophysics, v. 53, p. 1, 459-1,468.

Vozoff, Keeva, 1972, The magnetotelluric method in the exploration of sedimentary basins: Geophysics, v. 37, p. 980-1041.

Vozoff, Keeva, 1991, The magnetotelluric method, in Nabighian, M.N., Electromagnetic methods in applied geophysics: Tulsa, Okla., Society of Exploration Geophysicists, v. 2, pt. B, p. 641-711. 


\section{Appendix}

\section{Magnetotelluric Data Plots}

There are nine separate plots for each station:

1. Apparent Resistivity for the rotated maximum (x symbol) and minimum (o symbol) modes

2. Impedance Phase for the rotated maximum ( $\mathrm{x}$ symbol) and minimum (o symbol) modes

3. Rotation Angle for the impedance tensor (corresponds to the direction of maximum apparent resistivity)

4. Impedance Skew for the impedance tensor

5. Multiple Coherency for the rotated maximum (x symbol) and minimum (o symbol) modes of the electric field

6. Impedance Polar Plots (at 12 selected frequencies)

7. Tipper Magnitude for the vertical magnetic field

8. Tipper Strike for the vertical magnetic field

9. HzHx (x symbol) and HzHy (o symbol) Coherency

Refer to the "Magnetotelluric Data" section in this report for an explanation of these plots. The priorities listed on the plots were determined prior to data acquisition. During postprocessing the priority ranking was changed. 


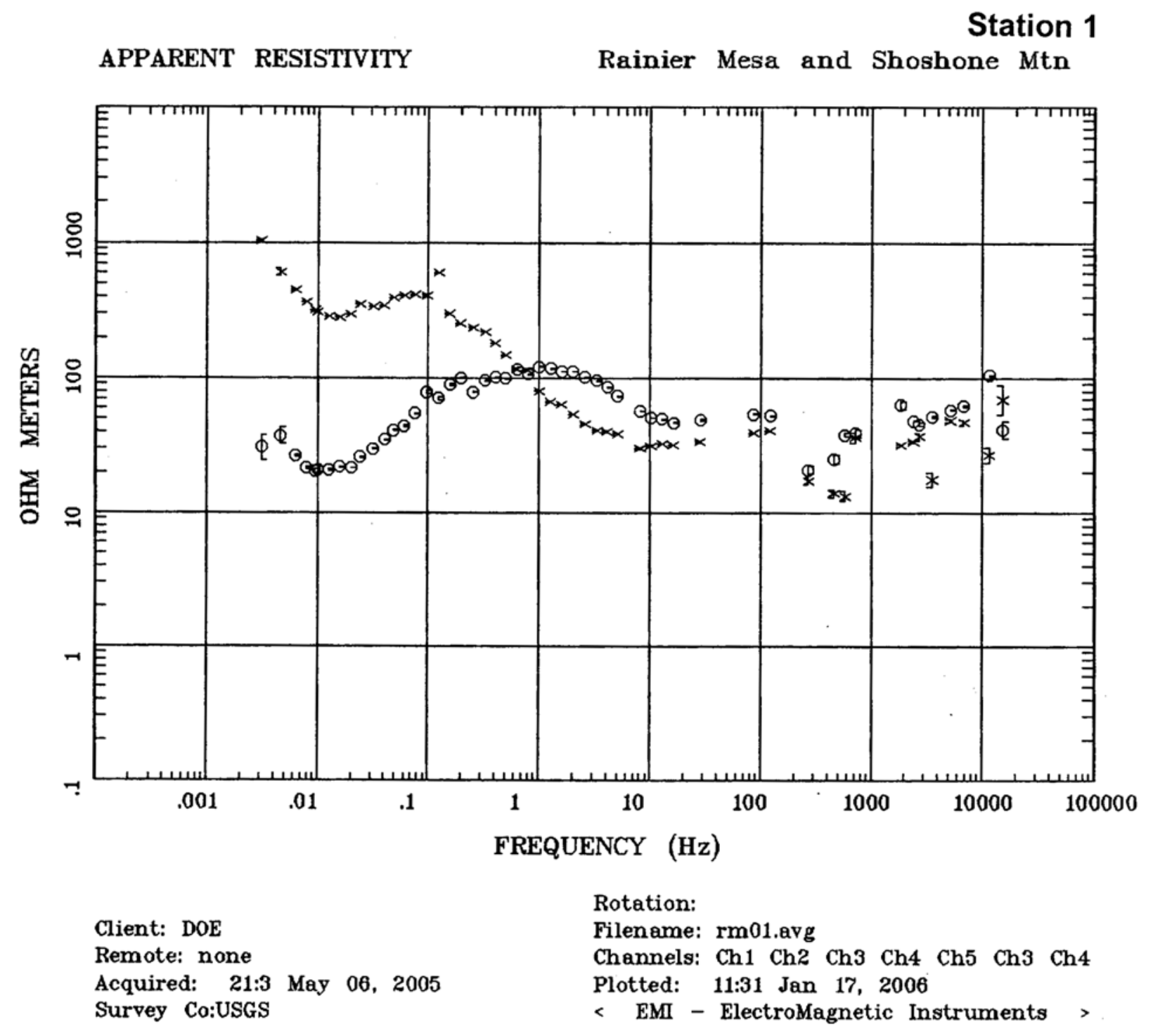




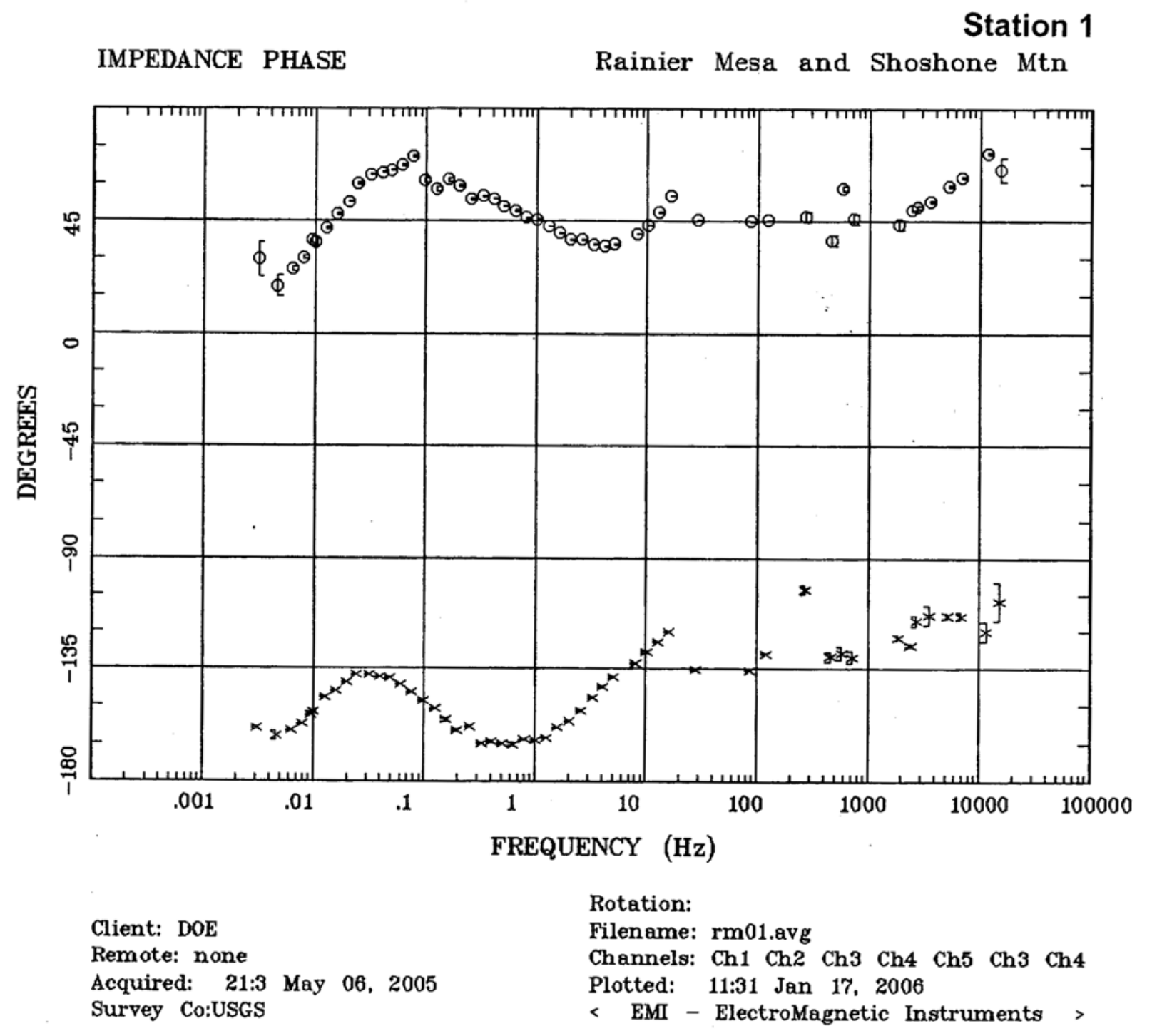




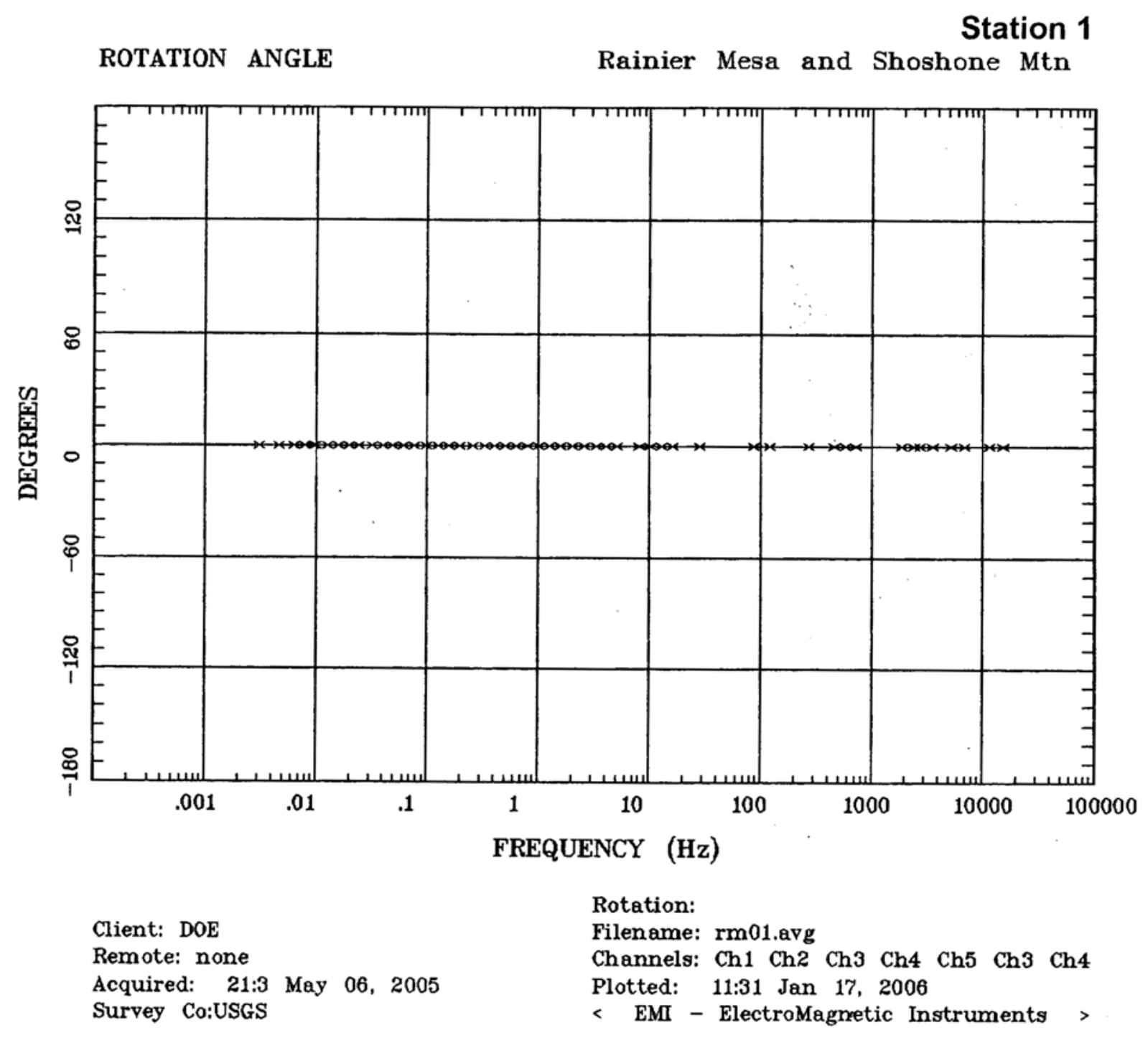




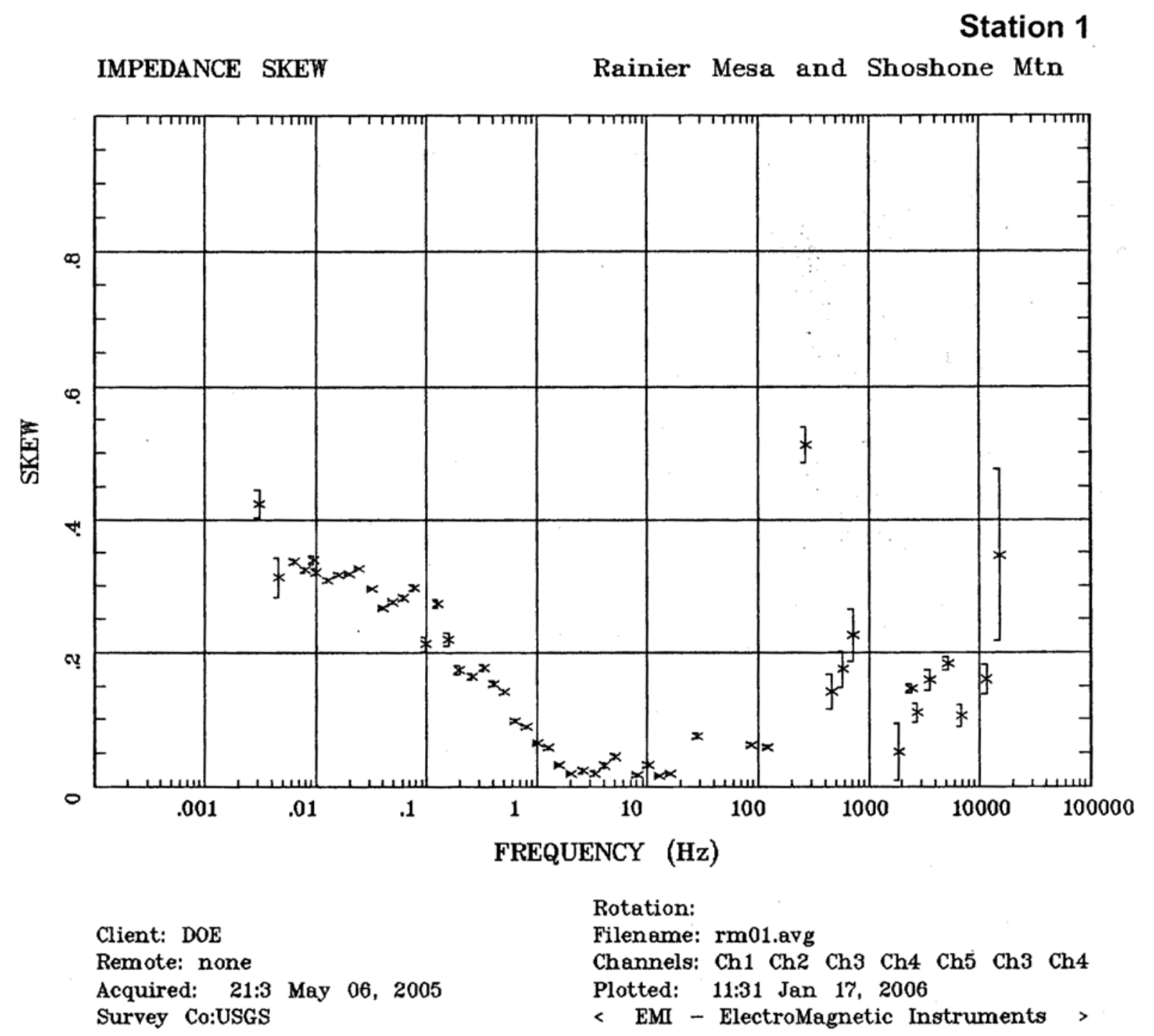




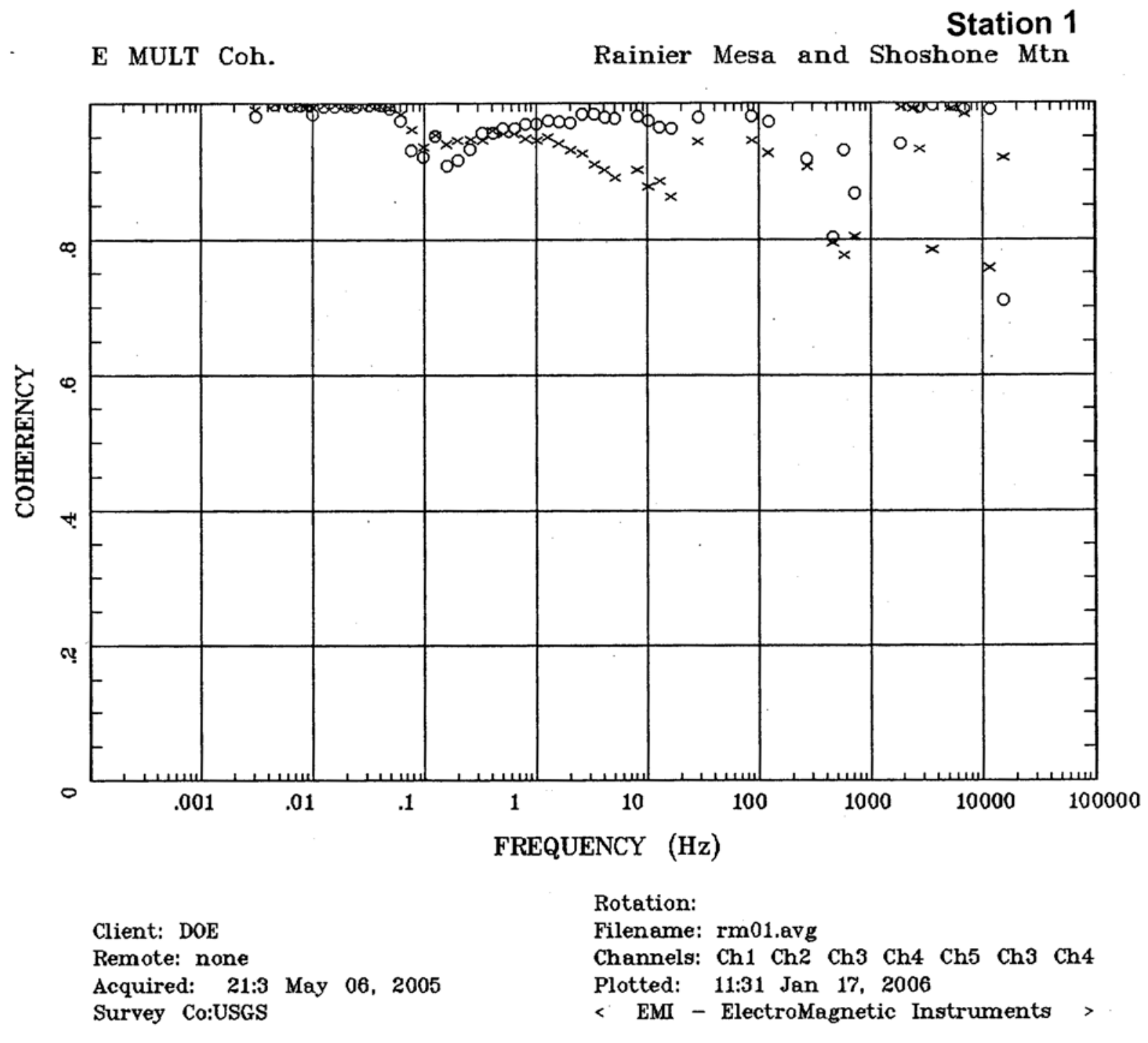


Station 1

POLAR PLOTS

Rainier Mesa and Shoshone Mtn

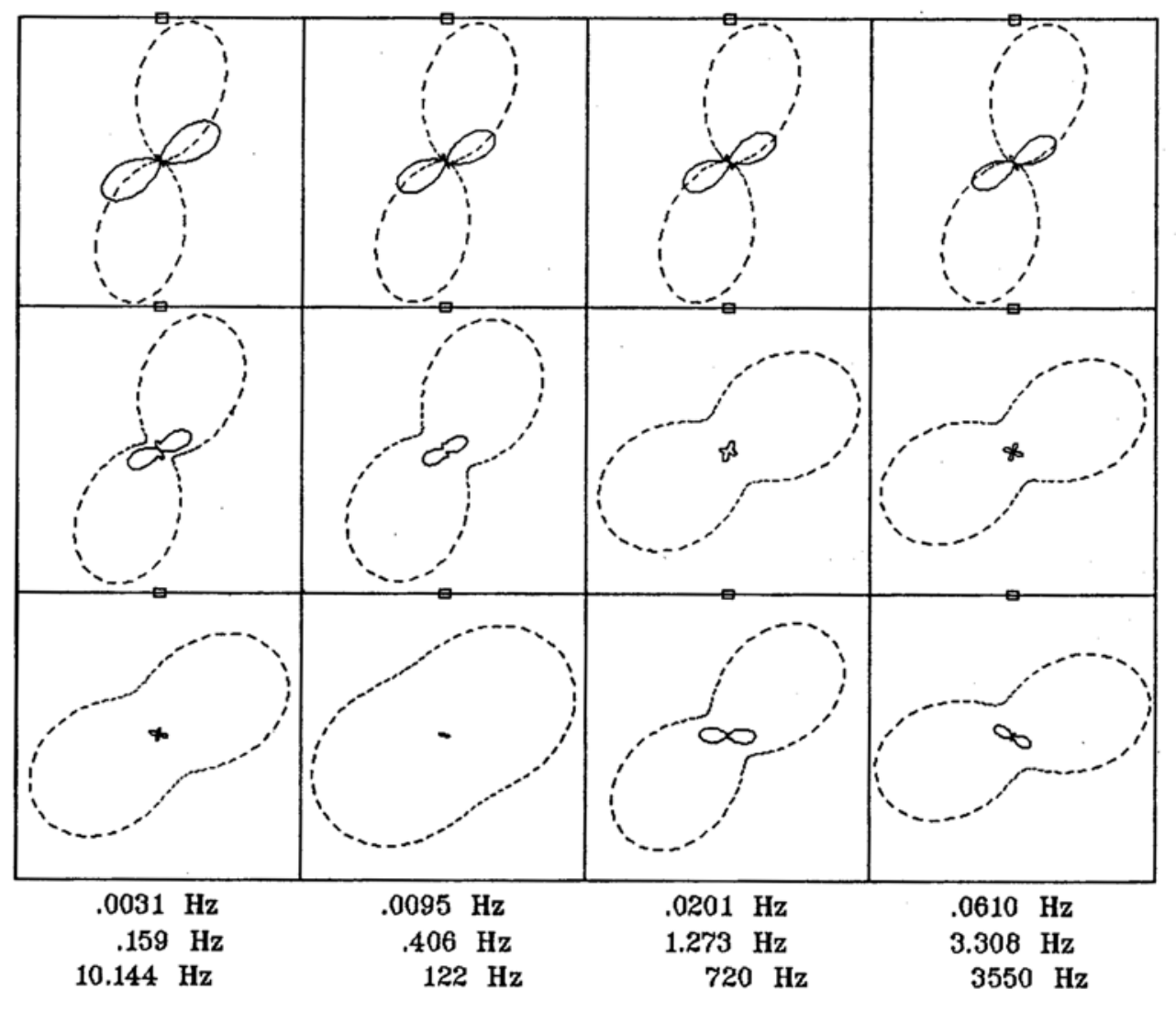

Client: DOE

Rotation:

Remote: none

Acquired: 21:3 May 06, 2005

Filename: rm01.avg

Channels: Ch1 Ch2 Ch3 Ch4 Ch5 Ch3 Ch4

Plotted: 11:31 Jan 17, 2006

Survey Co:USGS

< EMI - ElectroMagnetic Instruments > 


\section{Station 1}

TIPPER MAGNITUDE

Rainier Mesa and Shoshone Mtn

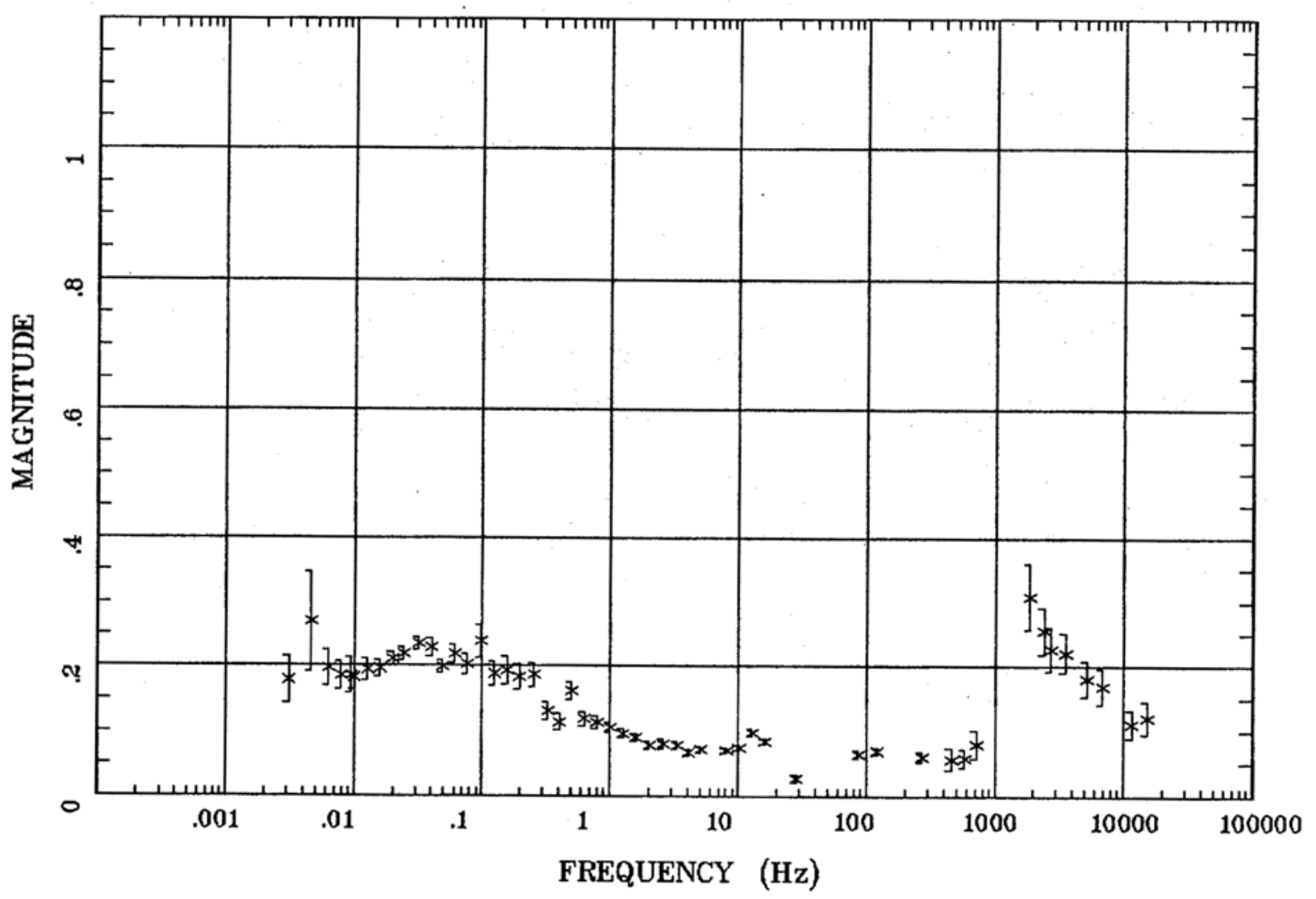

Client: DOE

Remote: none

Acquired: 21:3 May 06, 2005

Survey Co:USGS
Rotation:

Filename: rm01.avg

Channels: Ch1 Ch2 Ch3 Ch4 Ch5 Ch3 Ch4

Plotted: 11:32 Jan 17, 2006

< EMI - ElectroMagnetic Instruments > 


\section{Station 1}

TIPPER STRIKE

Rainier Mesa and Shoshone Mtn

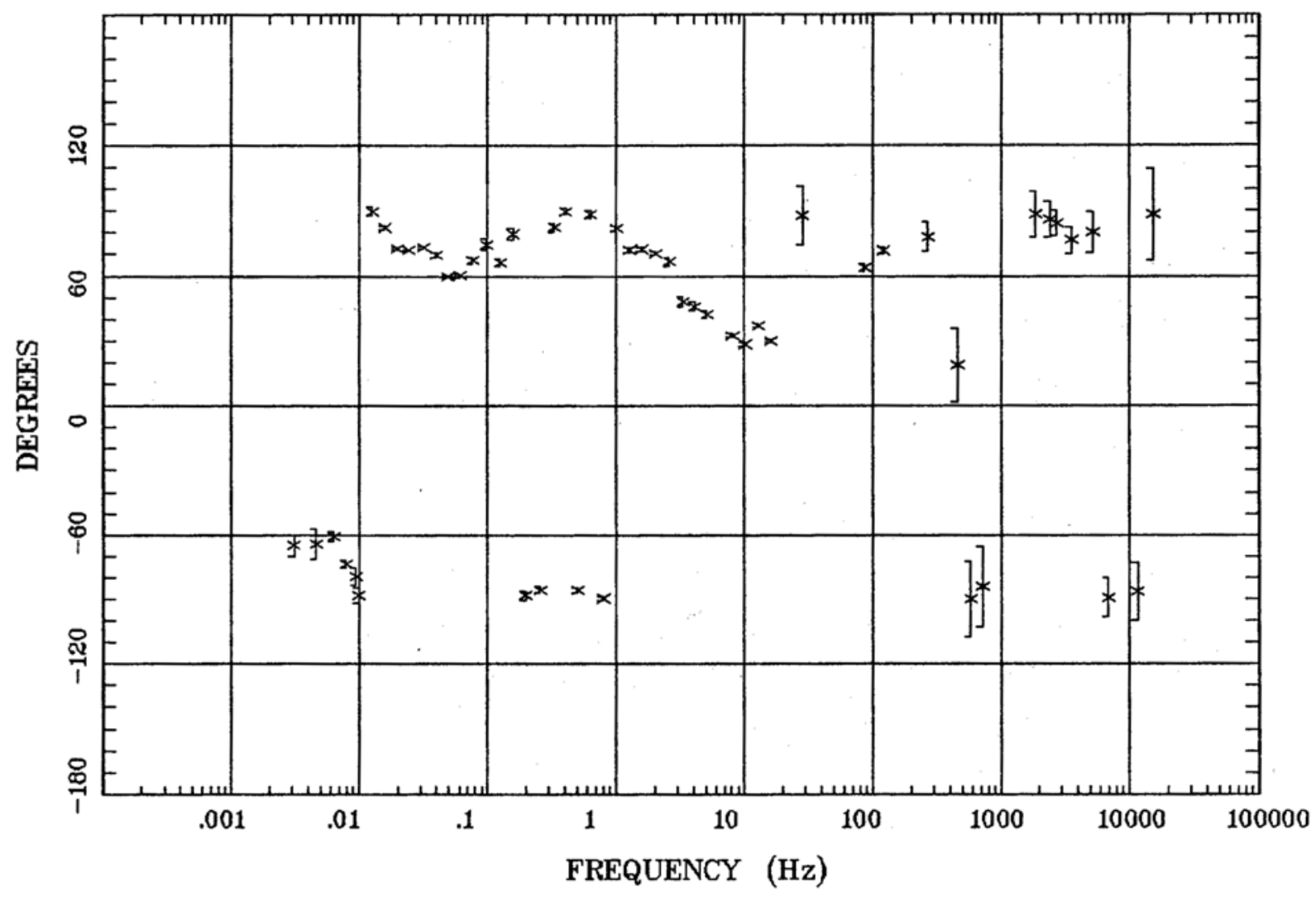

Client: DOE

Remote: none

Acquired: 21:3 May 06, 2005

Survey Co:USGS
Rotation:

Filename: rm01.avg

Channels: Ch1 Ch2 Ch3 Ch4 Ch5 Ch3 Ch4

Plotted: 11:32 Jan 17, 2006

< EMI - ElectroMagnetic Instruments > 


\section{Station 1}

HzHx.x Coh . HzHy.o

Rainier Mesa and Shoshone Mtn

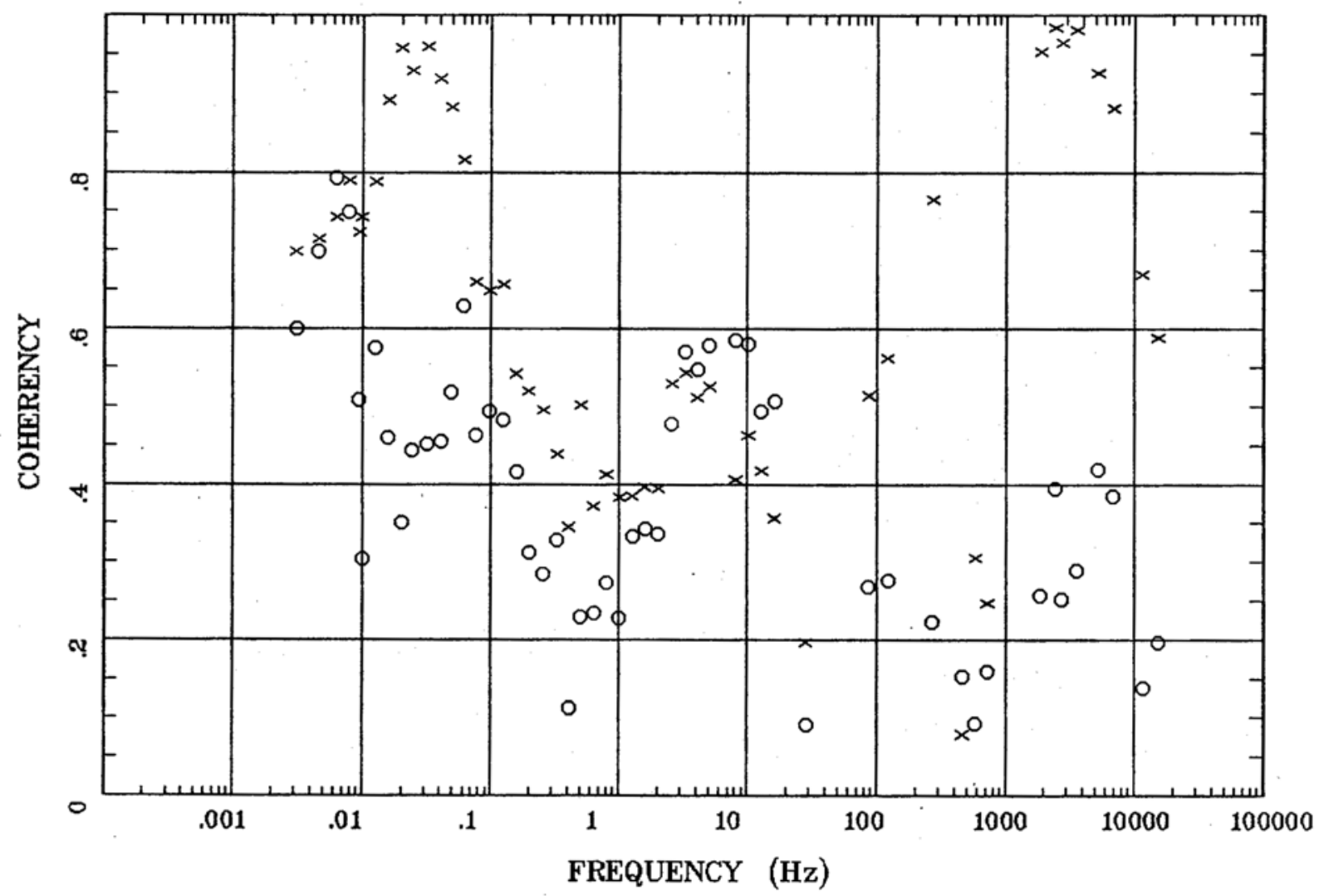

Client: DOE

Remote: none

Acquired: 21:3 May 06, 2005

Survey Co:USGS
Rotation:

Filename: rm01.avg

Channels: Ch1 Ch2 Ch3 Ch4 Ch5 Ch3 Ch4

Plotted: 11:32 Jan 17, 2006

< EMI - ElectroMagnetic Instruments > 


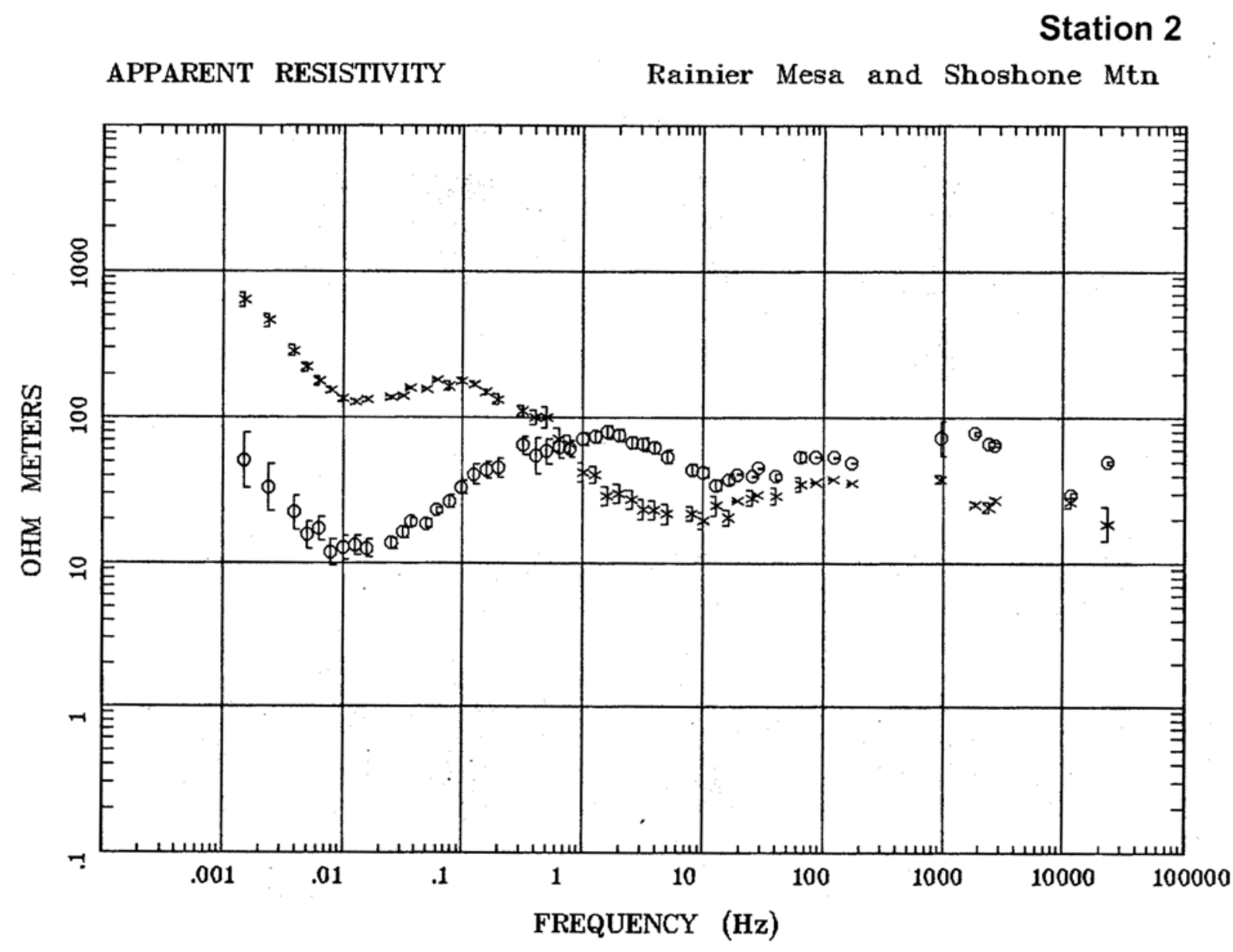

Client: DOE

Remote: none

Acquired: 02:0 May 06, 2005

Survey Co:USGS
Rotation:

Filename: rm02.avg

Channels: Ch1 Ch2 Ch3 Ch4 Ch5 Ch3 Ch4

Plotted: 10:59 Jan 17, 2006

< EMI - ElectroMagnetic Instruments > 


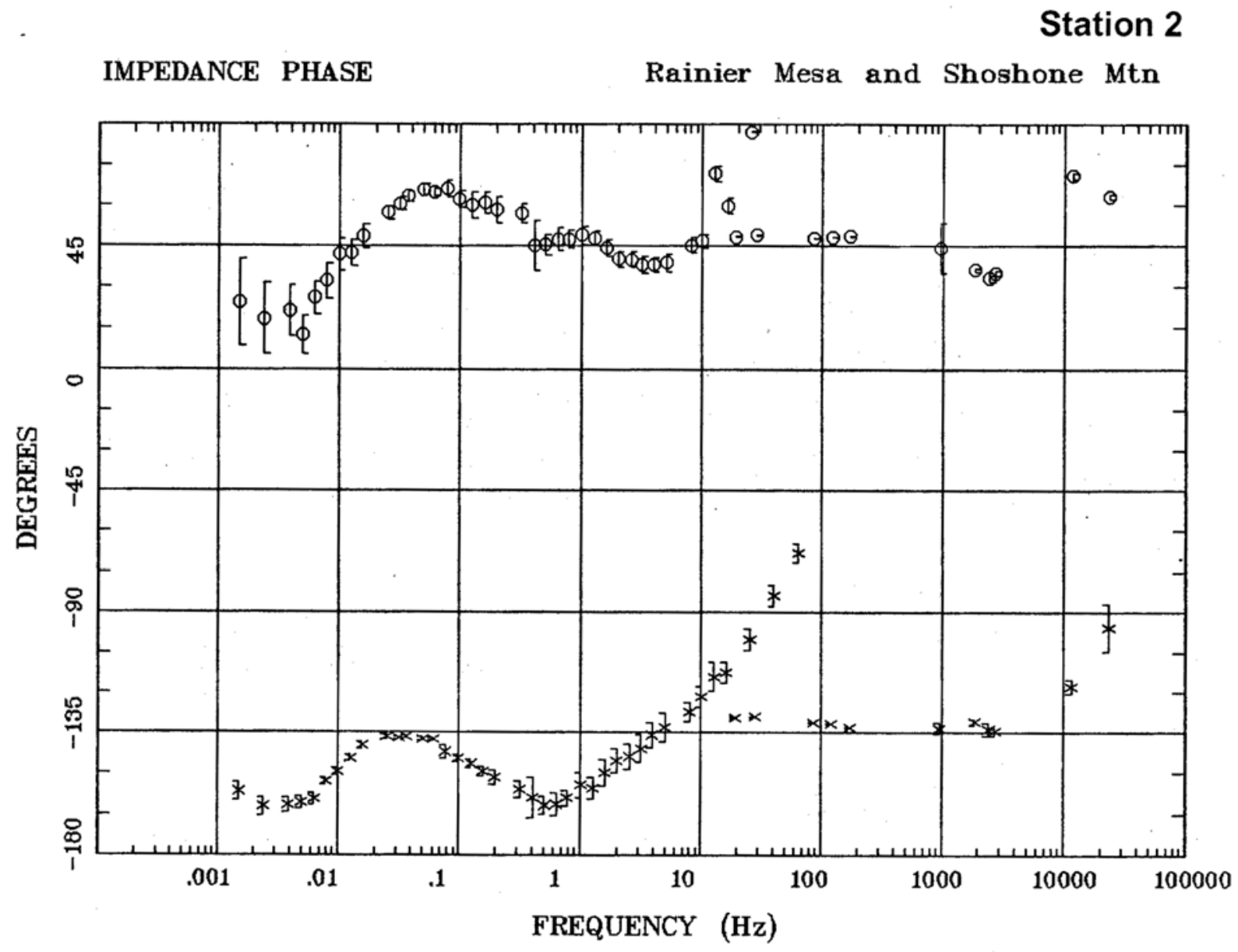

Client: DOE

Remote: none

Acquired: 02:0 May 06, 2005

Survey Co:USGS
Rotation:

Filename: rm02.avg

Channels: Ch1 Ch2 Ch3 Ch4 Ch5 Ch3 Ch4

Plotted: 10:59 Jan 17, 2006

< EMI - ElectroMagnetic Instruments > 


\section{Station 2}

\section{ROTATION ANGLE}

Rainier Mesa and Shoshone Mtn

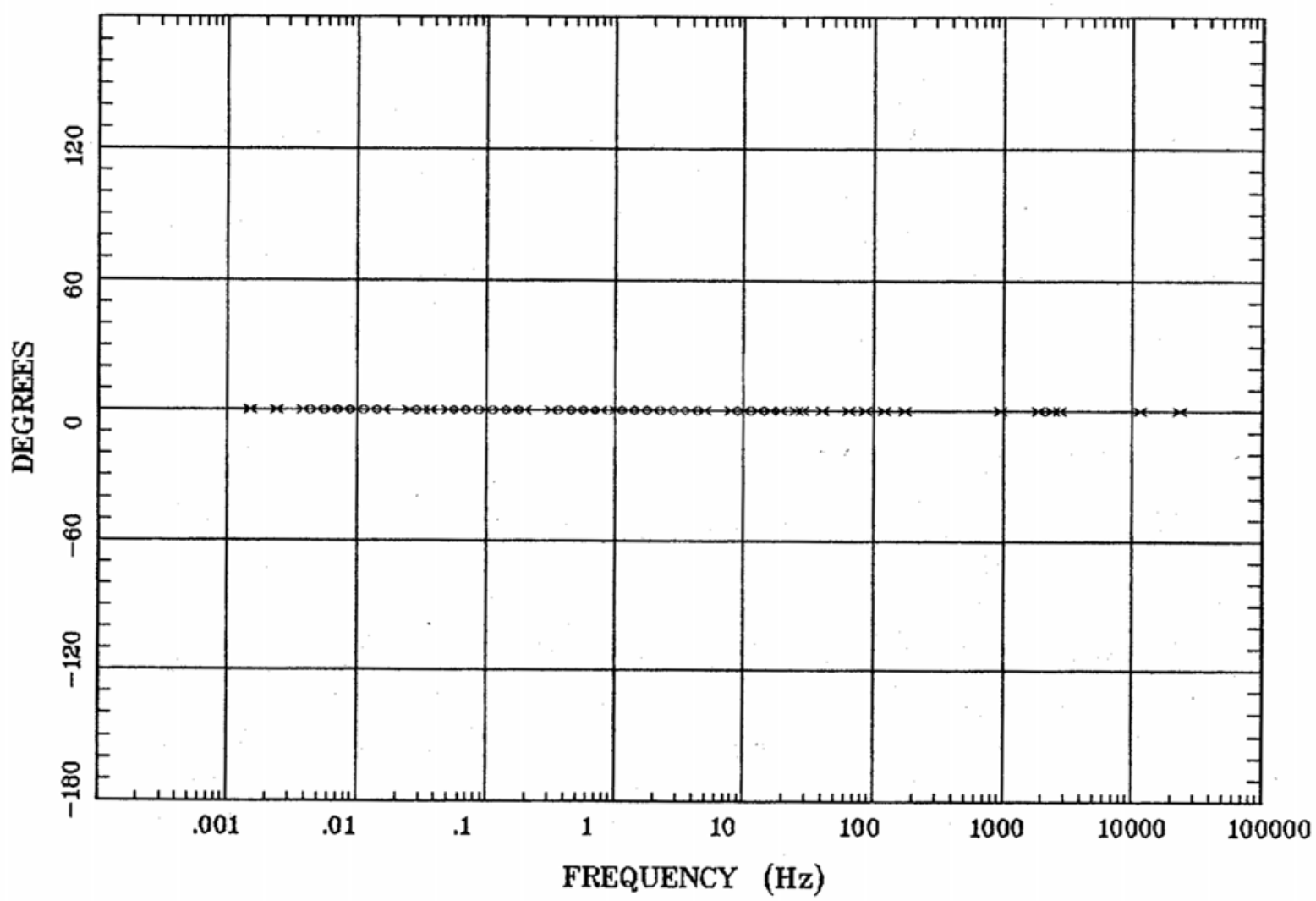

Client: DOE

Remote: none

Acquired: 02:0 May 06, 2005

Survey Co:USGS
Rotation:

Filename: rm02.avg

Channels: Ch1 Ch2 Ch3 Ch4 Ch5 Ch3 Ch4

Plotted: 10:59 Jan 17, 2006

< EMI - ElectroMagnetic Instruments > 


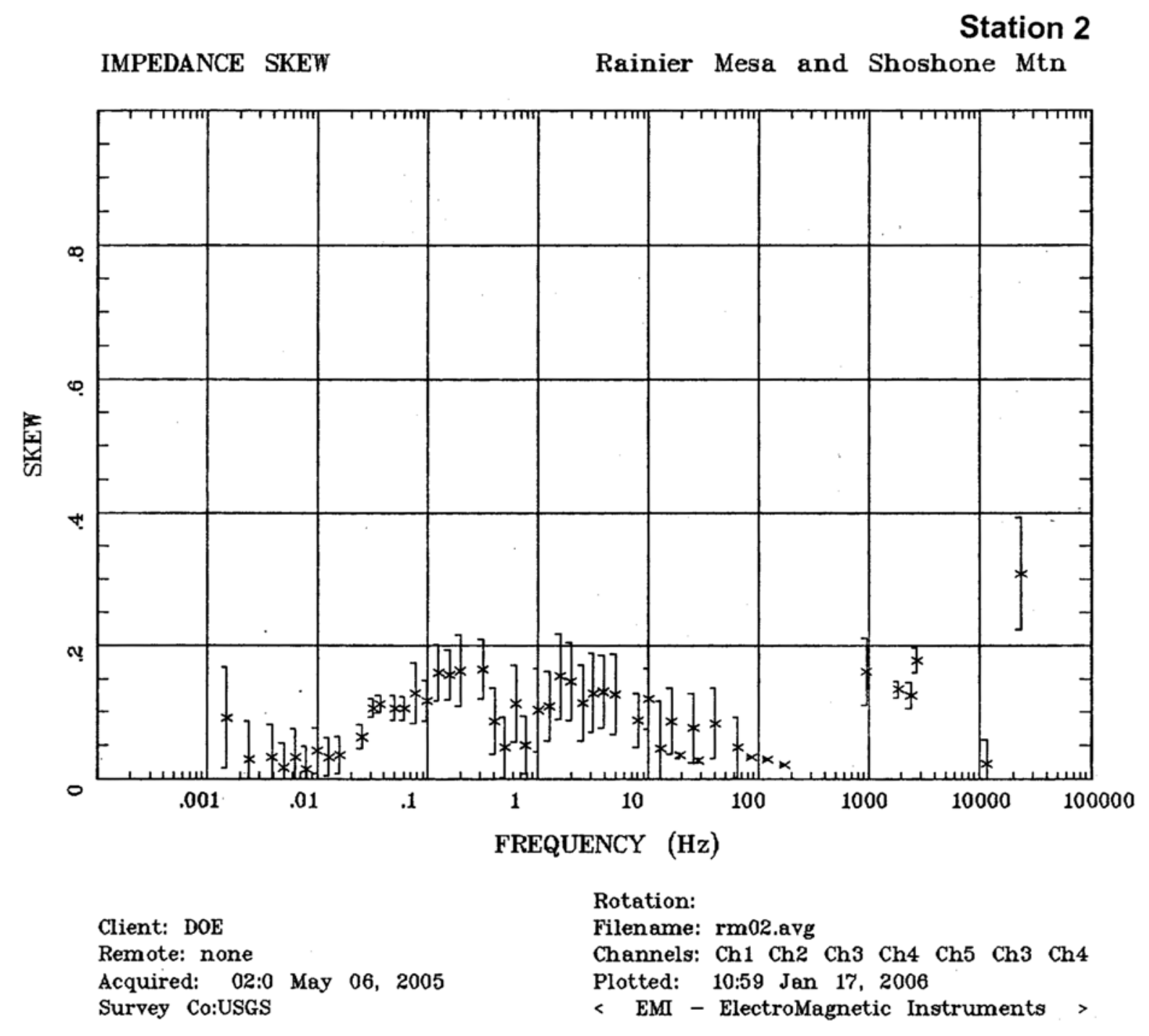




\section{Station 2}

E MULT Coh.

Rainier Mesa and Shoshone Mtn

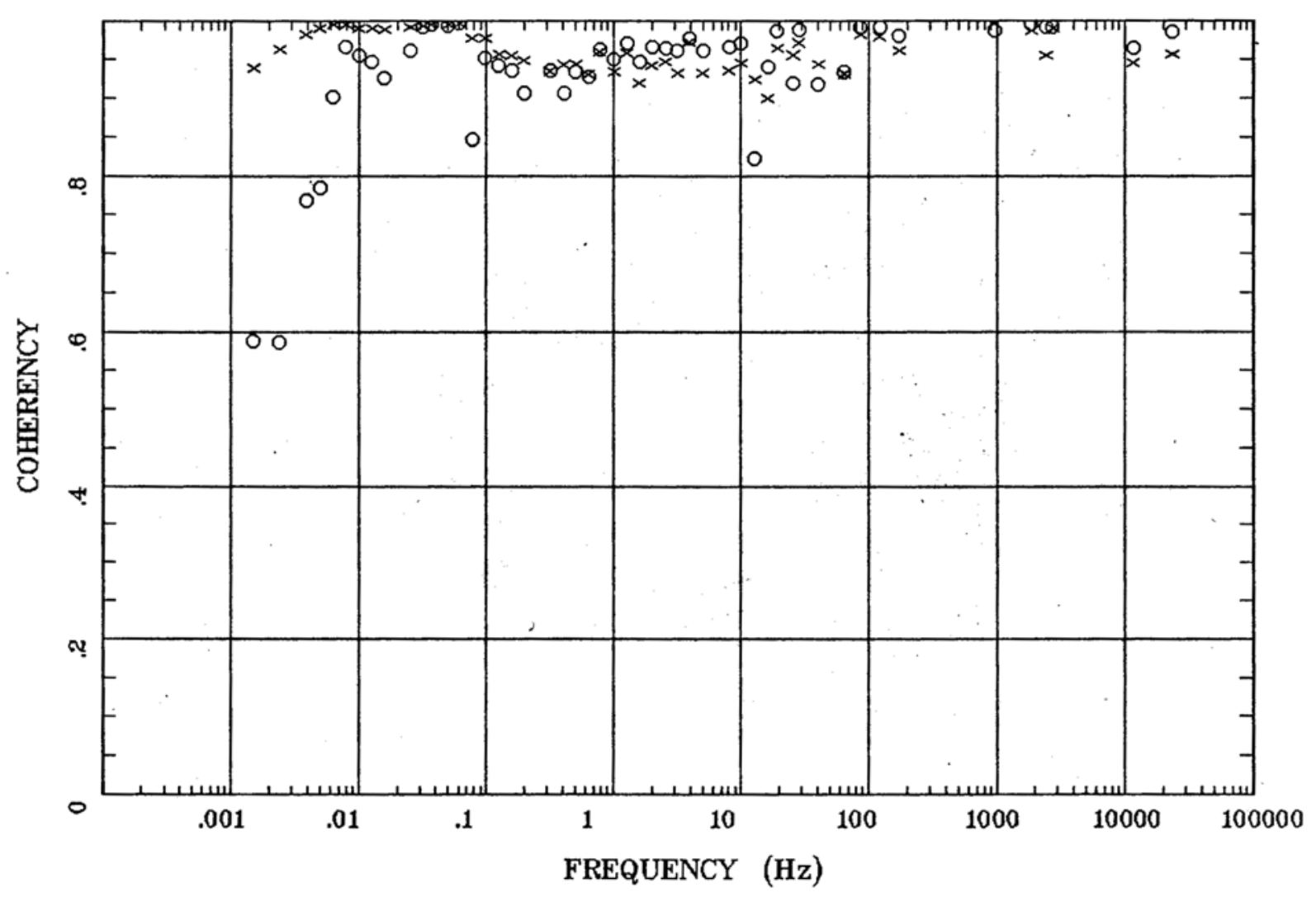

Client: DOE

Remote: none

Acquired: 02:0 May 06, 2005

Survey Co:USGS
Rotation:

Filename: rm02.avg

Channels: Ch1 Ch2 Ch3 Ch4 Ch5 Ch3 Ch4

Plotted: 10:59 Jan 17, 2006

< EMI - ElectroMagnetic Instruments > 
Station 2

POLAR PLOTS

Rainier Mesa and Shoshone Mtn

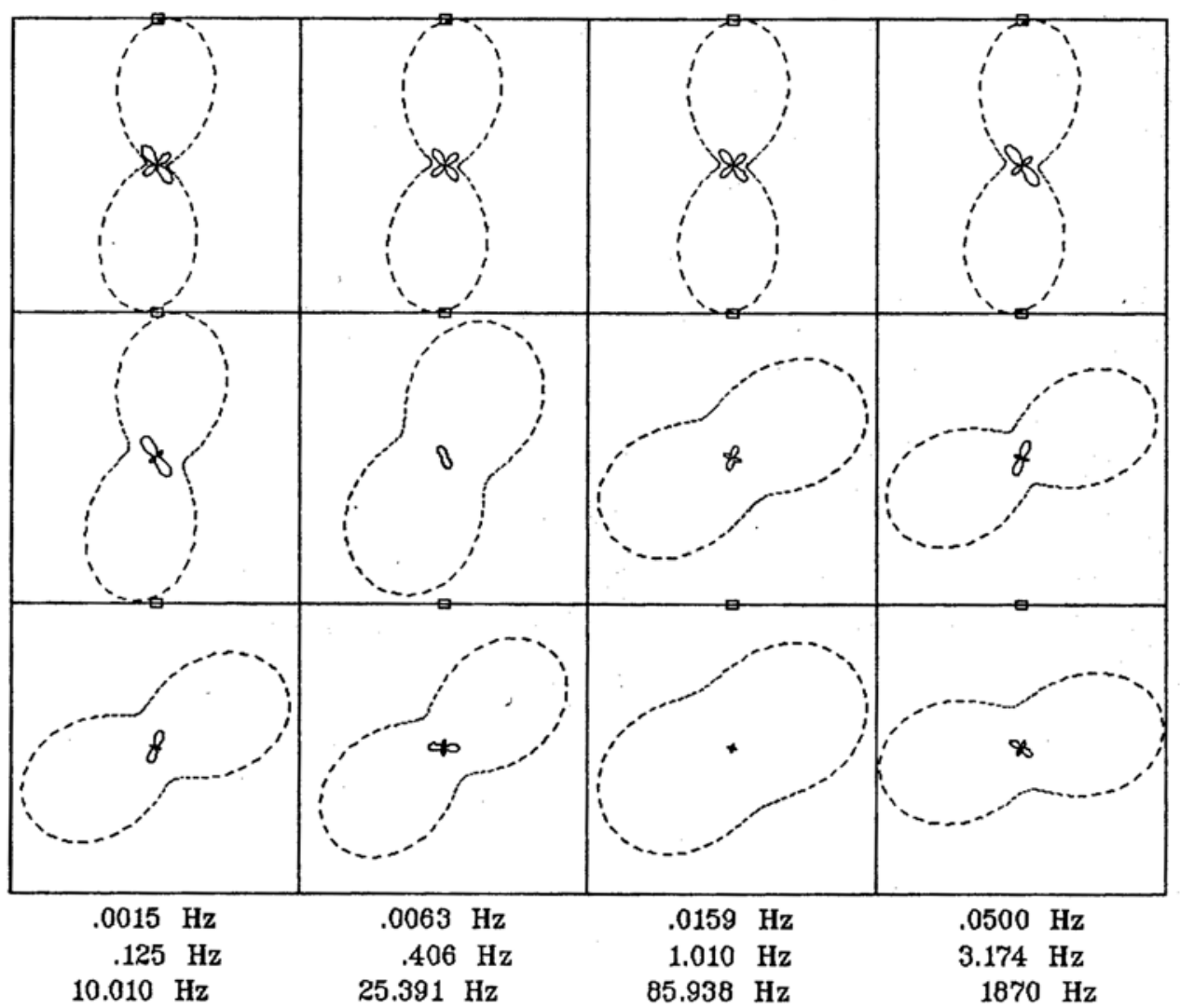

Client: DOE

Remote: none

Acquired: 02:0 May 06, 2005

Rotation

Filename: rm02.avg

Channels: Ch1 Ch2 Ch3 Ch4 Ch5 Ch3 Ch4

Plotted: 10:59 Jan 17, 2006

Survey Co:USGS

< EMI - ElectroMagnetic Instruments > 


\section{Station 2}

TIPPER MAGNITUDE

Rainier Mesa and Shoshone Mtn

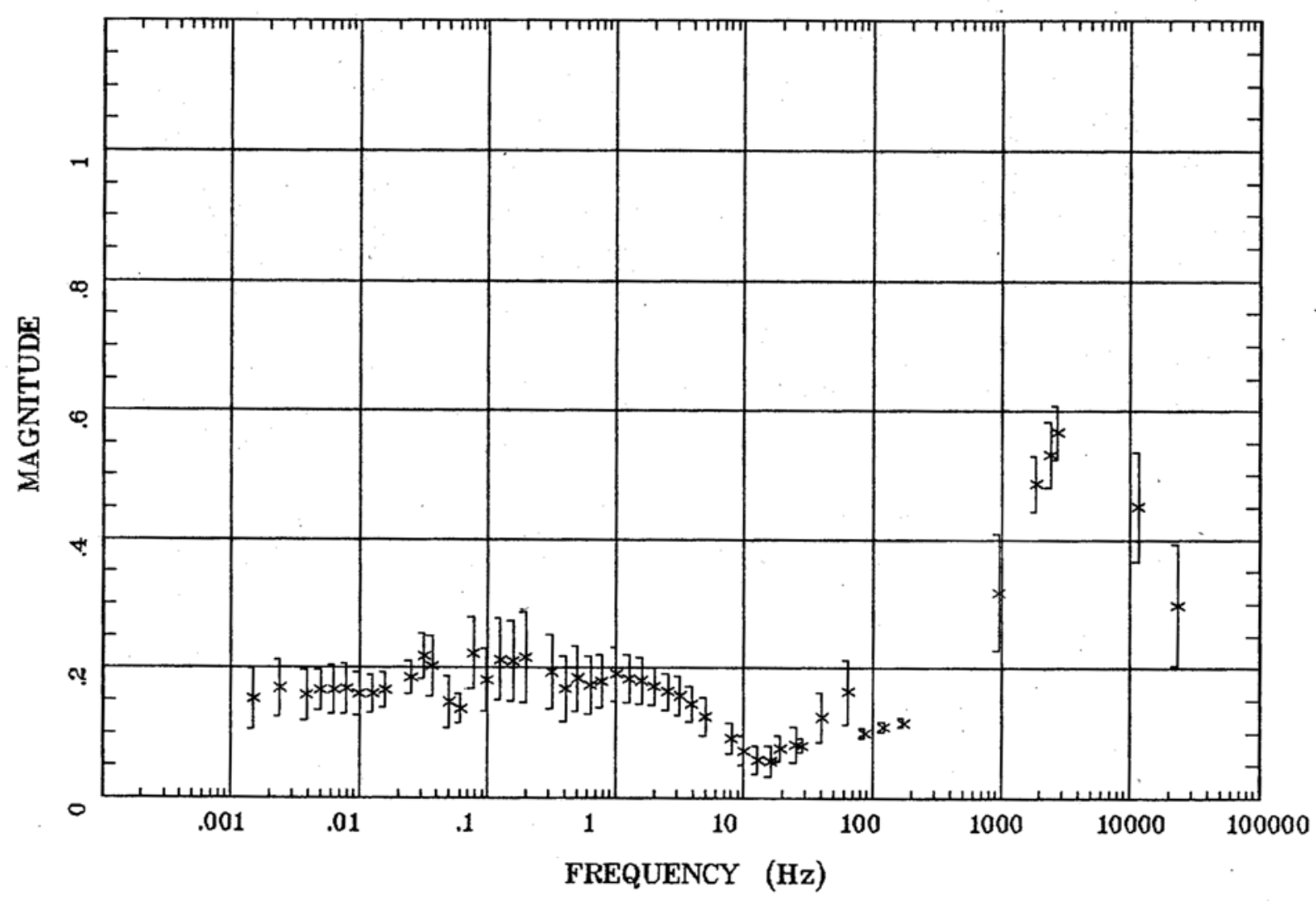

Client: DOE

Remote: none

Acquired: 02:0 May 06, 2005

Survey Co:USGS
Rotation:

Filename: rm02.avg

Channels: Ch1 Ch2 Ch3 Ch4 Ch5 Ch3 Ch4

Plotted: 10:59 Jan 17, 2006

< EMI - ElectroMagnetic Instruments > 


\section{Station 2}

TIPPER STRIKE

Rainier Mesa and Shoshone Mtn

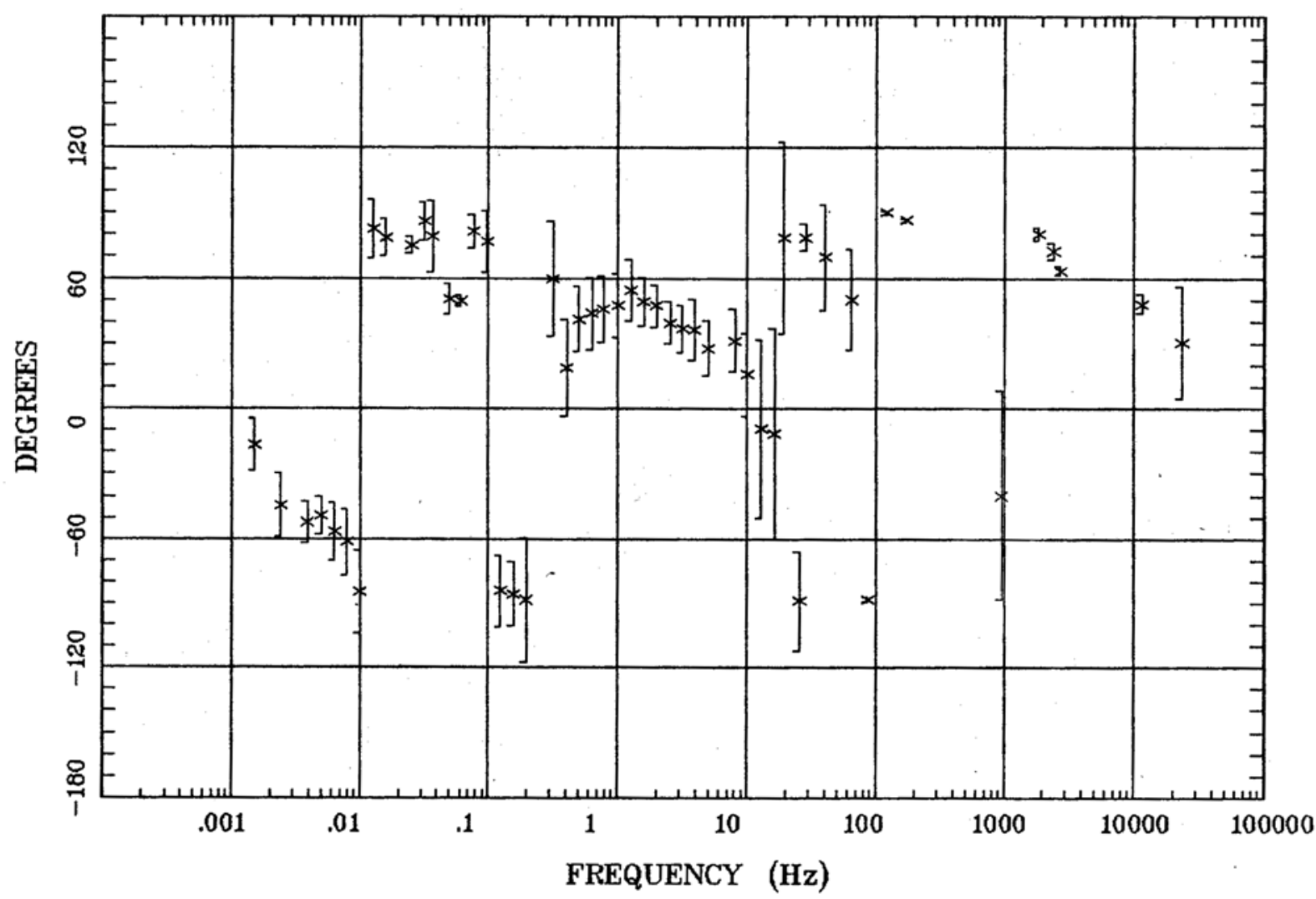

Client: DOE

Remote: none

Acquired: 02:0 May 06, 2005

Survey Co:USGS
Rotation:

Filename: rm02.avg

Channels: Ch1 Ch2 Ch3 Ch4 Ch5 Ch3 Ch4

Plotted: 10:59 Jan 17, 2006

< EMI - ElectroMagnetic Instruments > 
Station 2

HzHx.x Coh HzHy.o

Rainier Mesa and Shoshone Mtn

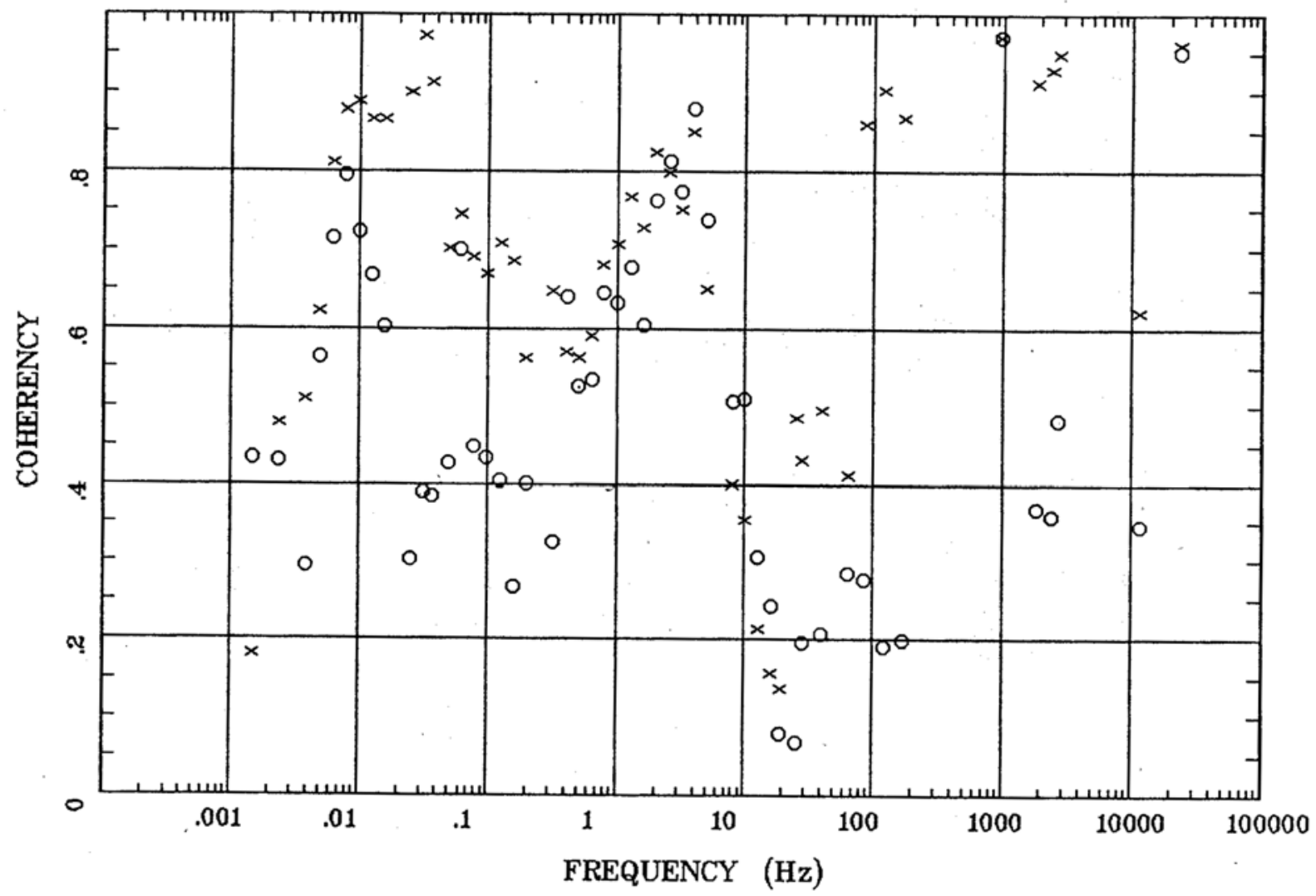

Client: DOE

Remote: none

Acquired: 02:0 May 06, 2005

Survey Co:USGS
Rotation:

Filename: rm02.avg

Channels: Ch1 Ch2 Ch3 Ch4 Ch5 Ch3 Ch4

Plotted: 10:59 Jan 17, 2006

< EMI - ElectroMagnetic Instruments > 
Station 3

APPARENT RESISTIVITY

Rainier Mesa and Shoshone Mtn

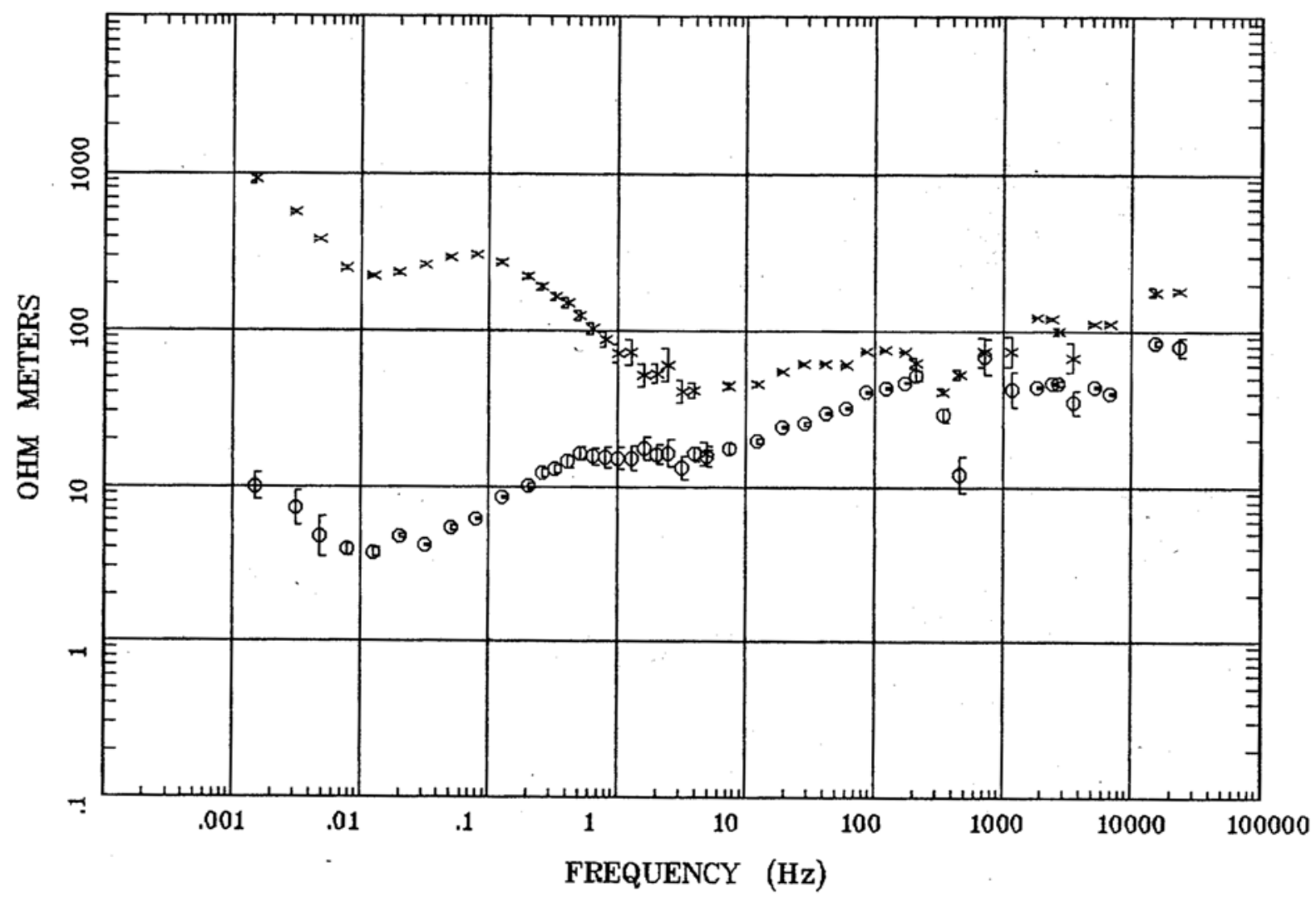

Client: DOE

Remote: none

Acquired: 22:2 May 06, 2005

Survey Co:USGS
Rotation:

Filename: rm03.avg

Channels: Ch1 Ch2 Ch3 Ch4 Ch5 Ch3 Ch4

Plotted: 11:00 Jan 17, 2006

< EMI - ElectroMagnetic Instruments > 


\section{Station 3}

IMPEDANCE PHASE

Rainier Mesa and Shoshone Mtn

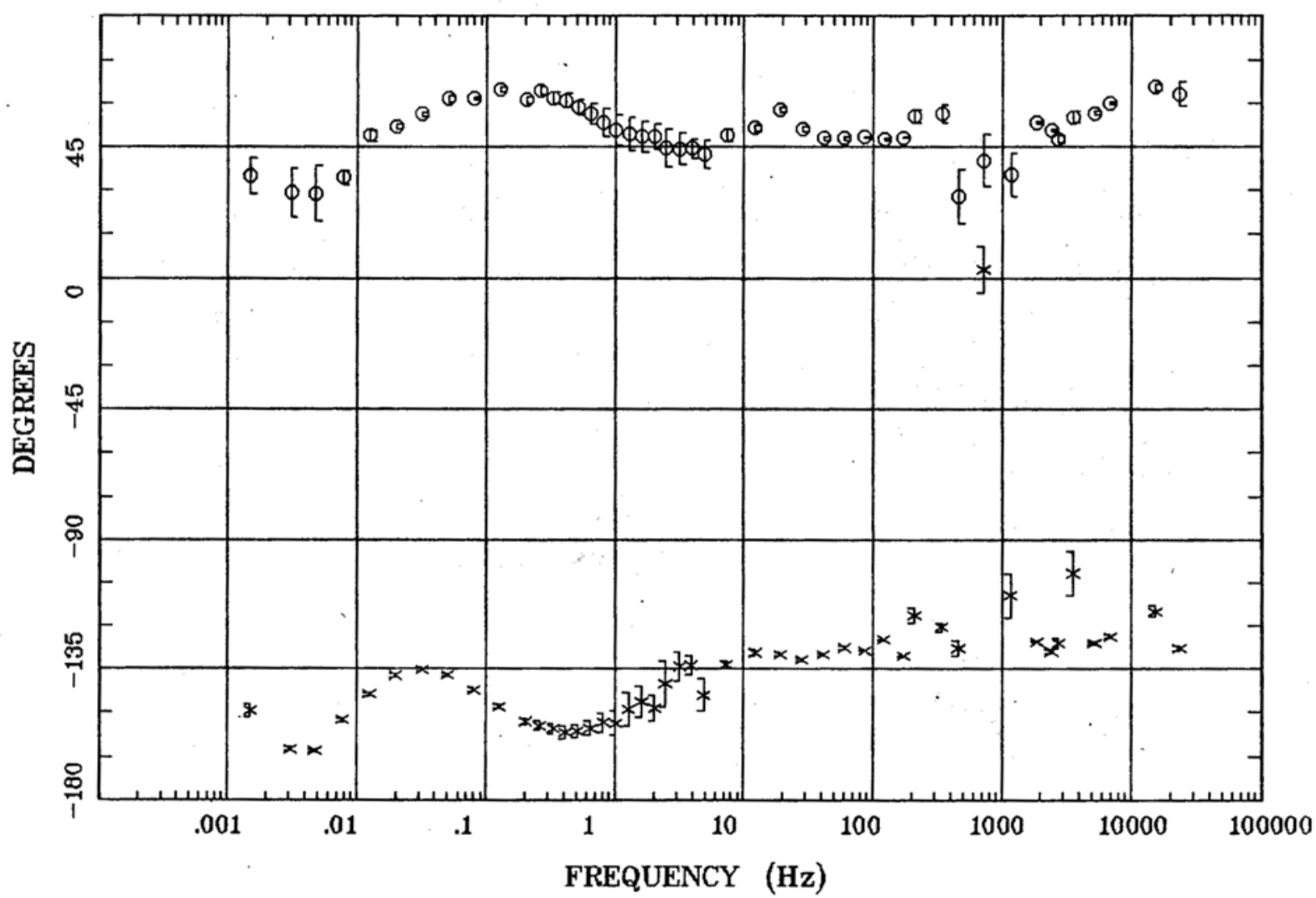

Client: DOE

Remote: none

Acquired: 22:2 May 06, 2005

Survey Co:USGS
Rotation:

Filename: rm03.avg

Channels: Ch1 Ch2 Ch3 Ch4 Ch5 Ch3 Ch4

Plotted: 11:00 Jan 17, 2006

< EMI - ElectroMagnetic Instruments > 


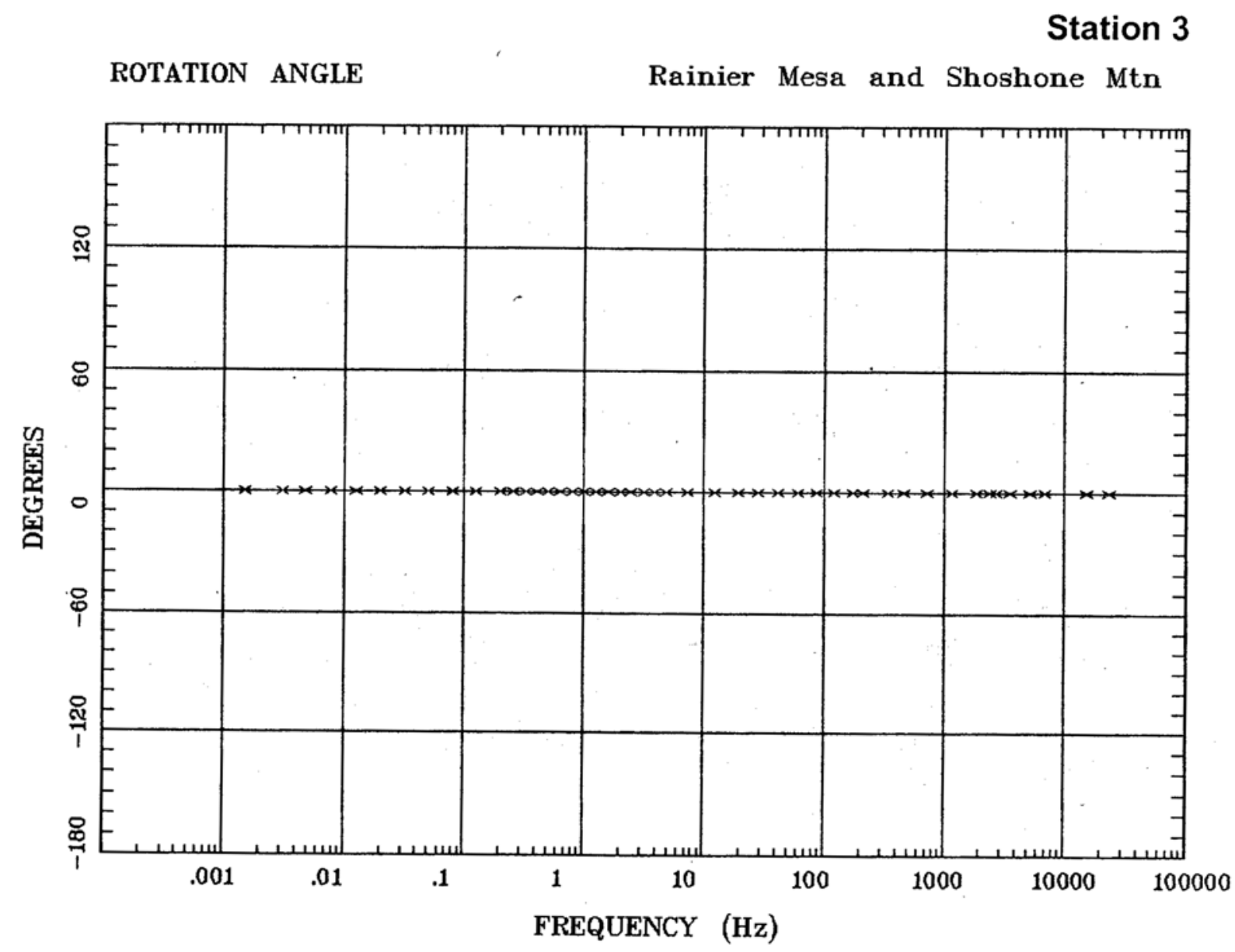

Client: DOE

Remote: none

Acquired: 22:2 May 06, 2005

Survey Co:USGS
Rotation:

Filename: rm03.avg

Channels: Ch1 Ch2 Ch3 Ch4 Ch5 Ch3 Ch4

Plotted: 11:00 Jan 17, 2006

< EMI - ElectroMagnetic Instruments > 


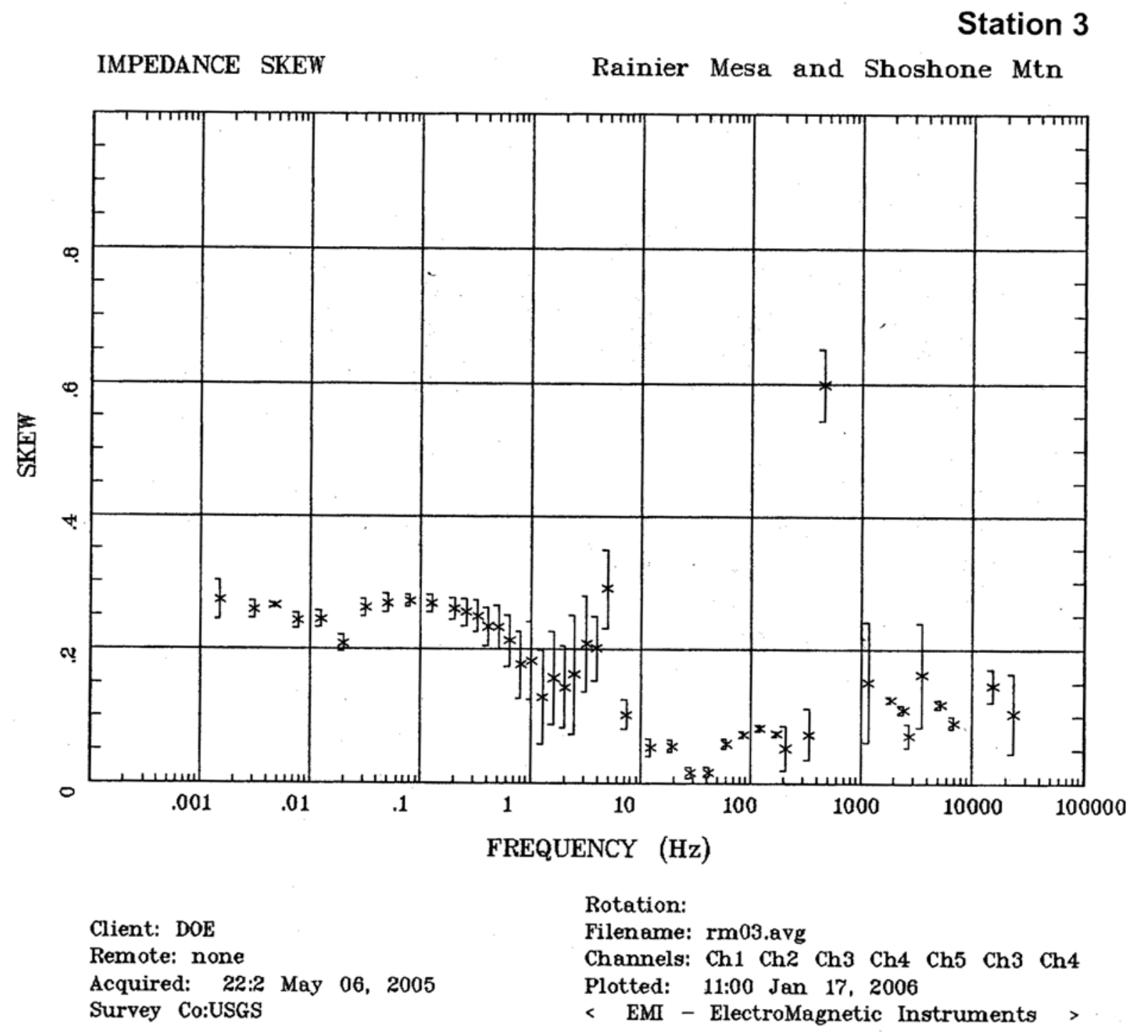


Station 3

E MULT Coh.

Rainier Mesa and Shoshone Mtn

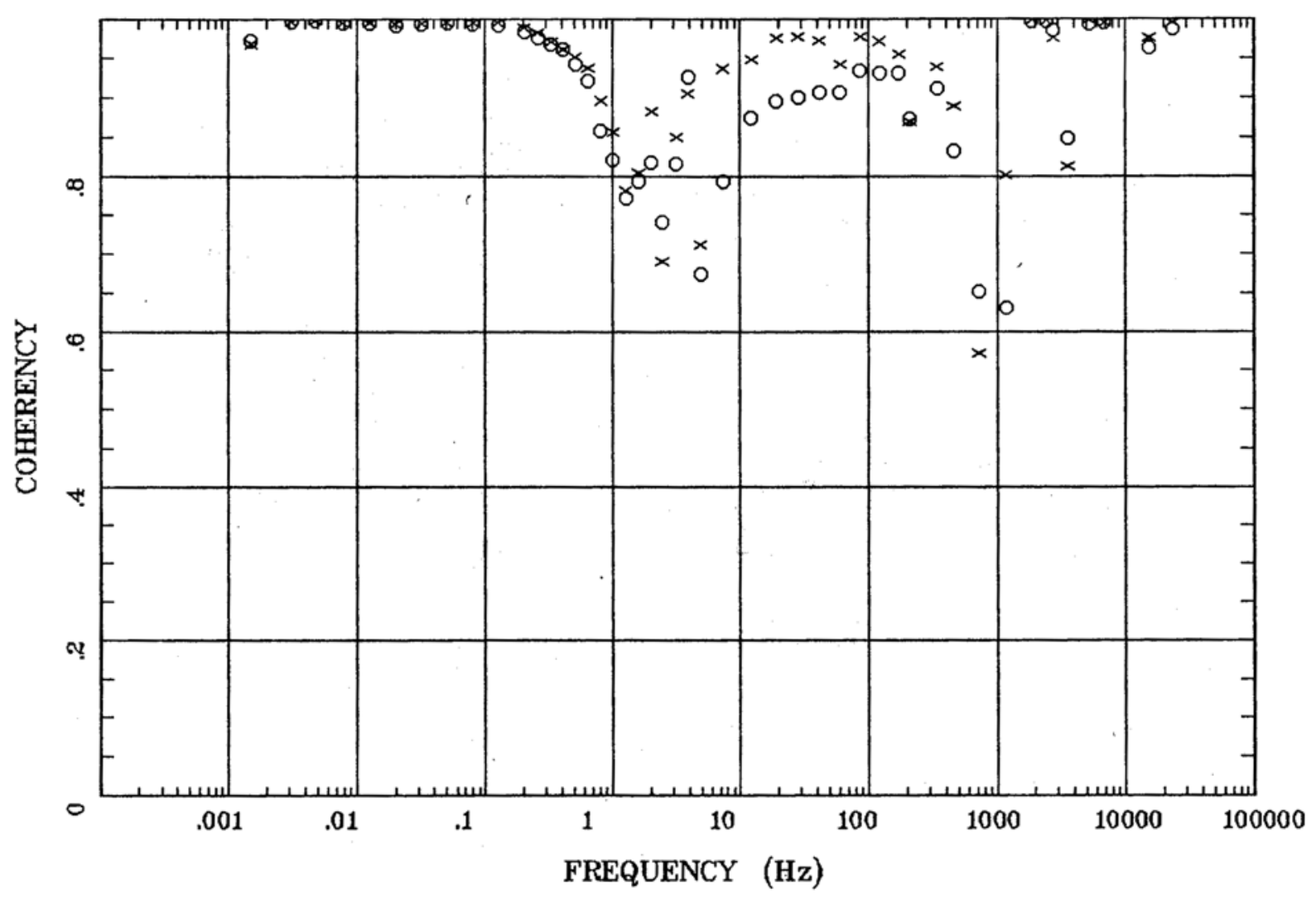

Client: DOE

Remote: none

Acquired: 22:2 May 06, 2005

Survey Co:USGS
Rotation:

Filename: rm03.avg

Channels: Ch1 Ch2 Ch3 Ch4 Ch5 Ch3 Ch4

Plotted: 11:00 Jan 17, 2006

< EMI - ElectroMagnetic Instruments > 
Station 3

POLAR PLOTS

Rainier Mesa and Shoshone Mtn

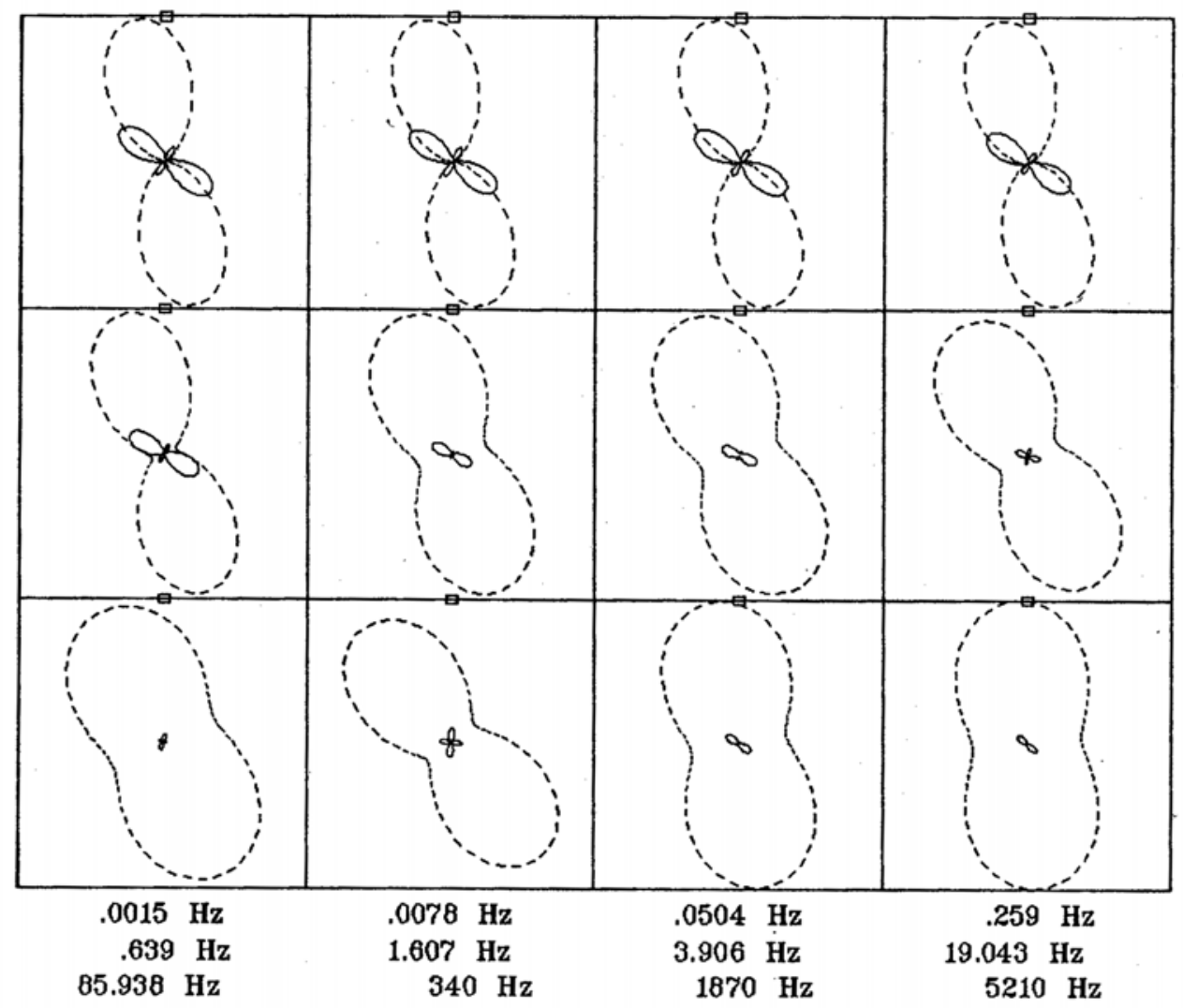

Client: DOE

Remote: none

Acquired: 22:2 May 06, 2005

Survey Co:USGS
Rotation:

Filename: rm03.avg

Channels: Ch1 Ch2 Ch3 Ch4 Ch5 Ch3 Ch4

Plotted: 11:00 Jan 17, 2006

< EMI - ElectroMagnetic Instruments > 


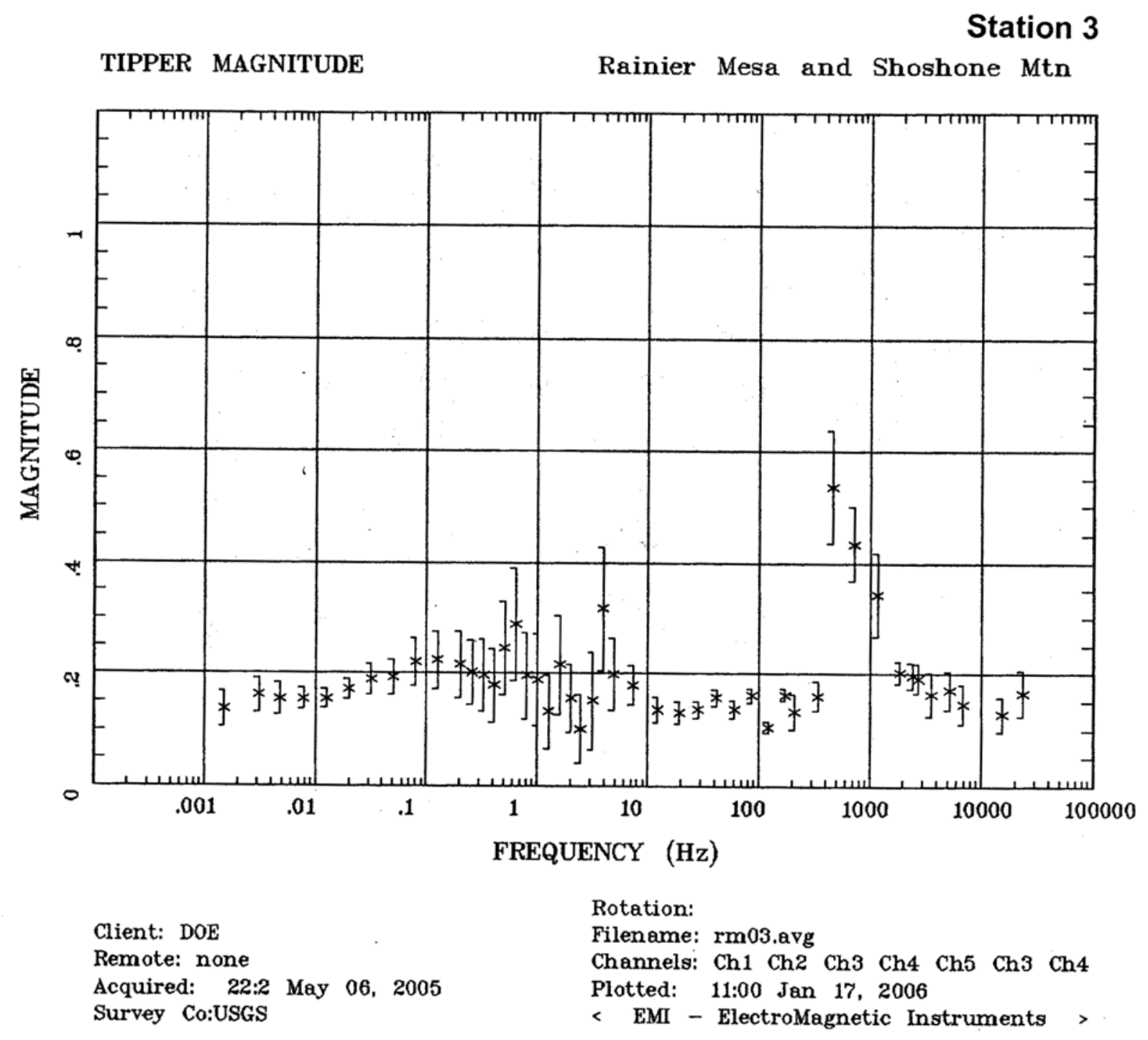




\section{Station 3}

TIPPER STRIKE

Rainier Mesa and Shoshone Mtn

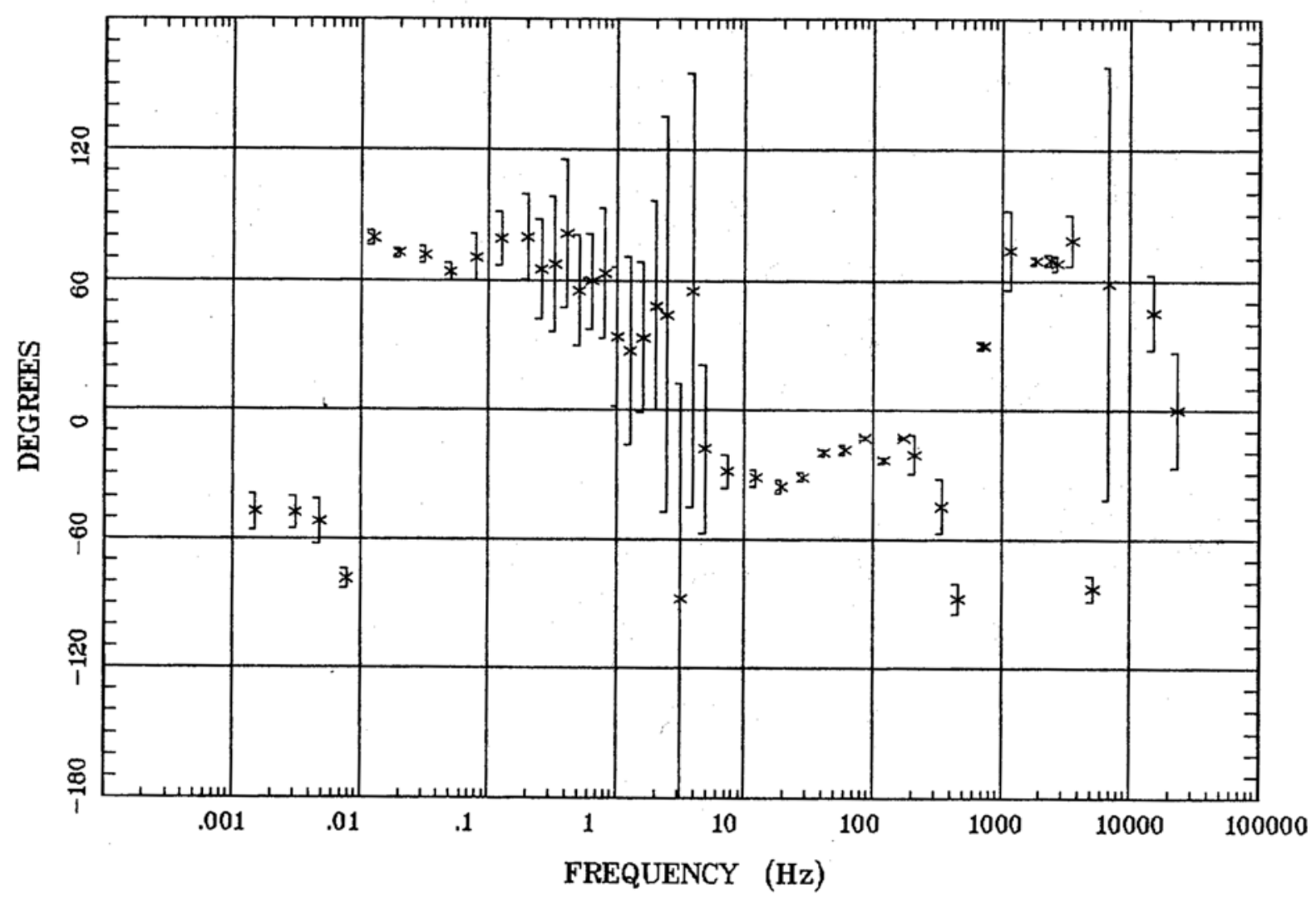

Client: DOE

Remote: none

Acquired: 22:2 May 06, 2005

Survey Co:USGS
Rotation:

Filename: rm03.avg

Channels: Ch1 Ch2 Ch3 Ch4 Ch5 Ch3 Ch4

Plotted: 11:00 Jan 17, 2006

< EMI - ElectroMagnetic Instruments > 


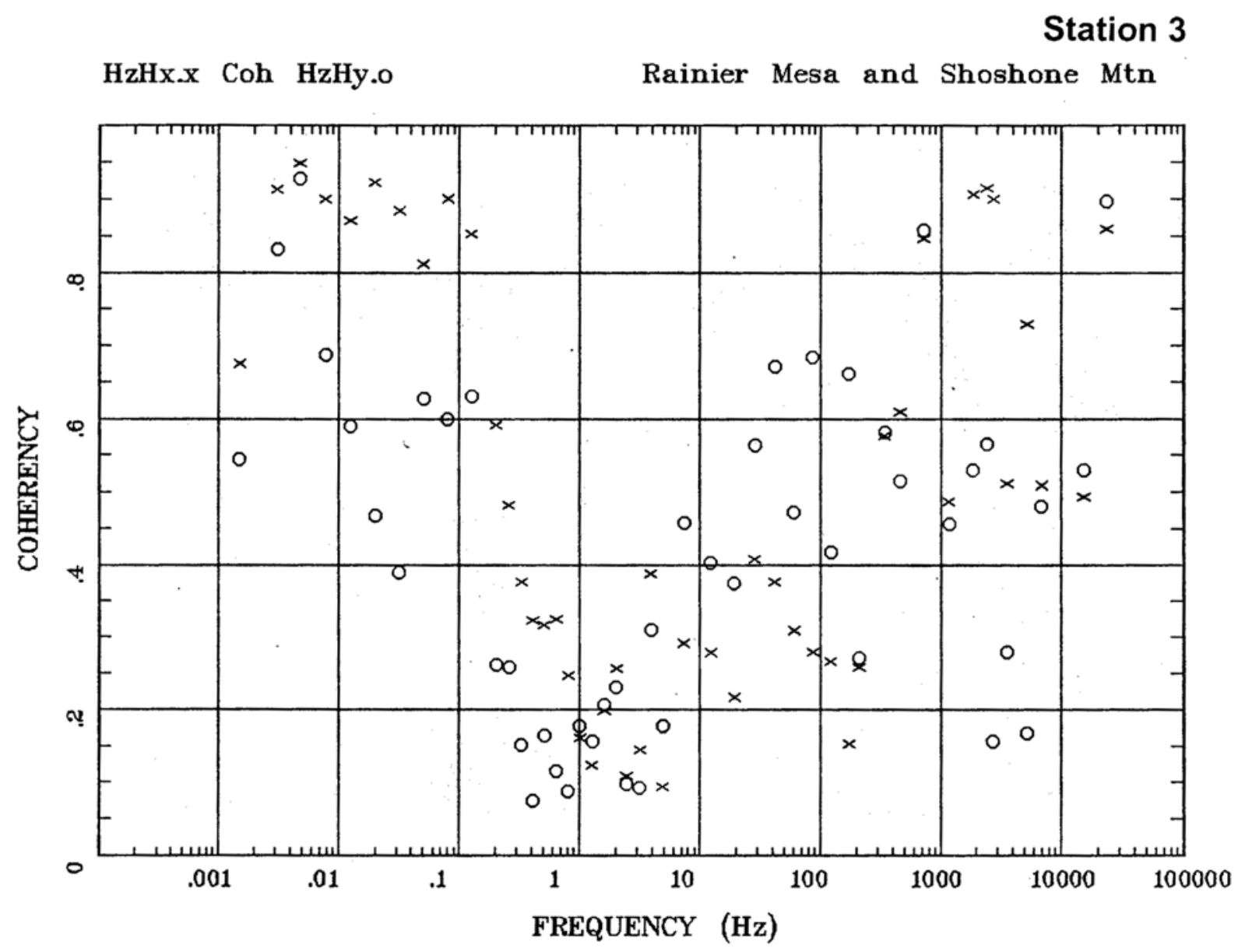

Client: $\mathrm{DOE}$

Remote: none

Acquired: 22:2 May 06, 2005

Survey Co:USGS
Rotation:

Filename: rm03.avg

Channels: Ch1 Ch2 Ch3 Ch4 Ch5 Ch3 Ch4

Plotted: 11:00 Jan 17, 2006

< EMI - ElectroMagnetic Instruments > 


\section{Station 4}

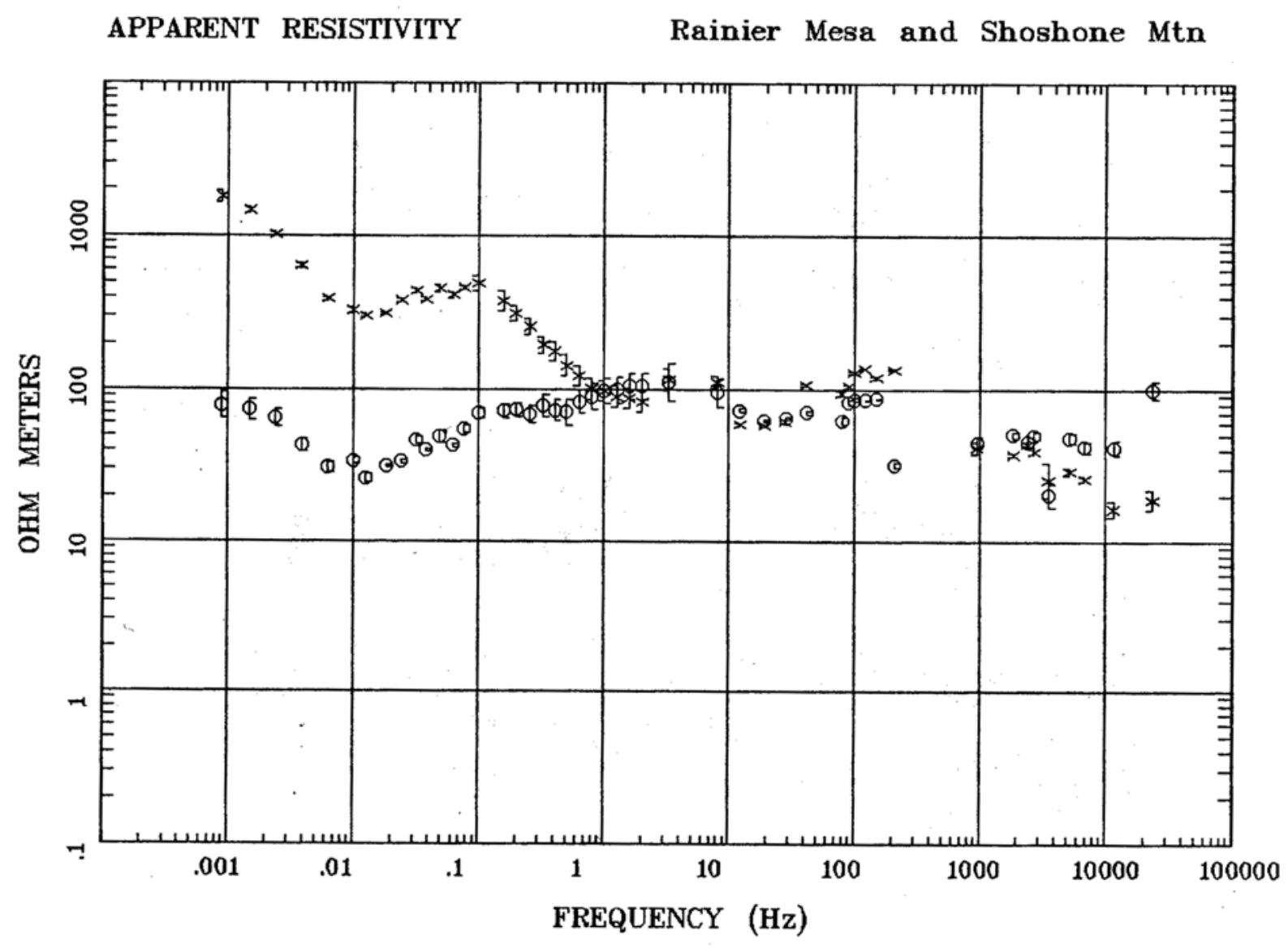

Client: DOE

Remote: none

Acquired: 21:5 May 07, 2005

Survey Co:USGS
Rotation:

Filename: rm04.avg

Channels: Ch1 Ch2 Ch3 Ch4 Ch5 Ch3 Ch4

Plotted: 11:03 Jan 17, 2006

< EMI - ElectroMagnetic Instruments > 


\section{Station 4}

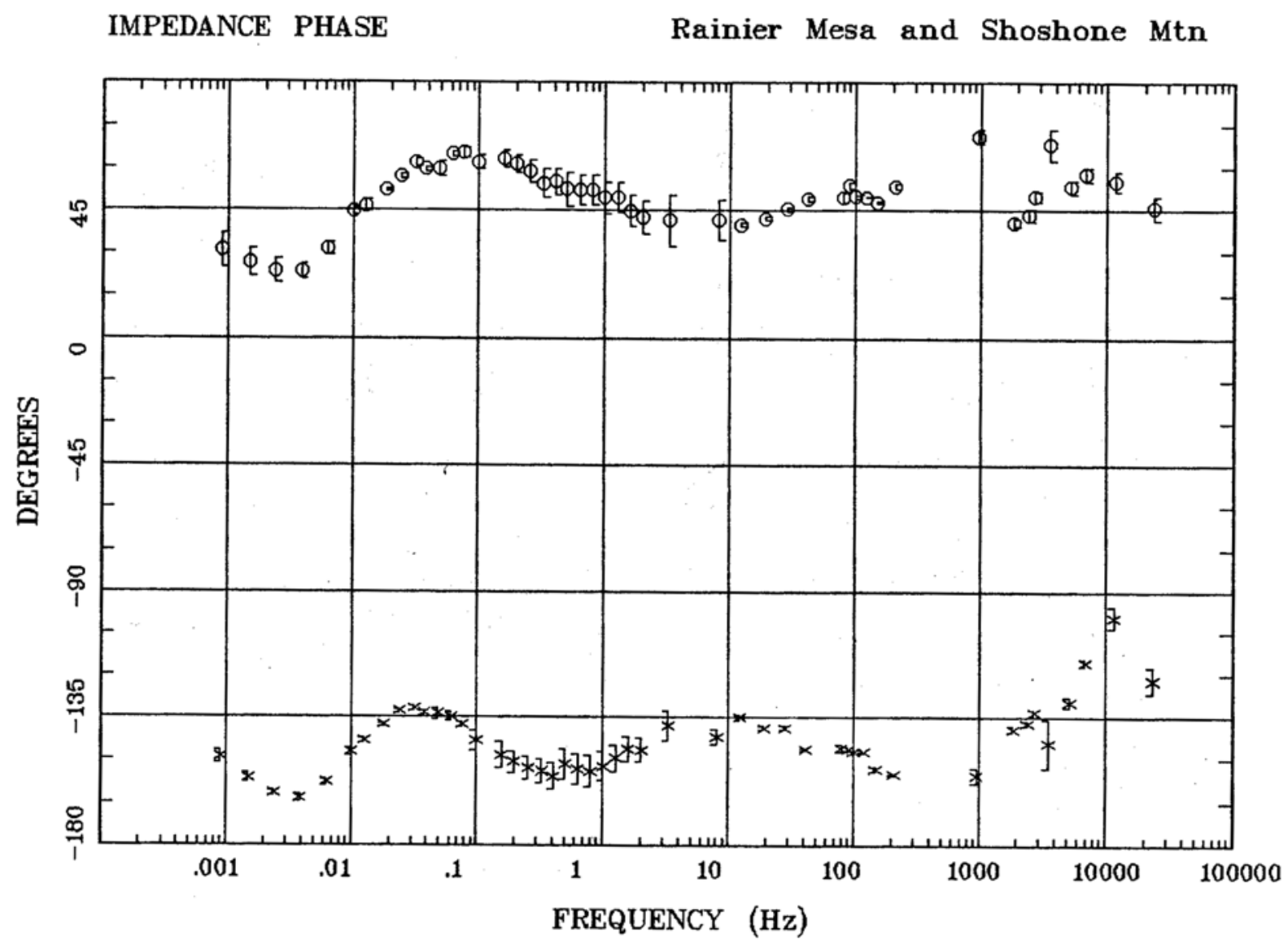

Client: DOE

Remote: none

Acquired: 21:5 May 07, 2005

Survey Co:USGS
Rotation:

Filename: rm04.avg

Channels: Ch1 Ch2 Ch3 Ch4 Ch5 Ch3 Ch4

Plotted: 11:03 Jan 17, 2006

< EMI - ElectroMagnetic Instruments > 


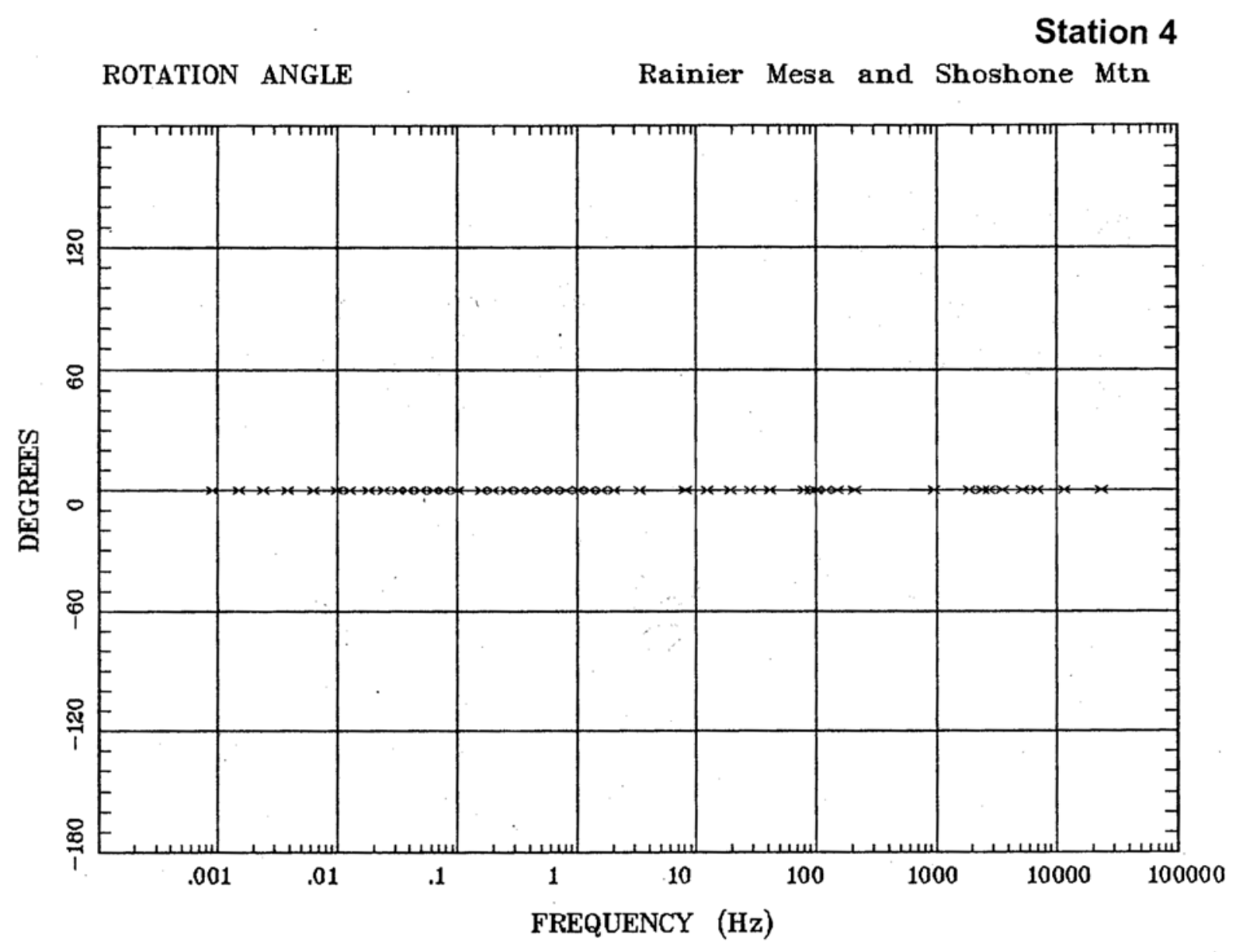

Client: DOE

Remote: none

Acquired: 21:5 May 07, 2005

Rotation:

Filename: rm04.avg

Channels: Ch1 Ch2 Ch3 Ch4 Ch5 Ch3 Ch4

Plotted: 11:03 Jan 17, 2006

Survey Co:USGS

< EMI - ElectroMagnetic Instruments > 


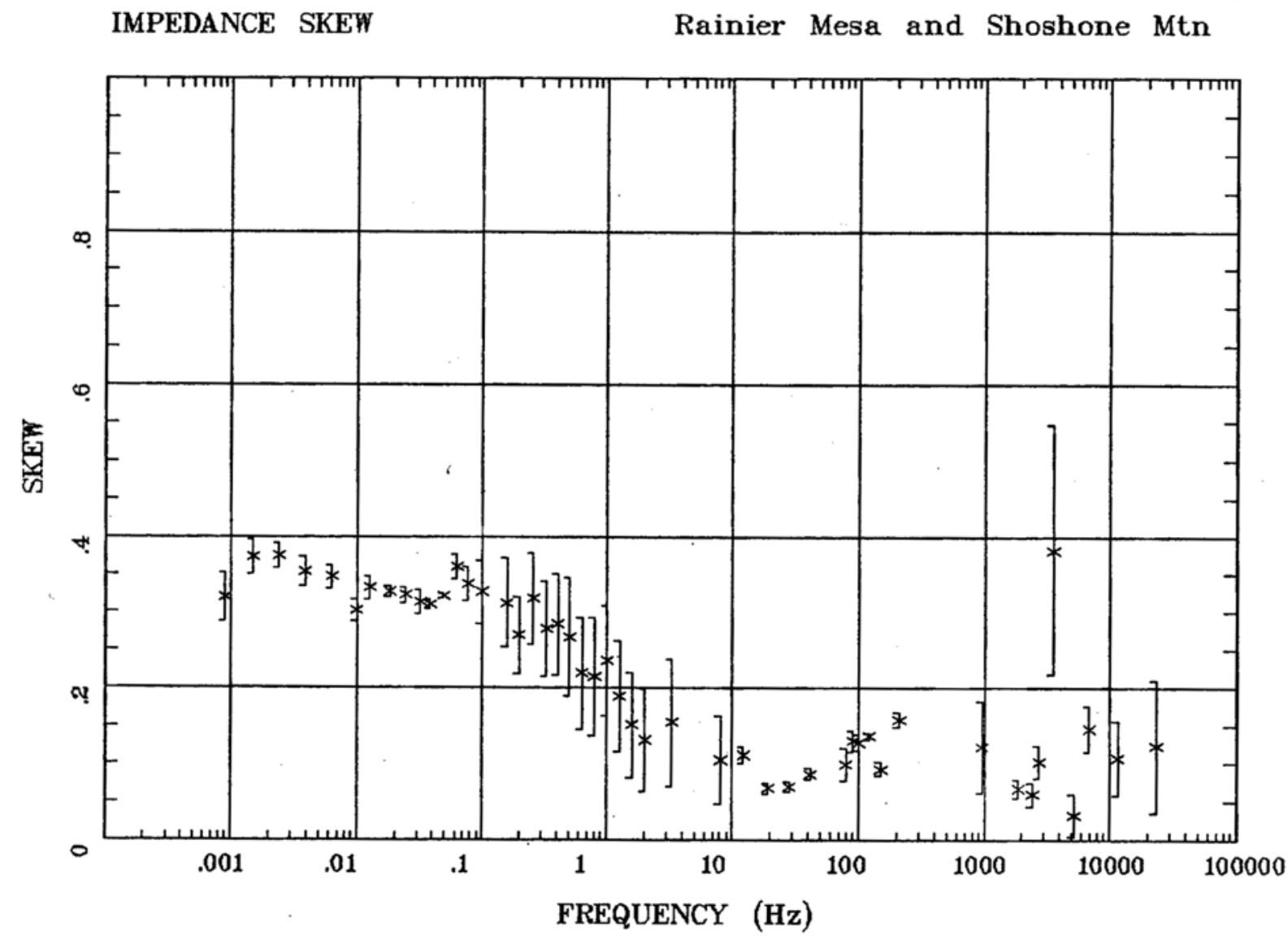

Client: DOE

Remote: none

Acquired: 21:5 May 07, 2005

Survey Co:USGS
Rotation:

Filename: rm04.avg

Channels: Ch1 Ch2 Ch3 Ch4 Ch5 Ch3 Ch4

Plotted: 11:03 Jan 17, 2006

< EMI - ElectroMagnetic Instruments > 


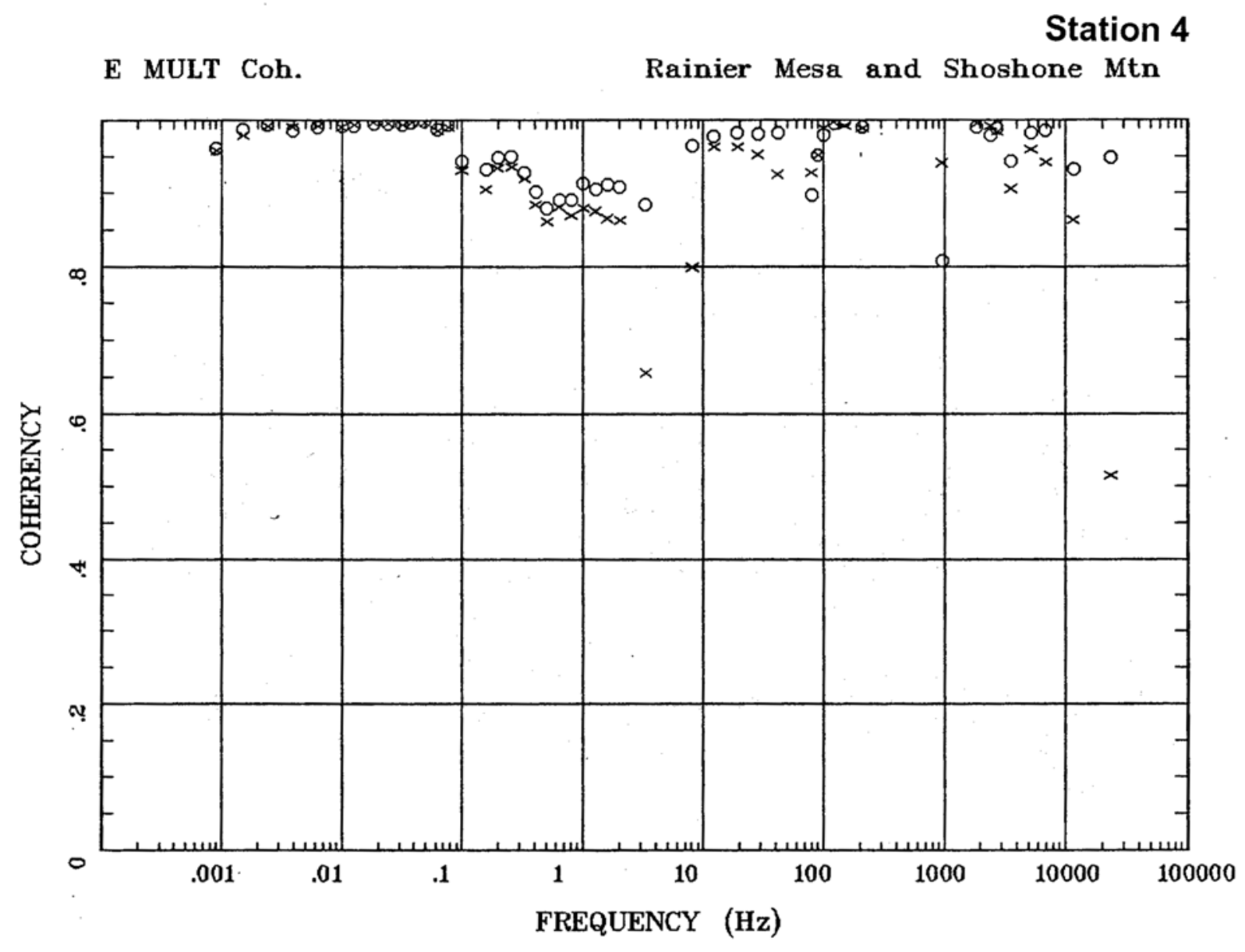

Client: DOE

Remote: none

Acquired: 21:5 May 07, 2005

Survey Co:USGS

Rotation:

Filename: rm04.avg

Channels: Ch1 Ch2 Ch3 Ch4 Ch5 Ch3 Ch4

Plotted: 11:03 Jan 17, 2006

< EMI - ElectroMagnetic Instruments > 
Station 4

POLAR PLOTS

Rainier Mesa and Shoshone Mtn

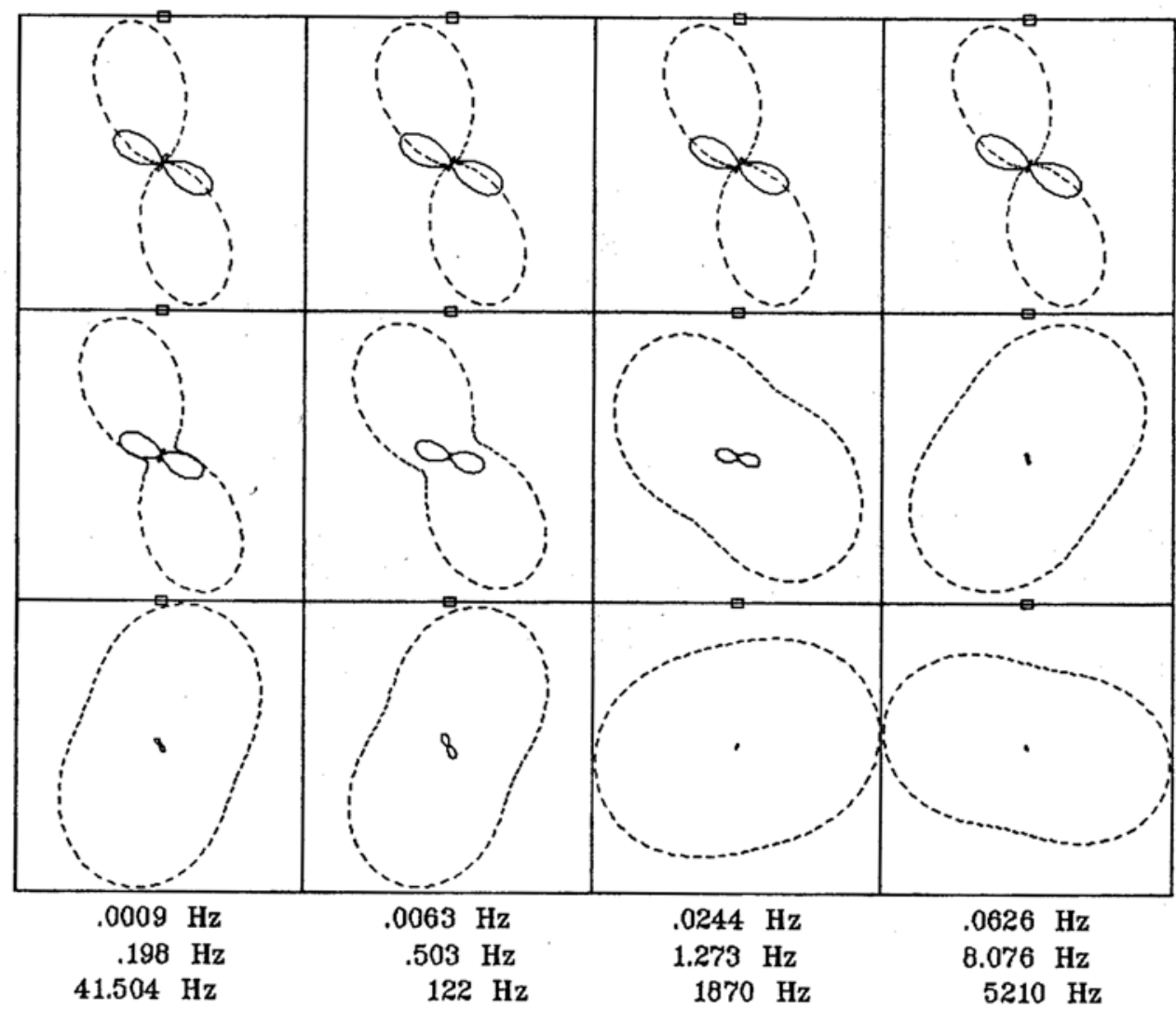

Client: DOE

Remote: none

Acquired: 21:5 May 07, 2005

Survey Co:USGS
Rotation:

Filename: rm04.avg

Channels: Ch1 Ch2 Ch3 Ch4 Ch5 Ch3 Ch4

Plotted: 11:03 Jan 17, 2006

< EMI - ElectroMagnetic Instruments > 
TIPPER MAGNITUDE

Station 4

Rainier Mesa and Shoshone Mtn

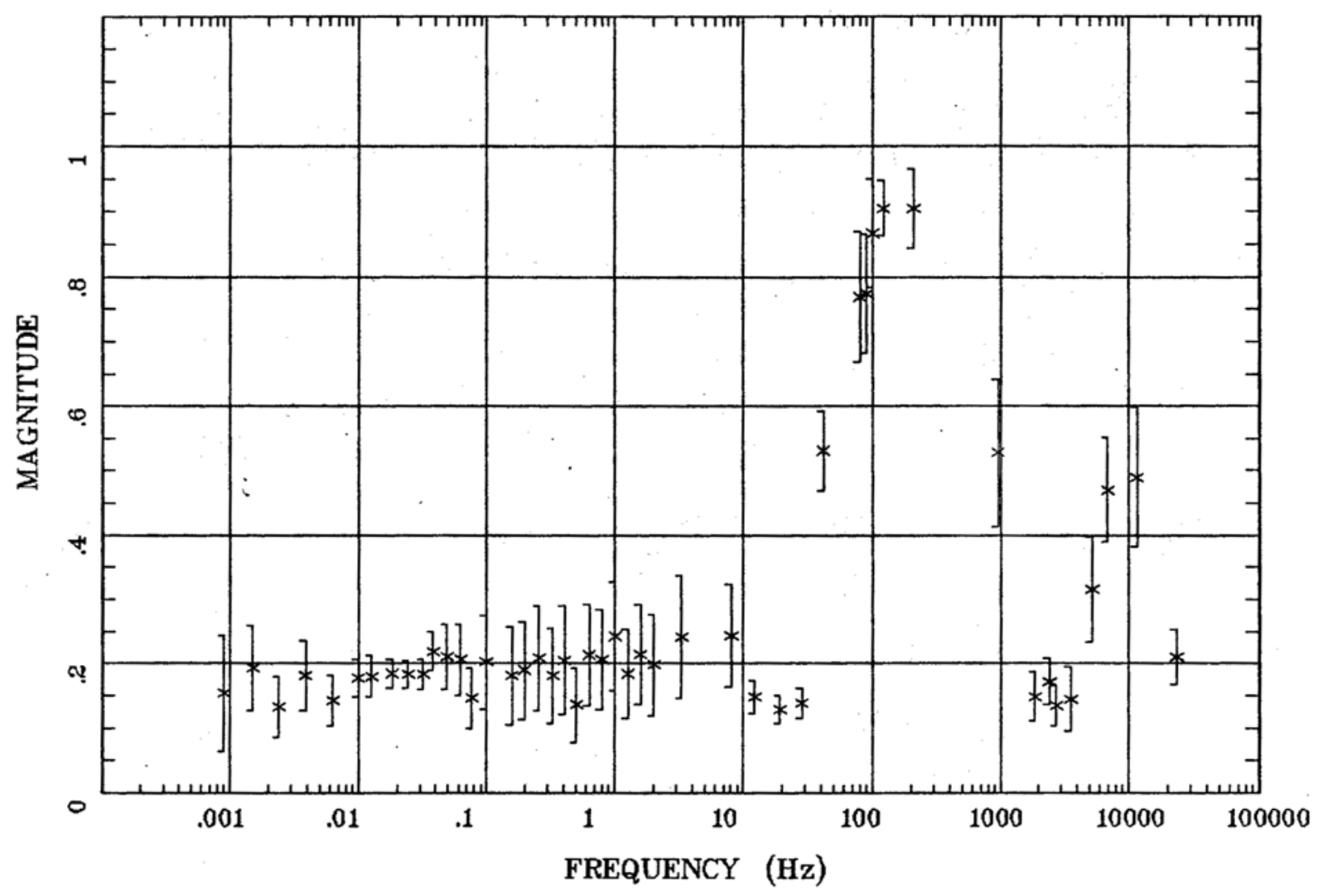

Client: DOE

Remote: none

Acquired: 21:5 May 07, 2005

Survey Co:USGS
Rotation:

Filename: rm04.avg

Channels: Ch1 Ch2 Ch3 Ch4 Ch5 Ch3 Ch4

Plotted: 11:03 Jan 17, 2006 .

< EMI - ElectroMagnetic Instruments > 


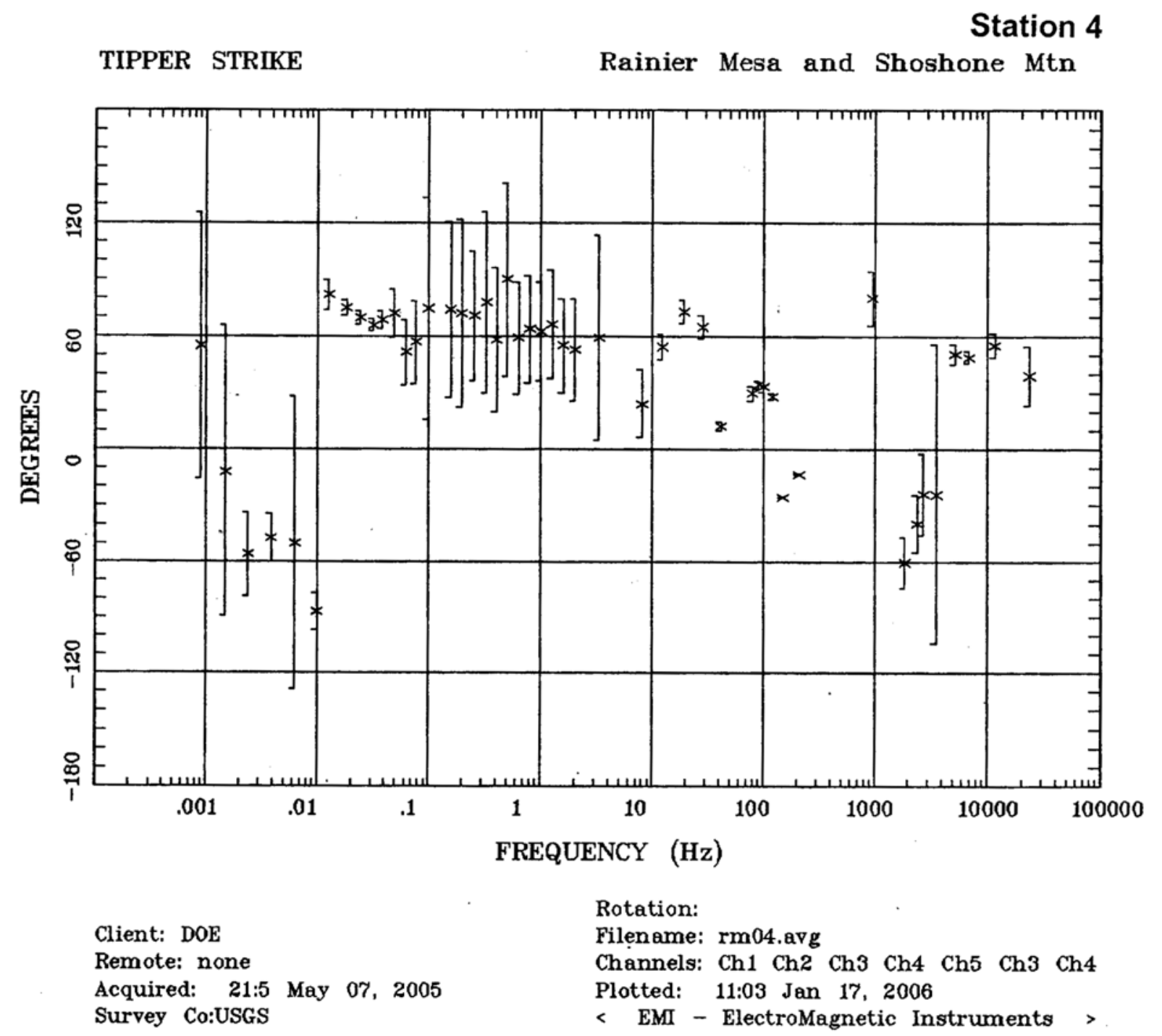


Station 4

HzHx.x Coh HzHy.o

Rainier Mesa and Shoshone Mtn

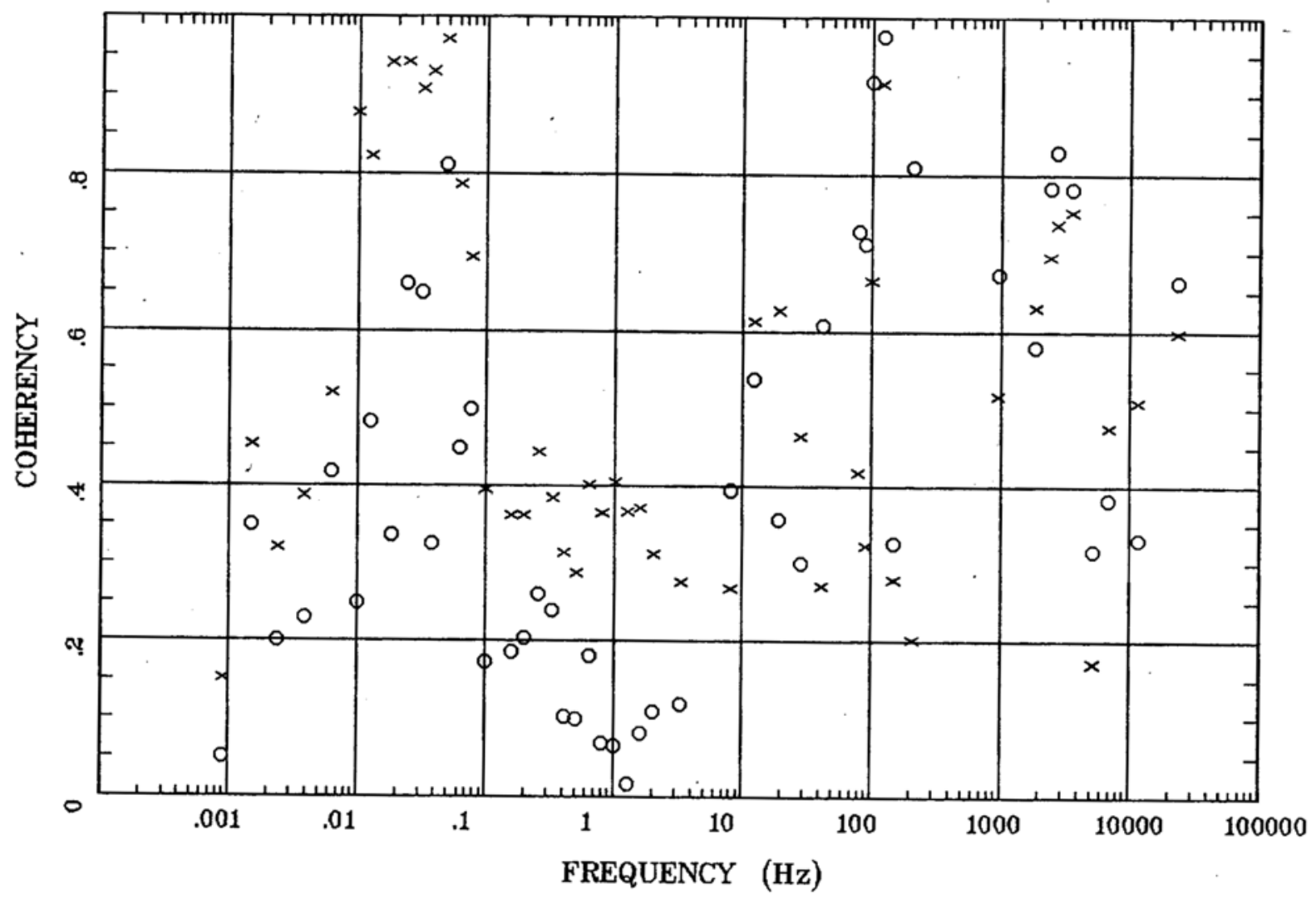

Client: DOE

Remote: none

Acquired: 21:5 May 07, 2005

Survey Co:USGS
Rotation:

Filename: rm04.avg

Channels: Ch1 Ch2 Ch3 Ch4 Ch5 Ch3 Ch4

Plotted: 11:03 Jan 17, 2006

< EMI - ElectroMagnetic Instruments > 


\section{Station 5}

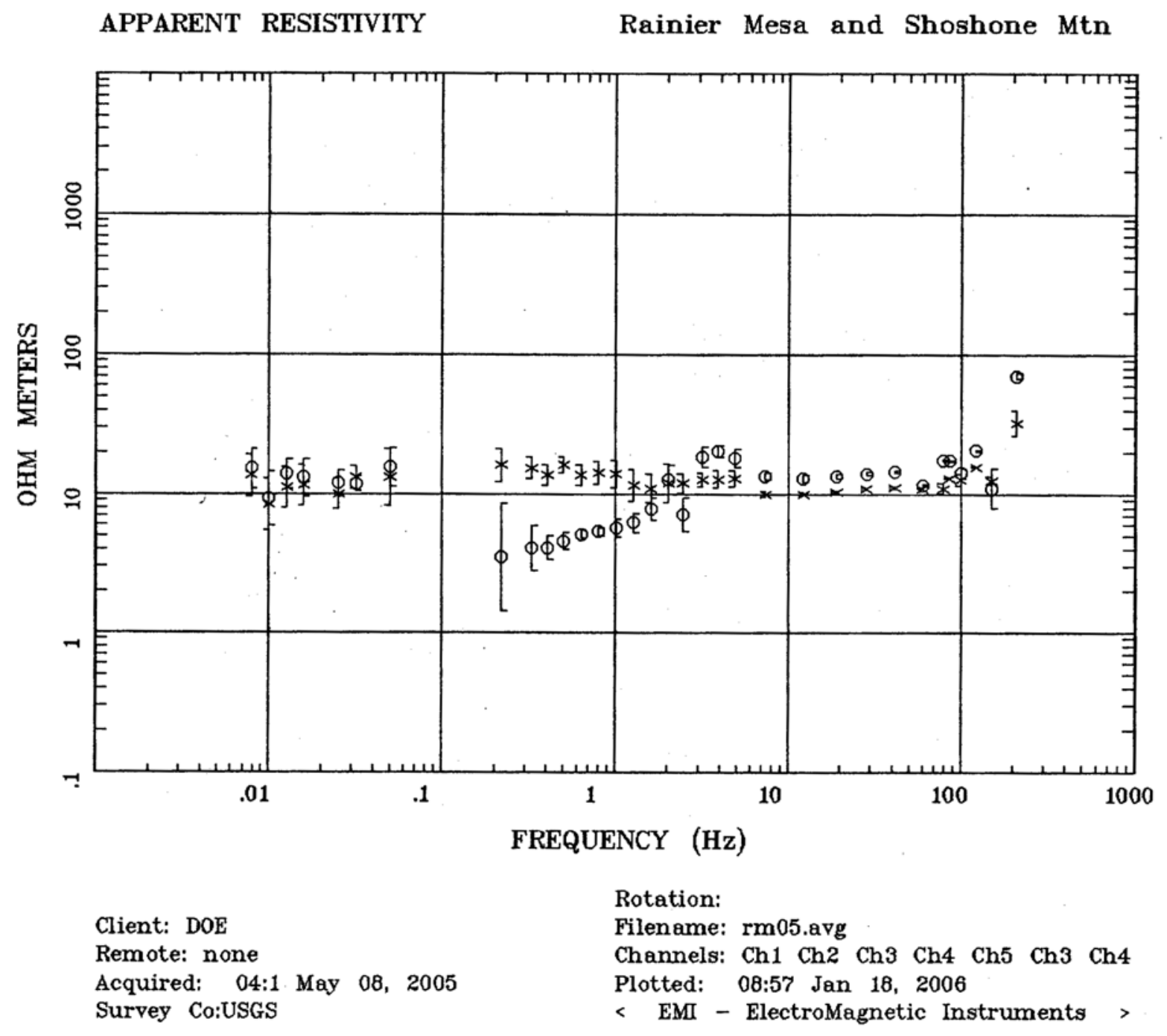




\section{Station 5}

IMPEDANCE PHASE

Rainier Mesa and Shoshone Mtn

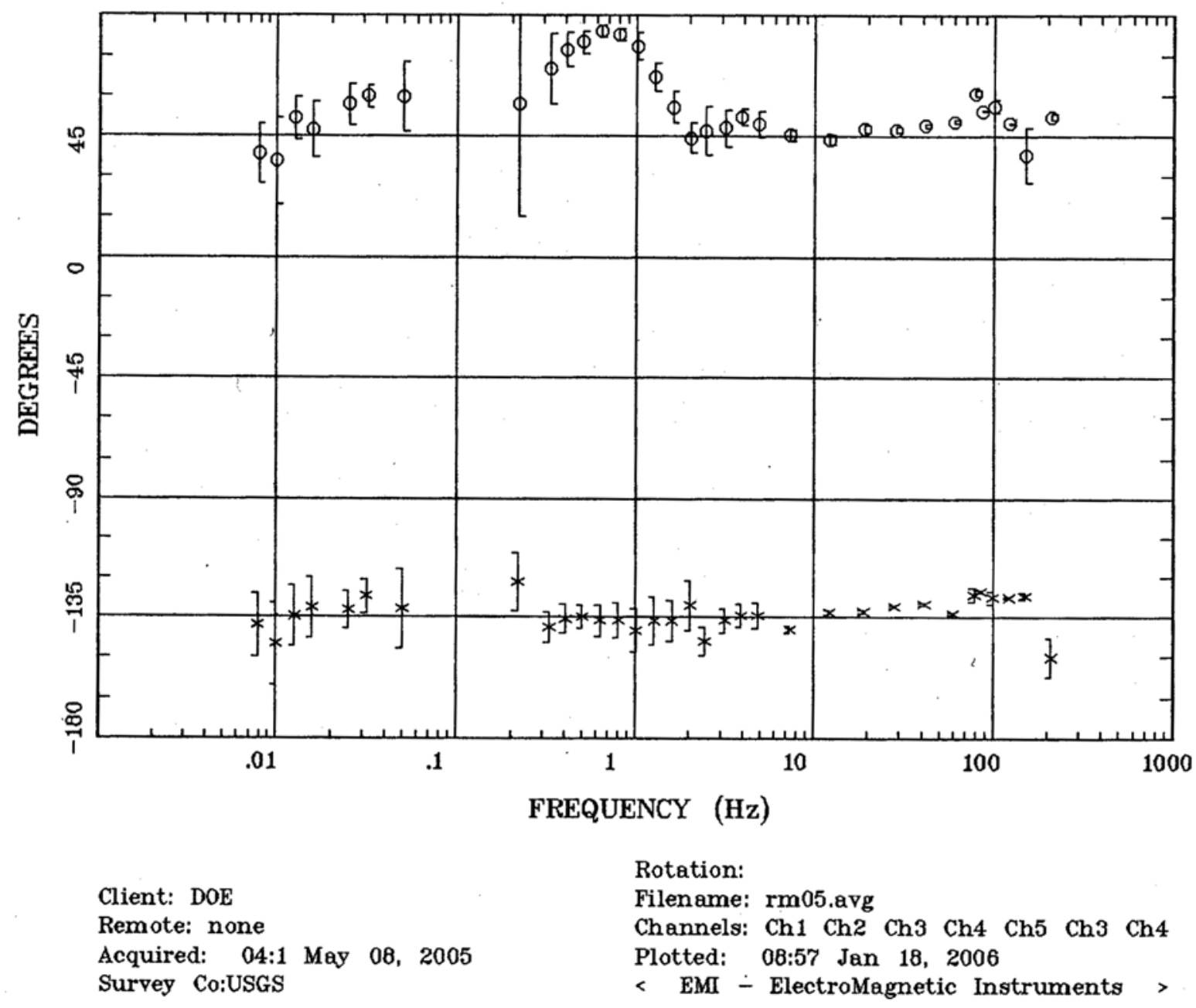


Station 5

ROTATION ANGLE

Rainier Mesa and Shoshone Mtn

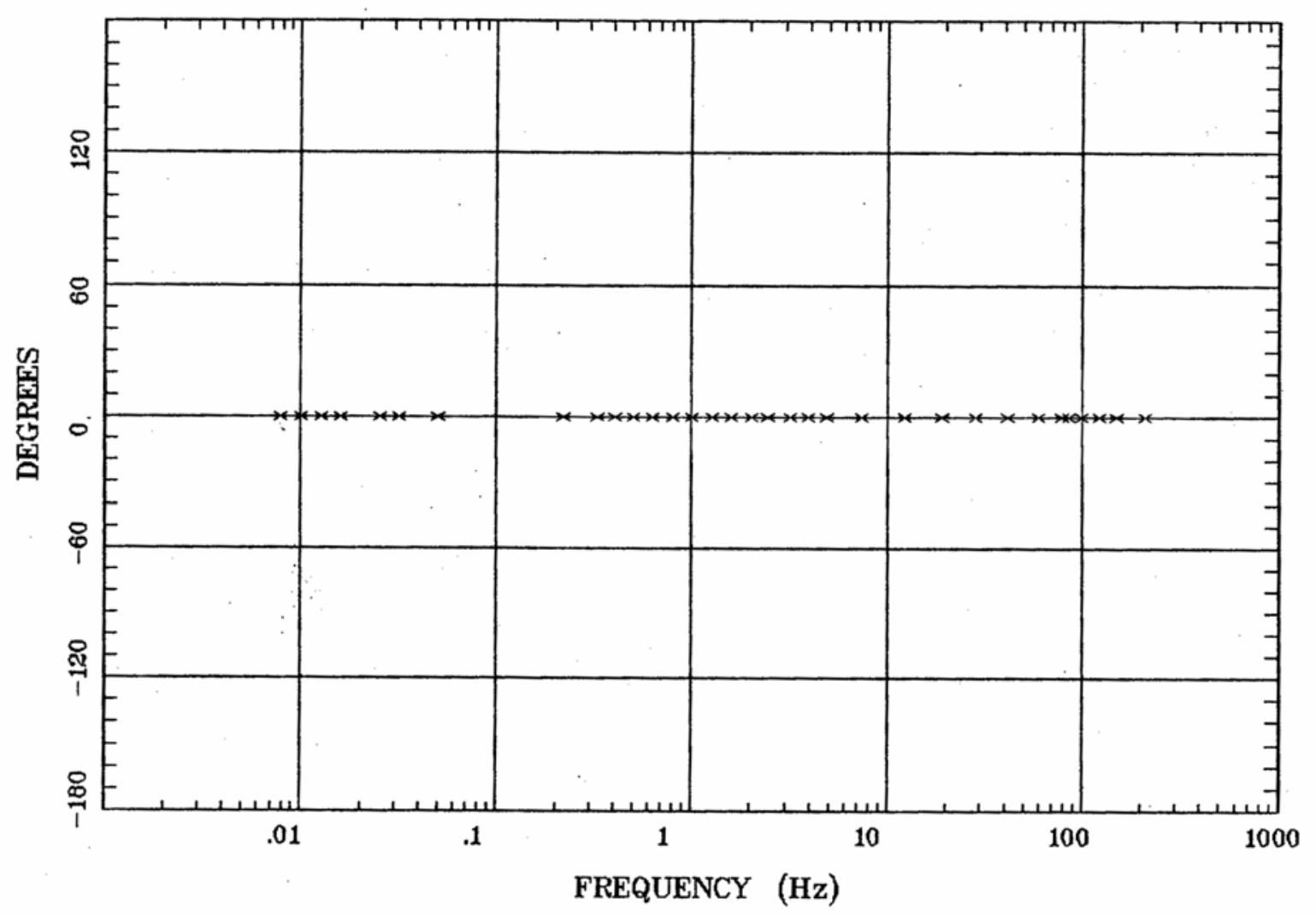

Client: DOE

Remote: none

Acquired: 04:1 May 08, 2005

Survey Co:USGS
Rotation:

Filename: rm05.avg

Channels: Ch1 Ch2 Ch3 Ch4 Ch5 Ch3 Ch4

Plotted: 08:57 Jan 18, 2006

< EMI - ElectroMagnetic Instruments > 
IMPEDANCE SKEW

Station 5

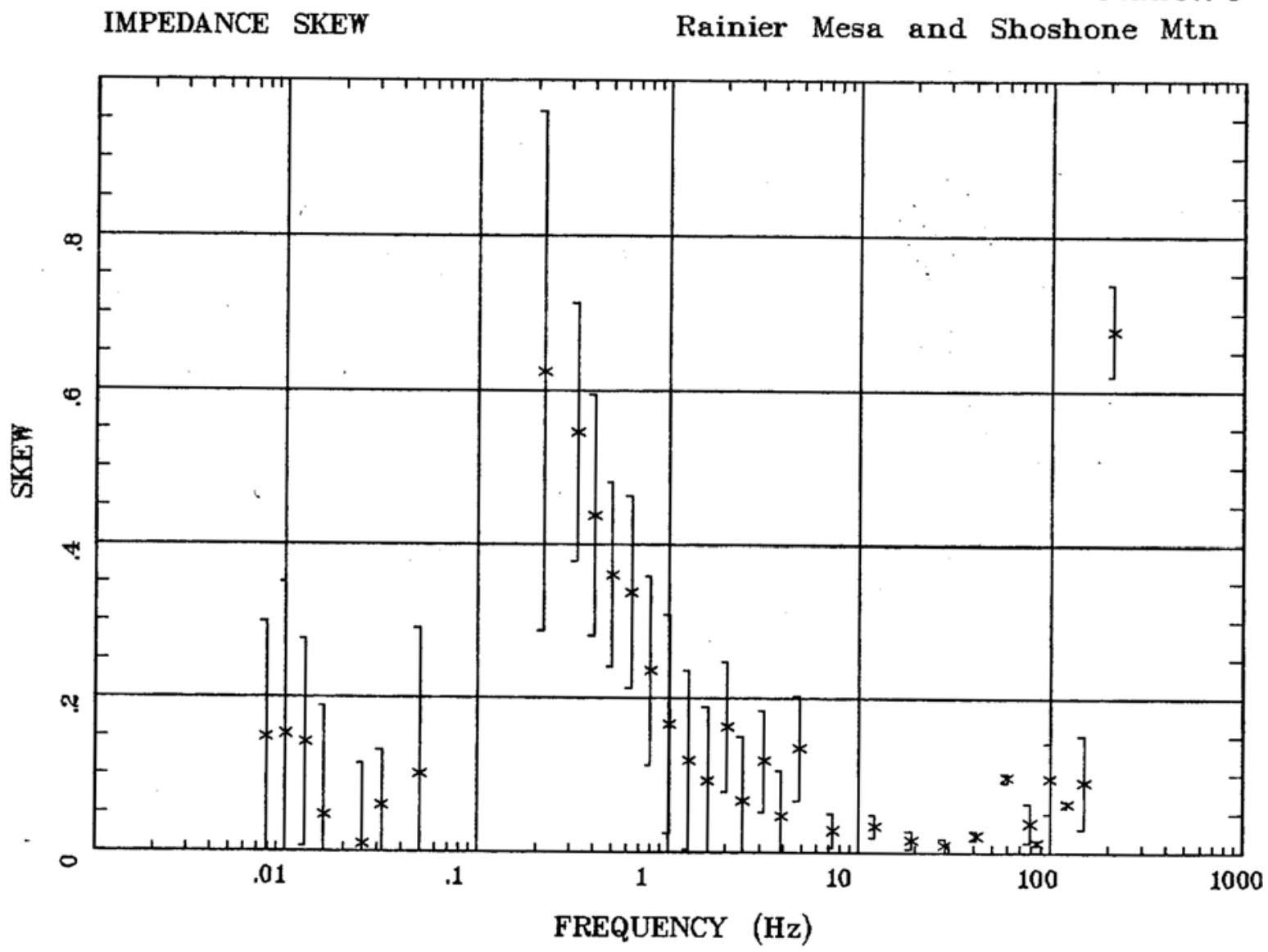

Client: DOE

Remote: none

Acquired: 04:1 May 08, 2005

Survey Co:USGS
Rotation:

Filename: rm05.avg

Channels: Ch1 Ch2 Ch3 Ch4 Ch5 Ch3 Ch4

Plotted: 08:57 Jan 18, 2006

< EMI - ElectroMagnetic Instruments > 
Station 5

E MULT Coh.

Rainier Mesa and Shoshone Mtn

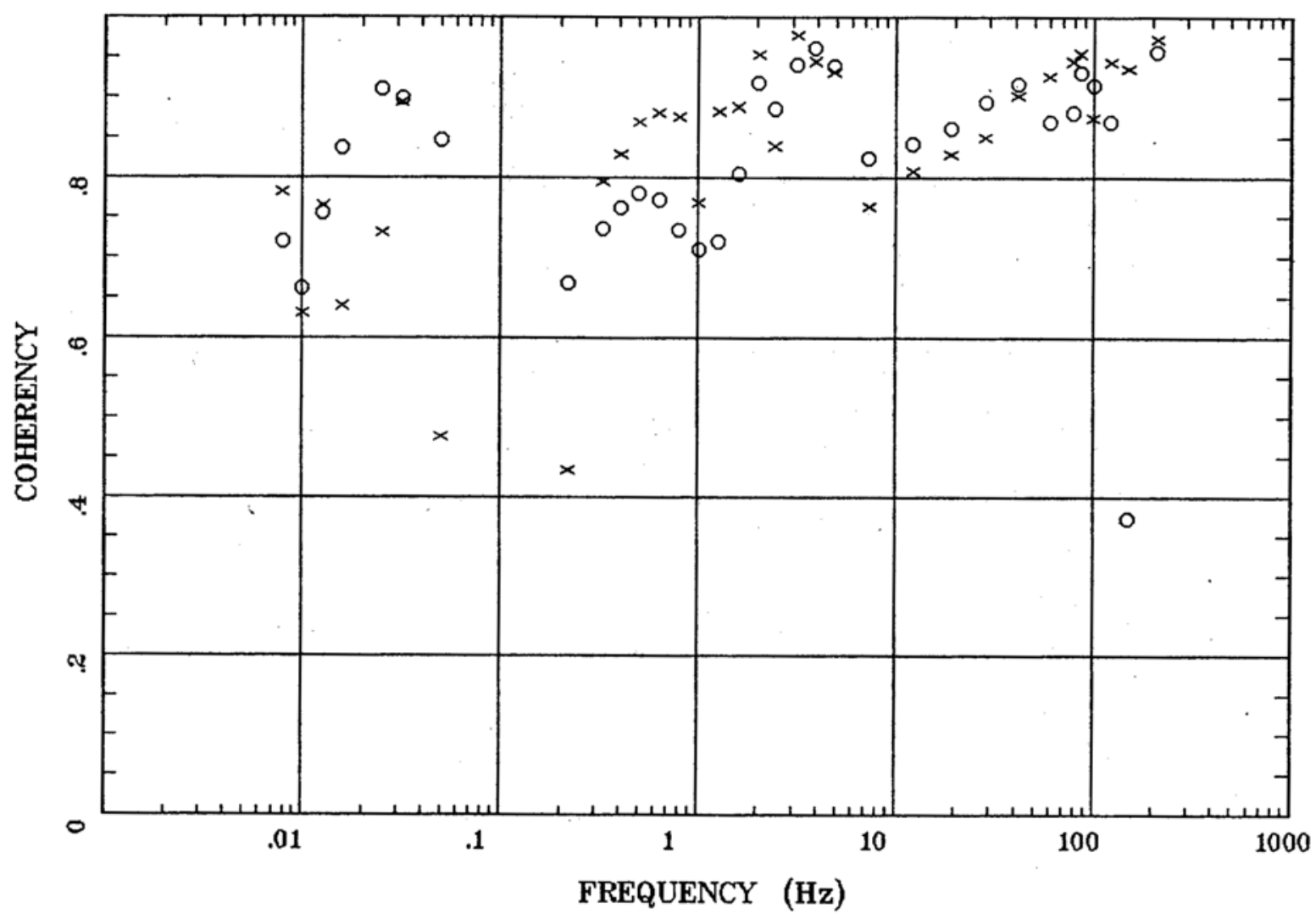

Client: DOE

Remote: none

Acquired: 04:1 May 08, 2005 Survey Co:USGS
Rotation:

Filename: rm05.avg Channels: Ch1 Ch2 Ch3 Ch4 Ch5 Ch3 Ch4 Plotted: 08:57 Jan 18, 2006

< EMI - ElectroMagnetic Instruments > 
Station 5

POLAR PLOTS

Rainier Mesa and Shoshone Mtn

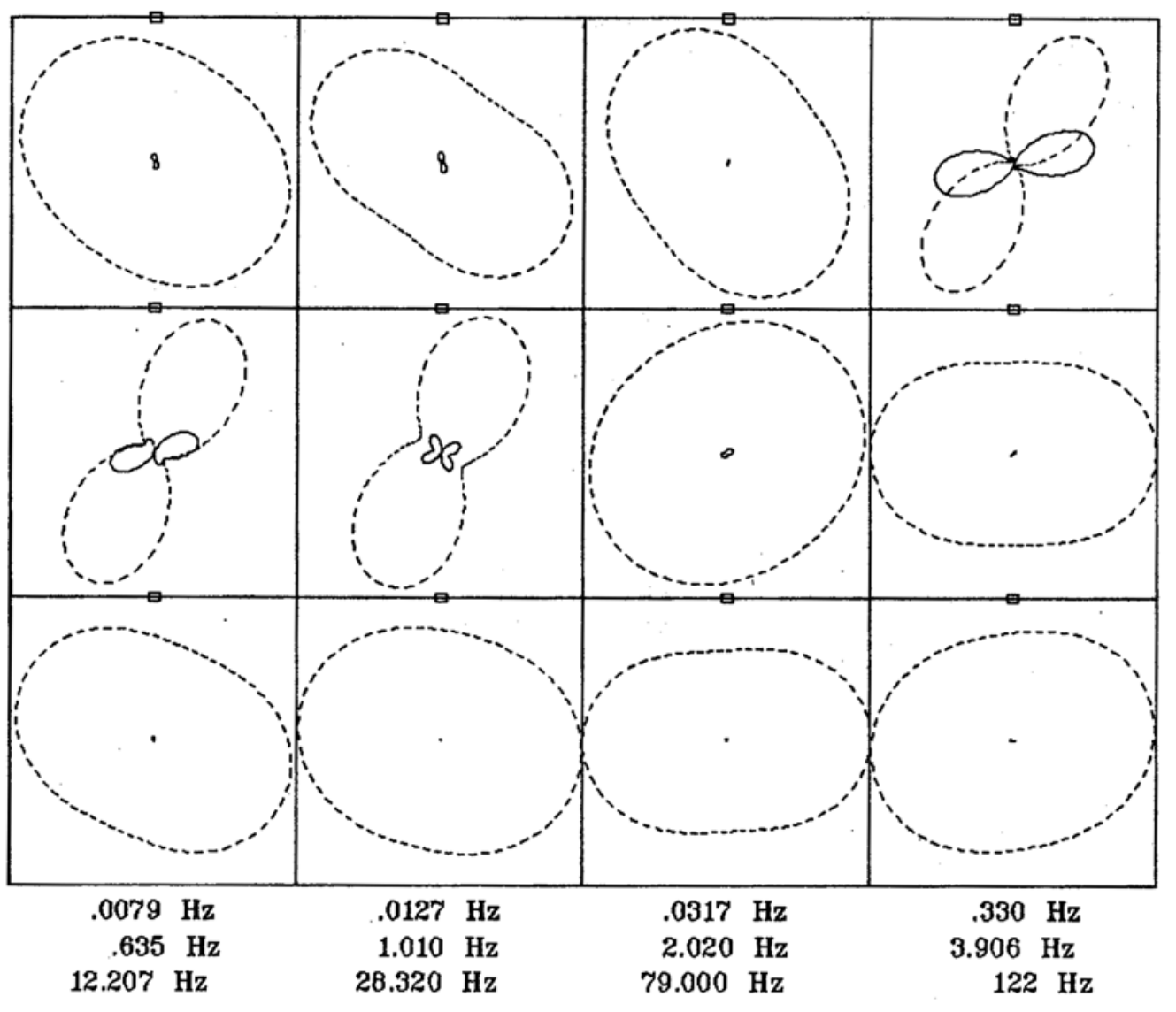

Client: DOE

Rotation:

Remote: none

Filename: rm05.avg

Acquired: 04:1 May 08, 2005

Channels: Ch1 Ch2 Ch3 Ch4 Ch5 Ch3 Ch4

Plotted: 08:57 Jan 18, 2006

Survey Co:USGS

< EMI - ElectroMagnetic Instruments > 


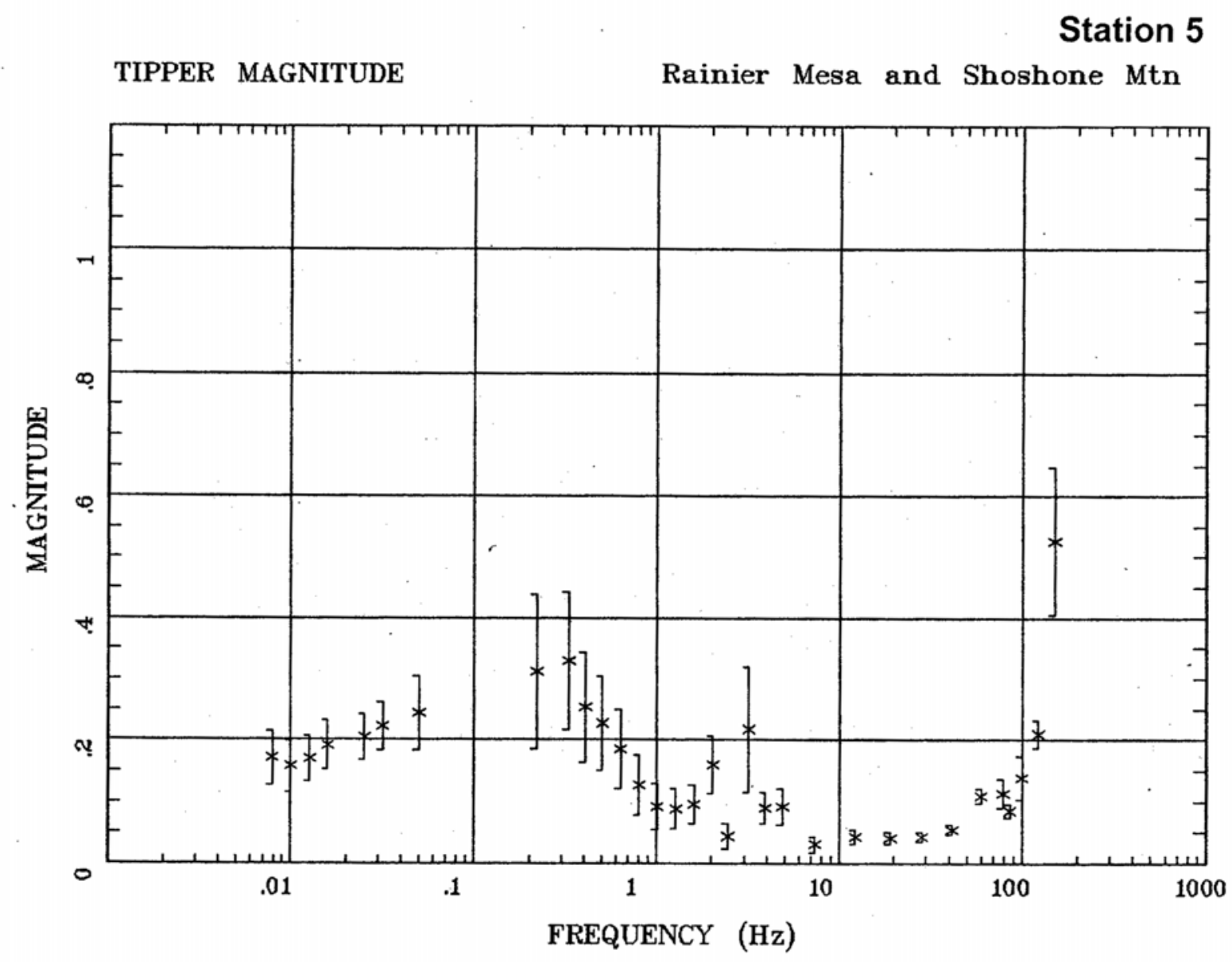

Client: DOE

Remote: none

Acquired: 04:1 May 08, 2005

Survey Co:USGS
Rotation:

Filename: rm05.avg

Channels: Ch1 Ch2 Ch3 Ch4 Ch5 Ch3 Ch4

Plotted: 08:57 Jan 18, 2006

< EMI - ElectroMagnetic Instruments > 
Station 5

TIPPER STRIKE

Rainier Mesa and Shoshone Mtn

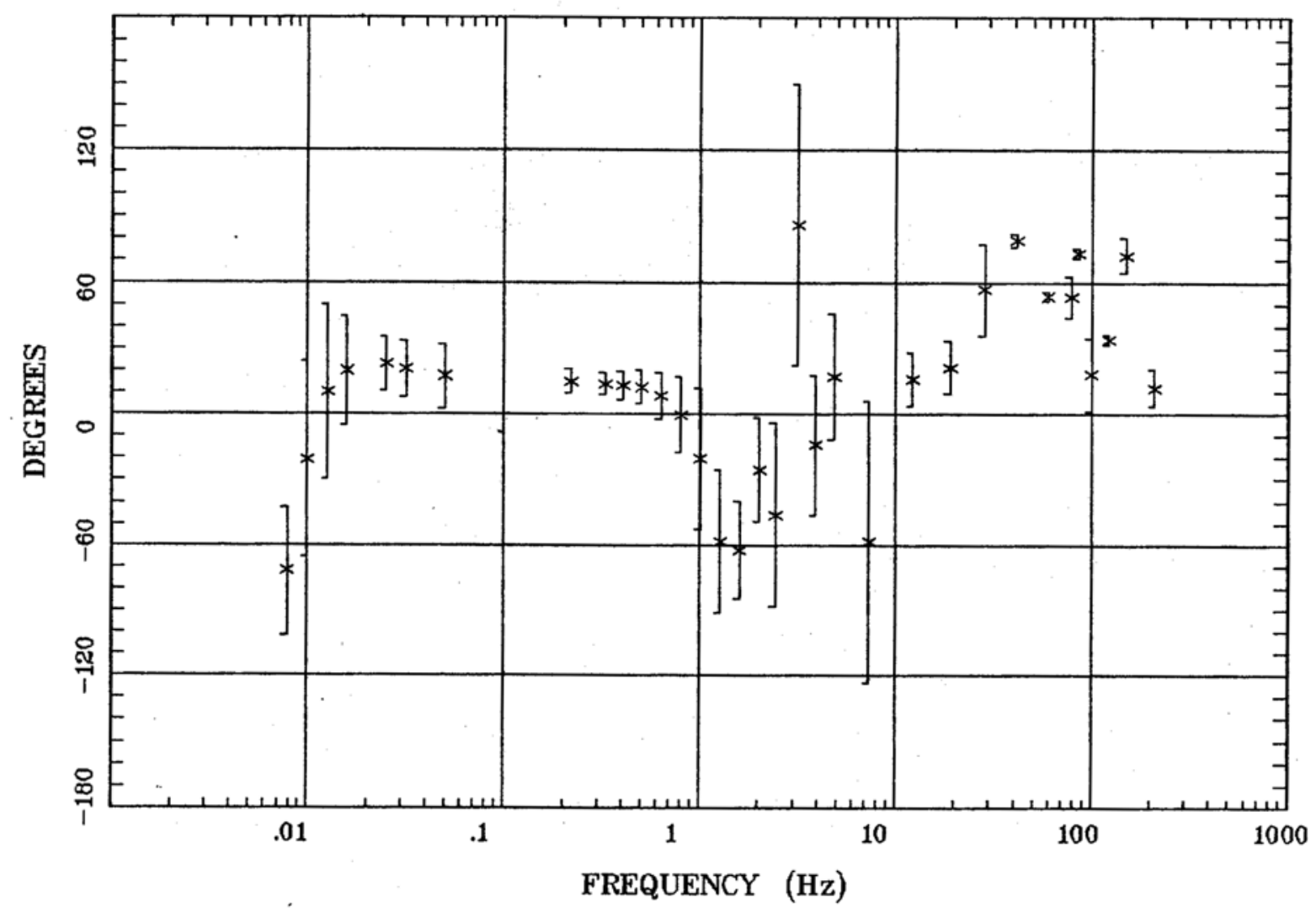

Client: DOE

Remote: none

Acquired: 04:1 May 08, 2005

Survey Co:USGS
Rotation:

Filenome: rm05.avg

Channels: Ch1 Ch2 Ch3 Ch4 Ch5 Ch3 Ch4

Plotted: 08:57 Jan 18, 2006

< EMI - ElectroMagnetic Instruments > 


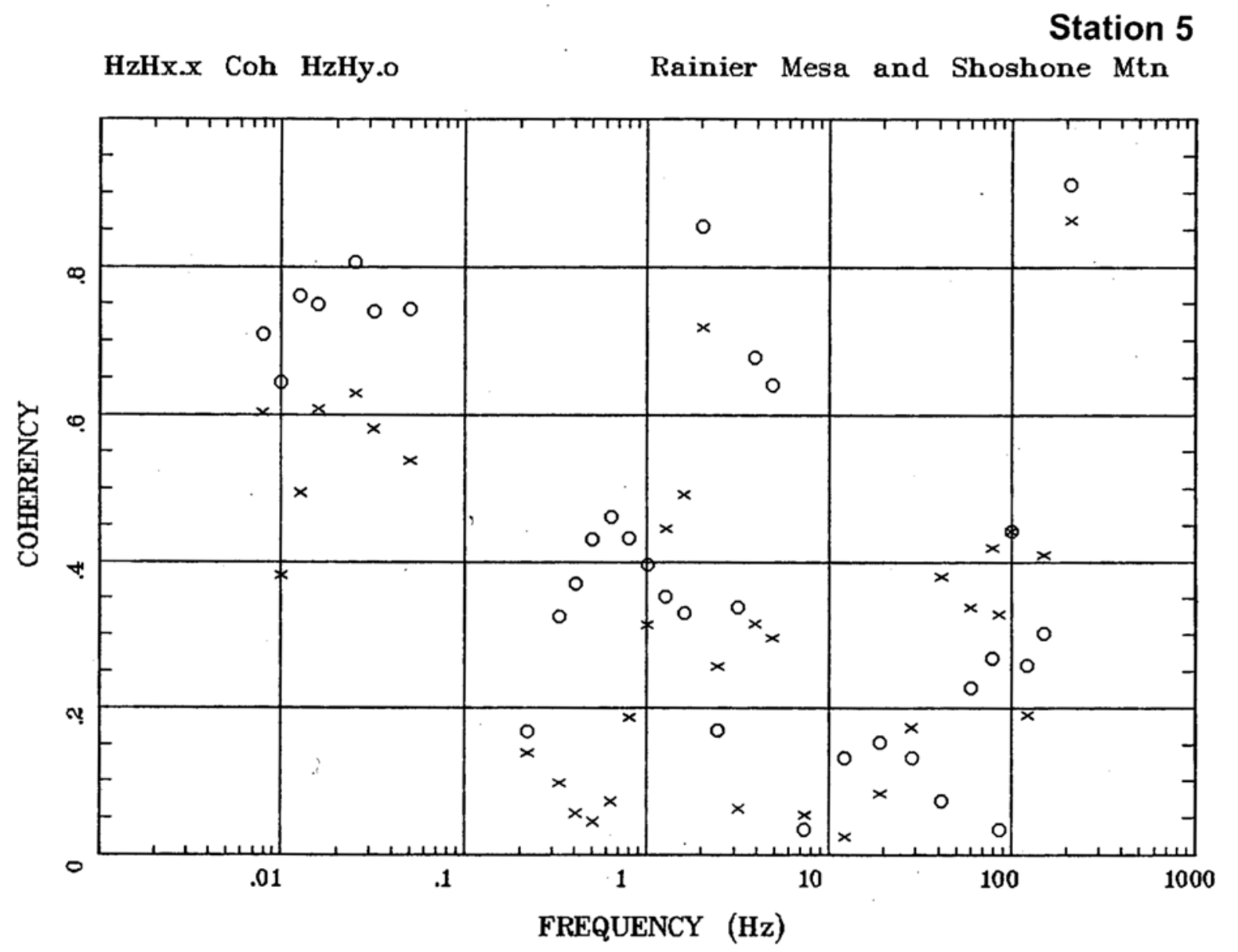

Client: DOE

Remote: none

Acquired: 04:1 May 08, 2005 Survey Co:USGS
Rotation:

Filename: rm05.avg

Channels: Ch1 Ch2 Ch3 Ch4 Ch5 Ch3 Ch4

Plotted: 08:57 Jan 18, 2006

< EMI - ElectroMagnetic Instruments > 


\section{Station 6}

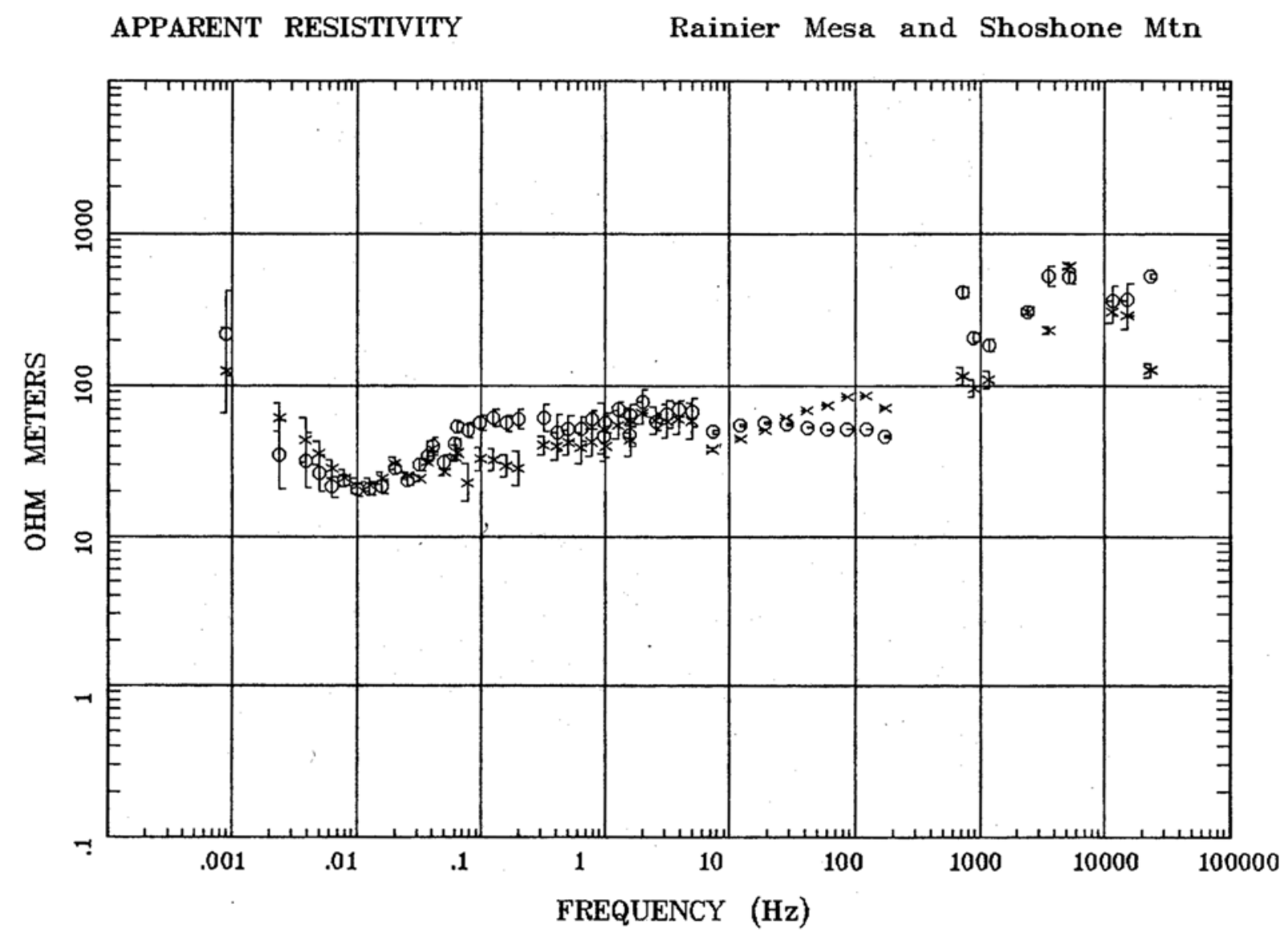

Client: DOE

Remote: none

Acquired: 04:4 May 05, 2005

Survey Co:USGS
Rotation:

Filename: rm06.avg

Channels: Ch1 Ch2 Ch3 Ch4 Ch5 Ch3 Ch4

Plotted: 11:30 Jan 17, 2006

< EMI - ElectroMagnetic Instruments > 


\section{Station 6}

IMPEDANCE PHASE

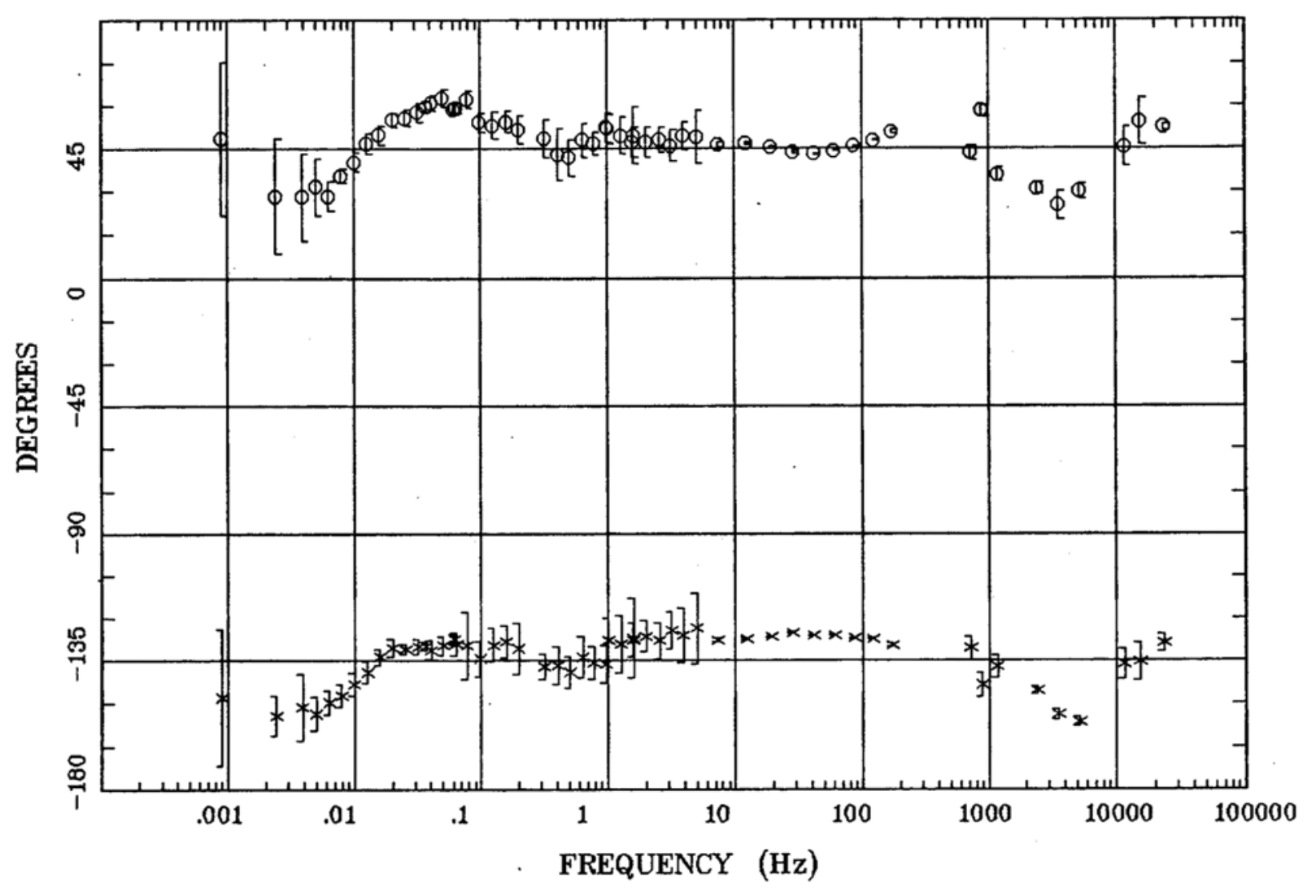

Client: DOE

Remote: none

Acquired: 04:4 May 05, 2005

Survey Co:USGS
Rotation:

Filename: rm06.avg

Channels: Ch1 Ch2 Ch3 Ch4 Ch5 Ch3 Ch4

Plotted: 11:31 Jan 17, 2006

< EMI - ElectroMagnetic Instruments > 


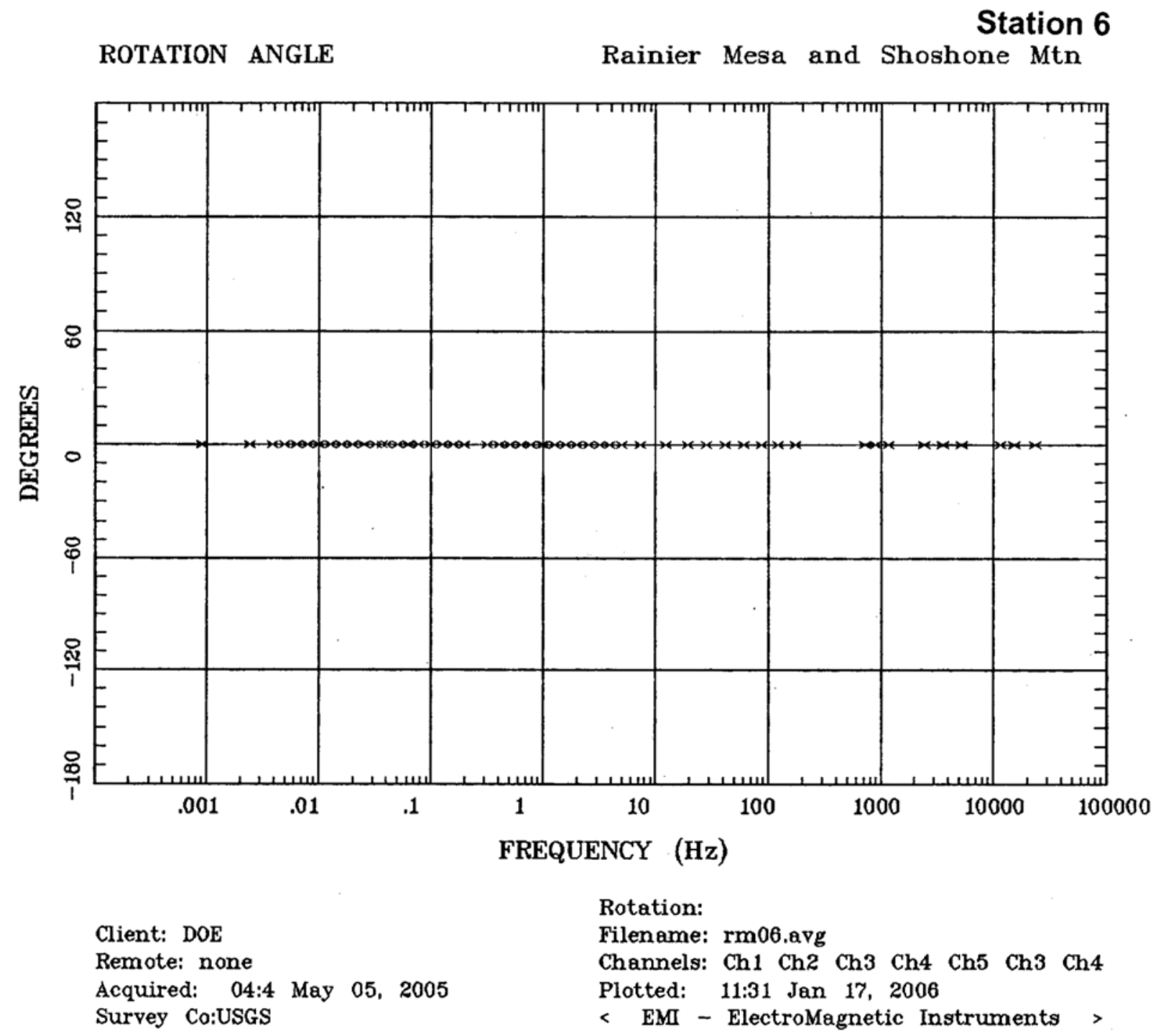




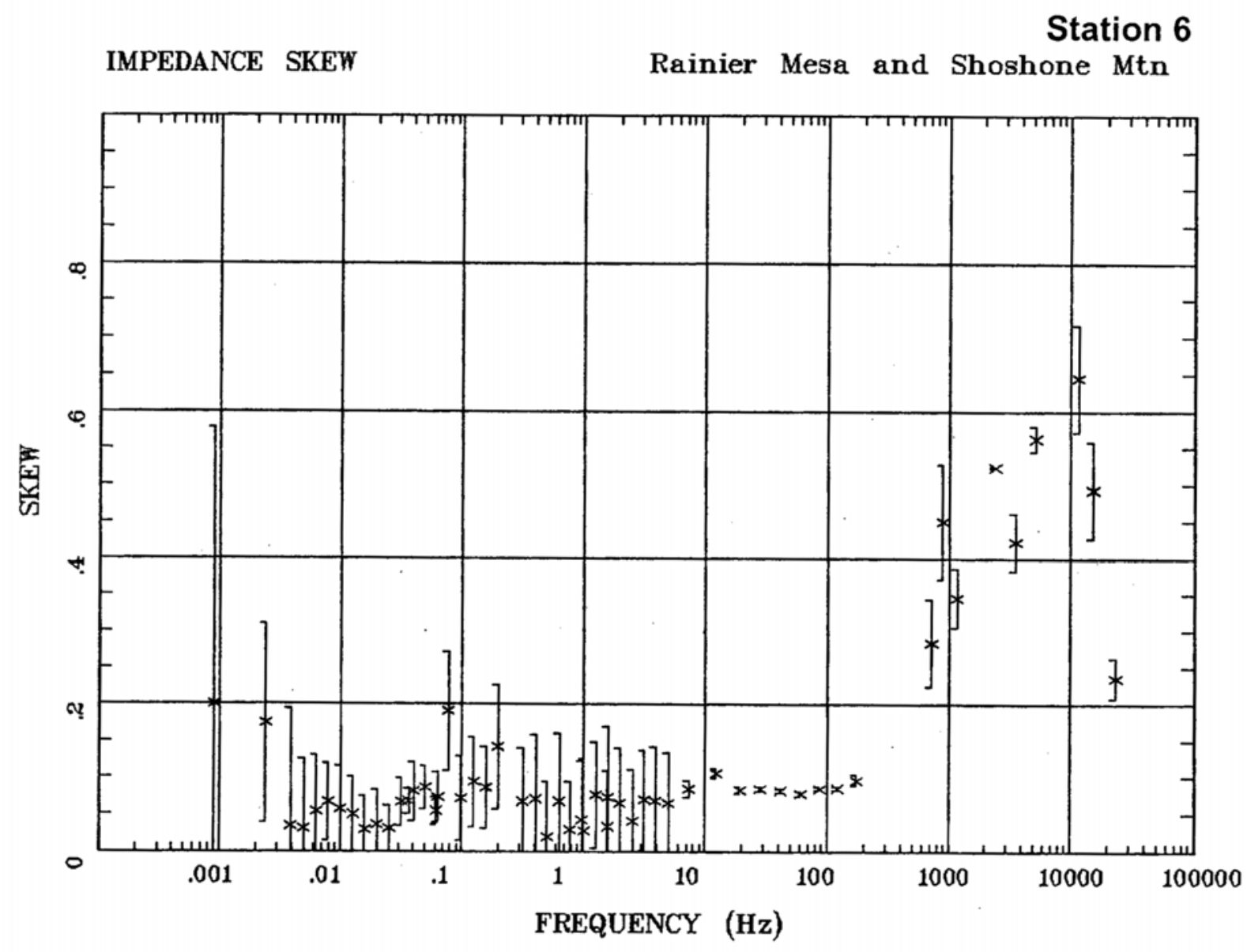

Client: DOE

Remote: none

Acquired: 04:4 May 05, 2005

Survey Co:USGS
Rotation:

Filename: rm06.avg

Channels: Ch1 Ch2 Ch3 Ch4 Ch5 Ch3 Ch4

Plotted: 11:31 Jan 17, 2006

< EMI - ElectroMagnetic Instruments > 


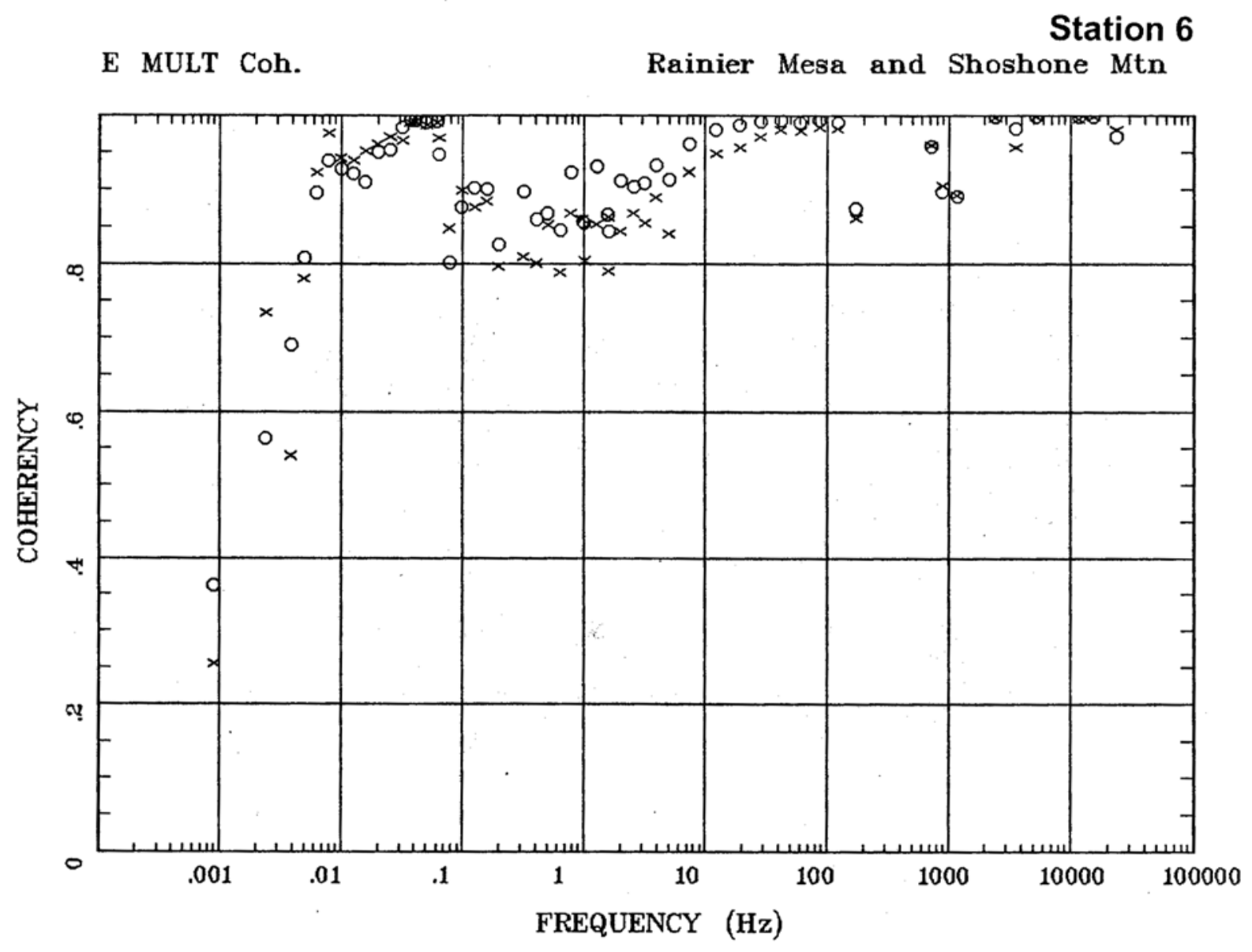

Client: DOE

Remote: none

Acquired: 04:4 May 05, 2005

Survey Co:USGS
Rotation:

Filename: rm06.avg

Channels: Ch1 Ch2 Ch3 Ch4 Ch5 Ch3 Ch4

Plotted: 11:31 Jan 17, 2006

< EMI - ElectroMagnetic Instruments > 
POLAR PLOTS

Rainier Mesa and Shoshone Station 6

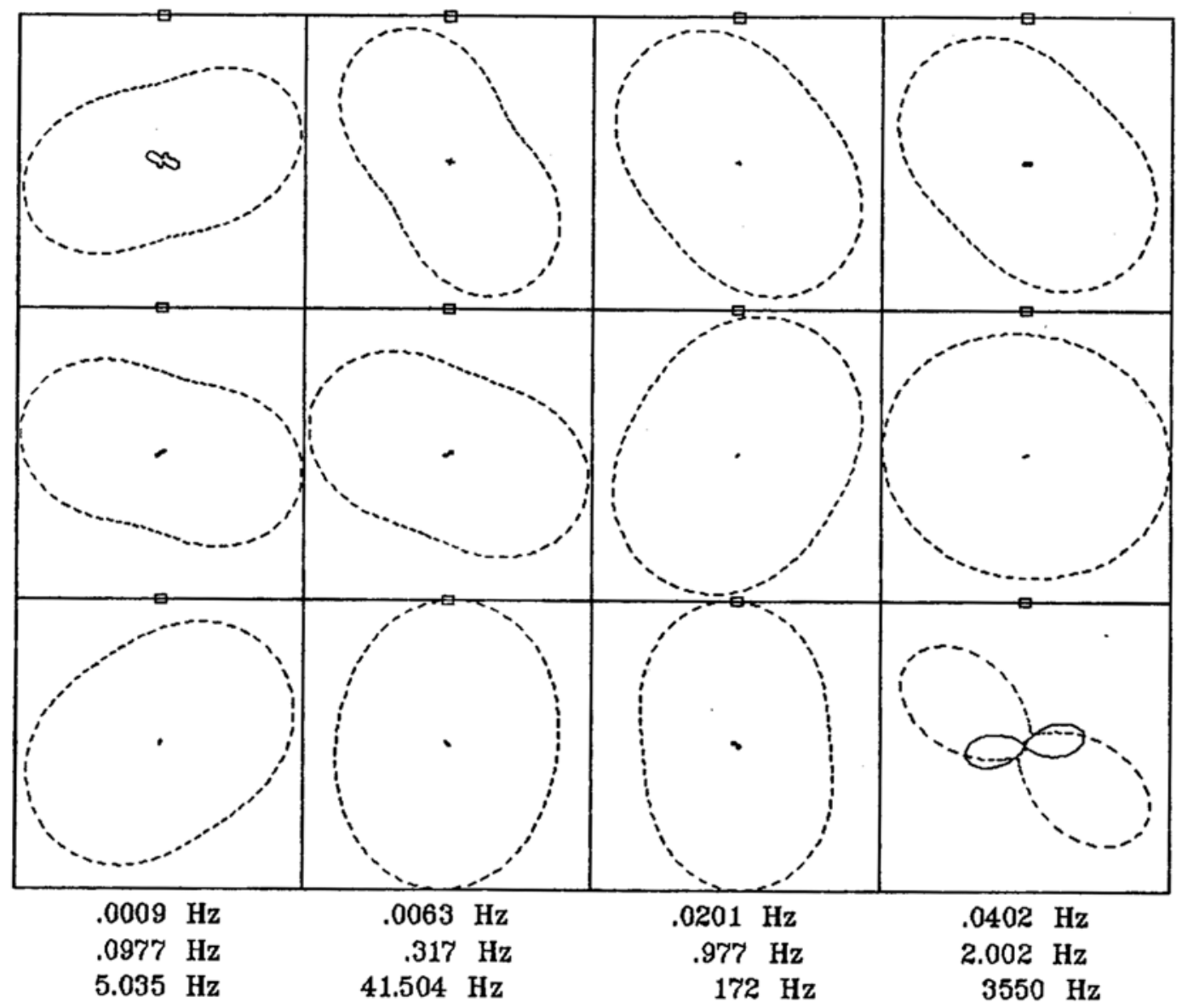

Client: DOE

Remote: none

Acquired: 04:4 May 05, 2005

Survey Co:USGS
Rotation:

Filename: rm06.avg

Channels: Ch1 Ch2 Ch3 Ch4 Ch5 Ch3 Ch4

Plotted: 11:31 Jan 17, 2006

< EMI - ElectroMagnetic Instruments > 


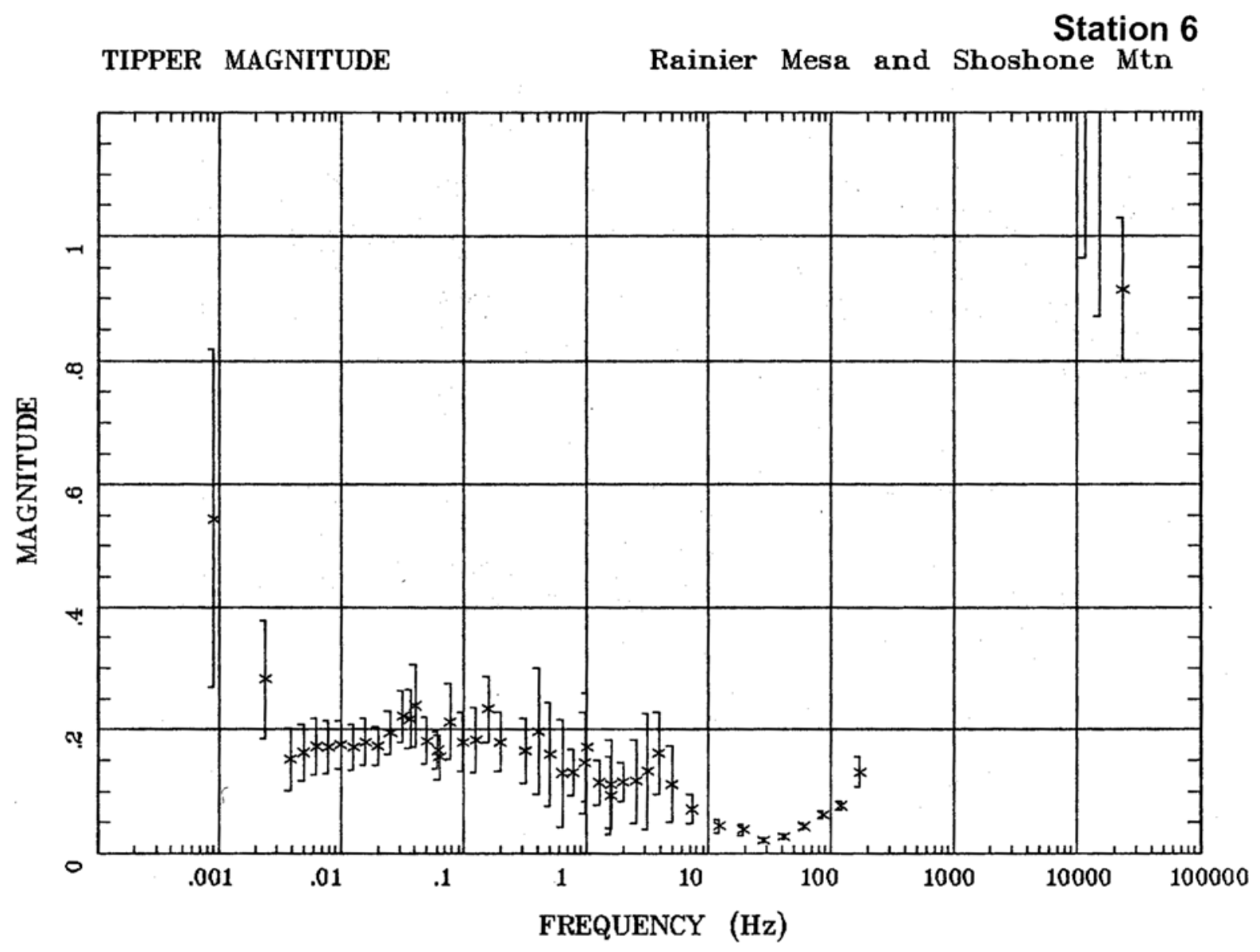

Client: DOE

Remote: none

Acquired: 04:4 May 05, 2005

Survey Co:USGS
Rotation:

Filename: rm06.avg

Channels: Ch1 Ch2 Ch3 Ch4 Ch5 Ch3 Ch4

Plotted: 11:31 Jan 17, 2006

< EMI - ElectroMagnetic Instruments > 


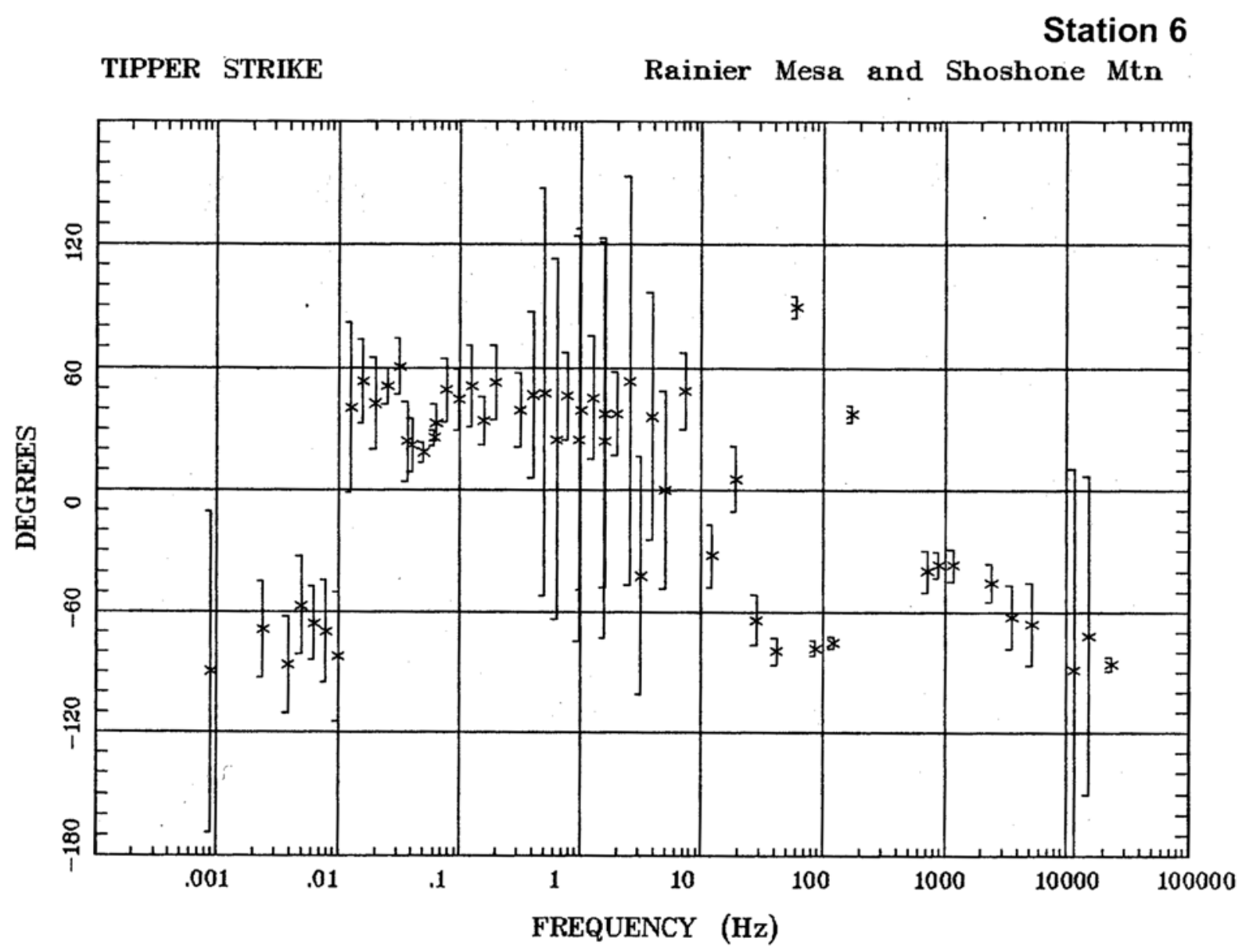

Client: DOE

Remote: none

Acquired: 04:4 May 05, 2005

Survey Co:USGS
Rotation:

Filename: rm06.avg

Channels: Ch1 Ch2 Ch3 Ch4 Ch5 Ch3 Ch4

Plotted: 11:31 Jan 17, 2006

< EMI - ElectroMagnetic Instruments > 


\section{Station 6}

HzHx.x Coh HzHy.o

Rainier Mesa and Shoshone Mtn

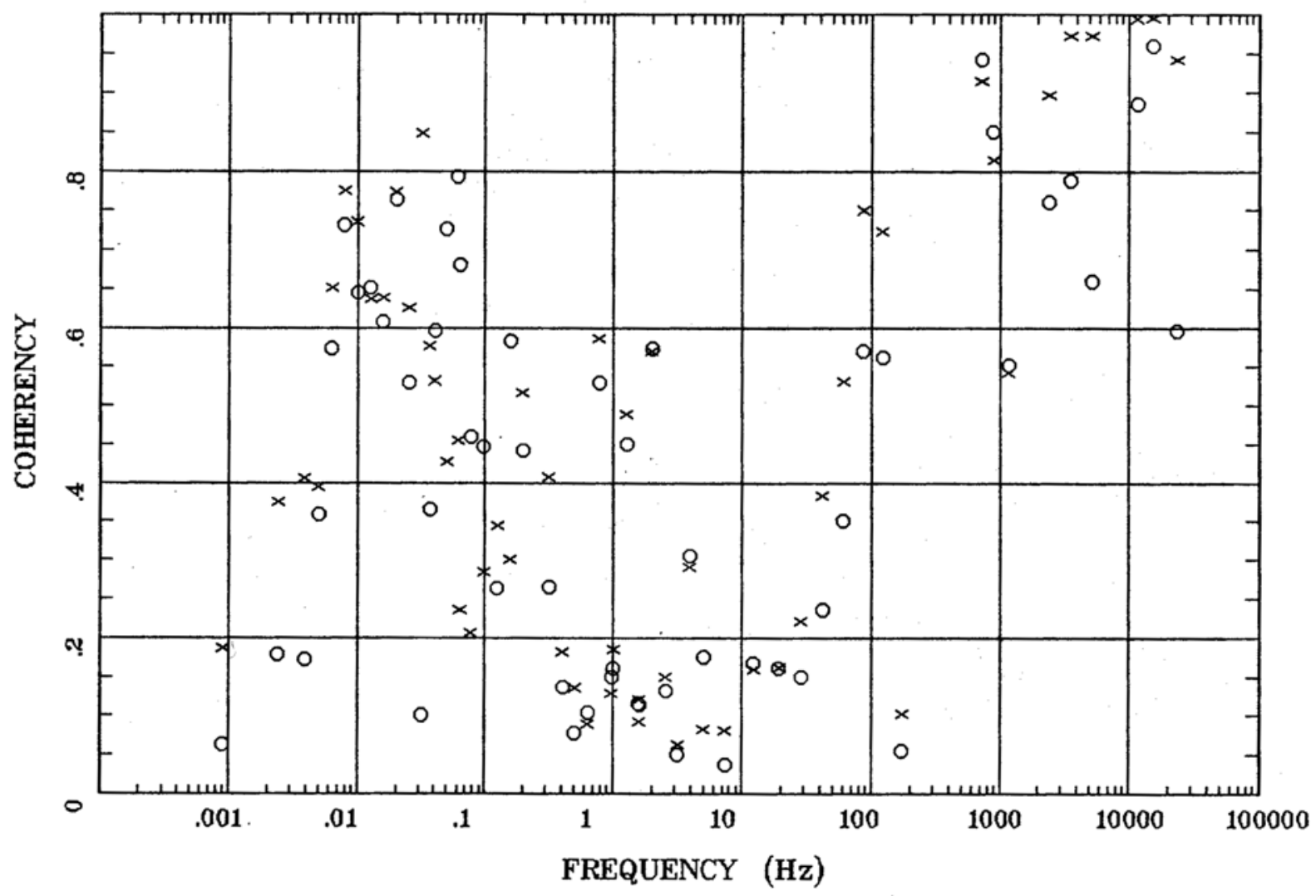

Client: $\mathrm{DOE}$

Remote: none

Acquired: 04:4 May 05, 2005

Survey Co:USGS
Rotation:

Filename: rm06.avg

Channels: Ch1 Ch2 Ch3 Ch4 Ch5 Ch3 Ch4

Plotted: 11:31 Jan 17, 2006

< EMI - ElectroMagnetic Instruments > 


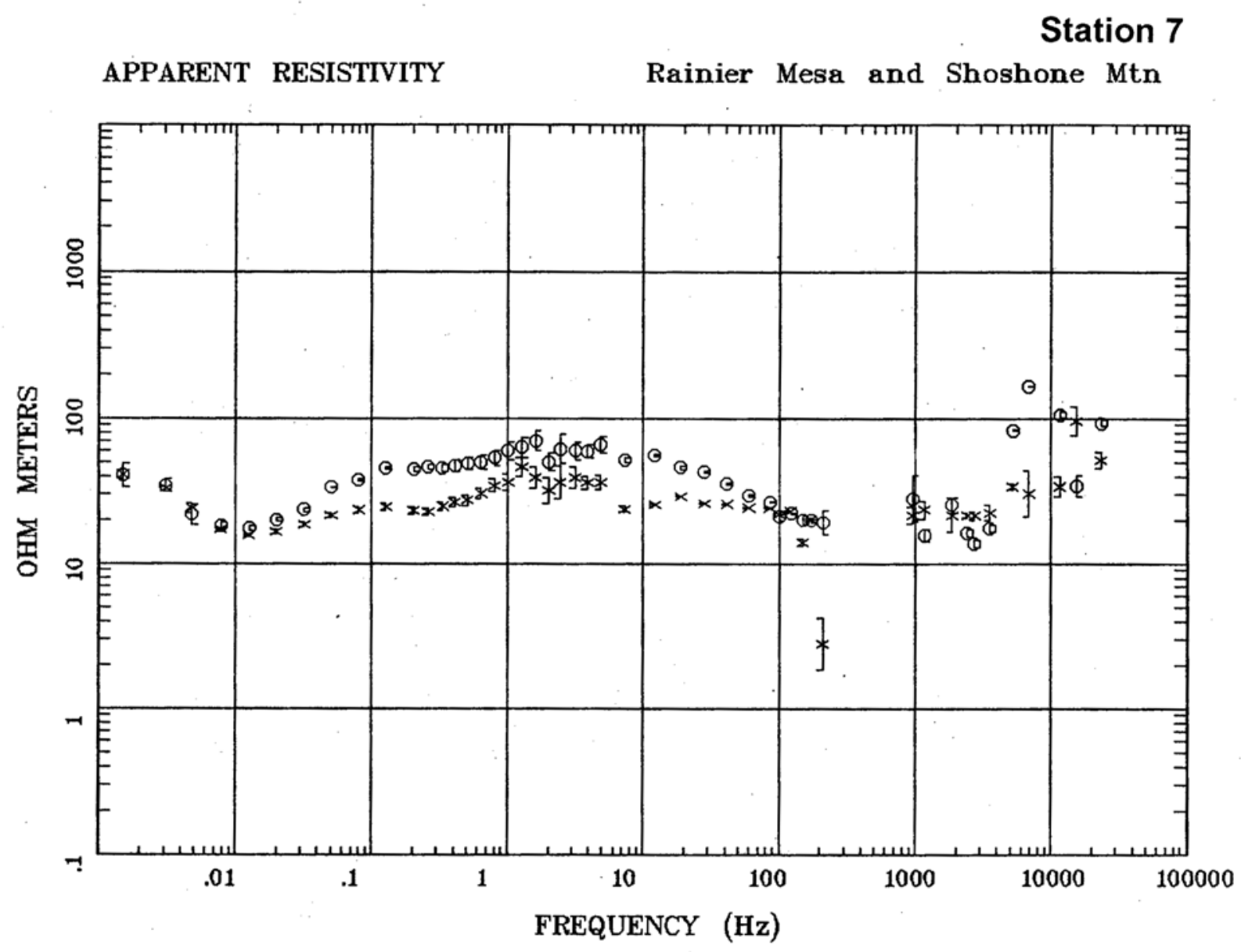

Client: DOE

Remote: none

Acquired: 22:0 May 11, 2005

Survey Co:USGS
Rotation:

Filename: rm07.avg

Channels: Ch1 Ch2 Ch3 Ch4 Ch5 Ch3 Ch4

Plotted: 08:59 Jan 18, 2006

< EMI - ElectroMagnetic Instruments > 


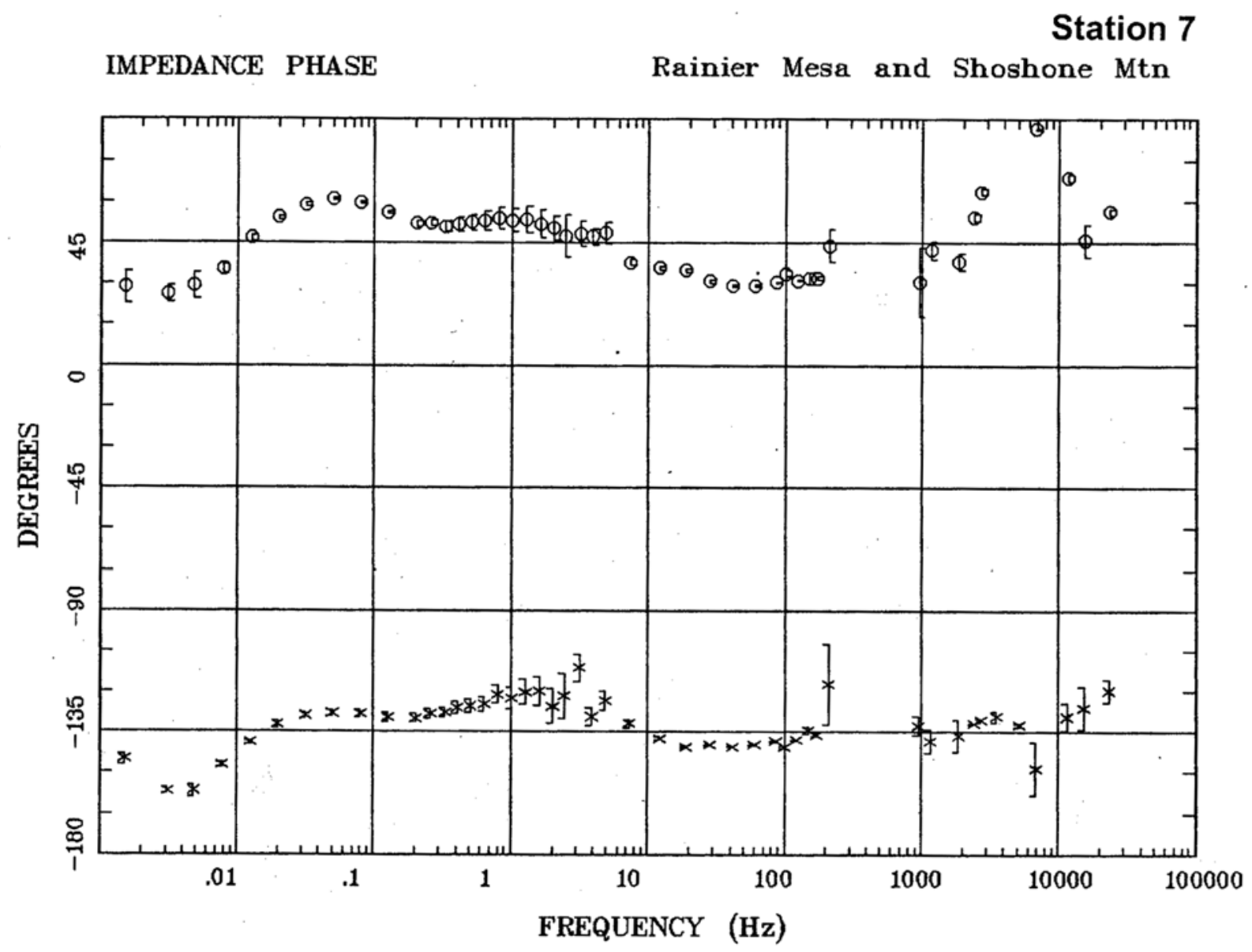

Client: $\mathrm{DOE}$

Remote: none

Acquired: 22:0 May 11, 2005

Survey Co:USGS
Rotation:

Filename: rm07.avg

Channels: Ch1 Ch2 Ch3 Ch4 Ch5 Ch3 Ch4

Plotted: 08:59 Jan 18, 2006

< EMI - ElectroMagnetic Instruments > 


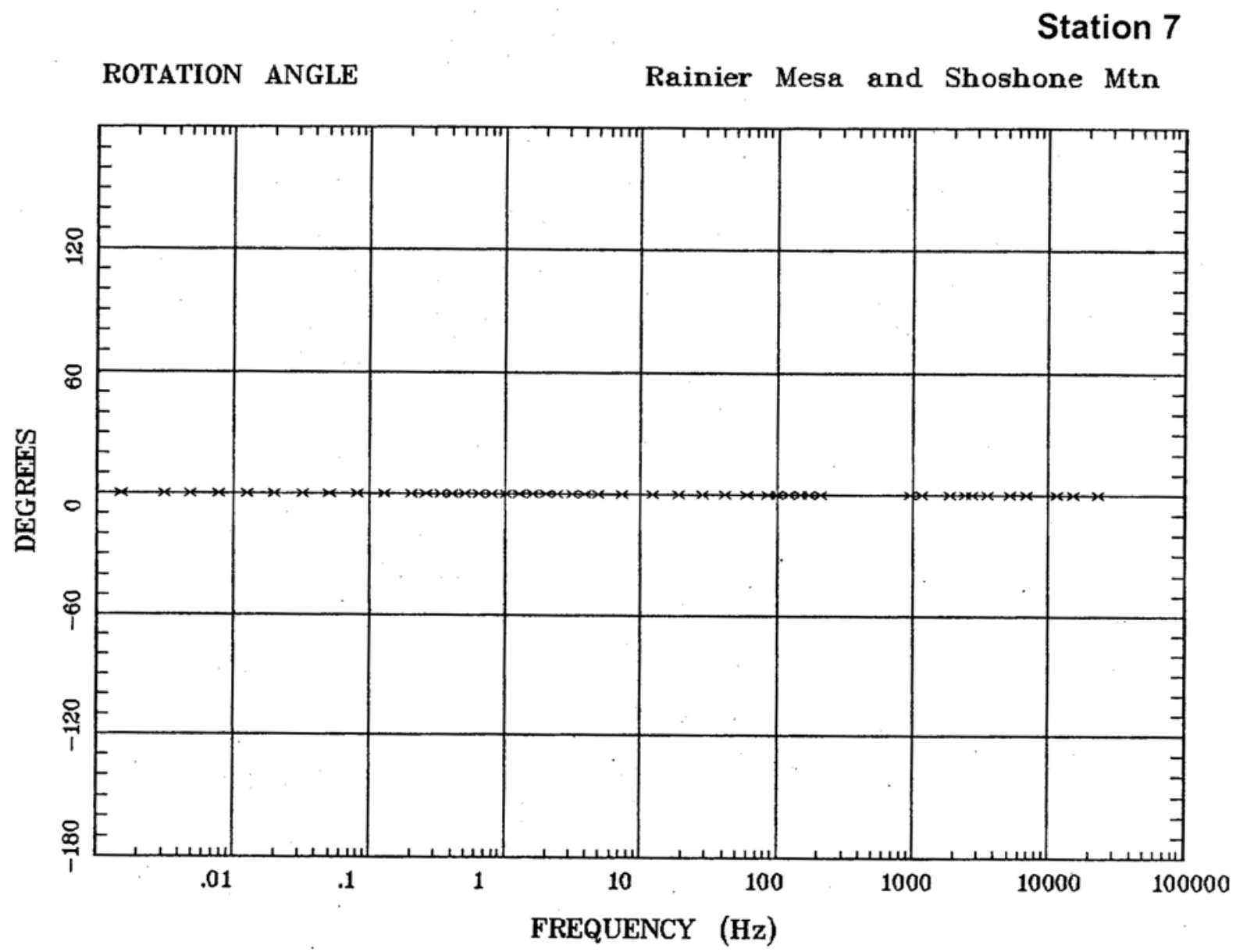

Client: DOE

Remote: none

Acquired: 22:0 May 11, 2005

Survey Co:USGS

Rotation:

Filename: rm07.avg

Channels: Ch1 Ch2 Ch3 Ch4 Ch5 Ch3 Ch4

Plotted: 08:59 Jan 18, 2006

< EMI - ElectroMagnetic Instruments > 


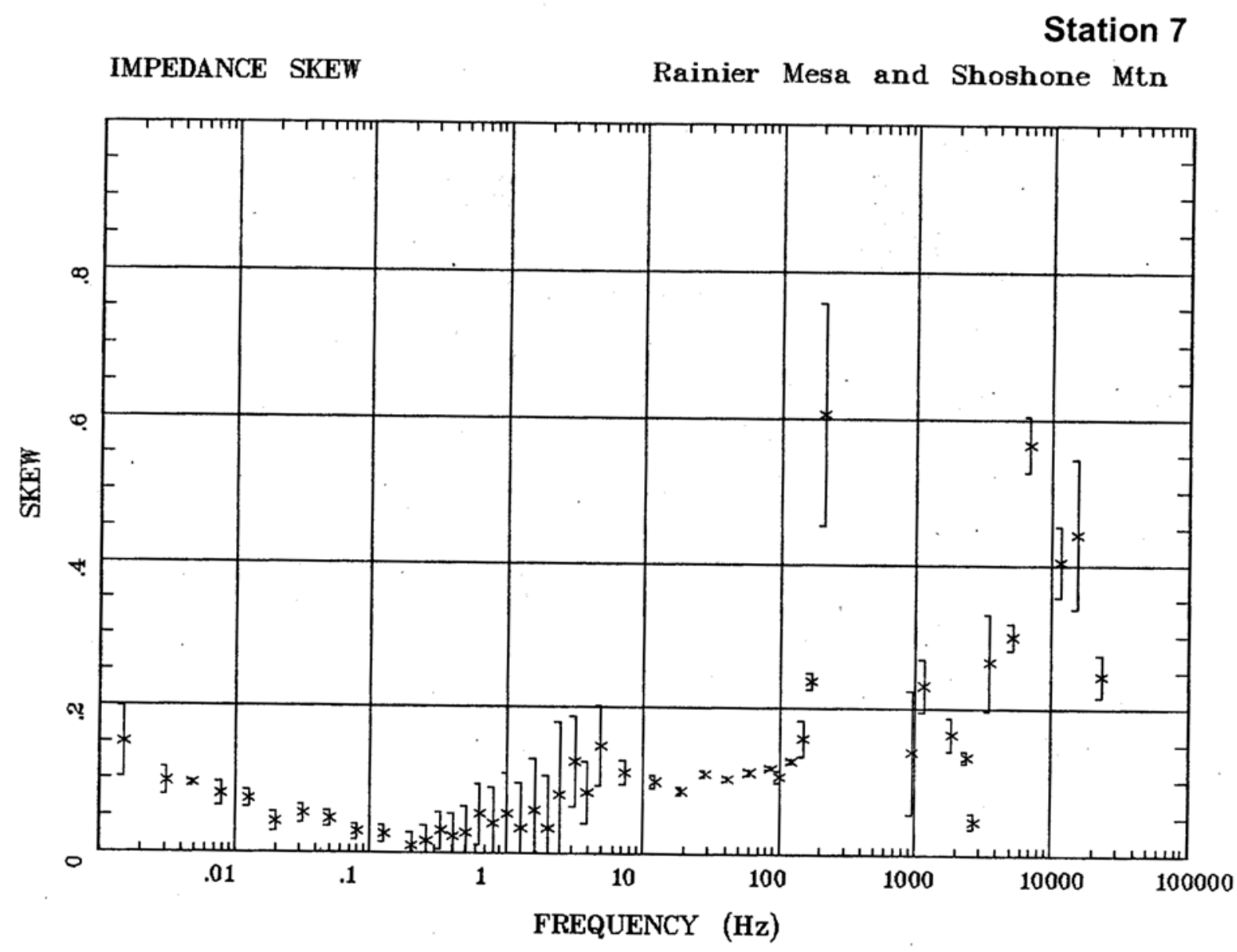

Client: DOE

Remote: none

Acquired: 22:0 May 11, 2005

Survey Co:USGS
Rotation:

Filename: rm07.avg

Channels: Ch1 Ch2 Ch3 Ch4 Ch5 Ch3 Ch4

Plotted: 08:59 Jan 18, 2006

< EMI - ElectroMagnetic Instruments > 


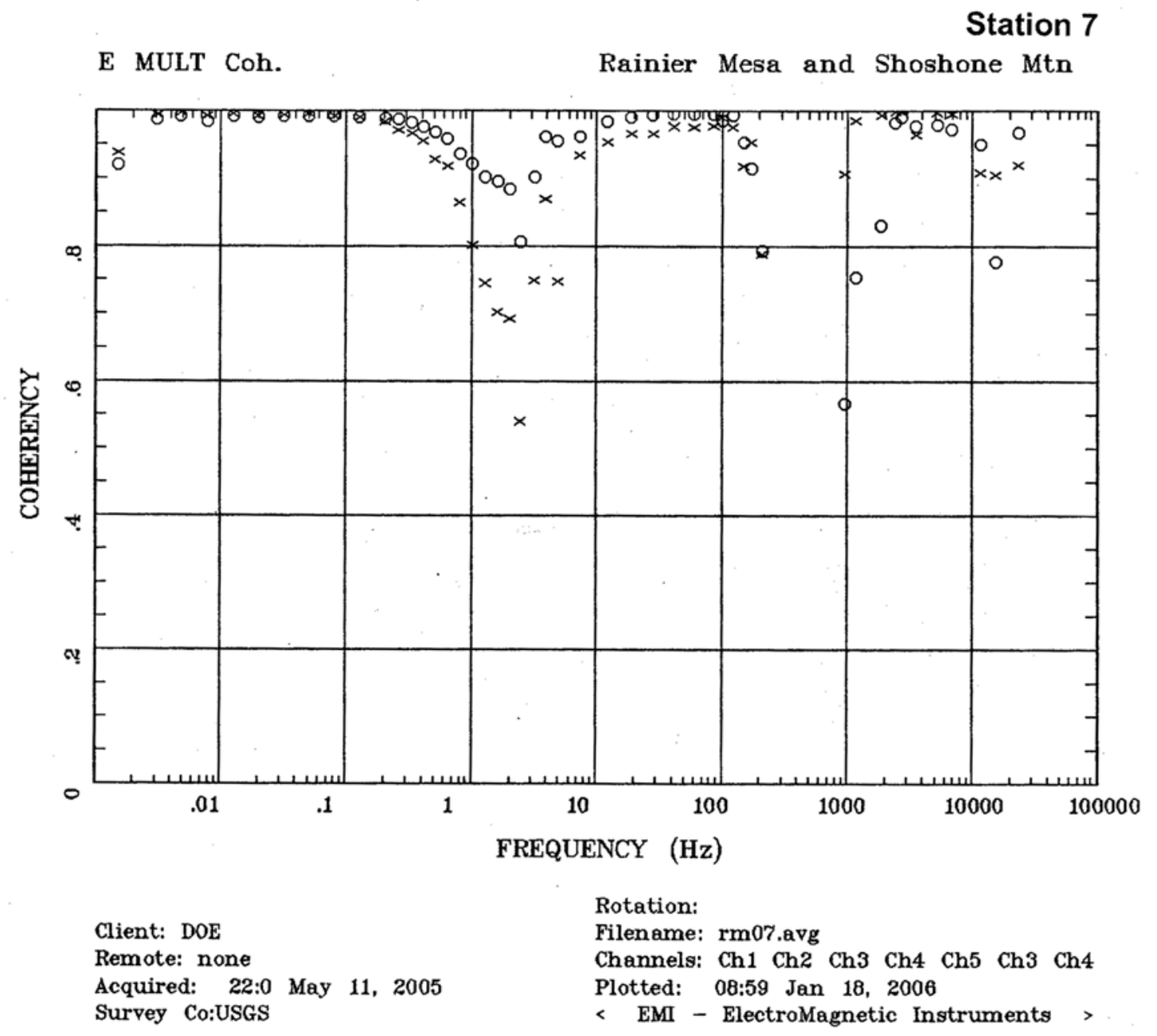




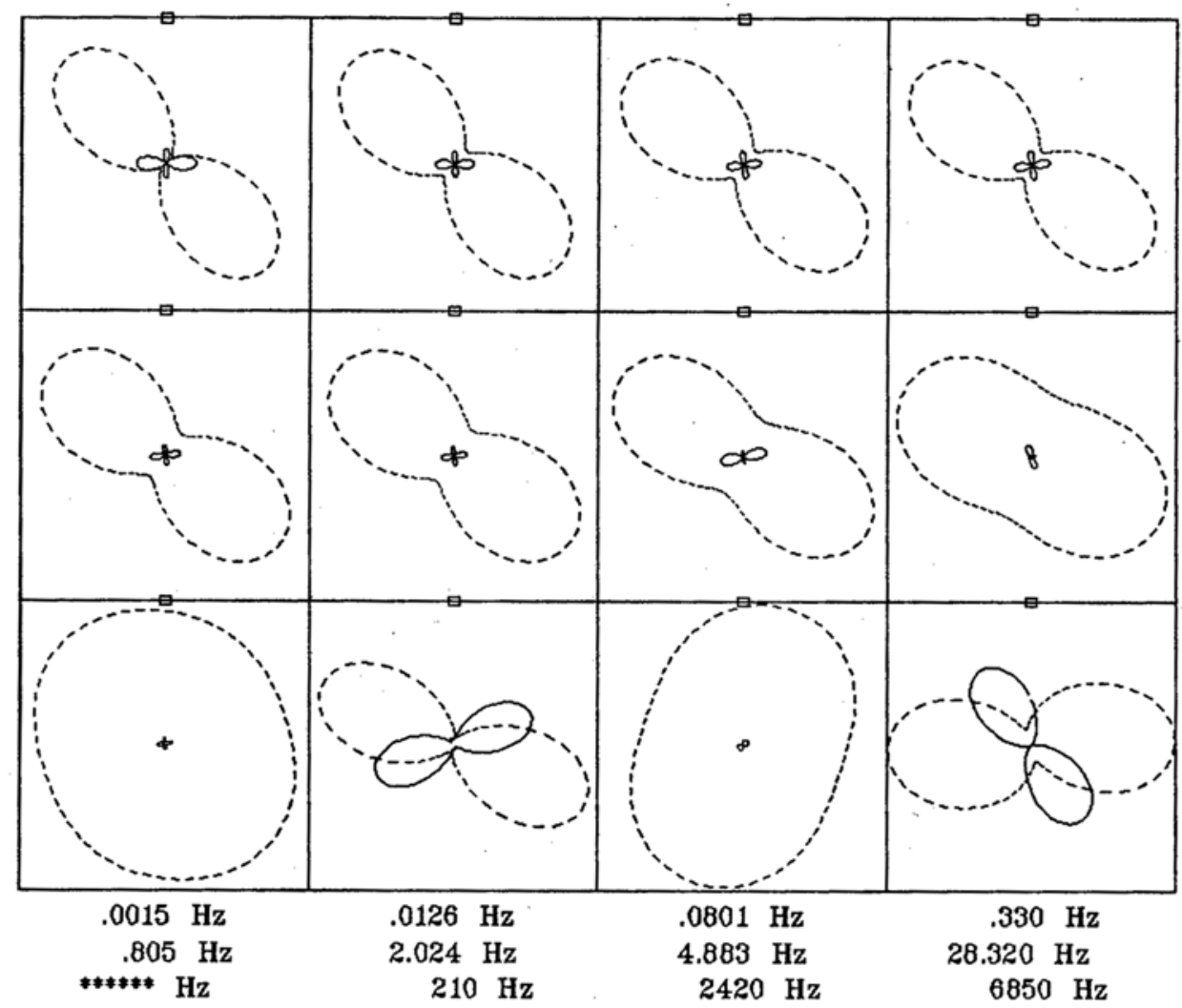

Client: DOE

Remote: none

Acquired: 22:0 May 11, 2005

Survey Co:USGS
Rotation:

Filename: rm07.avg

Channels: Ch1 Ch2 Ch3 Ch4 Ch5 Ch3 Ch4

Plotted: 08:59 Jan 18, 2006

< EMI - ElectroMagnetic Instruments > 


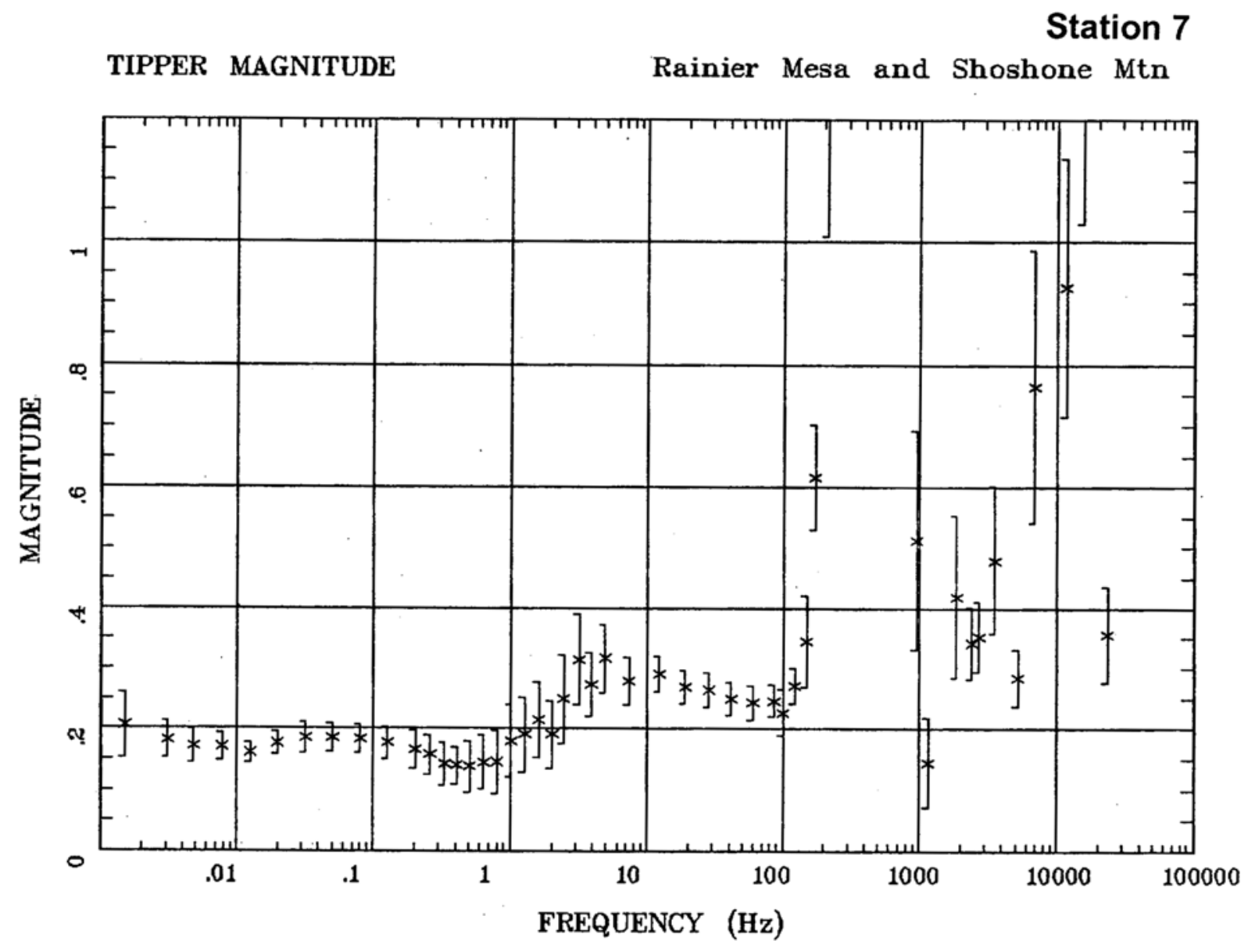

Client: DOE

Remote: none

Acquired: 22:0 May 11, 2005

Survey Co:USGS
Rotation:

Filename: rm07.avg

Channels: Ch1 Ch2 Ch3 Ch4 Ch5 Ch3 Ch4

Plotted: 08:59 Jan 18, 2006

< EMI - ElectroMagnetic Instruments > 
Station 7

TIPPER STRIKE

Rainier Mesa and Shoshone Mtn

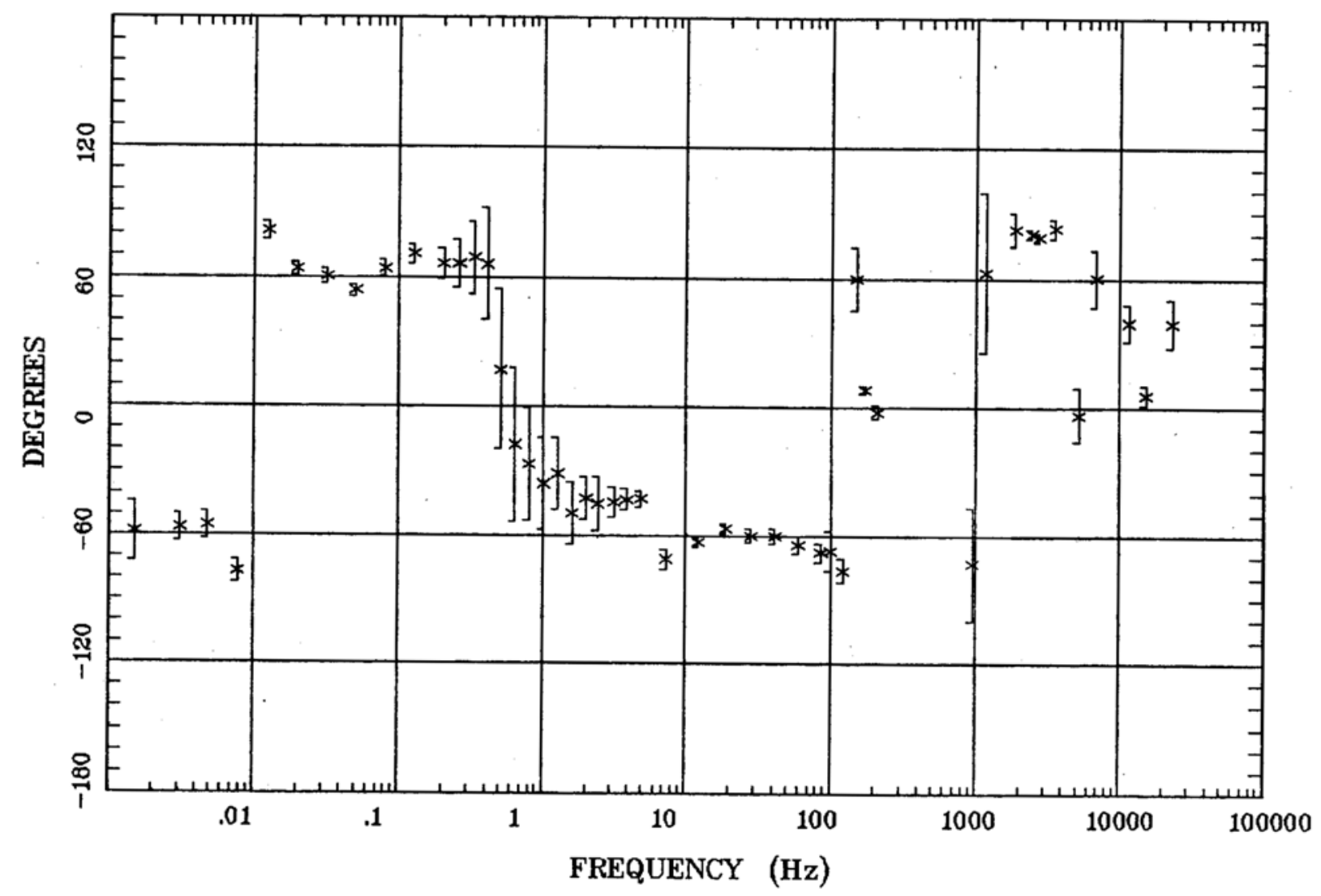

Client: DOE

Remote: none

Acquired: 22:0 May 11, 2005

Survey Co:USGS
Rotation:

Filename: rm07.avg

Channels: Ch1 Ch2 Ch3 Ch4 Ch5 Ch3 Ch4

Plotted: 08:59 Jan 18, 2006

< EMI - ElectroMagnetic Instruments > 
Station 7

HzHx.x Coh HzHy.o

Rainier Mesa and Shoshone Mtn

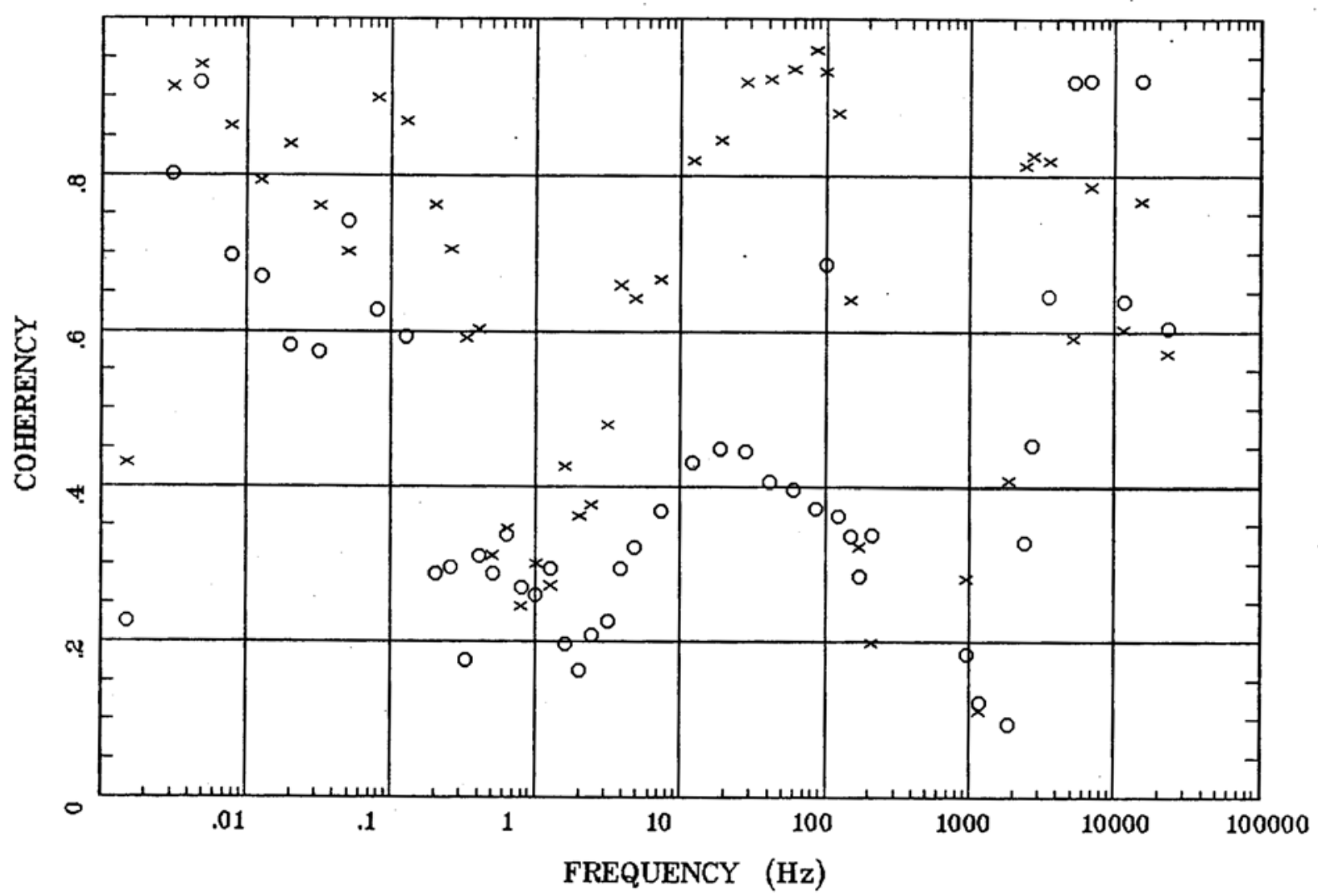

Client: DOE

Remote: none

Acquired: 22:0 May 11, 2005

Survey Co:USGS
Rotation:

Filename: rm07.avg

Channels: Ch1 Ch2 Ch3 Ch4 Ch5 Ch3 Ch4

Plotted: 08:59 Jan 18, 2006

< EMI - ElectroMagnetic Instruments > 


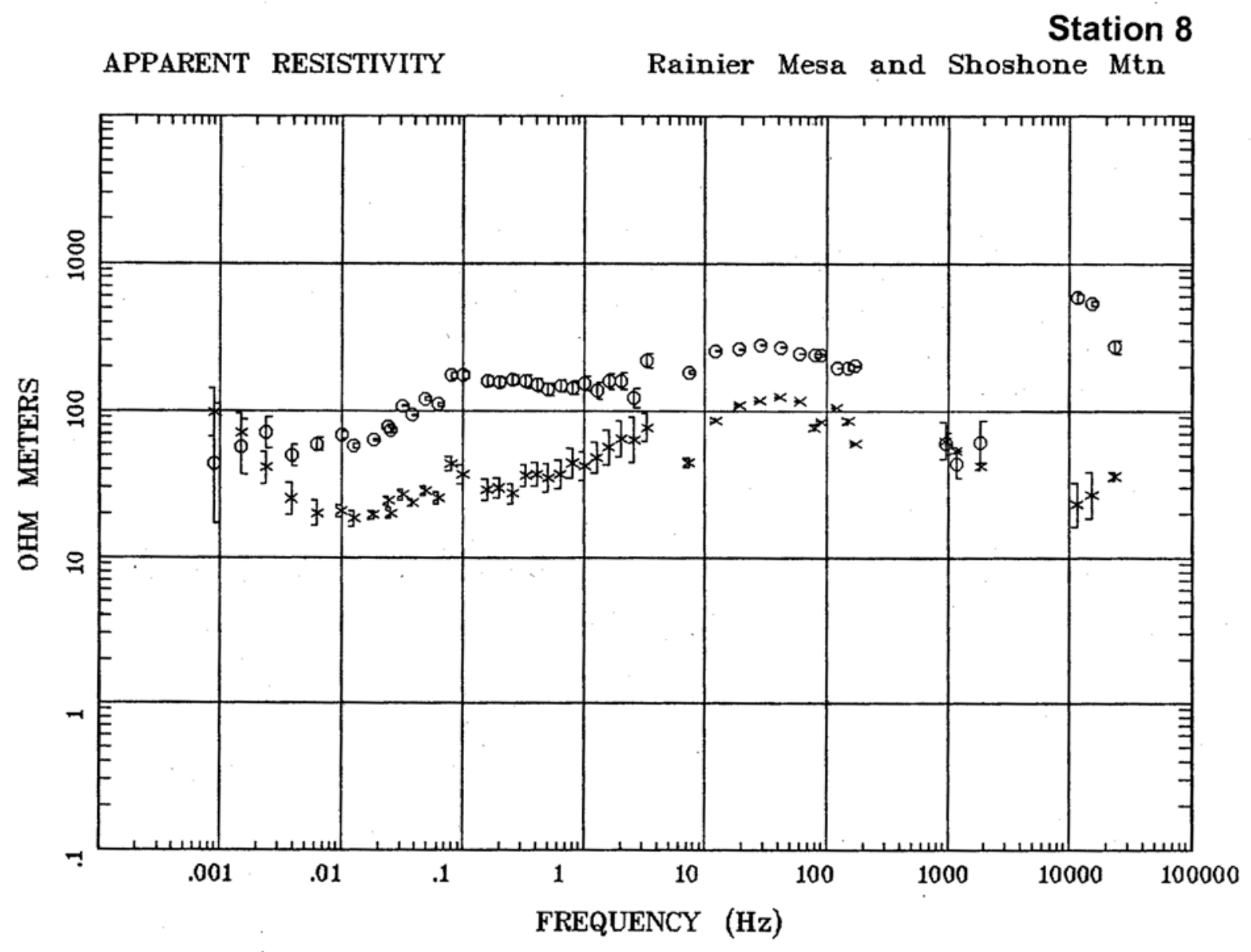

Client: DOE

Remote: none

Acquired: 01:3 May 08, 2005

Survey Co:USGS
Rotation:

Filename: rm08.avg

Channels: Ch1 Ch2 Ch3 Ch4 Ch5 Ch3 Ch4

Plotted: 12:52 Jan 17, 2006

< EMI - ElectroMagnetic Instruments > 


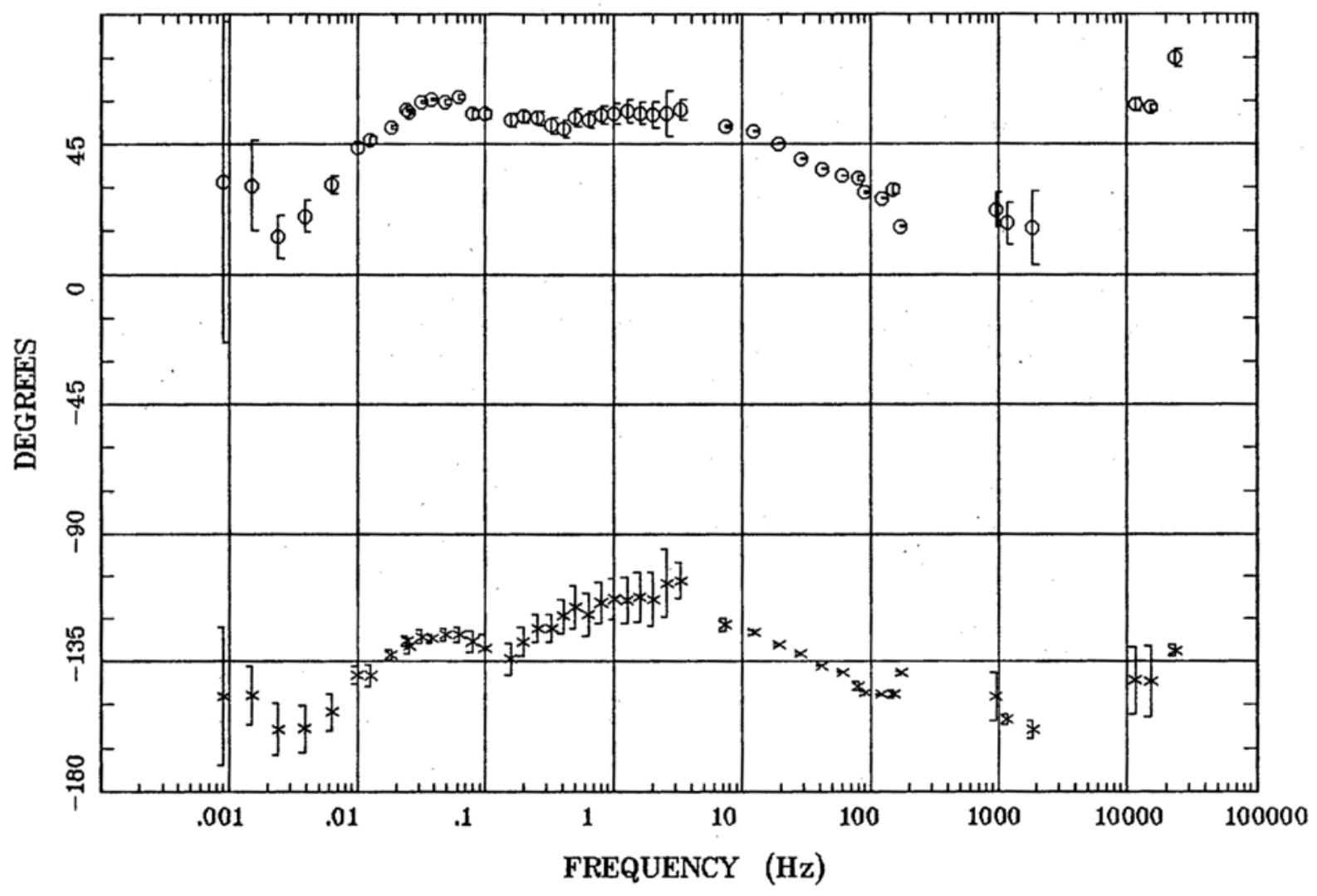

Client: $\mathrm{DOE}$

Remote: none

Acquired: 01:3 May 08, 2005

Survey Co:USGS
Rotation:

Filename: rm08.avg

Channels: Ch1 Ch2 Ch3 Ch4 Ch5 Ch3 Ch4

Plotted: 12:52 Jan 17, 2006

< EMI - ElectroMagnetic Instruments > 


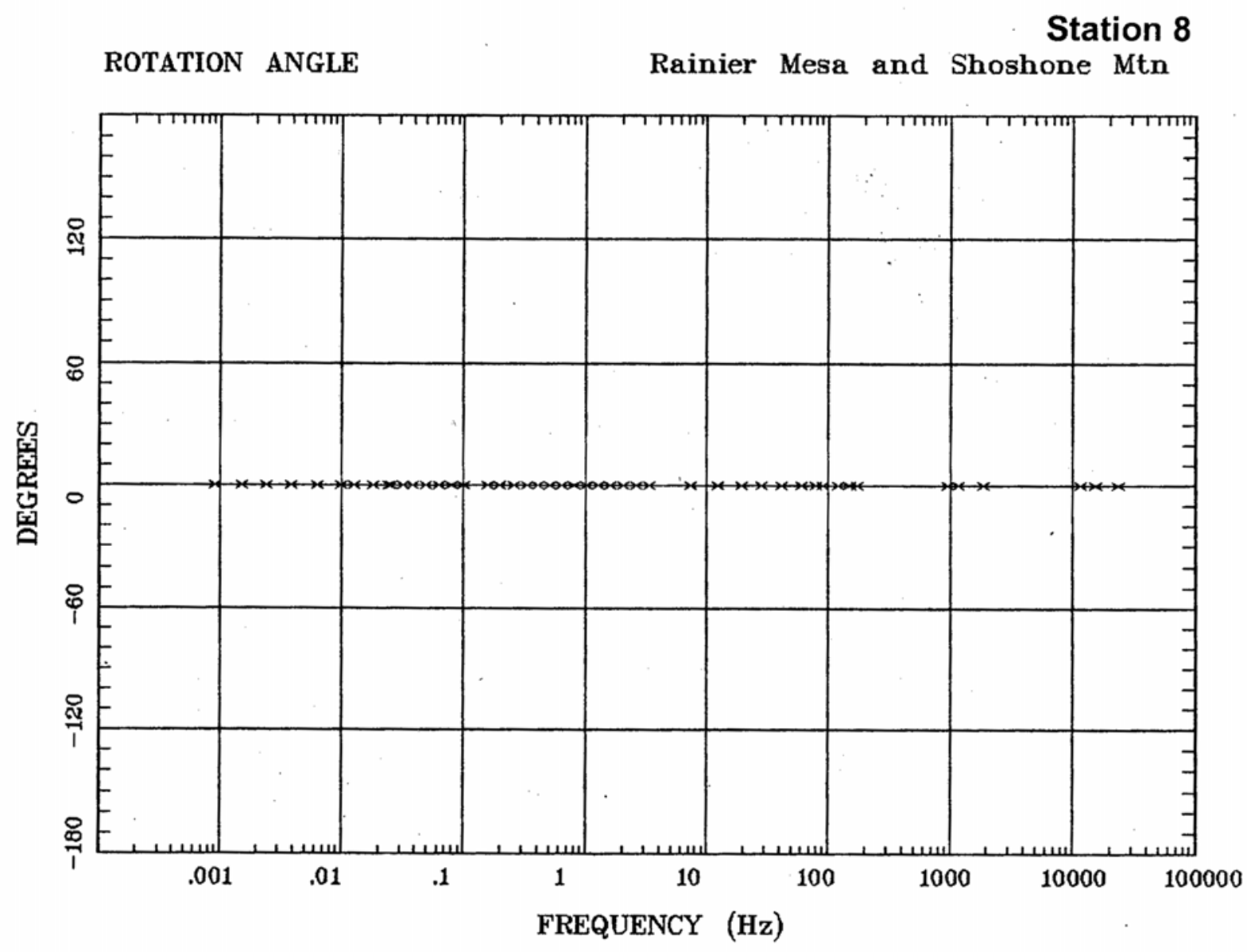

Client: DOE

Remote: none

Acquired: 01:3 May 08, 2005

Survey Co:USGS
Rotation:

Filename: rm08.avg

Channels: Ch1 Ch2 Ch3 Ch4 Ch5 Ch3 Ch4

Plotted: 12:52 Jan 17, 2006

< EMI - ElectroMagnetic Instruments > 


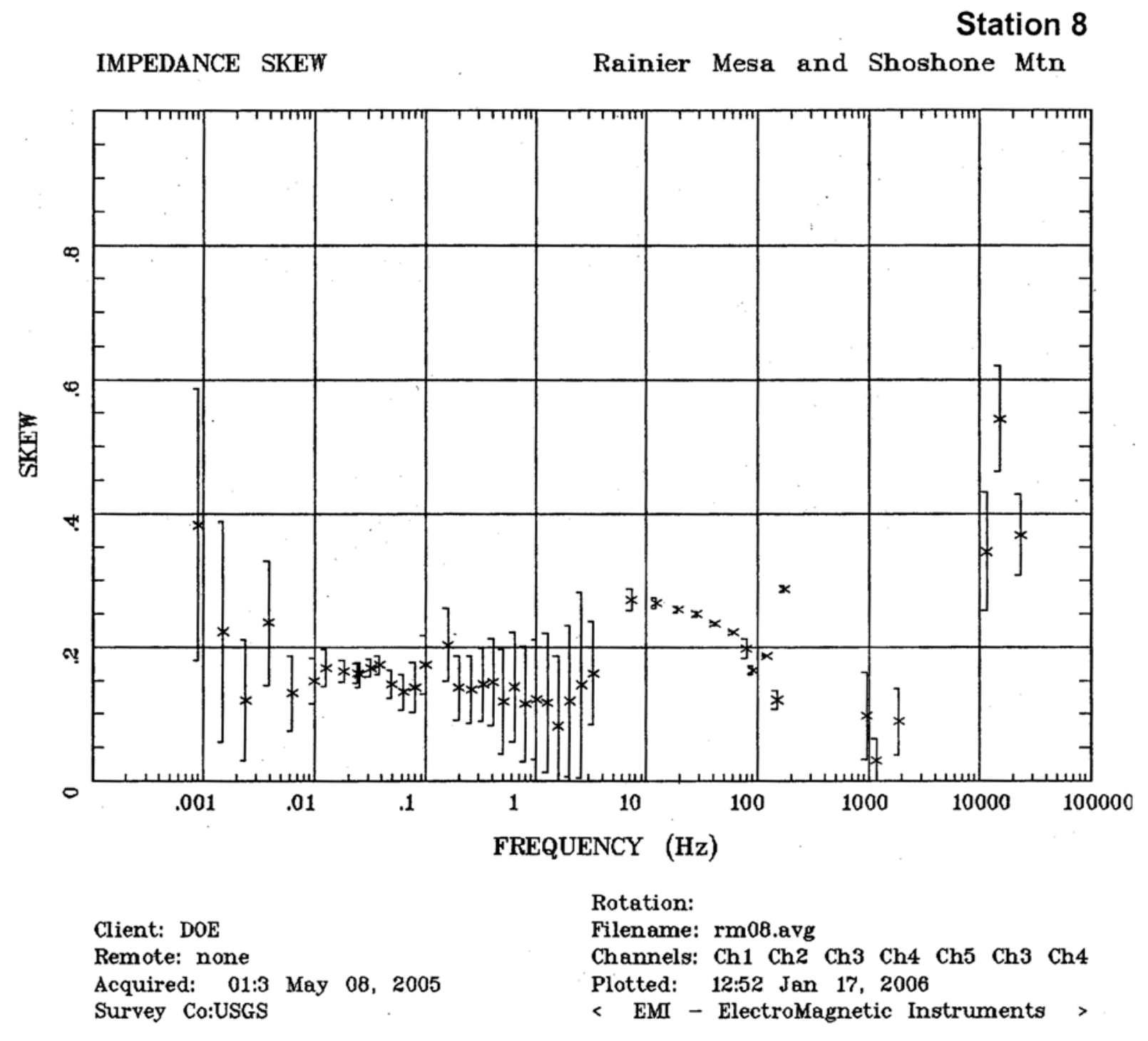




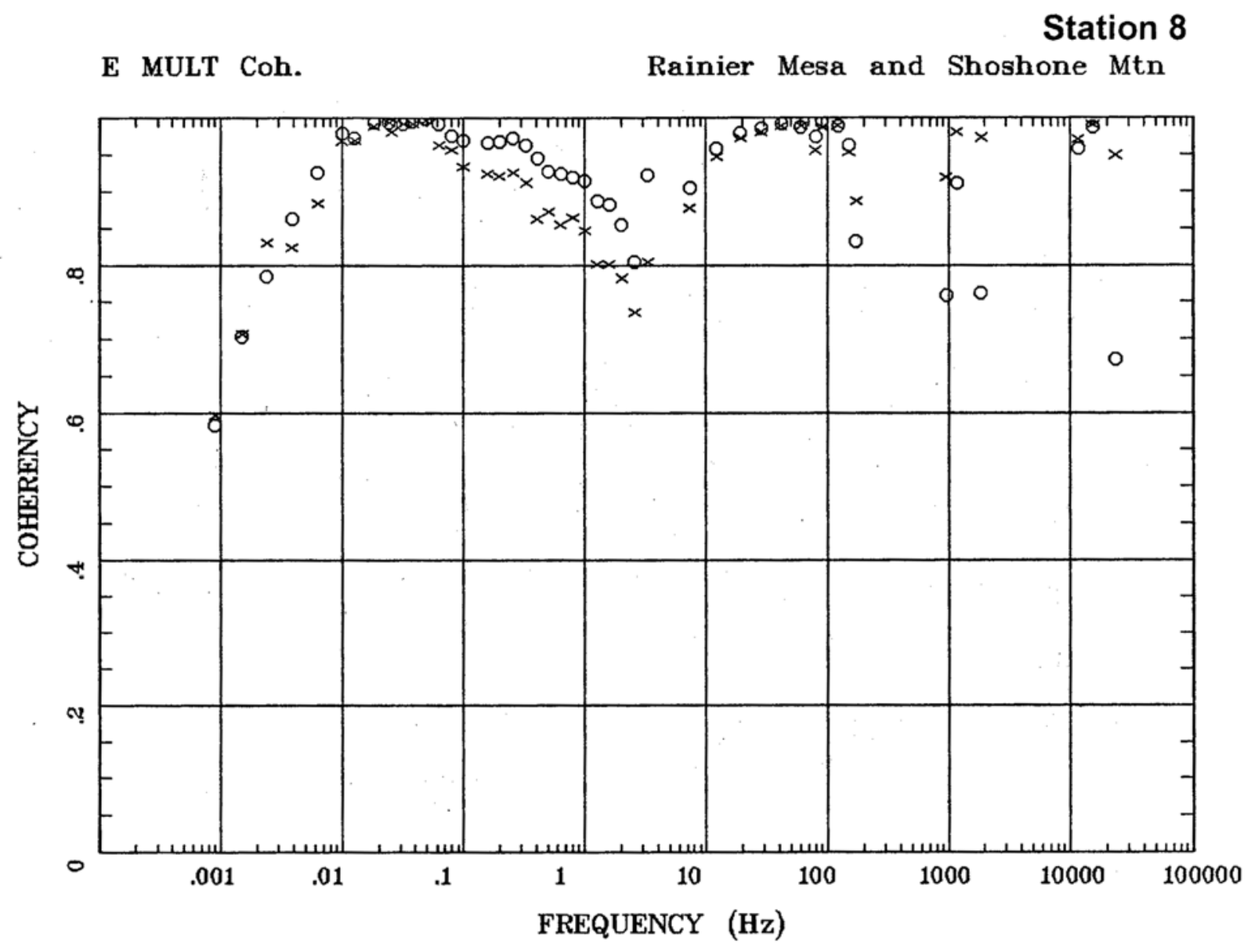

Client: DOE

Remote: none

Acquired: 01:3 May 08, 2005

Survey Co:USGS
Rotation:

Filename: rm08.avg

Channels: Ch1 Ch2 Ch3 Ch4 Ch5 Ch3 Ch4

Plotted: 12:52 Jan 17, 2006

< EMI - ElectroMagnetic Instruments > 


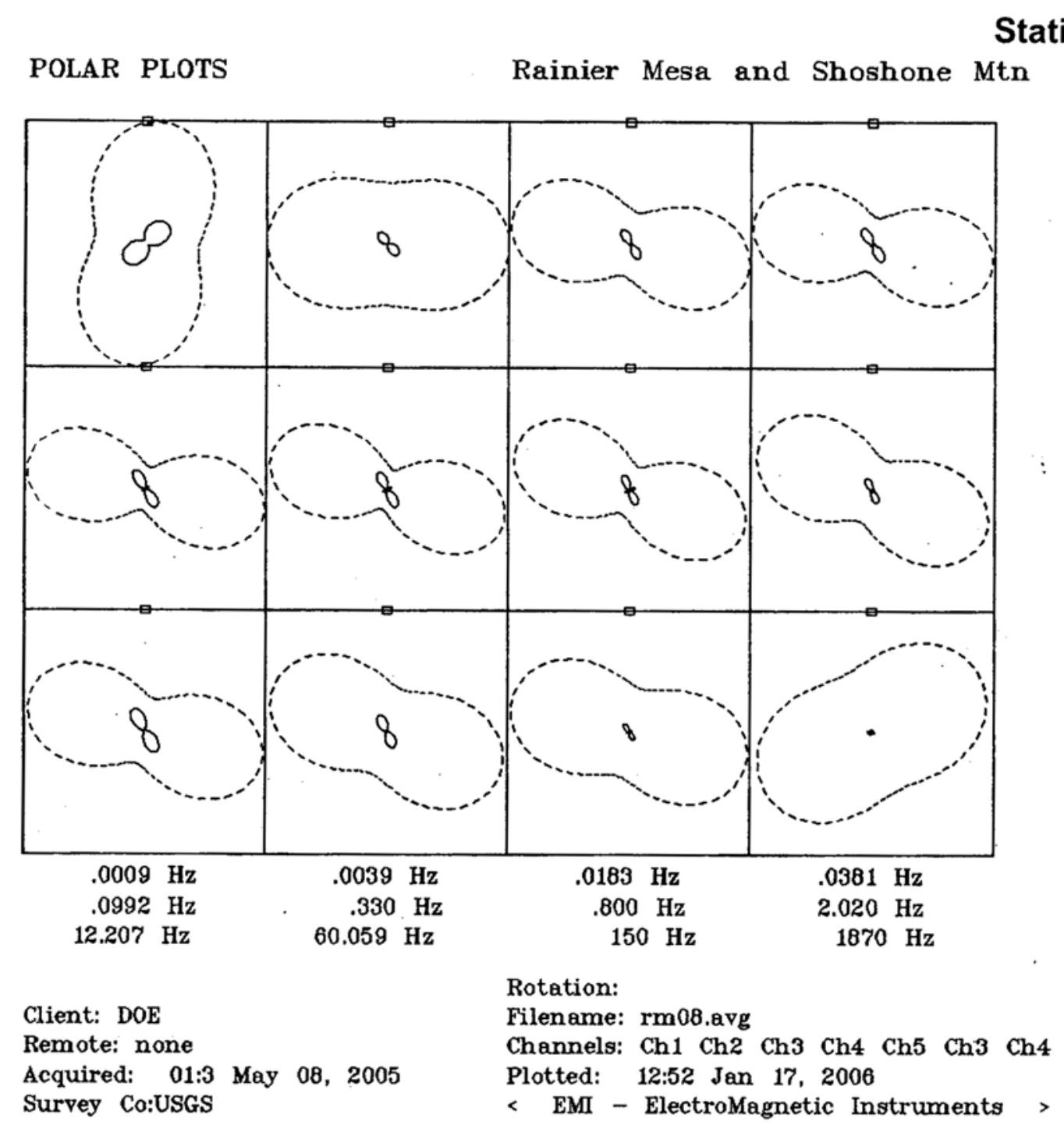




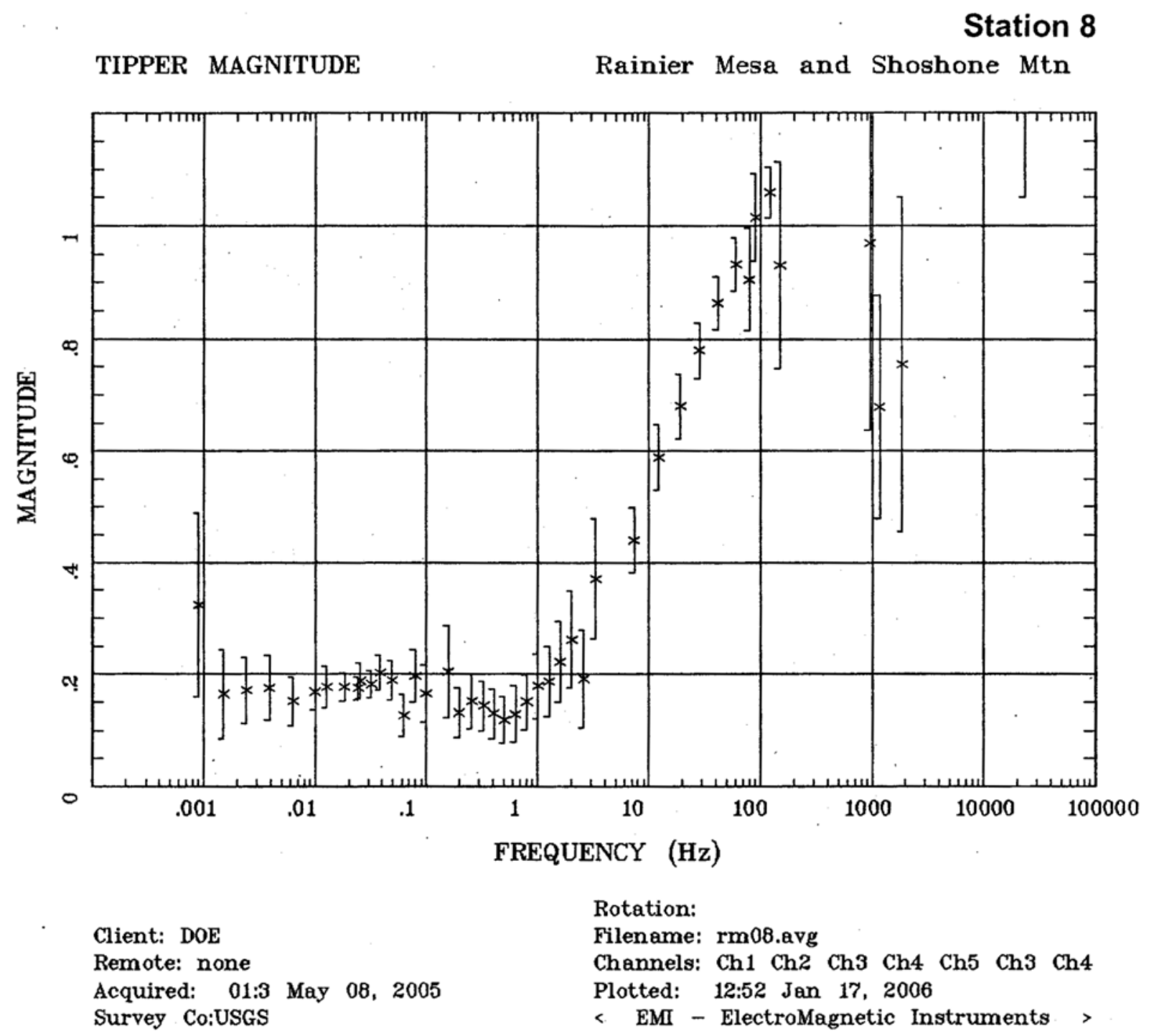




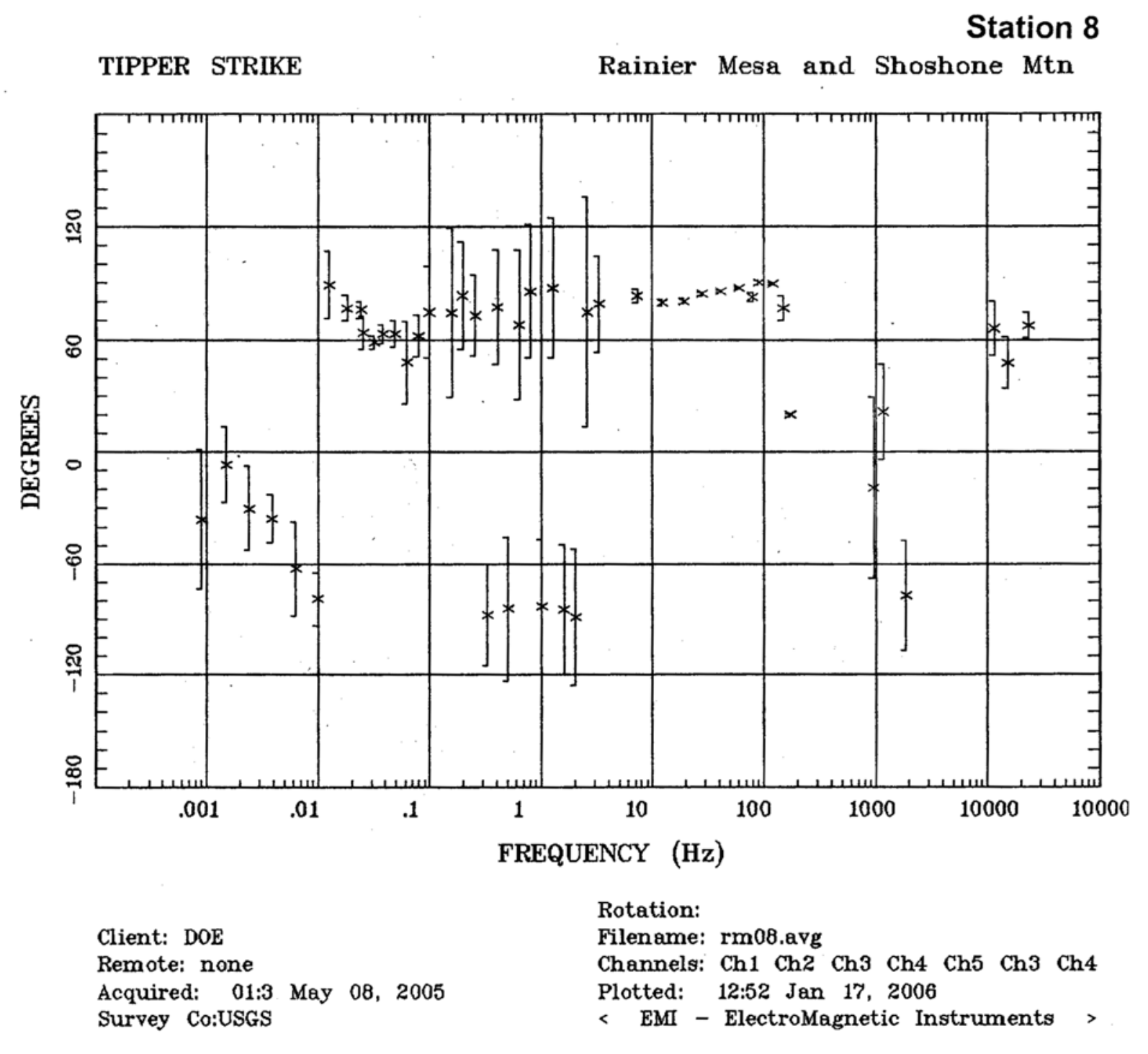




\section{HzHx.x Coh HzHy.o}

Station 8

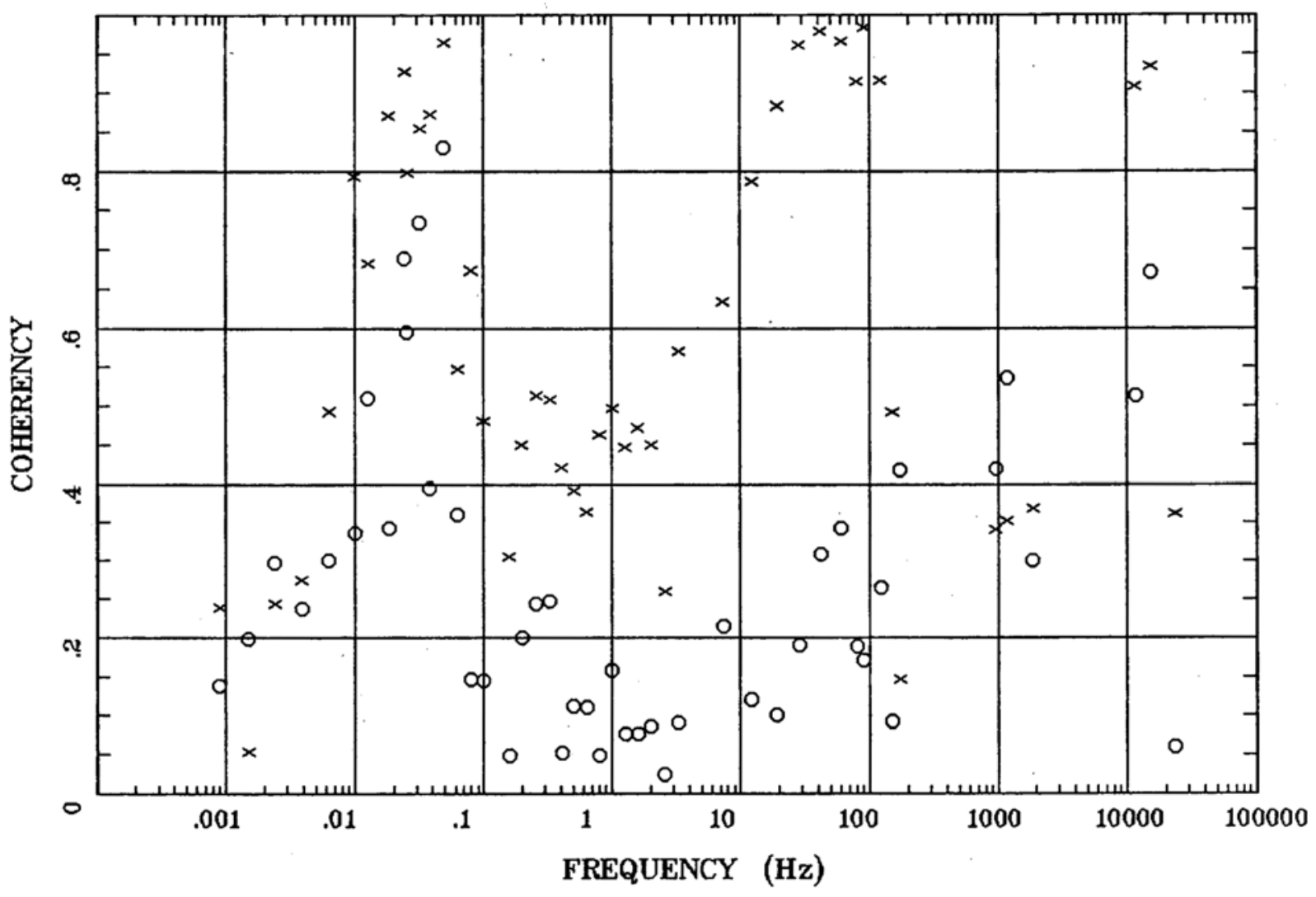

Client: DOE

Remote: none

Acquired: 01:3 May 08, 2005

Survey Co:USGS
Rainier Mesa and Shoshone Mtn

Rotation:

Filename: rm08.avg

Channels: Ch1 Ch2 Ch3 Ch4 Ch5 Ch3 Ch4

Plotted: 12:52 Jan 17, 2006

< EMI - ElectroMagnetic Instruments > 
Station 9

APPARENT RESISTIVITY

Rainier Mesa and Shoshone Mtn

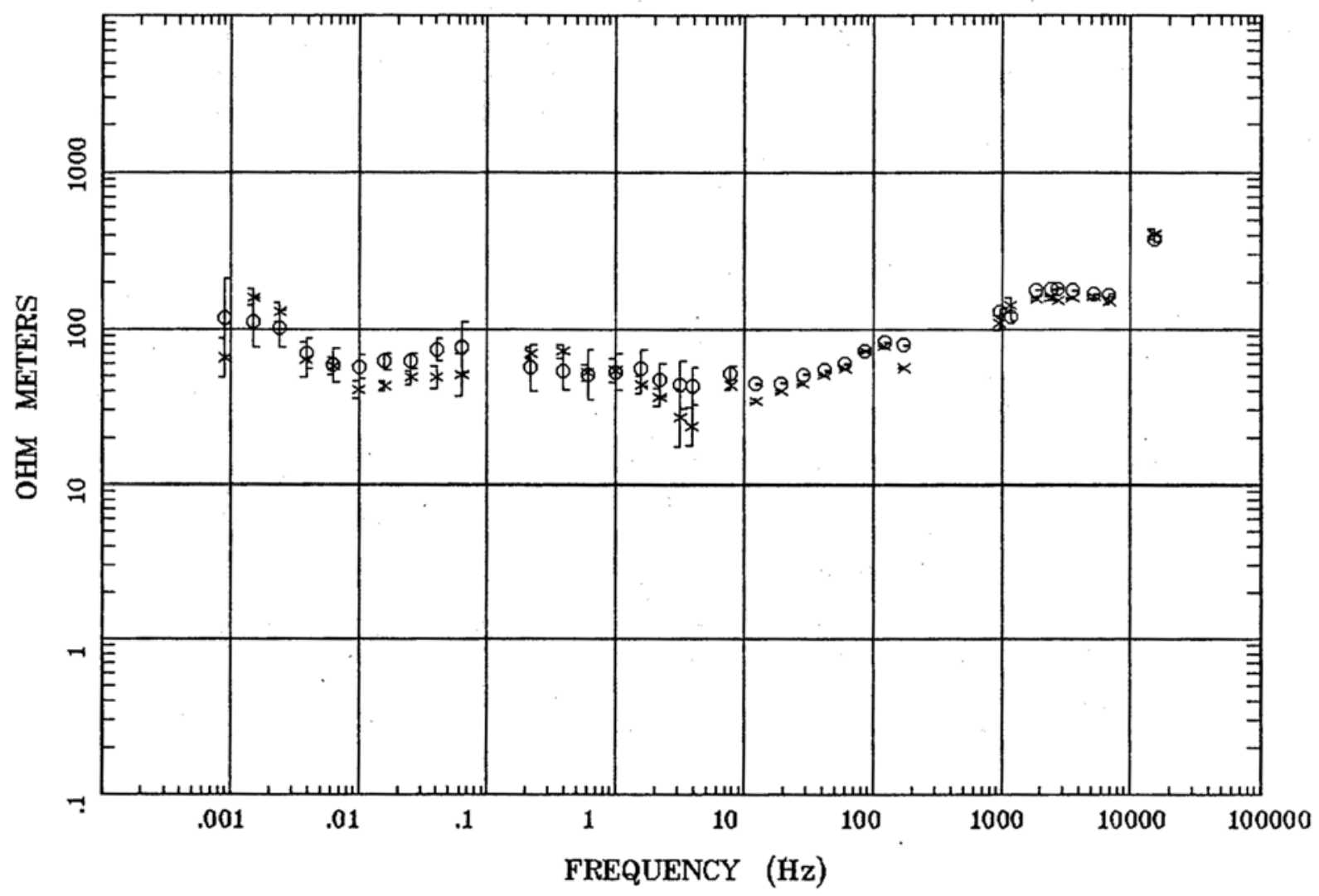

Client: DOE

Remote: none

Acquired: 22:0 May 12, 2005

Survey Co:USGS
Rotation:

Filename: rm09.avg

Channels: Ch1 Ch2 Ch3 Ch4 Ch5 Ch3 Ch4

Plotted: 12:54 Jan 17, 2006

< EMI - ElectroMagnetic Instruments > 


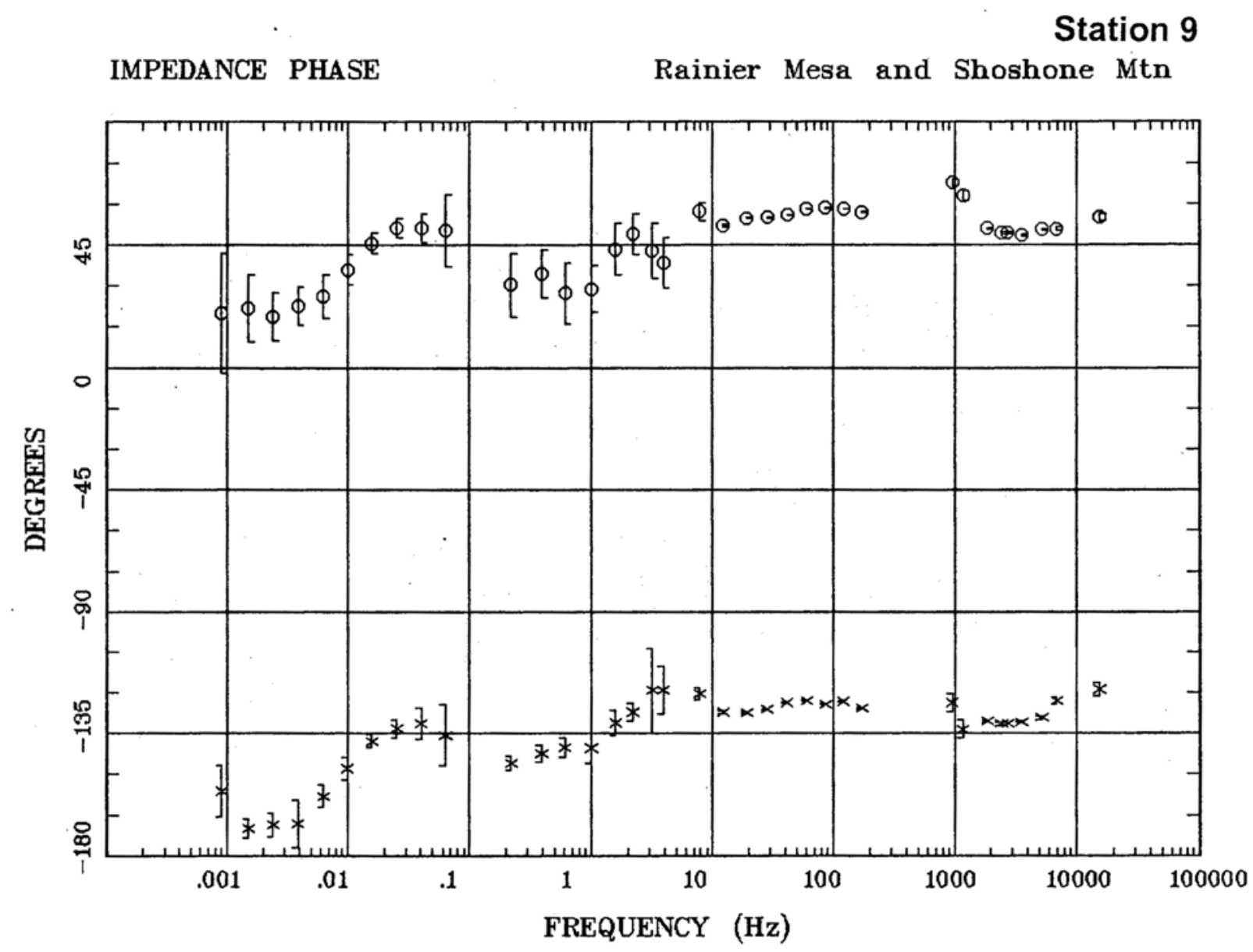

Client: DOE

Remote: none

Acquired: 22:0 May 12, 2005

Survey Co:USGS
Rotation:

Filename: rm09.avg

Channels: Ch1 Ch2 Ch3 Ch4 Ch5 Ch3 Ch4

Plotted: 12:54 Jan 17, 2006

< EMI - ElectroMagnetic Instruments > 


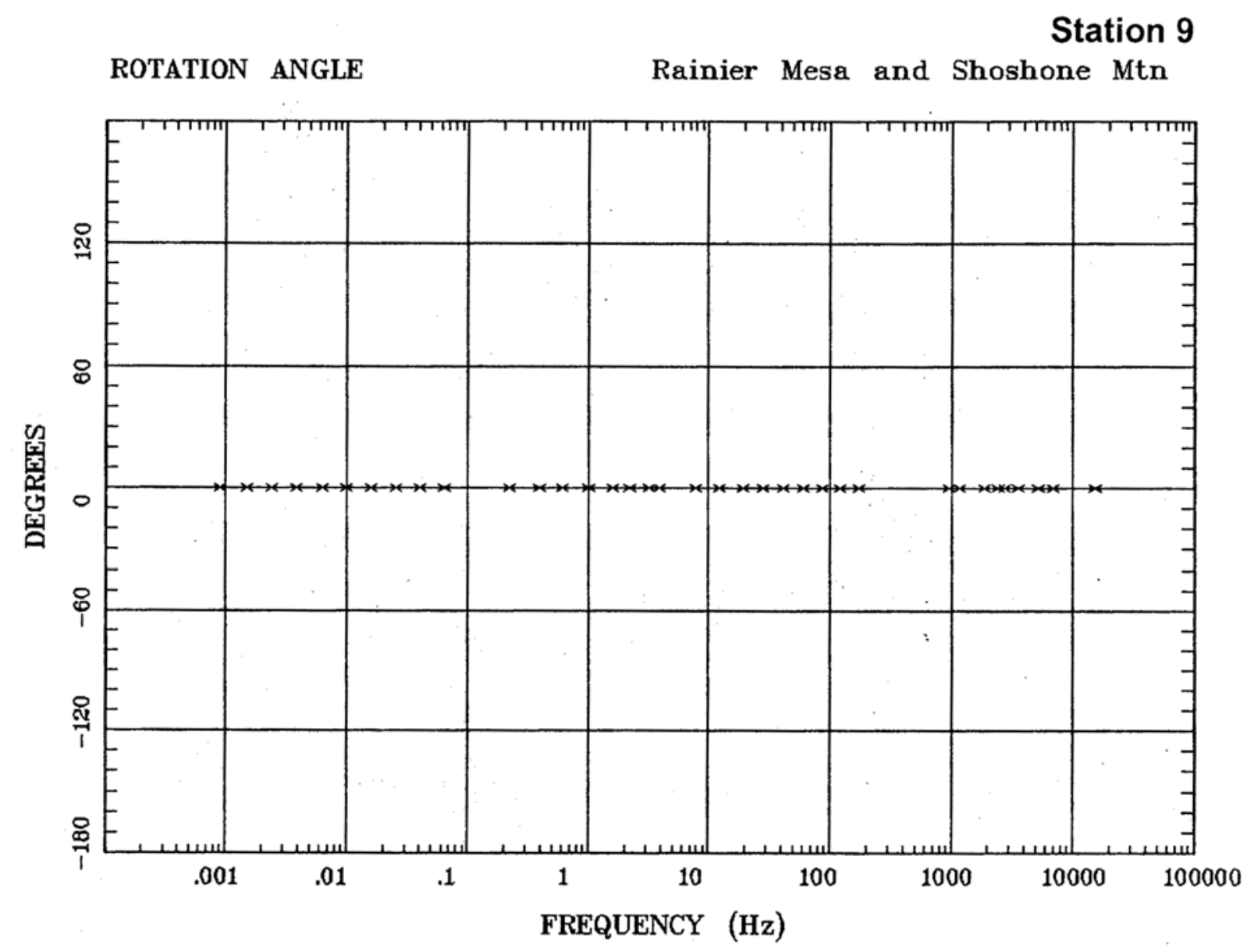

Client: DOE

Remote: none

Acquired: 22:0 May 12, 2005

Survey Co:USGS
Rotation:

Filename: rm09.avg

Channels: Ch1 Ch2 Ch3 Ch4 Ch5 Ch3 Ch4

Plotted: 12:54 Jan 17, 2006

< EMI - ElectroMagnetic Instruments > 


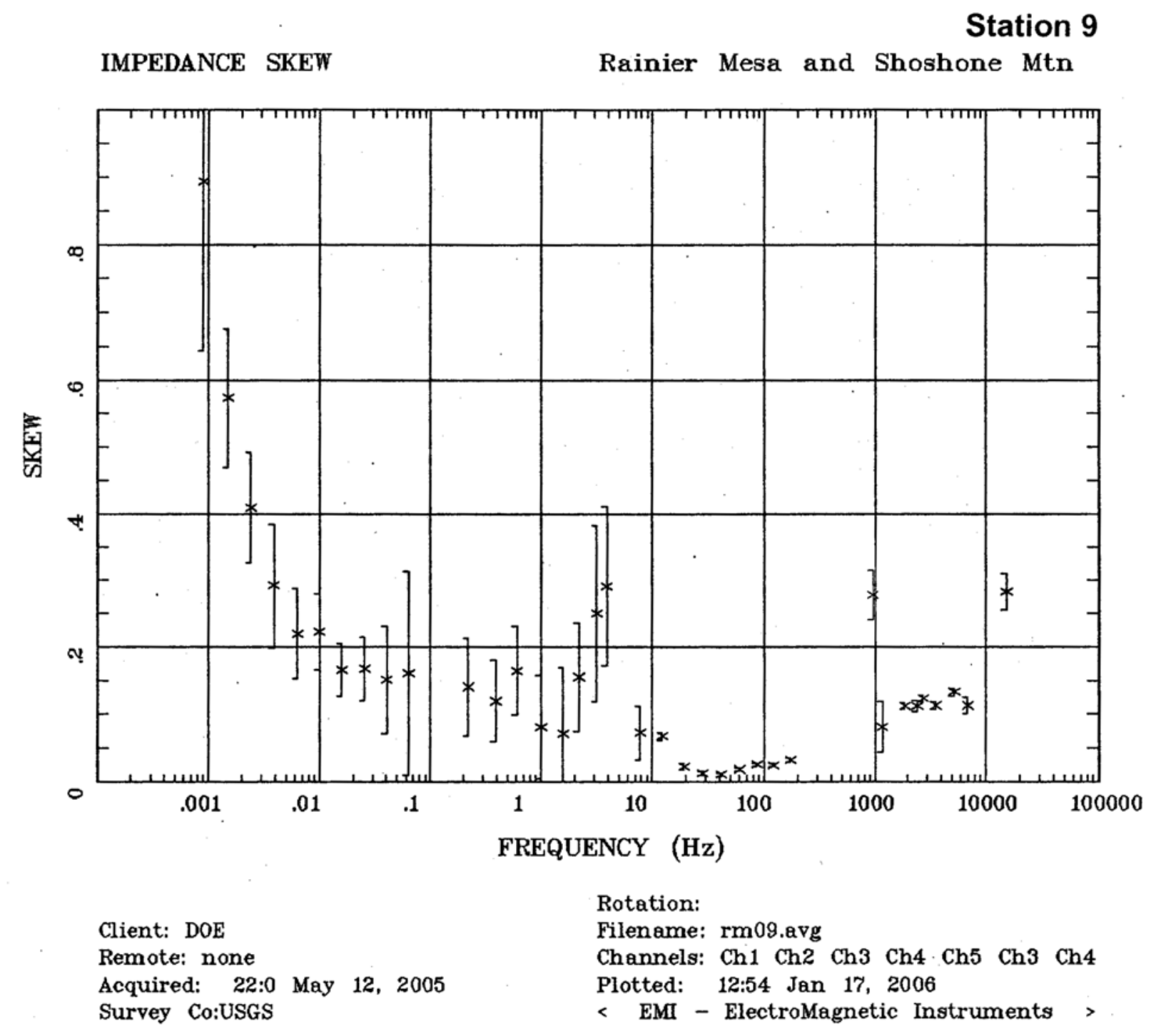




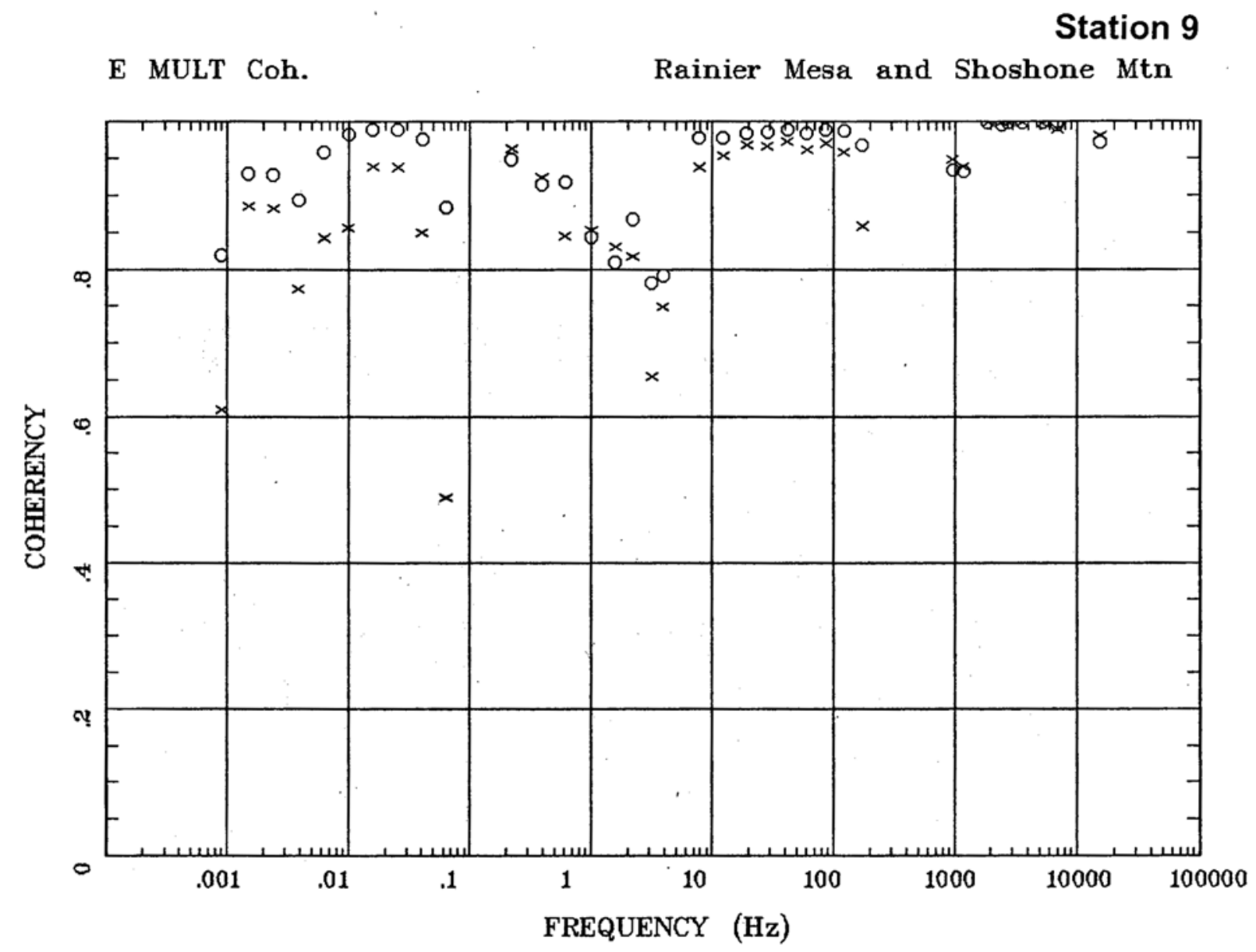

Client: $\mathrm{DOE}$

Remote: none

Acquired: 22:0 May 12, 2005

Survey Co:USGS
Rotation:

Filename: rm09.avg

Channels: Ch1 Ch2 Ch3 Ch4 Ch5 Ch3 Ch4

Plotted: 12:54 Jan 17, 2006

< EMI - ElectroMagnetic Instruments > 


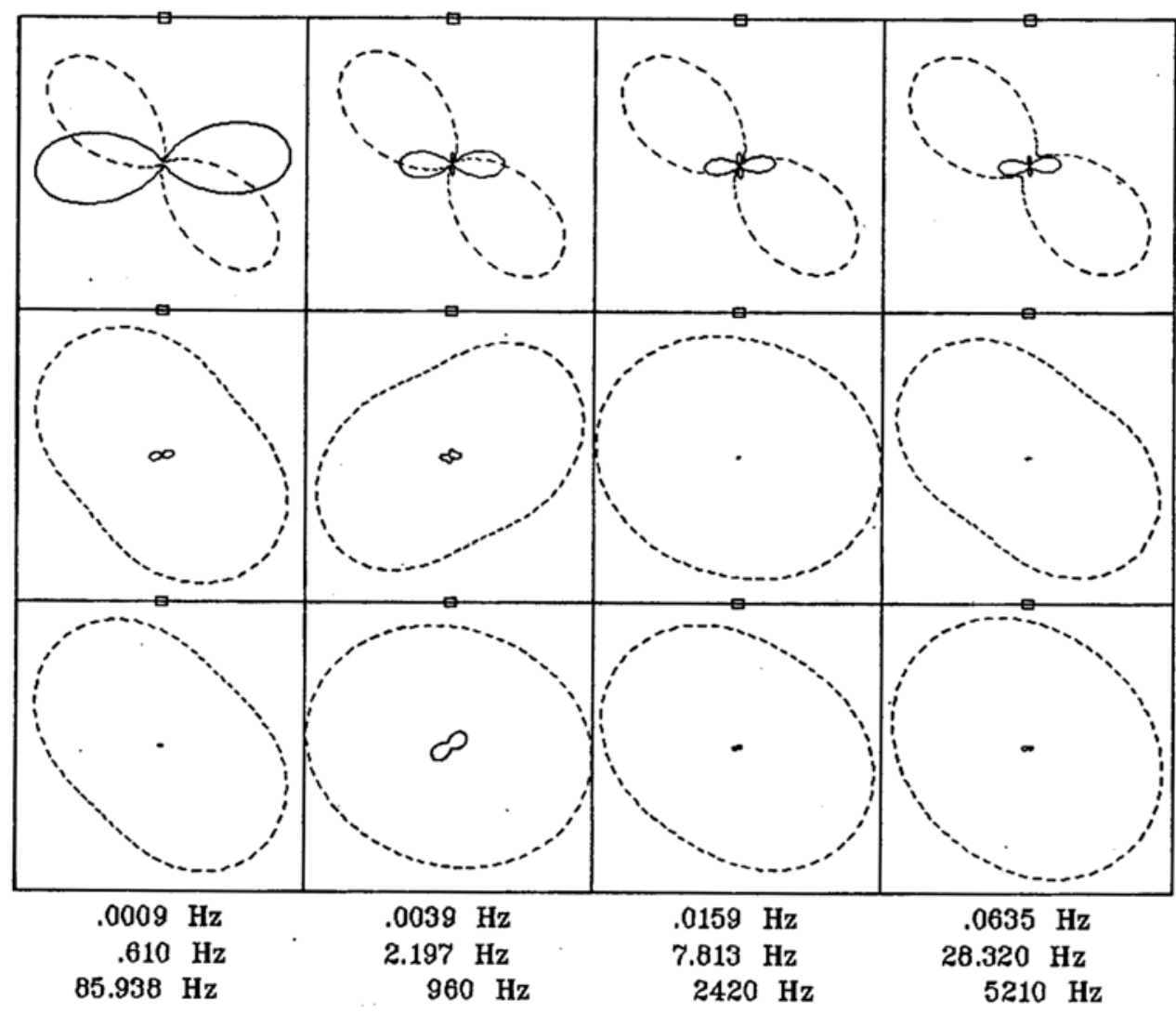

Client: DOE

Remote: none

Acquired: 22:0 May 12, 2005

Survey Co:USGS
Rotation:

Filename: rm09.avg

Channels: Ch1 Ch2 Ch3 Ch4 Ch5 Ch3 Ch4

Plotted: 12:54 Jan 17, 2006

< EMI - ElectroMagnetic Instruments > 


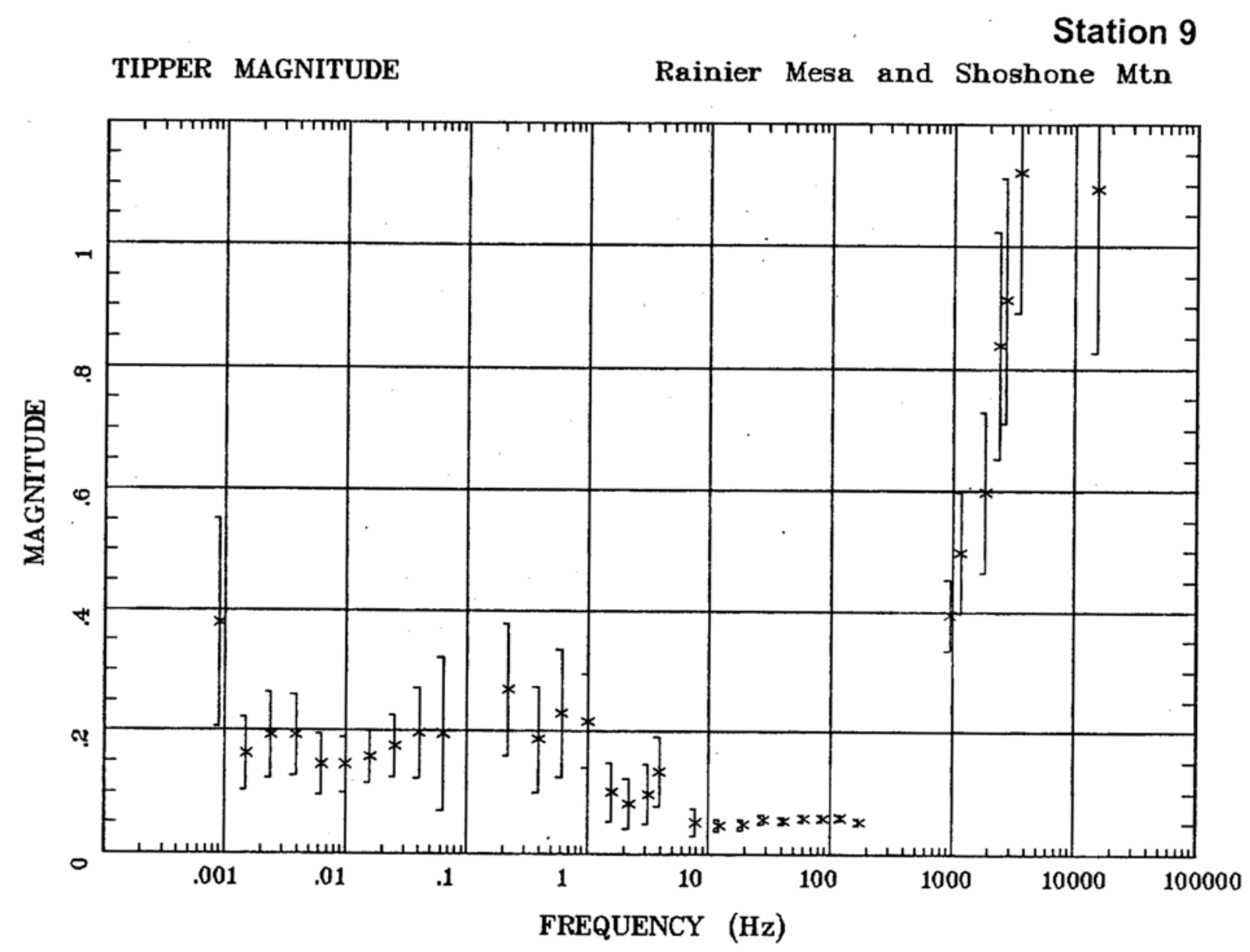

Client: DOE

Remote: none

Acquired: 22:0 May 12, 2005

Survey Co:USGS
Rotation:

Filename: rm09.avg

Channels: Ch1 Ch2 Ch3 Ch4 Ch5 Ch3 Ch4

Plotted: 12:54 Jan 17, 2006

< EMI - ElectroMagnetic Instruments > 


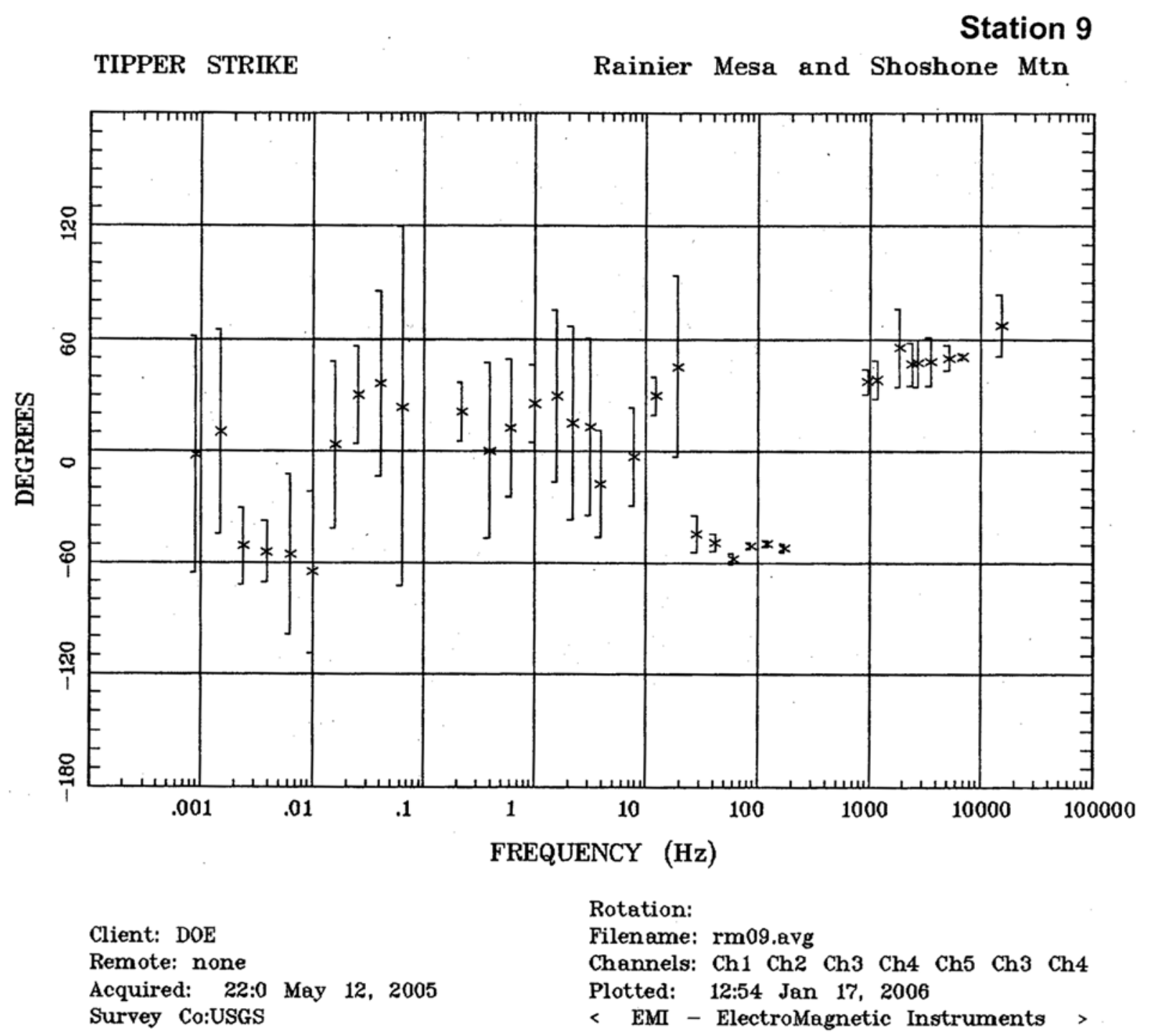




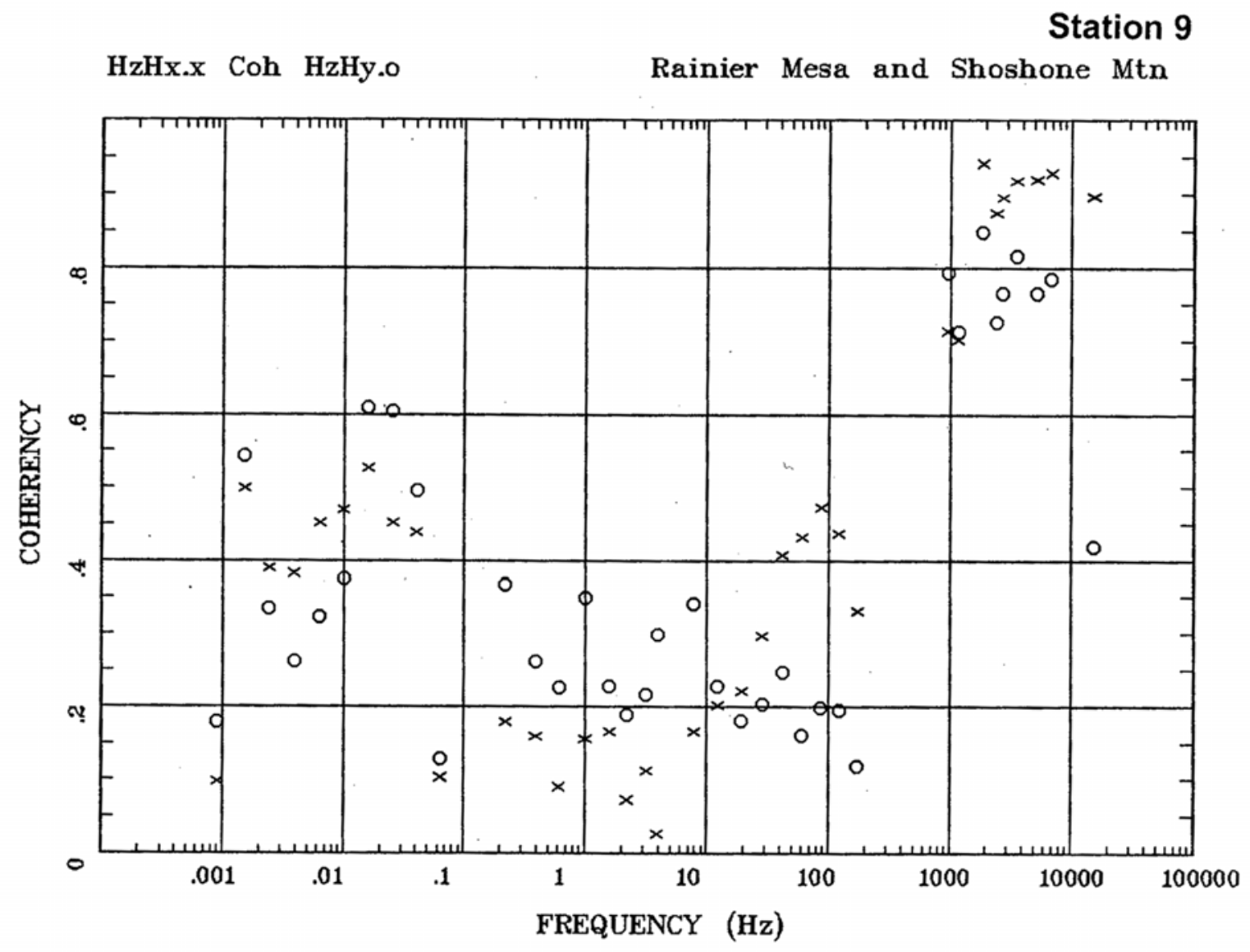

Client: DOE

Remote: none

Acquired: 22:0 May 12, 2005

Survey Co:USGS
Rotation:

Filename: rm09.avg

Channels: Ch1 Ch2 Ch3 Ch4 Ch5 Ch3 Ch4

Plotted: 12:54 Jan 17, 2006

< EMI - ElectroMagnetic Instruments > 


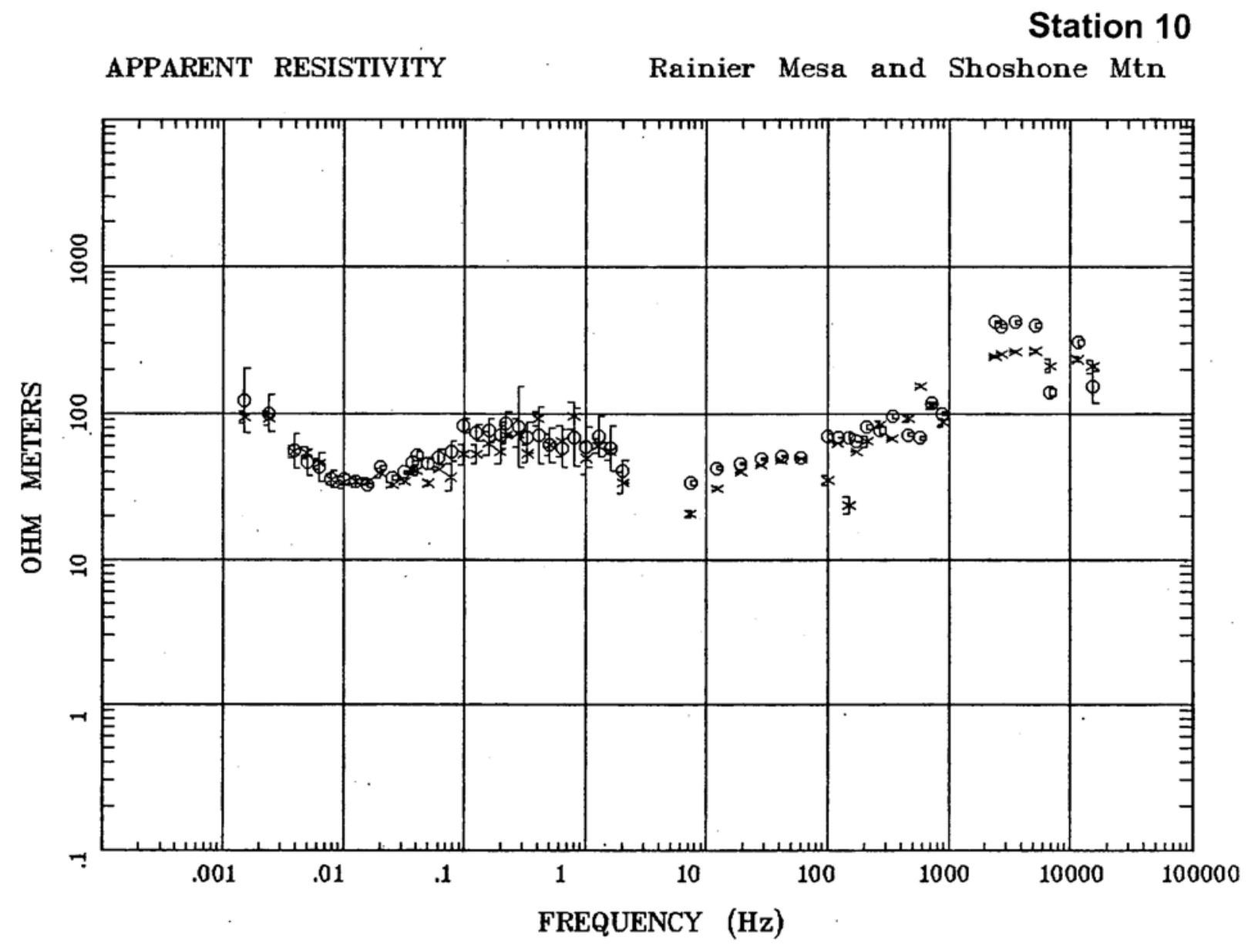

Client: DOE

Remote: none

Acquired: 00:3 May 13, 2005

Survey Co:USGS
Rotation:

Filename: rm10.avg

Channels: Ch1 Ch2 Ch3 Ch4 Ch5 Ch3 Ch4

Plotted: 12:55 Jan 17, 2006

< EMI - ElectroMagnetic Instruments > 
Station 10

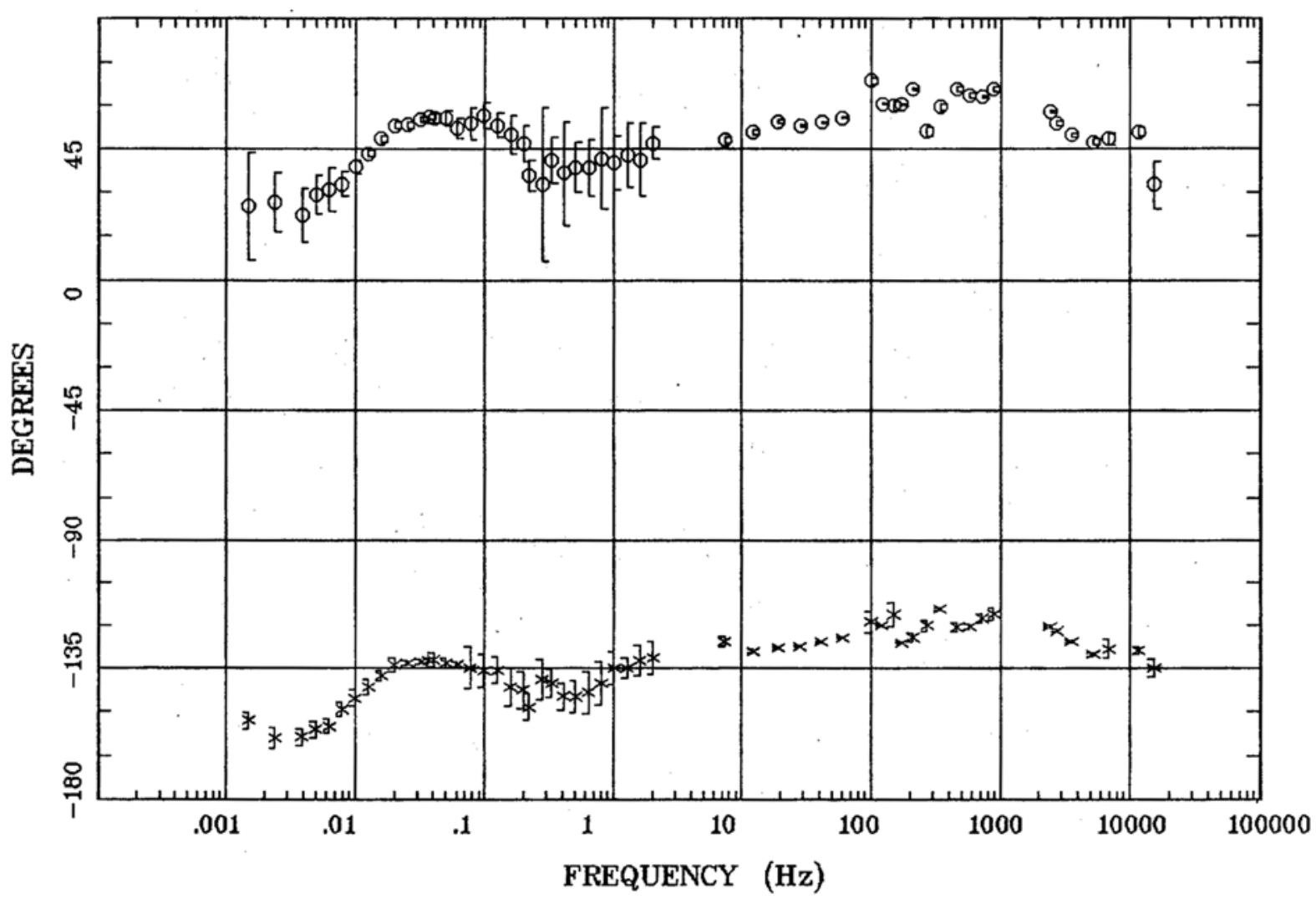

Client: DOE

Remote: none

Acquired: 00:3 May 13, 2005

Survey Co:USGS
Rotation:

Filename: rm10.avg

Channels: Ch1 Ch2 Ch3 Ch4 Ch5 Ch3 Ch4

Plotted: 12:55 Jan 17, 2006

< EMI - ElectroMagnetic Instruments > 
Station 10

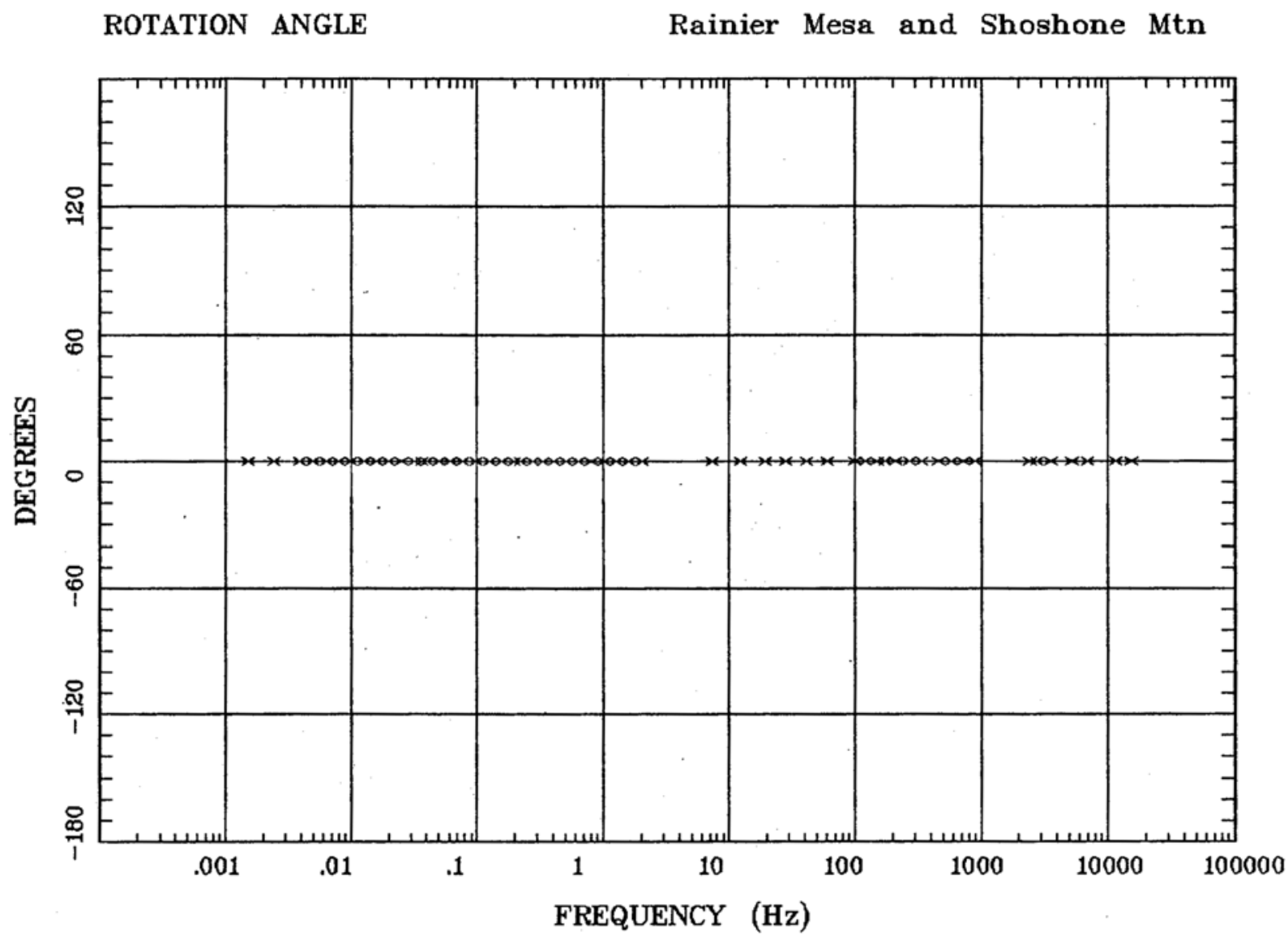

Client: DOE

Remote: none

Acquired: 00:3 May 13, 2005

Survey Co:USGS
Rotation:

Filename: rm10.avg

Channels: Ch1 Ch2 Ch3 Ch4 Ch5 Ch3 Ch4 Plotted: 12:55 Jan 17, 2006

< EMI - ElectroMagnetic Instruments > 


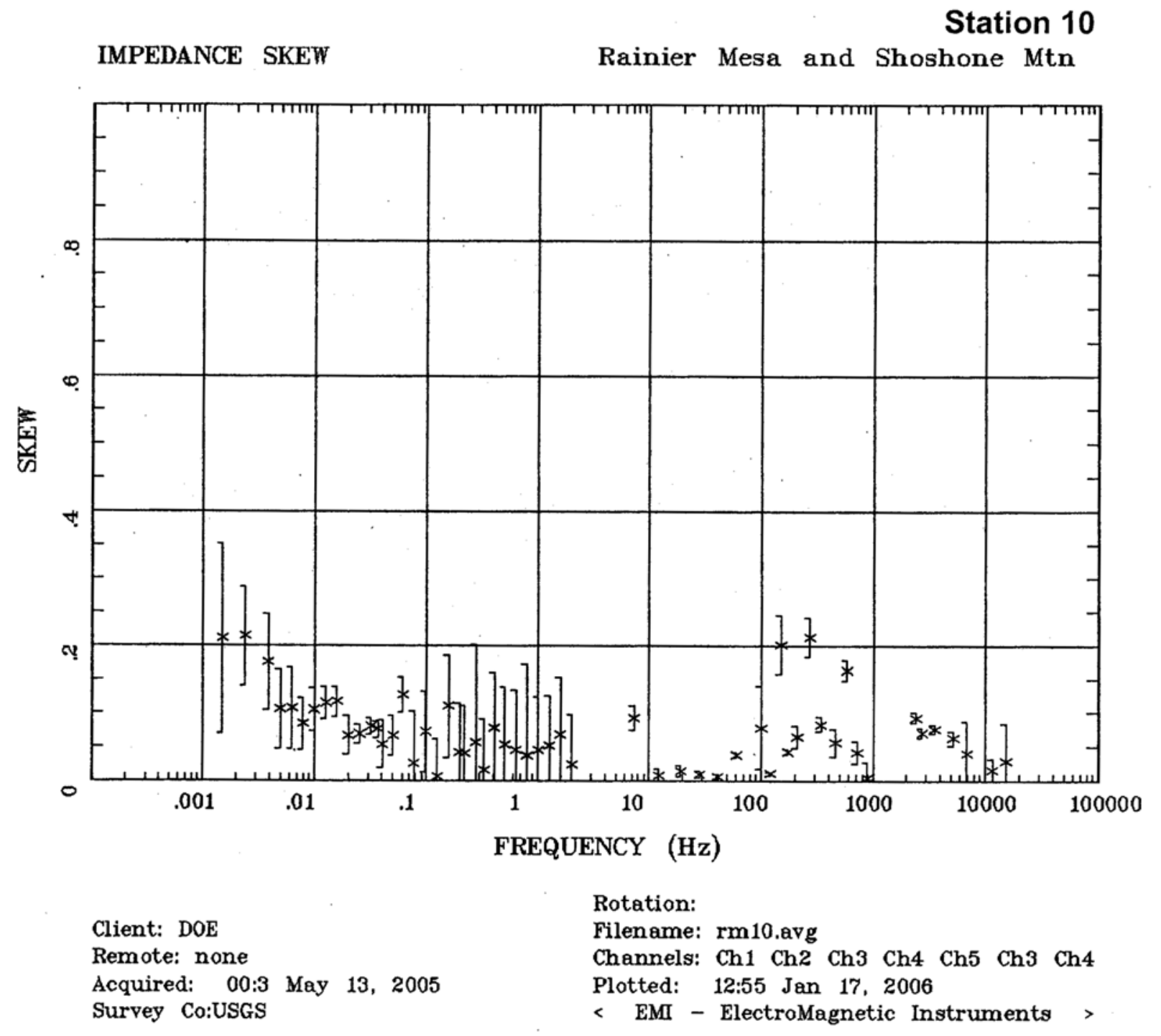


Station 10

E MULT Coh.

Rainier Mesa and Shoshone Mtn

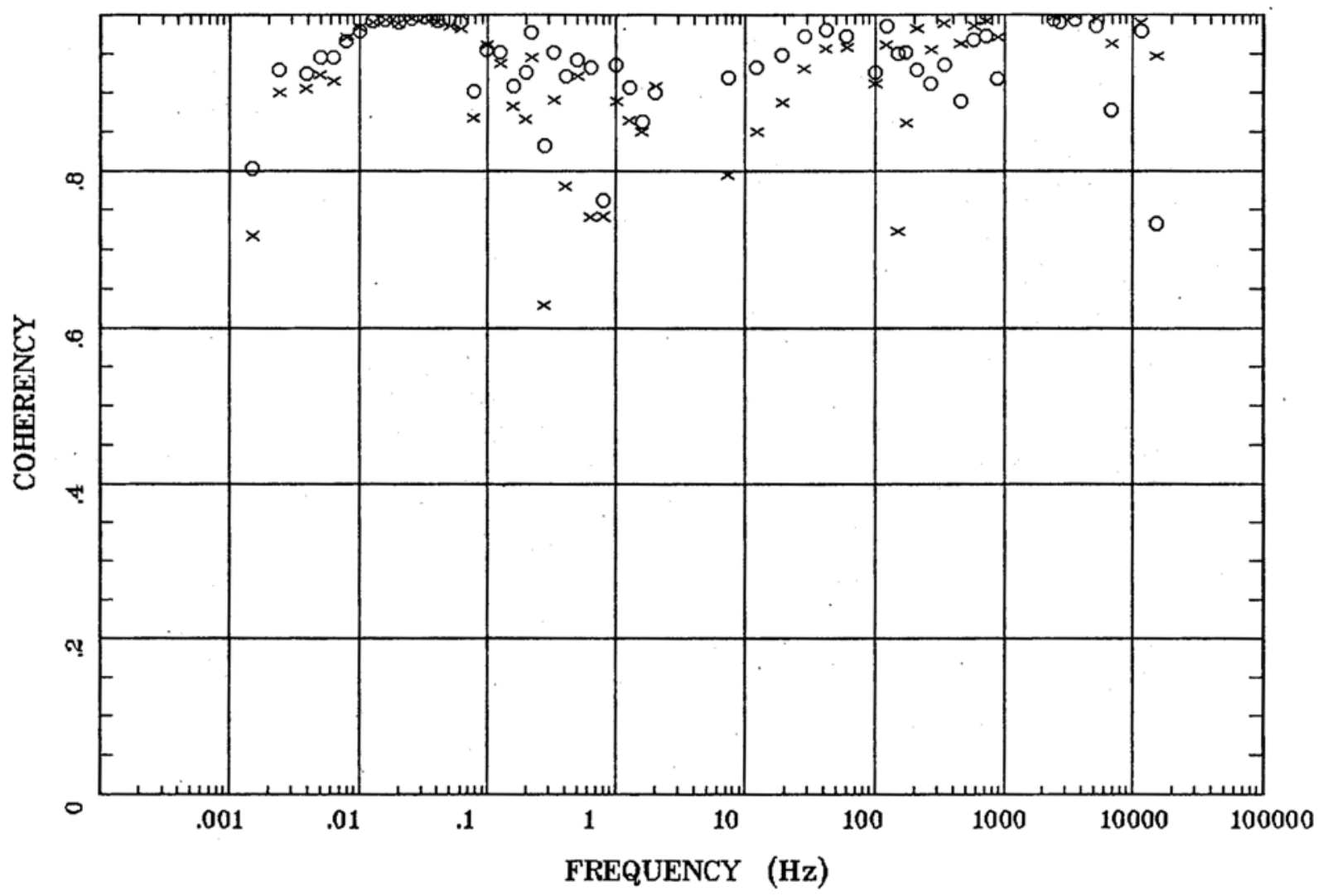

Client: DOE

Remote: none

Acquired: 00:3 May 13, 2005

Survey Co:USGS
Rotation:

Filename: rm10.avg

Channels: Ch1 Ch2 Ch3 Ch4 Ch5 Ch3 Ch4

Plotted: 12:55 Jan 17, 2006

< EMI - ElectroMagnetic Instruments > 
Station 10

POLAR PLOTS

Rainier Mesa and Shoshone Mtn

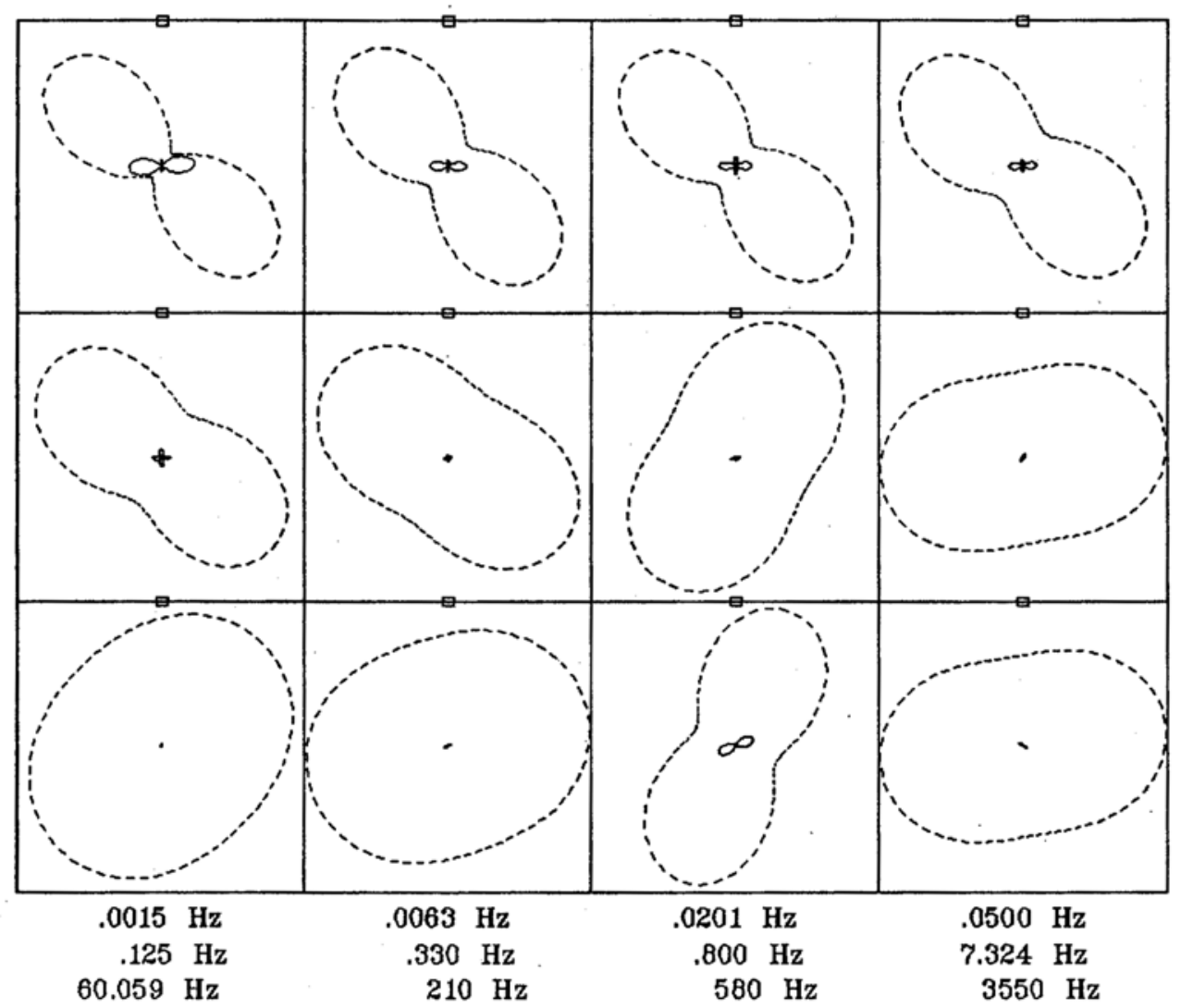

Rotation:

Client: DOE

Filename: rm10.avg

Remote: none

Channels: Ch1 Ch2 Ch3 Ch4 Ch5 Ch3 Ch4

Acquired: 00:3 May 13, 2005

Plotted: 12:55 Jan 17, 2006

Survey Co:USGS

< EMI - ElectroMagnetic Instruments > 
Station 10

TIPPER MAGNITUDE

Rainier Mesa and Shoshone Mtn

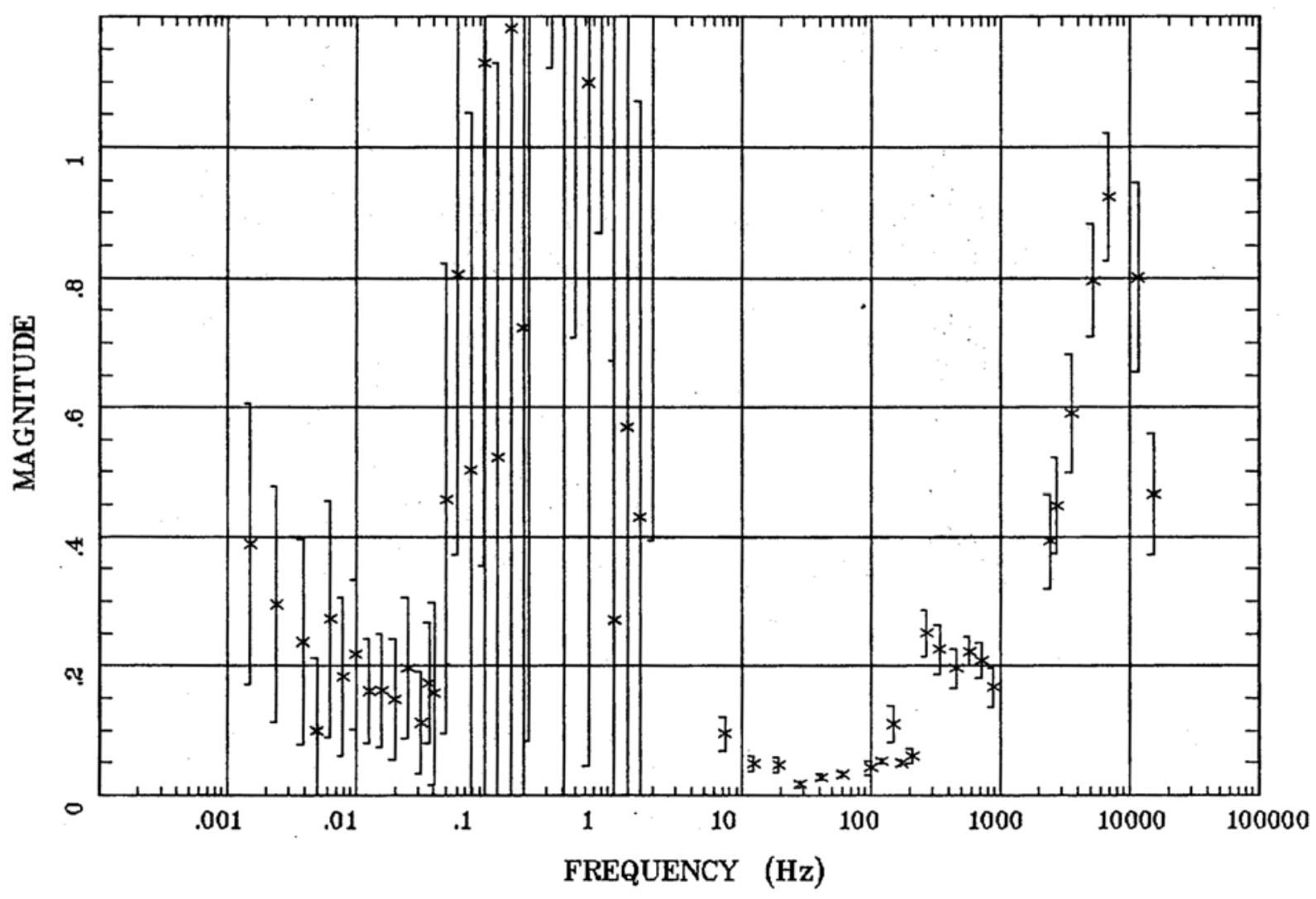

Client: DOE

Remote: none

Acquired: 00:3 May 13, 2005

Survey Co:USGS
Rotation:

Filename: rm10.avg

Channels: Ch1 Ch2 Ch3 Ch4 Ch5 Ch3 Ch4

Plotted: 12:55 Jan 17, 2006

< EMI - ElectroMagnetic Instruments > 
Station 10

TIPPER STRIKE

Rainier Mesa and Shoshone Mtn

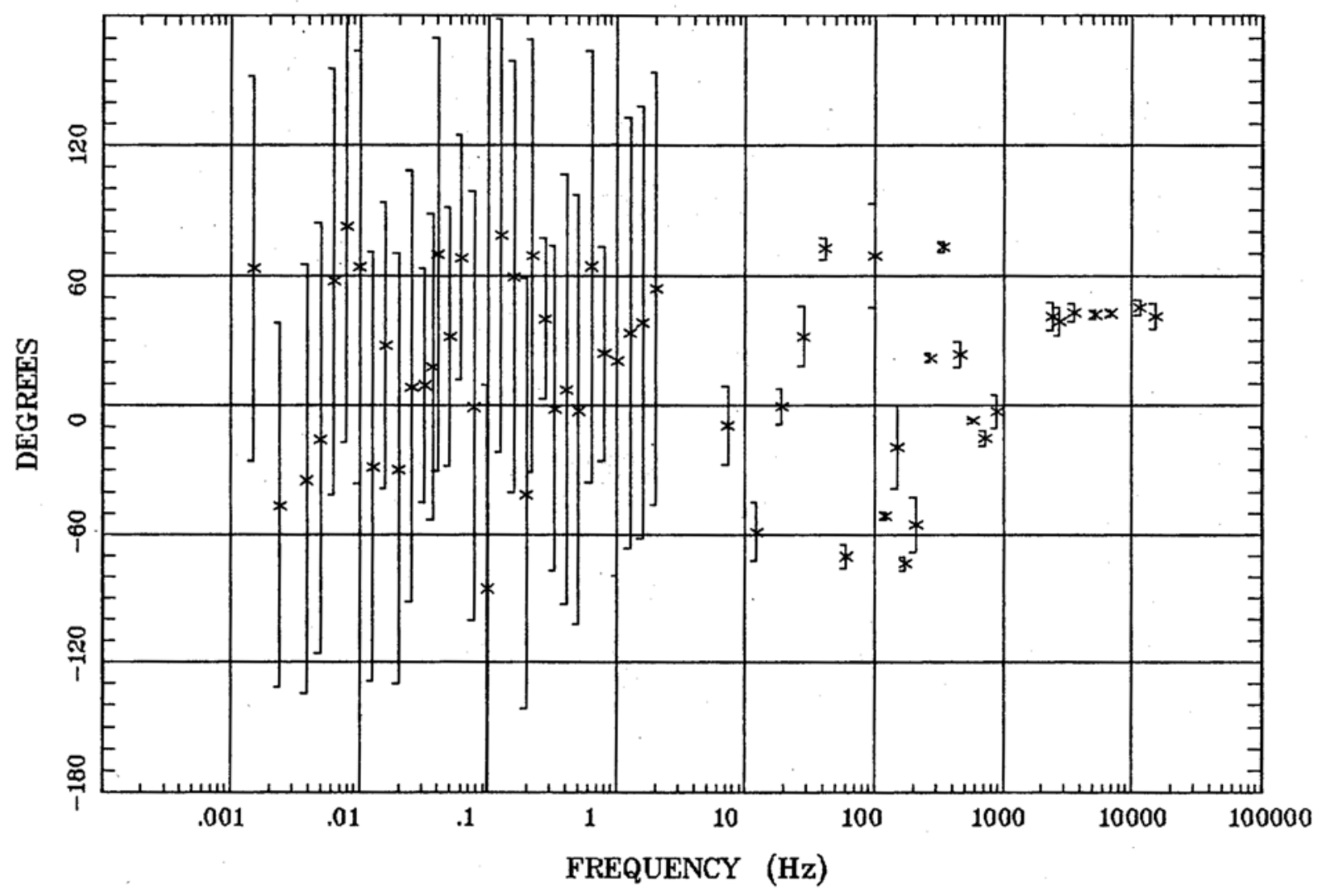

Client: DOE

Remote: none

Acquired: 00:3 May 13, 2005

Survey Co:USGS

Rotation:

Filename: rm10.avg

Channels: Ch1 Ch2 Ch3 Ch4 Ch5 Ch3 Ch4

Plotted: 12:55 Jan 17, 2006

< EMI - ElectroMagnetic Instruments > 


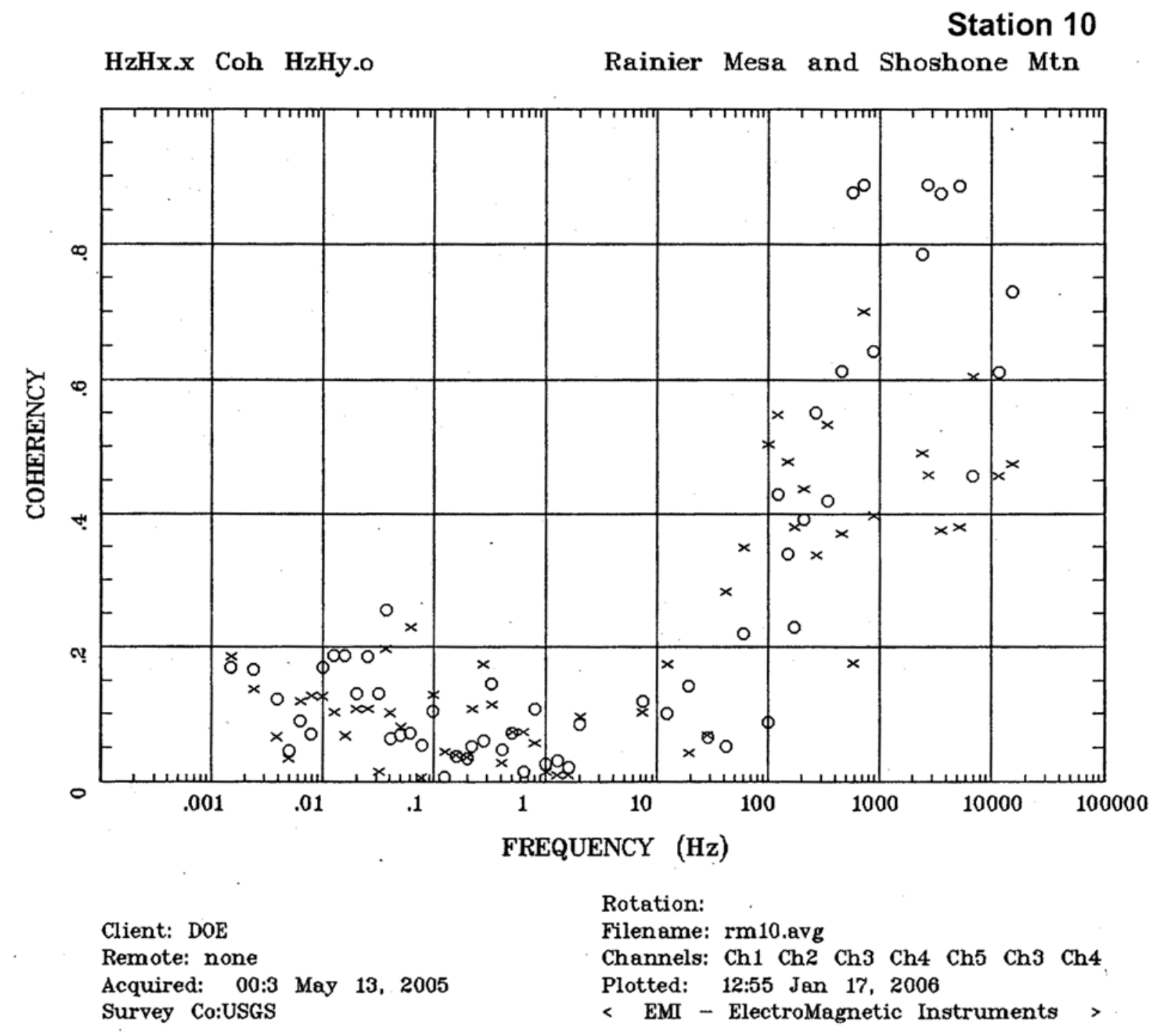




\section{Station 11}

APPARENT RESISTIVITY

Rainier Mesa and Shoshone Mtn

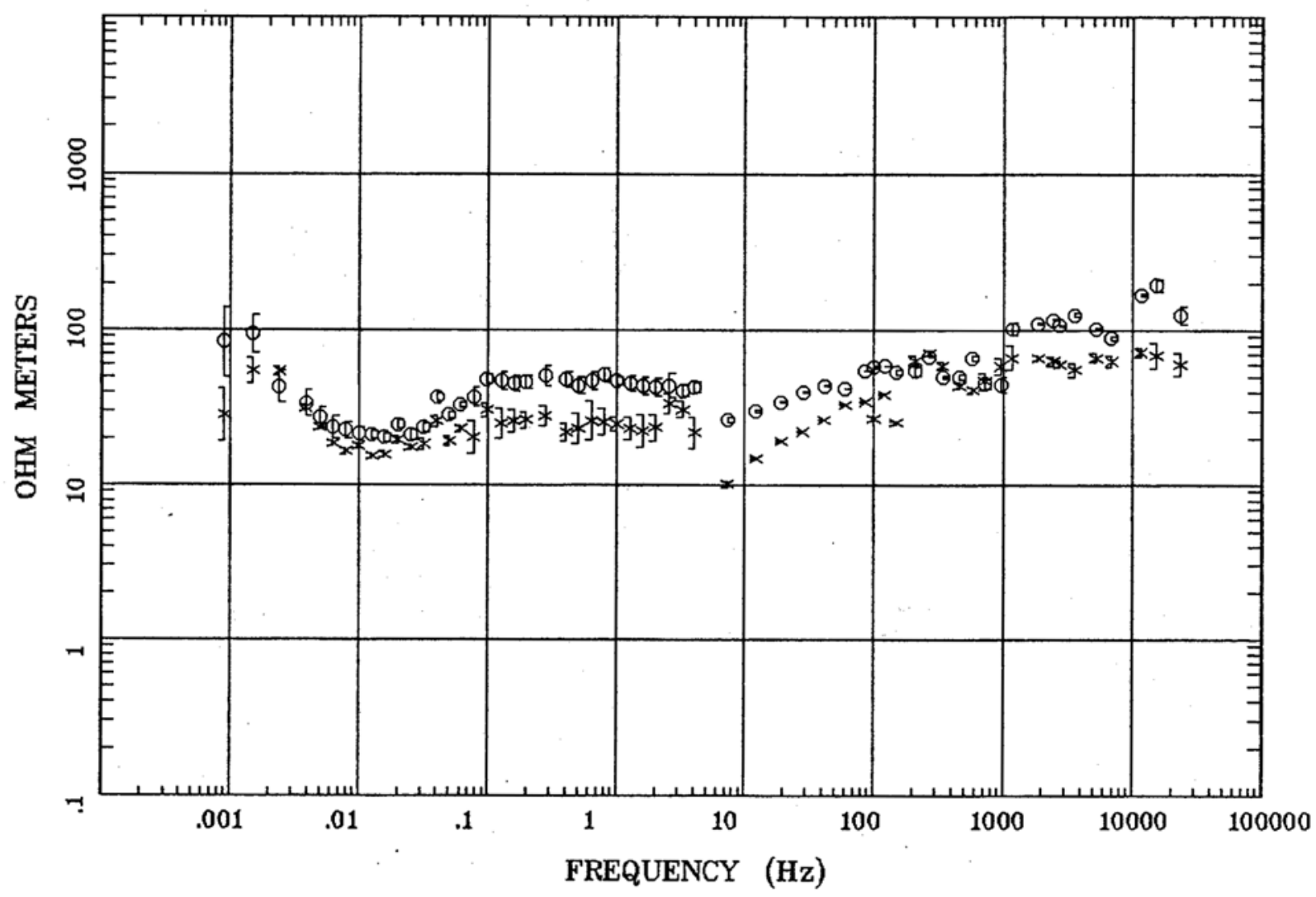

Client: DOE

Remote: none

Acquired: 03:1 May 13, 2005

Survey Co:USGS
Rotation:

Filename: rm11.avg

Channels: Ch1 Ch2 Ch3 Ch4 Ch5 Ch3 Ch4

Plotted: 13:08 Jan 17, 2006

< EMI - ElectroMagnetic Instruments > 


\section{Station 11}

IMPEDANCE PHASE

Rainier Mesa and Shoshone Mtn

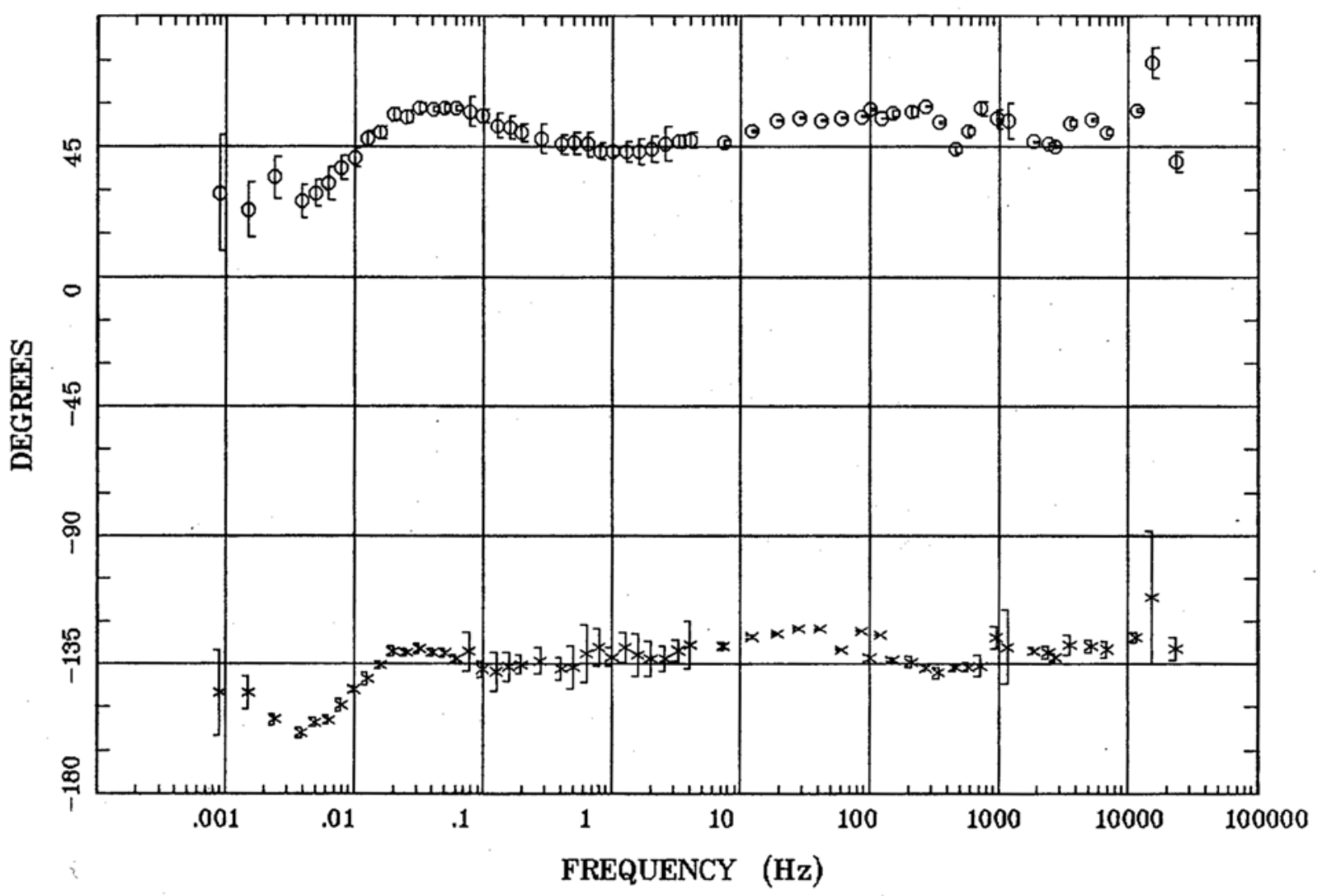

Client: DOE

Remote: none

Acquired: 03:1 May 13, 2005

Survey Co:USGS
Rotation:

Filename: rm11.avg

Channels: Ch1 Ch2 Ch3 Ch4 Ch5 Ch3 Ch4

Plotted: 13:08 Jan 17, 2006

< EMI - ElectroMagnetic Instruments > 


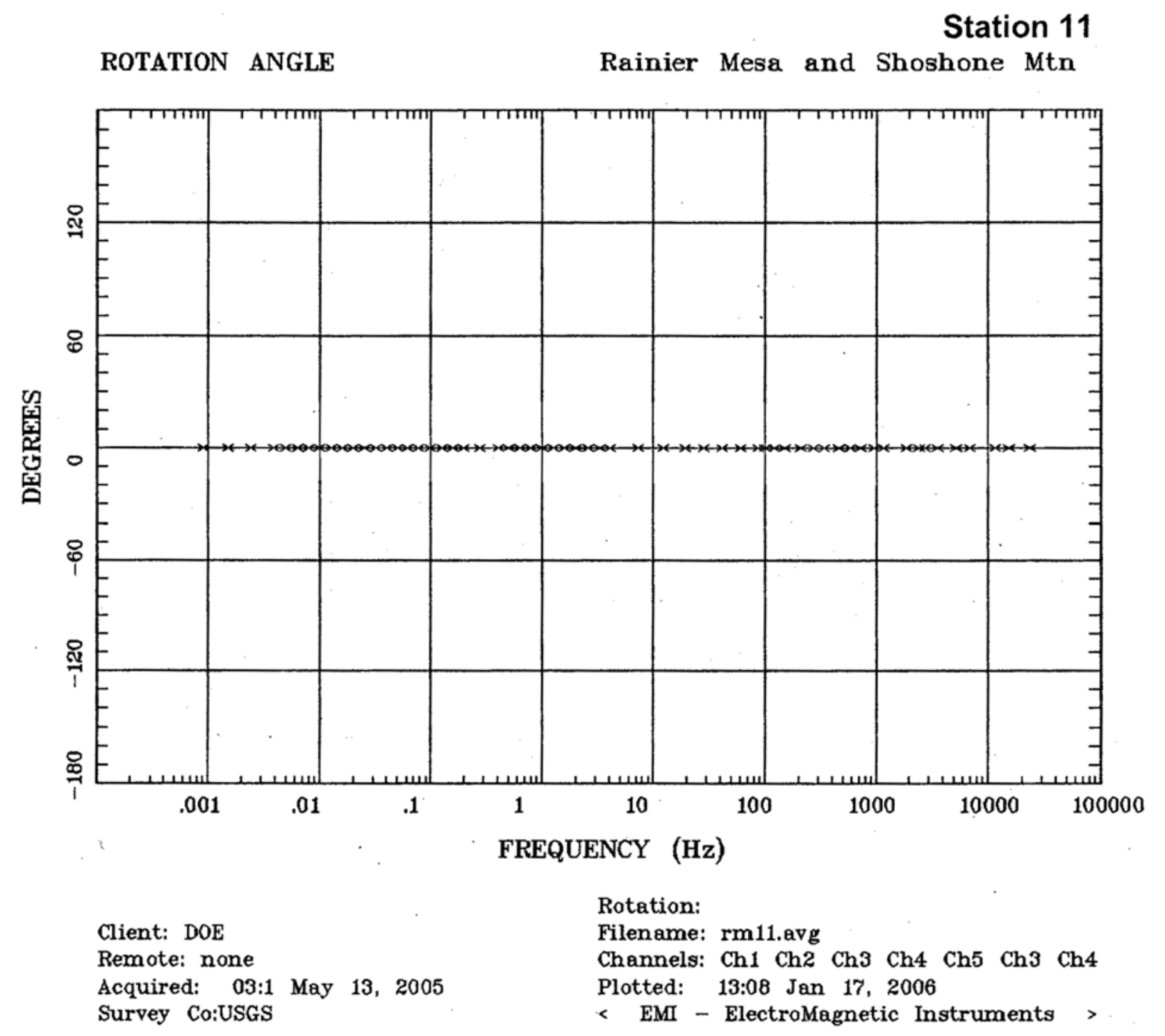




\section{Station 11}

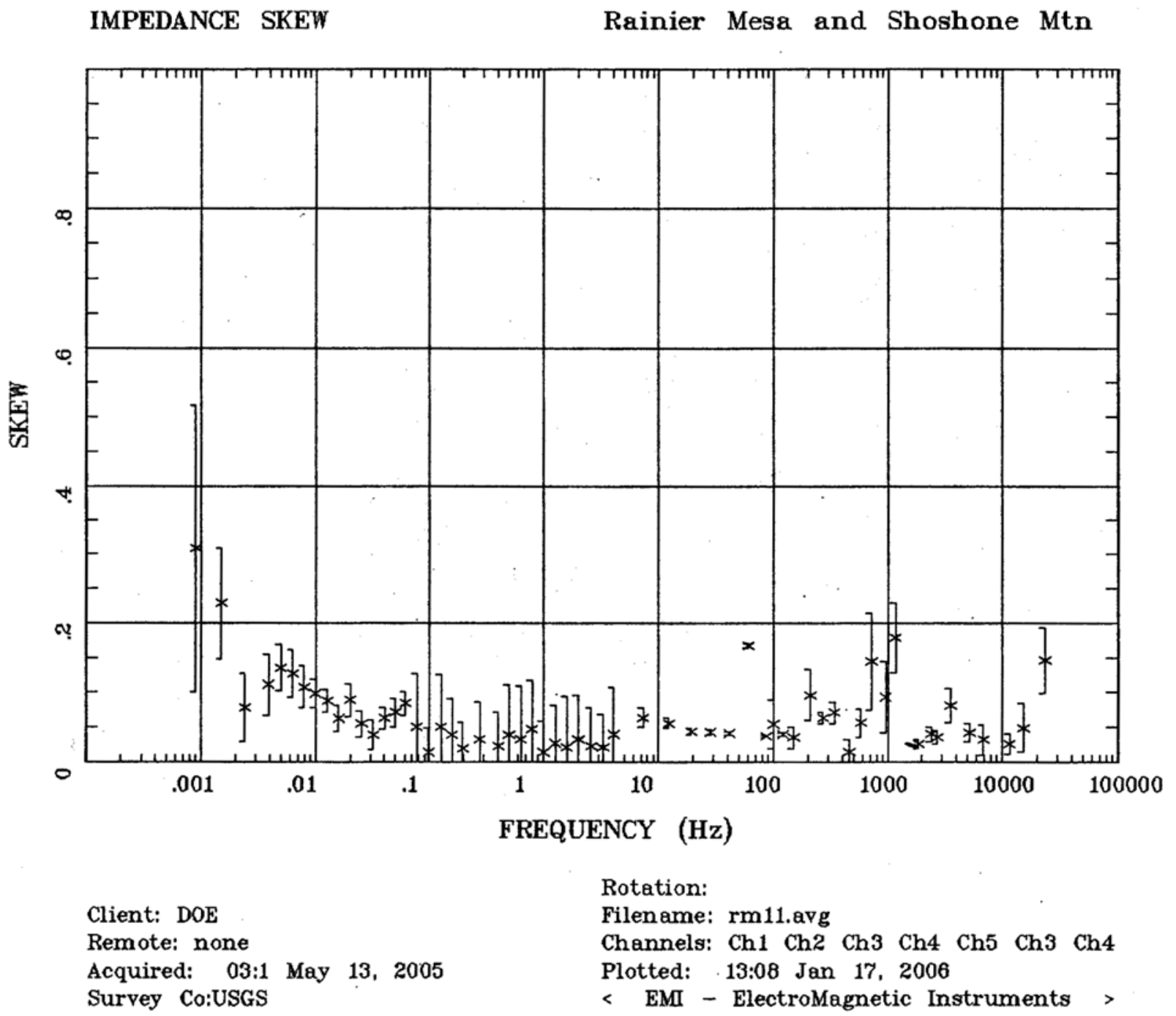




\section{Station 11}

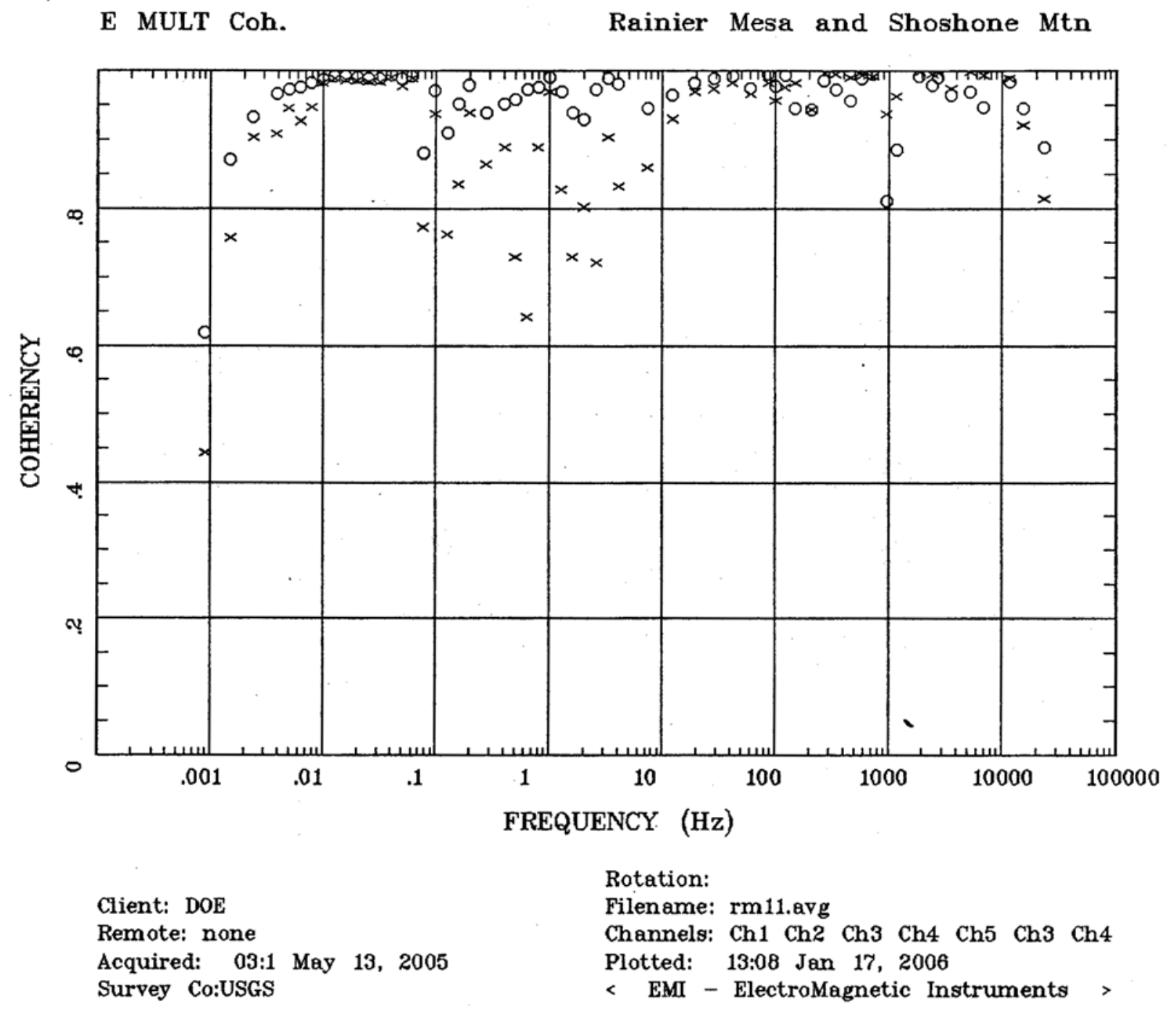


Station 11

POLAR PLOTS Rainier Mesa and Shoshone Mtn

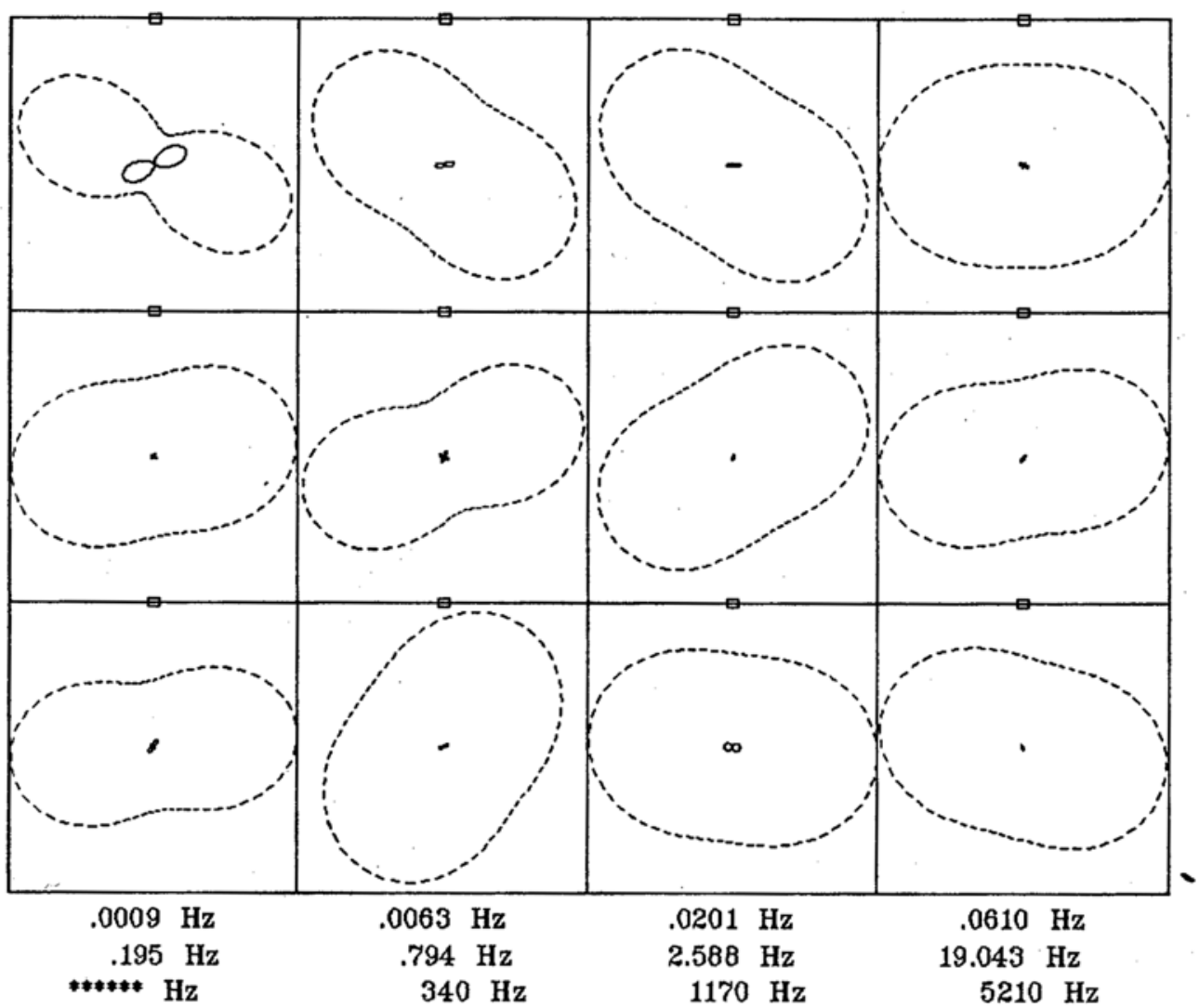

Client: DOE

Remote: none

Rotation:

Filename: rm11.avg

Channels: Ch1 Ch2 Ch3 Ch4 Ch5 Ch3 Ch4

Acquired: 03:1 May 13, 2005

Plotted: 13:08 Jan 17, 2006

Survey Co:USGS

< EMI - ElectroMagnetic Instruments > 


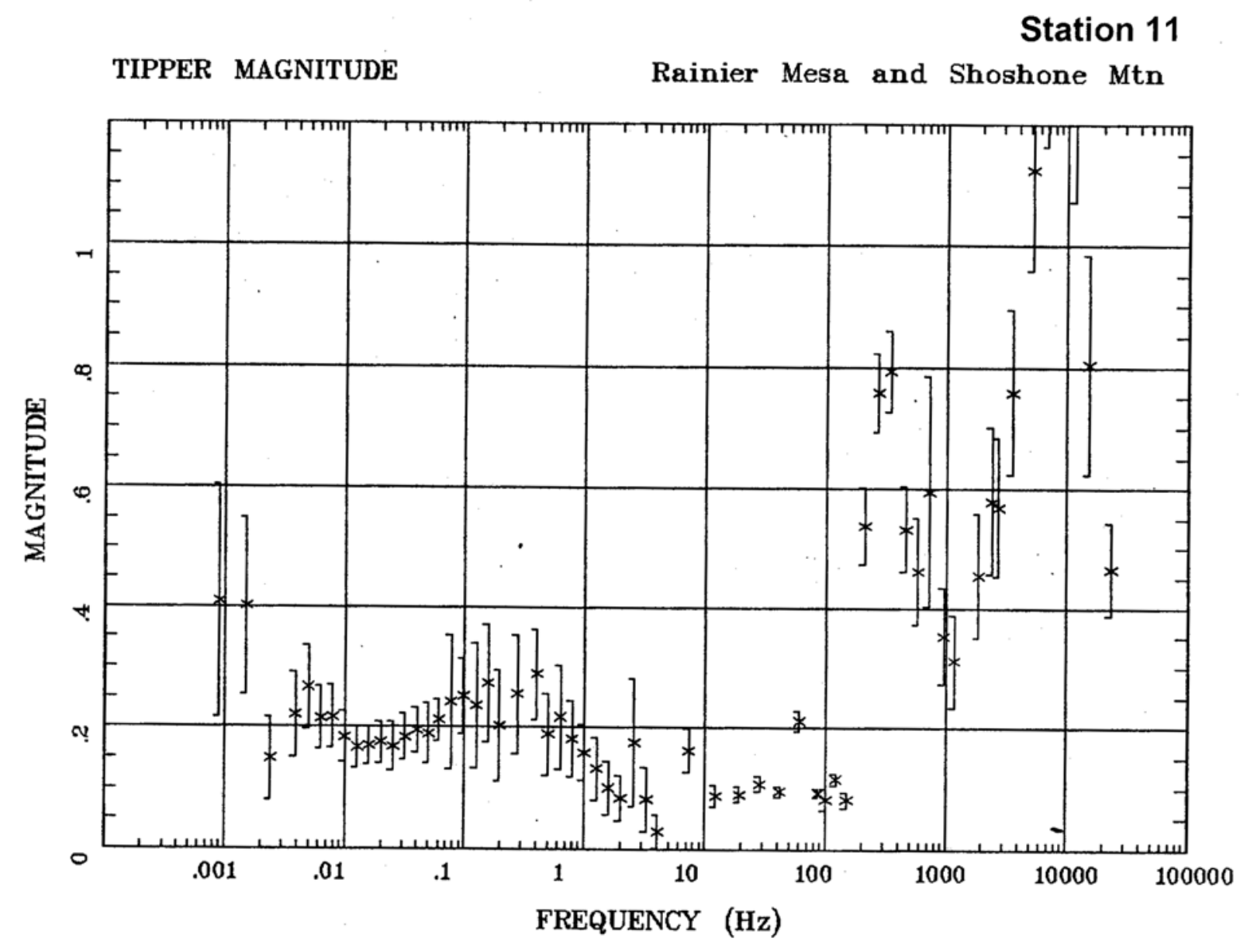

Client: DOE

Remote: none

Acquired: 03:1 May 13, 2005

Survey Co:USGS
Rotation:

Filename: rm11.avg

Channels: Ch1 Ch2 Ch3 Ch4 Ch5 Ch3 Ch4

Plotted: 13:08 Jan 17, 2006

< EMI - ElectroMagnetic Instruments > 


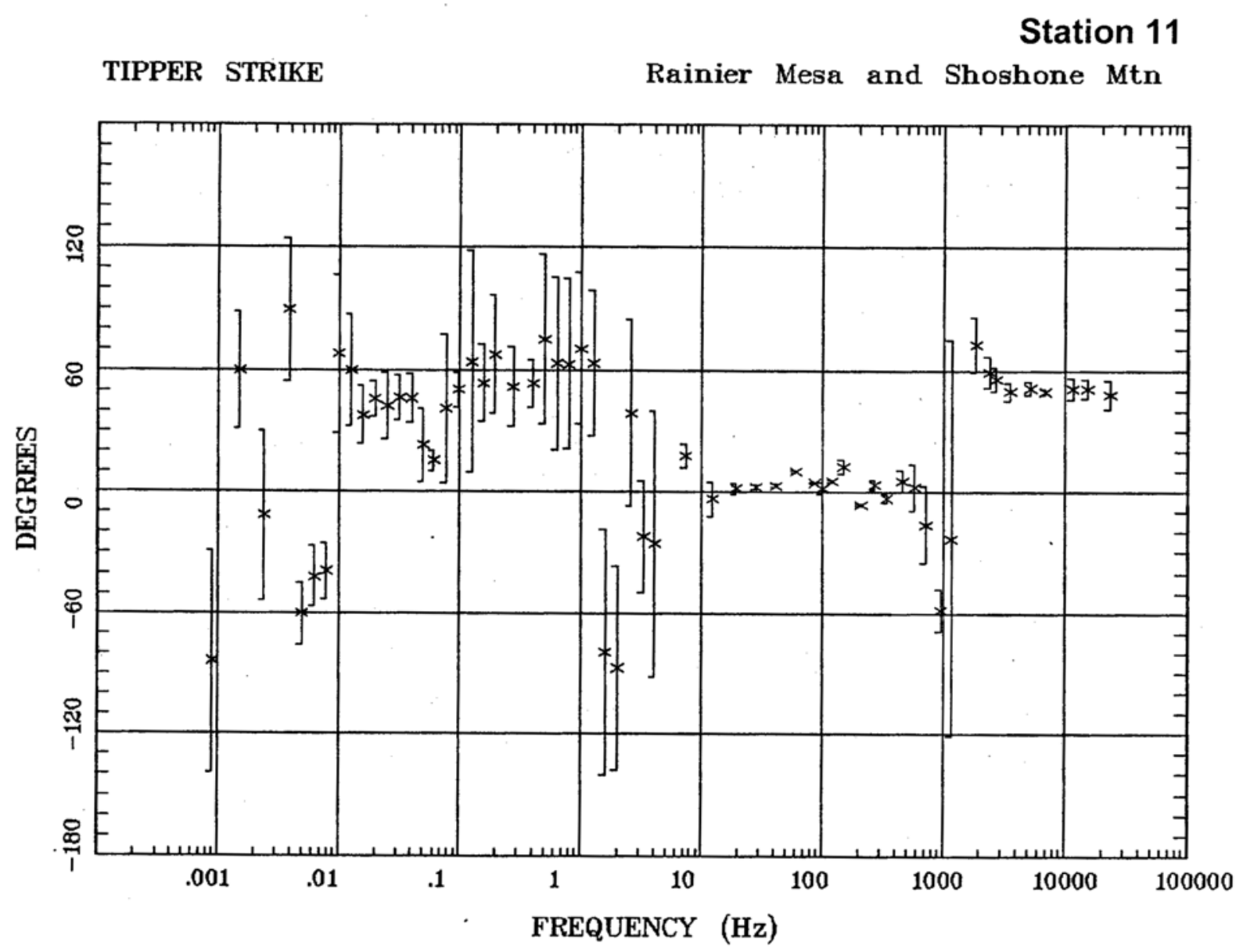

Client: DOE

Remote: none

Acquired: 03:1 May 13, 2005

Survey Co:USGS
Rotation:

Filename: rm11.avg

Channels: Ch1 Ch2 Ch3 Ch4 Ch5 Ch3 Ch4

Plotted: 13:08 Jan 17, 2006

< EMI - ElectroMagnetic Instruments > 


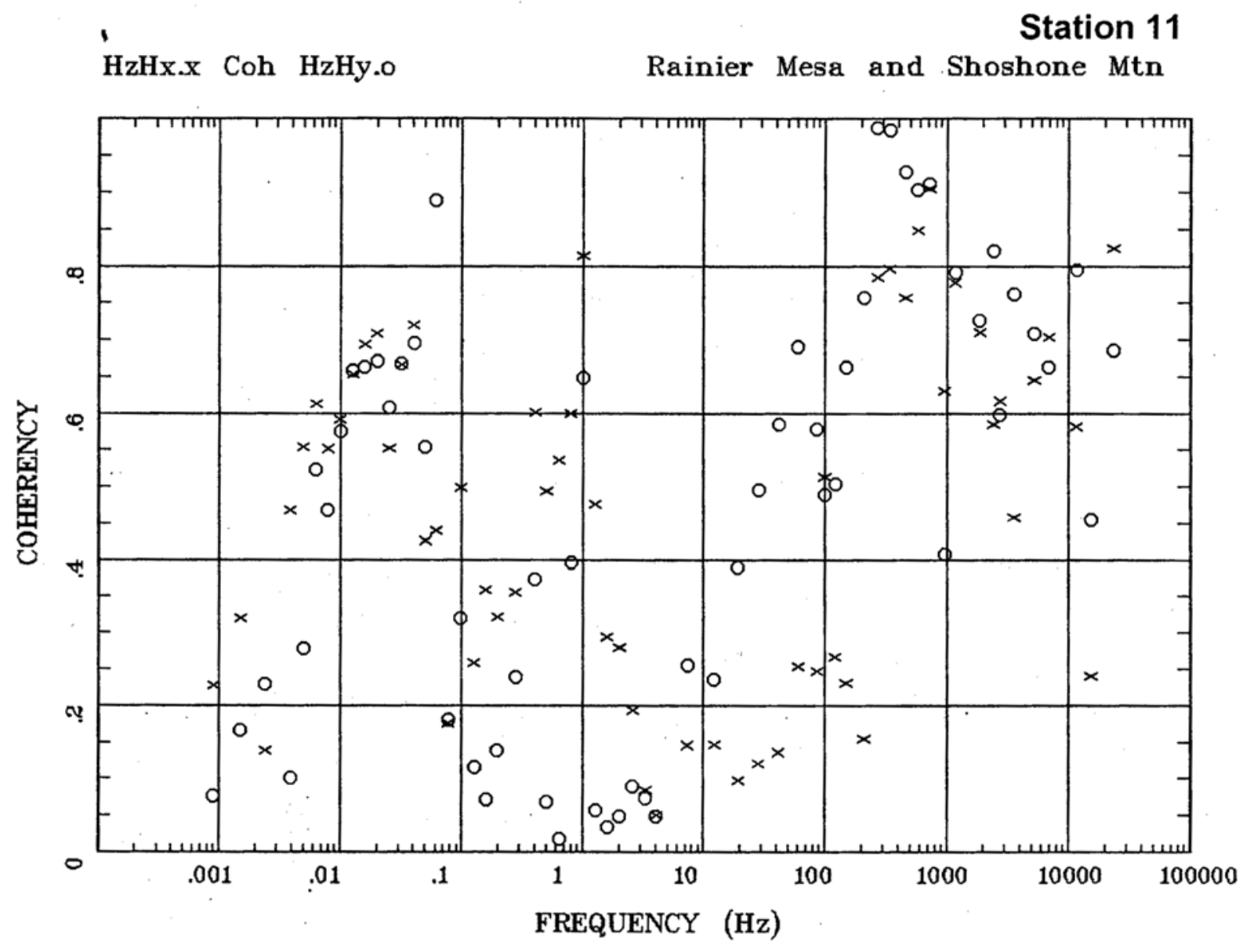

Client: $\mathrm{DOE}$

Remote: none

Acquired: 03:1 May 13, 2005

Survey Co:USGS
Rotation:

Filename: rm11.avg

Channels: Ch1 Ch2 Ch3 Ch4 Ch5 Ch3 Ch4

Plotted: 13:08 Jan 17, 2006

< EMI - ElectroMagnetic Instruments > 


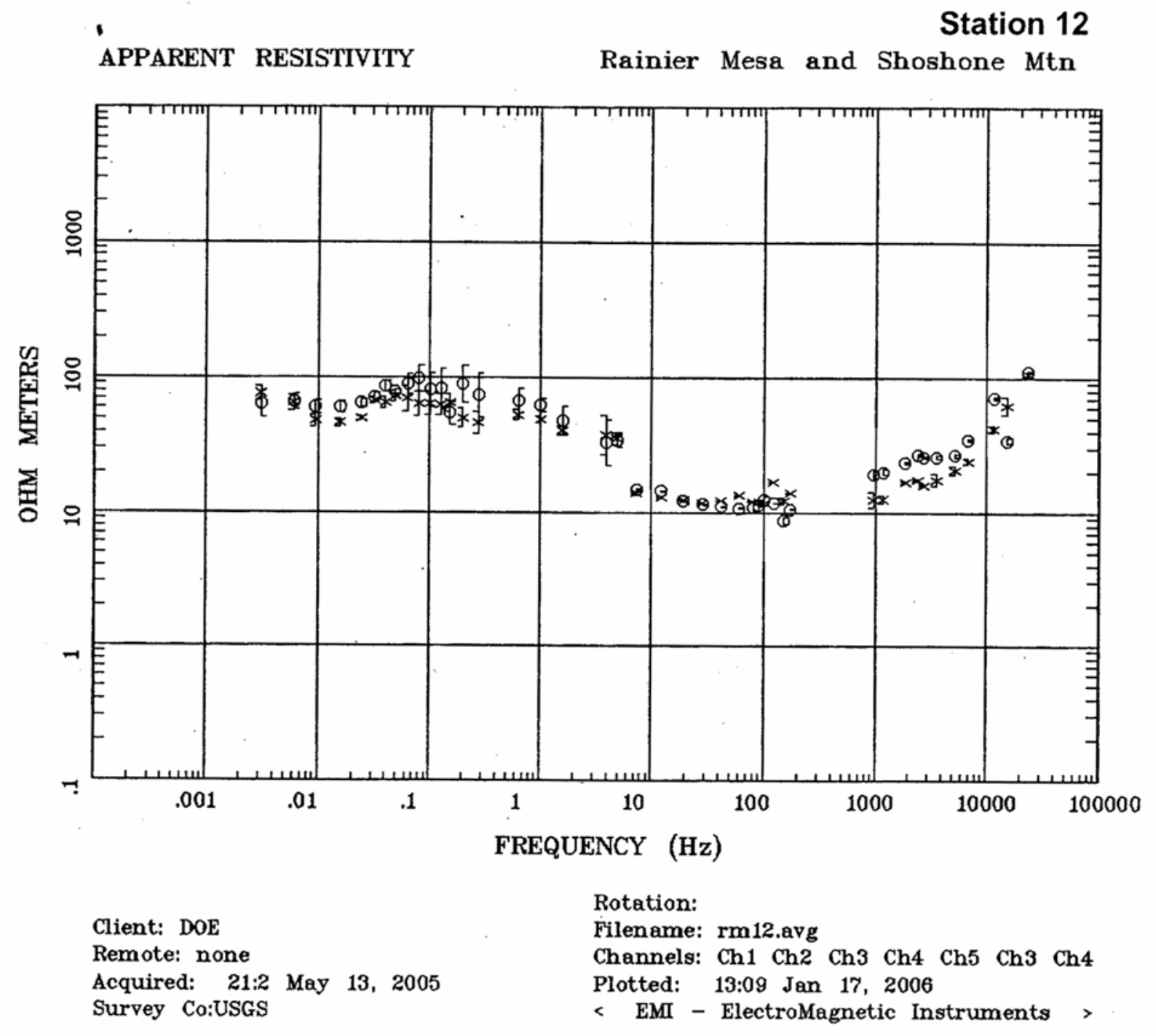


v

IMPEDANCE PHASE

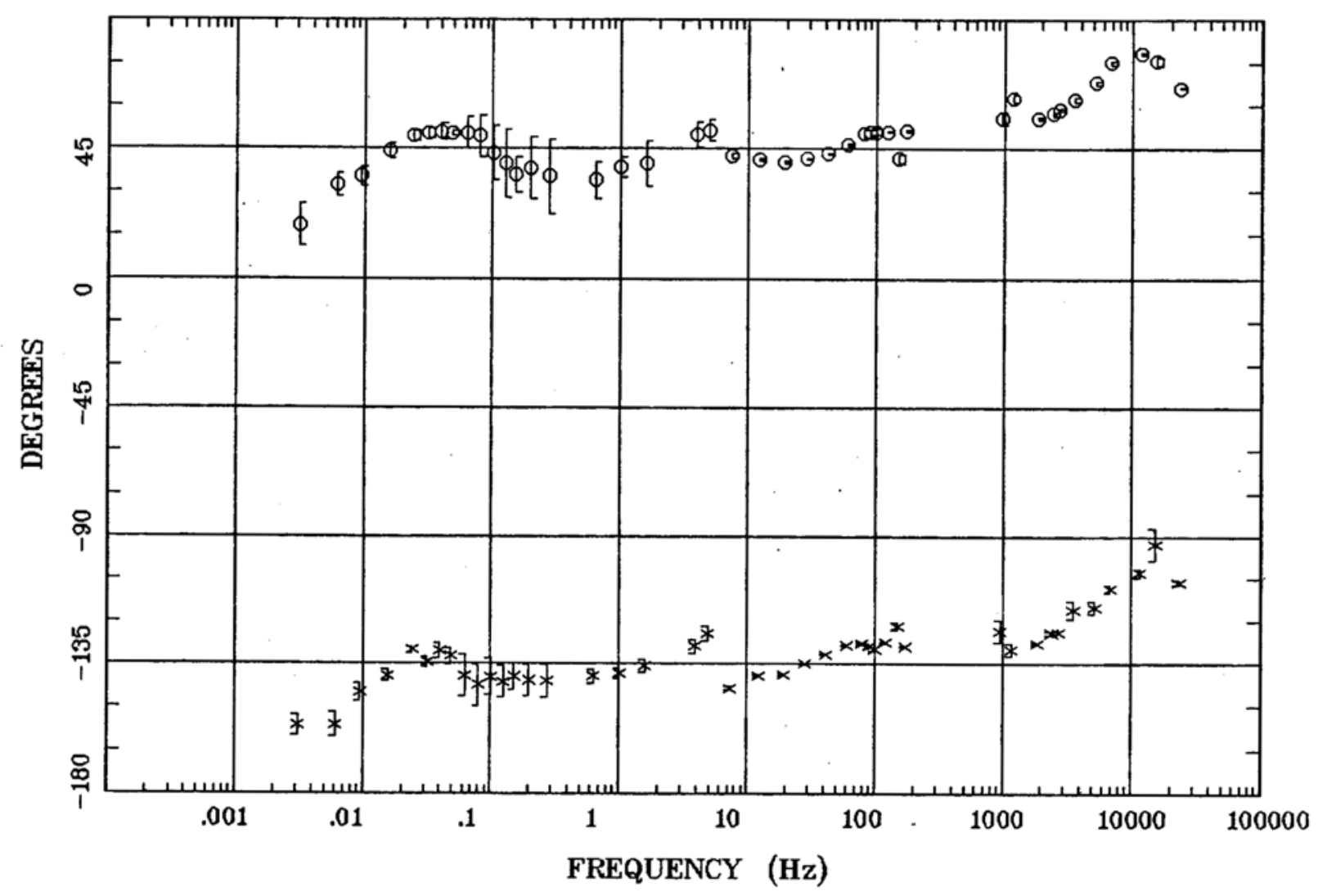

Station 12

Rainier Mesa and Shoshone Mtn
Client: DOE

Remote: none

Acquired: 21:2 May 13, 2005

Survey Co:USGS 


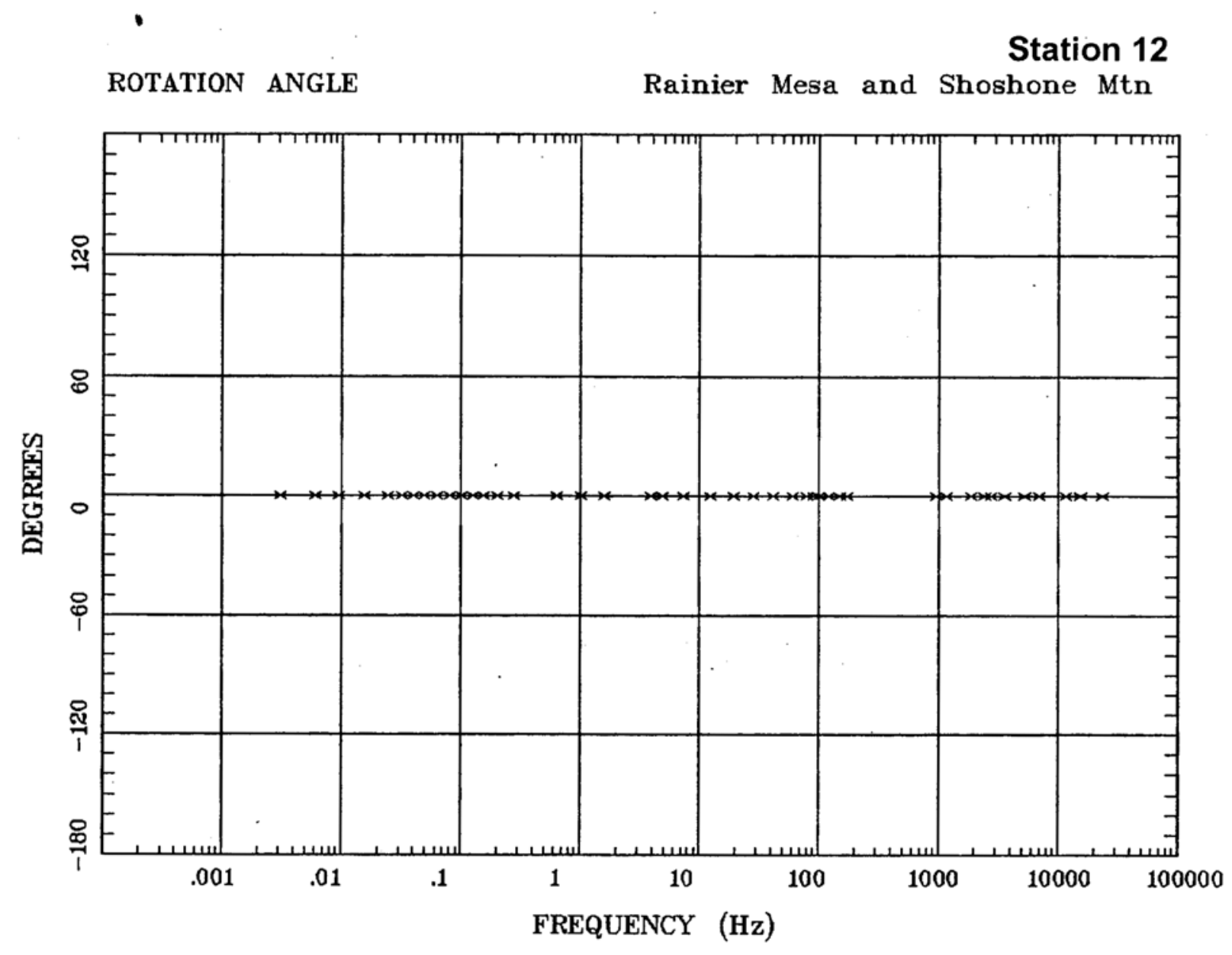

Client: DOE

Remote: none

Acquired: 21:2 May 13, 2005

Survey Co:USGS
Rotation:

Filename: rm12.avg

Channels: Ch1 Ch2 Ch3 Ch4 Ch5 Ch3 Ch4

Plotted: 13:09 Jan 17, 2006

< EMI - ElectroMagnetic Instruments > 


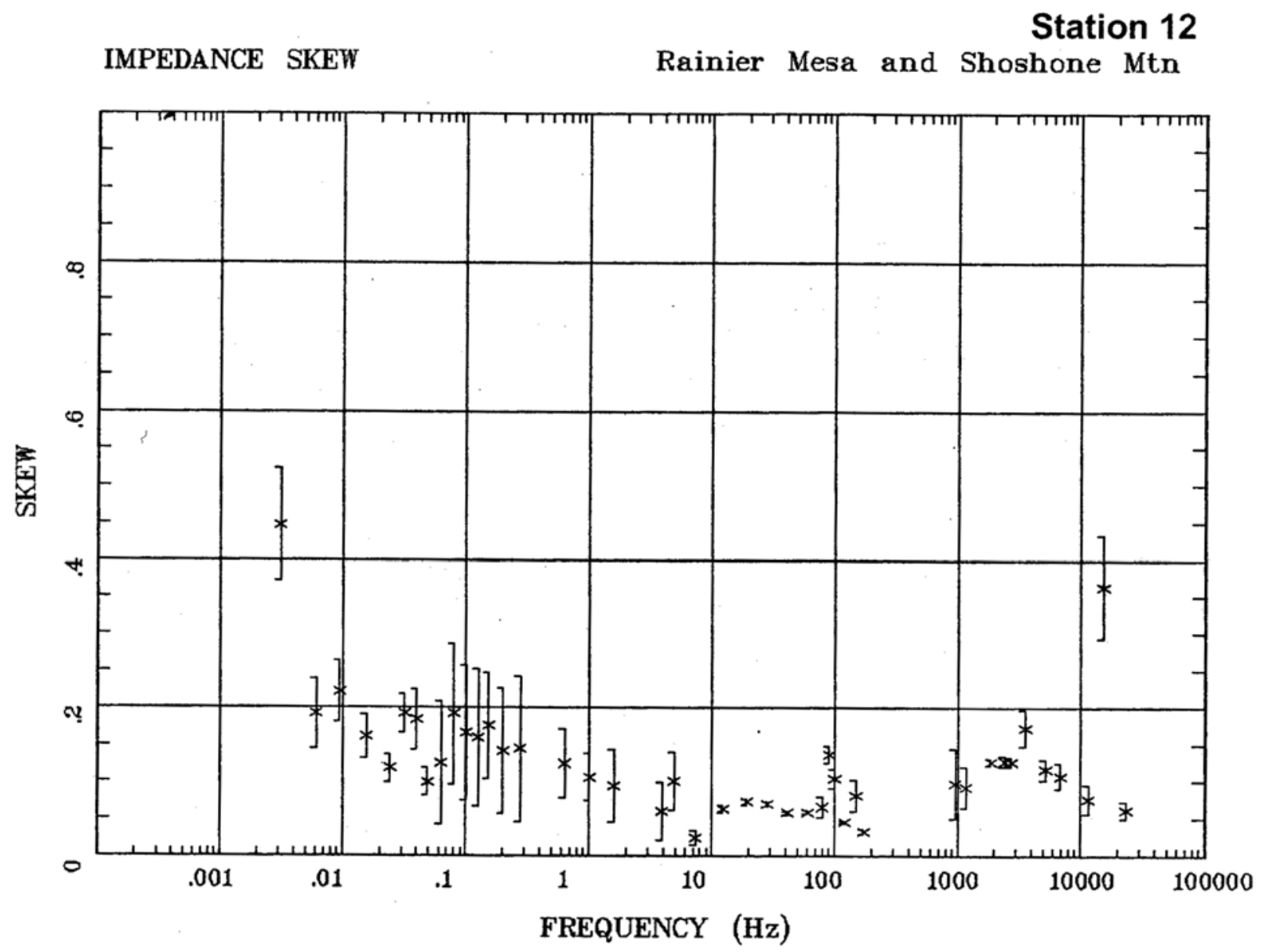

Client: DOE

Remote: none

Acquired: 21:2 May 13, 2005

Survey Co:USGS
Rotation:

Filename: rm12.avg

Channels: Ch1 Ch2 Ch3 Ch4 Ch5 Ch3 Ch4

Plotted: 13:09 Jan 17, 2006

< EMI - ElectroMagnetic Instruments > 
Station 12

E MULT Coh.

Rainier Mesa and Shoshone Mtn

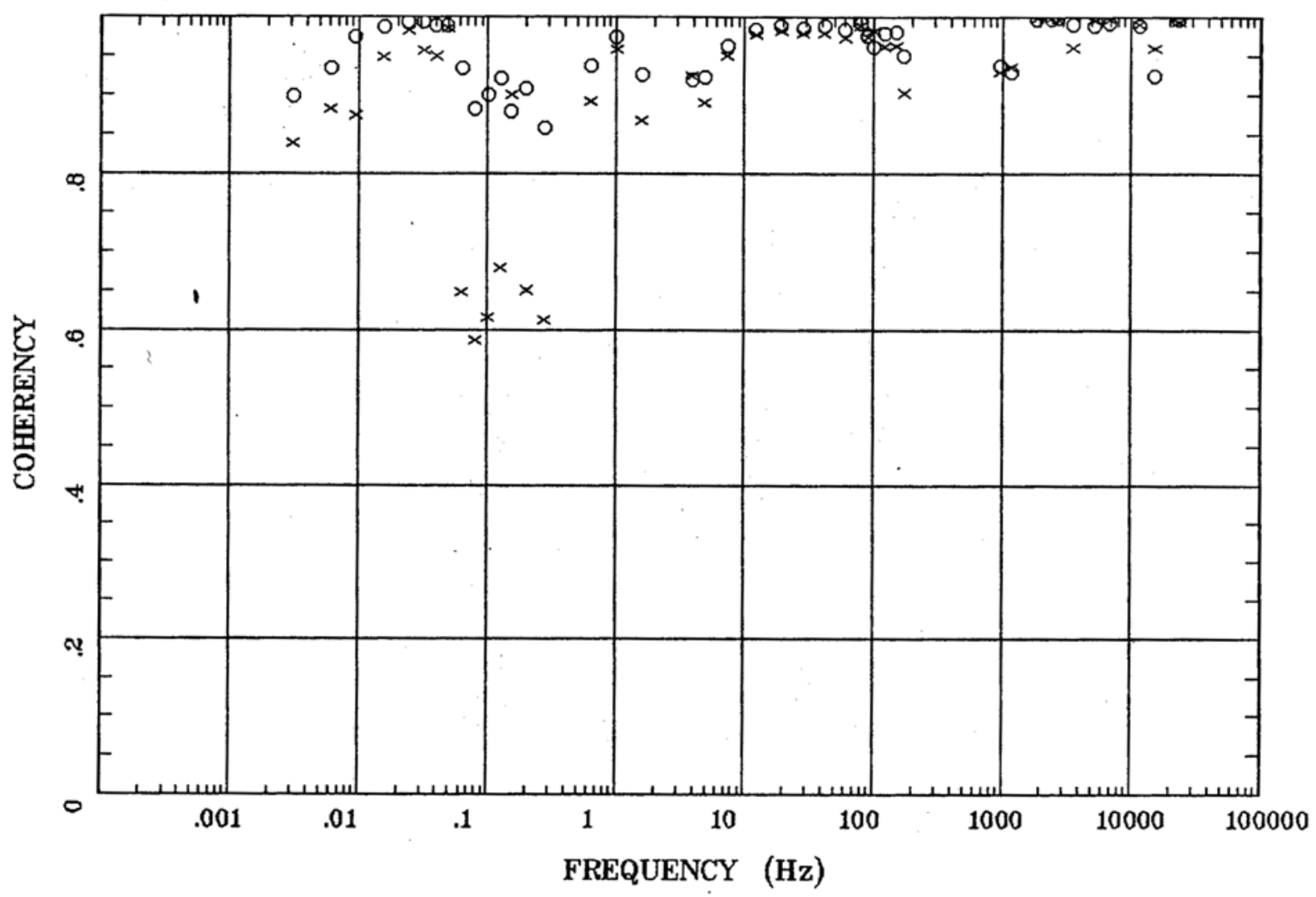

Client: DOE

Remote: none

Acquired: 21:2 May 13, 2005

Survey Co:USGS
Rotation:

Filename: rm12.avg

Channels: Ch1 Ch2 Ch3 Ch4 Ch5 Ch3 Ch4

Plotted: 13:09 Jan 17, 2006

< EMI - ElectroMagnetic Instruments > 


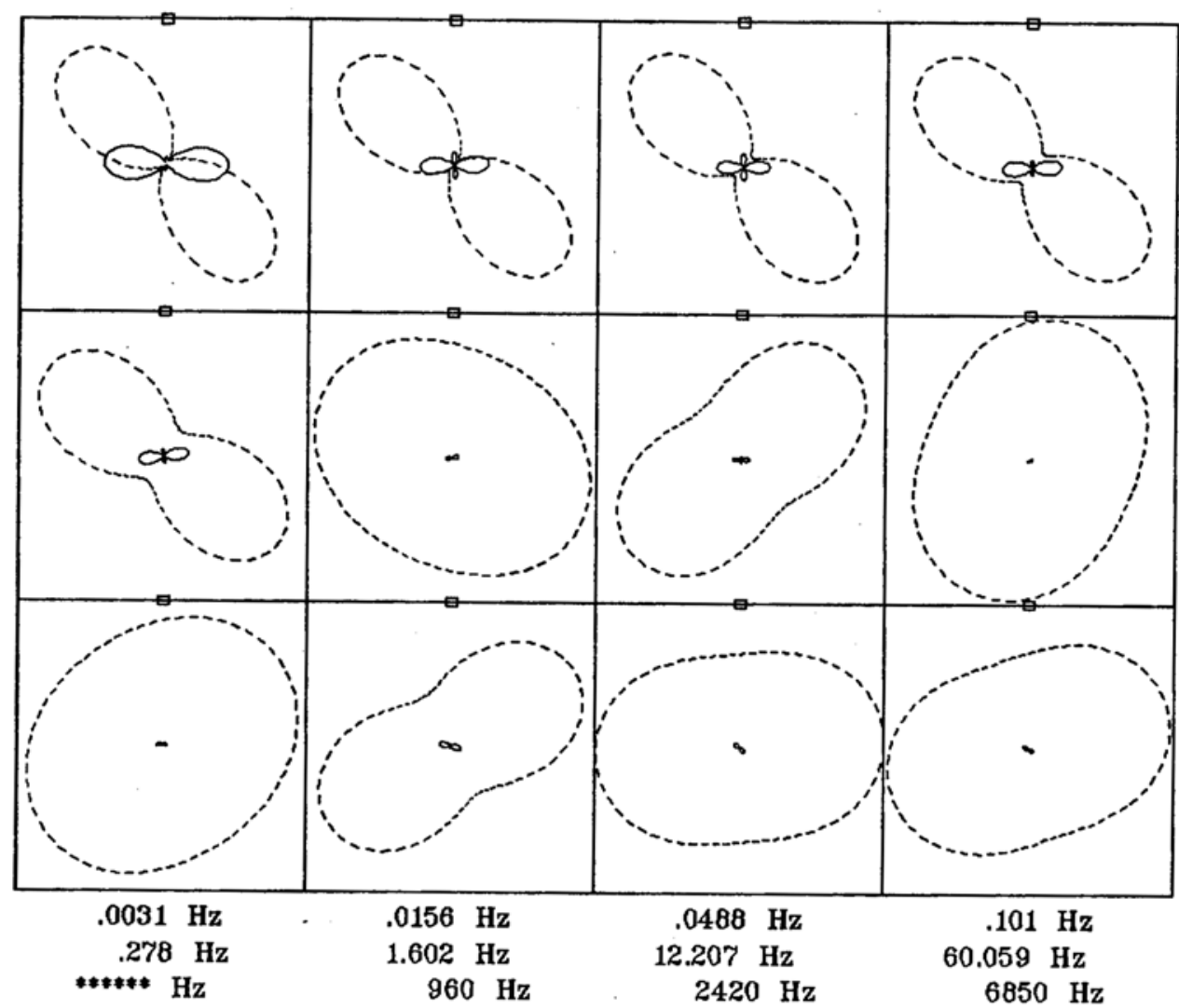

Client: DOE

Remote: none

Acquired: 21:2 May 13, 2005

Survey Co:USGS
Rainier Mesa and Shoshone Mtn

Rotation:

Filename: rm12.avg

Channels: Ch1 Ch2 Ch3 Ch4 Ch5 Ch3 Ch4

Plotted: 13:09 Jan 17, 2006

< EMI - ElectroMagnetic Instruments > 
Station 12

TIPPER MAGNITUDE

Rainier Mesa and Shoshone Mtn

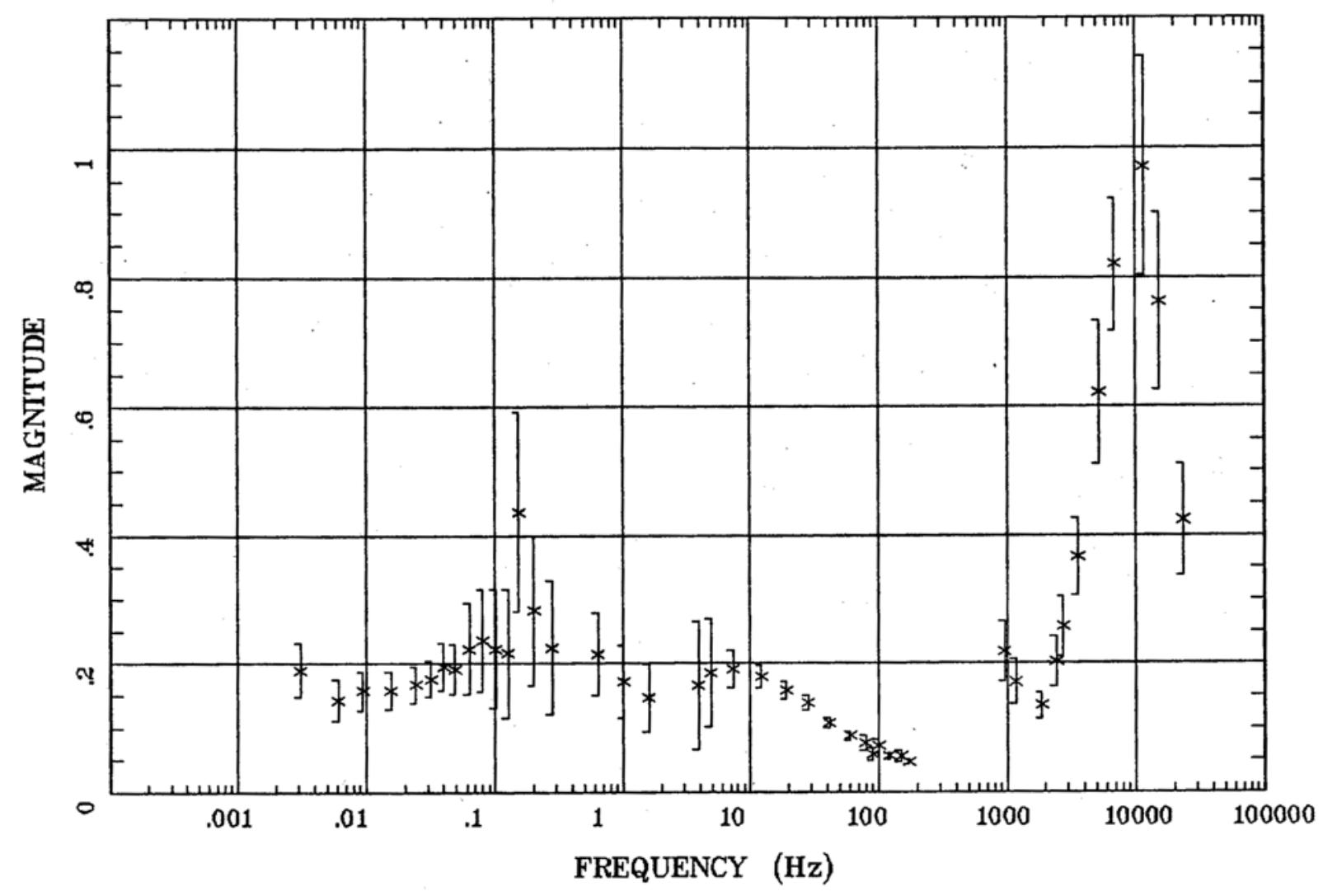

Client: DOE

Remote: none

Acquired: 21:2 May 13, 2005

Survey Co:USGS
Rotation:

Filename: rm12.avg

Channels: Ch1 Ch2 Ch3 Ch4 Ch5 Ch3 Ch4

Plotted: 13:09 Jan 17, 2006

< EMI - ElectroMagnetic Instruments > 
TIPPER STRIKE

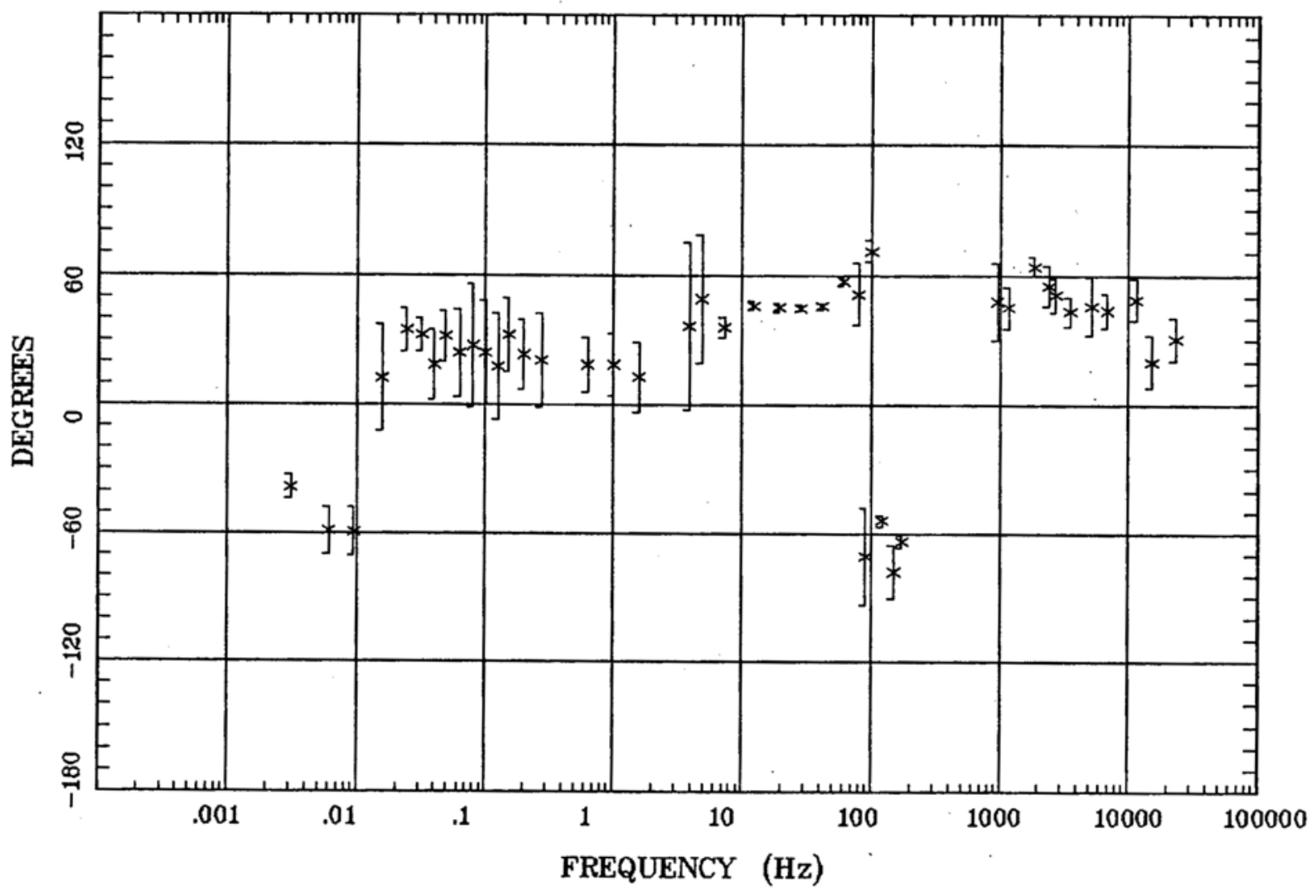

Client: DOE

Remote: none

Acquired: 21:2 May 13, 2005

Survey Co:USGS

Rainier Mesa and Shoshone Mtn

Station 12 


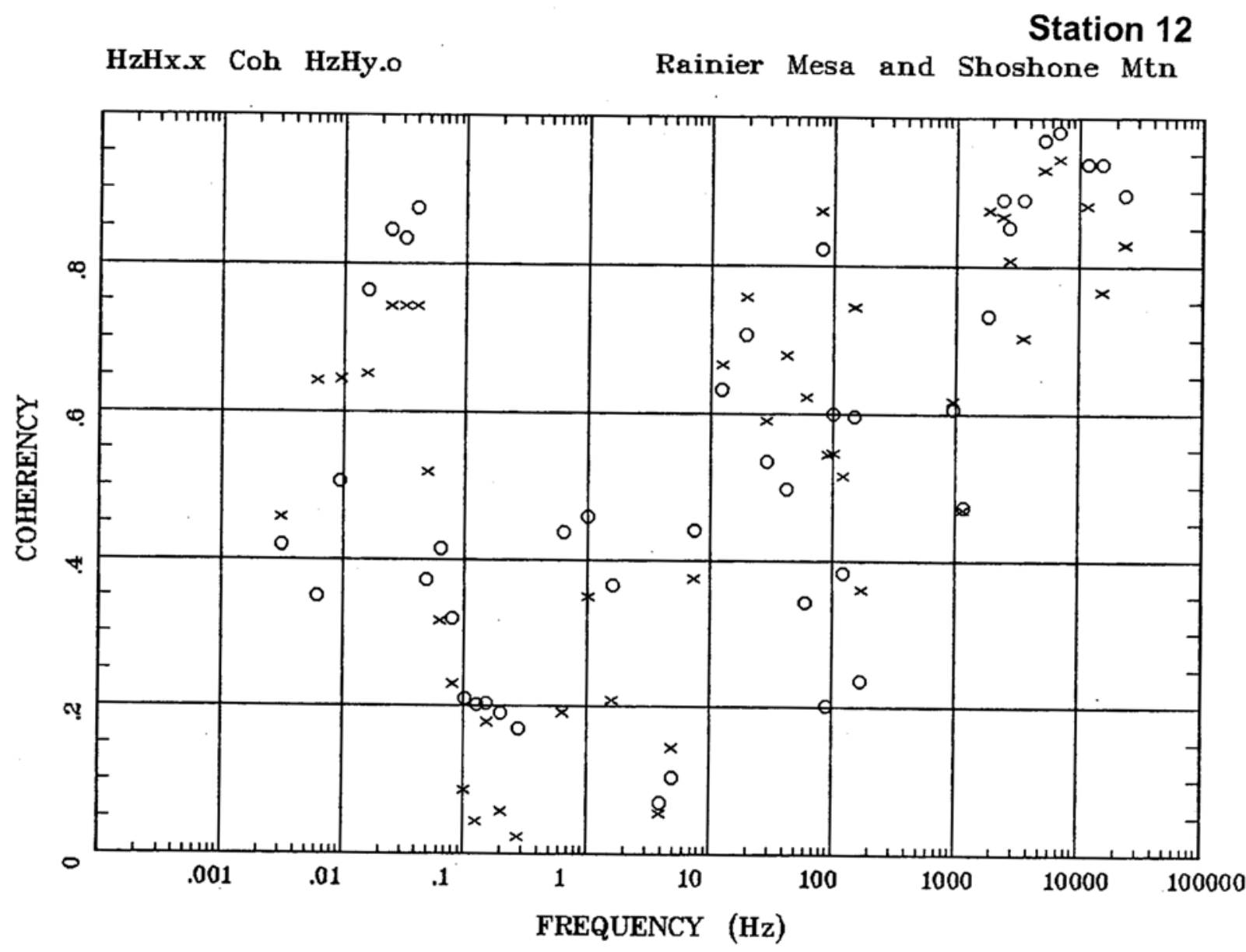

Client: DOE

Remote: none

Acquired: 21:2 May 13, 2005

Survey Co:USGS
Rotation:

Filename: rm12.avg

Channels: Ch1 Ch2 Ch3 Ch4 Ch5 Ch3 Ch4

Plotted: 13:09 Jan 17, 2006

< EMI - ElectroMagnetic Instruments > 
Station 13

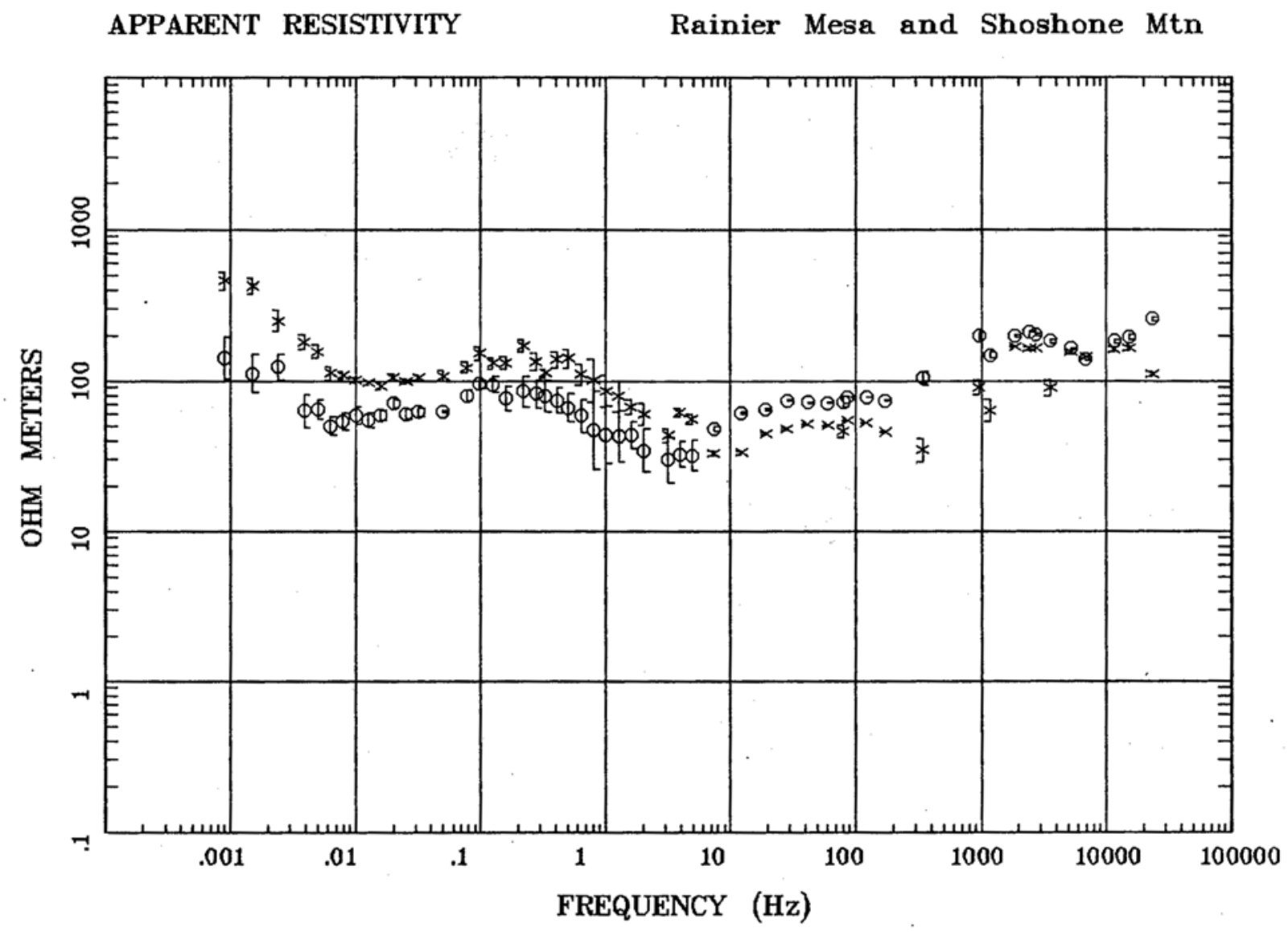

Client: DOE

Remote: none

Acquired: 00:4 May 12, 2005

Survey Co:USGS
Rotation:

Filename: rm13.avg

Channels: Ch1 Ch2 Ch3 Ch4 Ch5 Ch3 Ch4

Plotted: 13:19 Jan 17, 2006

< EMI - ElectroMagnetic Instruments > 
Station 13

IMPEDANCE PHASE

Rainier Mesa and Shoshone Mtn

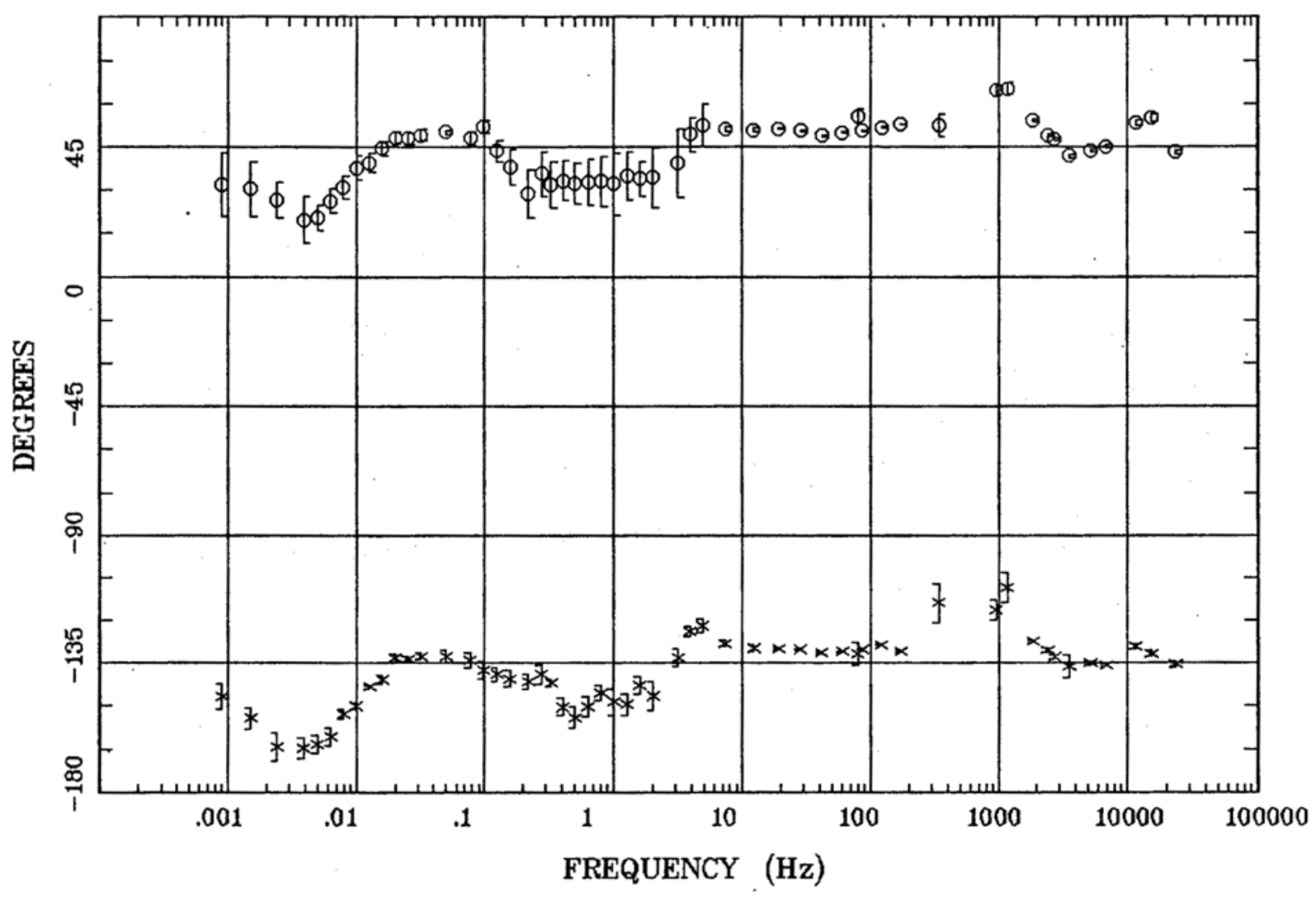

Client: DOE

Remote: none

Acquired: 00:4 May 12, 2005

Survey Co:USGS
Rotation:

Filename: rm13.avg

Channels: Ch1 Ch2 Ch3 Ch4 Ch5 Ch3 Ch4

Plotted: 13:19 Jan 17, 2006

< EMI - ElectroMagnetic Instruments > 


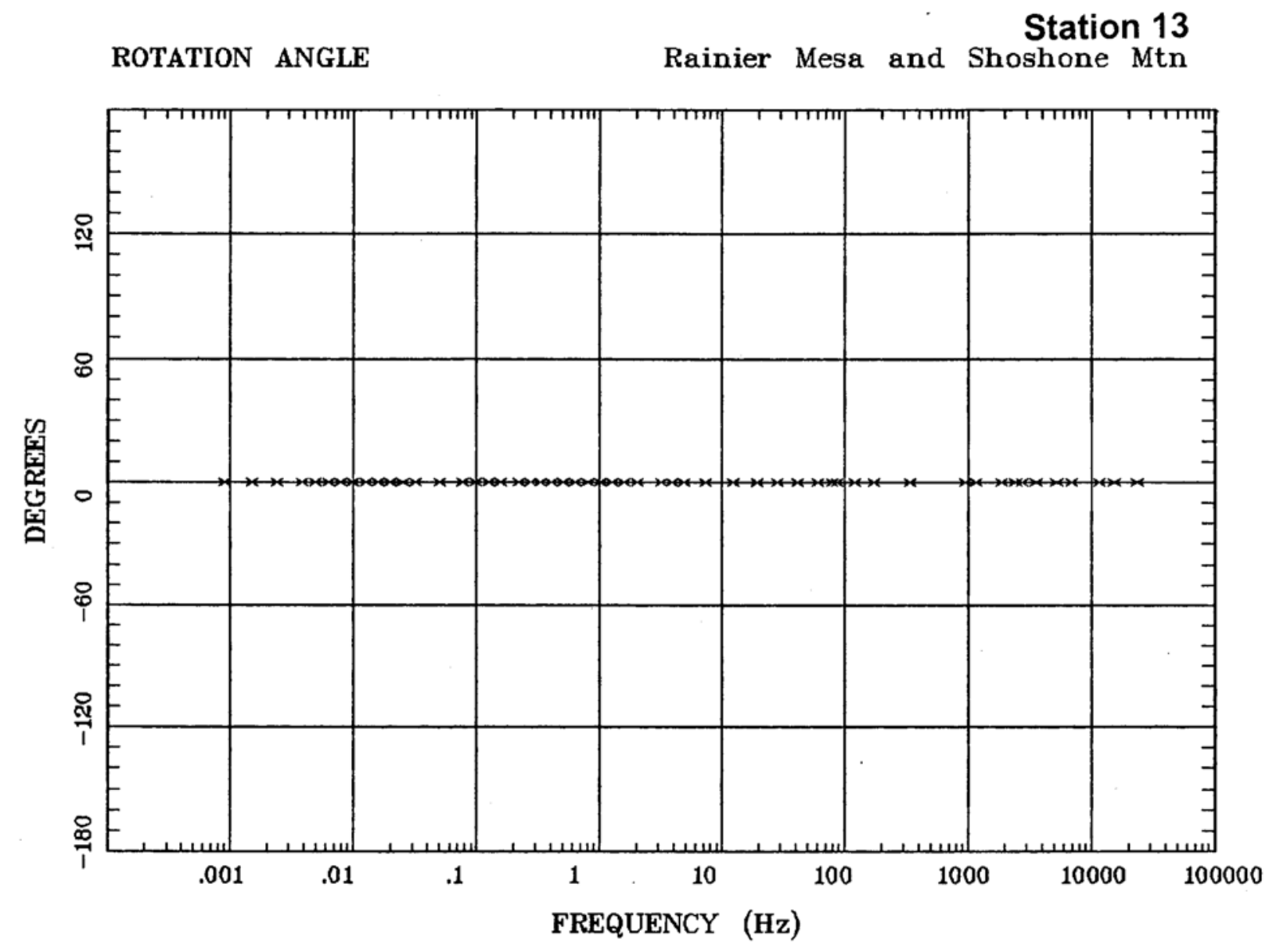

Client: DOE

Remote: none

Acquired: 00:4 May 12, 2005

Survey Co:USGS
Rotation:

Filename: rm13.avg

Channels: Ch1 Ch2 Ch3 Ch4 Ch5 Ch3 Ch4

Plotted: 13:19 Jan 17, 2006

< EMI - ElectroMagnetic Instruments > 


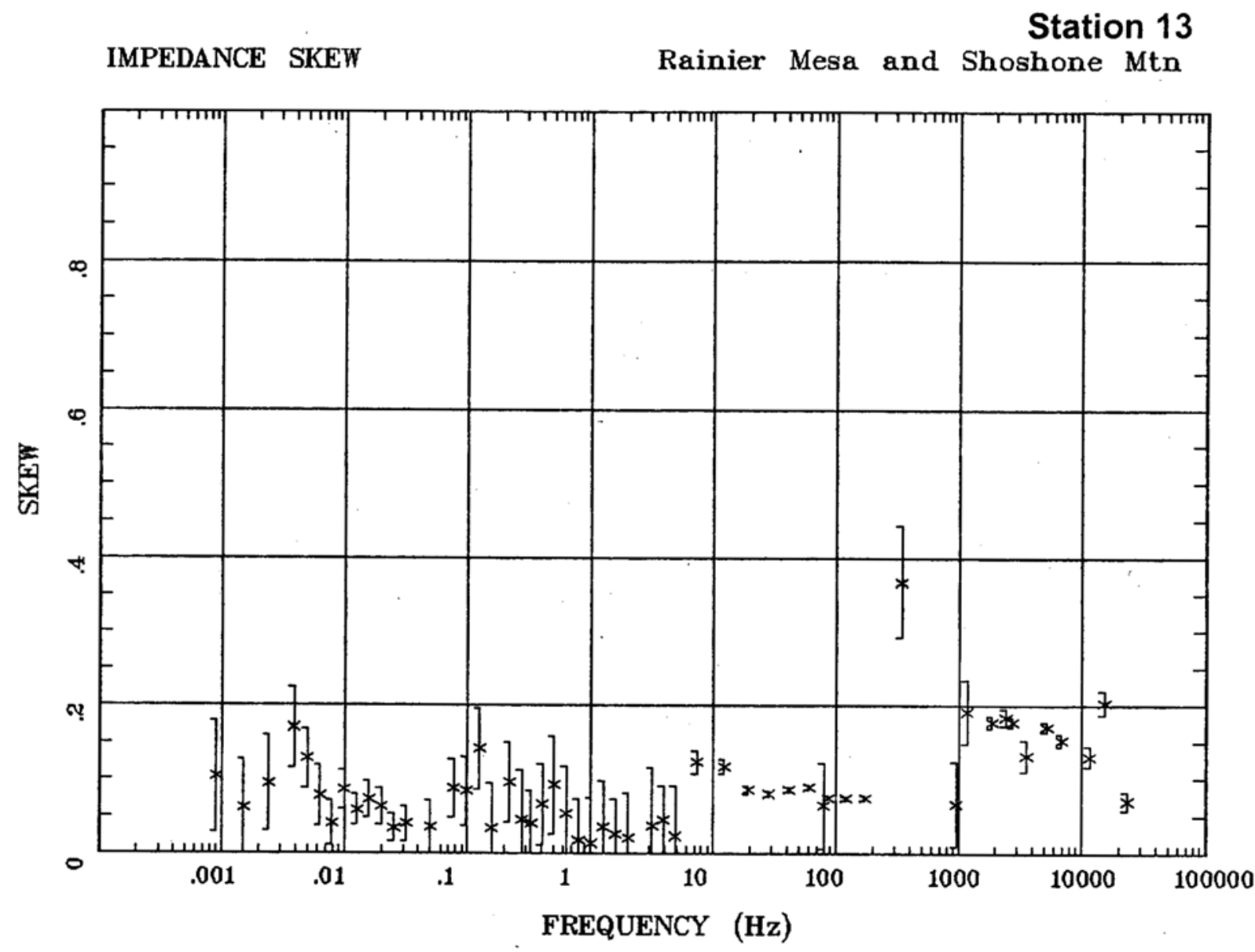

Client: DOE

Remote: none

Acquired: 00:4 May 12, 2005

Survey Co:USGS
Rotation:

Filename: rm13.avg

Channels: Ch1 Ch2 Ch3 Ch4 Ch5 Ch3 Ch4

Plotted: 13:19 Jan 17, 2006

< EMI - ElectroMagnetic Instruments > 
Station 13

E MULT Coh.

Rainier Mesa and Shoshone Mtn

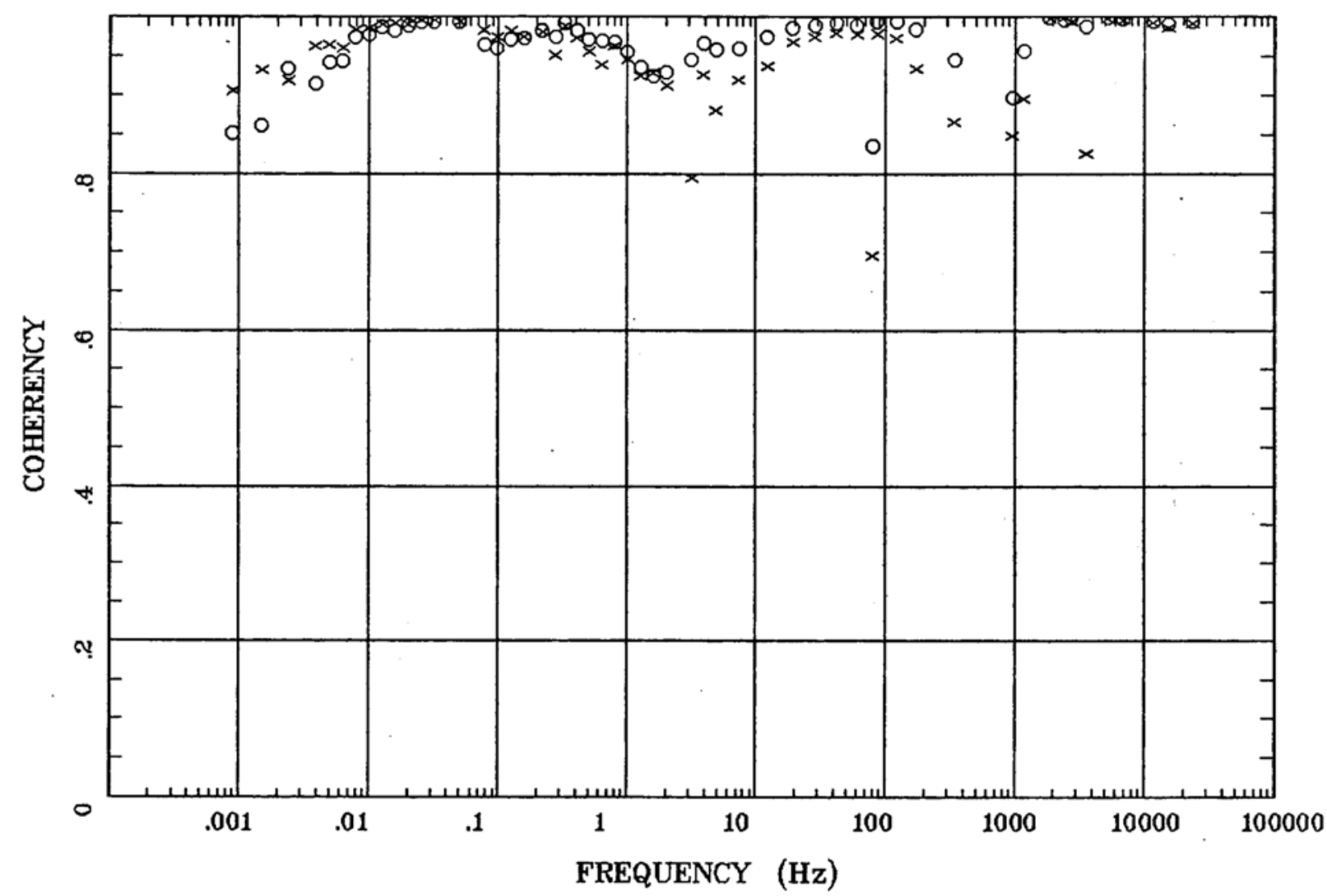

Client: DOE

Remote: none

Acquired: 00:4 May 12, 2005

Survey Co:USGS
Rotation:

Filename: rm13.avg

Channels: Ch1 Ch2 Ch3 Ch4 Ch5 Ch3 Ch4

Plotted: 13:19 Jan 17, 2006

< EMI - ElectroMagnetic Instruments > 
Station 13

POLAR PLOTS

Rainier Mesa and Shoshone Mtn

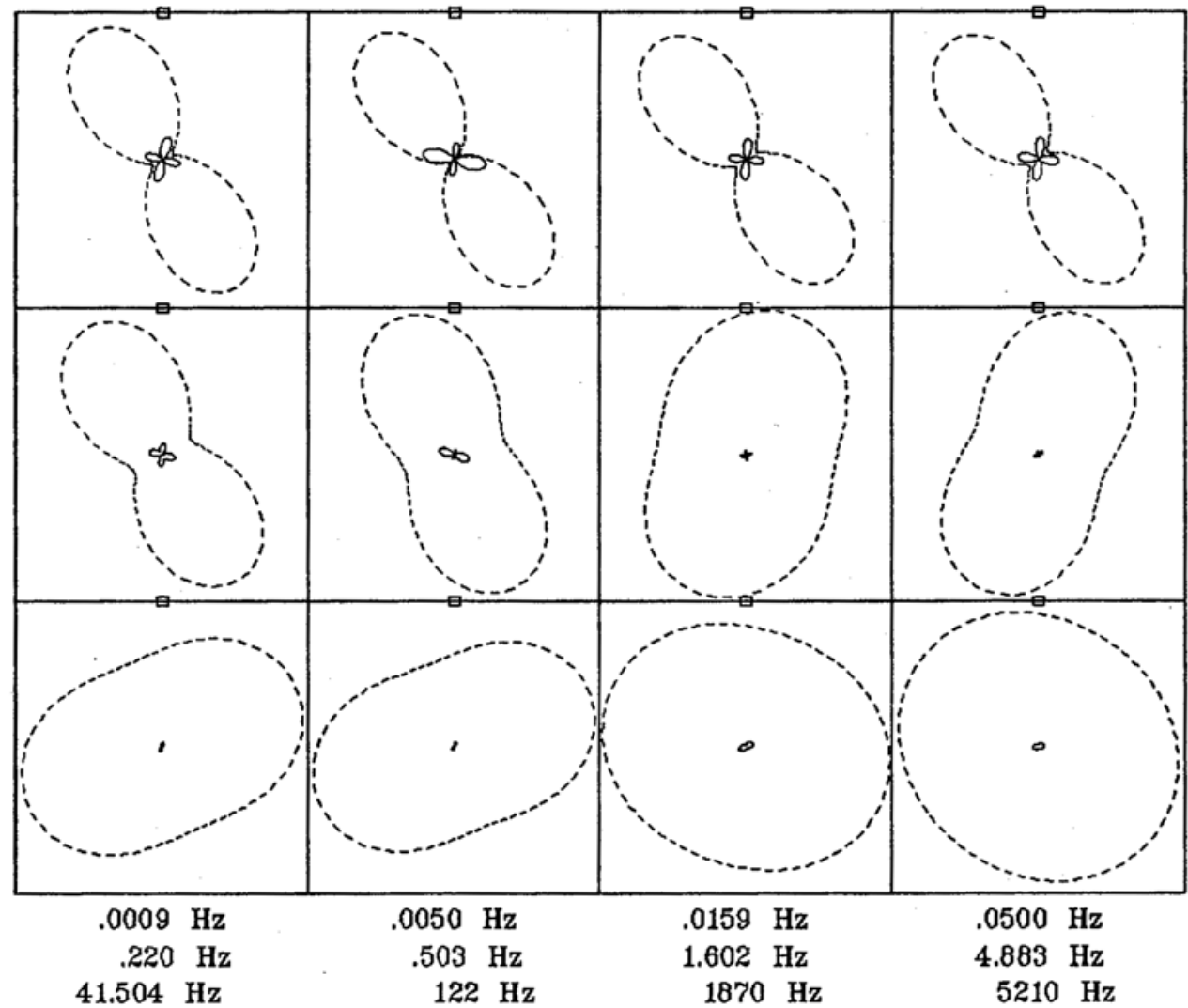

Client: DOE

Remote: none

Acquired: 00:4 May 12, 2005

Survey Co:USGS
Rotation:

Filename: rm13.avg

Channels: Ch1 Ch2 Ch3 Ch4 Ch5 Ch3 Ch4

Plotted: 13:19 Jan 17, 2006

< EMI - ElectroMagnetic Instruments > 
Station 13

TIPPER MAGNITUDE

Rainier Mesa and Shoshone Mtn

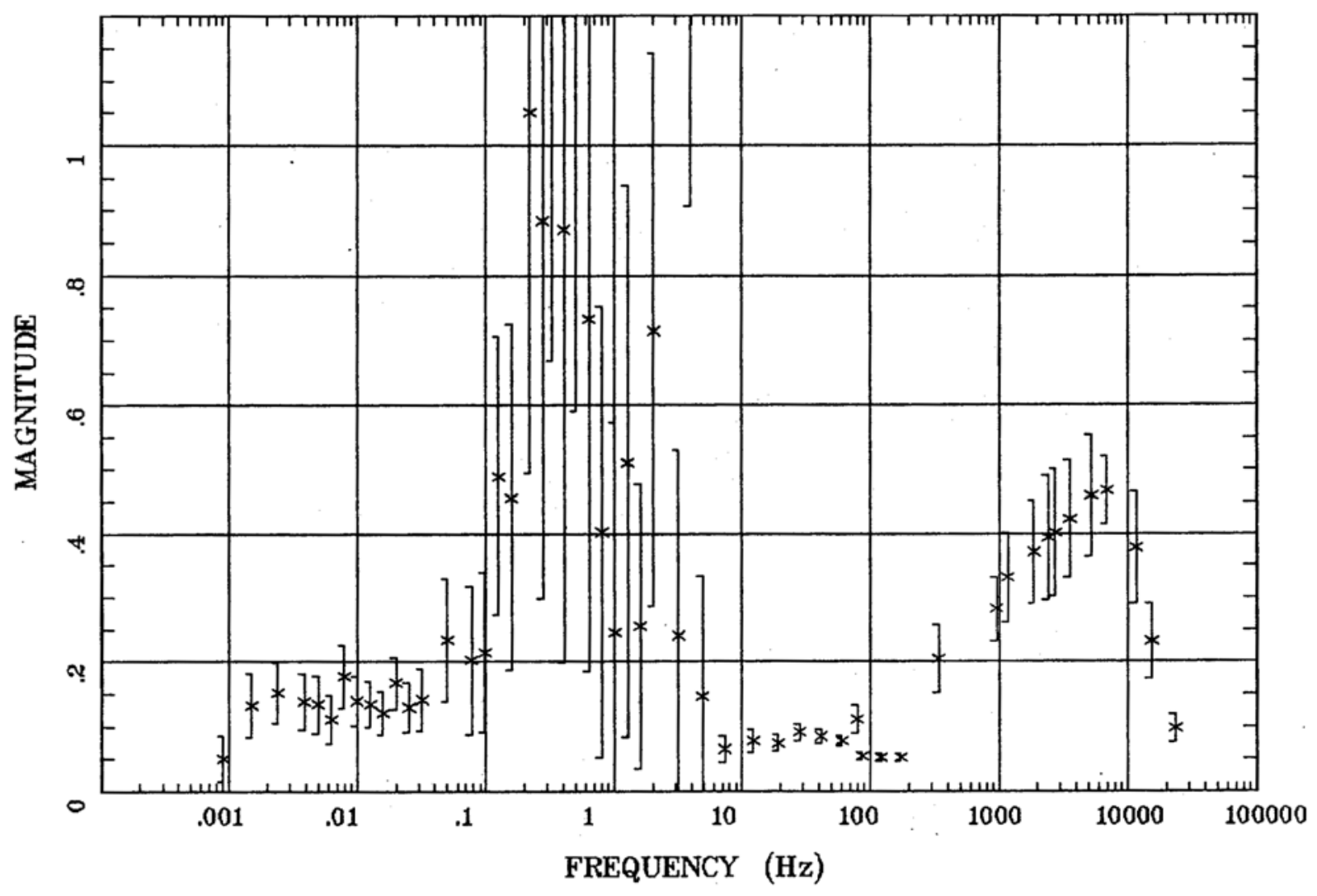

Client: DOE

Remote: none

Acquired: 00:4 May 12, 2005

Survey Co:USGS
Rotation:

Filename: rm13.avg

Channels: Ch1 Ch2 Ch3 Ch4 Ch5 Ch3 Ch4

Plotted: 13:19 Jan 17, 2006

< EMI - ElectroMagnetic Instruments > 


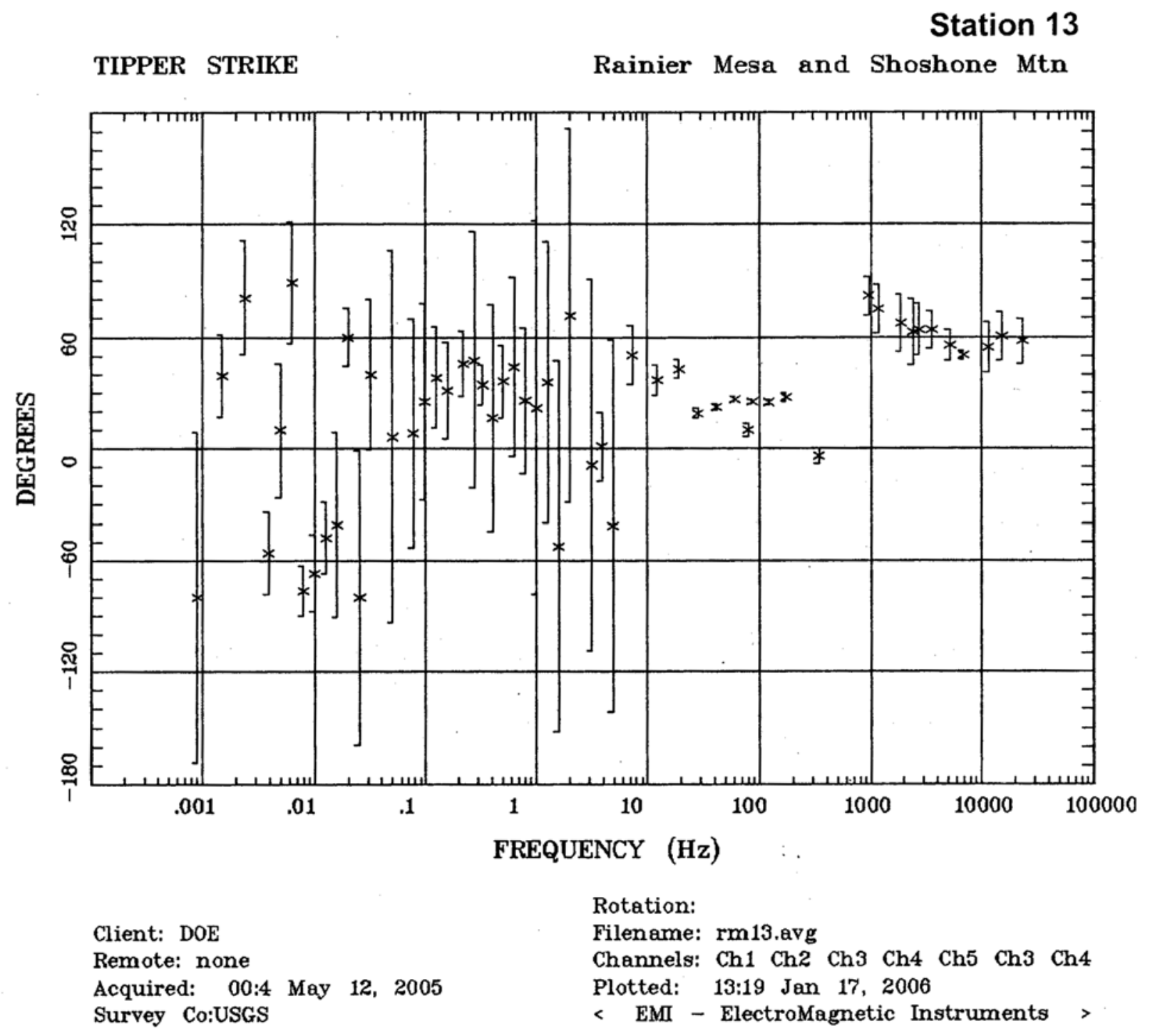


HzHx.x Coh HzHy.o

Station 13

Rainier Mesa and Shoshone Mtn

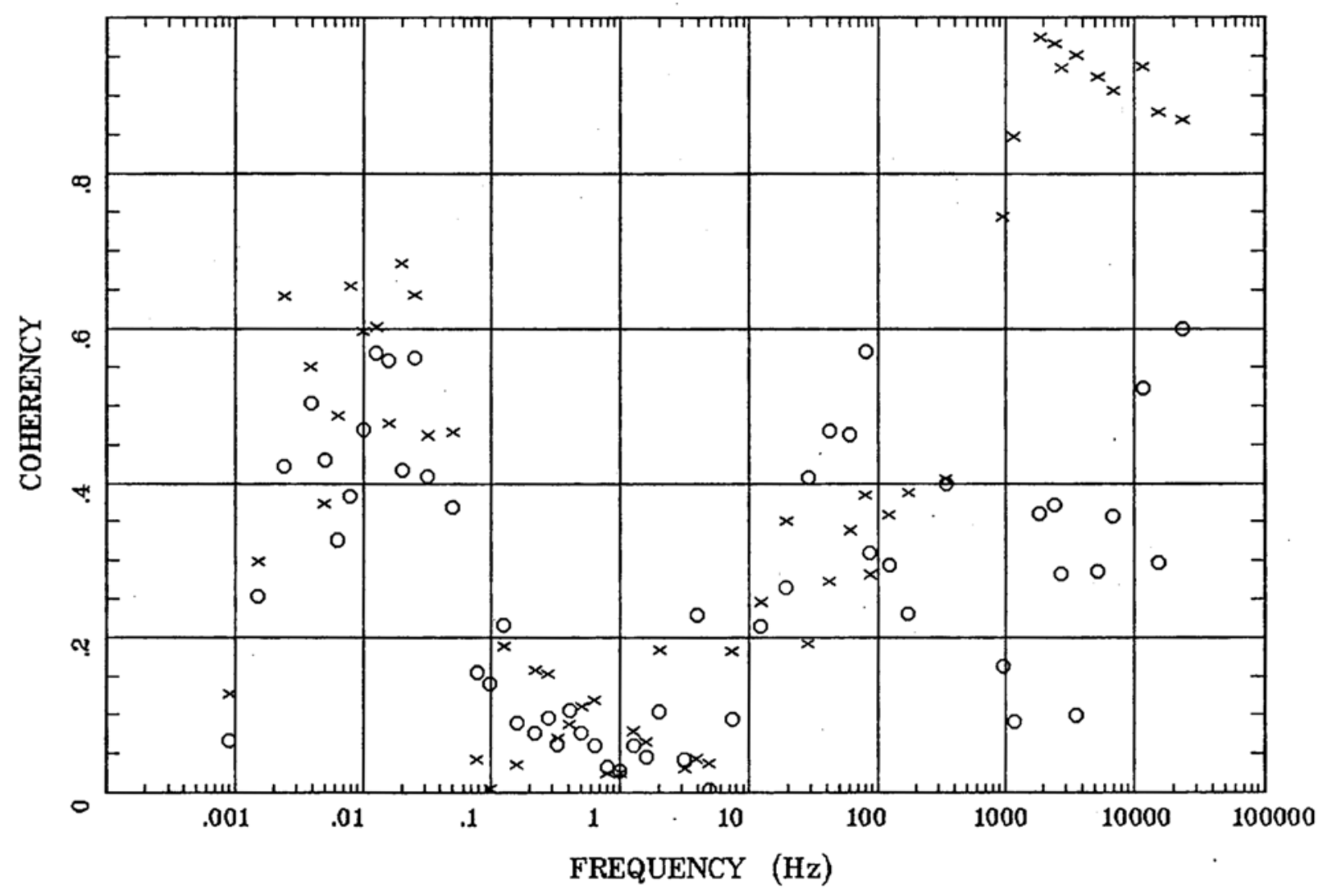

Client: DOE

Remote: none

Acquired: 00:4 May 12, 2005

Survey Co:USGS
Rotation:

Filename: rm13.avg

Channels: Ch1 Ch2 Ch3 Ch4 Ch5 Ch3 Ch4

Plotted: 13:19 Jan 17, 2006

< EMI - ElectroMagnetic Instruments > 


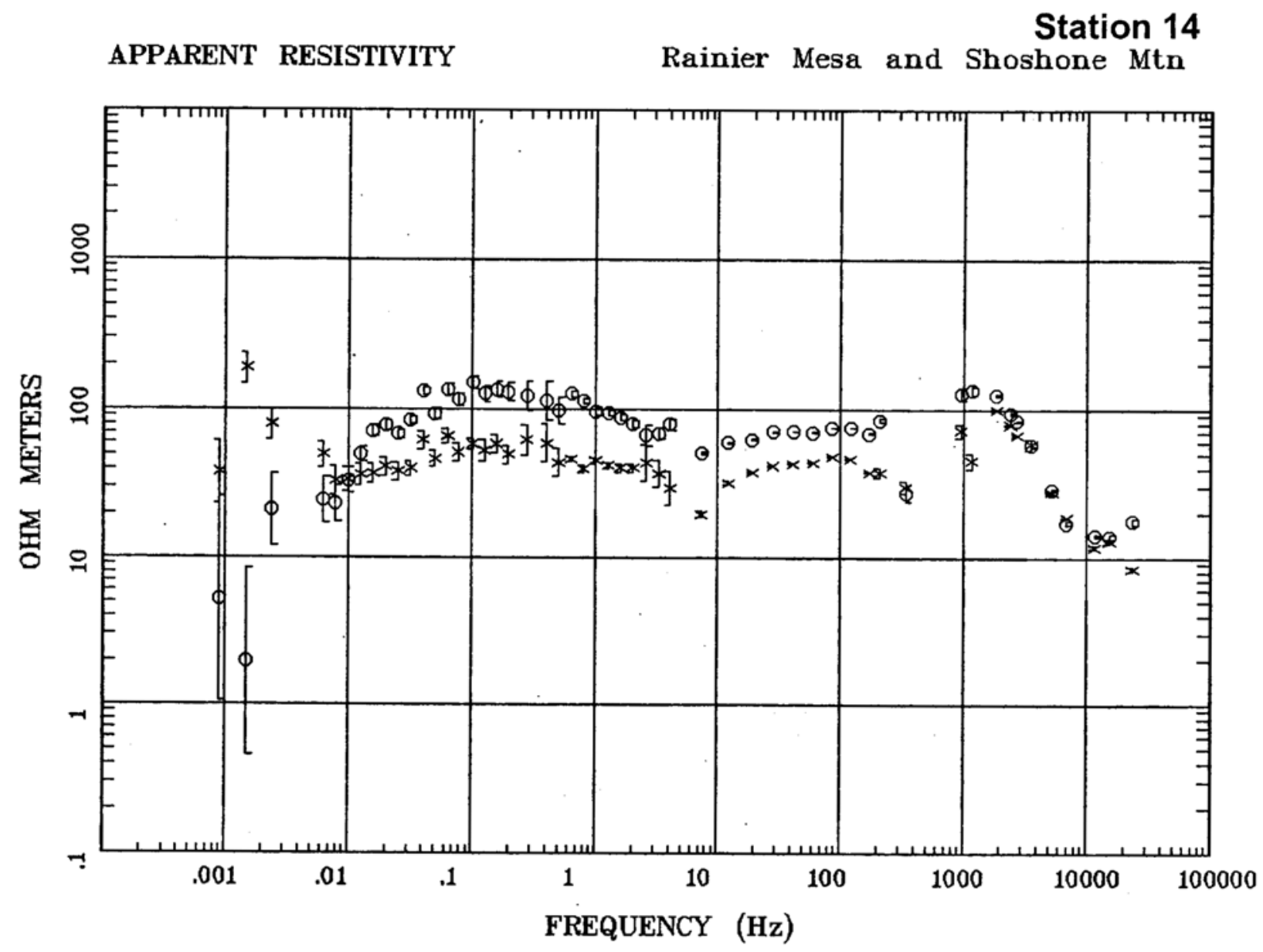

Client: DOE

Remote: none

Acquired: 21:5 May 10, 2005

Survey Co:USGS
Rotation:

Filename: rm14.avg

Channels: Ch1 Ch2 Ch3 Ch4 Ch5 Ch3 Ch4

Plotted: 13:21 Jan 17, 2006

< EMI - ElectroMagnetic Instruments > 
Station 14

IMPEDANCE PHASE

Rainier Mesa and Shoshone Mtn

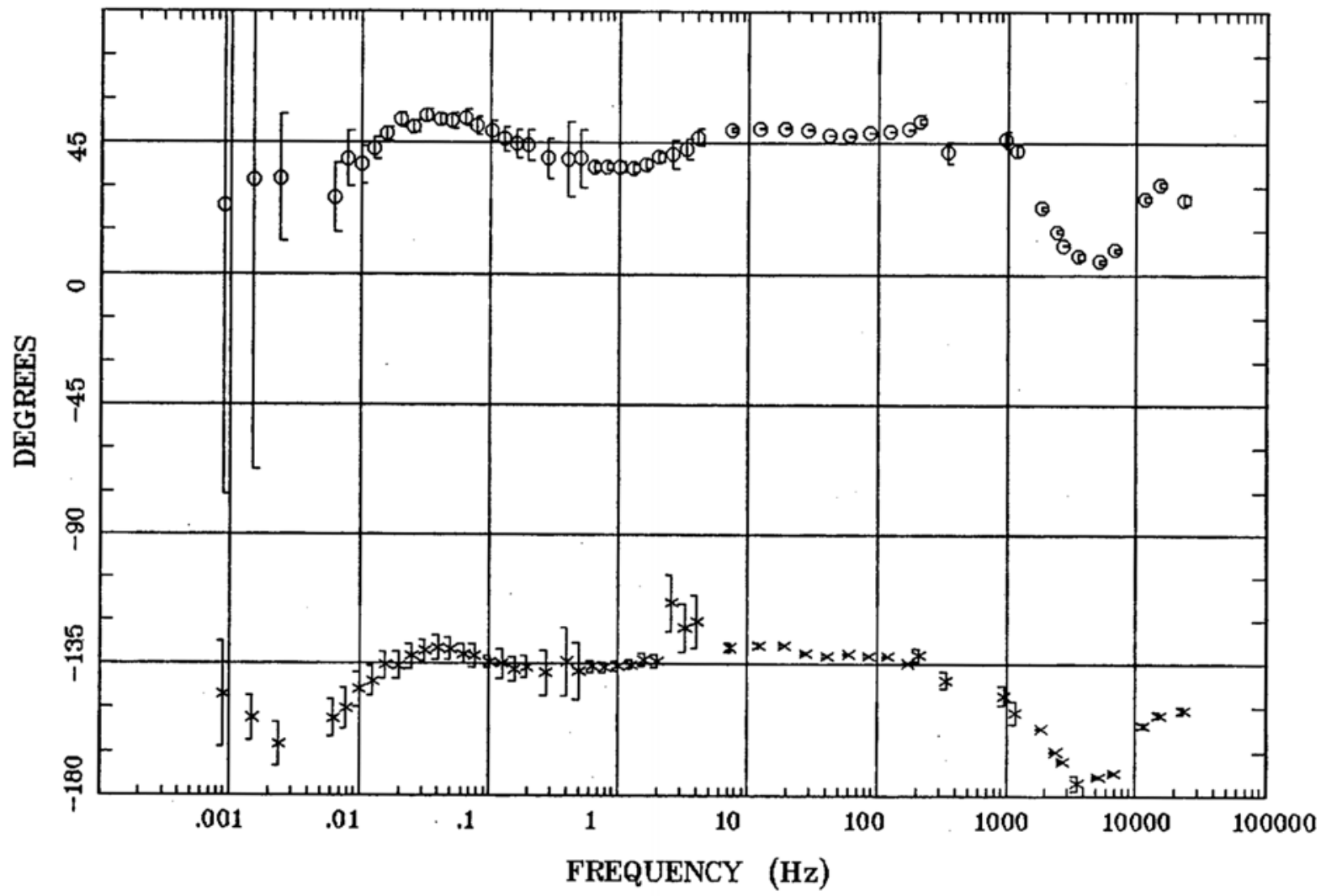

Client: DOE

Remote: none

Acquired: 21:5 May 10, 2005

Survey Co:USGS
Rotation:

Filename: rm14.avg

Channels: Ch1 Ch2 Ch3 Ch4 Ch5 Ch3 Ch4

Plotted: 13:21 Jan 17, 2006

< EMI - ElectroMagnetic Instruments > 


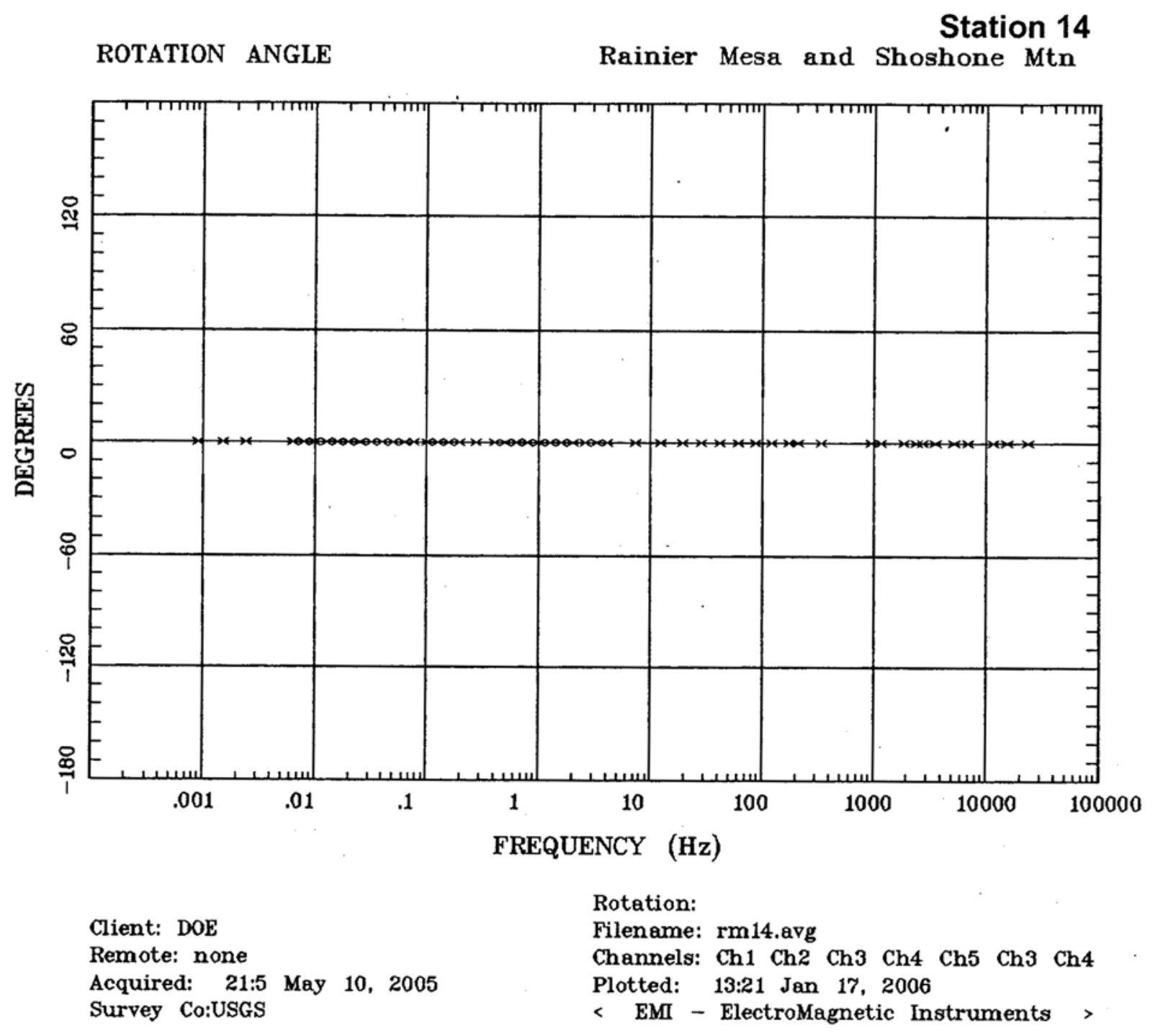




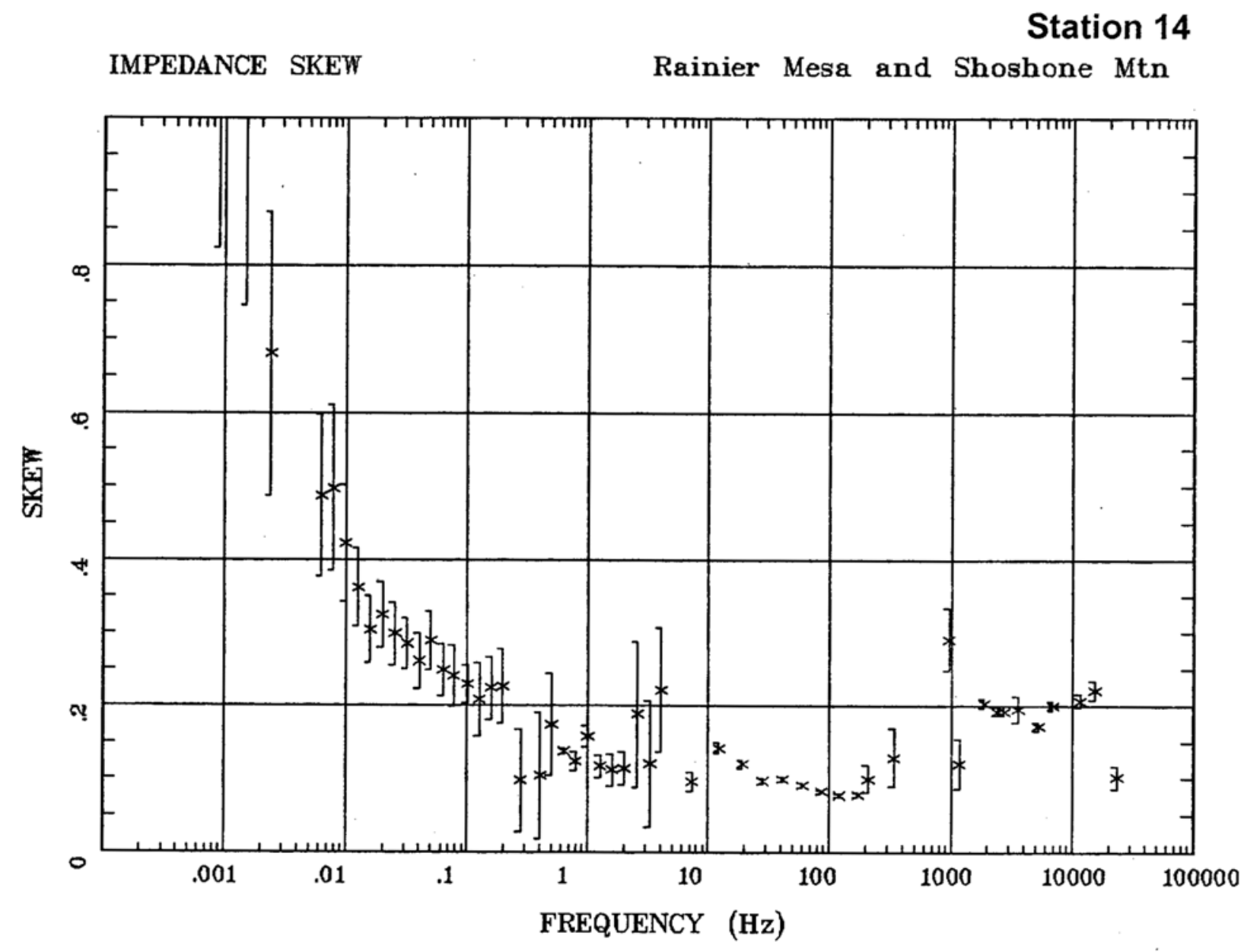

Client: DOE

Remote: none

Acquired: 21:5 May 10, 2005

Survey Co:USGS
Rotation:

Filename: rm14.avg

Channels: Ch1 Ch2 Ch3 Ch4 Ch5 Ch3 Ch4

Plotted: 13:21 Jan 17, 2006

< EMI - ElectroMagnetic Instruments > 
E MULT Coh.

Station 14

Rainier Mesa and Shoshone Mtn

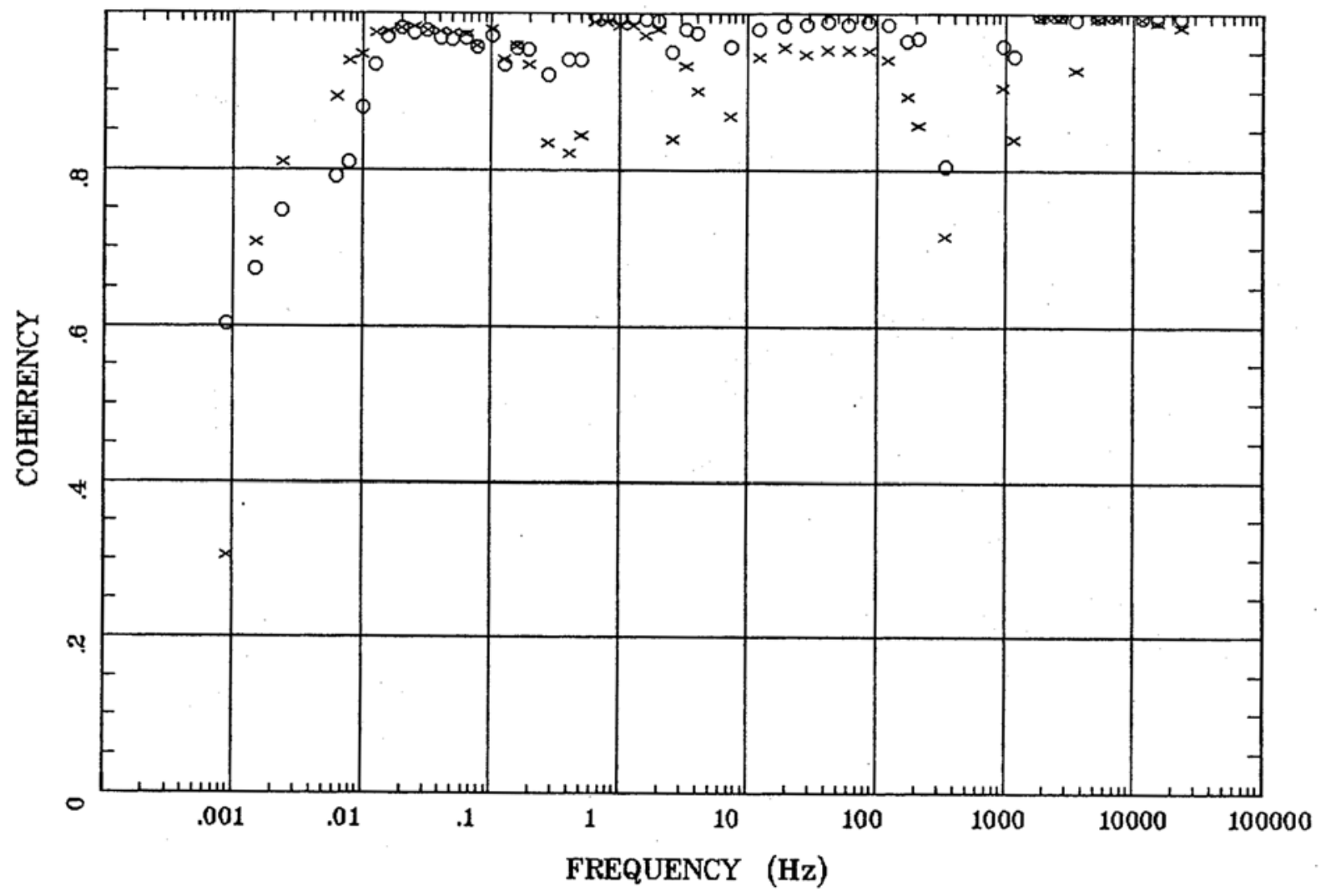

Client: DOE

Remote: none

Acquired: 21:5 May 10, 2005

Survey Co:USGS
Rotation:

Filename: rm14.avg

Channels: Ch1 Ch2 Ch3 Ch4 Ch5 Ch3 Ch4

Plotted: 13:21 Jan 17, 2006

< EMI - ElectroMagnetic Instruments > 
Station 14

POLAR PLOTS

Rainier Mesa and Shoshone Mtn

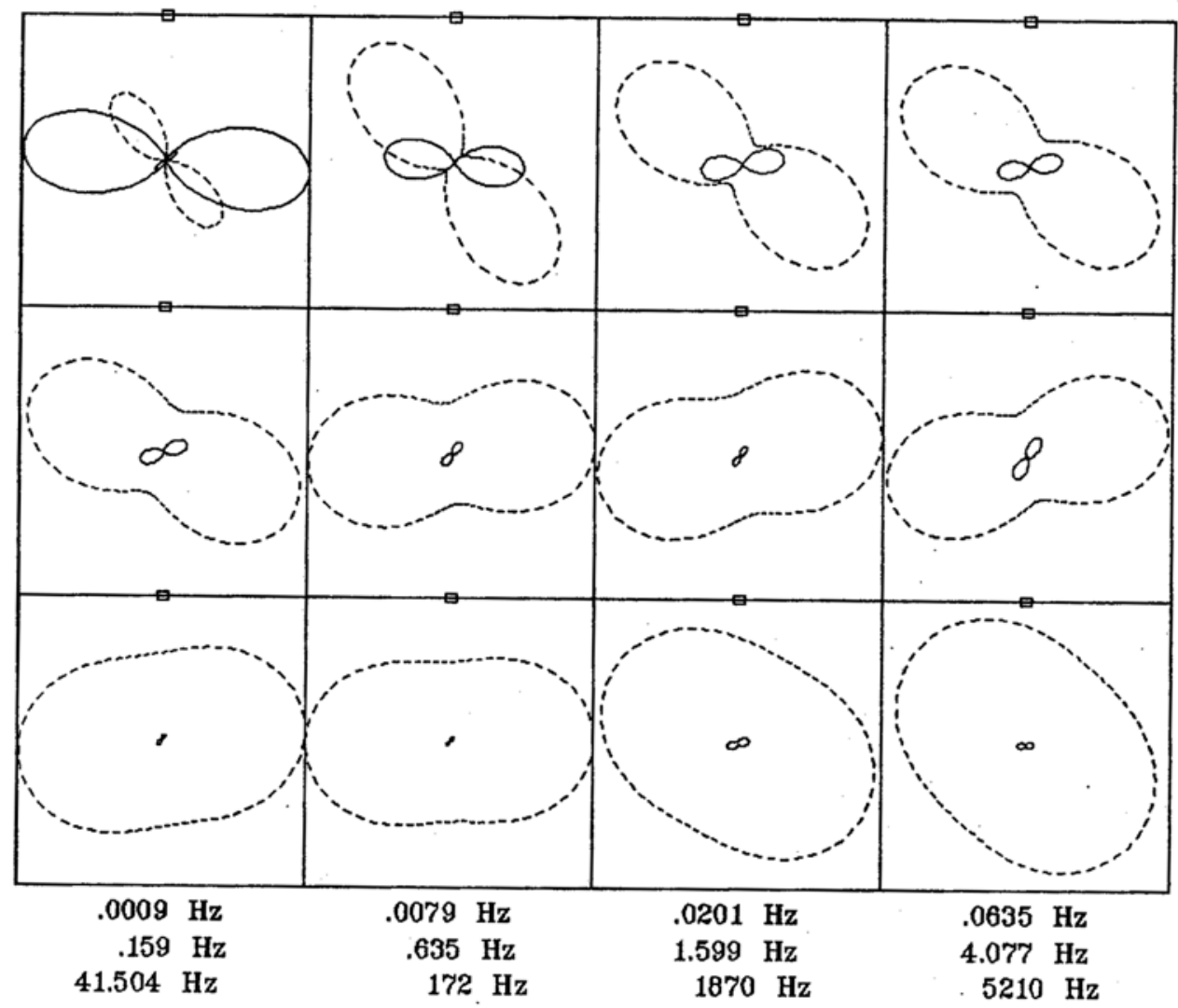

Client: DOE

Rotation:

Remote: none

Filename: rm14.avg

Channels: Ch1 Ch2 Ch3 Ch4 Ch5 Ch3 Ch4

Plotted: 13:21 Jan 17, 2006

Acquired: 21:5 May 10, 2005

Survey Co:USGS

< EMI - ElectroMagnetic Instruments > 
TIPPER MAGNITUDE

Station 14

Rainier Mesa and Shoshone Mtn

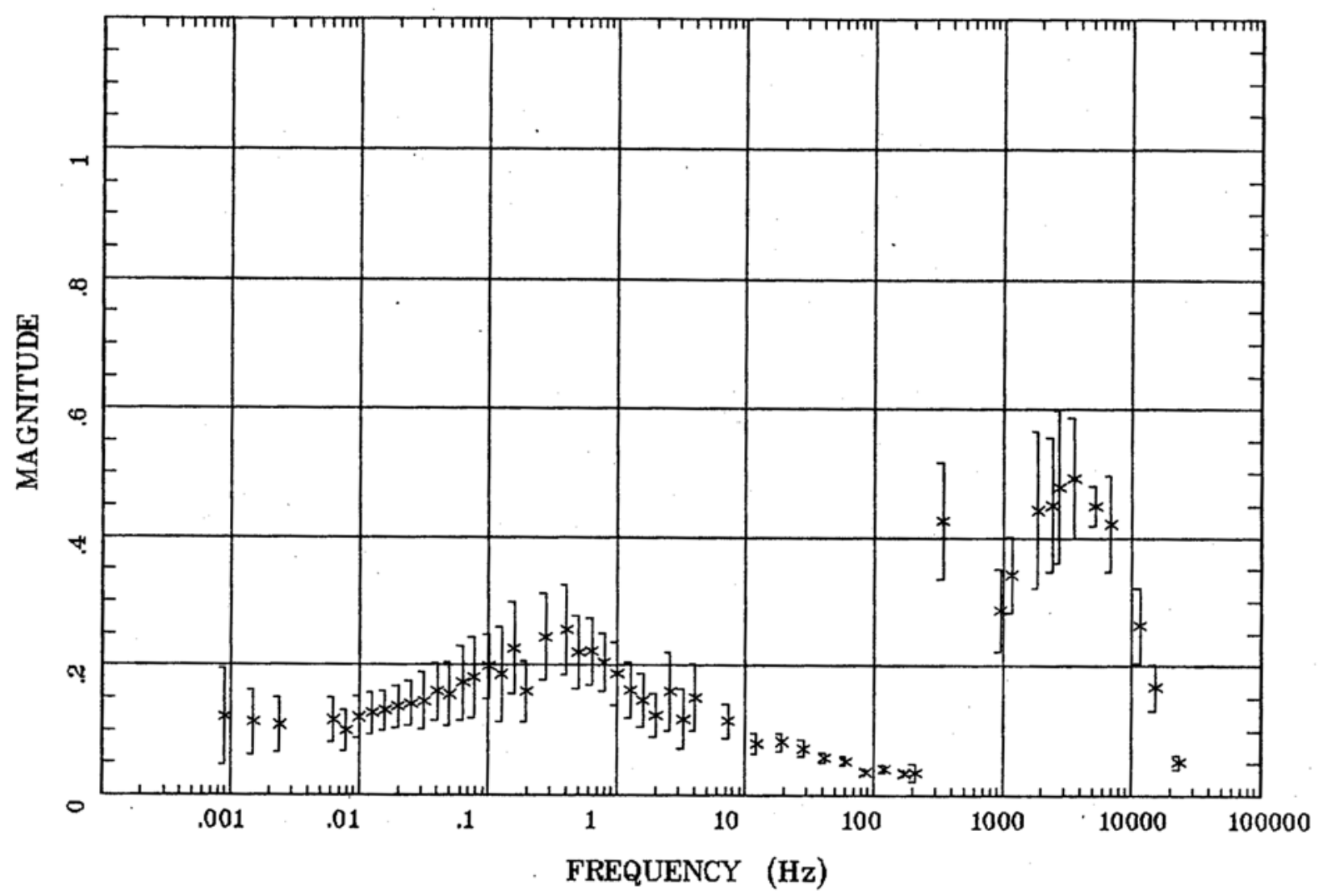

Client: DOE

Remote: none

Acquired: 21:5 May 10, 2005

Survey Co:USGS
Rotation:

Filename: rm14.avg

Channels: Ch1 Ch2 Ch3 Ch4 Ch5 Ch3 Ch4

Plotted: 13:21 Jan 17, 2006

< EMI - ElectroMagnetic Instruments > 
Station 14

TIPPER STRIKE

Rainier Mesa and Shoshone Mtn

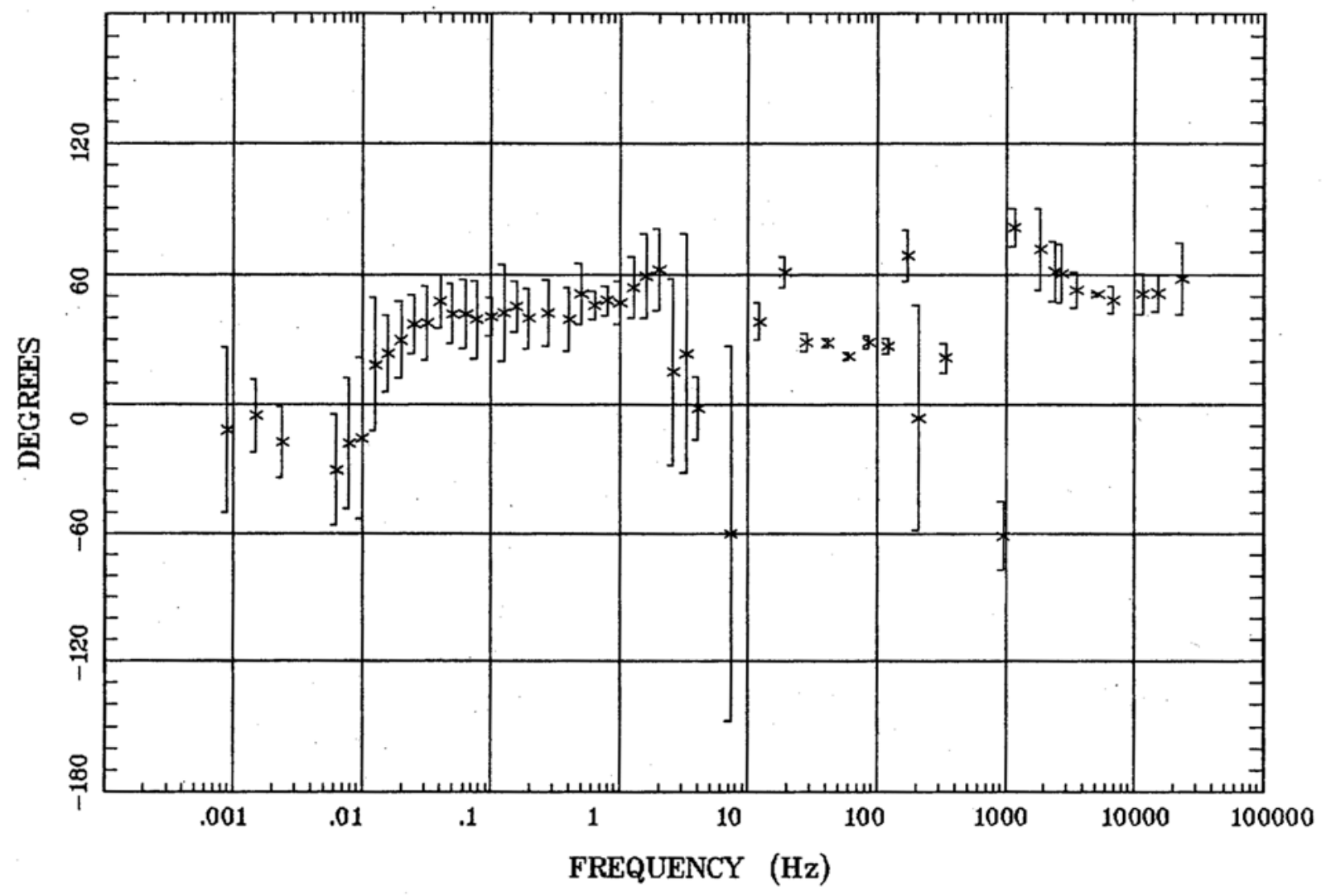

Client: DOE

Remote: none

Acquired: 21:5 May 10, 2005

Survey Co:USGS
Rotation:

Filename: rm14.avg

Channels: Ch1 Ch2 Ch3 Ch4 Ch5 Ch3 Ch4

Plotted: 13:21 Jan 17, 2006

< EMI - ElectroMagnetic Instruments > 
HzHx.x Coh HzHy.o

Station 14

Rainier Mesa and Shoshone Mtn

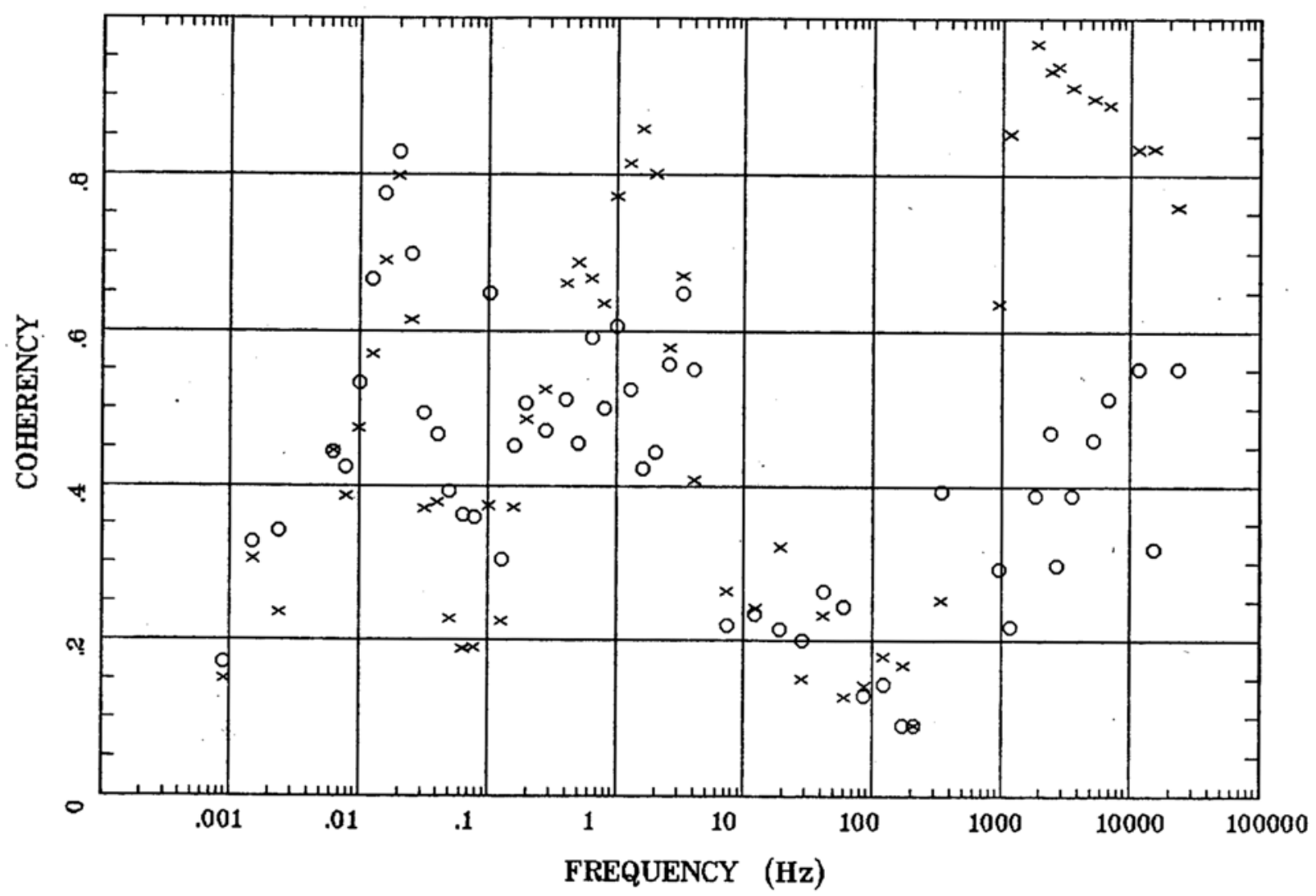

Client: $\mathrm{DOE}$

Remote: none

Acquired: 21:5 May 10, 2005

Survey Co:USGS
Rotation:

Filename: rm14.avg

Channels: Ch1 Ch2 Ch3 Ch4 Ch5 Ch3 Ch4

Plotted: 13:21 Jan 17, 2006

< EMI - ElectroMagnetic Instruments > 


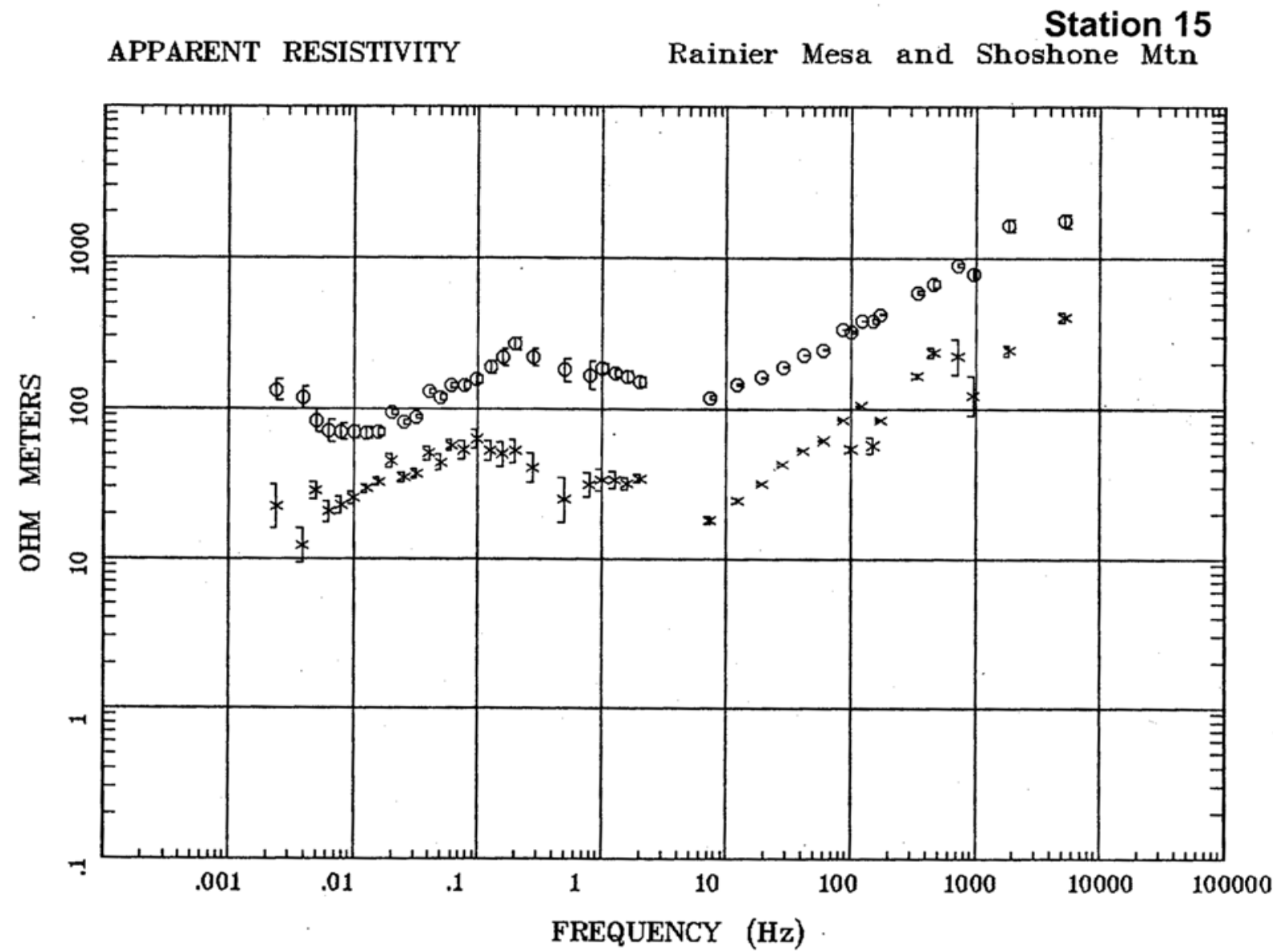

Client: DOE Remote: none Acquired: 03:3 May 12, 2005 Survey Co:USGS
Rotation:

Filename: rm15.avg

Channels: Ch1 Ch2 Ch3 Ch4 Ch5 Ch3 Ch4 Plotted: 13:22 Jan 17, 2006

< EMI - ElectroMagnetic Instruments > 


\section{Station 15}

IMPEDANCE PHASE

Rainier Mesa and Shoshone Mtn

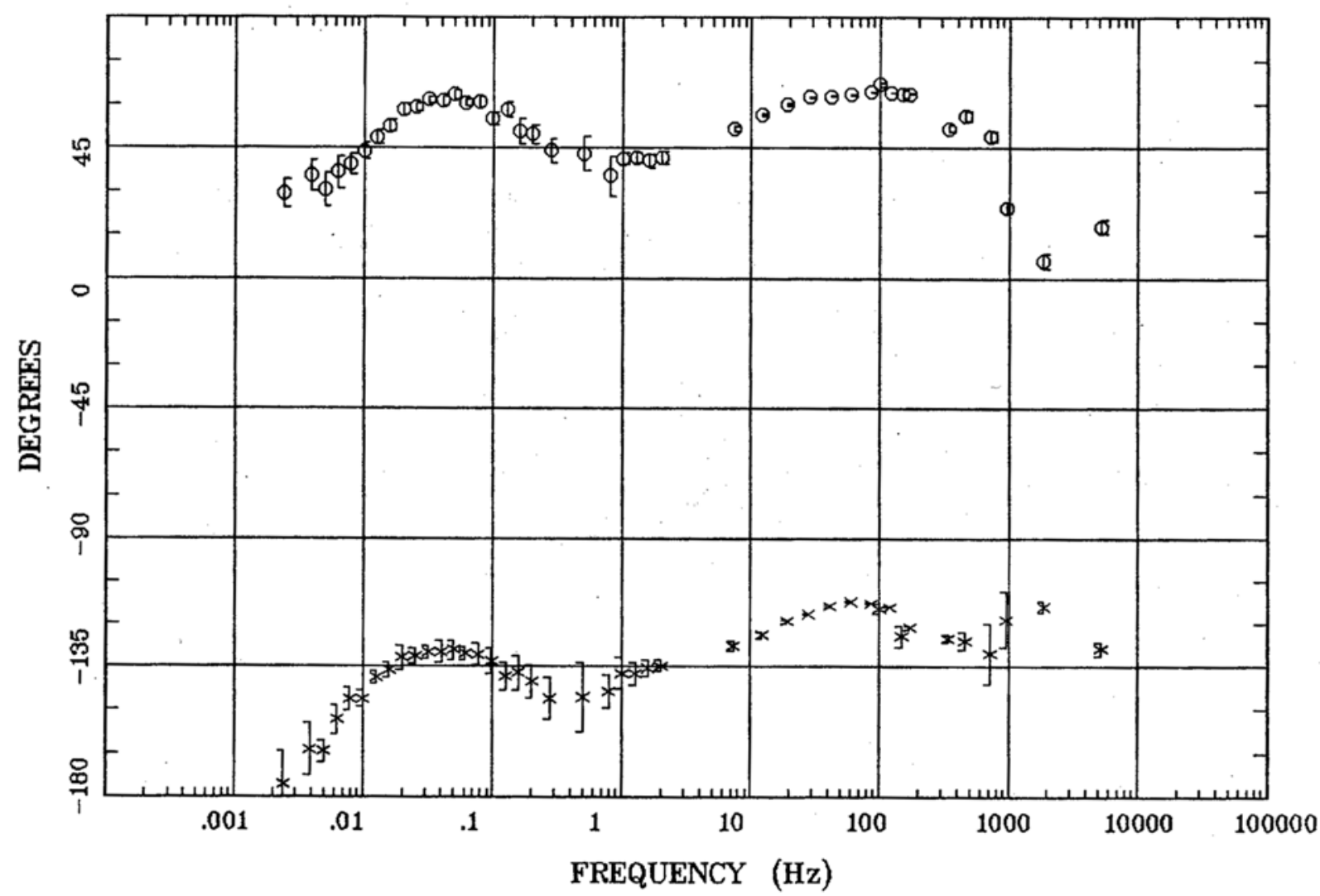

Client: DOE

Remote: none

Acquired: 03:3 May 12, 2005

Survey Co:USGS
Rotation:

Filename: rm15.avg

Channels: Ch1 Ch2 Ch3 Ch4 Ch5 Ch3 Ch4

Plotted: 13:22 Jan 17, 2006

< EMI - ElectroMagnetic Instruments > 
Station 15

ROTATION ANGLE

Rainier Mesa and Shoshone Mtn

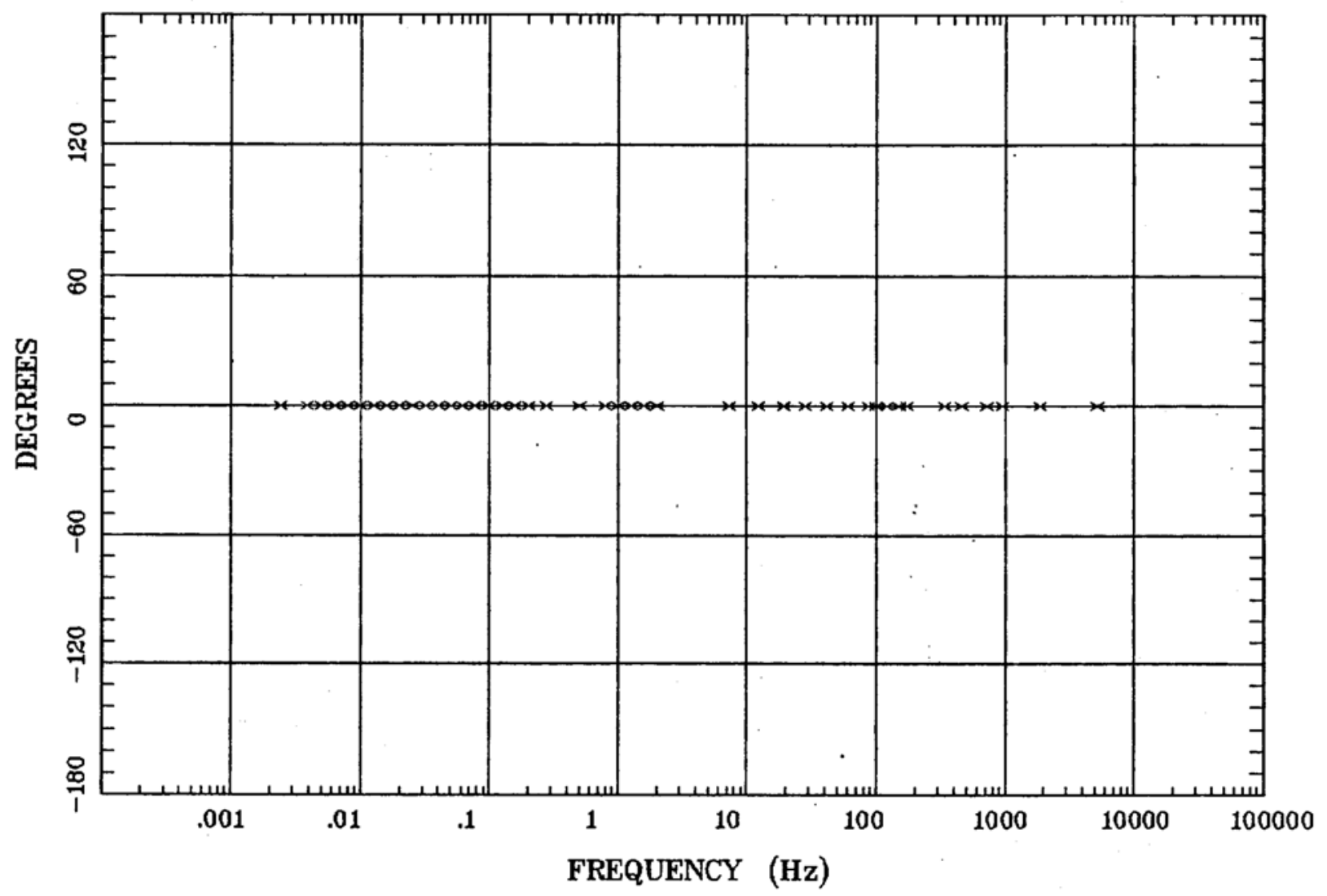

Client: DOE

Remote: none

Acquired: 03:3 May 12, 2005

Survey Co:USGS
Rotation:

Filename: rm15.avg

Channels: Ch1 Ch2 Ch3 Ch4 Ch5 Ch3 Ch4

Plotted: 13:22 Jan 17, 2006

< EMI - ElectroMagnetic Instruments > 


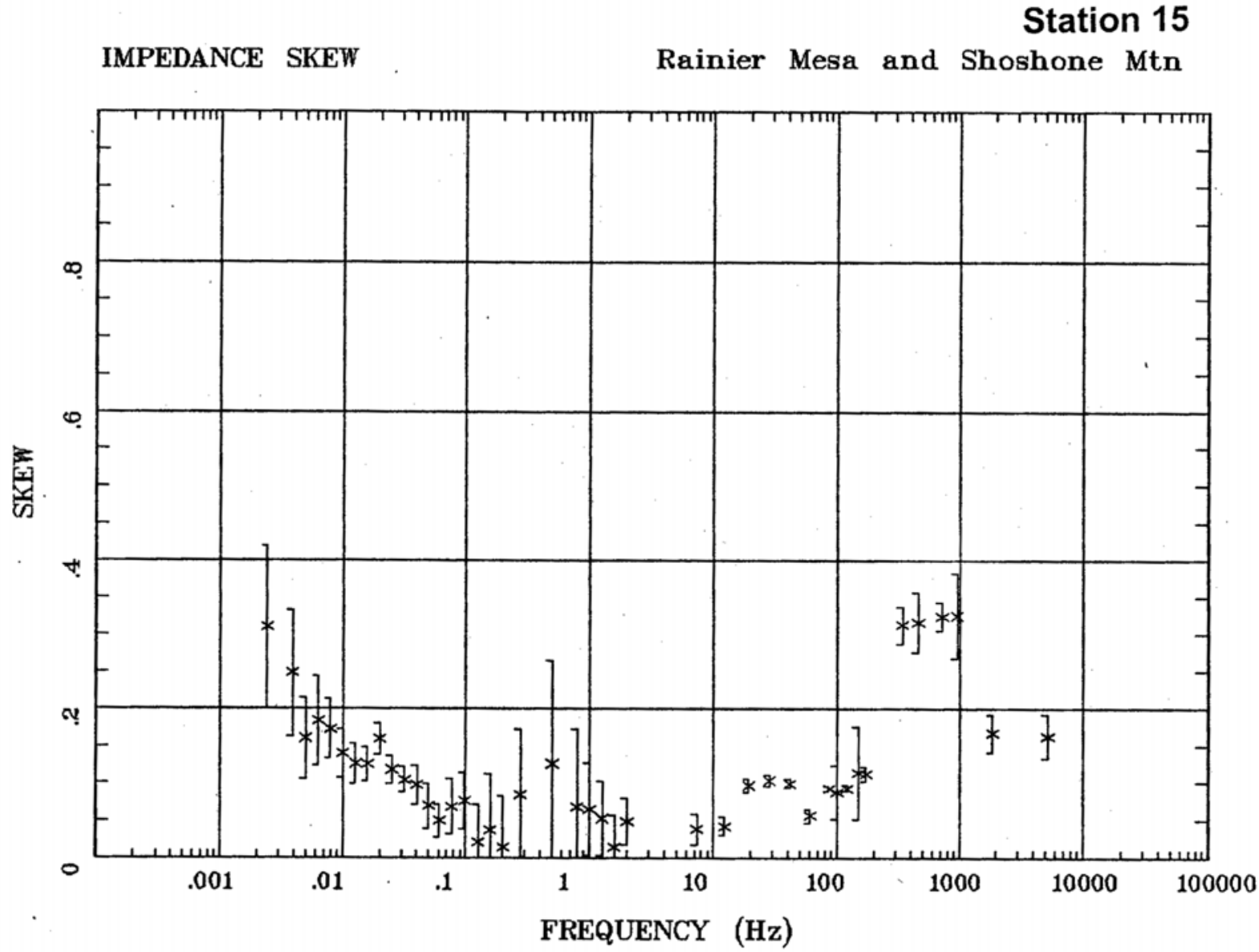

Client: $\mathrm{DOE}$

Remote: none

Acquired: 03:3 May 12, 2005

Survey Co:USGS
Rotation:

Filename: rm15.avg

Channels: Ch1 Ch2 Ch3 Ch4 Ch5 Ch3 Ch4

Plotted: 13:22 Jan 17, 2006

< EMI - ElectroMagnetic Instruments > 
E MULT Coh.

Station 15

Rainier Mesa and Shoshone Mtn

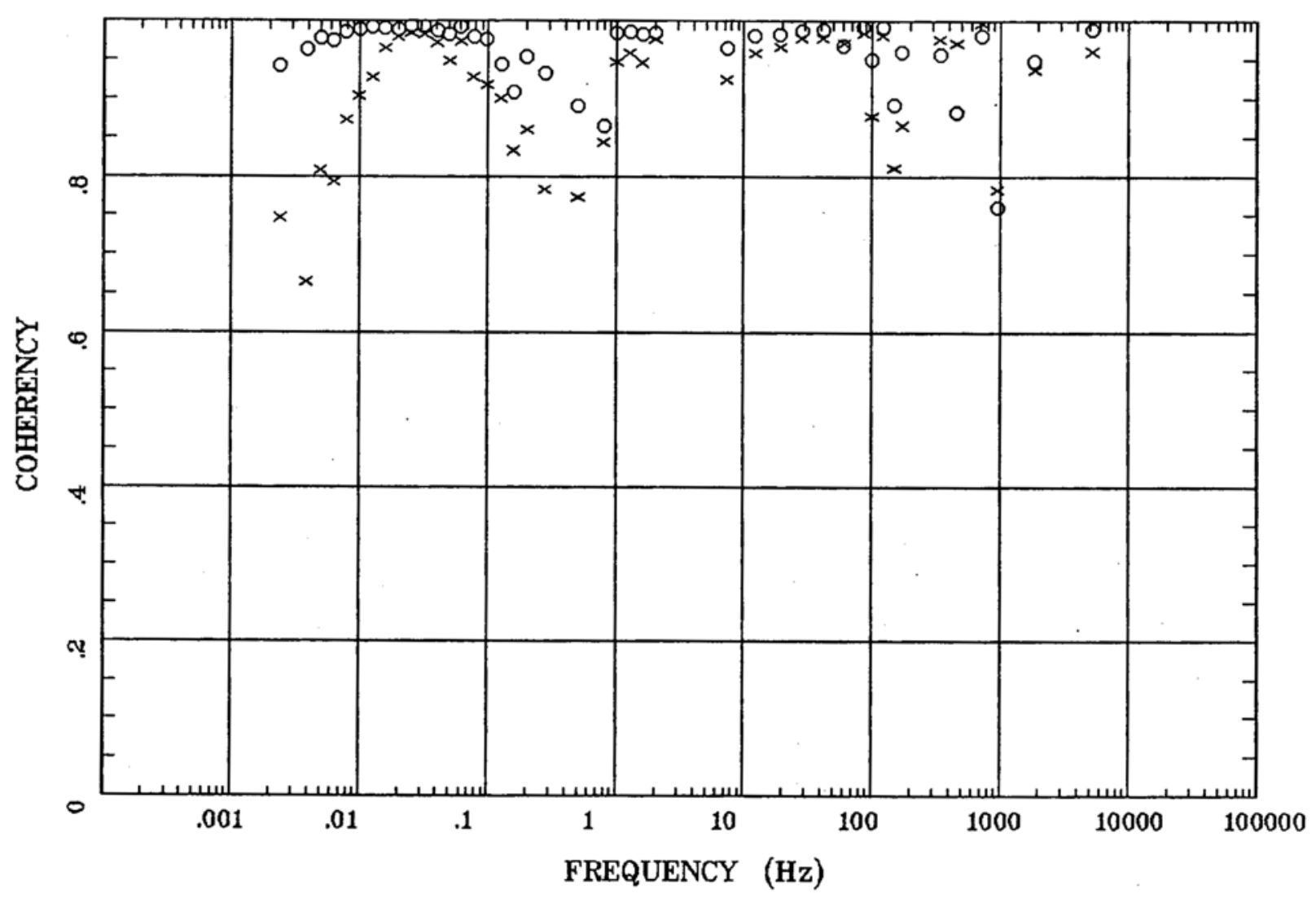

Client: $\mathrm{DOE}$

Remote: none

Acquired: 03:3 May 12, 2005

Survey Co:USGS
Rotation:

Filename: rm15.avg

Channels: Ch1 Ch2 Ch3 Ch4 Ch5 Ch3 Ch4

Plotted: 13:22 Jan 17, 2006

< EMI - ElectroMagnetic Instruments > 
Station 15

POLAR PLOTS

Rainier Mesa and Shoshone Mtn

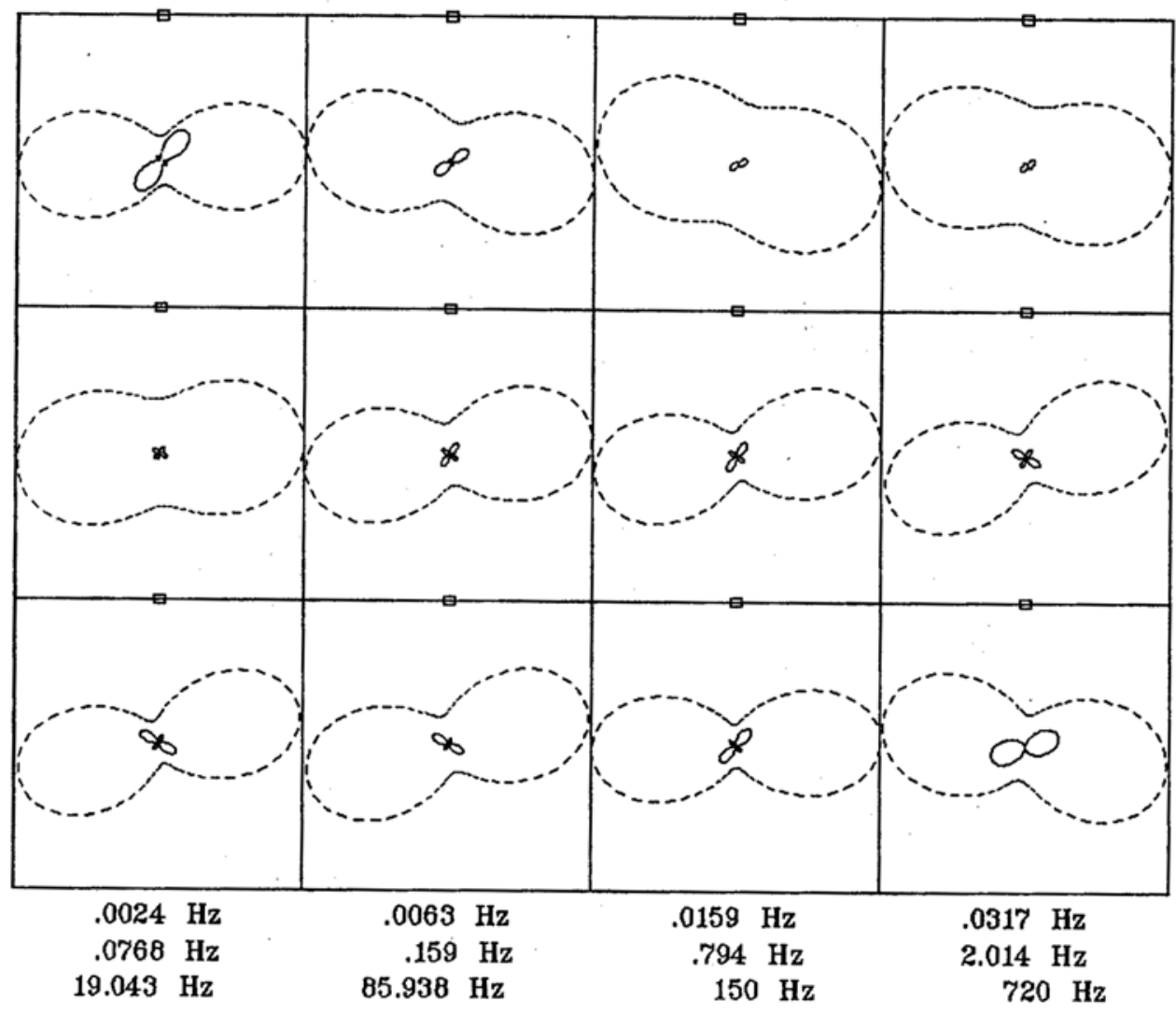

Client: DOE

Remote: none

Acquired: 03:3 May 12, 2005

Survey Co:USGS
Rotation:

Filename: rm15.avg

Channels: Ch1 Ch2 Ch3 Ch4 Ch5 Ch3 Ch4

Plotted: 13:22 Jan 17, 2006

< EMI - ElectroMagnetic Instruments > 
TIPPER MAGNITUDE

Station 15

Rainier Mesa and Shoshone Mtn

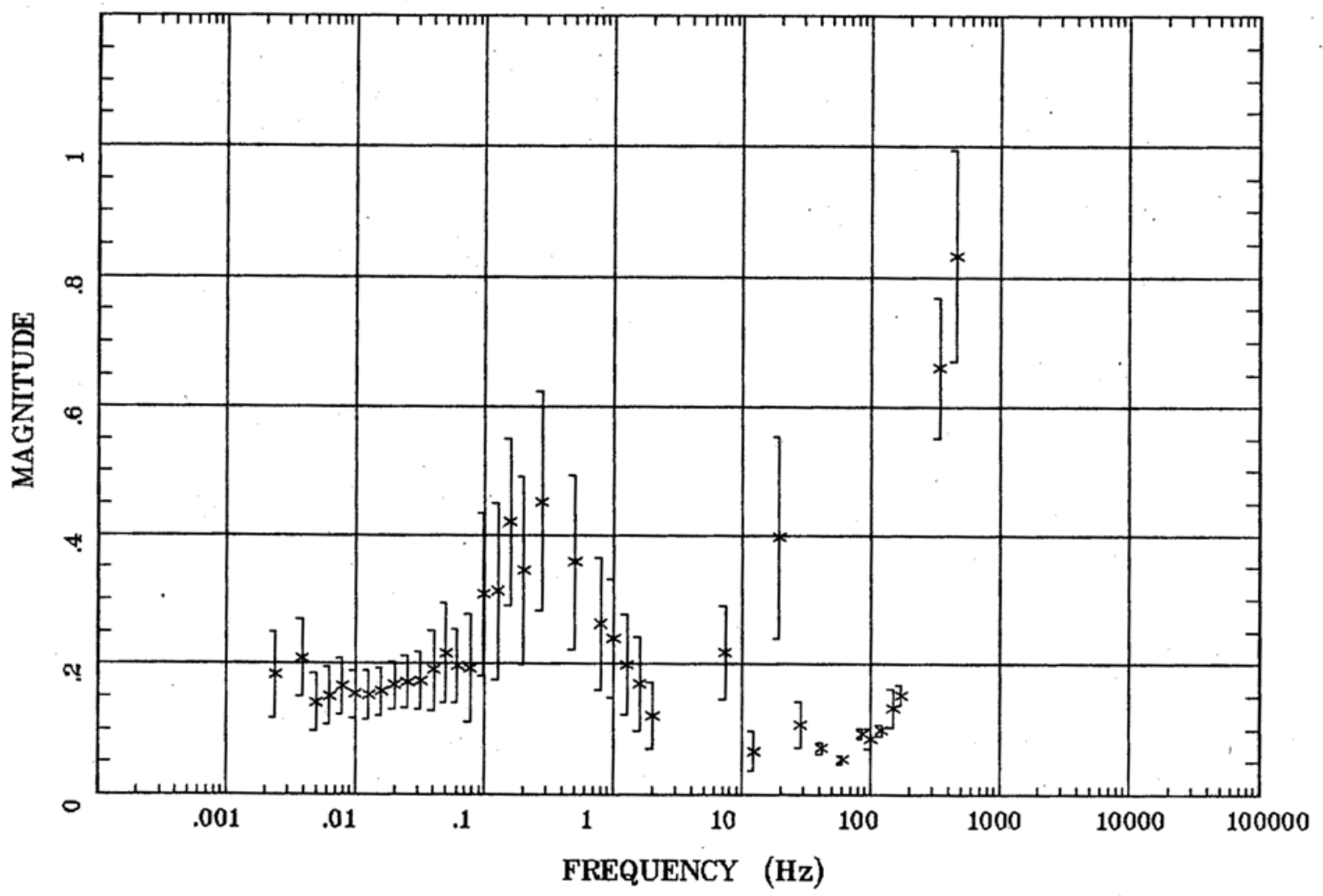

Client: DOE

Remote: none

Acquired: 03:3 May 12, 2005

Survey Co:USGS
Rotation:

Filename: rm15.avg

Channels: Ch1 Ch2 Ch3 Ch4 Ch5 Ch3 Ch4

Plotted: 13:22 Jan 17, 2006

< EMI - ElectroMagnetic Instruments > 
Station 15

TIPPER STRIKE

Rainier Mesa and Shoshone Mtn

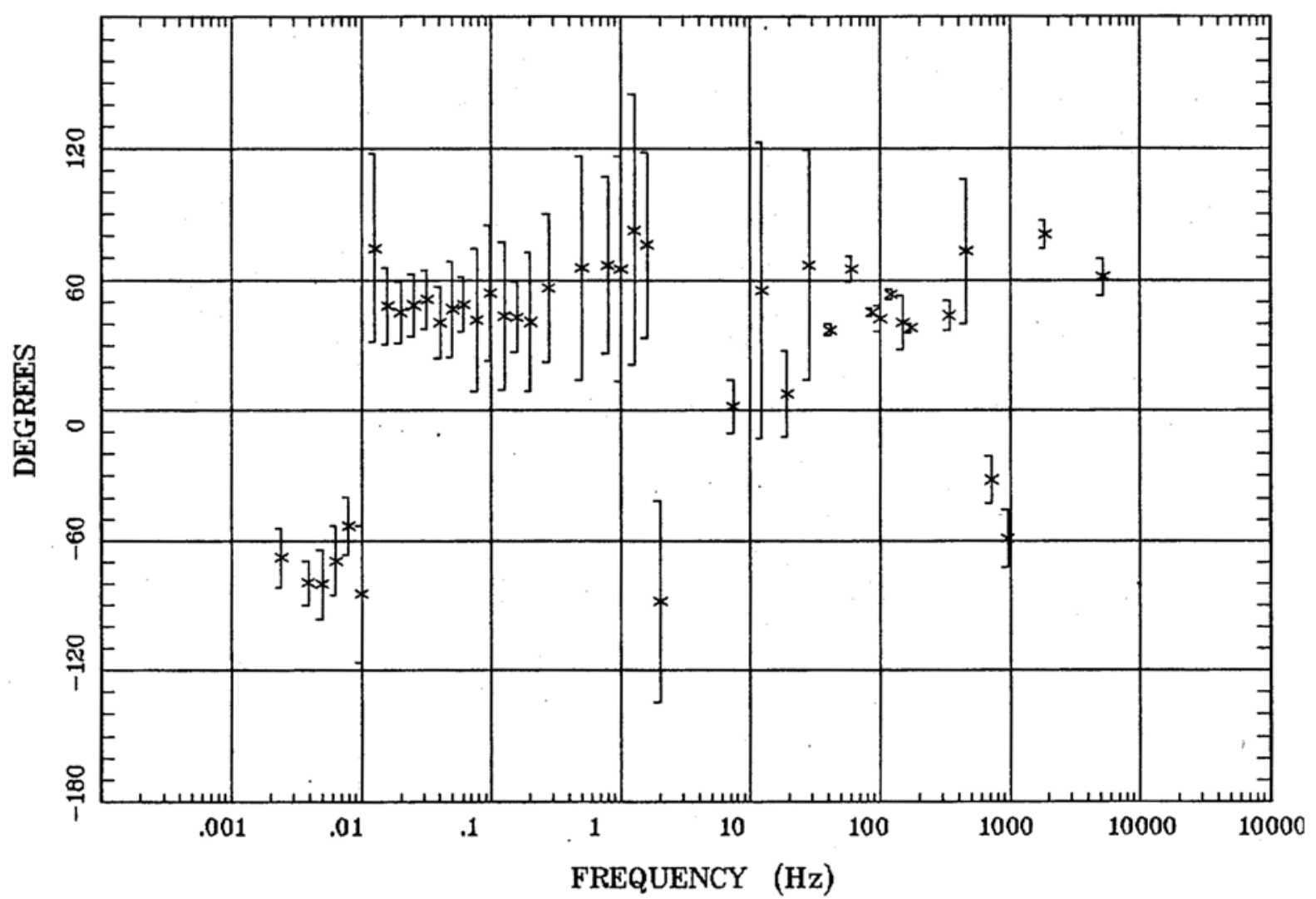

Client: DOE

Remote: none

Acquired: 03:3 May 12, 2005

Survey Co:USGS
Rotation:

Filename: rm15.avg

Channels: Ch1 Ch2 Ch3 Ch4 Ch5 Ch3 Ch4 Plotted: 13:22 Jan 17, 2006

< EMI - ElectroMagnetic Instruments > 
Station 15

HzHx.x Coh HzHy.o

Rainier Mesa and Shoshone Mtn

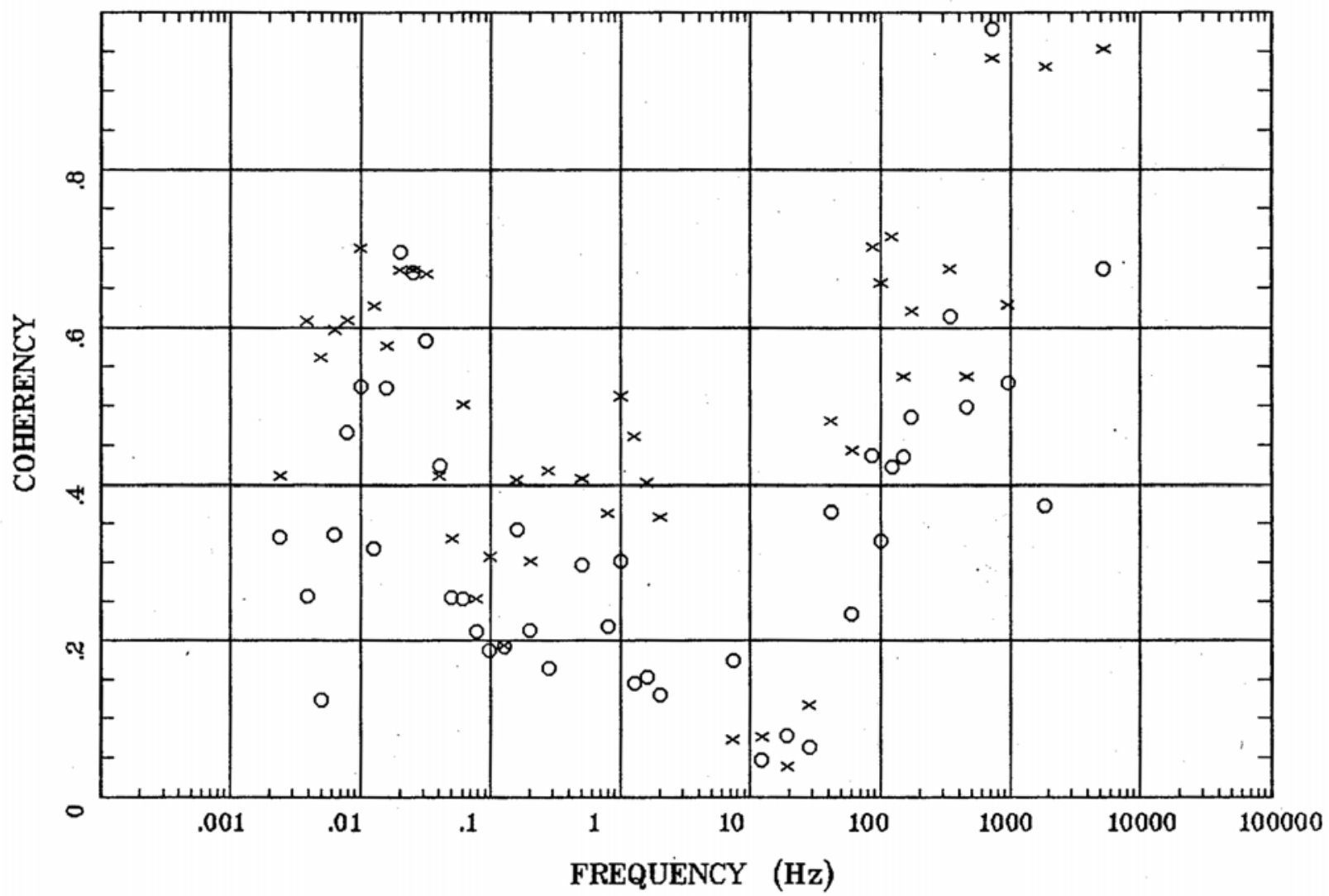

Client: DOE

Remote: none

Acquired: 03:3 May 12, 2005

Survey Co:USGS
Rotation:

Filename: rm15.avg

Channels: Ch1 Ch2 Ch3 Ch4 Ch5 Ch3 Ch4

Plotted: 13:22 Jan 17, 2006

< EMI - ElectroMagnetic Instruments > 


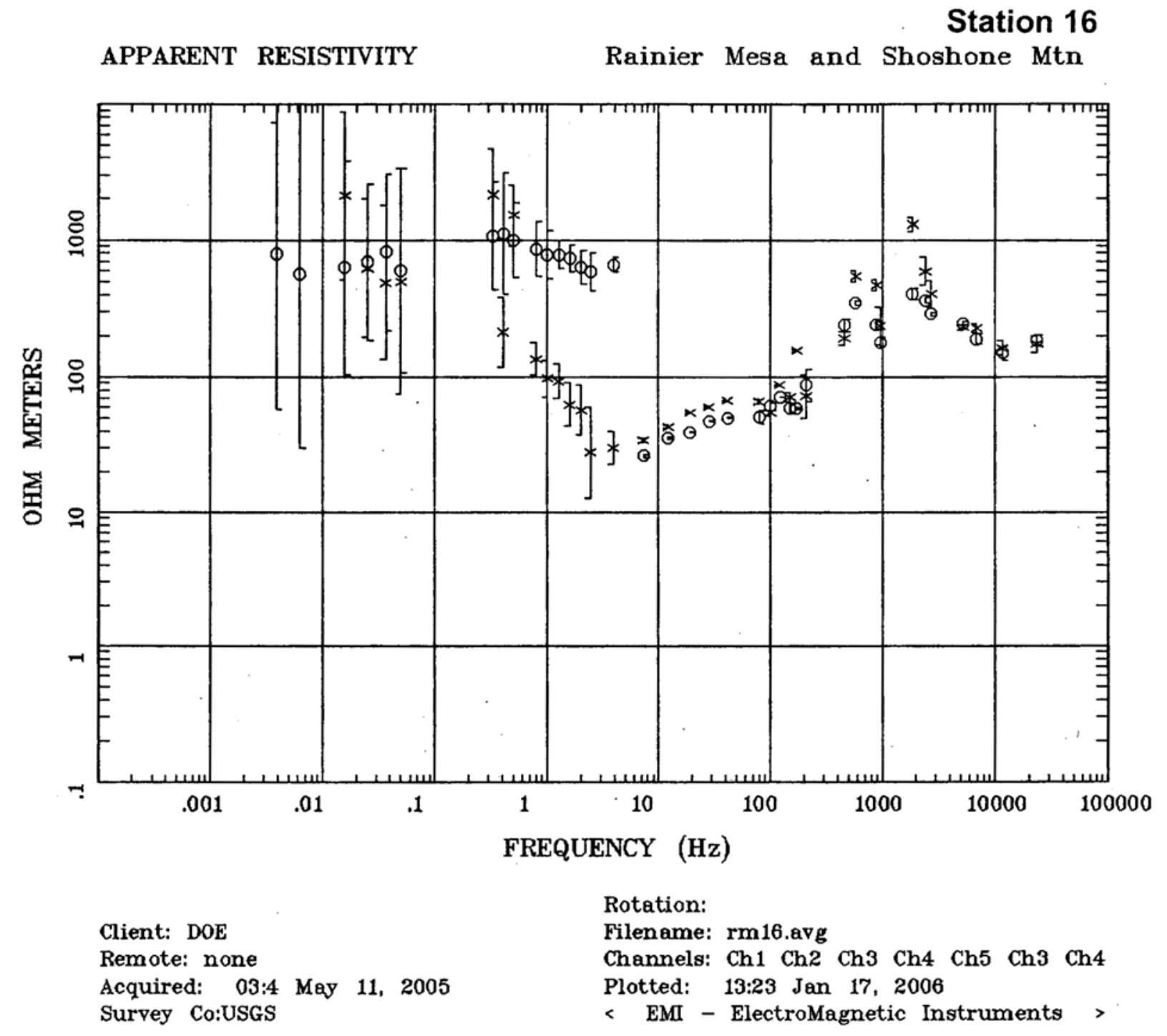




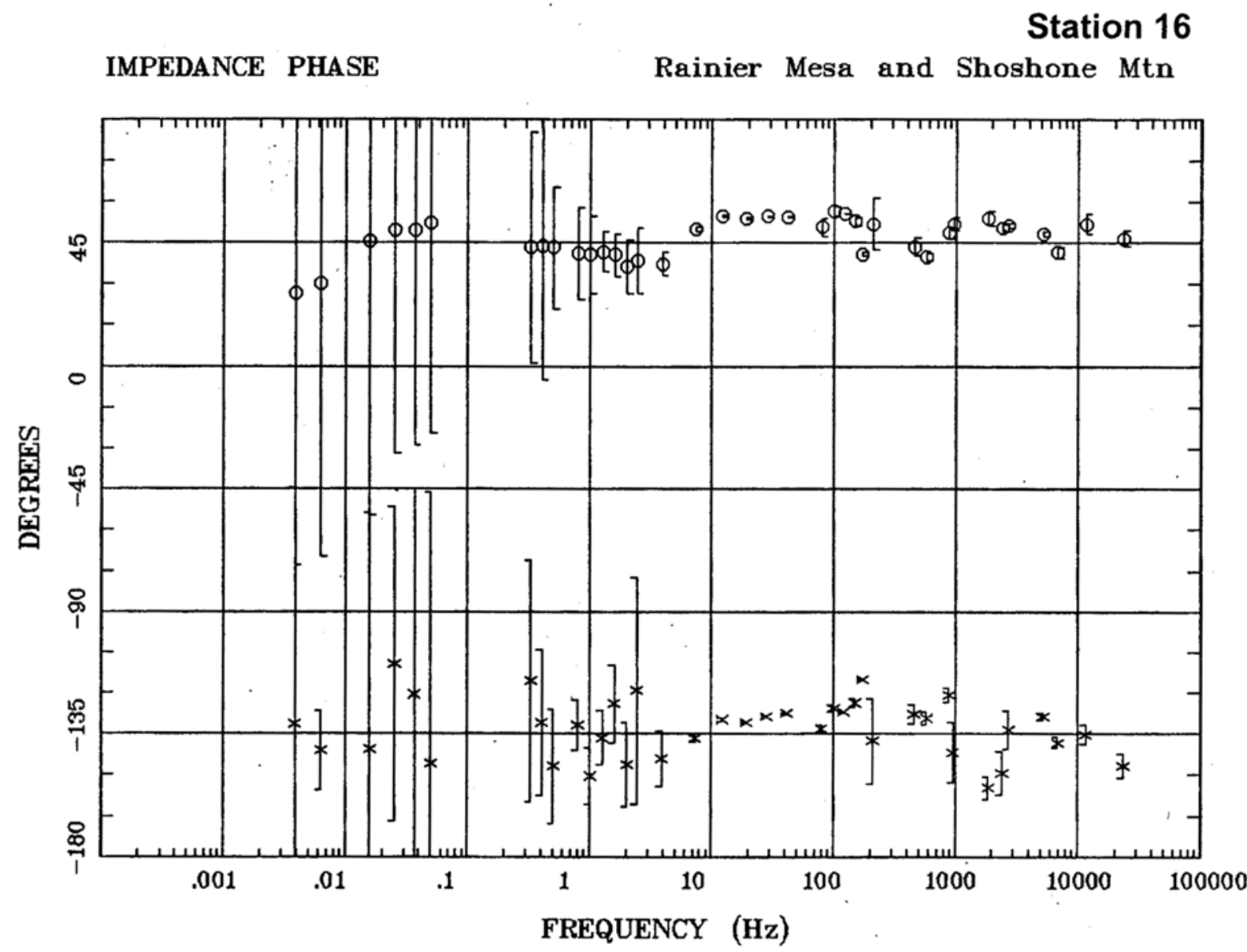

Client: DOE

Remote: none

Acquired: 03:4 May 11, 2005 Survey Co:USGS
Rotation:

Filename: rm16.avg

Channels: Ch1 Ch2 Ch3 Ch4 Ch5 Ch3 Ch4

Plotted: 13:23 Jan 17, 2006

< EMI - ElectroMagnetic Instruments > 
Station 16

ROTATION ANGLE

Rainier Mesa and Shoshone Mtn

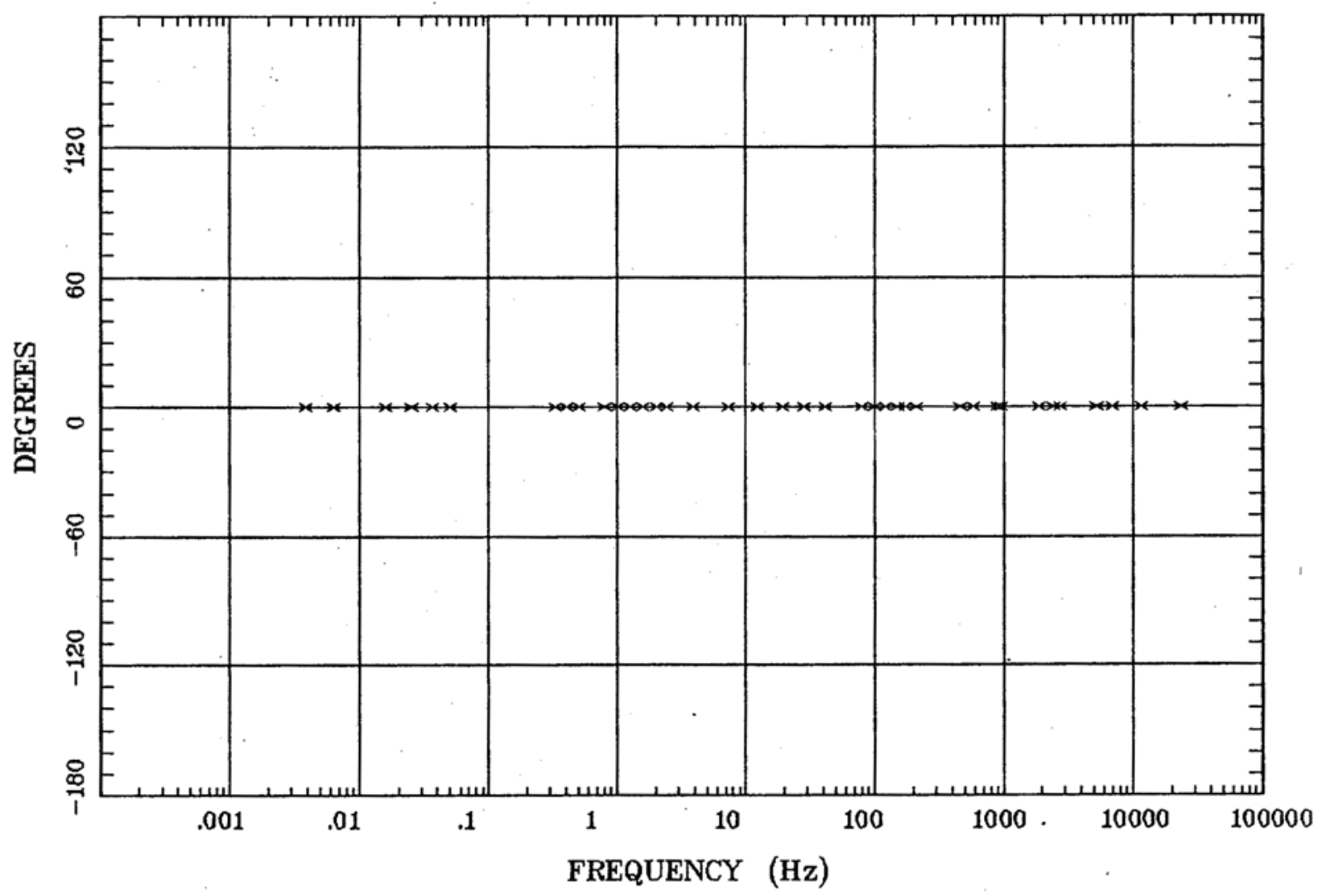

Client: $\mathrm{DOE}$

Remote: none

Acquired: 03:4 May 11, 2005

Survey Co:USGS
Rotation:

Filename: rm16.avg

Channels: Ch1 Ch2 Ch3 Ch4 Ch5 Ch3 Ch4

Plotted: 13:23 Jan 17, 2006

< EMI - ElectroMagnetic Instruments > 
Station 16

IMPEDANCE SKEW

Rainier Mesa and Shoshone Mtn

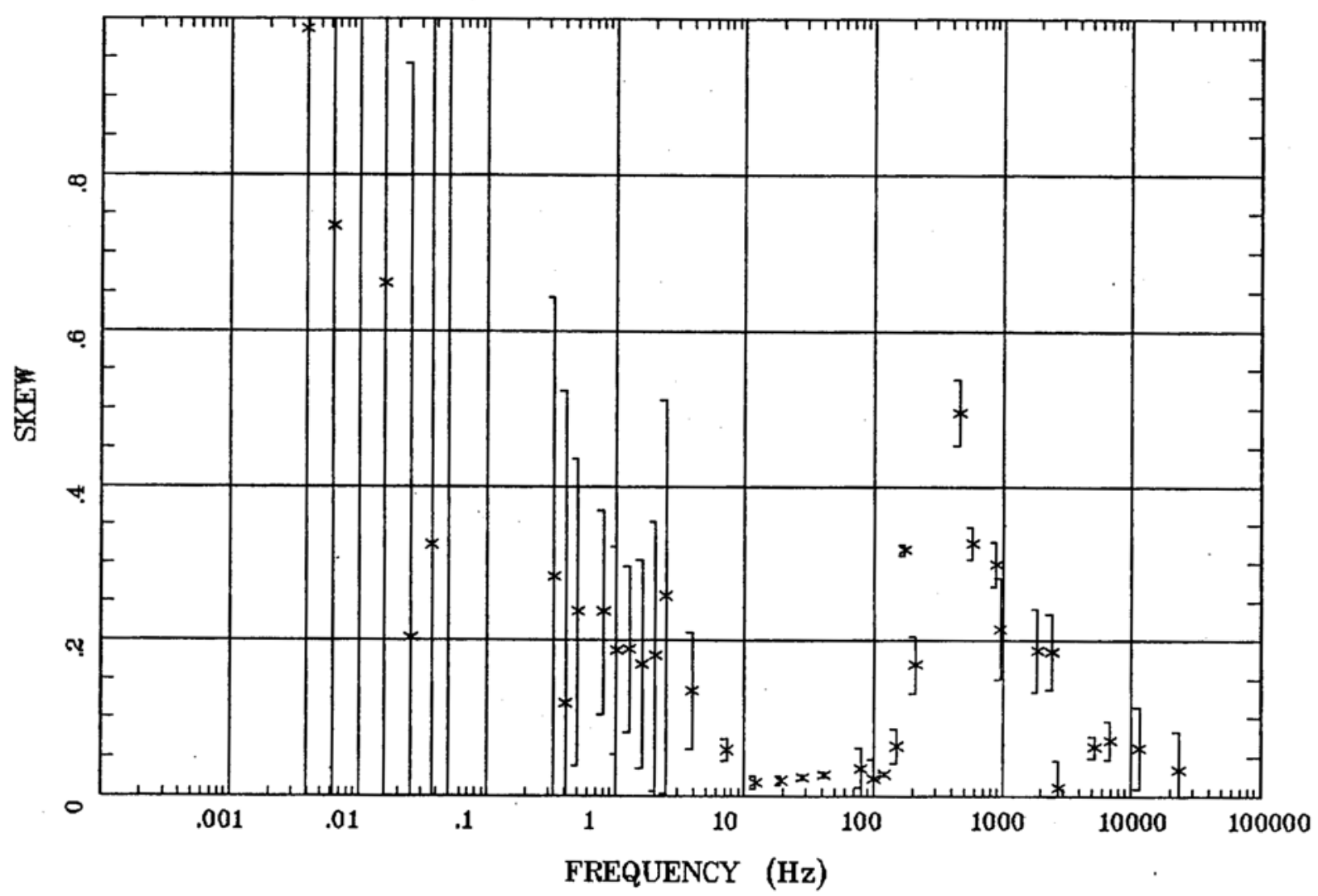

Client: DOE

Remote: none

Acquired: 03:4 May 11, 2005

Survey Co:USGS

Rotation:

Filename: rm16.avg

Channels: Ch1 Ch2 Ch3 Ch4 Ch5 Ch3 Ch4

Plotted: 13:23 Jan 17, 2006

< EMI - ElectroMagnetic Instruments > 
E MULT Coh.

Station 16

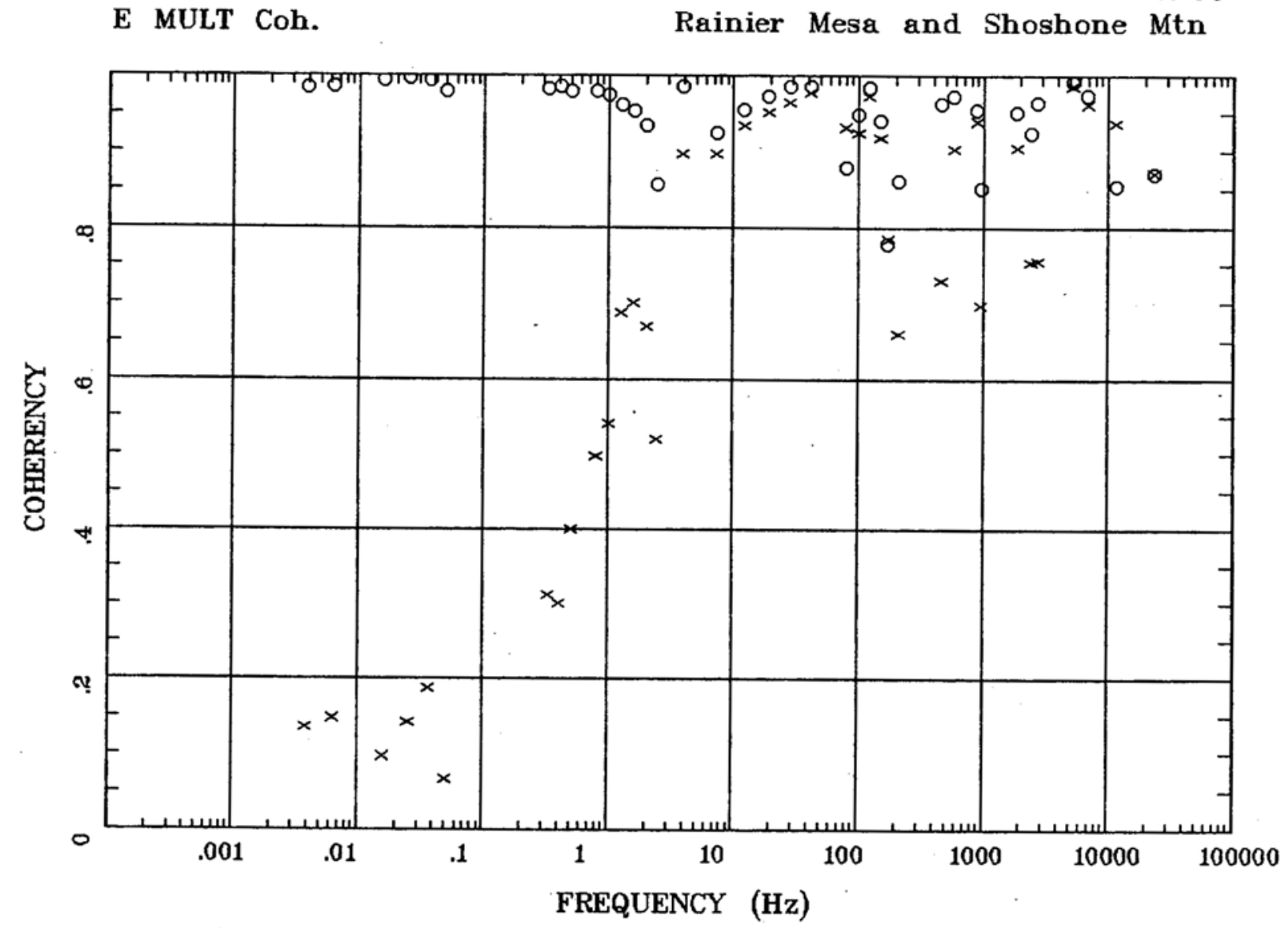

Client: DOE

Remote: none

Acquired: 03:4 May 11, 2005

Survey Co:USGS
Rotation:

Filename: rm16.avg

Channels: Ch1 Ch2 Ch3 Ch4 Ch5 Ch3 Ch4

Plotted: 13:23 Jan 17, 2006

< EMI - ElectroMagnetic Instruments > 


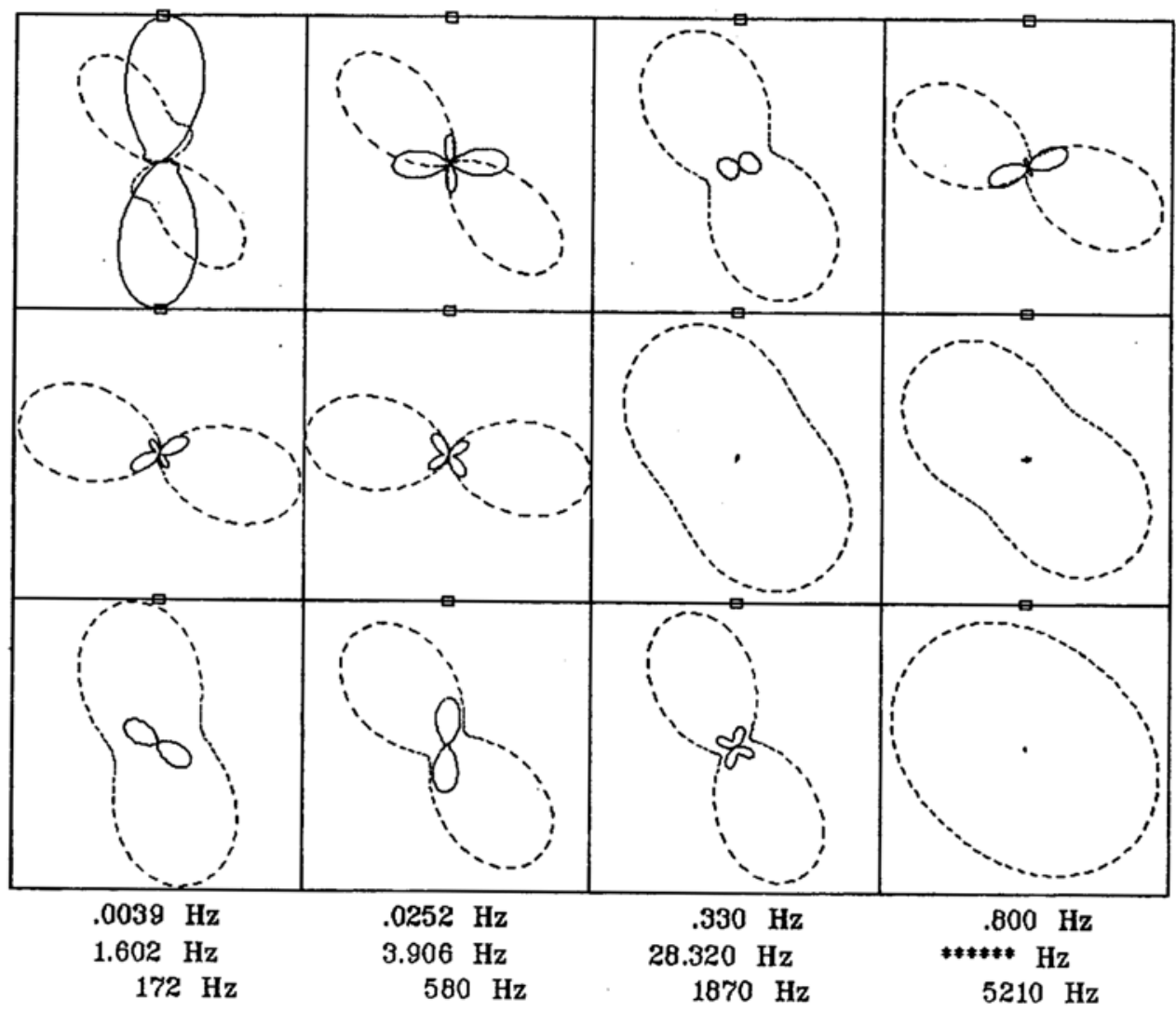

Client: $\mathrm{DOE}$

Remote: none

Acquired: 03:4 May 11, 2005

Survey Co:USGS
Rainier Mesa and Shoshone Mtn

Rotation:

Filename: rm16.avg

Channels: Ch1 Ch2 Ch3 Ch4 Ch5 Ch3 Ch4

Plotted: 13:23 Jan 17, 2006

< EMI - ElectroMagnetic Instruments > 


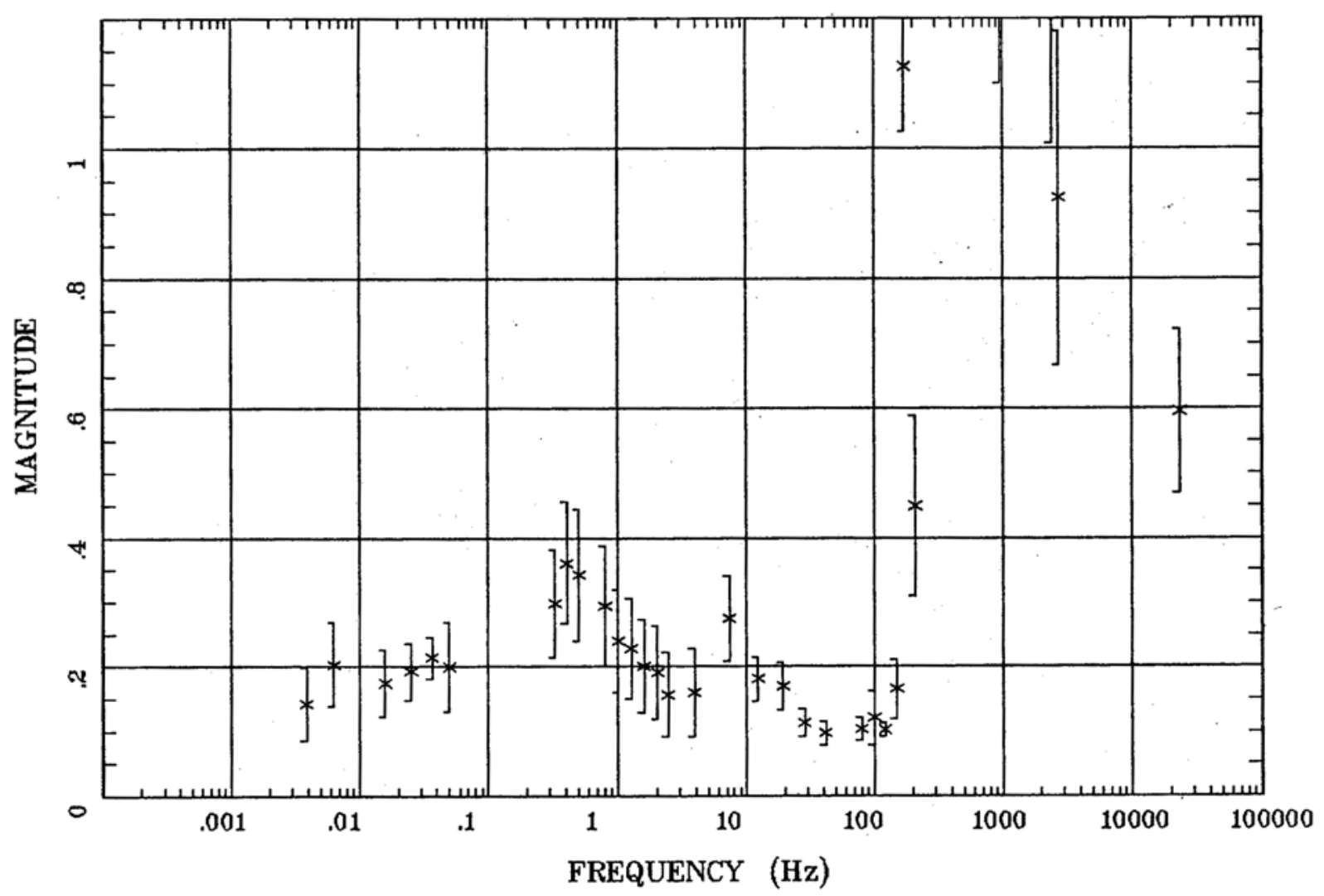

Client: DOE

Remote: none

Acquired: 03:4 May 11, 2005

Survey Co:USGS
Rotation:

Filename: rm16.avg

Channels: Ch1 Ch2 Ch3 Ch4 Ch5 Ch3 Ch4

Plotted: 13:23 Jan 17, 2006

< EMI - ElectroMagnetic Instruments > 


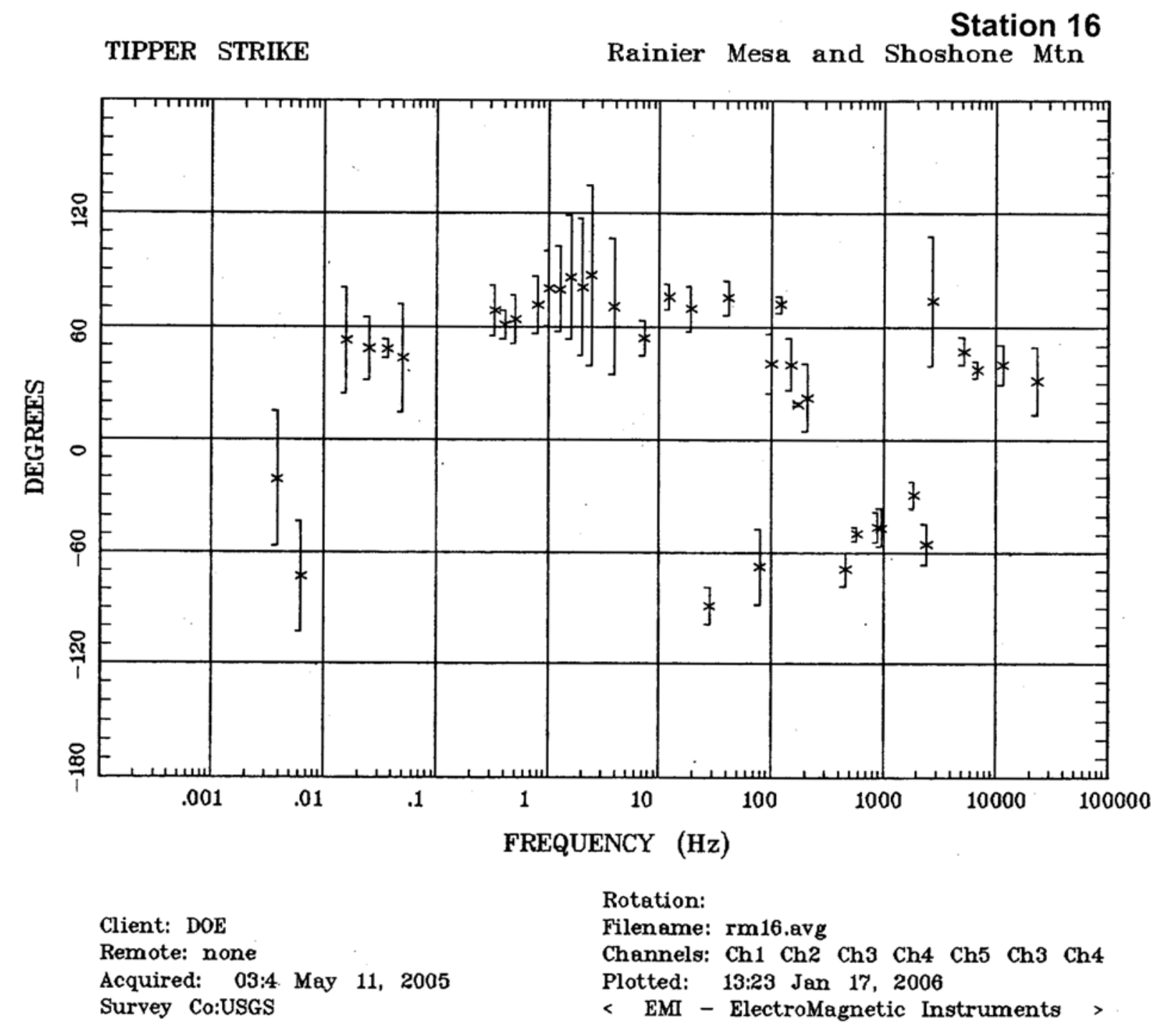




\section{Station 16}

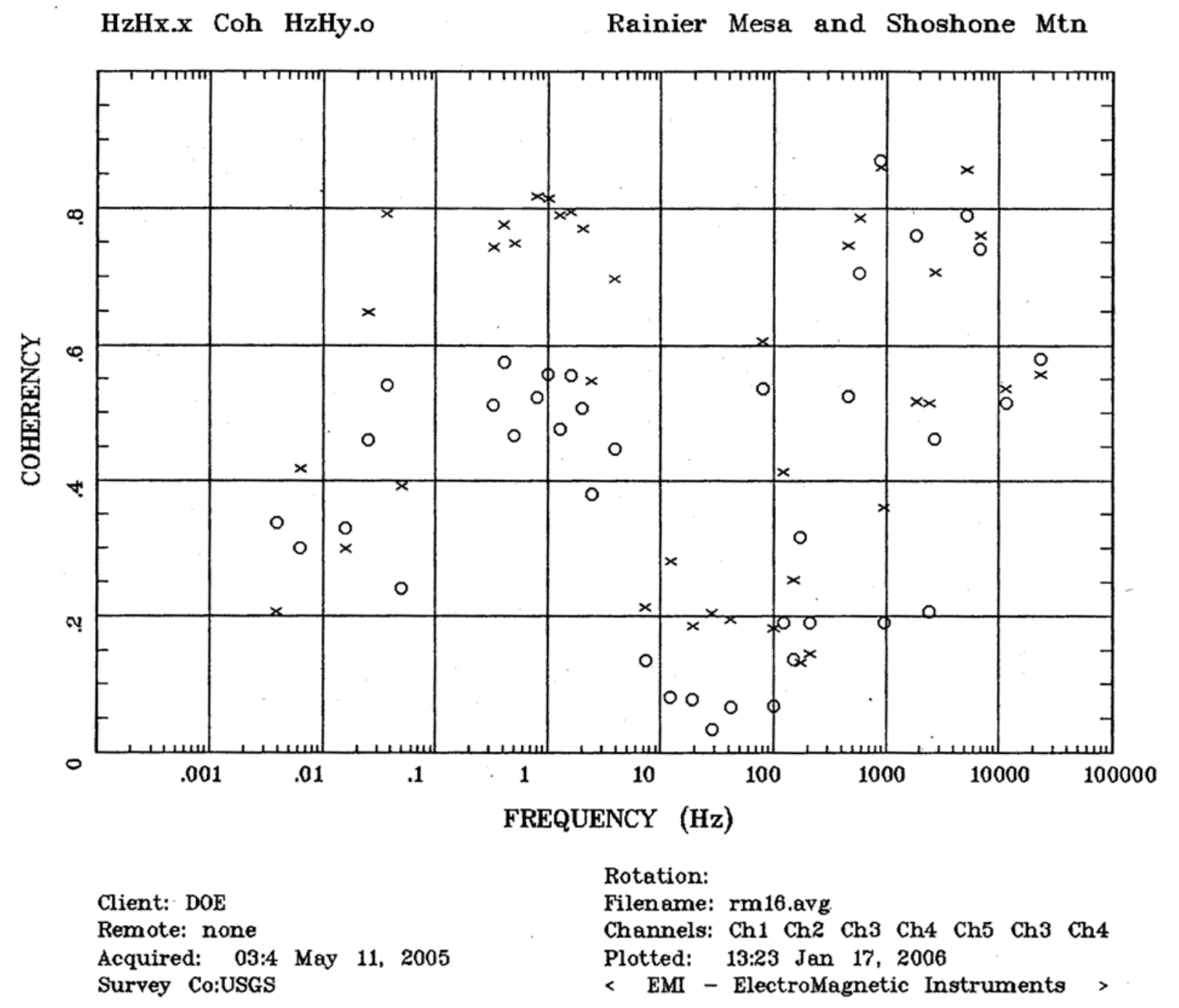




\section{Station 17}

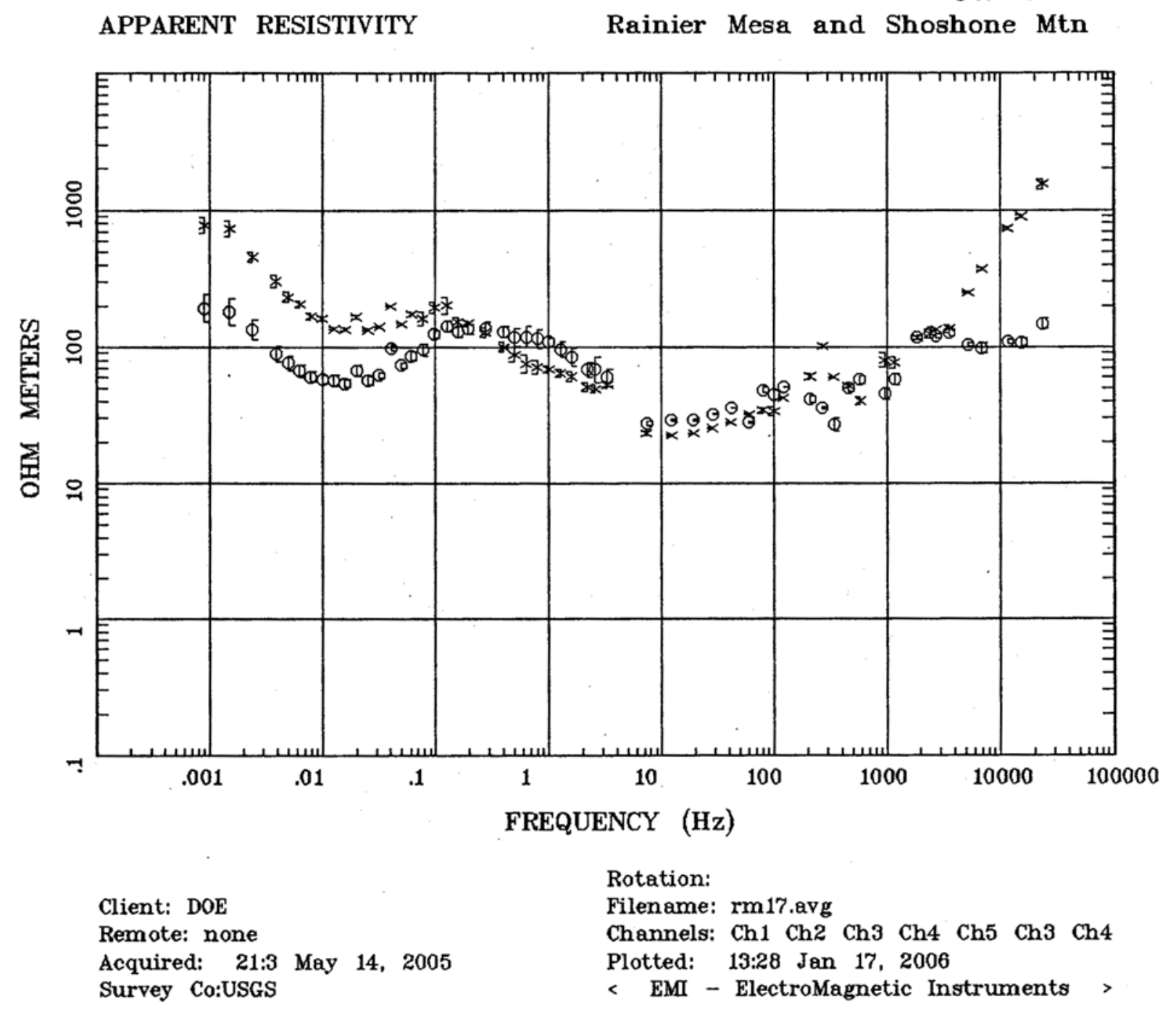




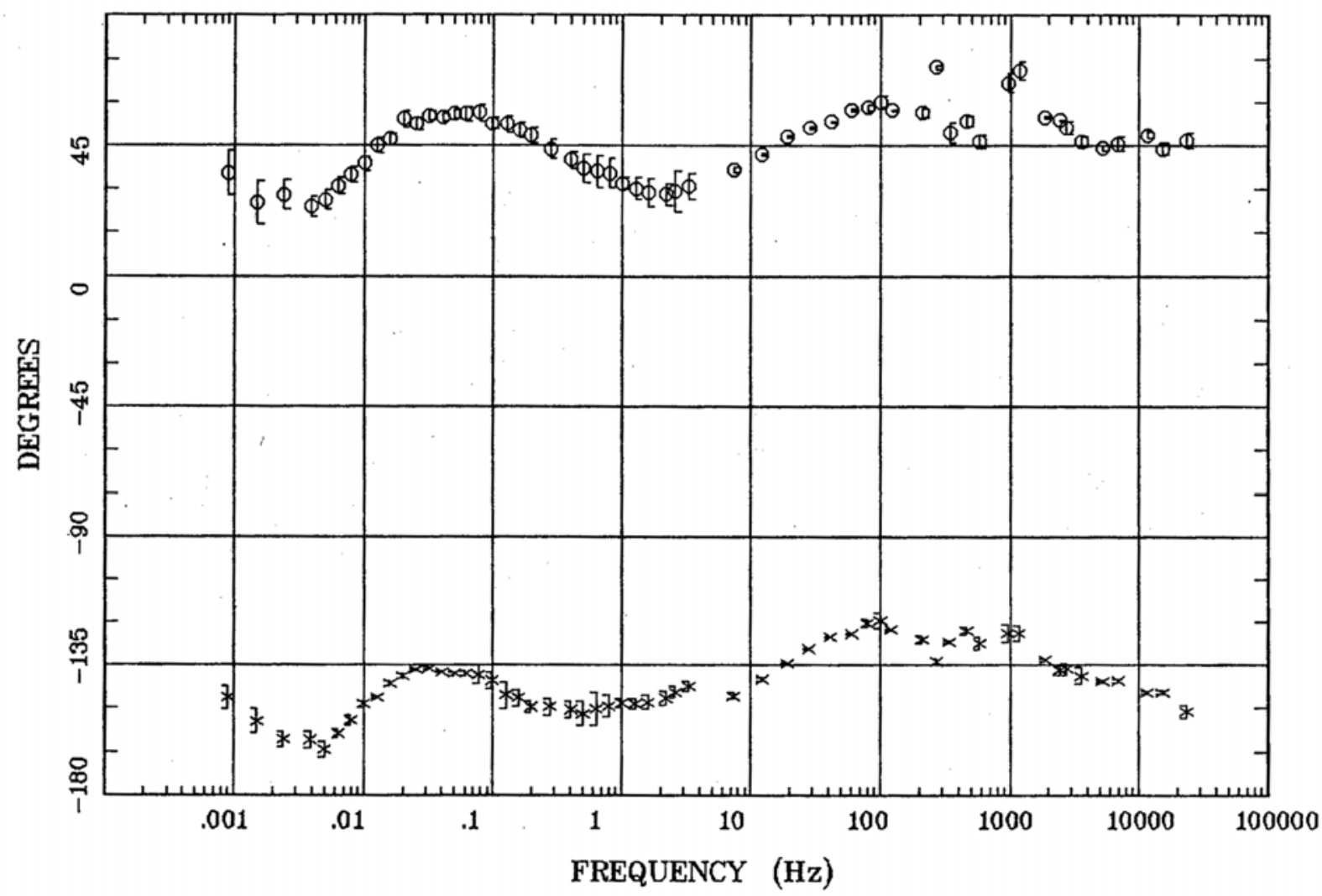

Client: DOE

Remote: none

Acquired: 21:3 May 14, 2005

Survey Co:USGS
Rotation:

Filename: rm17.avg

Channels: Ch1 Ch2 Ch3 Ch4 Ch5 Ch3 Ch4

Plotted: 13:28 Jan 17, 2006

$<$ EMI - ElectroMagnetic Instruments > 


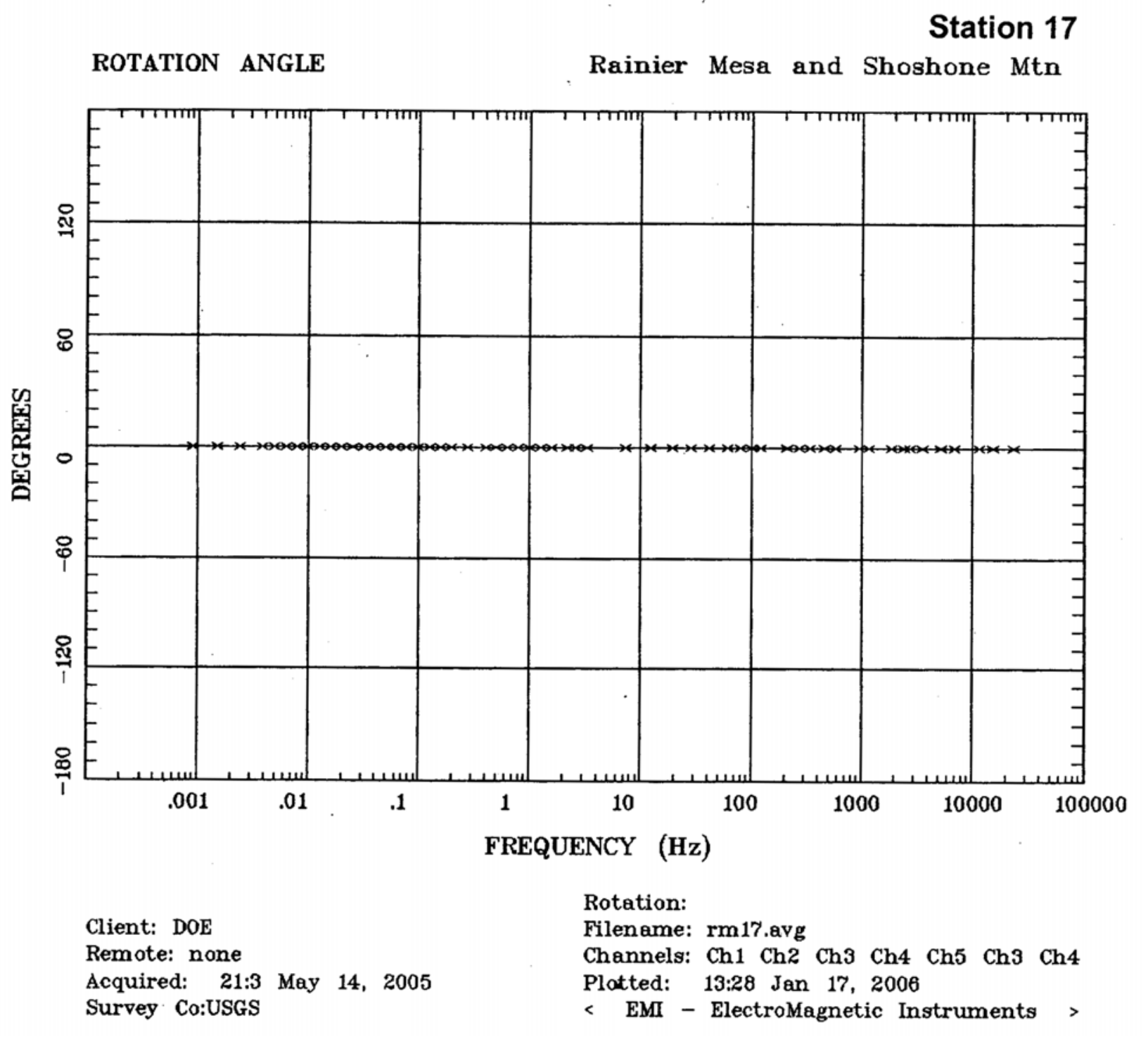




\section{Station 17}

IMPEDANCE SKEW

Rainier Mesa and Shoshone Mtn

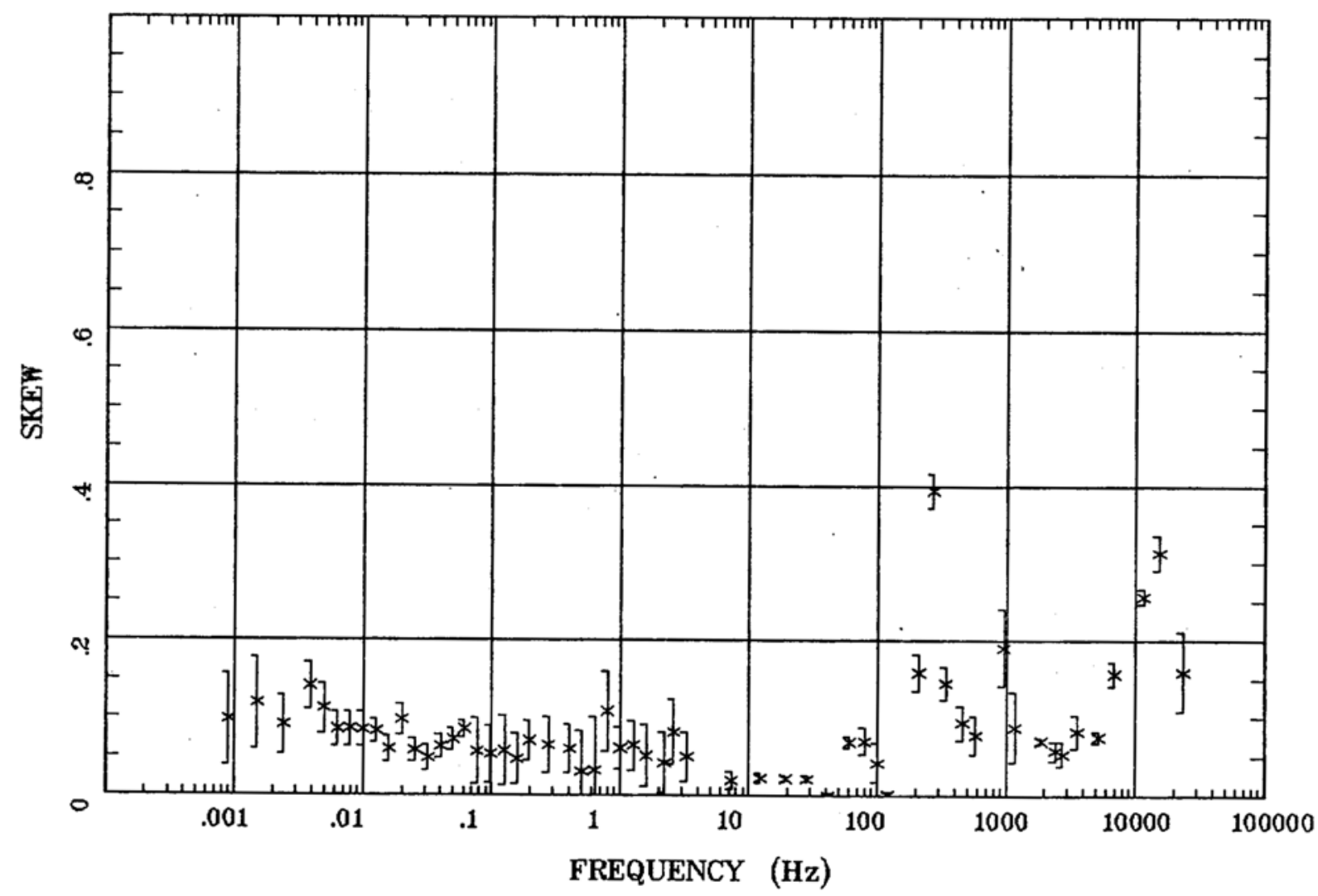

Client: DOE

Remote: none

Acquired: 21:3 May 14, 2005

Survey Co:USGS
Rotation:

Filename: rm17.avg

Channels: Ch1 Ch2 Ch3 Ch4 Ch5 Ch3 Ch4

Plotted: 13:28 Jan 17, 2006

< EMI - ElectroMagnetic Instruments > 


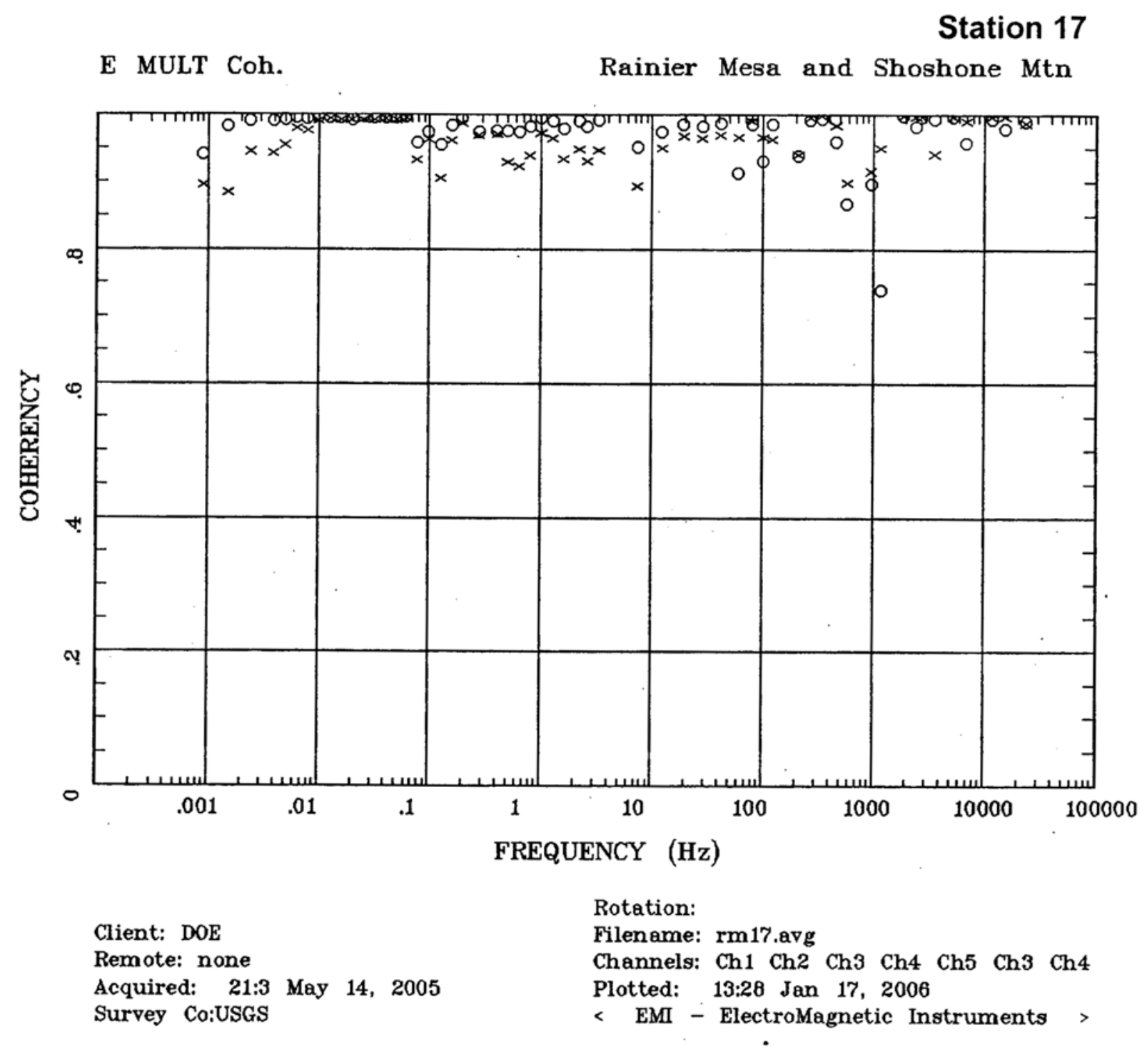


Station 17

POLAR PLOTS

Rainier Mesa and Shoshone Mtn

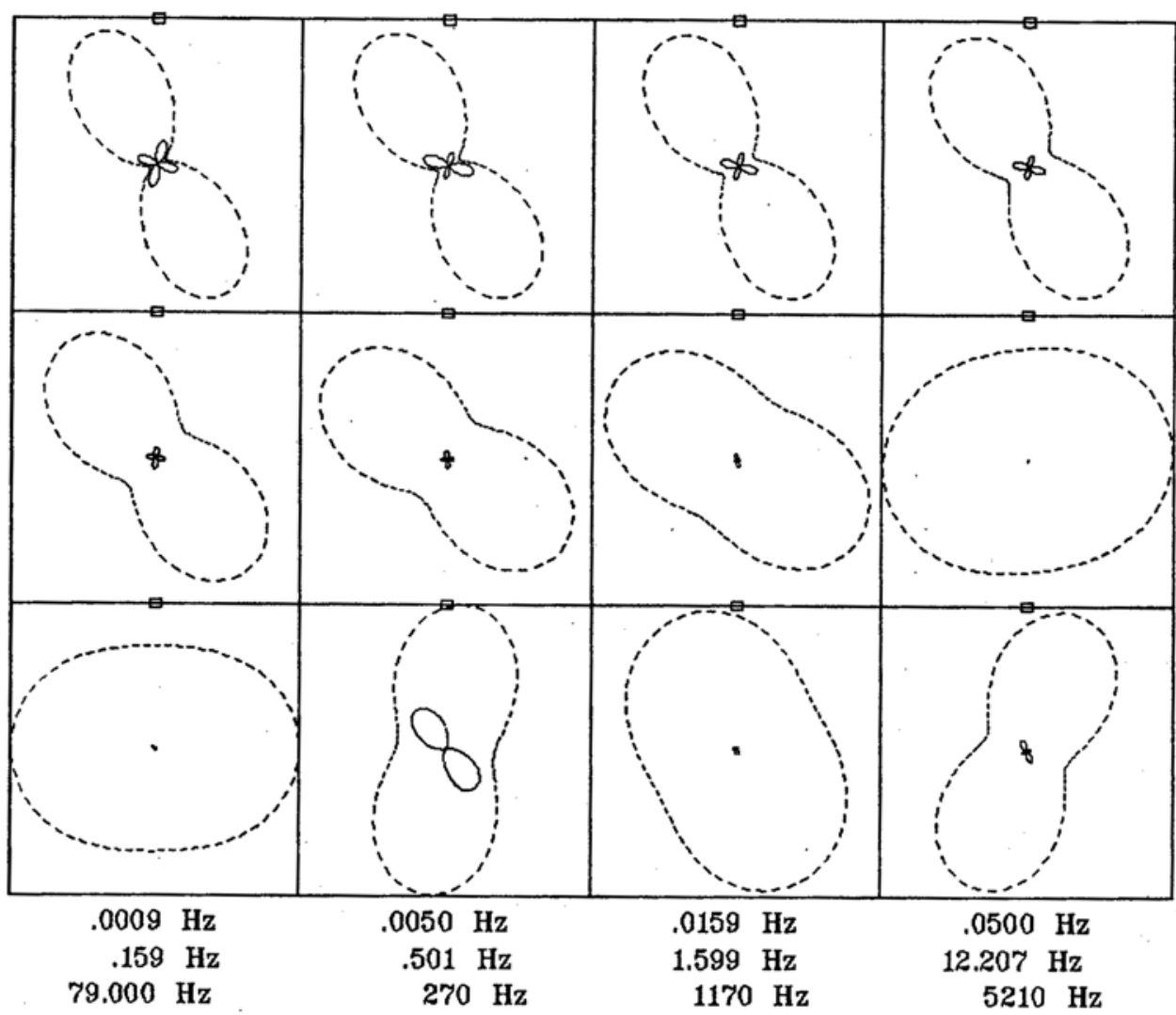

Client: DOE

Rotation:

Remote: none

Acquired: 21:3 May 14, 2005

Filename: rm17.avg

Channels: Ch1 Ch2 Ch3 Ch4 Ch5 Ch3 Ch4

Plotted: 13:28 Jan 17, 2006

Survey Co:USGS

< EMI - ElectroMagnętic Instruments > 
Station 17

TIPPER MAGNITUDE

Rainier Mesa and Shoshone Mtn

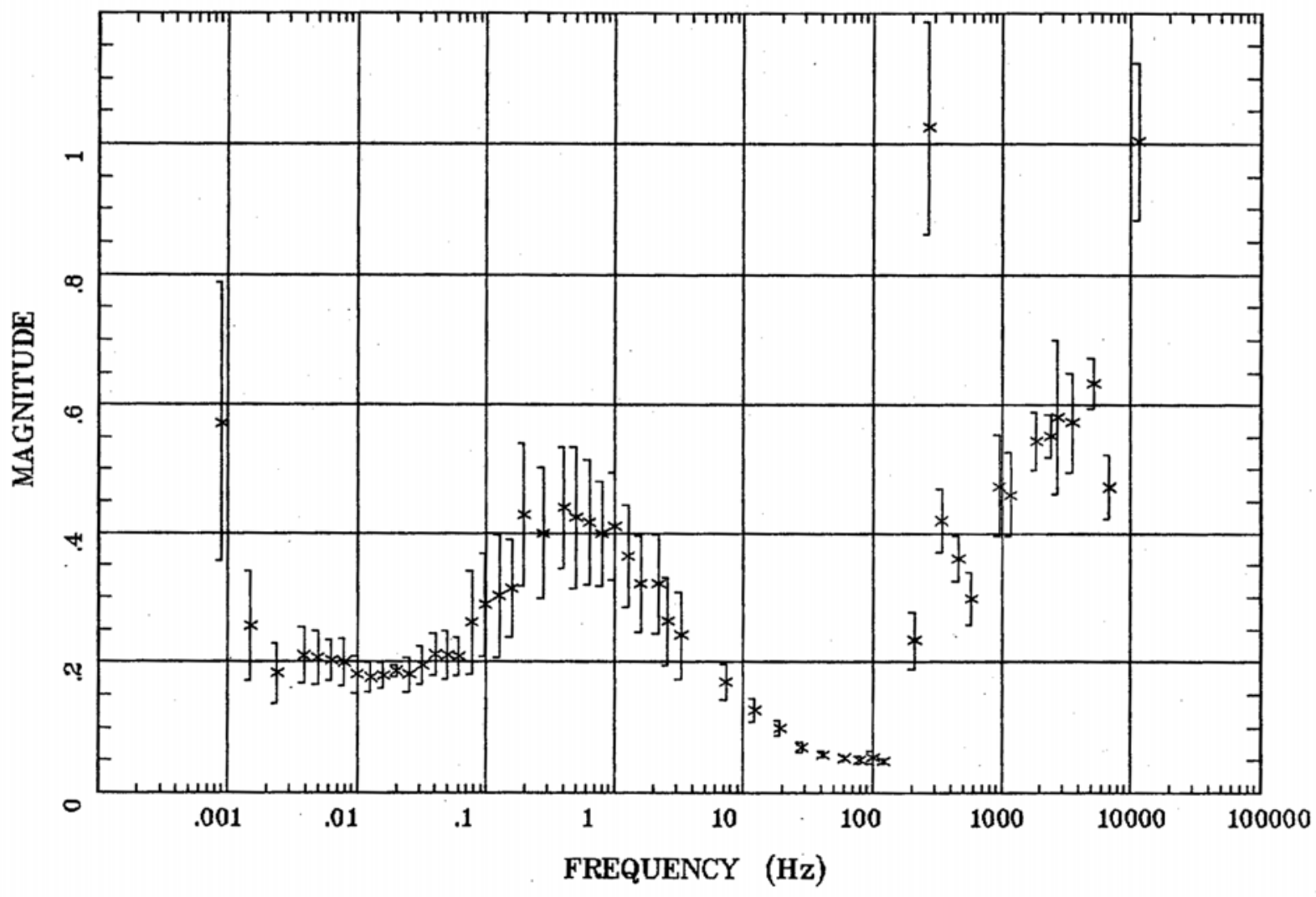

Client: DOE

Remote: none

Acquired: 21:3 May 14, 2005

Survey Co:USGS

Rotation:

Filename: rm17.avg

Channels: Ch1 Ch2 Ch3 Ch4 Ch5 Ch3 Ch4

Plotted: 13:28 Jan 17, 2006

< EMI - ElectroMagnetic Instruments > 


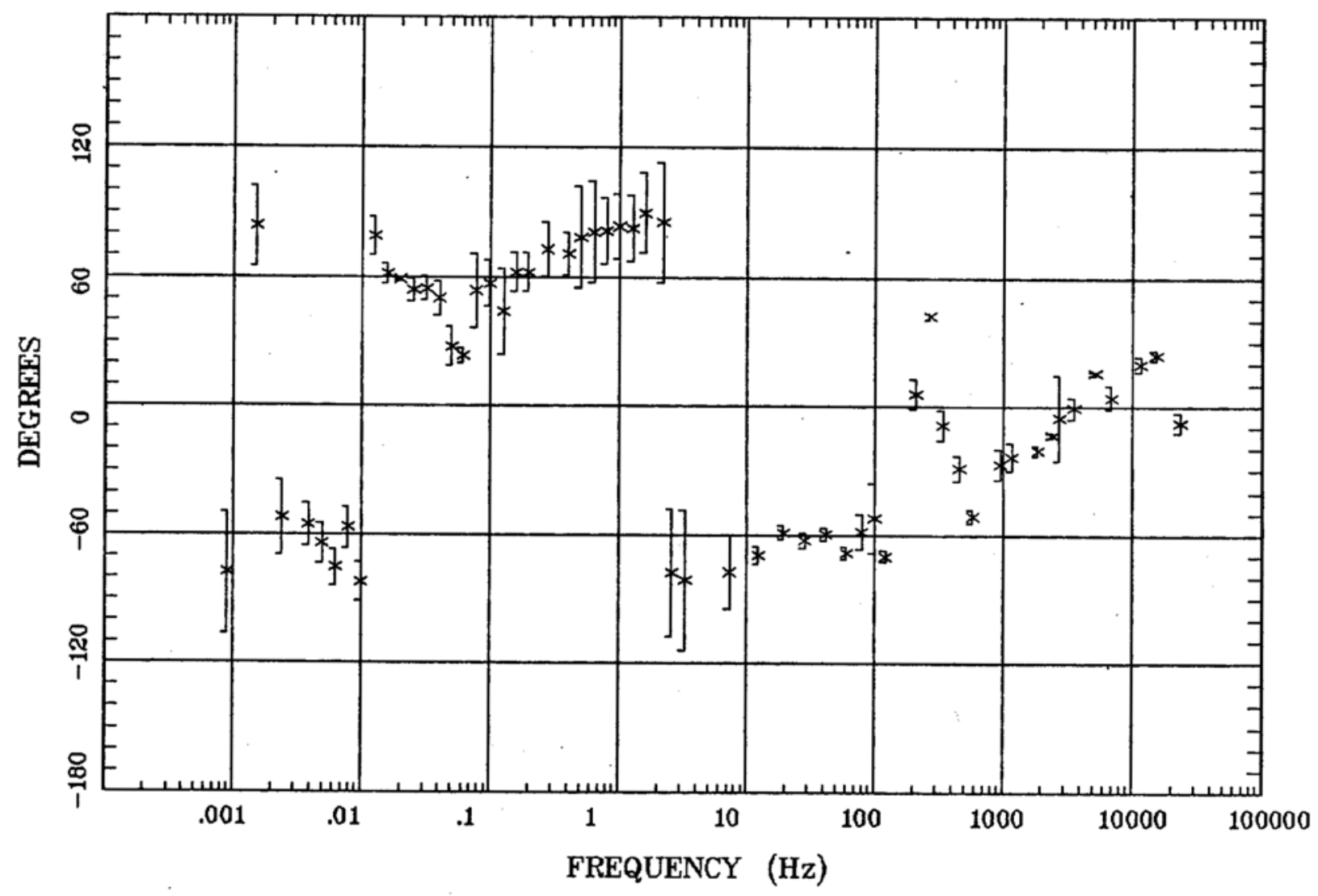

Client: DOE

Remote: none

Acquired: 21:3 May 14, 2005

Survey Co:USGS
Rotation:

Filename: rm17.avg

Channels: Ch1 Ch2 Ch3 Ch4 Ch5 Ch3 Ch4

Plotted: 13:28 Jan 17, 2006

< EMI - ElectroMagnetic Instruments > 
HzHx.x Coh HzHy.o

Station 17

Rainier Mesa and Shoshone Mtn

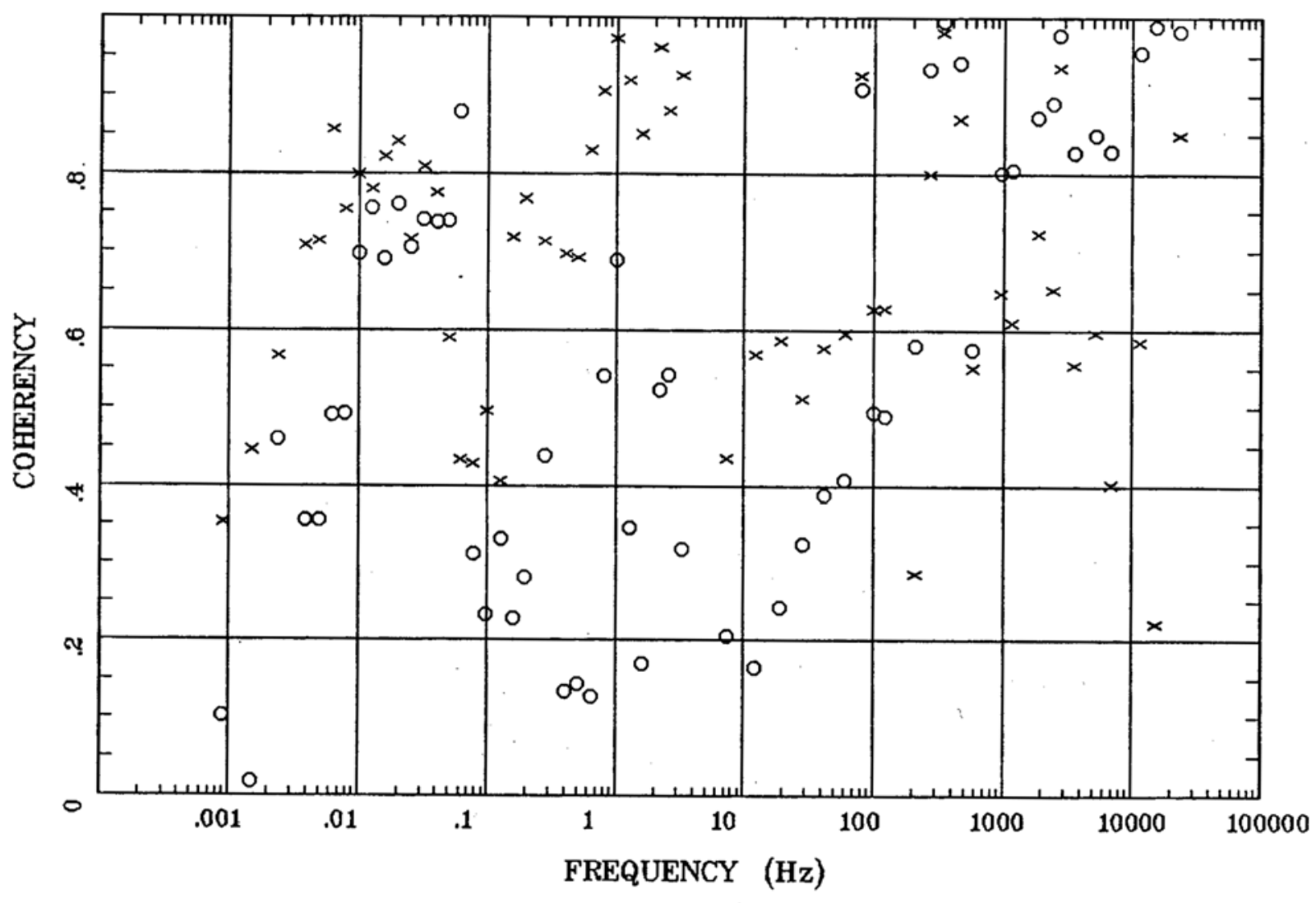

Client: DOE

Remote: none

Acquired: 21:3 May 14, 2005

Survey Co:USGS
Rotation:

Filename: rm17.avg

Channels: Ch1 Ch2 Ch3 Ch4 Ch5 Ch3 Ch4

Plotted: 13:28 Jan 17, 2006

< EMI - ElectroMagnetic Instruments > 


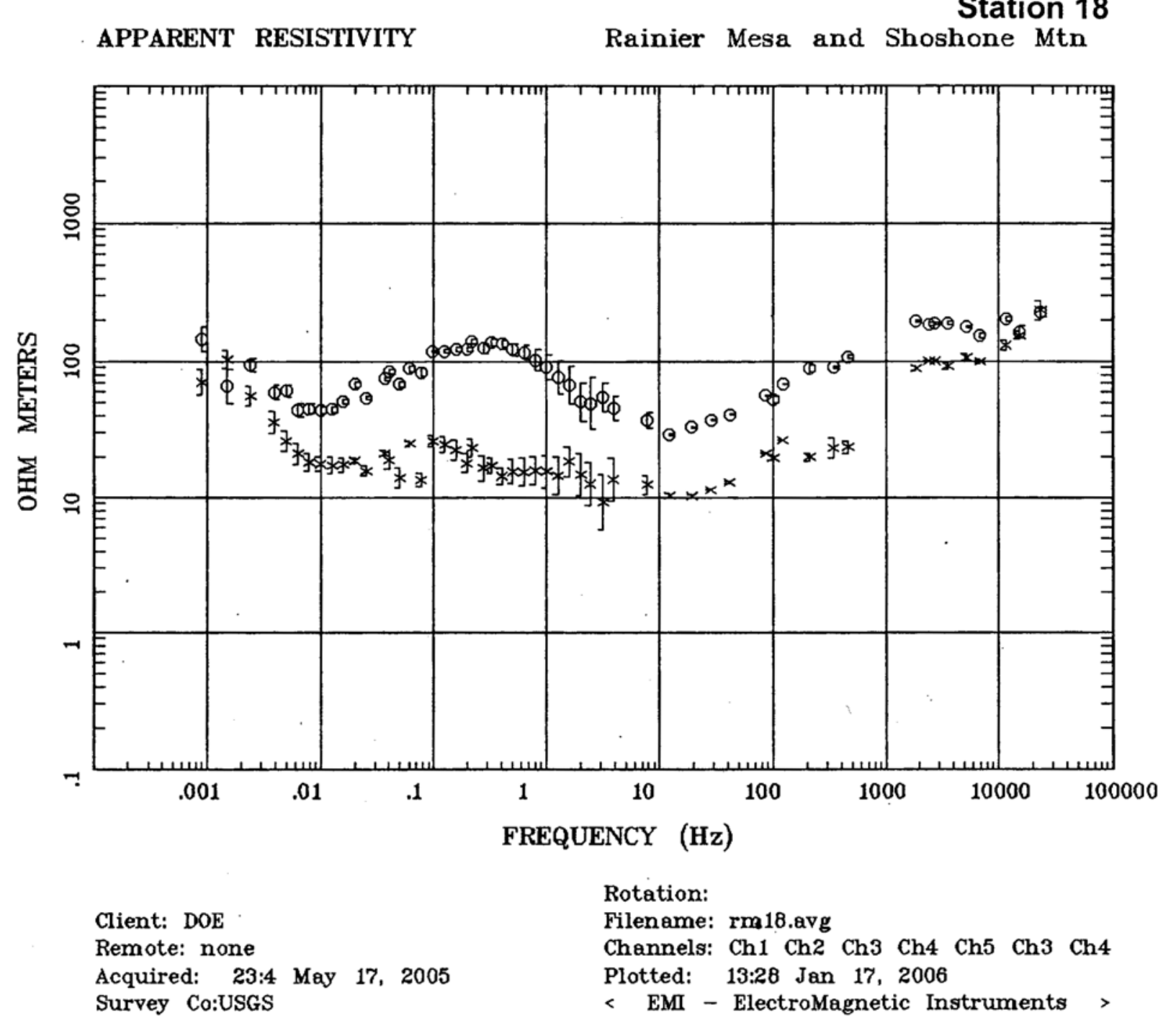




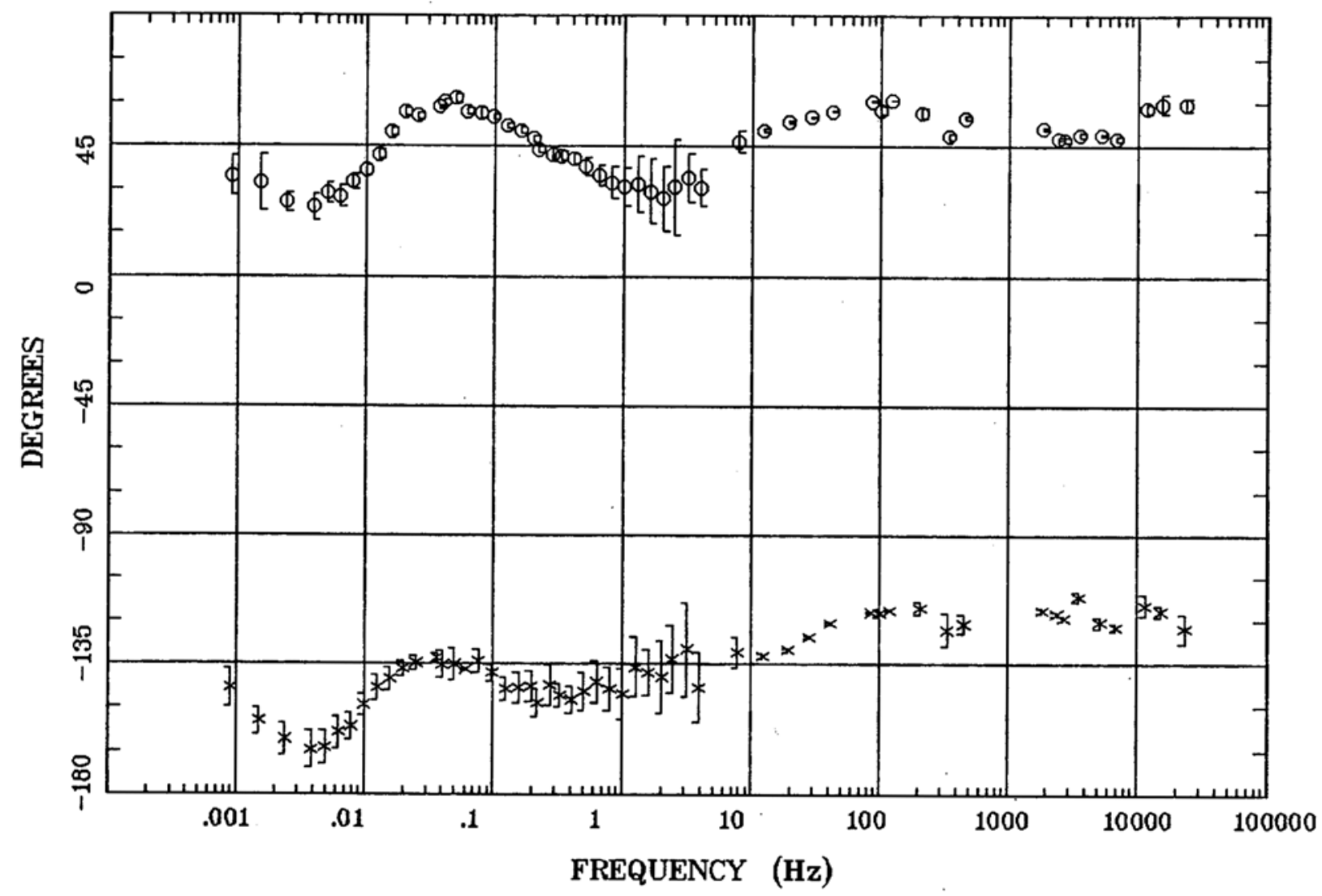

Client: $\mathrm{DOE}$

Remote: none

Acquired: 23:4 May 17, 2005

Survey Co:USGS

Rotation:

Filename: rm18.avg

Channels: Ch1 Ch2 Ch3 Ch4 Ch5 Ch3 Ch4

Plotted: 13:28 Jan 17, 2006

< EMI - ElectroMagnetic Instruments > 


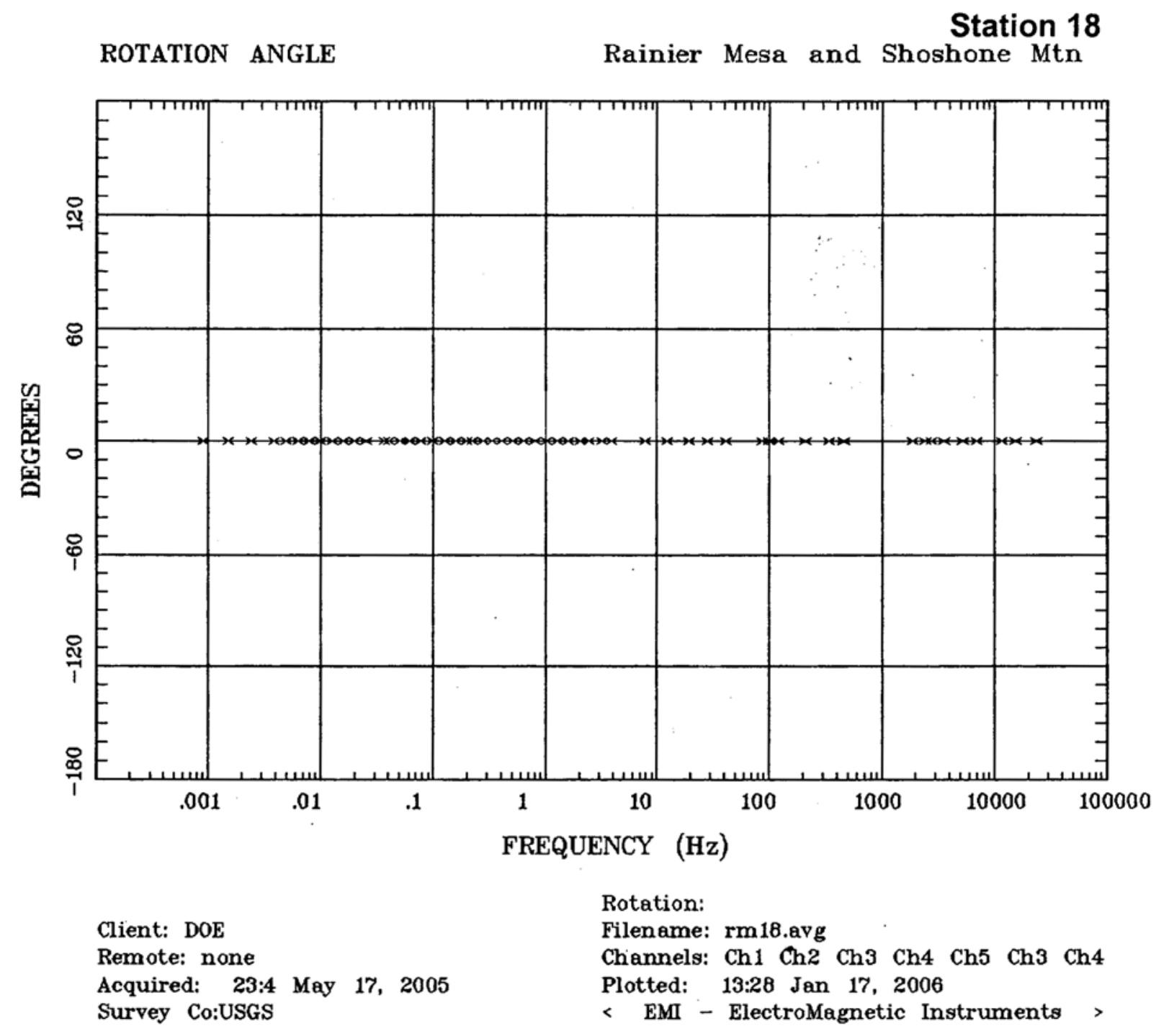




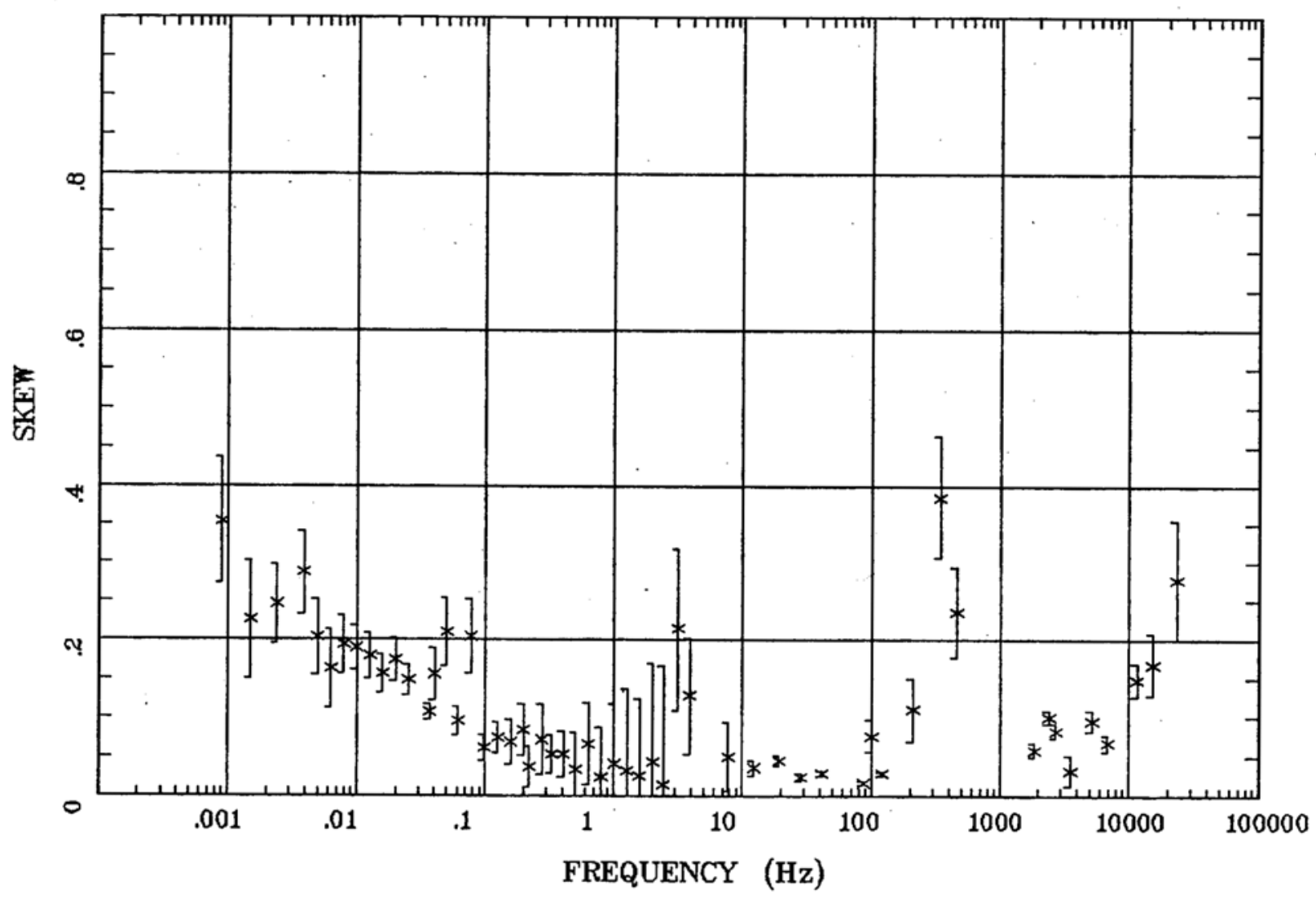

Client: DOE

Remote: none

Acquired: 23:4 May 17, 2005

Survey Co:USGS
Rotation:

Filename: rm18.avg.

Channels: Ch1 Ch2 Ch3 Ch4 Ch5 Ch3 Ch4 Plotted: 13:29 Jan 17, 2006

< EMI - ElectroMagnetic Instruments > 
E MULT Coh.

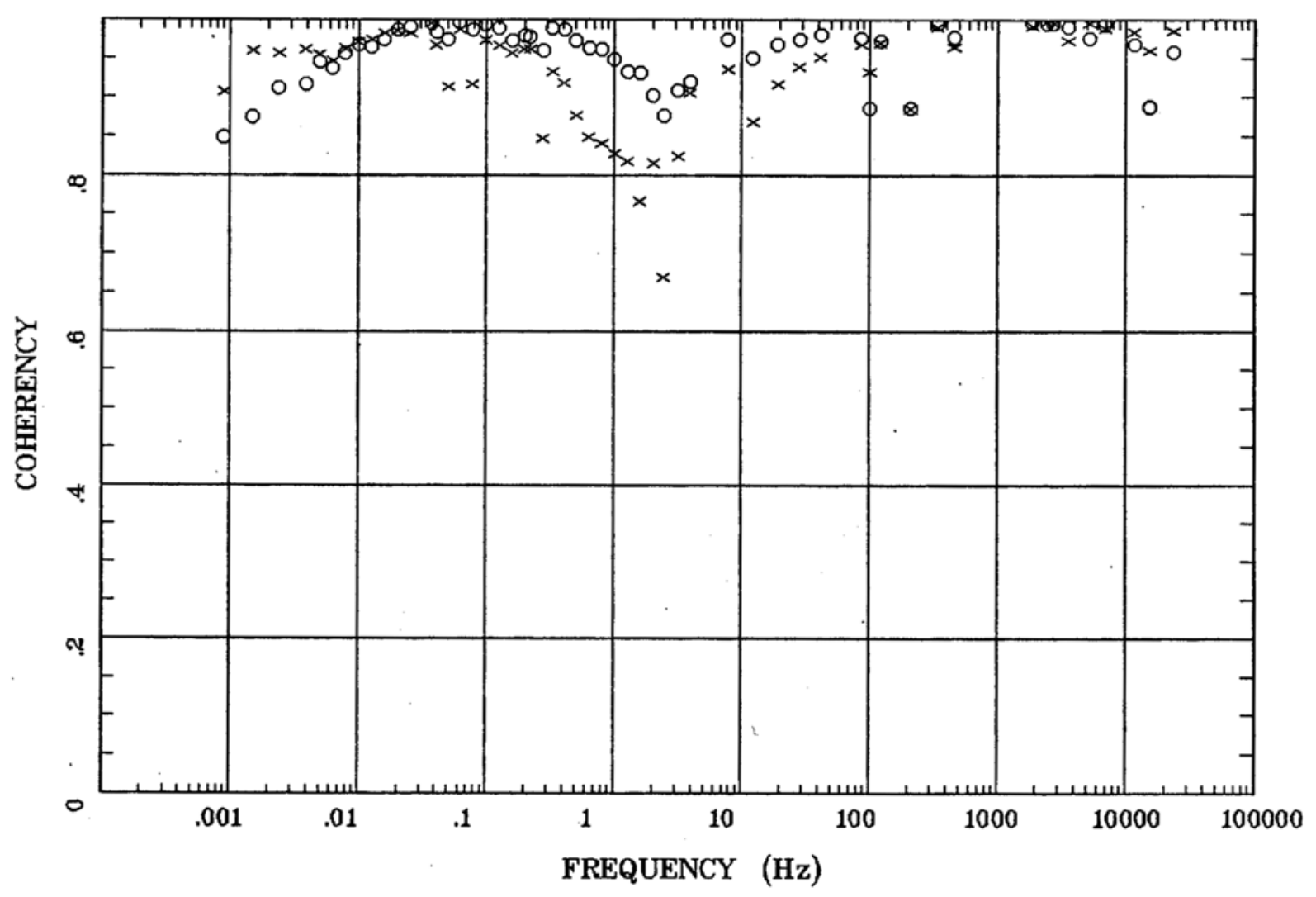

Client: DOE

Remote: none

Acquired: 23:4 May 17, 2005

Survey Co:USGS
Station 18

Rainier Mesa and Shoshone Mtn 


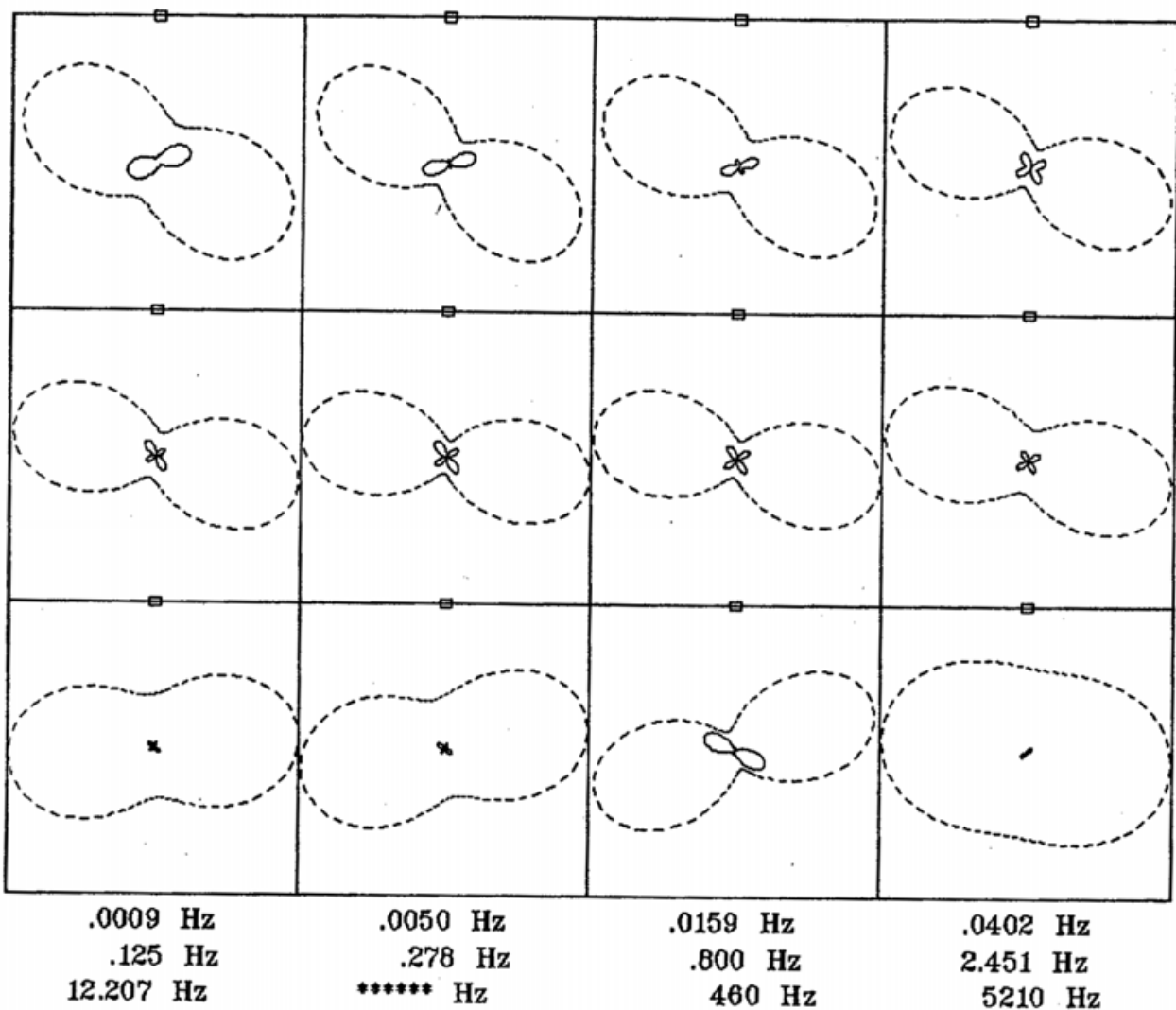

Client: DOE

Remote: none

Acquired: 23:4 May 17, 2005

Survey Co:USGS
Rainier Mesa and Shoshone Mtn

Rotation:

Filename: rm18.avg

Channels: Ch1 Ch2 Ch3 Ch4 Ch5 Ch3 Ch4

Plotted: 13:29 Jan 17, 2006

< EMI - ElectroMagnetic Instruments > 


\section{TIPPER MAGNITUDE}

\section{Station 18}

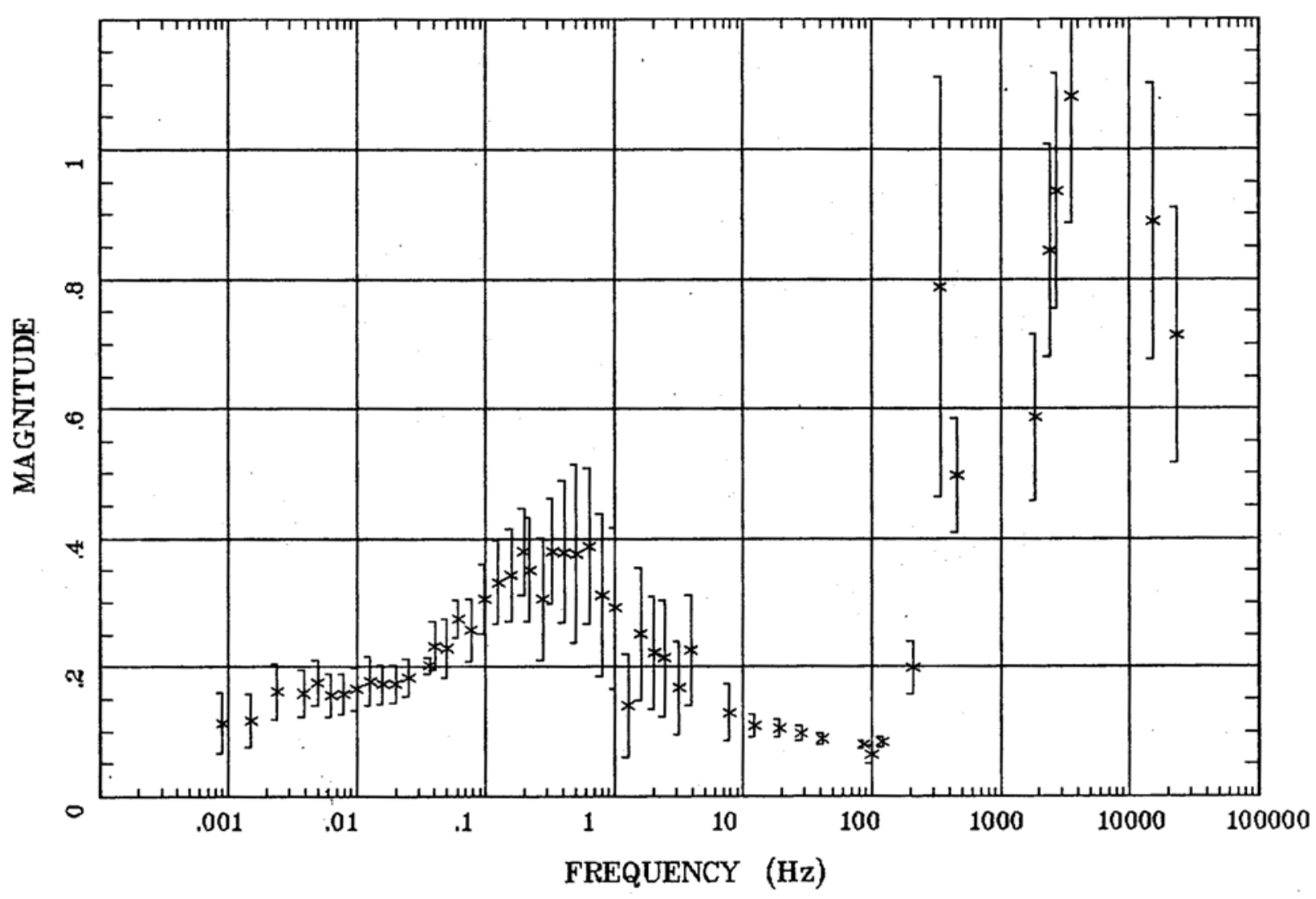

Client: DOE

Remote: none

Acquired: 23:4 May 17, 2005

Survey Co:USGS
Rainier Mesa and Shoshone Mtn

Rotation:

Filename: rm18.avg.

Channels: Ch1 Ch2 Ch3 Ch4 Ch5 Ch3 Ch4

Plotted: 13:29 Jan 17, 2006

< EMI - ElectroMagnetic Instruments > 


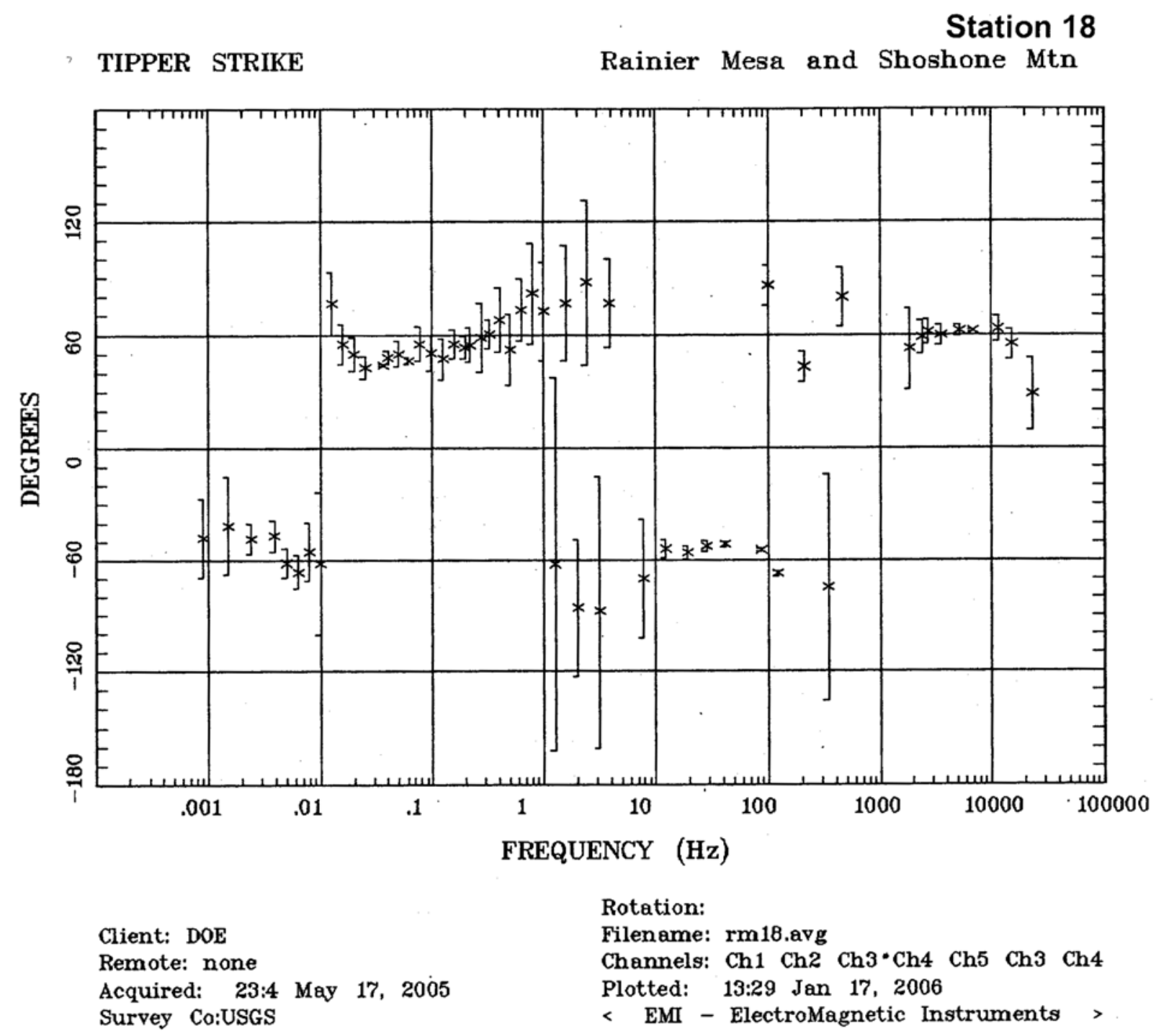


HzHx.x Coh HzHy.o

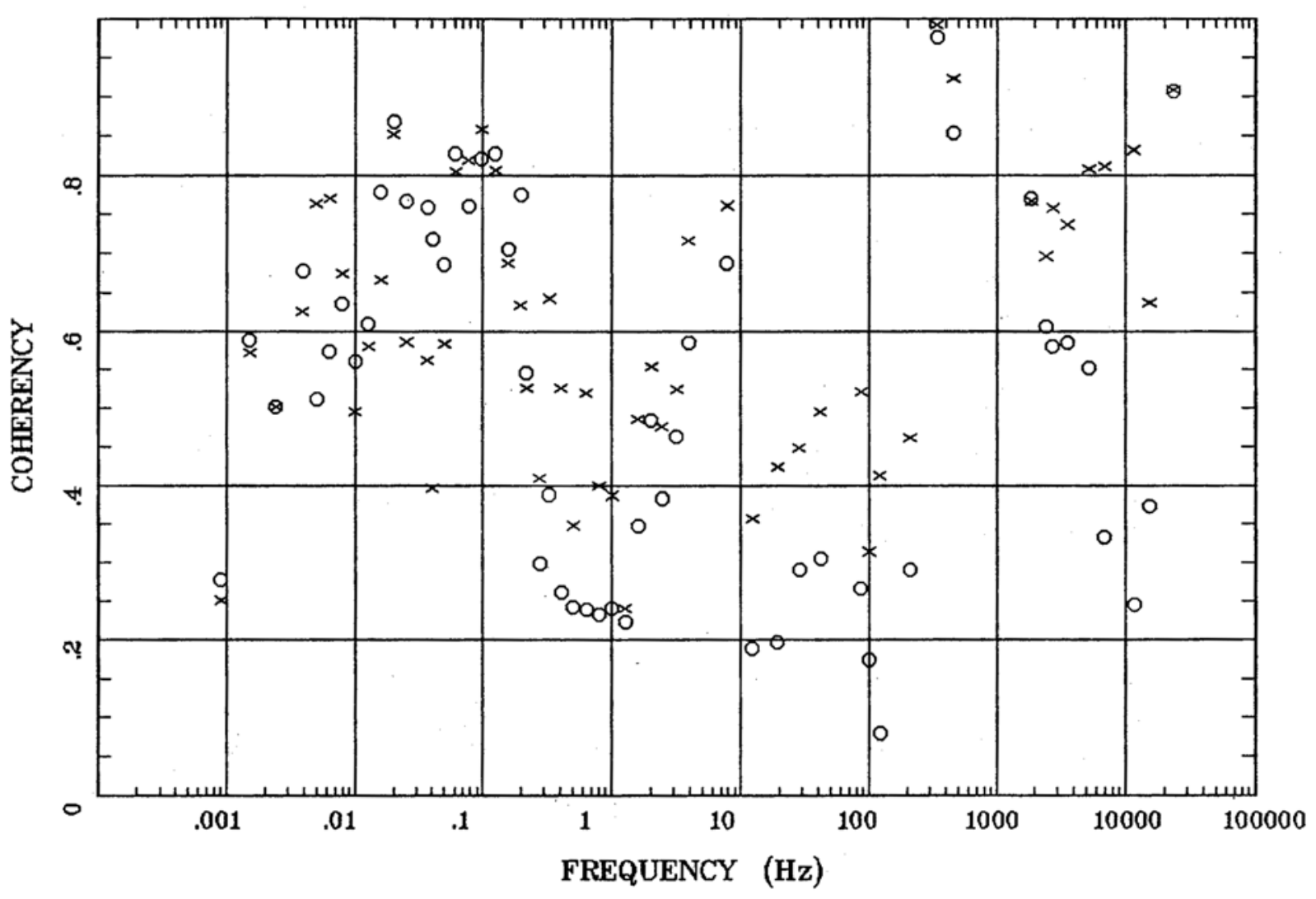

Client: DOE

Remote: none

Acquired: 23:4 May 17, 2005

Survey Co:USGS
Station 18

Rainier Mesa and Shoshone Mtn

Rotation:

Filename: rm18.avg

Channels: Ch1 Ch2 Ch3 Ch4 Ch5 Ch3 Ch4

Plotted: 13:29 Jan 17, 2006

< EMI - ElectroMagnetic Instruments > 
Station 19

APPARENT RESISTIVITY

Rainier Mesa and Shoshone Mtn

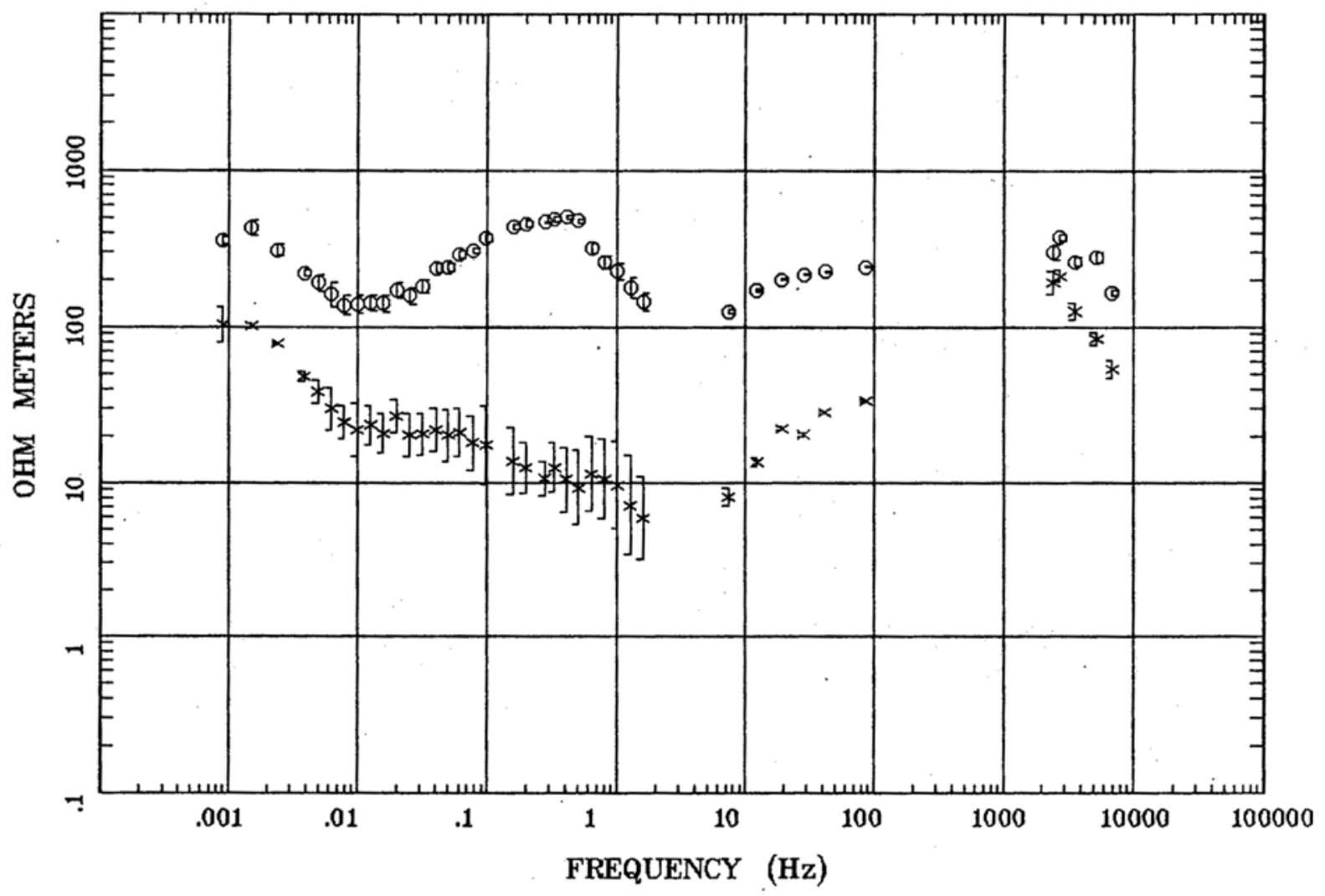

Client: DOE

Remote: none

Acquired: 01:3 May 16, 2005

Survey Co:USGS
Rotation:

Filename: rm19.avg "

Channels: Ch1 Ch2 Ch3 Ch4 Ch5 Ch3 Ch4

Plotted: 13:30 Jan 17, 2006

< EMI - ElectroMagnetic Instruments > 
Station 19

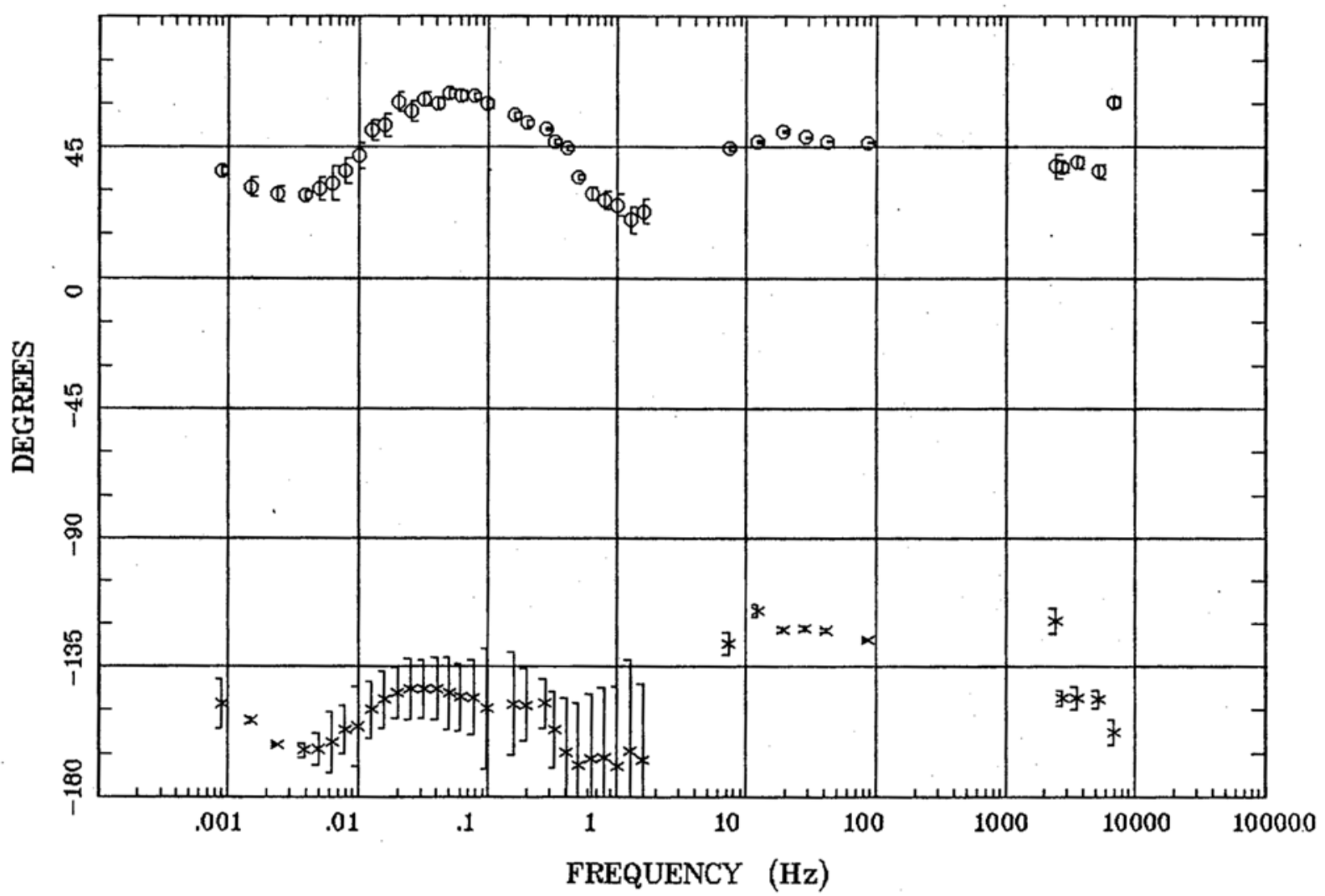

Client: $\mathrm{DOE}$

Remote: none

Acquired: 01:3 May 16, 2005

Survey Co:USGS
Rotation:

Filename: rm19.avg

Channels: Ch1 Ch2 Ch3 Ch4 Ch5 Ch3 Ch4

Plotted: 13:30 Jan 17, 2006

< EMI - ElectroMagnetic Instruments > 
Station 19

ROTATION ANGLE

Rainier Mesa and Shoshone Mtn

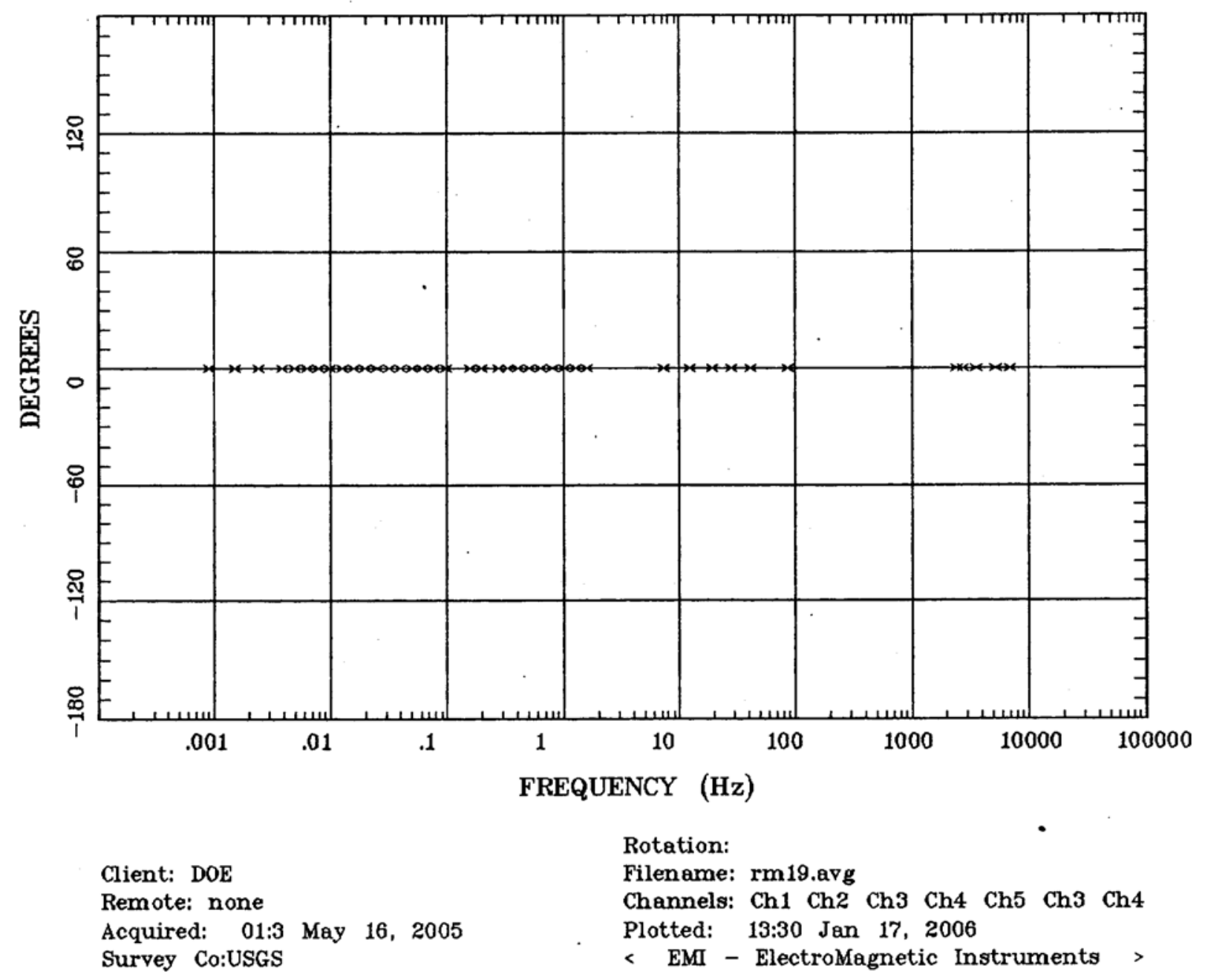




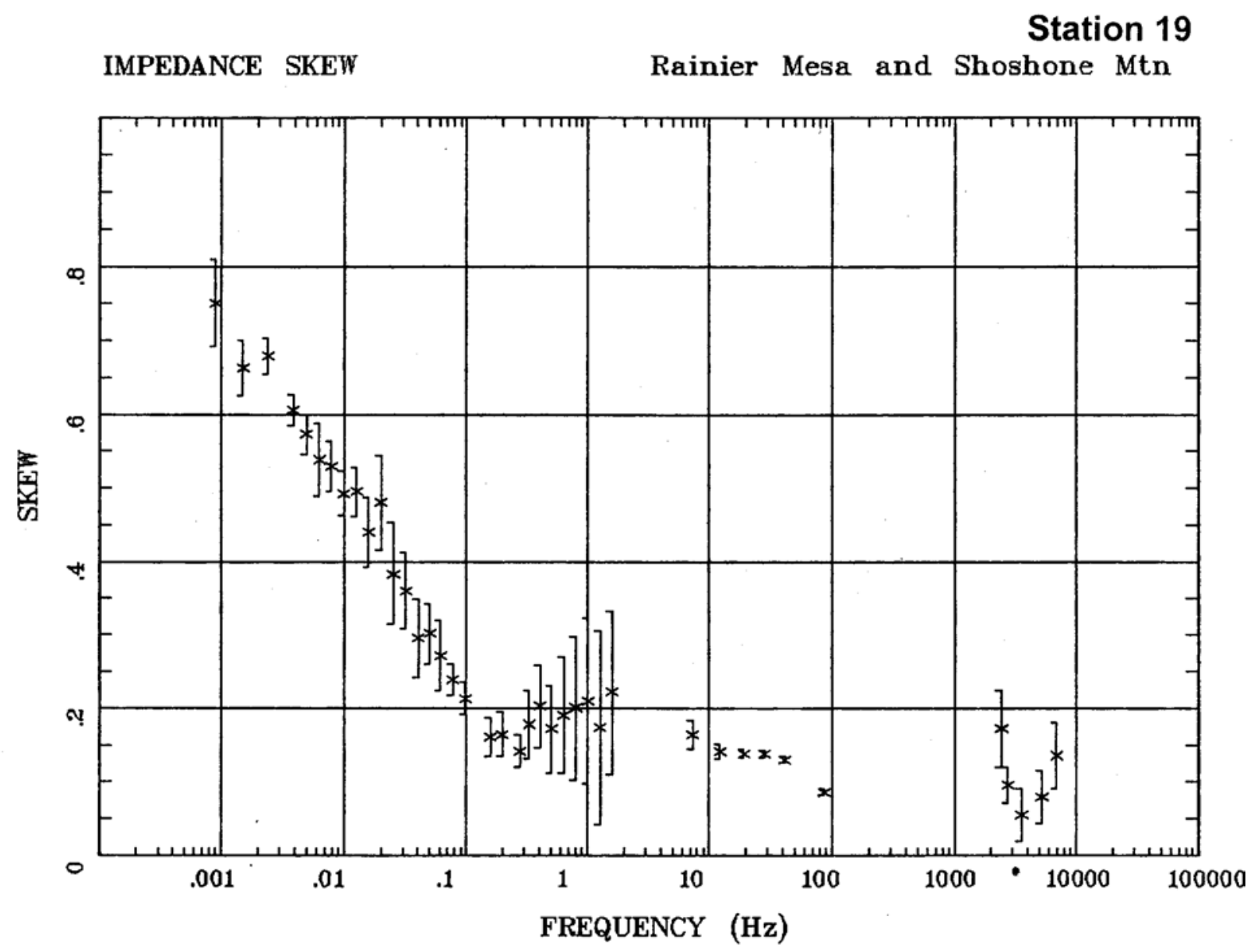

Client: DOE

Remote: none

Acquired: 01:3 May 16, 2005

Survey Co:USGS
Rotation:

Filename: rm19.avg

Channels: Ch1 Ch2 Ch3 Ch4 Ch5 Ch3 Ch4

Plotted: 13:30 Jan 17, 2006

< EMI - ElectroMagnetic Instruments > 
Station 19

E MULT Coh.

Rainier Mesa and Shoshone Mtn

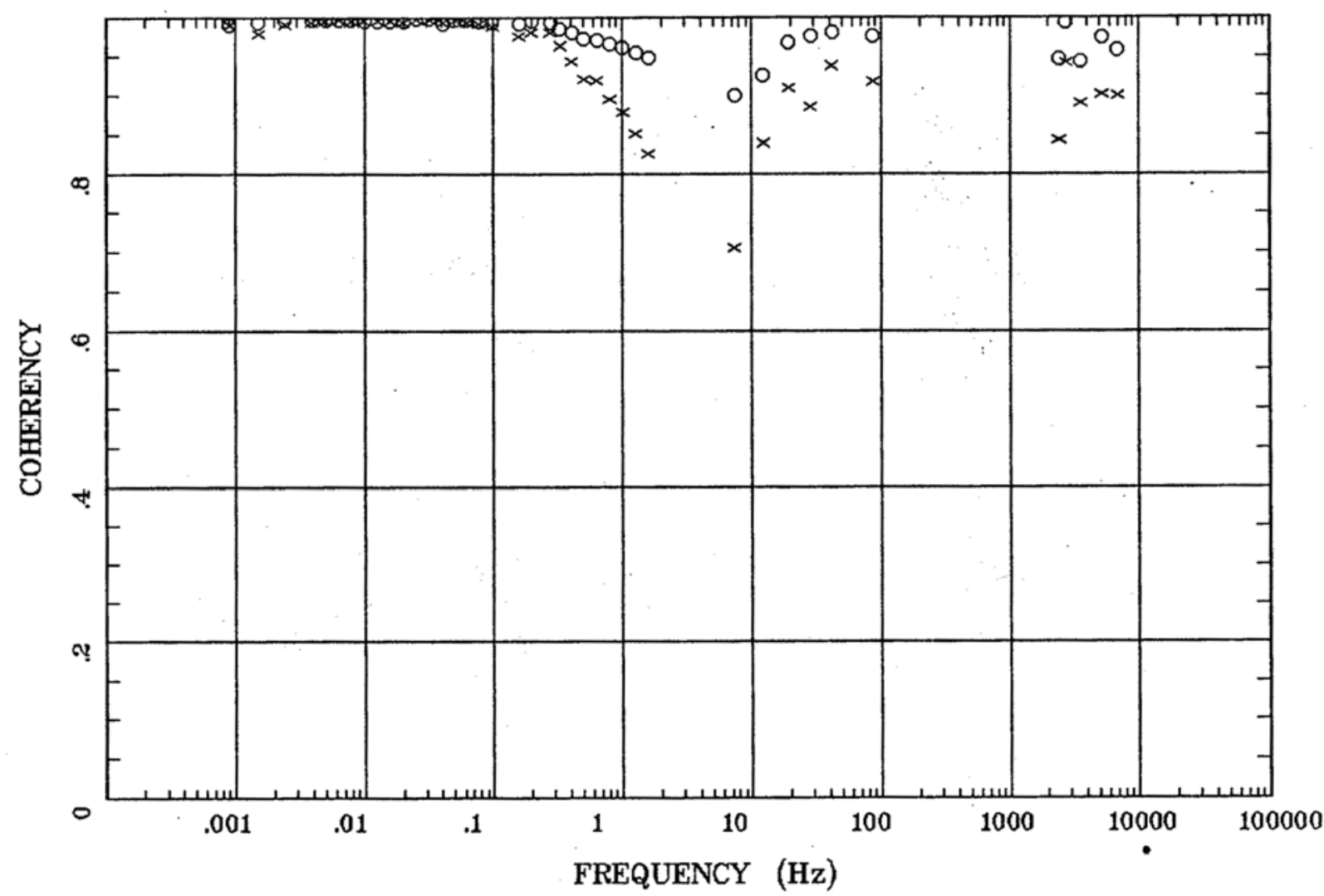

Client: DOE

Remote: none

Acquired: 01:3 May 16, 2005

Survey Co:USGS
Rotation:

Filename: rm19.avg

Channels: Ch1 Ch2 Ch3 Ch4 Ch5 Ch3 Ch4

Plotted: 13:30 Jan 17, 2006

< EMI - ElectroMagnetic Instruments > 


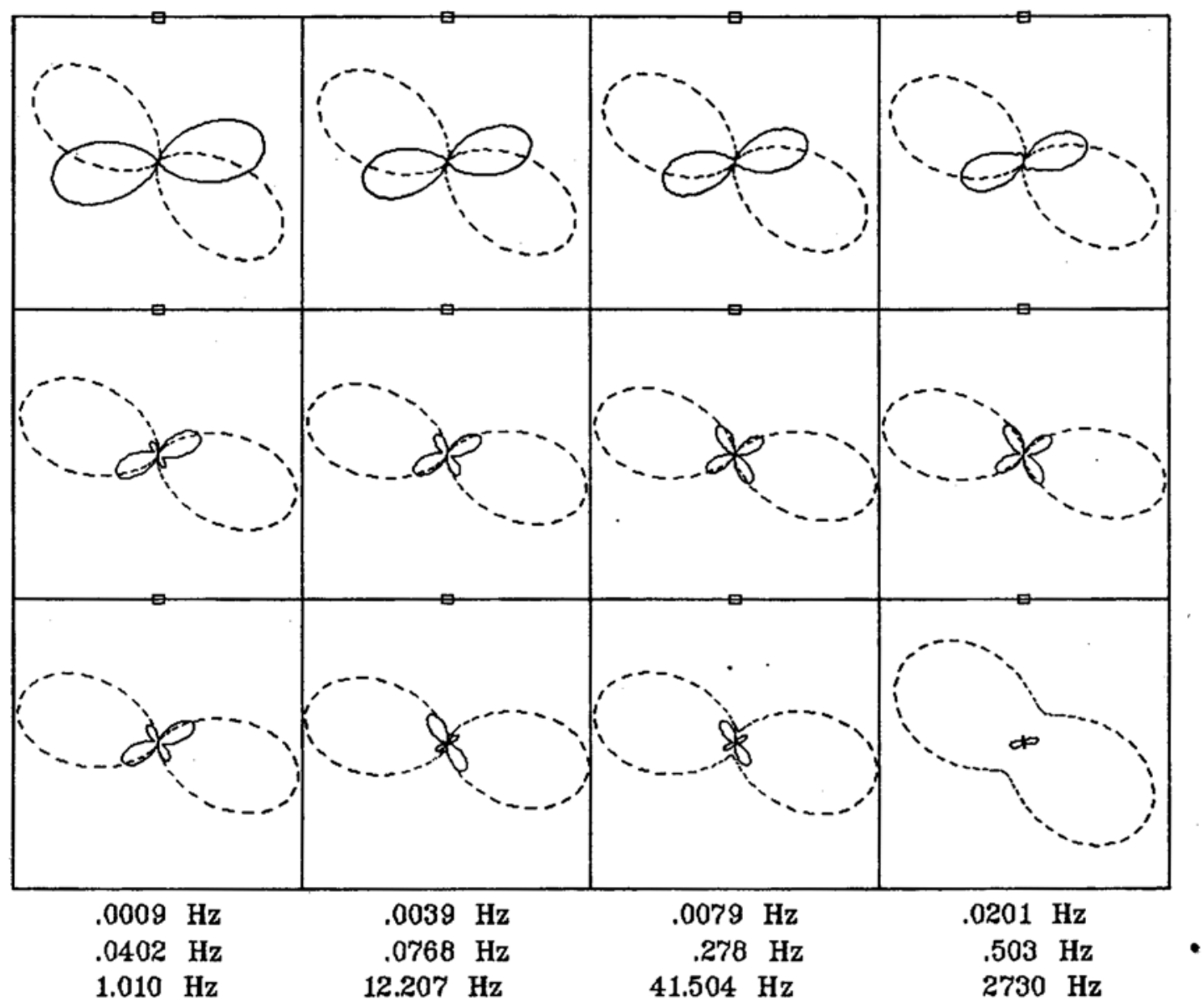

Client: DOE

Remote: none

Acquired: 01:3 May 16, 2005

Survey Co:USGS
Rotation:

Filename: rm19.avg

Channels: Ch1 Ch2 Ch3 Ch4 Ch5 Ch3 Ch4

Plotted: 13:30 Jan 17, 2006

< EMI - ElectroMagnetic Instruments > 
TIPPER MAGNITUDE

Station 19

Rainier Mesa and Shoshone Mtn

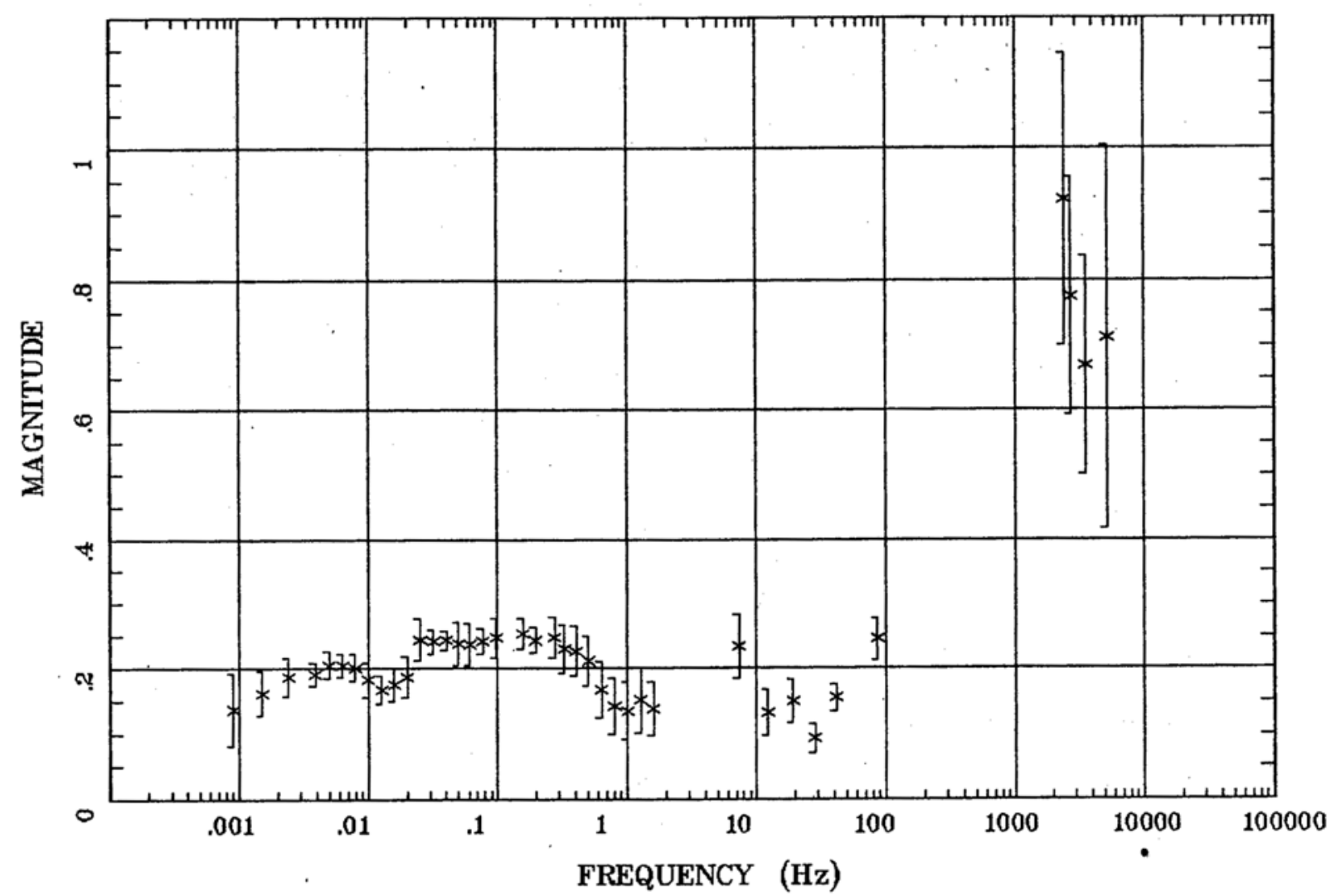

Client: DOE

Remote: none

Acquired: 01:3 May 16, 2005

Survey Co:USGS
Rotation:

Filename: rm19.avg

Channels: Ch1 Ch2 Ch3 Ch4 Ch5 Ch3 Ch4

Plotted: 13:30 Jan 17, 2006

< EMI - ElectroMagnetic Instruments > 


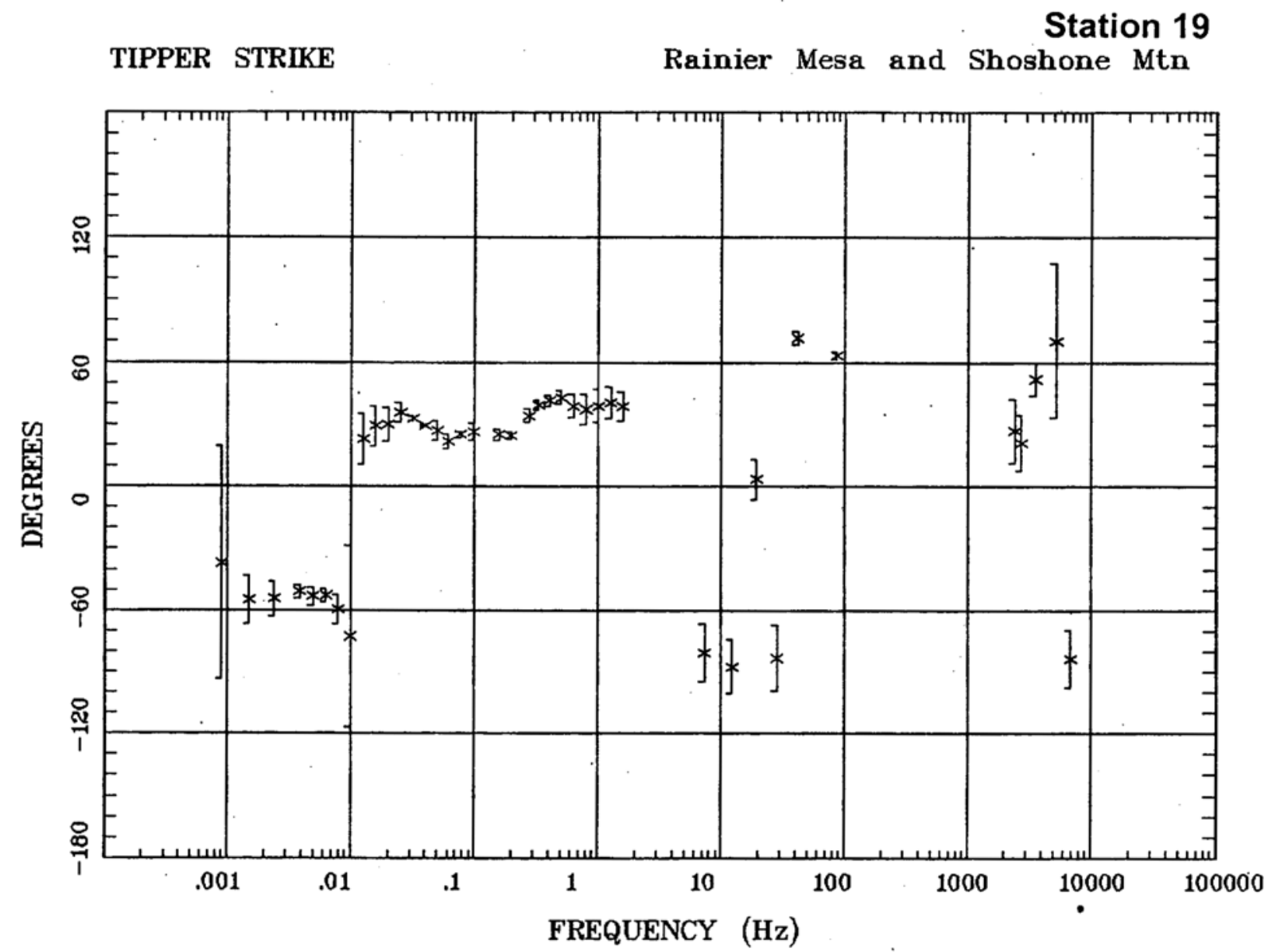

Client: DOE

Remote: none

Acquired: 01:3 May 16, 2005

Survey Co:USGS
Rotation:

Filename: rm19.avg

Channels: Ch1 Ch2 Ch3 Ch4 Ch5 Ch3 Ch4

Plotted: 13:30 Jan 17, 2006

< EMI - ElectroMagnetic Instruments > 
HzHx.x Coh HzHy.o

Station 19

Rainier Mesa and Shoshone Mtn

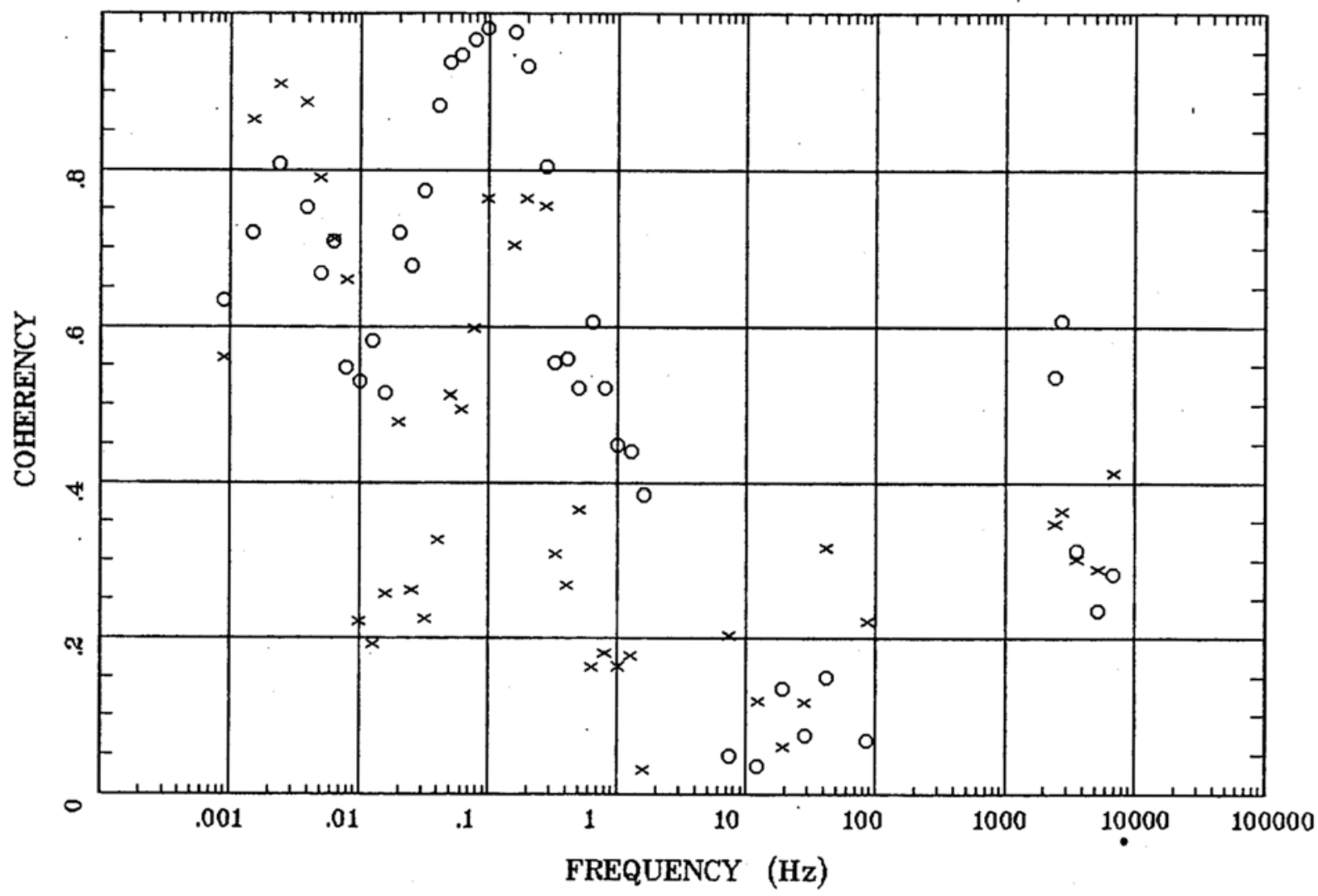

Client: DOE

Remote: none

Acquired: 01:3 May 16, 2005 Survey Co:USGS
Rotation:

Filename: rm19.avg

Channels: Ch1 Ch2 Ch3 Ch4 Ch5 Ch3 Ch4

Plotted: 13:30 Jan 17, 2006

< EMI - ElectroMagnetic Instruments > 


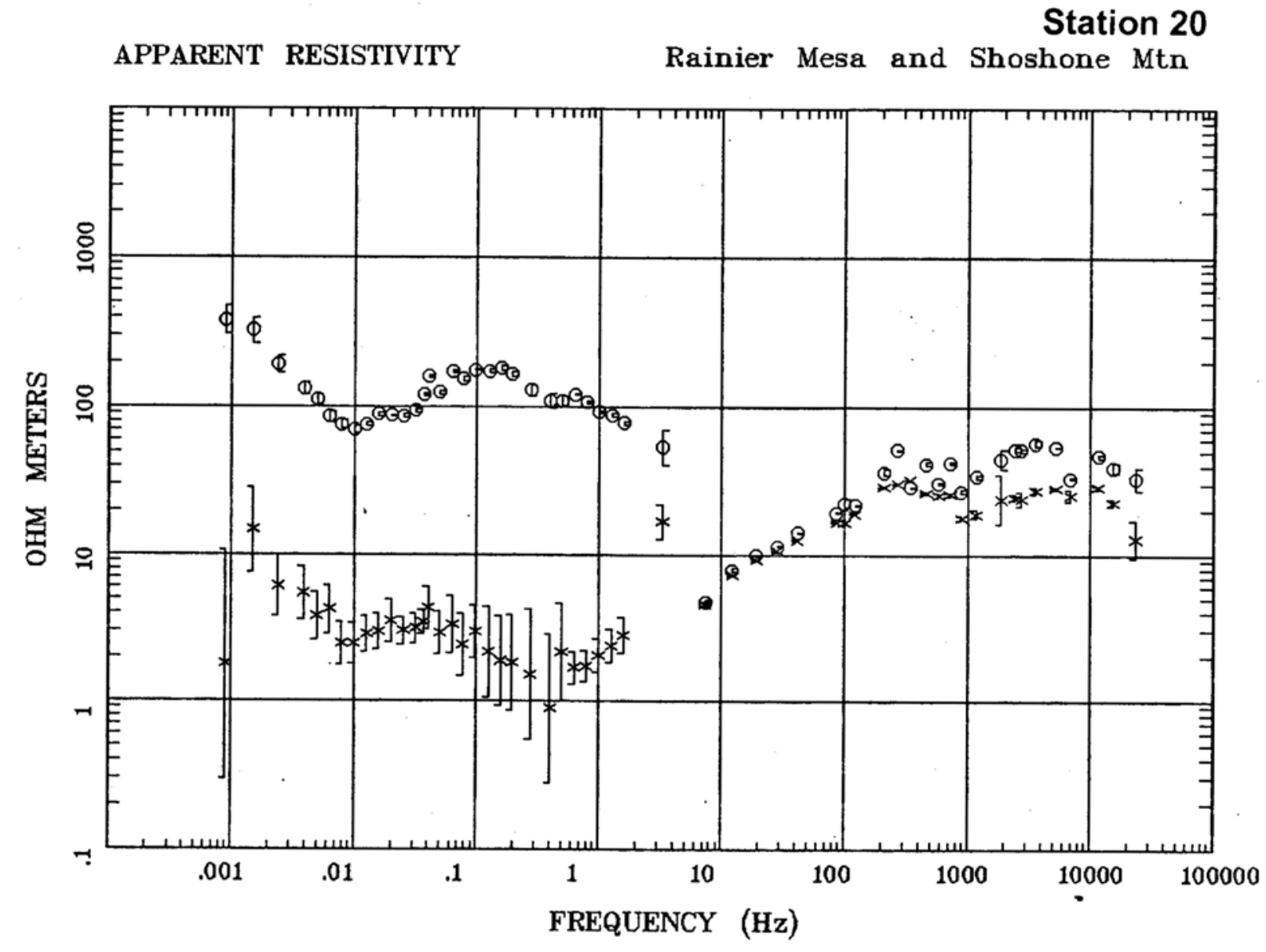

Client: DOE

Remote: none

Acquired: 04:0 May 16, 2005

Survey $\cdot$ Co:USGS
Rotation:

Filename: rm20.avg

Channels: Ch1 Ch2 Ch3 Ch4 Ch5 Ch3 Ch4

Plotted: 13:45 Jan 17, 2006

< EMI - ElectroMagnetic Instruments > 


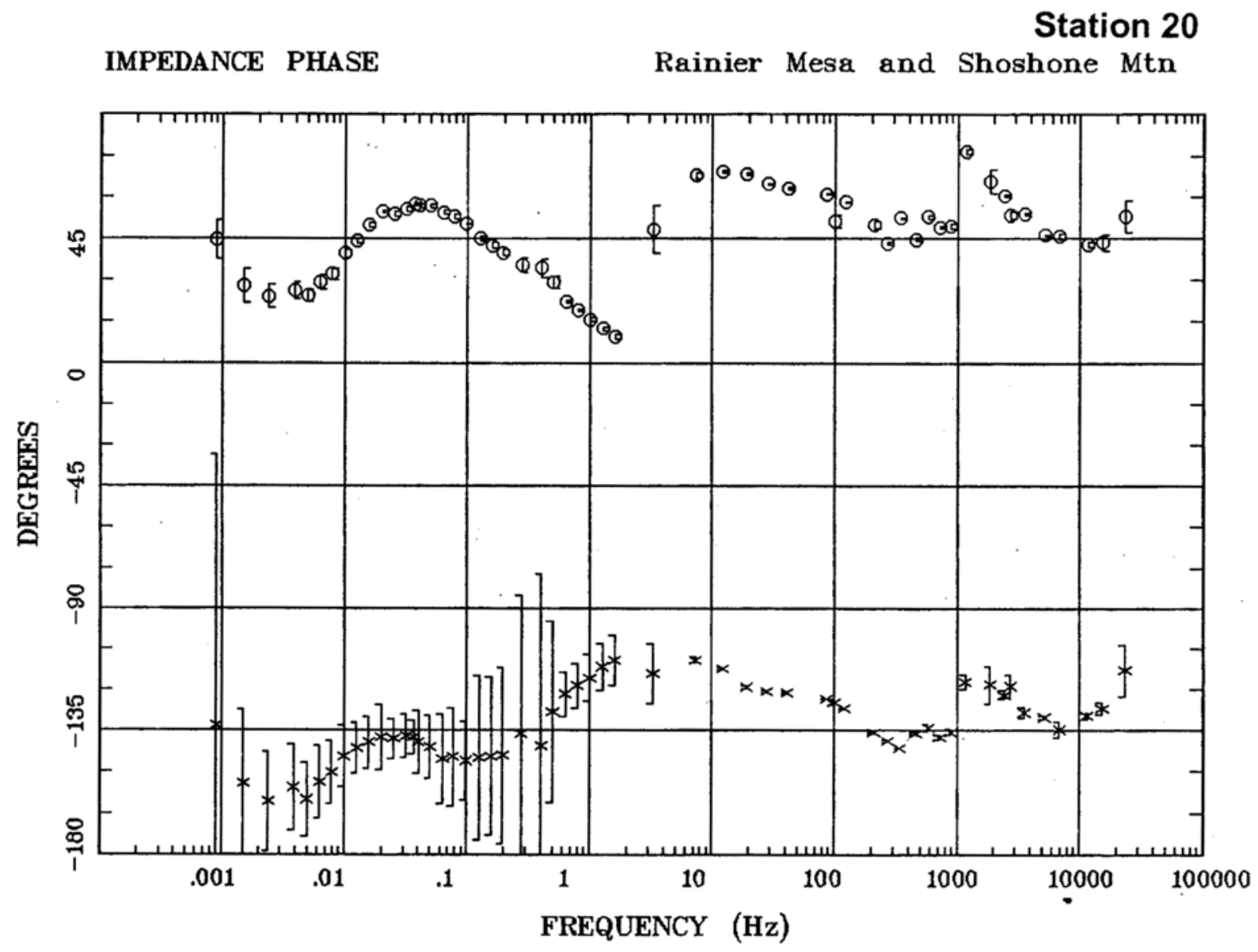

Client: DOE

Remote: none

Acquired: 04:0 May 16, 2005 Survey Co:USGS
Rotation:

Filename: rm20.avg

Channels: Ch1 Ch2 Ch3 Ch4 Ch5 Ch3 Ch4

Plotted: 13:45 Jan 17, 2006

< EMI - ElectroMagnetic Instruments > 


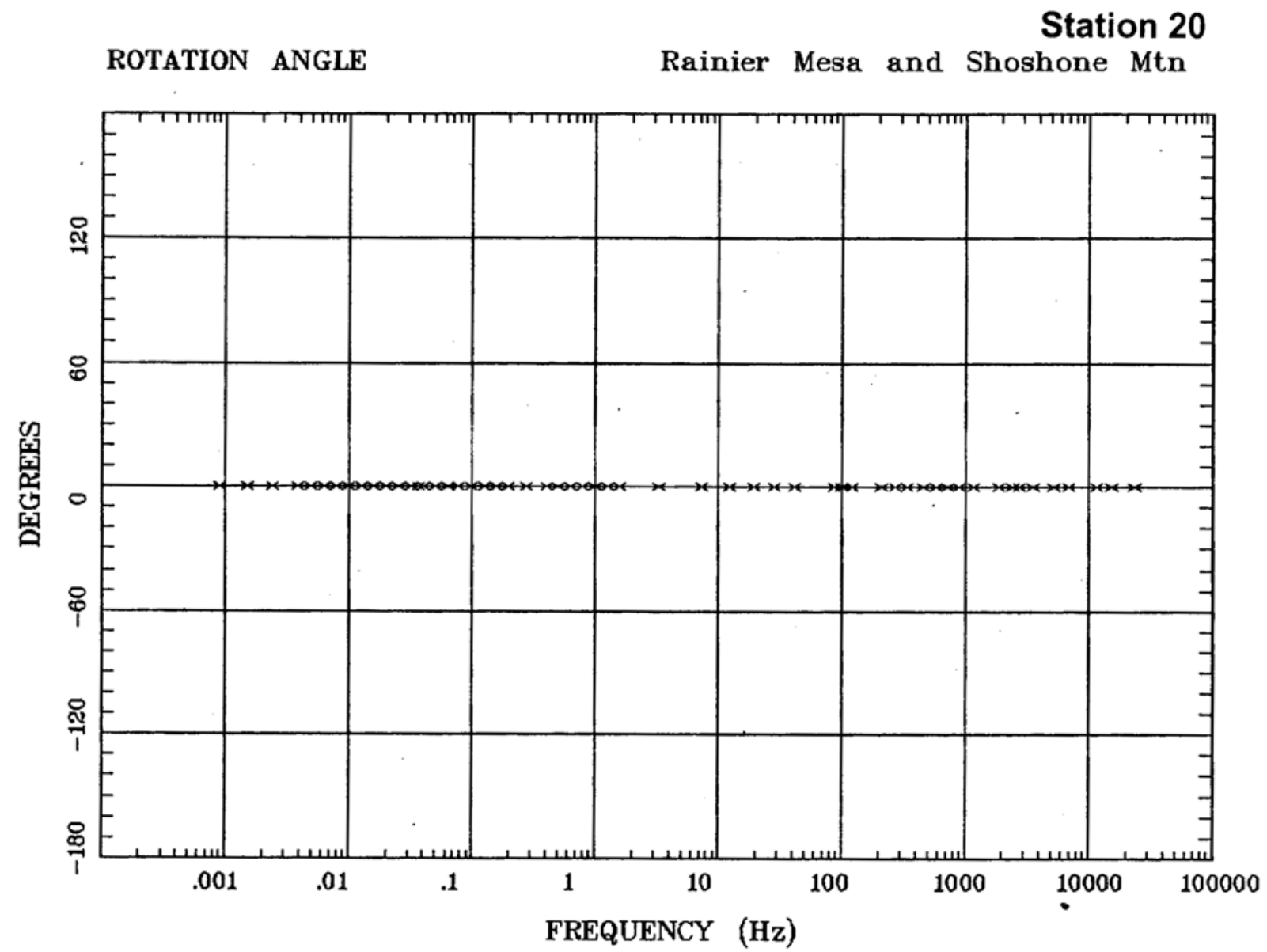

Client: DOE

Remote: none

Acquired: 04:0 May 16, 2005

Survey Co:USGS
Rotation:

Filename: rm20.avg

Channels: Ch1 Ch2 Ch3 Ch4 Ch5 Ch3 Ch4

Plotted: 13:45 Jan 17, 2006

< EMI - ElectroMagnetic Instruments > 
Station 20

IMPEDANCE SKEW

Rainier Mesa and Shoshone Mtn

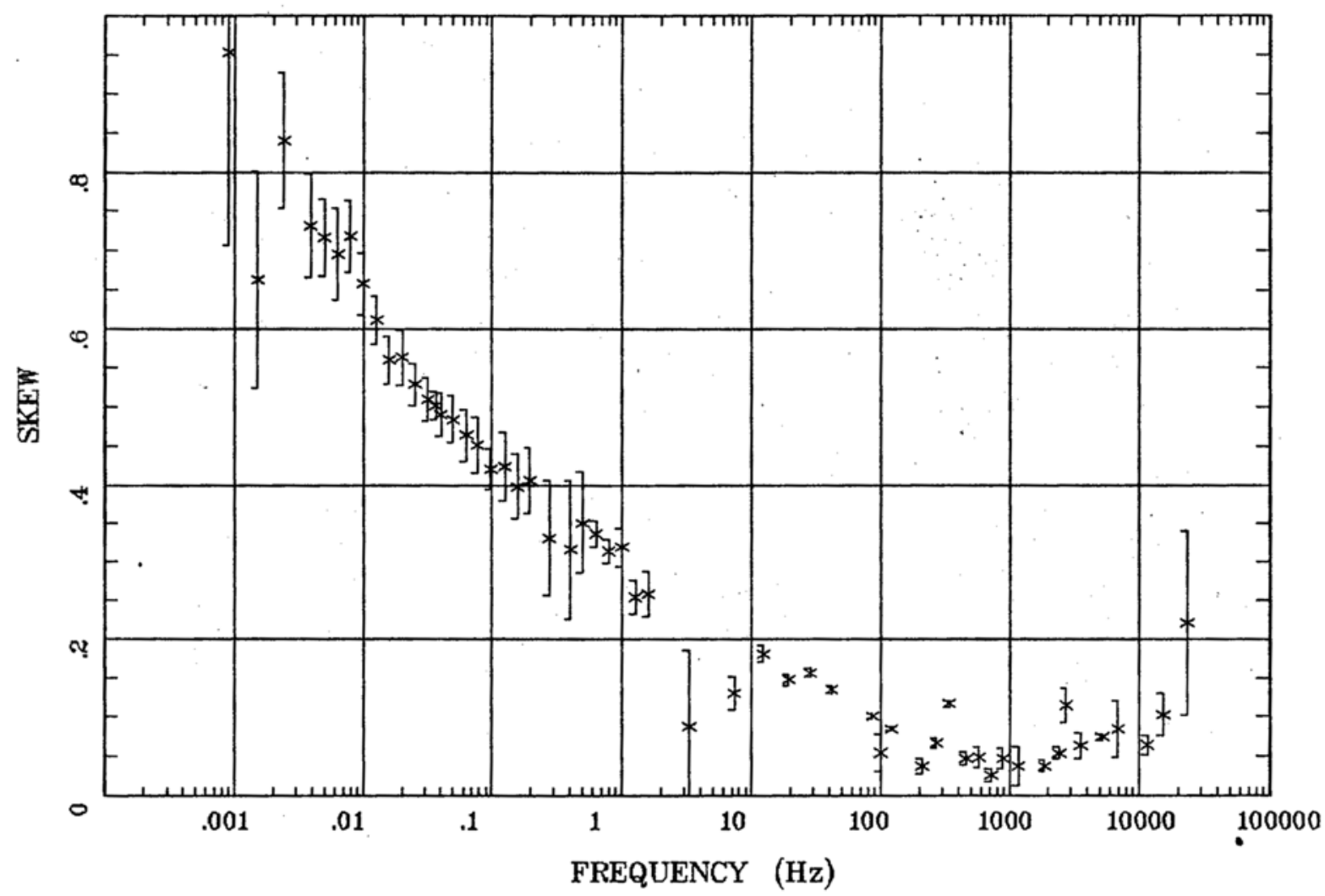

Client: DOE

Remote: none

Acquired: 04:0 May 16, 2005

Survey Co:USGS
Rotation:

Filename: rm20.avg

Channels: Ch1 Ch2 Ch3 Ch4 Ch5 Ch3 Ch4

Plotted: 13:45 Jan 17, 2006

< EMI - ElectroMagnetic Instruments > 
Station 20

E MULT Coh.

Rainier Mesa and Shoshone Mtn

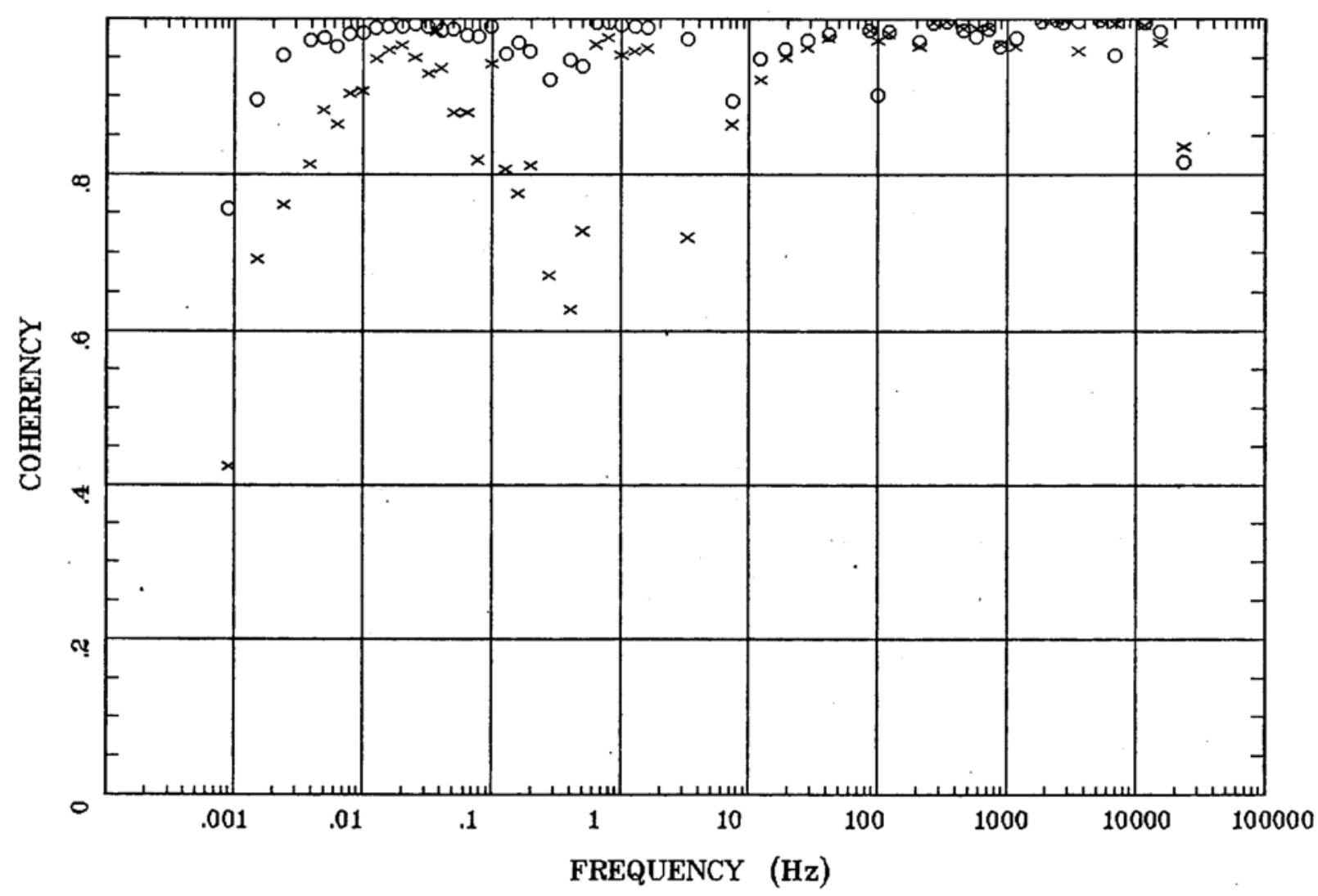

Client: DOE

Remote: none

Acquired: 04:0 May 16, 2005

Survey Co:USGS
Rotation:

Filename: rm20.avg

Channels: Ch1 Ch2 Ch3 Ch4 Ch5 Ch3 Ch4

Plotted: 13:45 Jan 17, 2006

< EMI - ElectroMagnetic Instruments > 
POLAR PLOTS

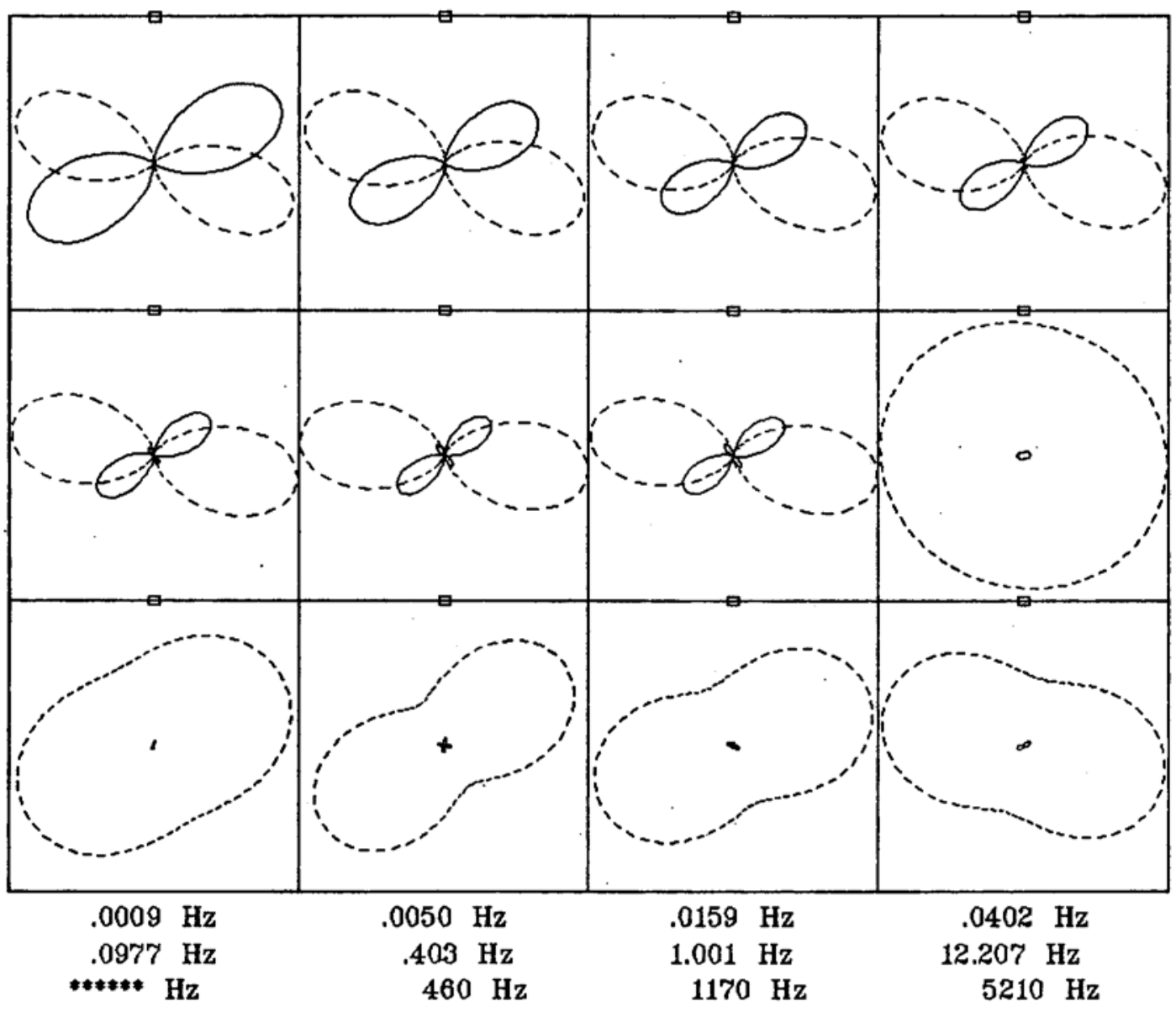

Client: $\mathrm{DOE}$

Remote: none

Acquired: 04:0 May 16, 2005 Survey Co:USGS
Rainier Mesa and Shoshone Mtn

Rotation:

Station 20

Filename: rm20.avg

Channels: Ch1 Ch2 Ch3 Ch4 Ch5 Ch3 Ch4

Plotted: 13:45 Jan 17, 2006

< EMI - ElectroMagnetic Instruments > 
TIPPER MAGNITUDE

Station 20

Rainier Mesa and Shoshone Mtn

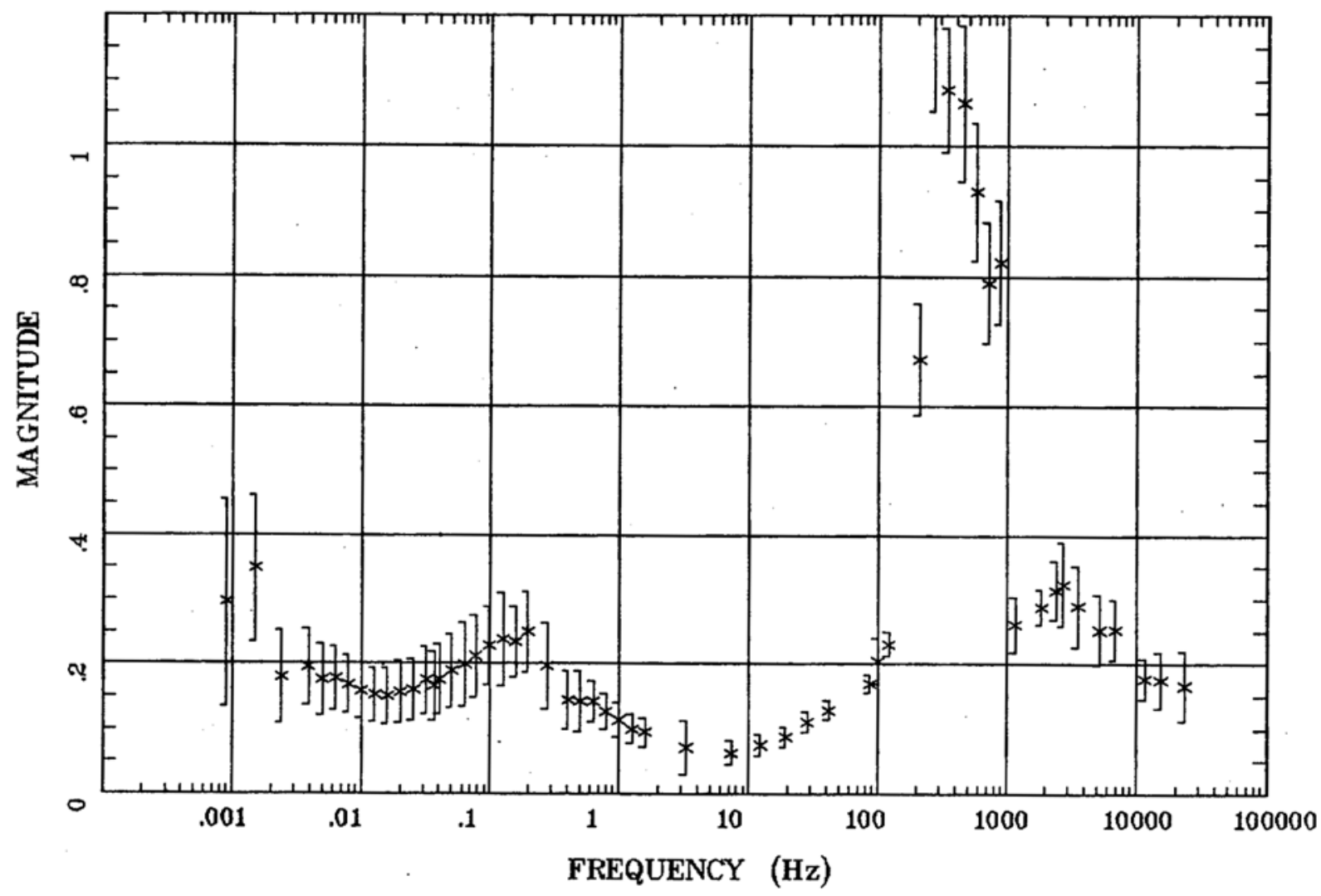

Client: DOE

Remote: none

Acquired: 04:0 May 16, 2005

Survey Co:USGS
Rotation:

Filename: rm20.avg

Channels: Ch1 Ch2 Ch3 Ch4 Ch5 Ch3 Ch4

Plotted: 13:45 Jan 17, 2006

< EMI - ElectroMagnetic Instruments > 


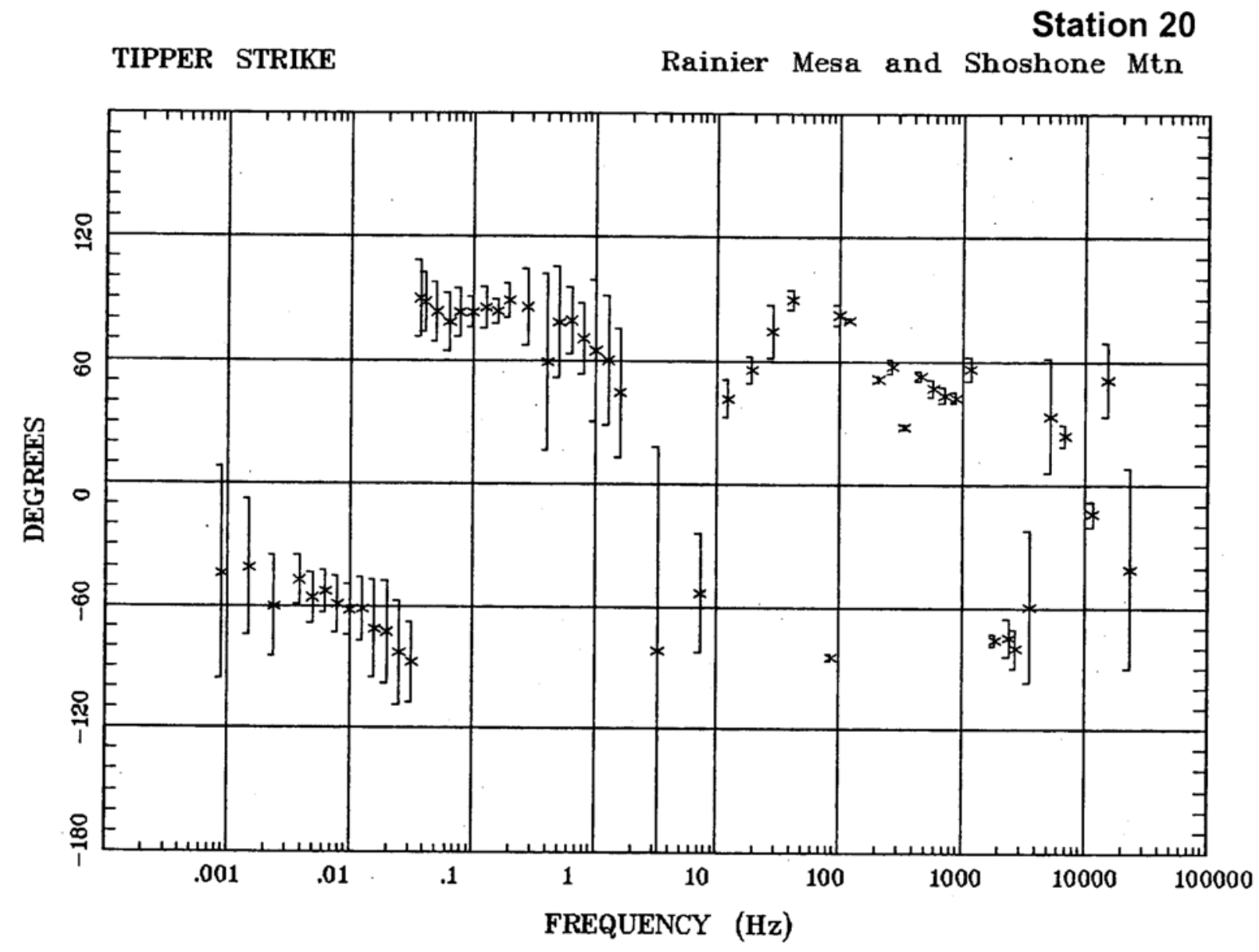

Client: DOE

Remote: none

Acquired: 04:0 May 16, 2005

Survey Co:USGS
Rotation:

Filename: rm20.avg

Channels: Ch1 Ch2 Ch3 Ch4 Ch5 Ch3 Ch4

Plotted: 13:45 Jan 17, 2006

< EMI - ElectroMagnetic Instruments > 


\section{Station 20}

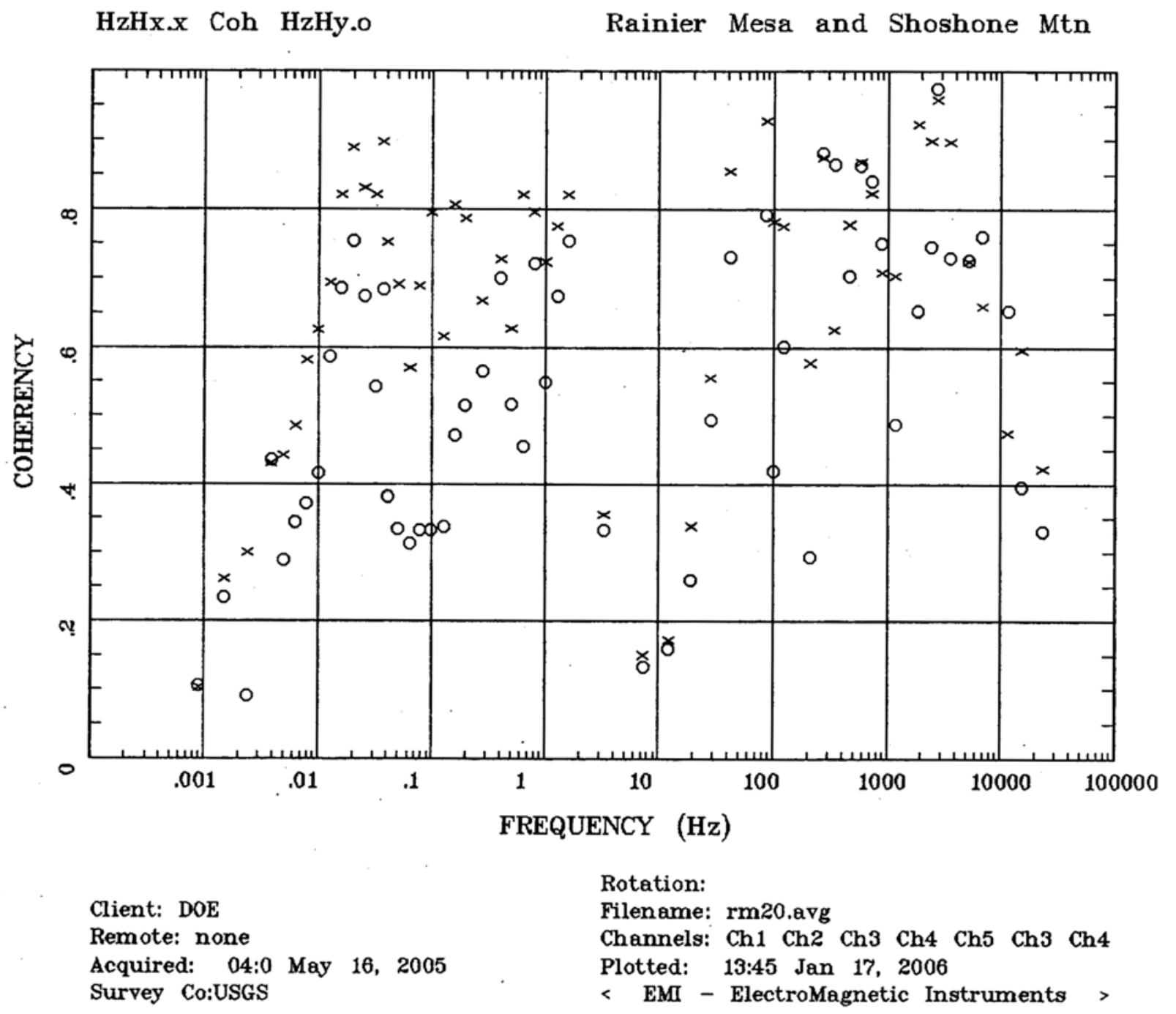




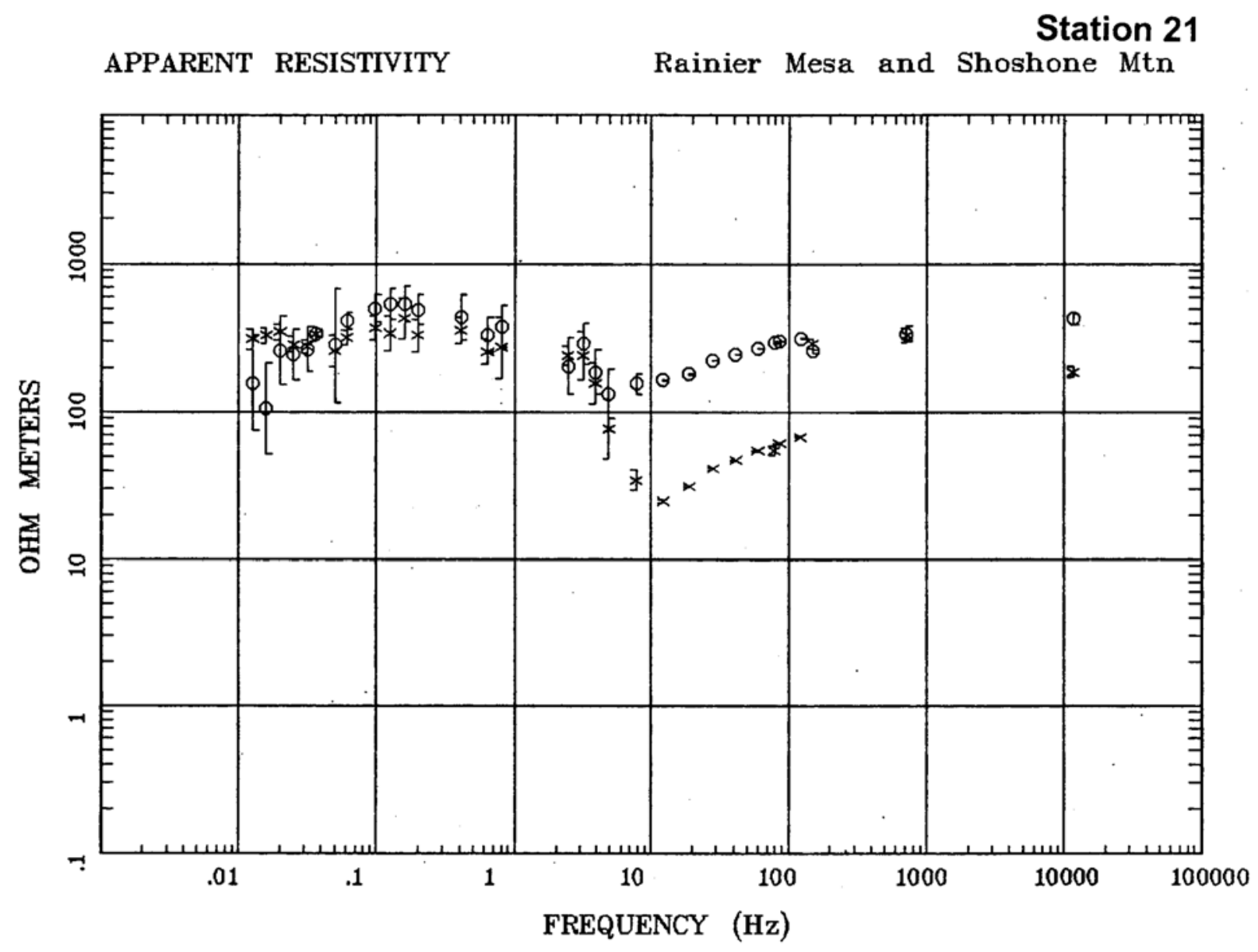

Client: DOE

Remote: none

Acquired: 01:0 May 14, 2005

Survey Co:USGS
Rotation:

Filename: rm21.avg

Channels: Ch1 Ch2 Ch3 Ch4 Ch5 Ch3 Ch4

Plotted: 08:58 Jan 18, 2006

< EMI - ElectroMagnetic Instruments > 


\section{Station 21}

\section{IMPEDANCE PHASE}

Rainier Mesa and Shoshone Mtn

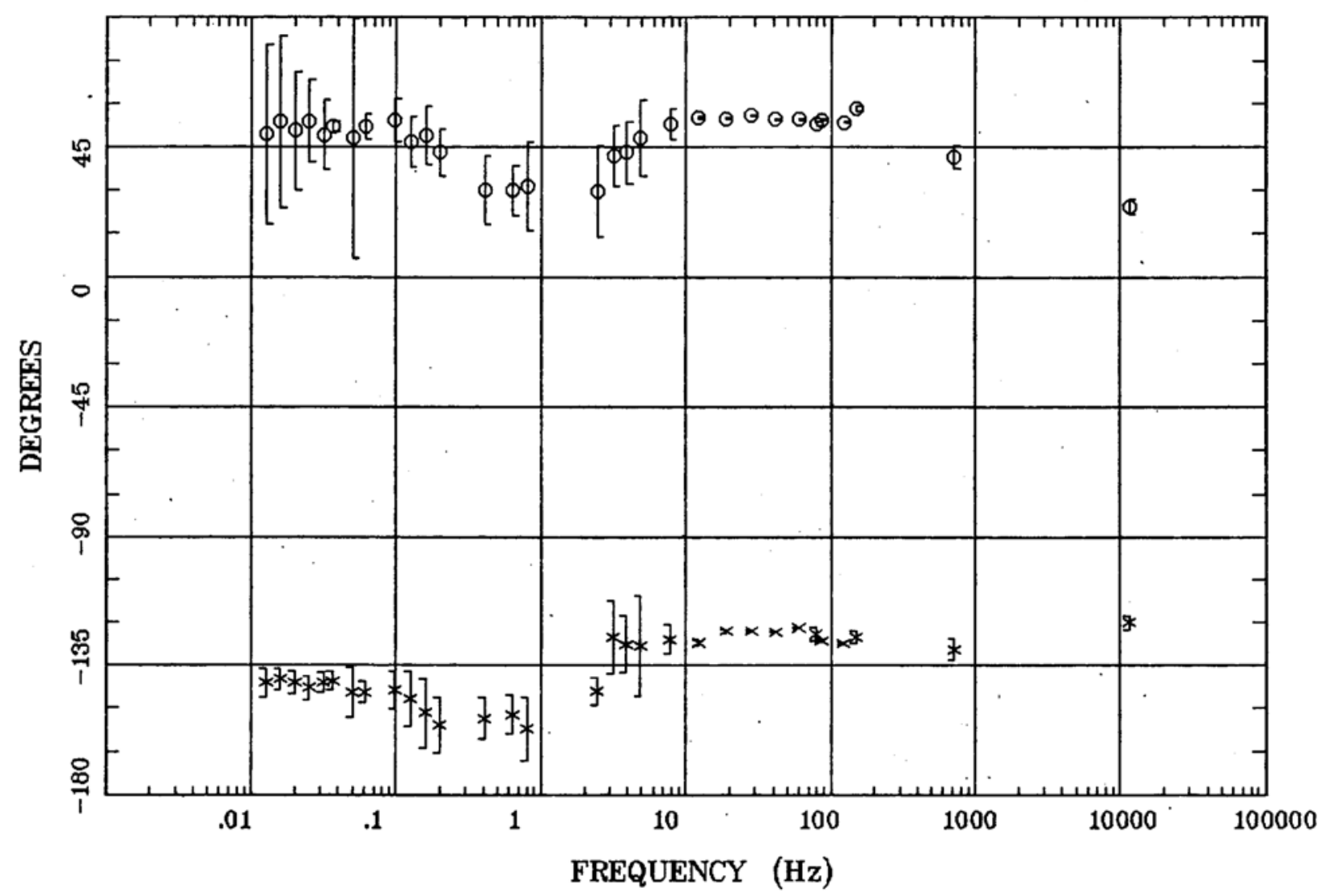

Client: DOE

Remote: none

Acquired: 01:0 May 14, 2005

Survey Co:USGS
Rotation:

Filename: rm21.avg

Channels: Ch1 Ch2 Ch3 Ch4 Ch5 Ch3 Ch4

Plotted: 08:58 Jan 18, 2006

< EMI - ElectroMagnetic Instruments > 


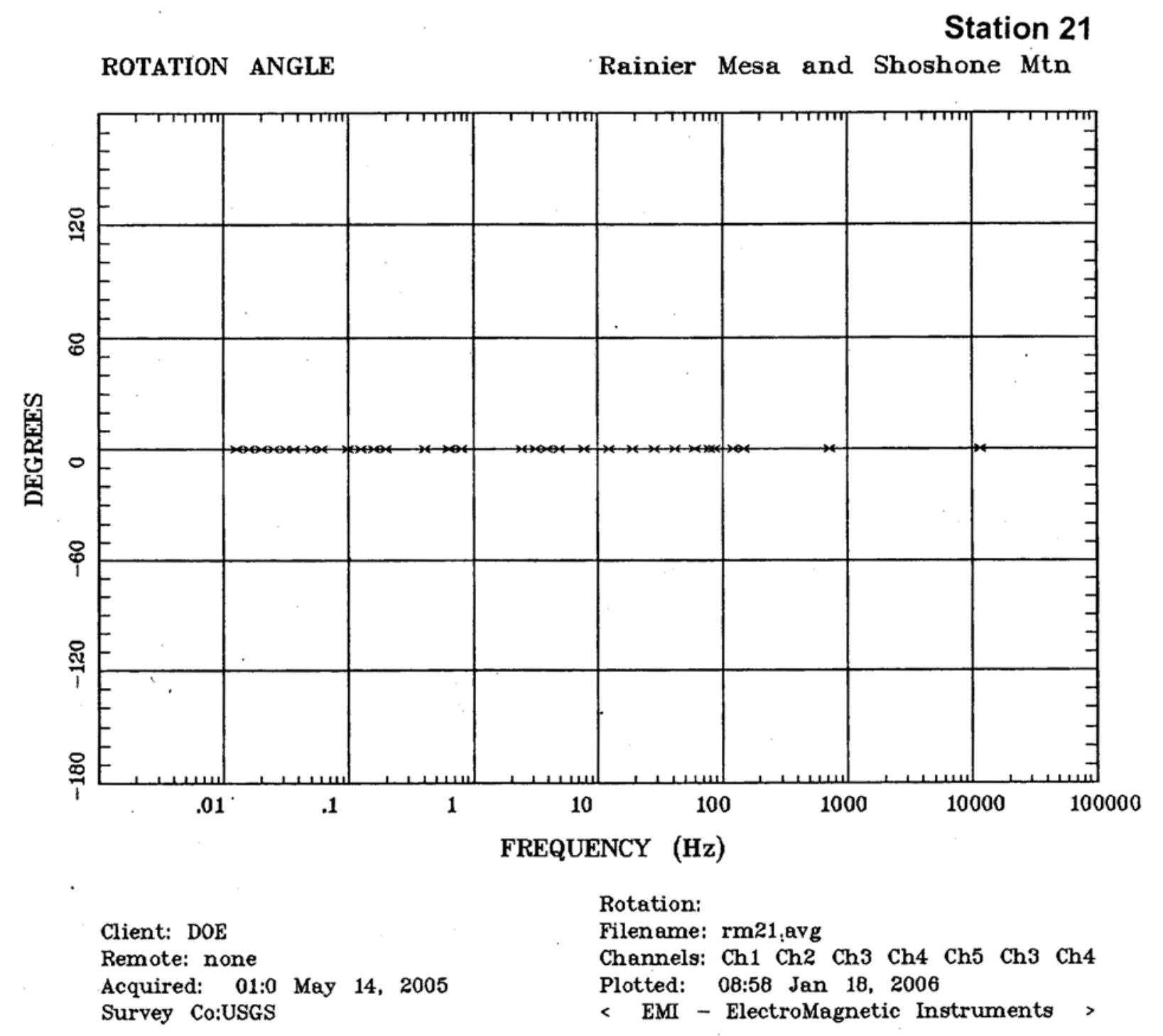


Station 21

IMPEDANCE SKEW

Rainier Mesa and Shoshone Mtn

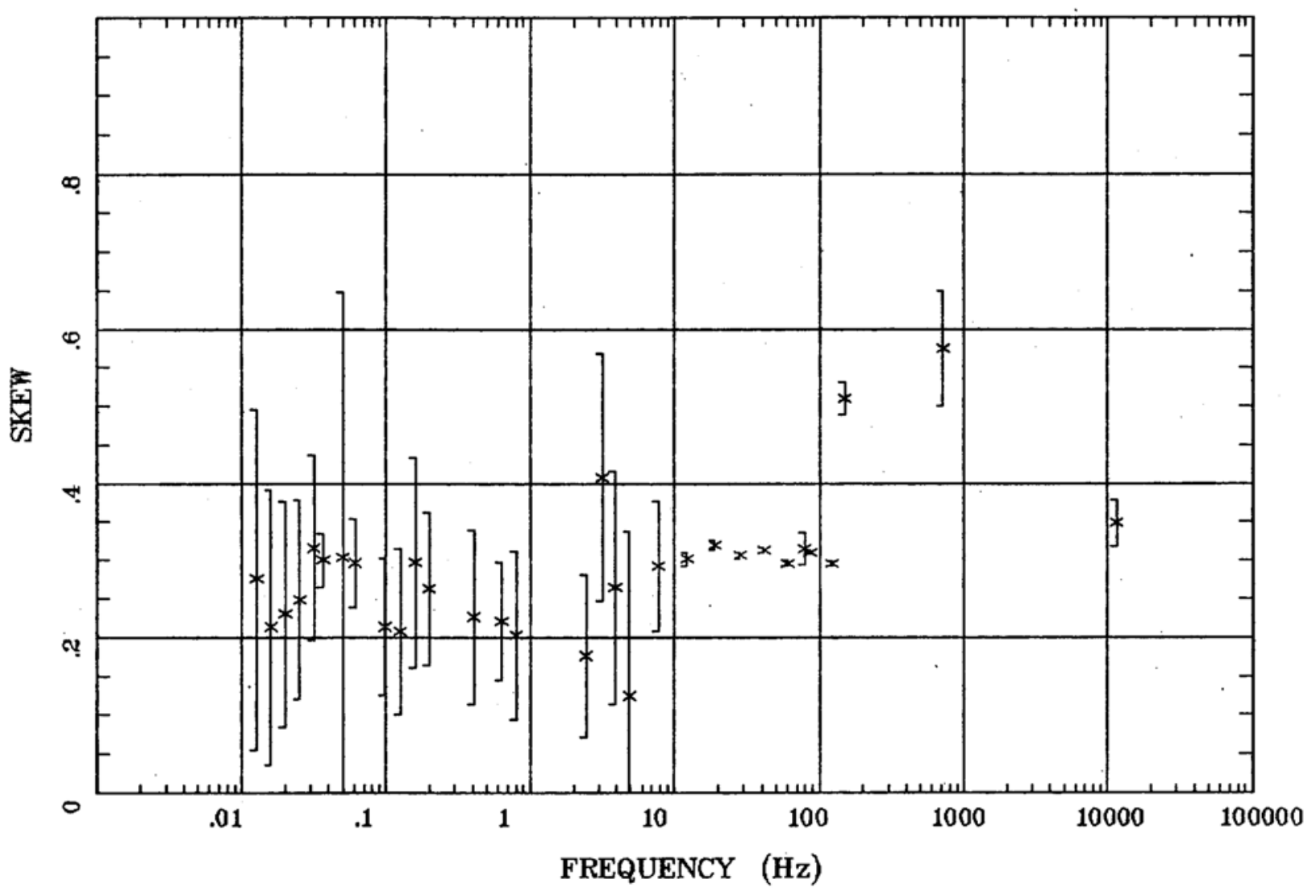

Client: DOE

Remote: none

Acquired: 01:0 May 14, 2005

Survey Co:USGS
Rotation:

Filename: rm21.avg

Channels: $\mathrm{Ch} 1 \mathrm{Ch} 2 \mathrm{Ch} 3 \mathrm{Ch} 4 \mathrm{Ch} 5 \mathrm{Ch} 3 \mathrm{Ch} 4$ Plotted: 08:58 Jan 18, 2006

< EMI - ElectroMagnetic Instruments > 
Station 21

E MULT Coh.

Rainier Mesa and Shoshone Mtn

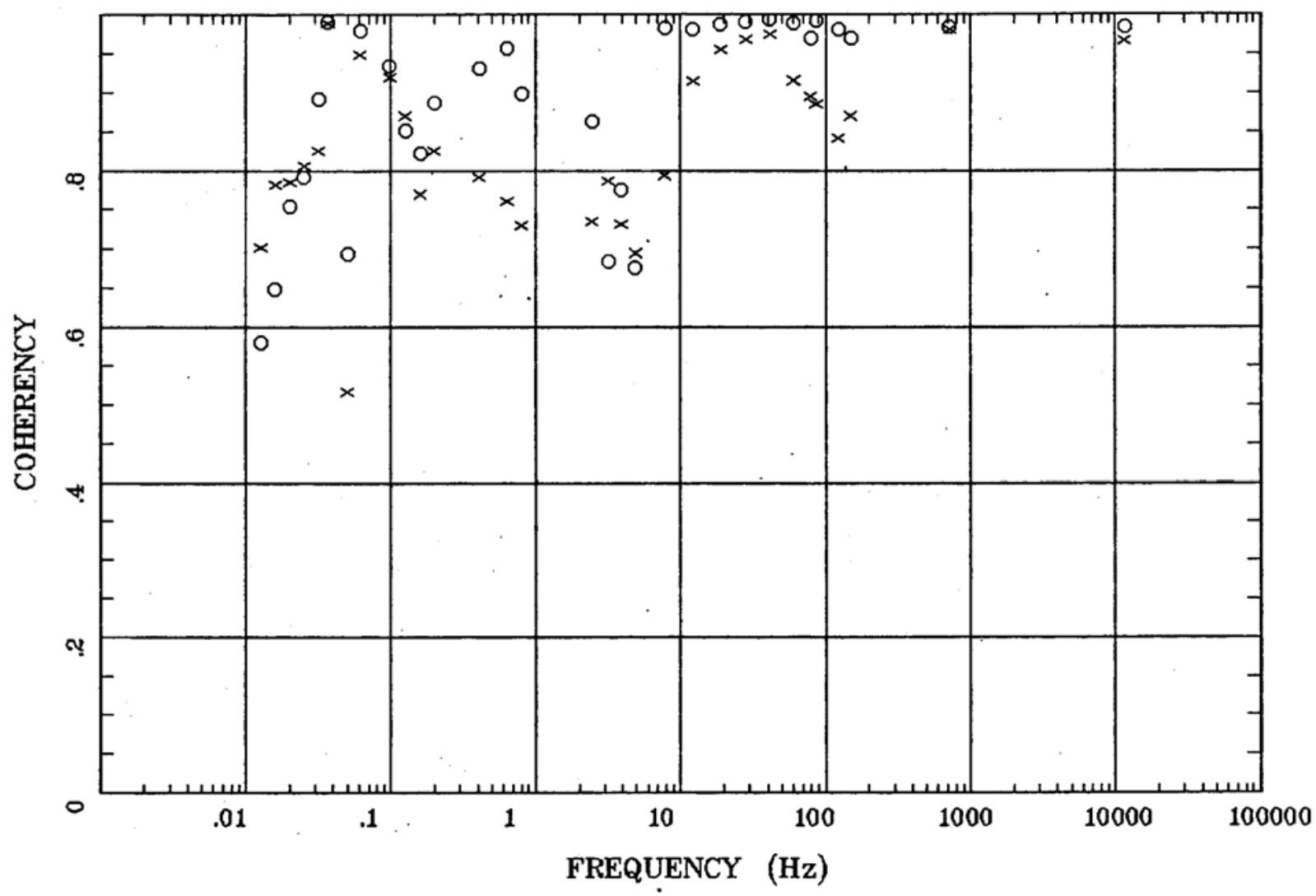

Client: DOE

Remote: none

Acquired: 01:0 May 14, 2005

Survey Co:USGS
Rotation:

Filename: rm21.avg

Channels: Ch1 Ch2 Ch3 Ch4 Ch5 Ch3 Ch4 Plotted: 08:58 Jan 18, 2006

< EMI - ElectroMagnetic Instruments > 
Station 21

POLAR PLOTS

Rainier Mesa and Shoshone Mtn

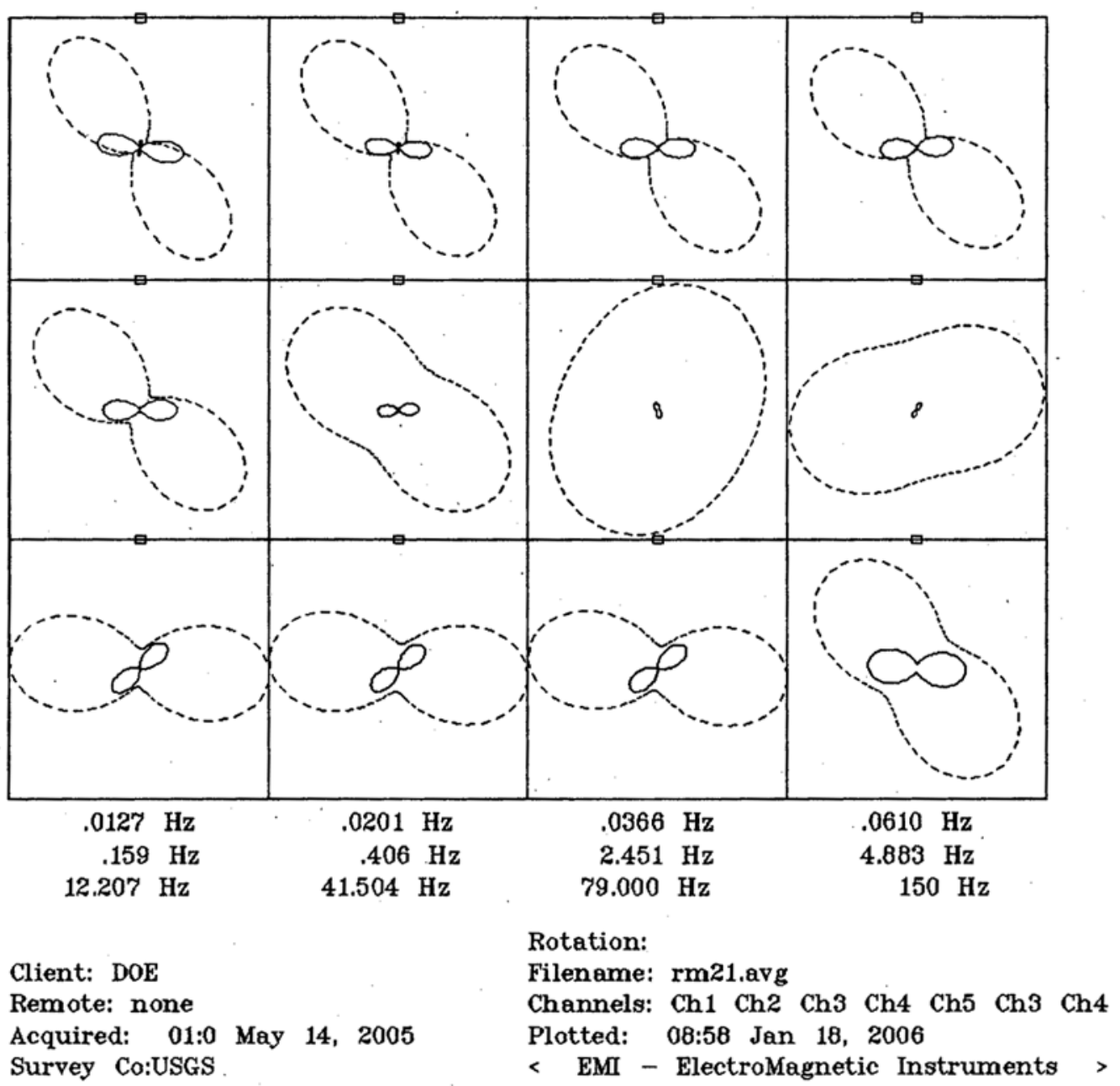


TIPPER MAGNITUDE

Station 21

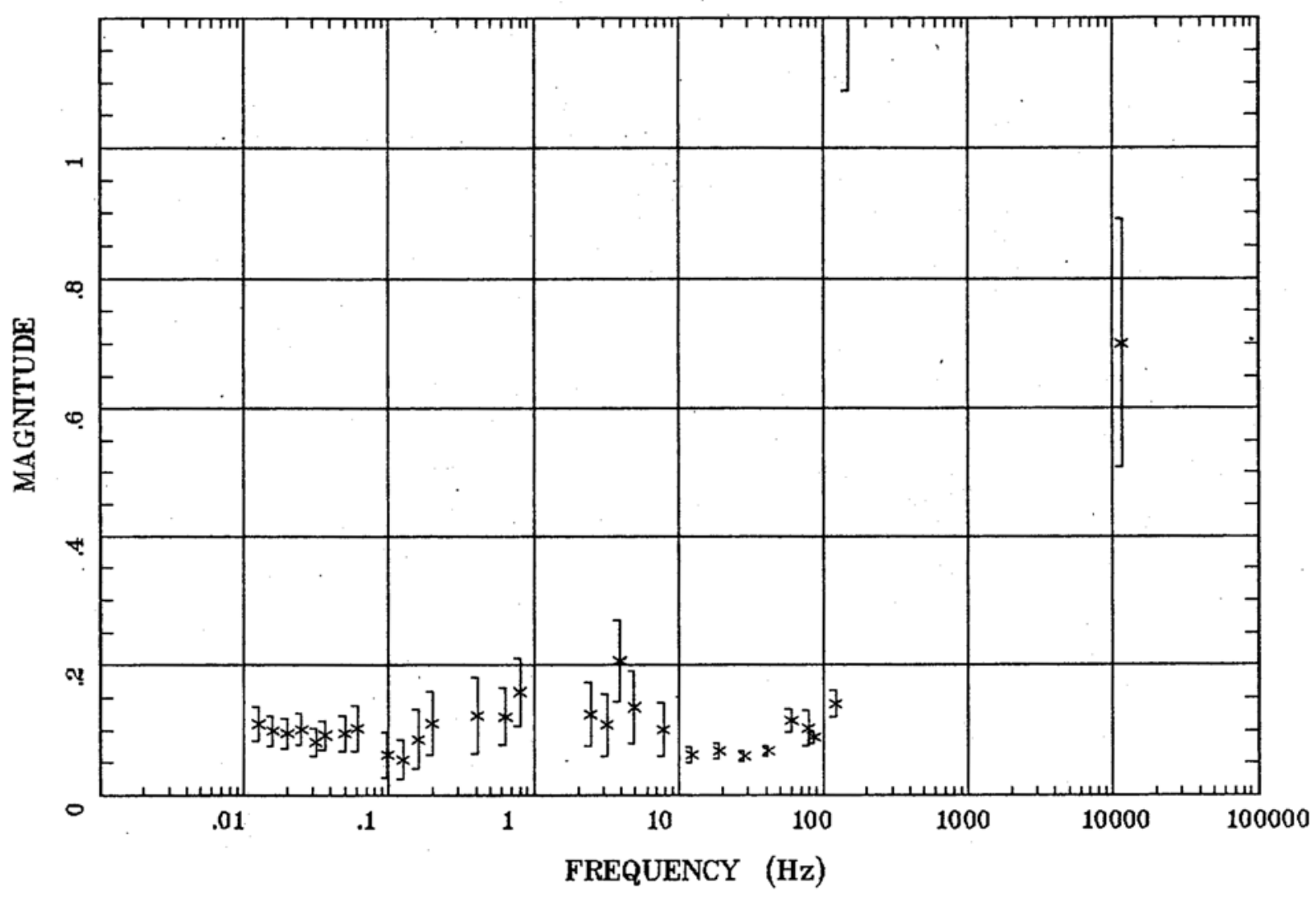

Client: DOE

Remote: none

Acquired: 01:0 May 14, 2005

Survey Co:USGS
Rainier Mesa and Shoshone Mtn

Rotation:

Filename: rm21.avg

Channels: Ch1 Ch2 Ch3 Ch4 Ch5 Ch3 Ch4

Plotted: 08:58 Jan 18, 2006

< EMI - ElectroMagnetic Instruments > 


\section{Station 21}

TIPPER STRIKE

Rainier Mesa and Shoshone Mtn

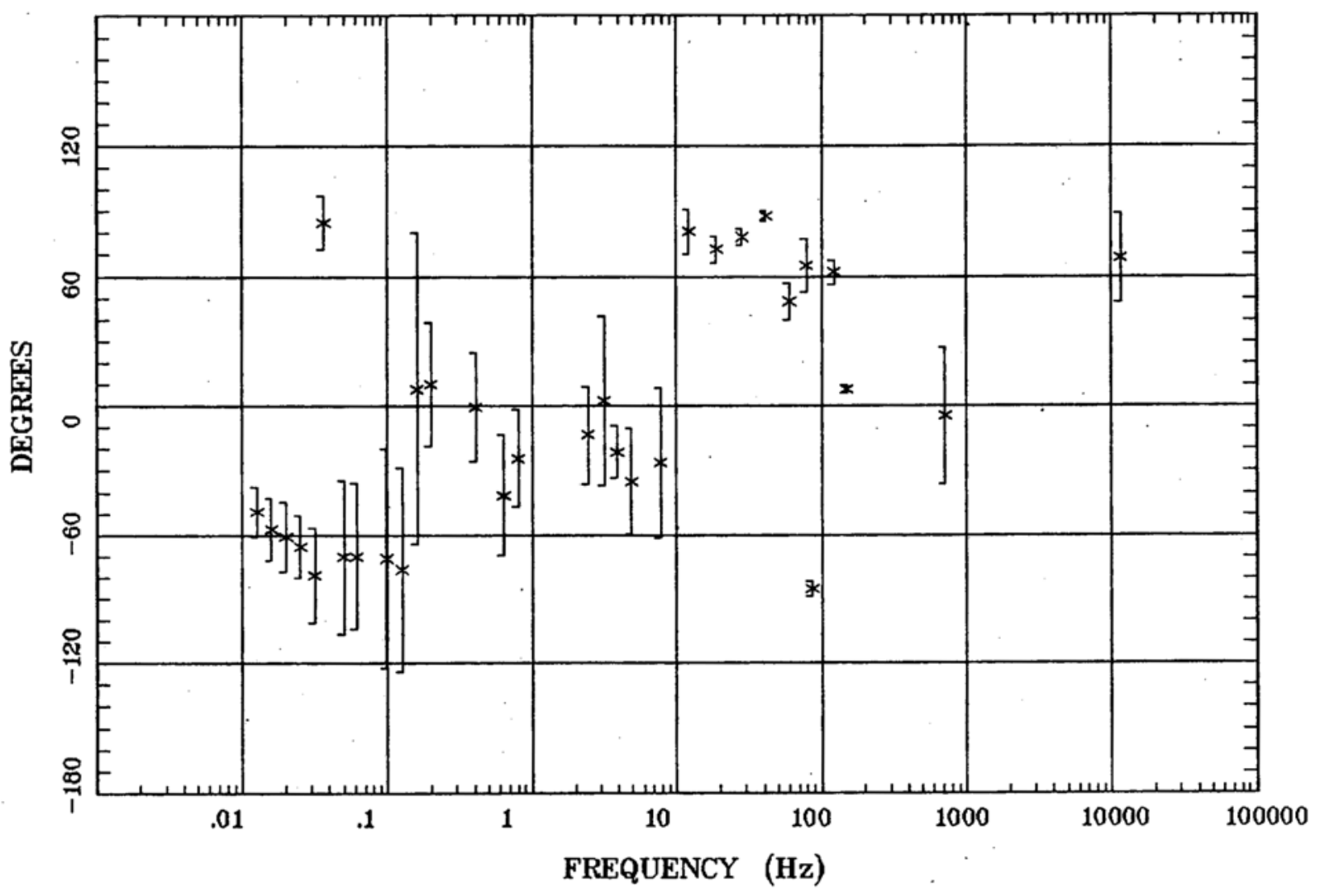

Client: DOE

Remote: none

Acquired: 01:0 May 14, 2005

Survey Co:USGS
Rotation:

Filename: rm21.avg

Channels: Ch1 Ch2 Ch3 Ch4 Ch5 Ch3 Ch4

Plotted: 08:58 Jan 18, 2006

< EMI - ElectroMagnetic Instruments > 


\section{Station 21}

HzHx.x Coh HzHy.o

Rainier Mesa and Shoshone Mtn

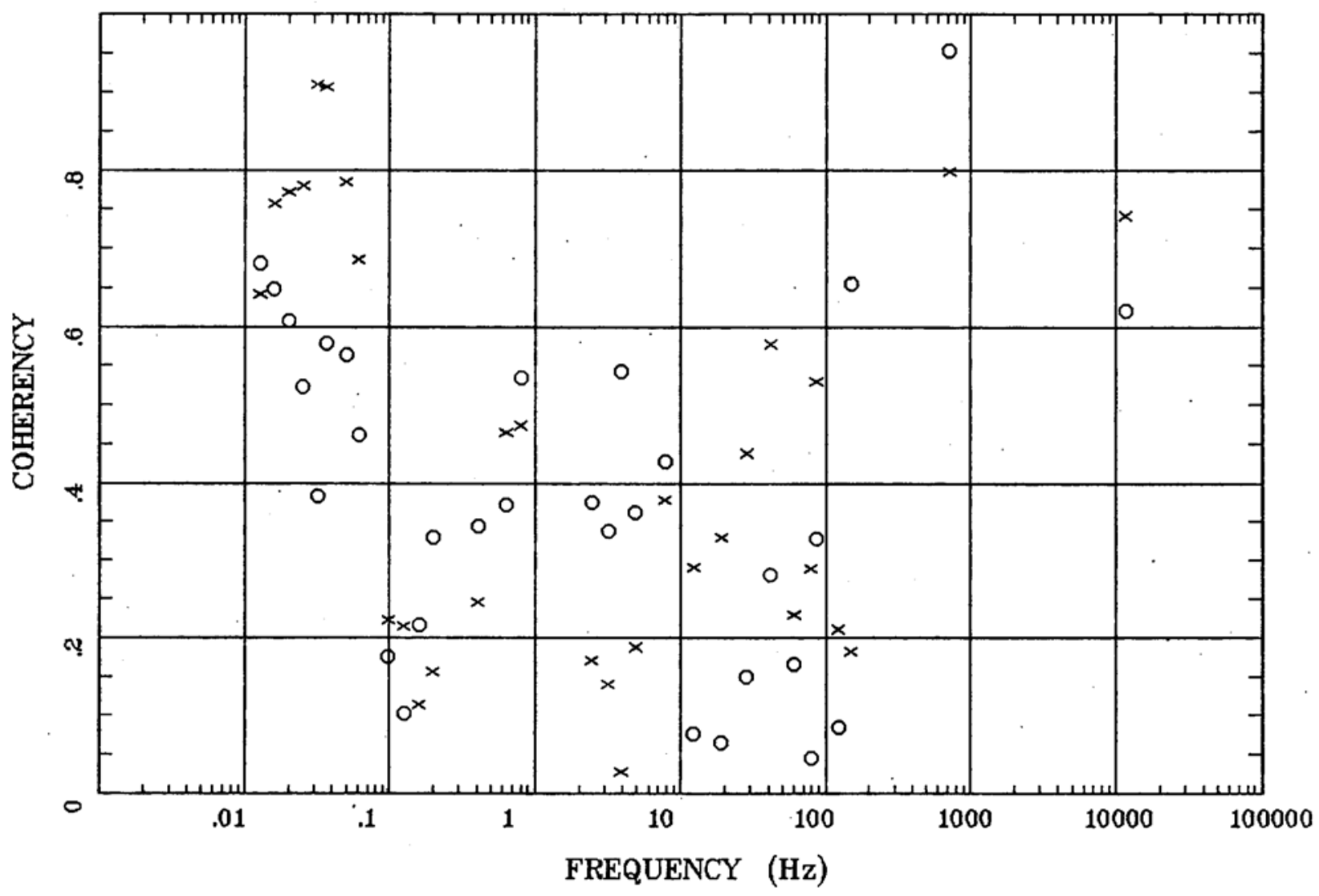

Client: DOE

Remote: none

Acquired: 01:0 May 14, 2005

Survey Co:USGS
Rotation:

Filename: rm21.avg

Channels: Ch1 Ch2 Ch3 Ch4 Ch5 Ch3 Ch4

Plotted: 08:58 Jan 18, 2006

< EMI - ElectroMagnetic Instruments > 
Station 22

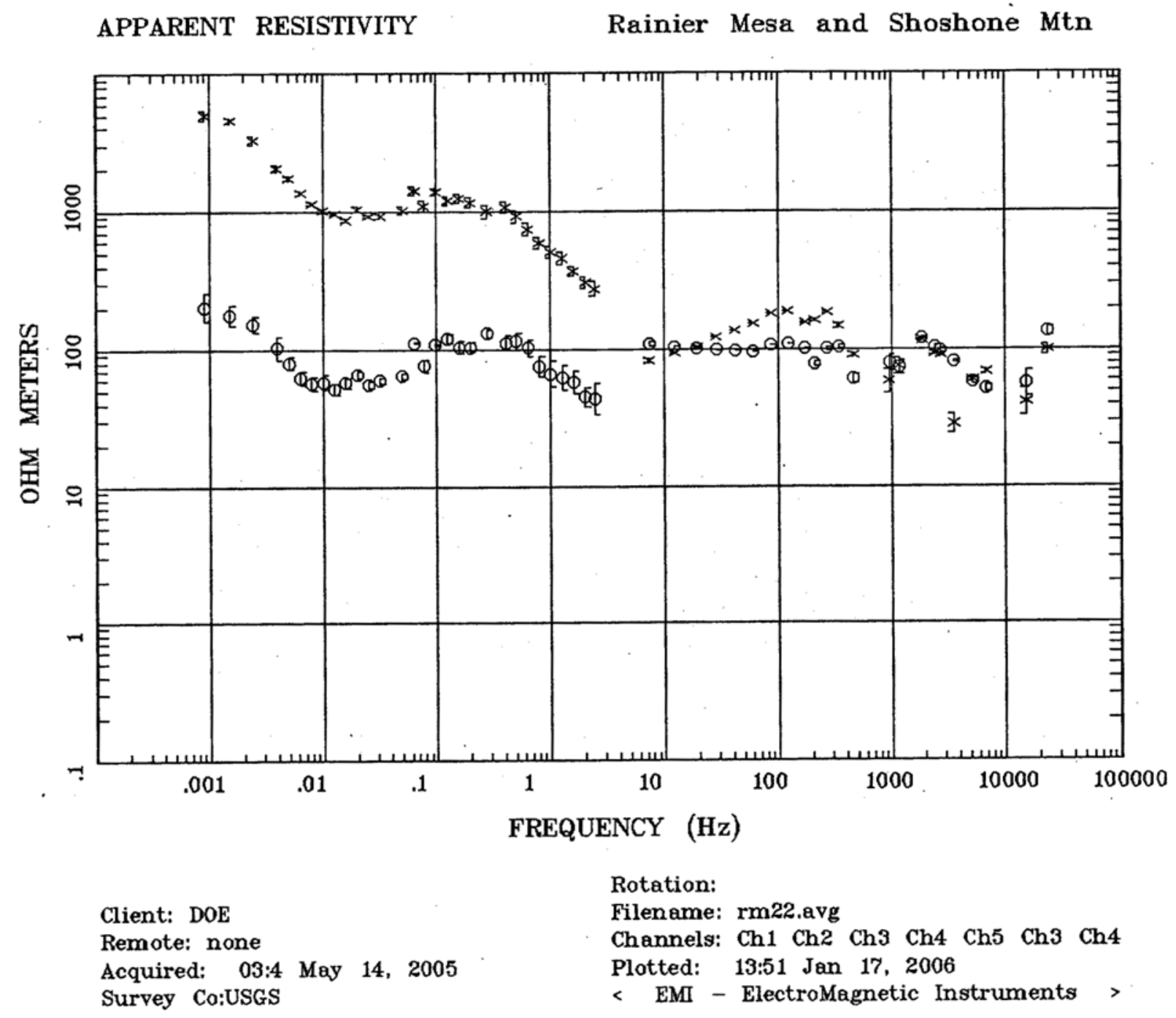


Station 22

IMPEDANCE PHASE

Rainier Mesa and Shoshone Mtn

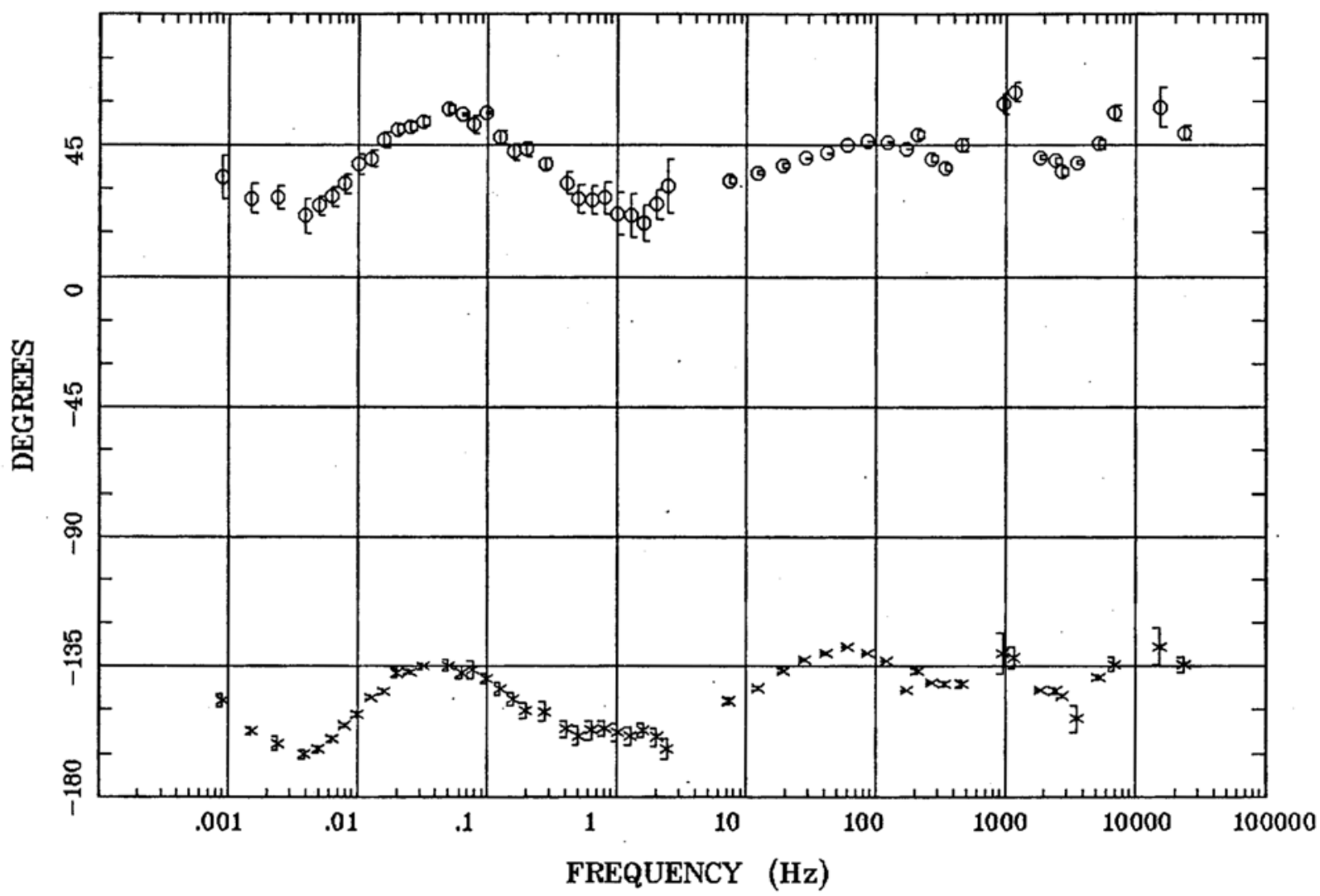

Client: DOE

Remote: none

Acquired: 03:4 May 14, 2005

Survey Co:USGS
Rotation:

Filename: rm22.avg

Channels: Ch1 Ch2 Ch3 Ch4 Ch5 Ch3 Ch4

Plotted: 13:51 Jan 17, 2006

< EMI - ElectroMagnetic Instruments > 
Station 22

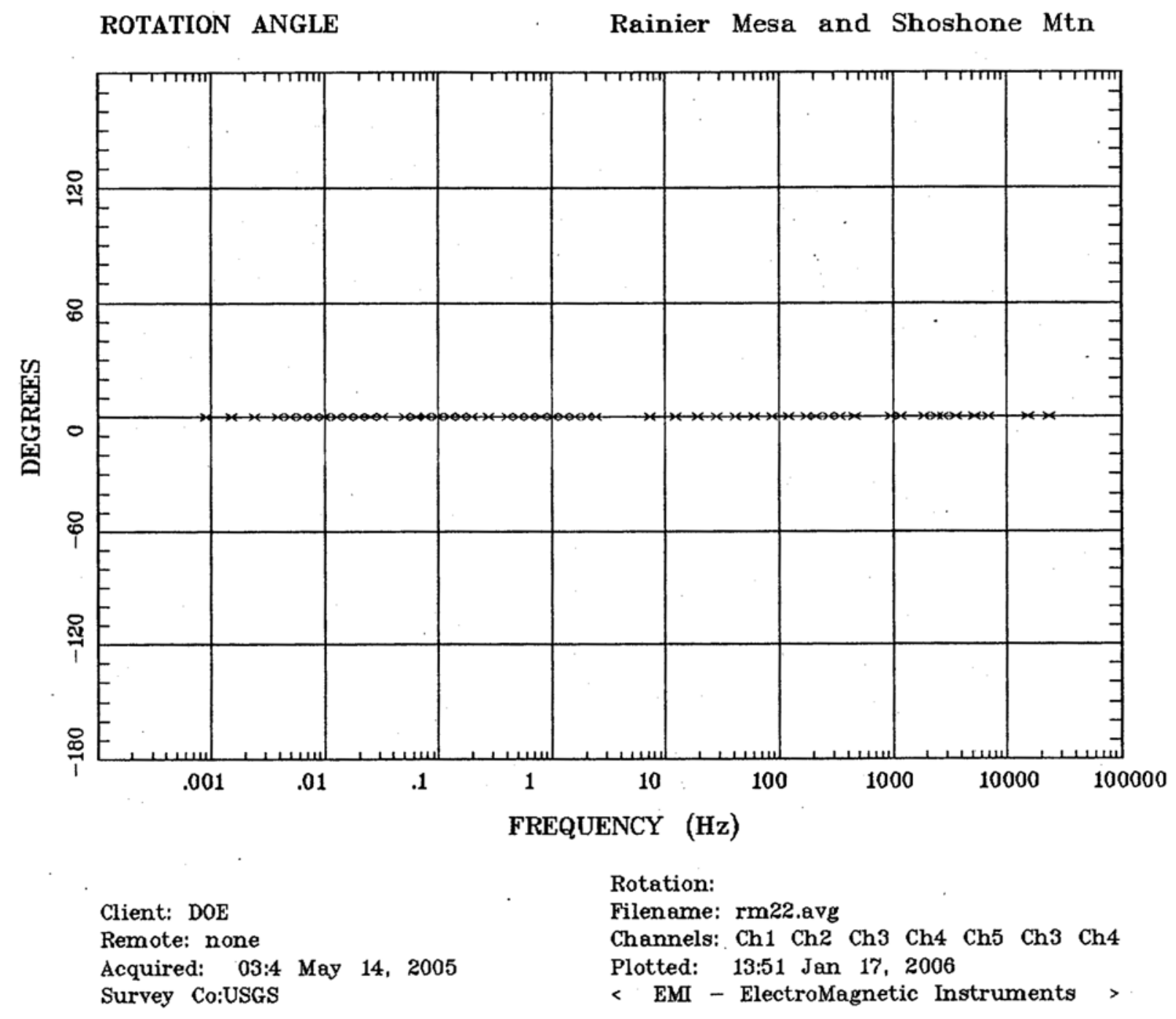


Station 22

IMPEDANCE SKEW

Rainier Mesa and Shoshone Mtn

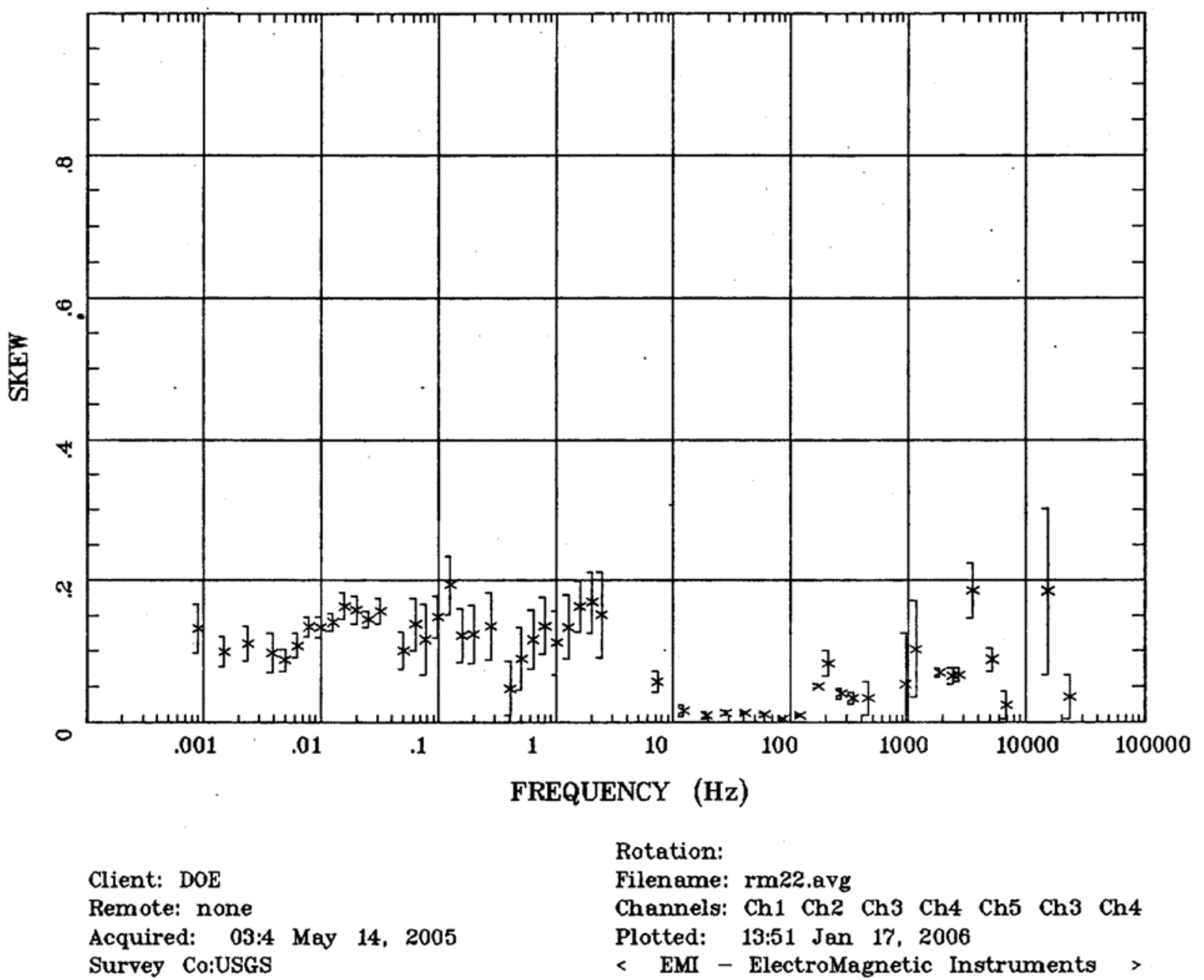




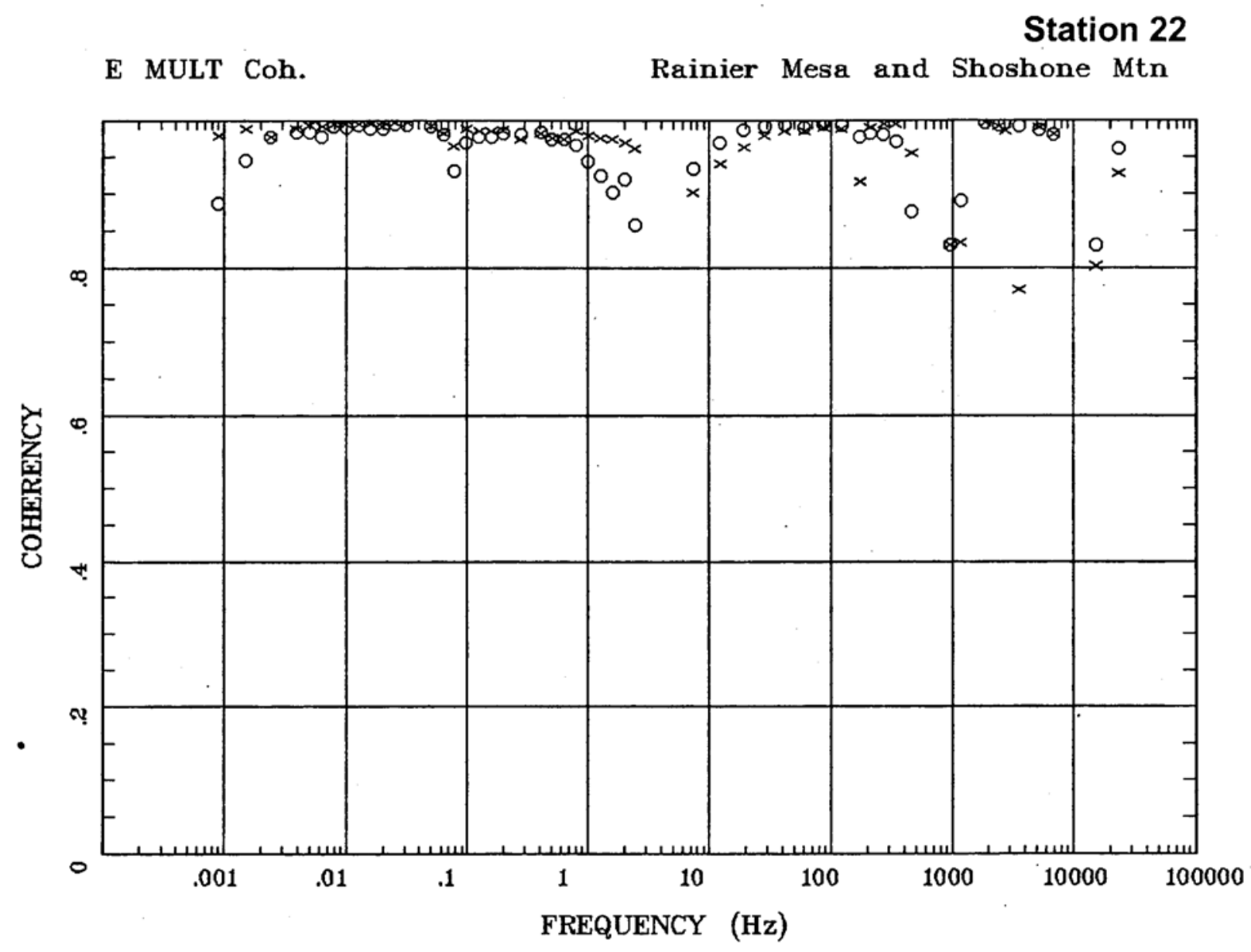

Client: DOE

Remote: none

Acquired: 03:4 May 14, 2005

Survey Co:USGS
Rotation:

Filename: rm22.avg

Channels: Ch1 Ch2 Ch3 Ch4 Ch5 Ch3 Ch4

Plotted: 13:51 Jan 17, 2006

< EMI - ElectroMagnetic Instruments > 
Station 22

POLAR PLOTS

Rainier Mesa and Shoshone Mtn

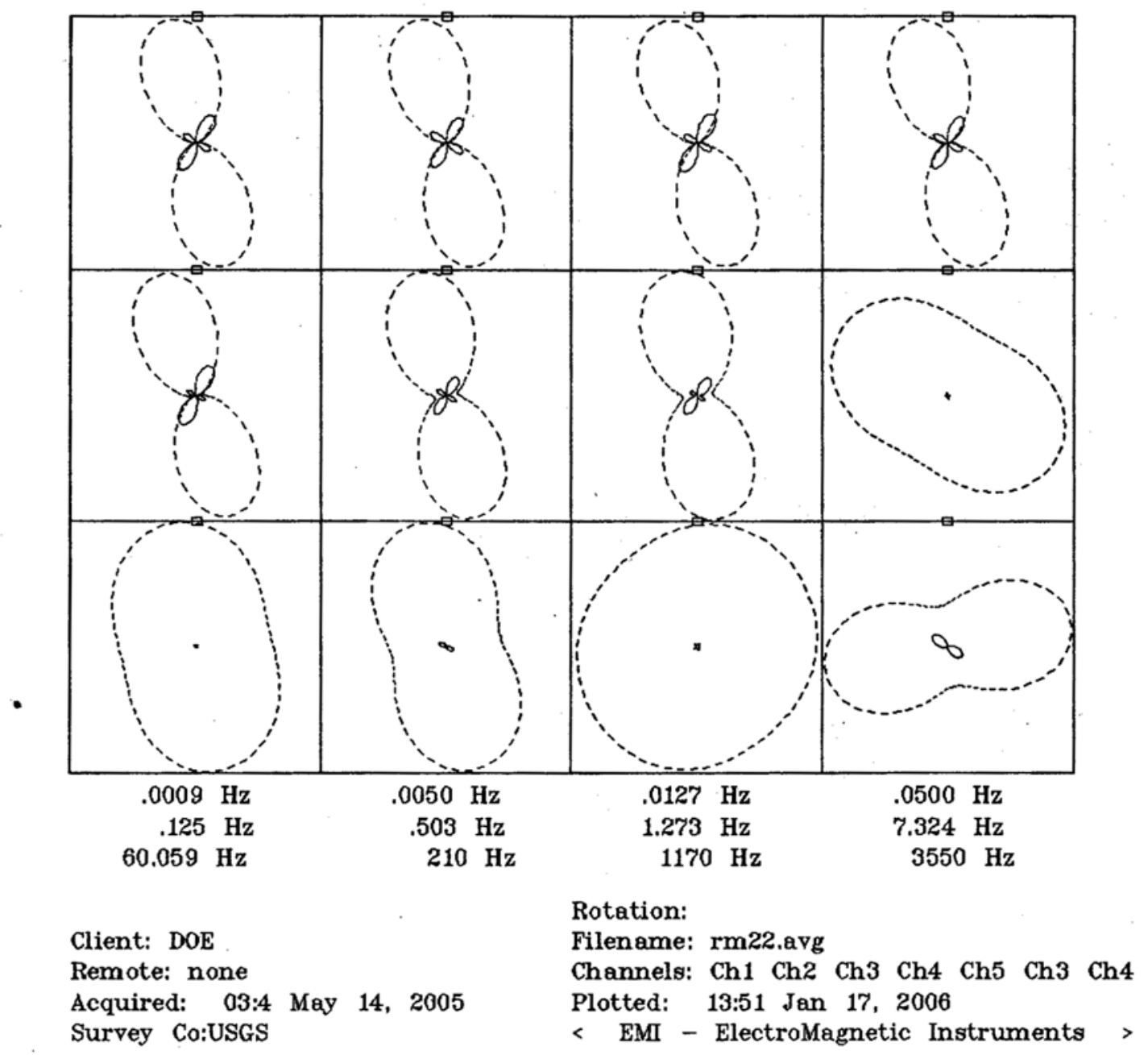




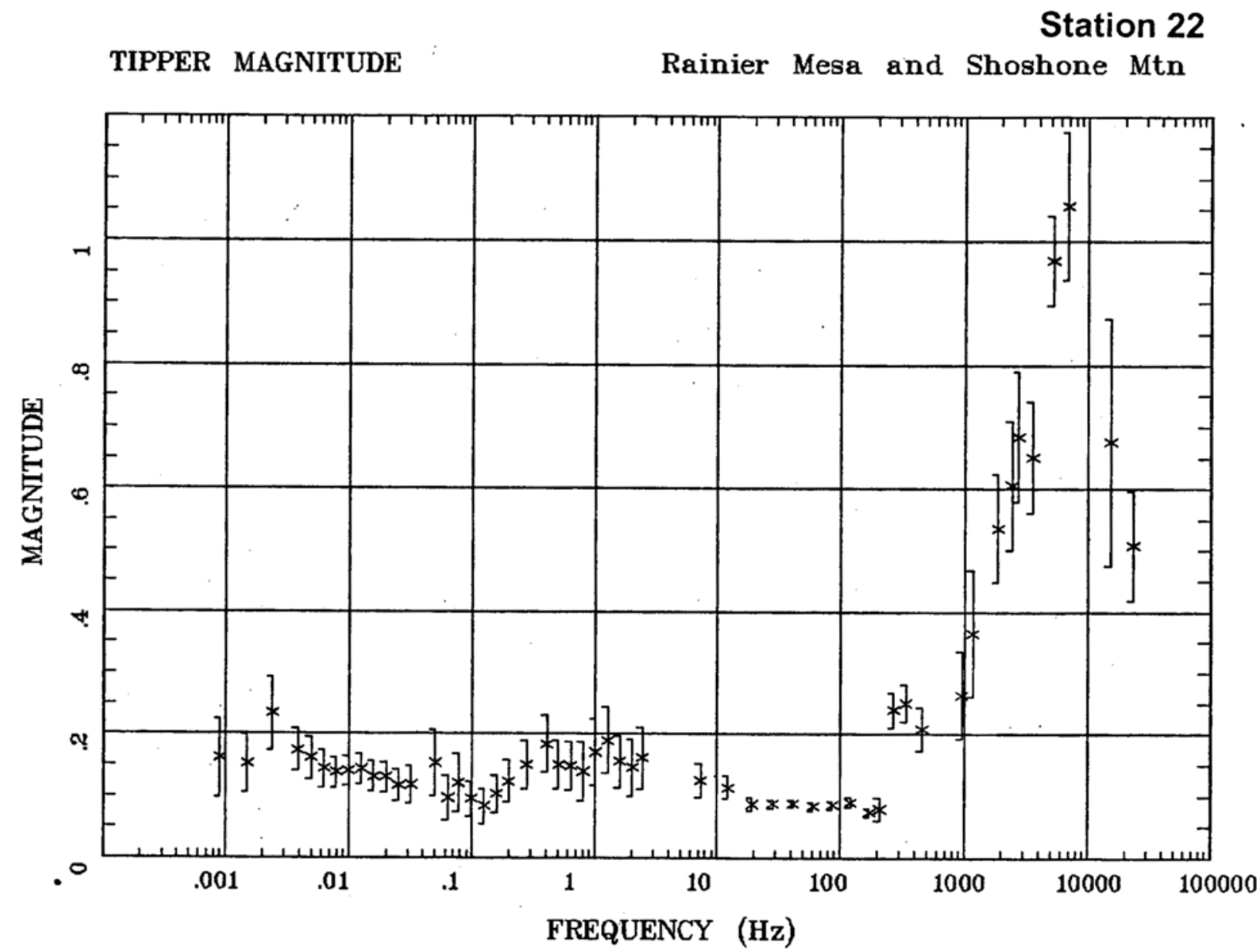

Client: DOE

Remote: none

Acquired: 03:4 May 14, 2005

Survey Co:USGS
Rotation:

Filename: rm22.avg

Channels: Ch1 Ch2 Ch3 Ch4 Ch5 Ch3 Ch4

Plotted: 13:51 Jan 17, 2006

< EMI - ElectroMagnetic Instruments > 
TIPPER STRIKE

Station 22

Rainier Mesa and Shoshone Mtn

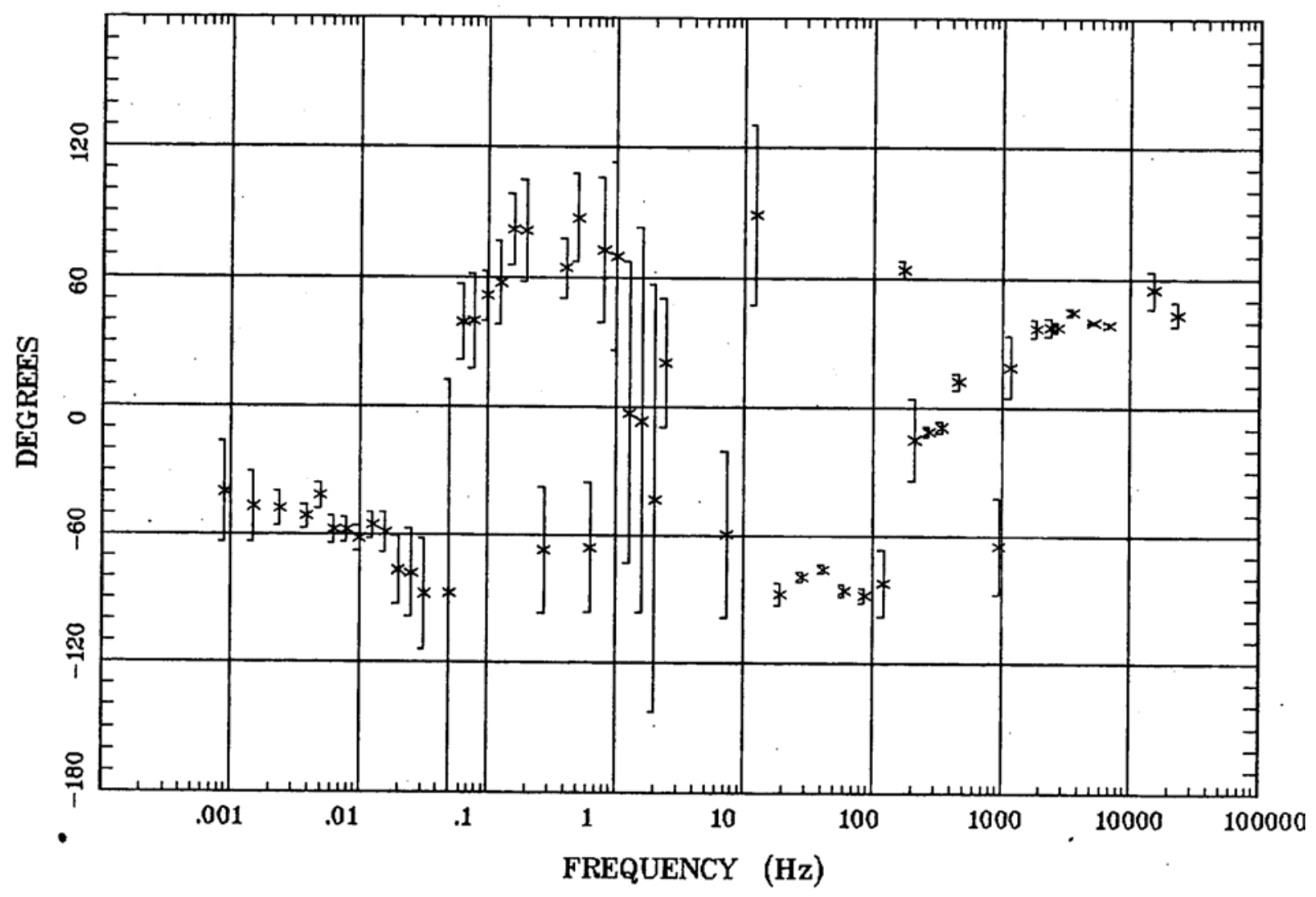

Client: DOE

Remote: none

Acquired: 03:4 May 14, 2005

Survey Co:USGS
Rotation:

Filename: rm22.avg

Channels: Ch1 Ch2 Ch3 Ch4 Ch5 Ch3 Ch4

Plotted: 13:51 Jan 17, 2006

< EMI - ElectroMagnetic Instruments > 
Station 22

HzHx.x Coh HzHy.o

Rainier Mesa and Shoshone Mtn

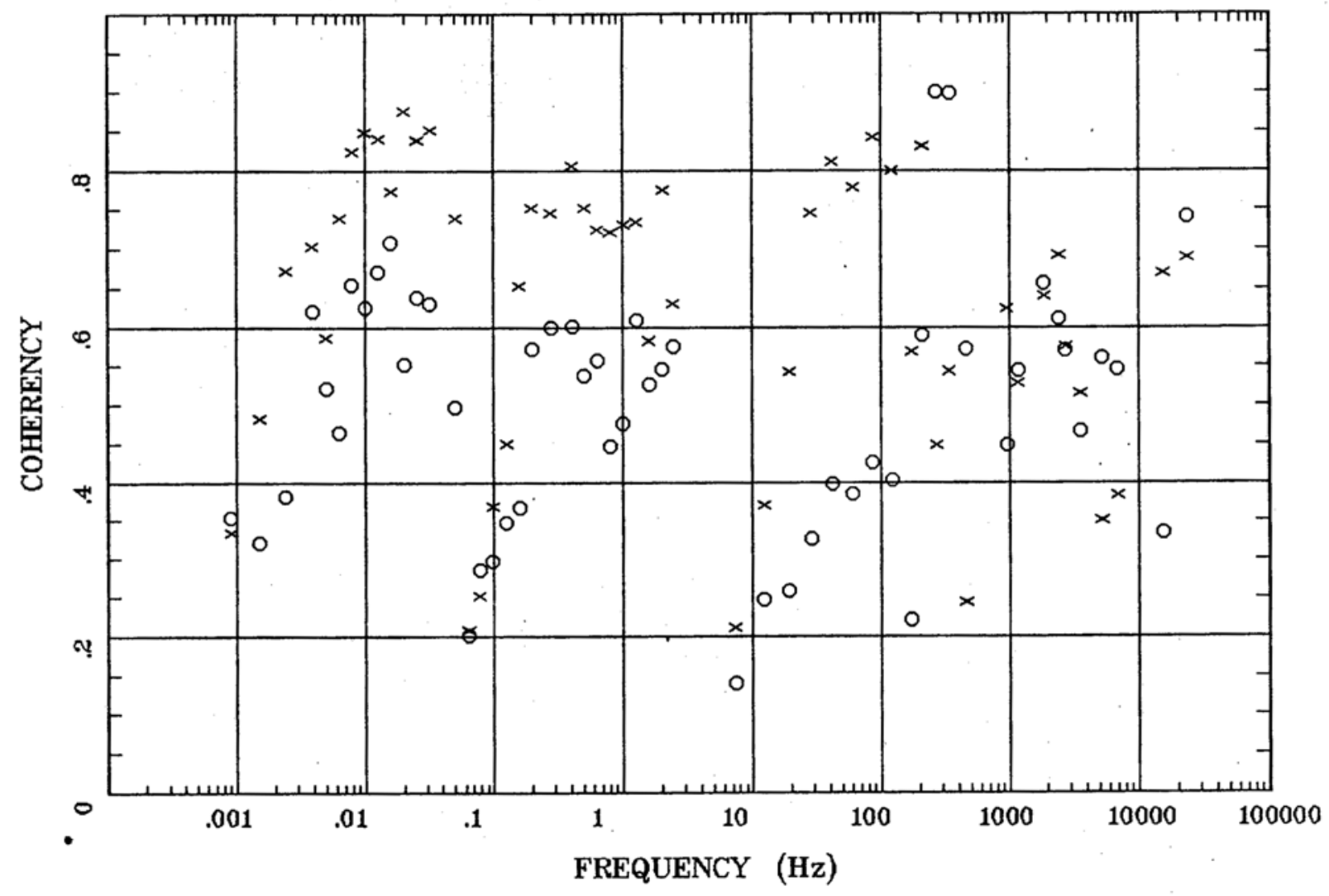

Client: DOE

Remote: none

Acquired: 03:4 May 14, 2005

Survey Co:USGS
Rotation:

Filename: rm22.avg

Channels: Ch1 Ch2 Ch3 Ch4 Ch5 Ch3 Ch4

Plotted: 13:51 Jan 17, 2006

< EMI - ElectroMagnetic Instruments > 


\section{Station 23}

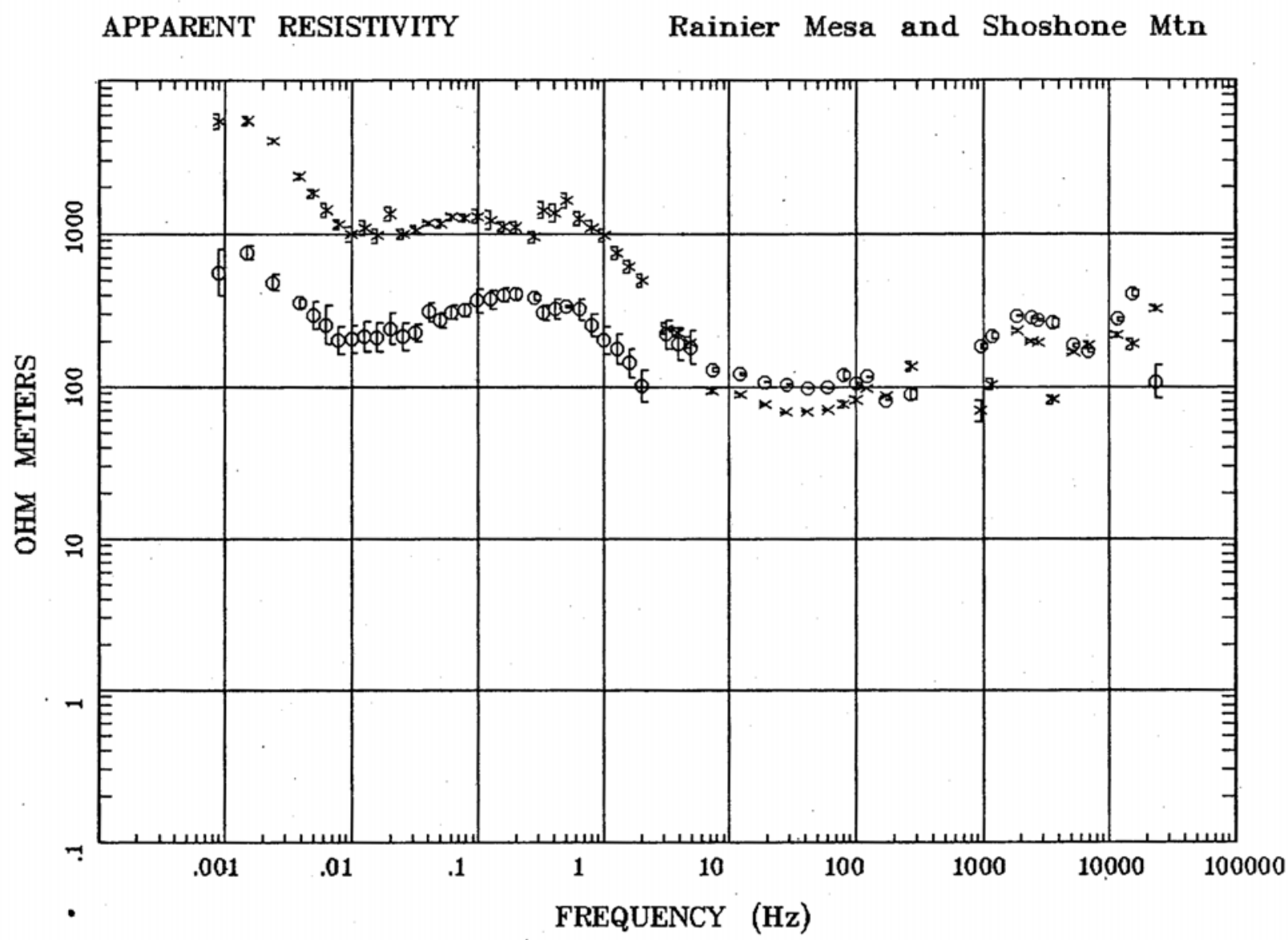

Client: DOE

Remote: none

Acquired: 22:0 May 16, 2005

Survey Co:USGS

Rotation:

Filename: rm23.avg

Channels: Ch1 Ch2 Ch3 Ch4 Ch5 Ch3 Ch4

Plotted: 13:52 Jan 17, 2006

< EMI - ElectroMagnetic Instruments > 
Station 23

IMPEDANCE PHASE

Rainier Mesa and Shoshone Mtn

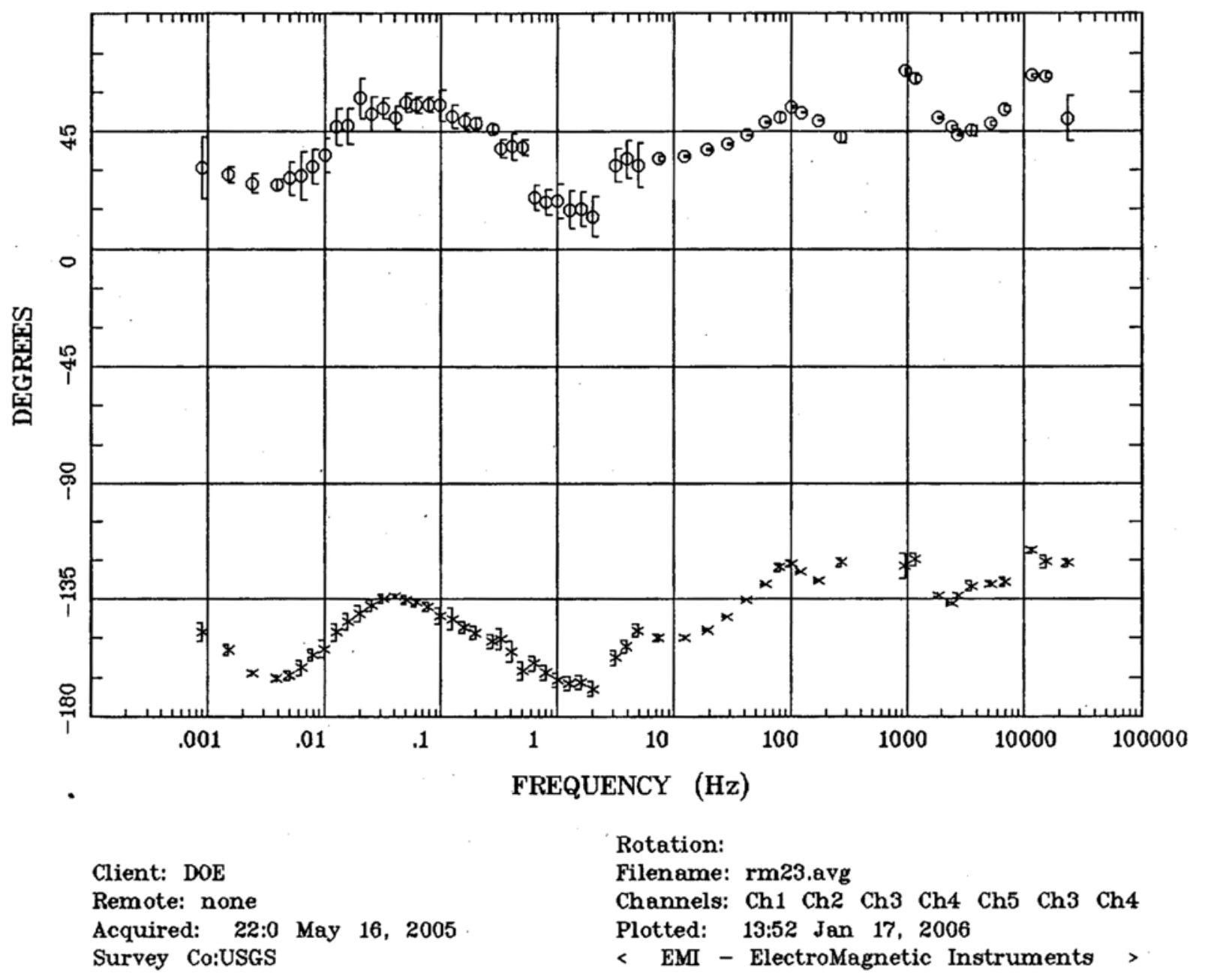


Station 23

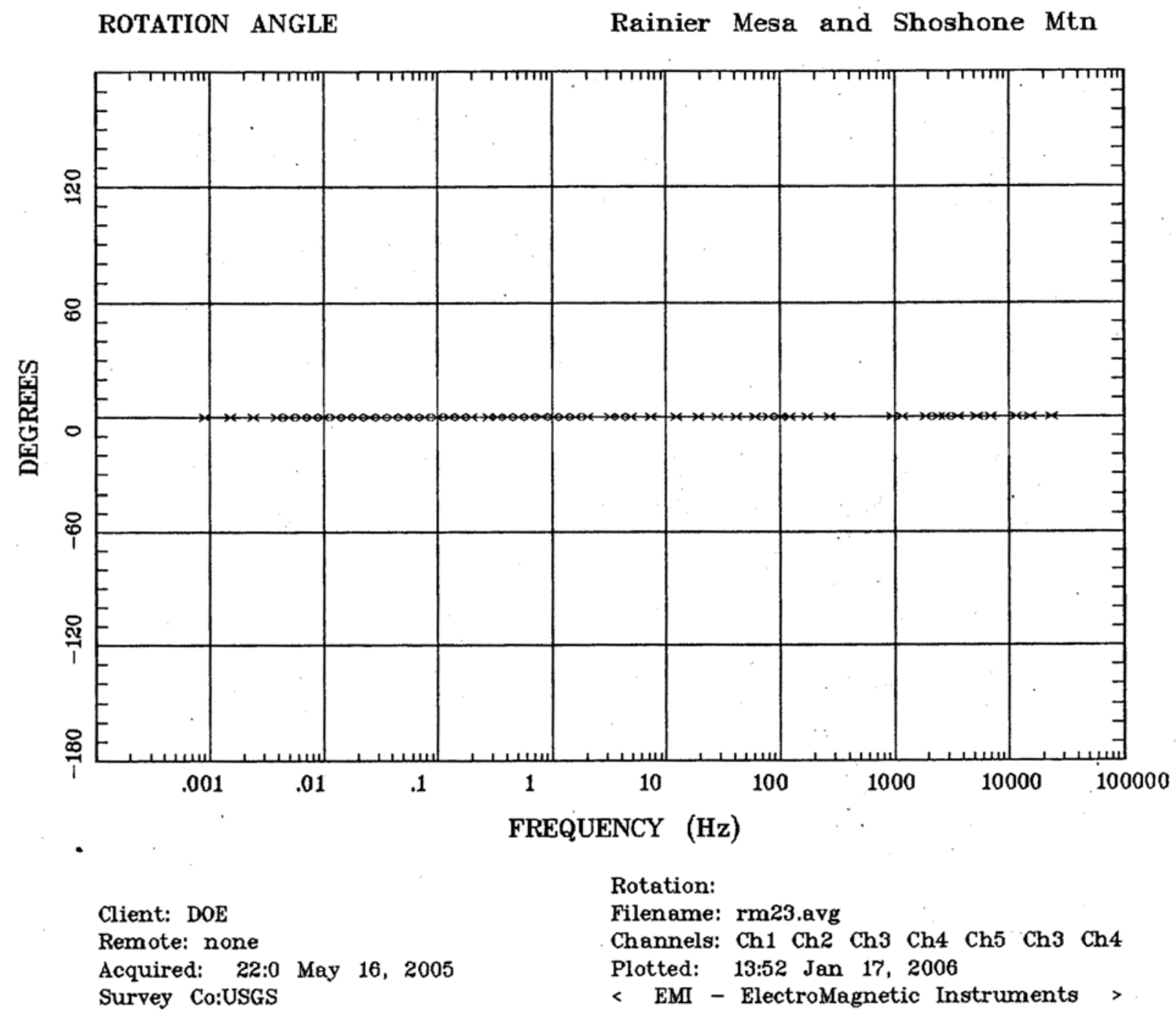


Station 23

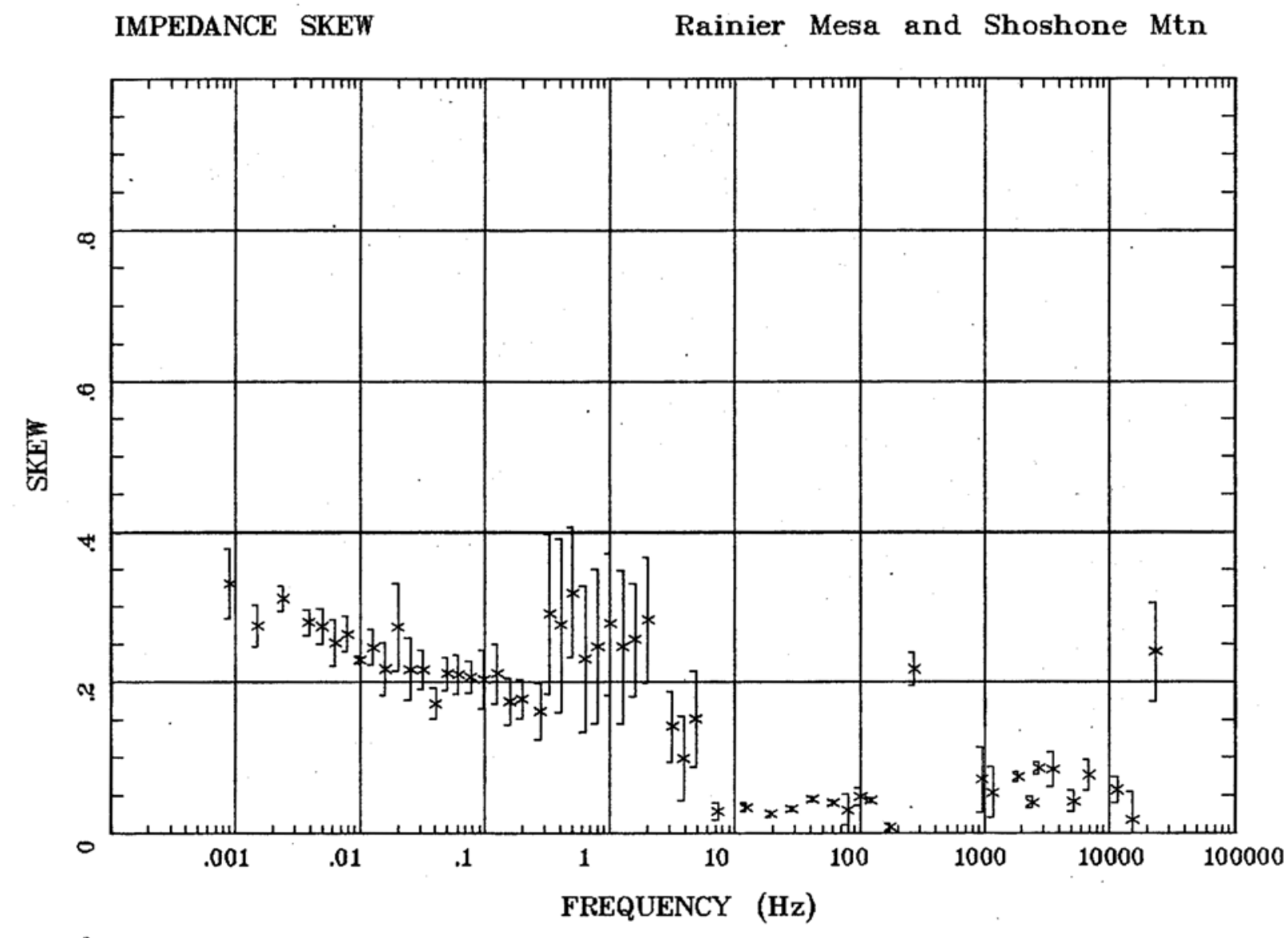

Client: $\mathrm{DOE}$

Remote: none

Acquired: 22:0 May 16, 2005

Survey Co:USGS
Rotation:

Filename: rm23.avg

Channels: Ch1 Ch2 Ch3 Ch4 Ch5 Ch3 Ch4

Plotted: 13:52 Jan 17, 2006

< EMI - ElectroMagnetic Instruments > 
Station 23

E MULT Coh.

Rainier Mesa and Shoshone Mtn

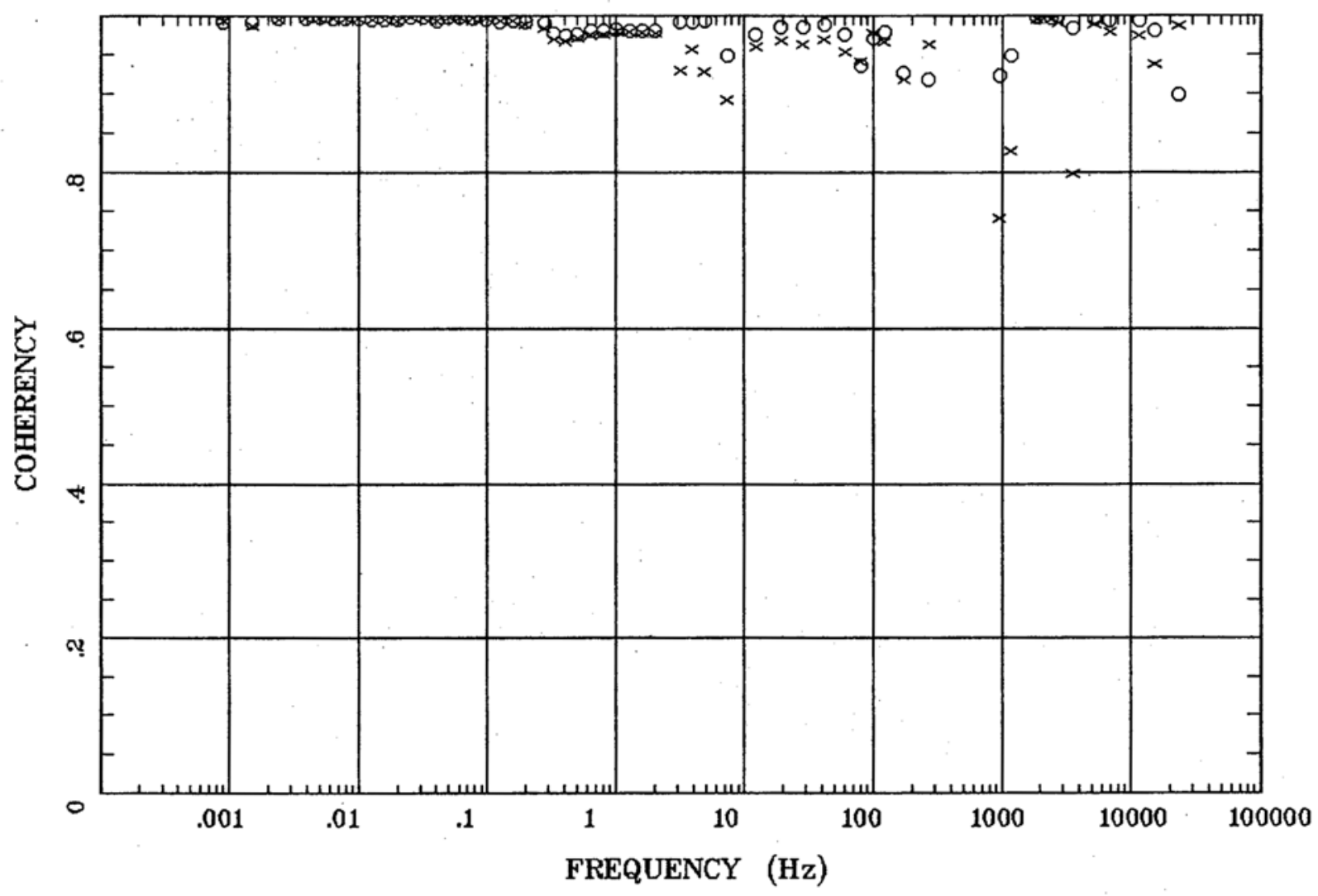

Client: DOE

Rotation:

Remote: none

Acquired: 22:0 May 16, 2005

Survey Co:USGS

Filename: rm23.avg

Channels: Ch1 Ch2 Ch3 Ch4 Ch5 Ch3 Ch4

Plotted: 13:52 Jan 17, 2006

< EMI - ElectroMagnetic Instruments > 


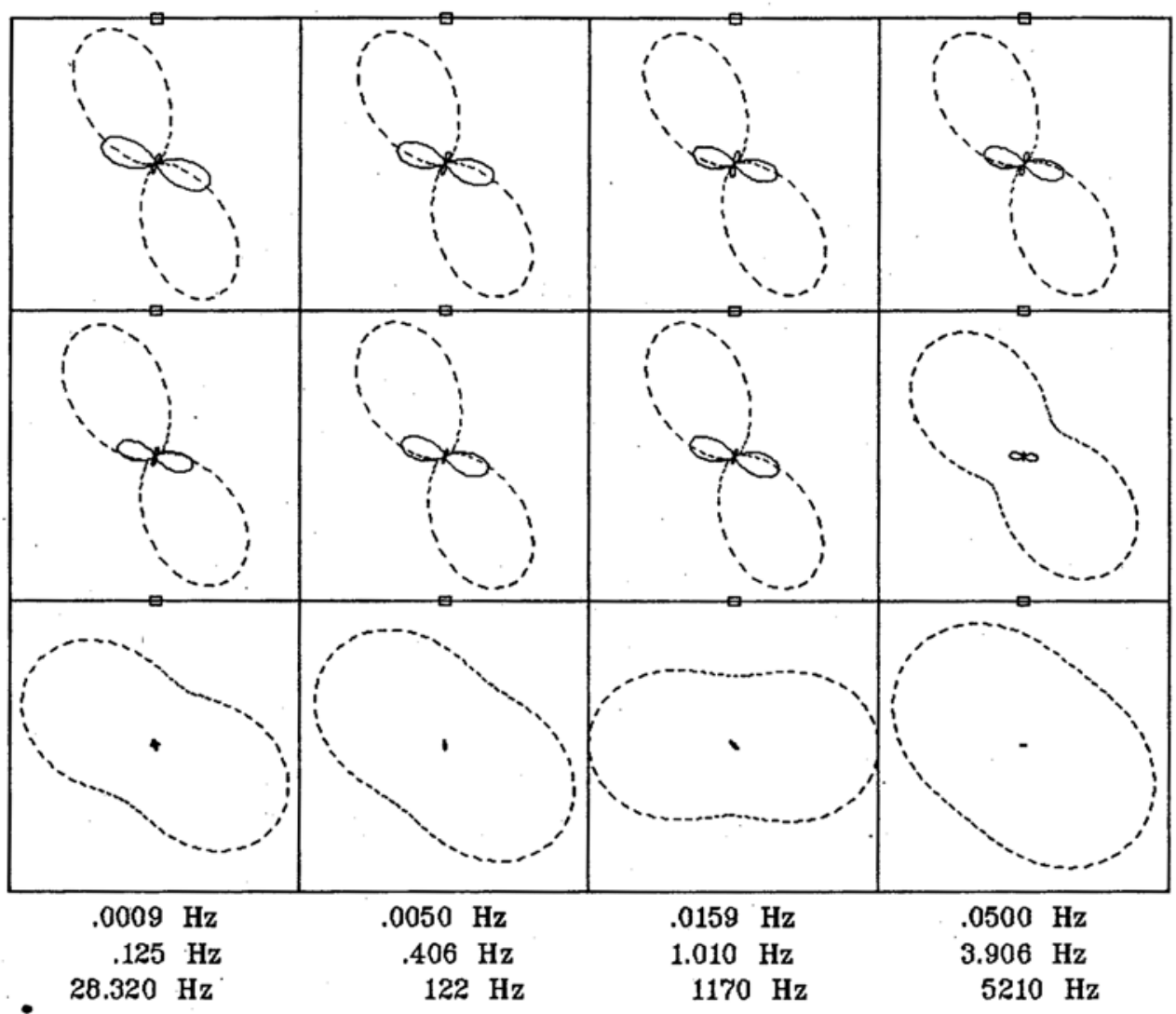

Client: $\mathrm{DOE}$

Remote: none

Acquired: 22:0 May 16, 2005

Survey Co:USGS
Rotation:

Filename: rm23.avg

Channels: Ch1 Ch2 Ch3 Ch4 Ch5 Ch3 Ch4

Plotted: 13:52 Jan 17, 2006

< EMI - ElectroMagnetic Instruments > 


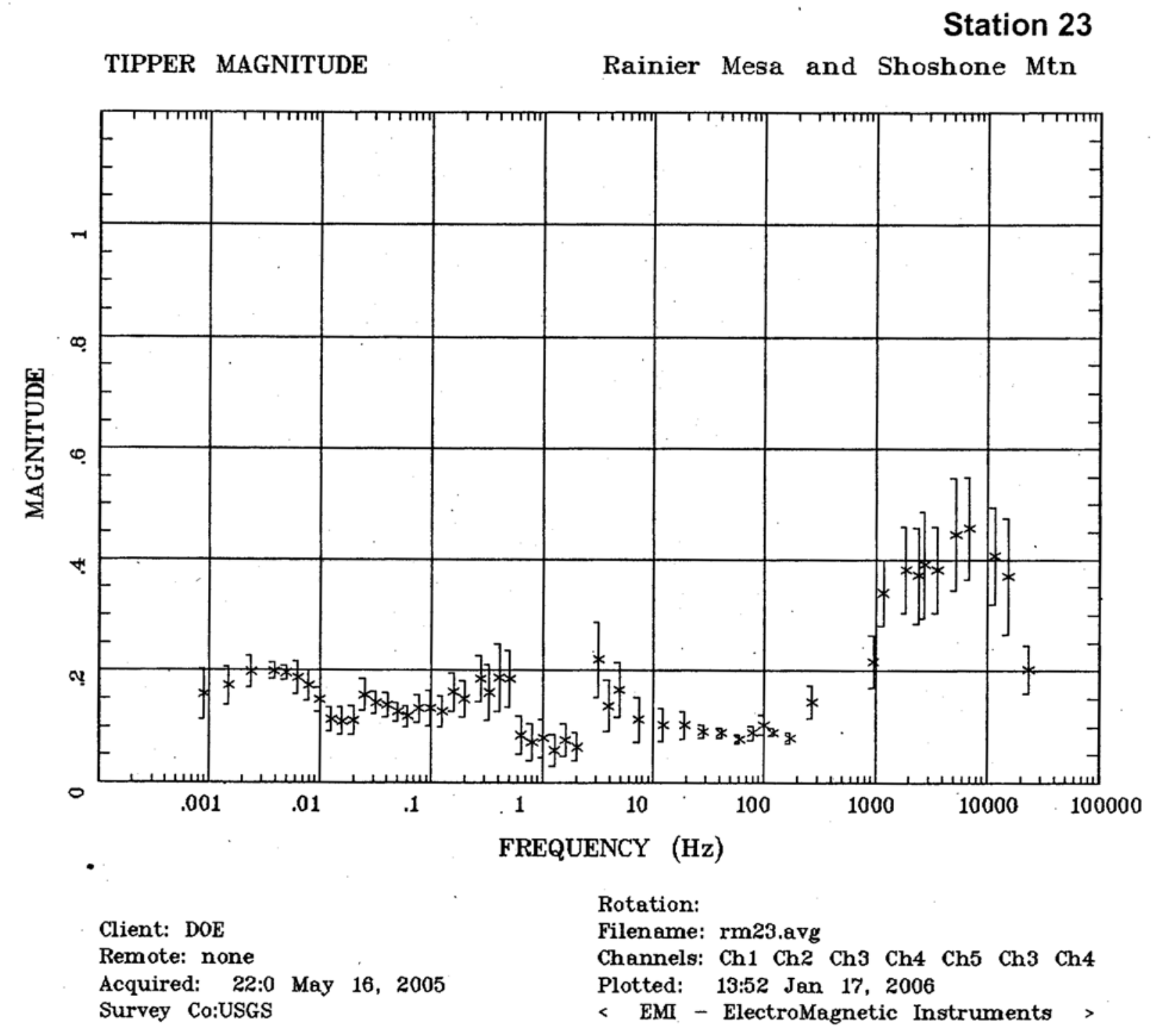


Station 23

TIPPER STRIKE

Rainier Mesa and Shoshone Mtn

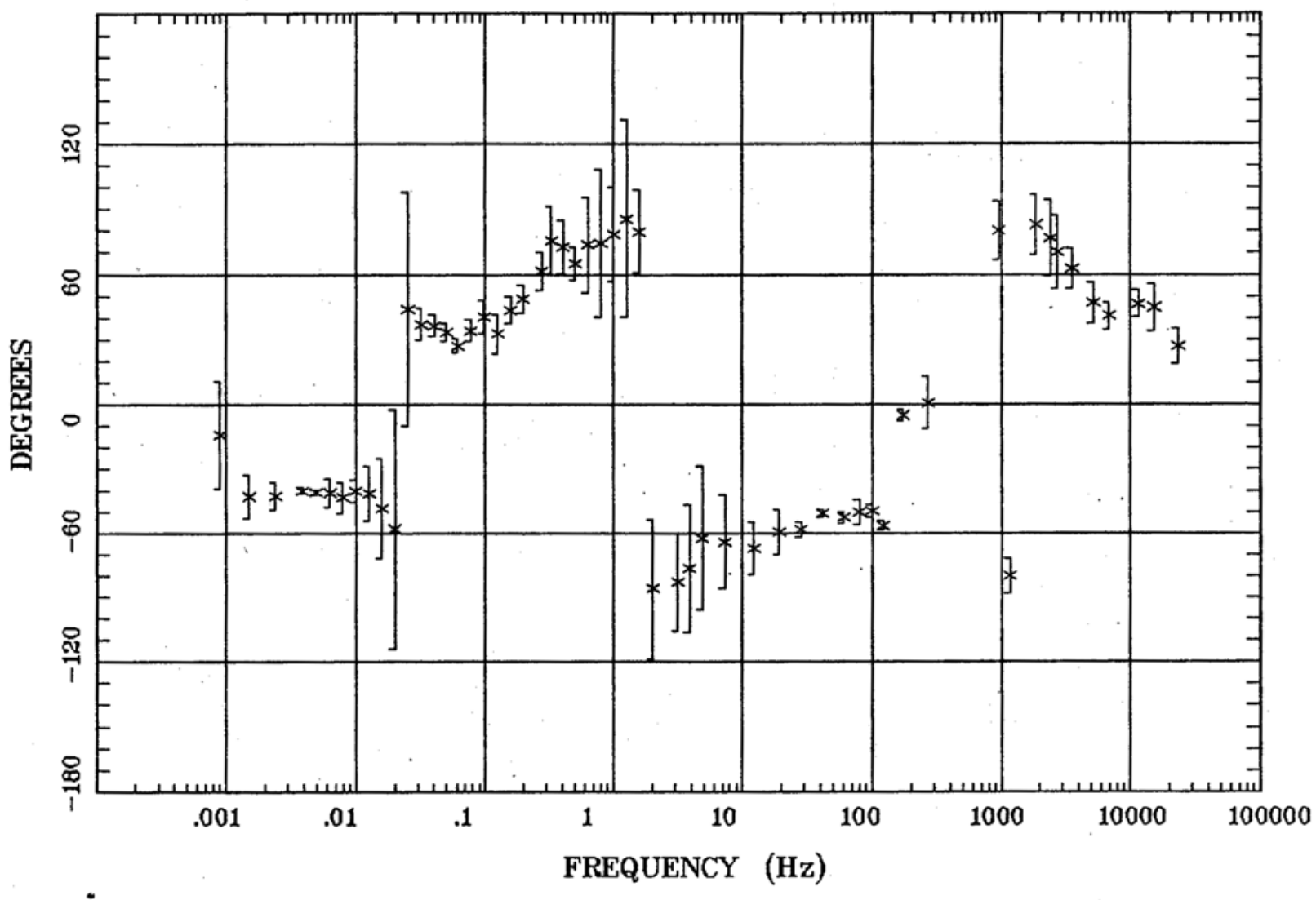

Client: DOE

Remote: none

Acquired: 22:0 May 16, 2005

Survey Co:USGS
Rotation:

Filename: rm23.avg

Channels: Ch1 Ch2 Ch3 Ch4 Ch5 Ch3 Ch4

Plotted: 13:52 Jan 17, 2006

< EMI - ElectroMagnetic Instruments > 
Station 23

HzHx.x Coh HzHy.o

Rainier Mesa and Shoshone Mtn

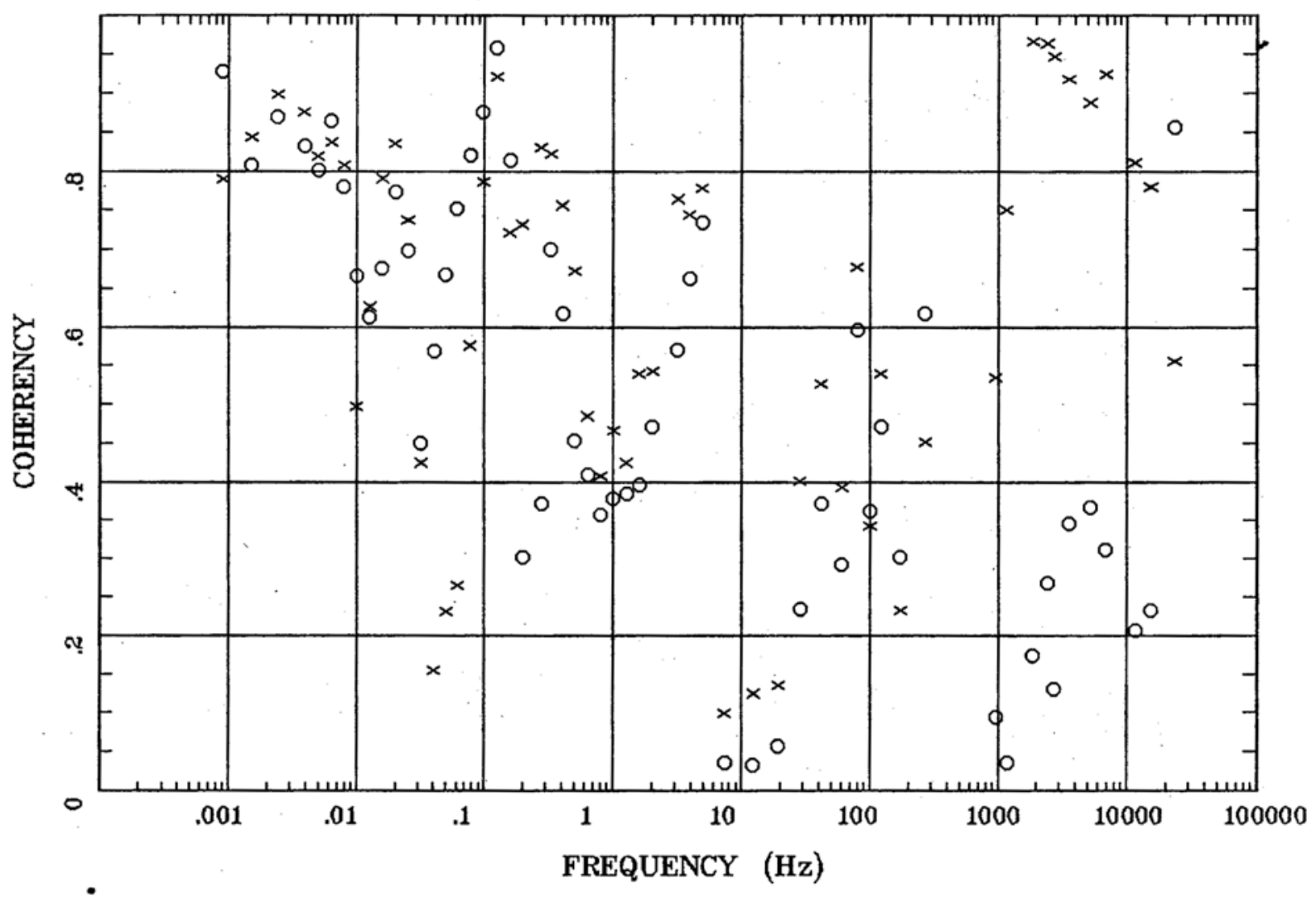

Client: DOE

Rotation:

Filename: rm23.avg

Channels: Ch1 Ch2 Ch3 Ch4 Ch5 Ch3 Ch4

Remote: none

Acquired: 22:0 May 16, 2005

Plotted: 13:52 Jan 17, 2006

Survey Co:USGS

< EMI - ElectroMagnetic Instruments > 
Station 24

APPARENT RESISTIVITY

Rainier Mesa and Shoshone Mtn

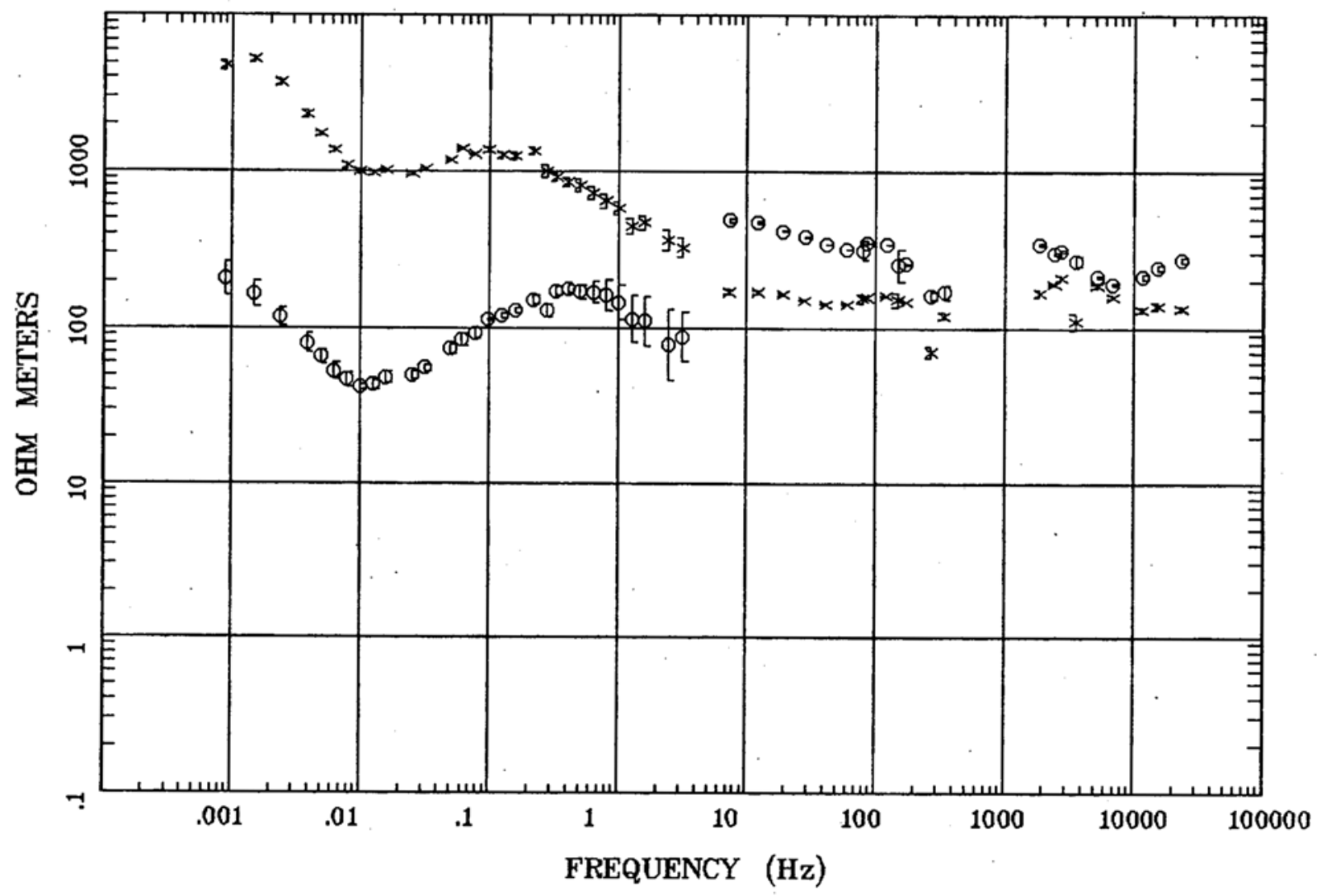

Client: DOE

Remote: none

Acquired: 00:1 May 17, 2005

Survey Co:USGS
Rotation:

Filename: rm24.avg

Channels: Ch1 Ch2 Ch3 Ch4 Ch5 Ch3 Ch4

Plotted: 13:53 Jan 17, 2006

< EMI - ElectroMagnetic Instruments > 


\section{Station 24}

IMPEDANCE PHASE

Rainier Mesa and Shoshone Mtn

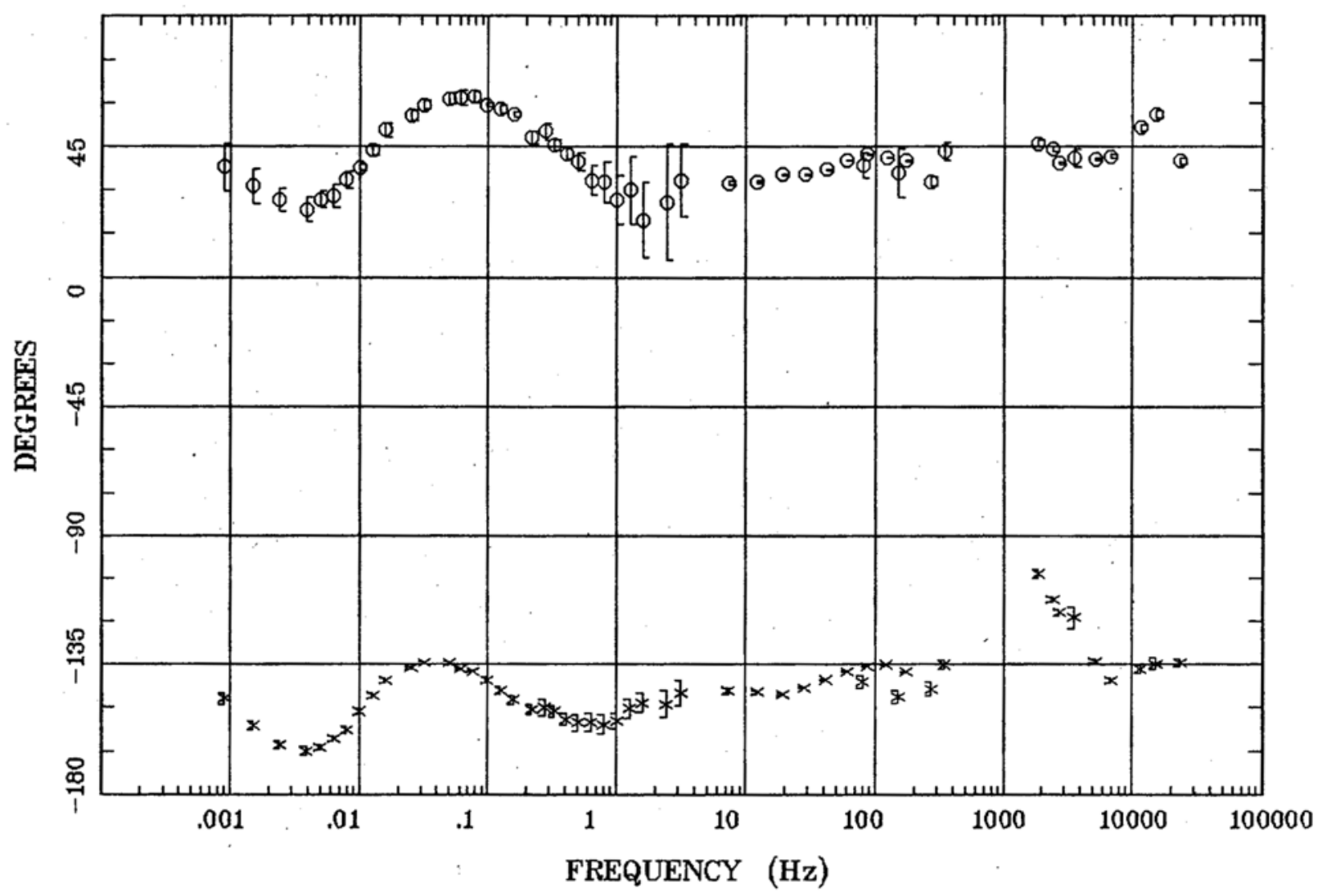

Client: DOE

Remote: none

Acquired: 00:1 May 17, 2005

Survey Co:USGS
Rotation:

Filename: rm24.avg

Channels: Ch1 Ch2 Ch3 Ch4 Ch5 Ch3 Ch4

Plotted: 13:53 Jan 17, 2006

< EMI - ElectroMagnetic Instruments > 


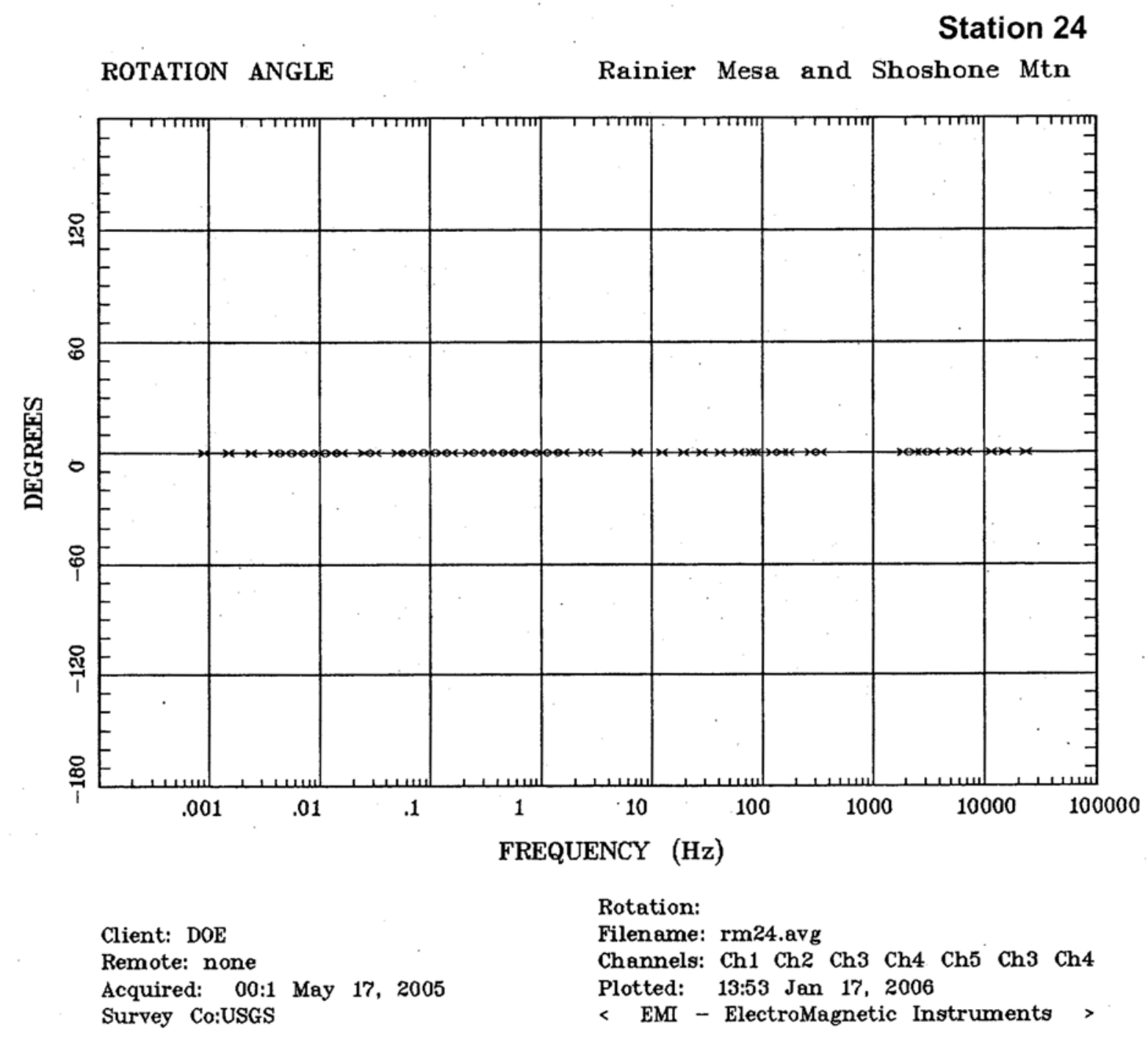


Station 24

IMPEDANCE SKEW

Rainier Mesa and Shoshone Mtn

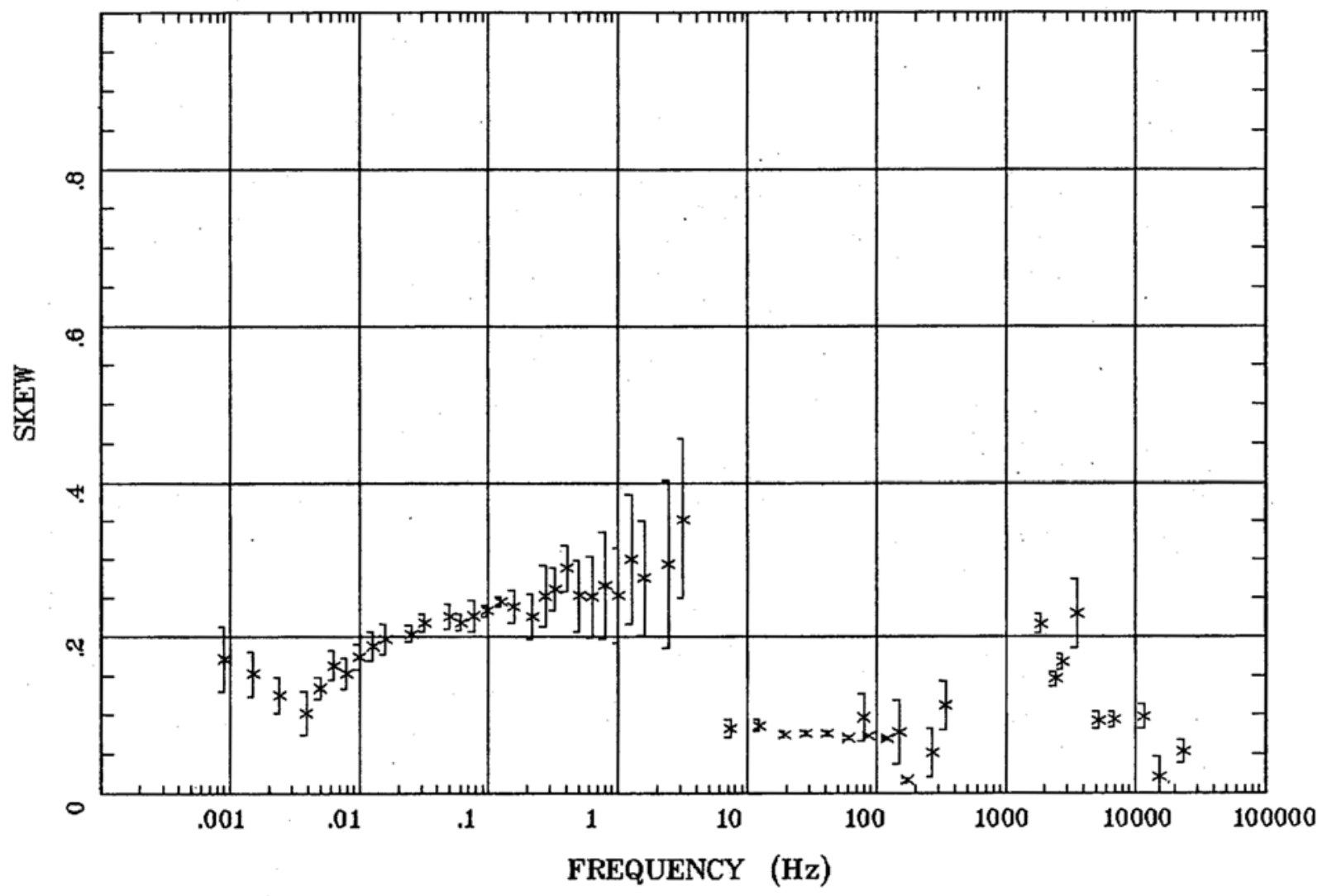

Client: DOE

Remote: none

Acquired: 00:1 May 17, 2005

Survey Co:USGS
Rotation:

Filename: rm24.avg

Channels: Ch1 ${ }^{\circ} \mathrm{Ch} 2 \mathrm{Ch} 3 \mathrm{Ch} 4$ Ch5 Ch3 Ch4

Plotted: 13:53 Jan 17, 2006

< EMI - ElectroMagnetic Instruments > 


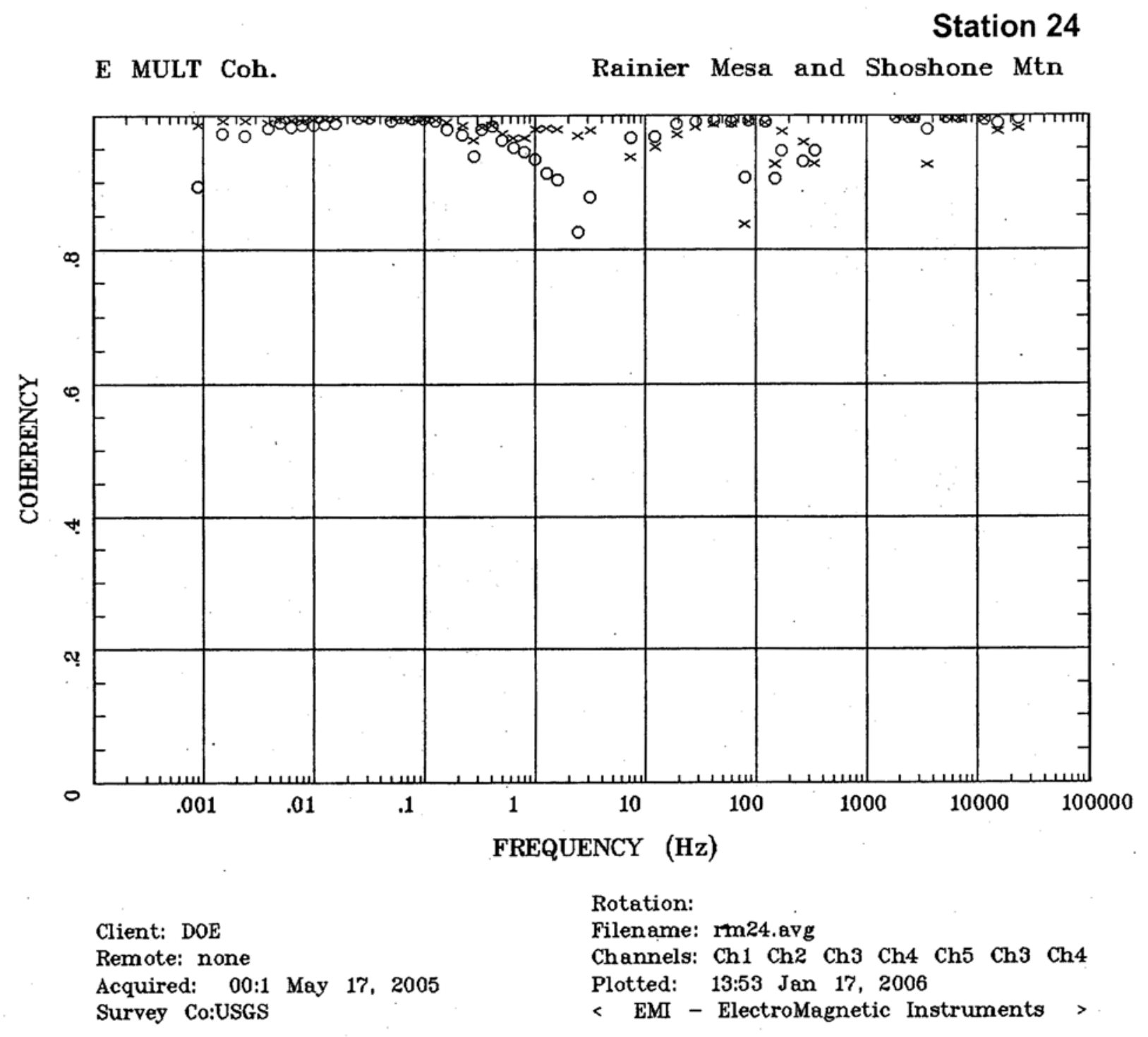


Station 24

POLAR PLOTS

Rainier Mesa and Shoshone Mtn

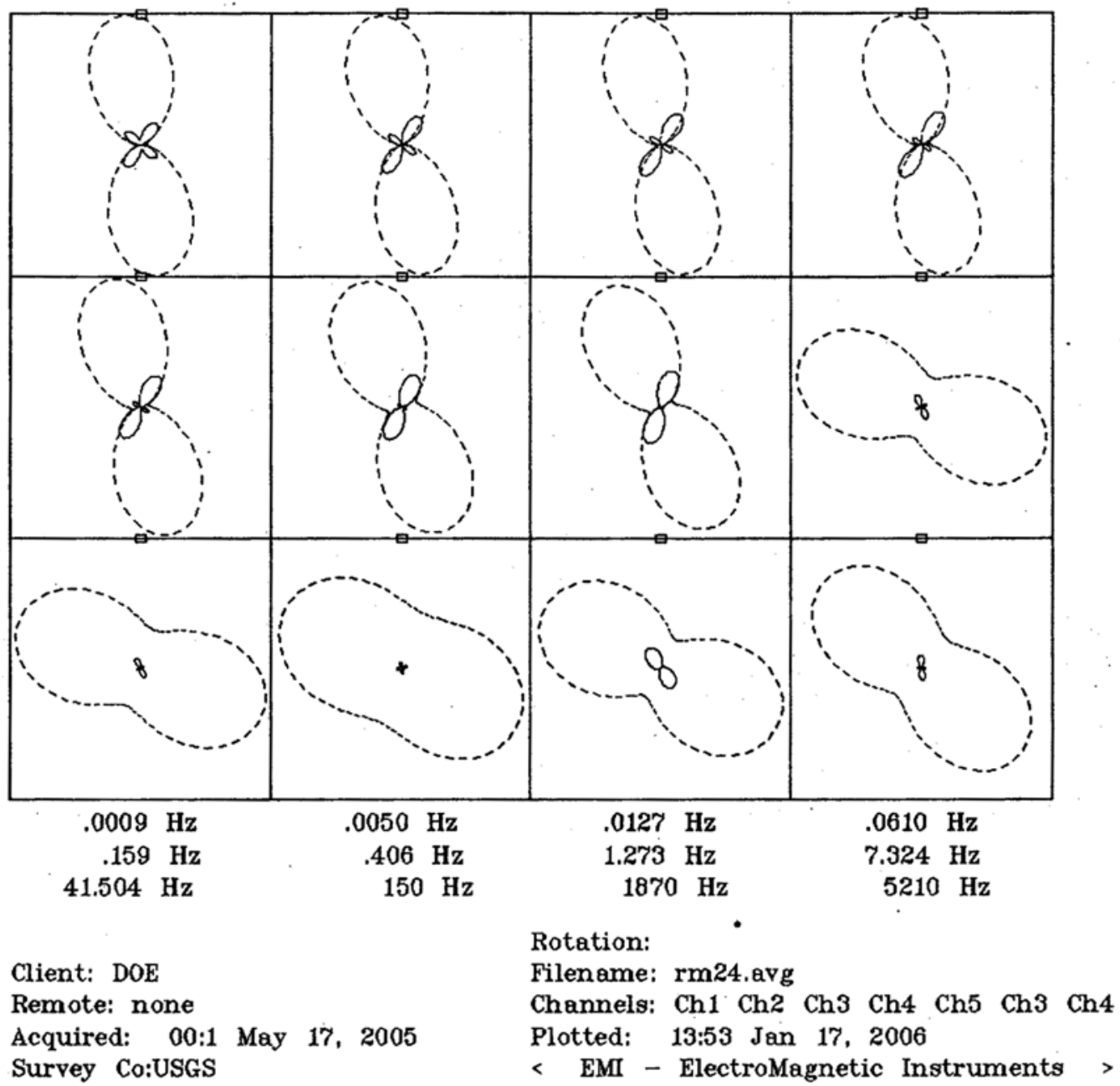




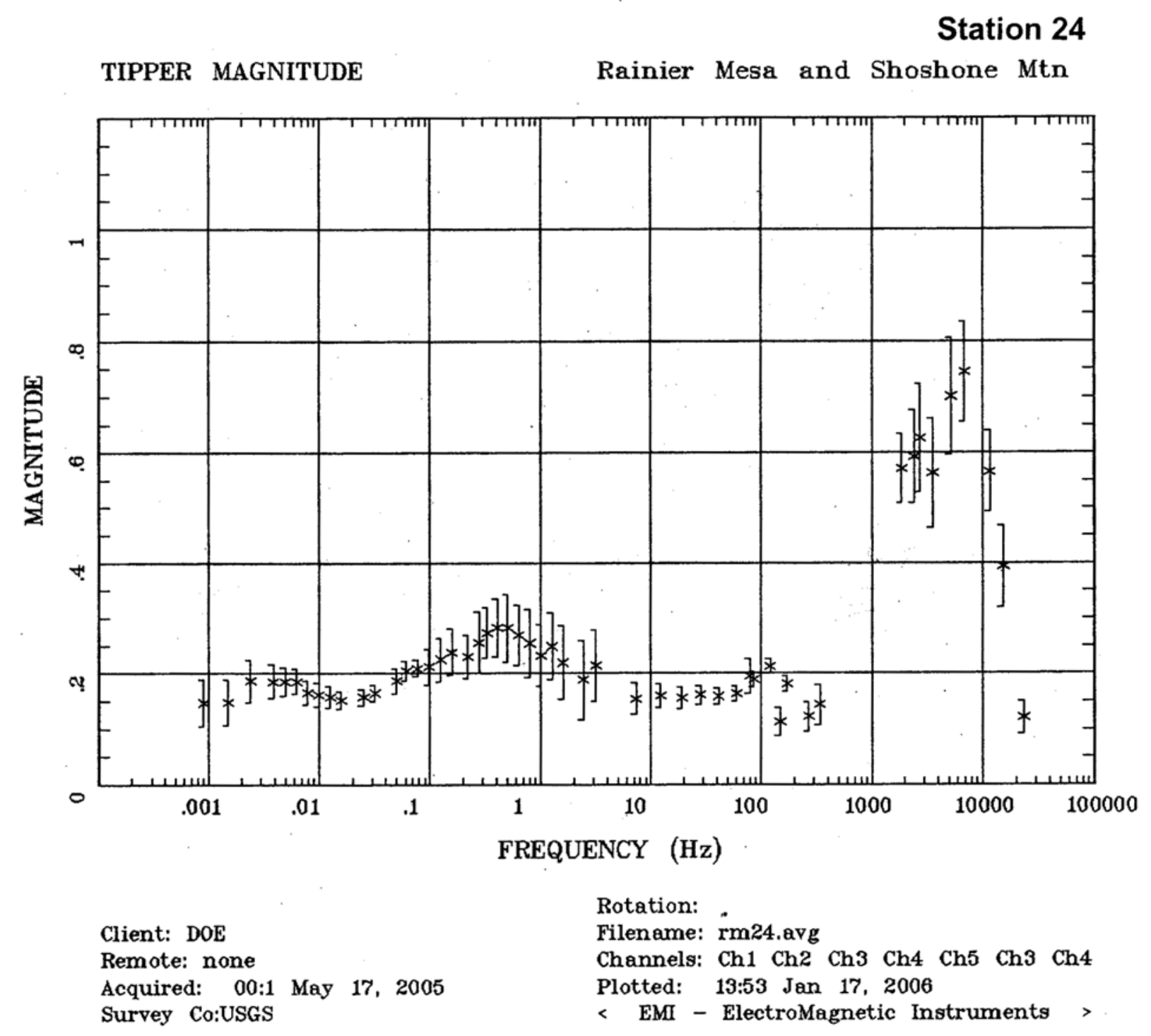


Station 24

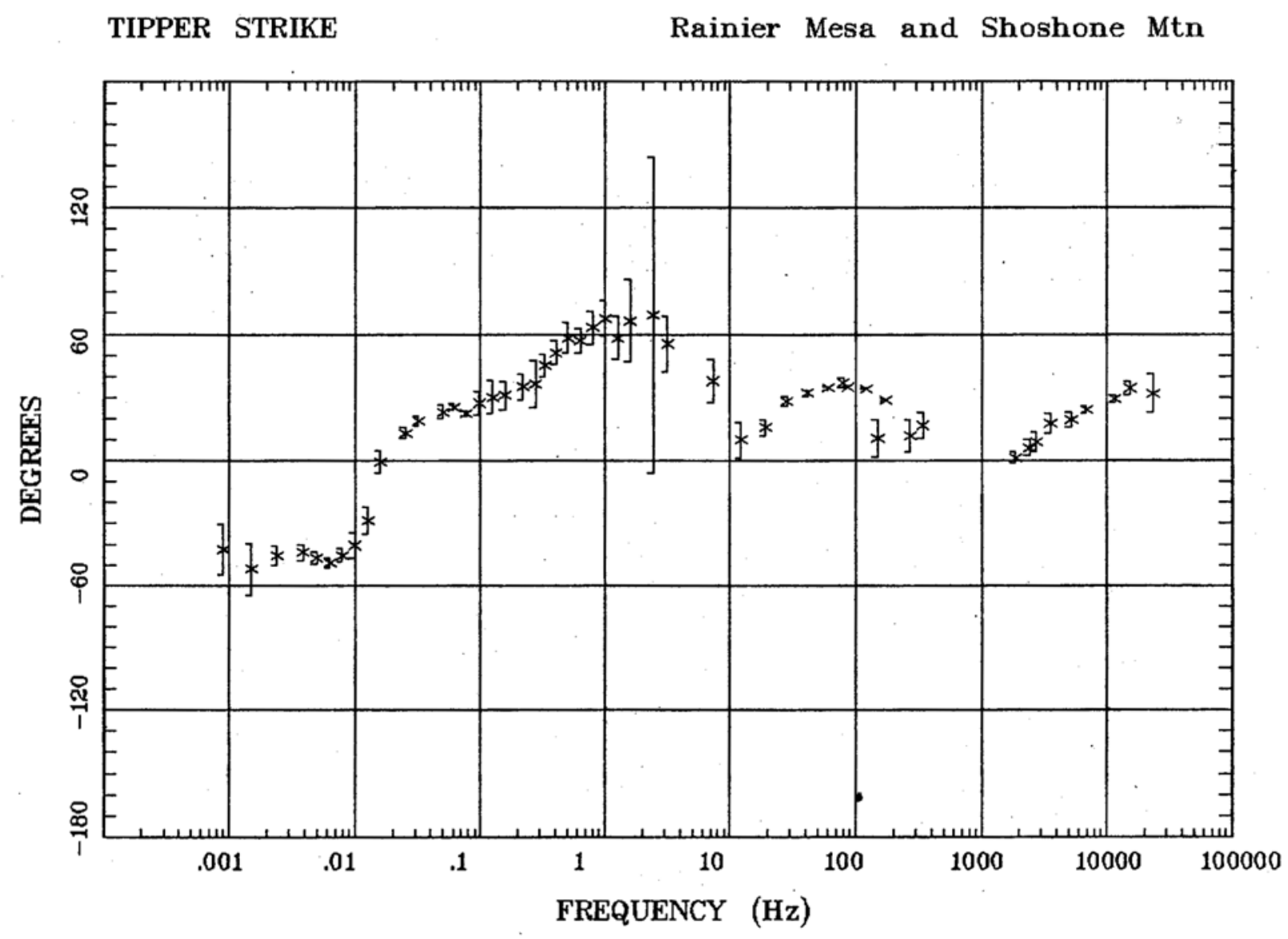

Client: DOE

Remote: none

Acquired: 00:1 May 17, 2005

Survey Co:USGS
Rotation:

Filename: rm24.avg

Channels: Ch1 Ch2 Ch3 Ch4 Ch5 Ch3 Ch4

Plotted: 13:53 Jan 17, 2006

< EMI - ElectroMagnetic Instruments > 
Station 24

HzHx.x Coh HzHy.o

Rainier Mesa and Shoshone Mtn

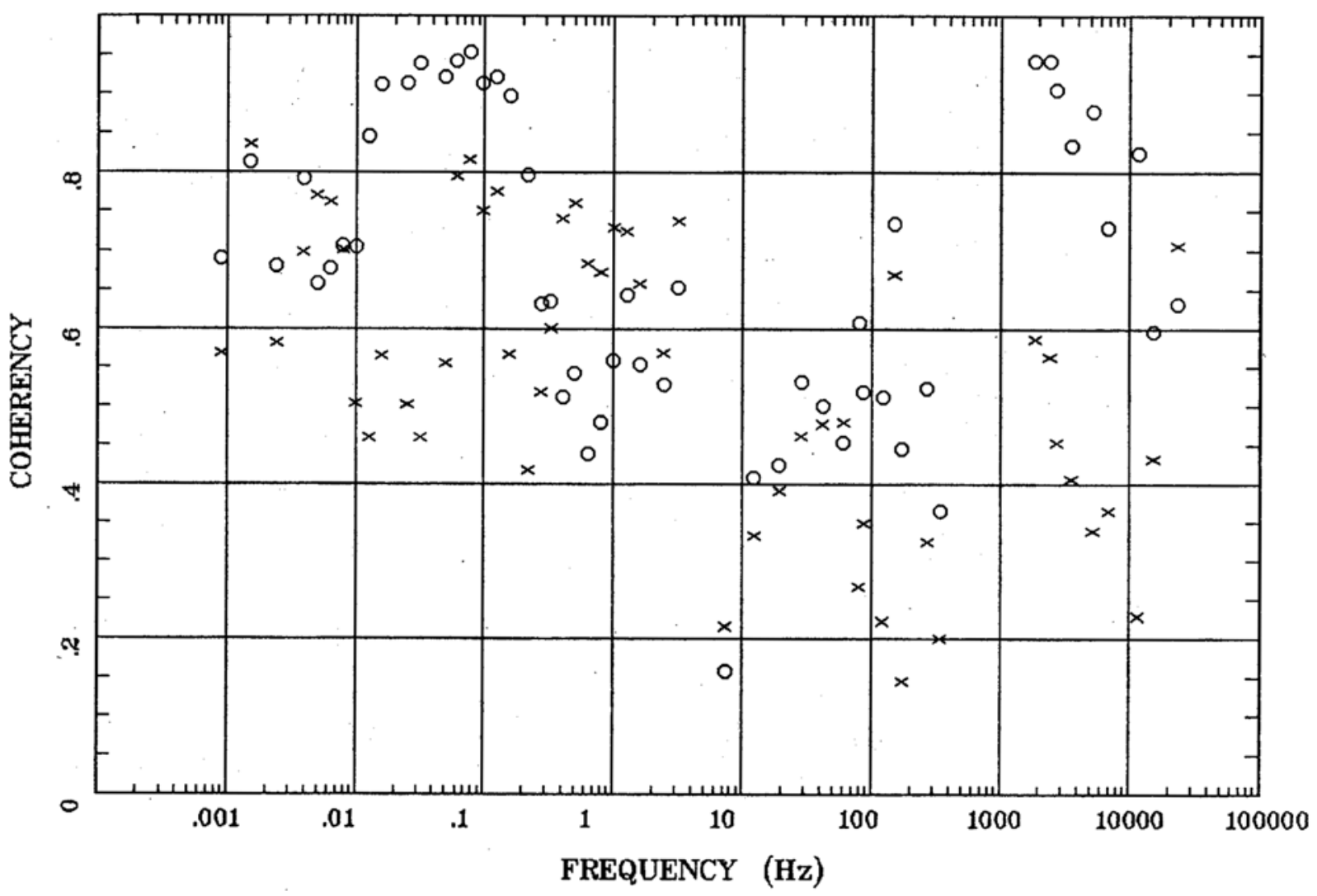

Client: DOE

Remote: none

Acquired: 00:1 May 17, 2005

Survey Co:USGS

Rotation:

Filename: rm24.avg

Channels: Ch1 Ch2 Ch3 Ch4 Ch5 Ch3 Ch4

Plotted: 13:53 Jan 17, 2006

< EMI - ElectroMagnetic Instruments > 


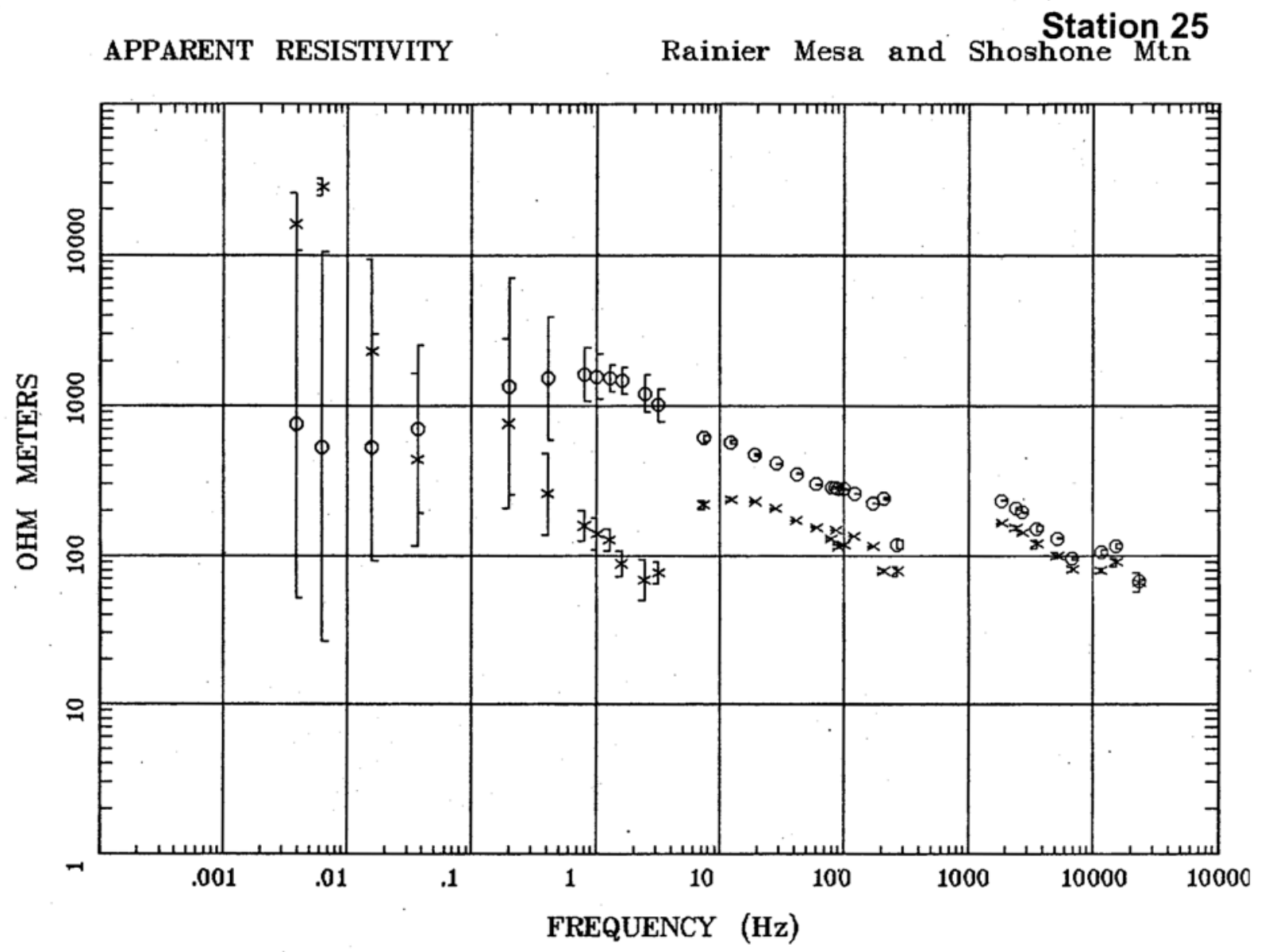

Client: DOE

Remote: none

Acquired: 00:5 May 15, 2005

Survey Co:USGS
Rotation:

Filename: rm25.avg

Channels: Ch1 Ch2 Ch3 Ch4 Ch5 Ch3 Ch4

Plotted: 13:54 Jan 17, 2006

< EMI - ElectroMagnetic Instruments > 


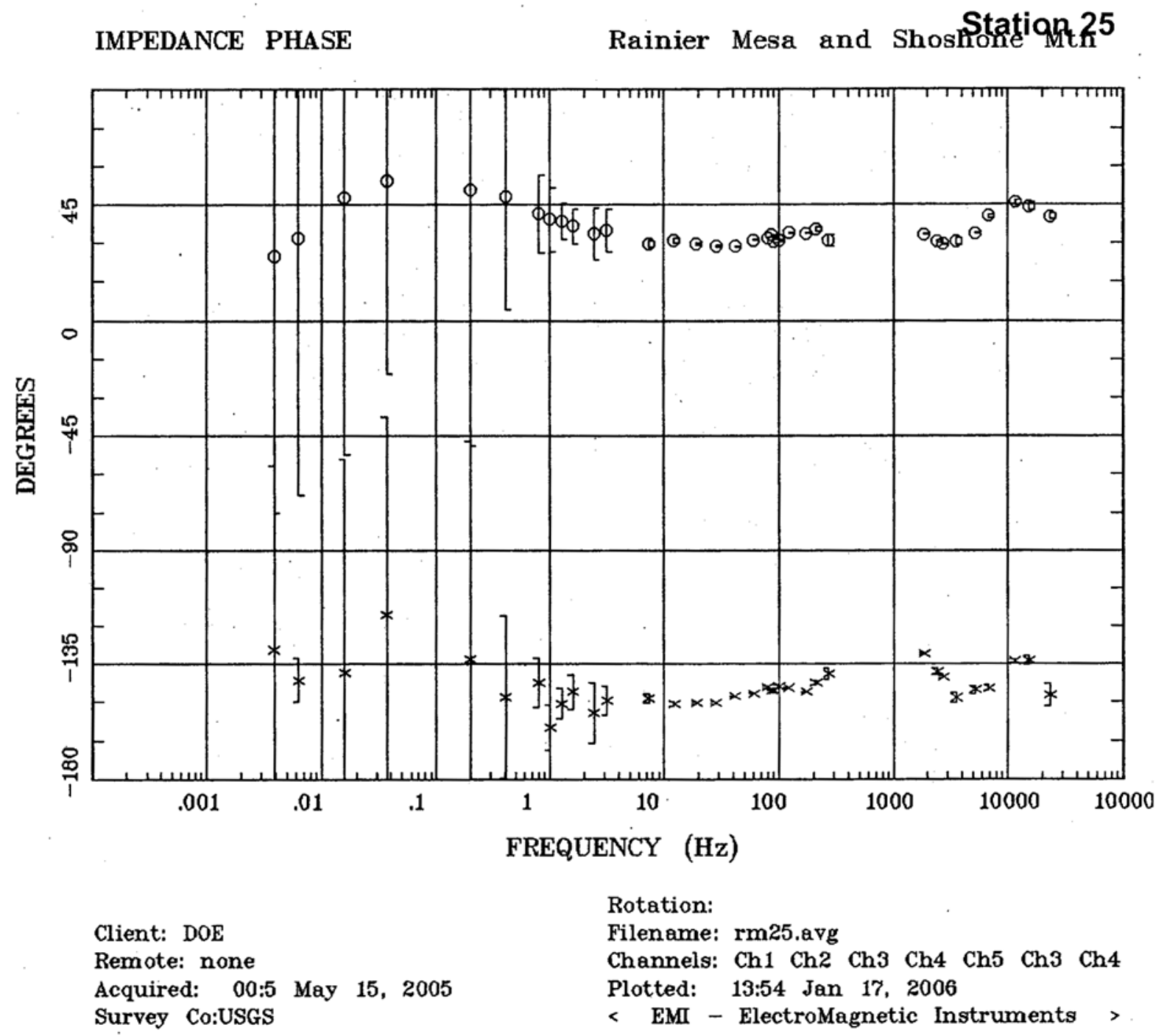




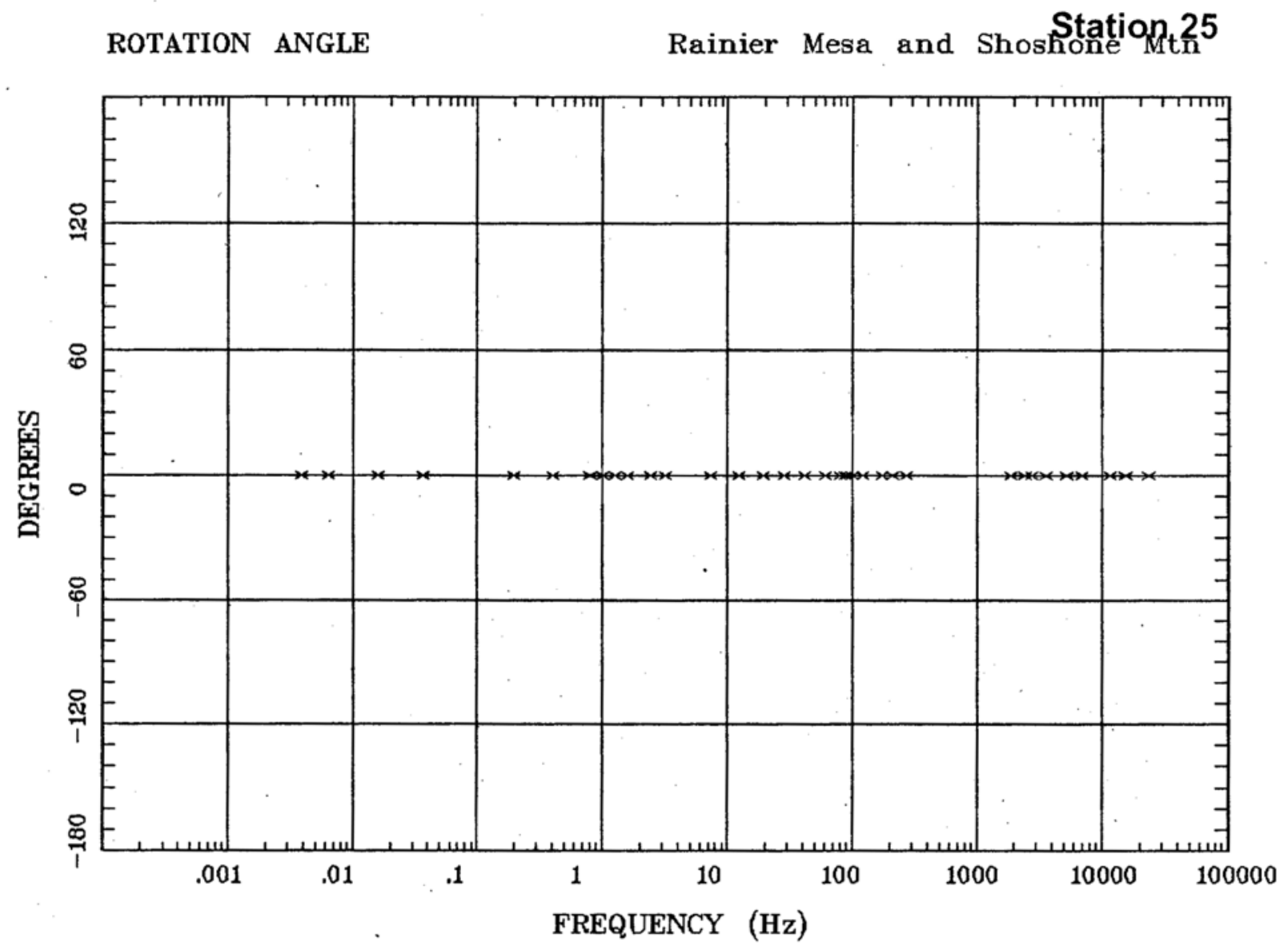

Client: DOE

Remote: none

Acquired: 00:5 May 15, 2005

Survey Co:USGS
Rotation:

Filename: rm25.avg

Channels: Ch1 Ch2 Ch3 Ch4 Ch5 Ch3 Ch4

Plotted: 13:54 Jan 17, 2006

< EMI - ElectroMagnetic Instruments > 


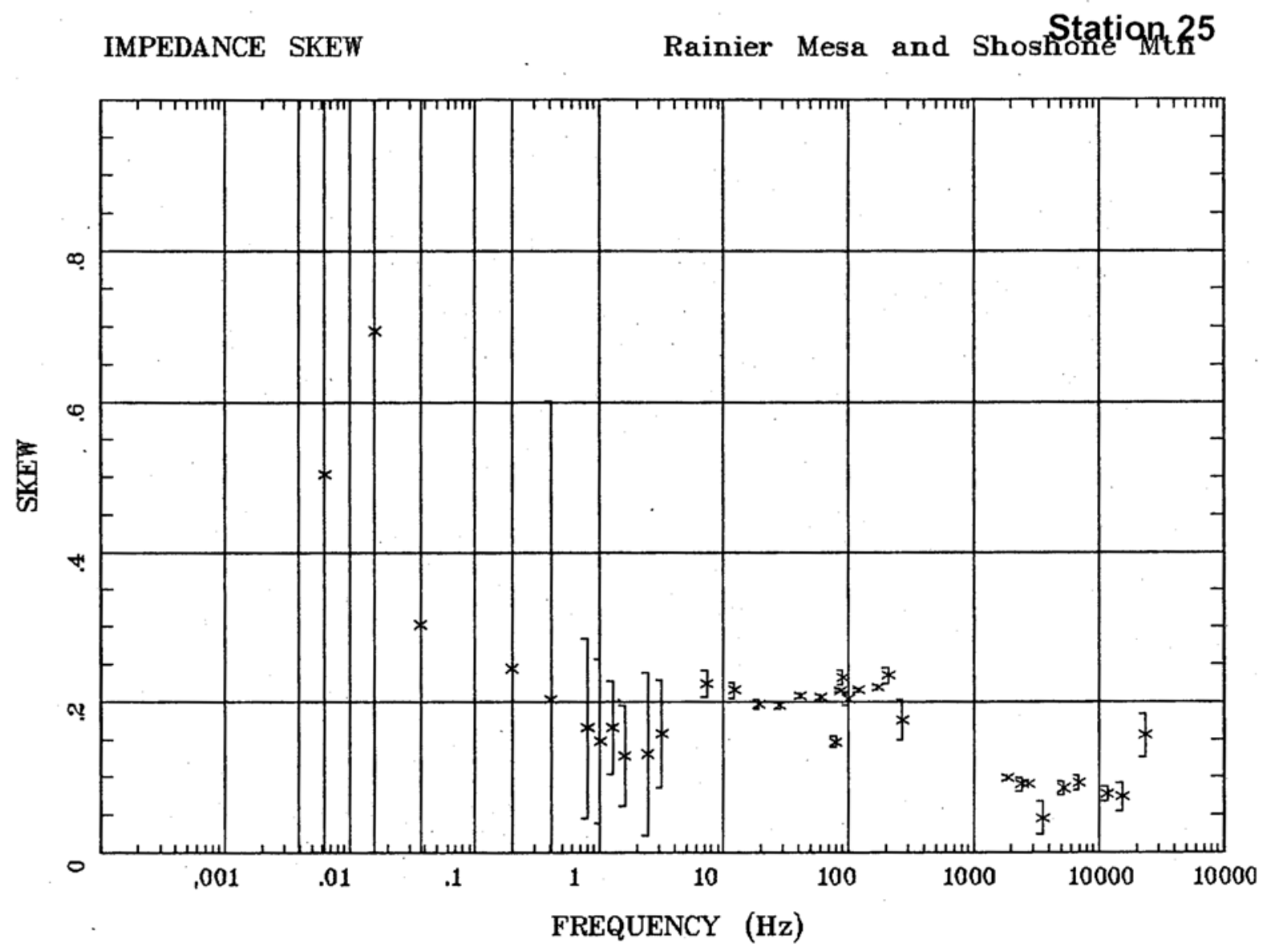

Client: DOE

Remote: none

Acquired: 00:5 May 15, 2005

Survey Co:USGS

Rotation:

Filename: rm25.avg

Channels: Ch1 Ch2 Ch3 Ch4 Ch5 Ch3 Ch4

Plotted: 13:54 Jan 17, 2006

< EMI - ElectroMagnetic Instruments > 


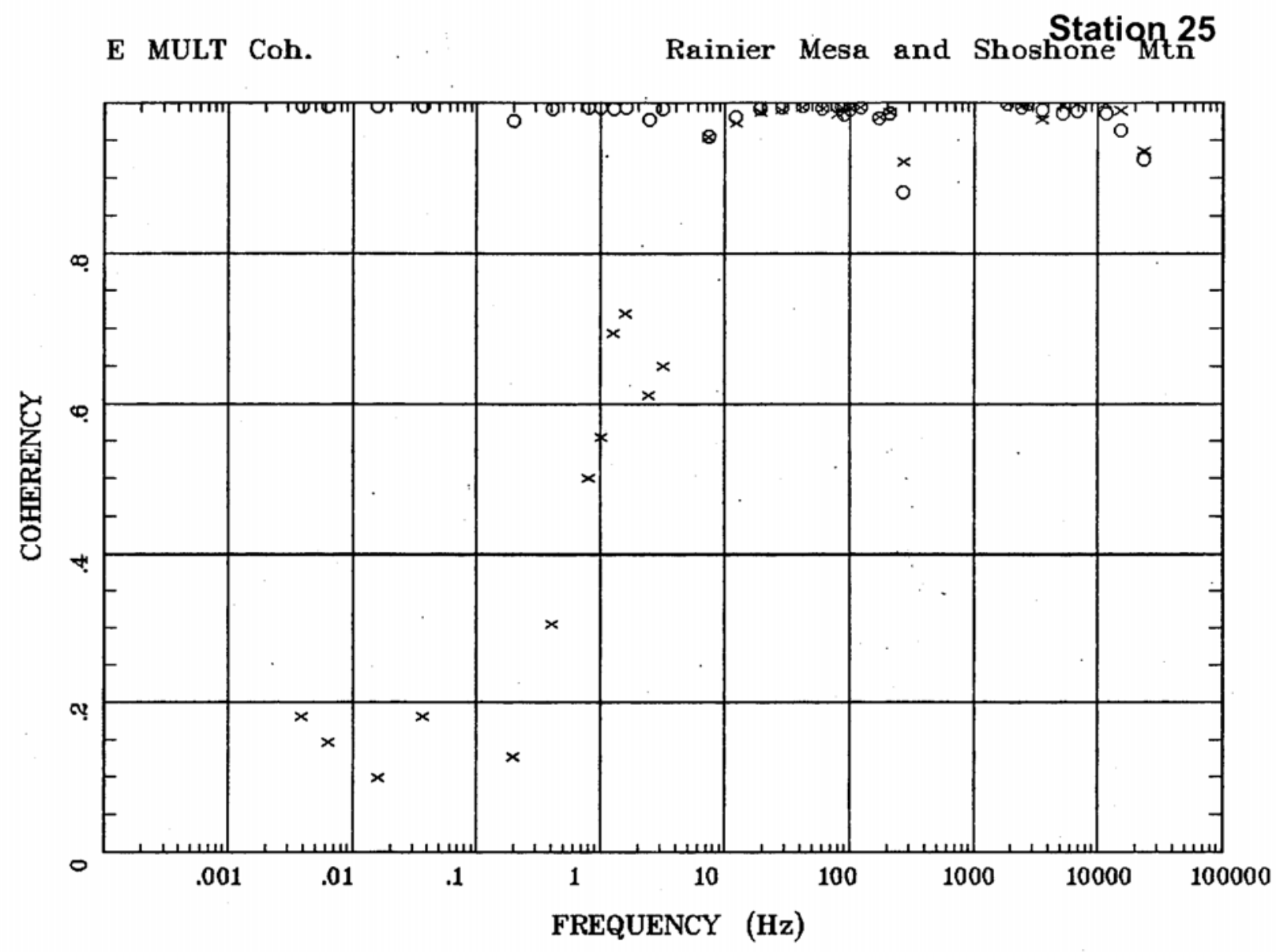

Client: DOE

Remote: none

Acquired: 00:5 May 15, 2005

Survey Co:USGS

Rotation:

Filename: rm25.avg

Channels: Ch1 Ch2 Ch3 Ch4 Ch5 Ch3 Ch4

Plotted: 13:54 Jan 17, 2006

< EMI - ElectroMagnetic Instruments > 


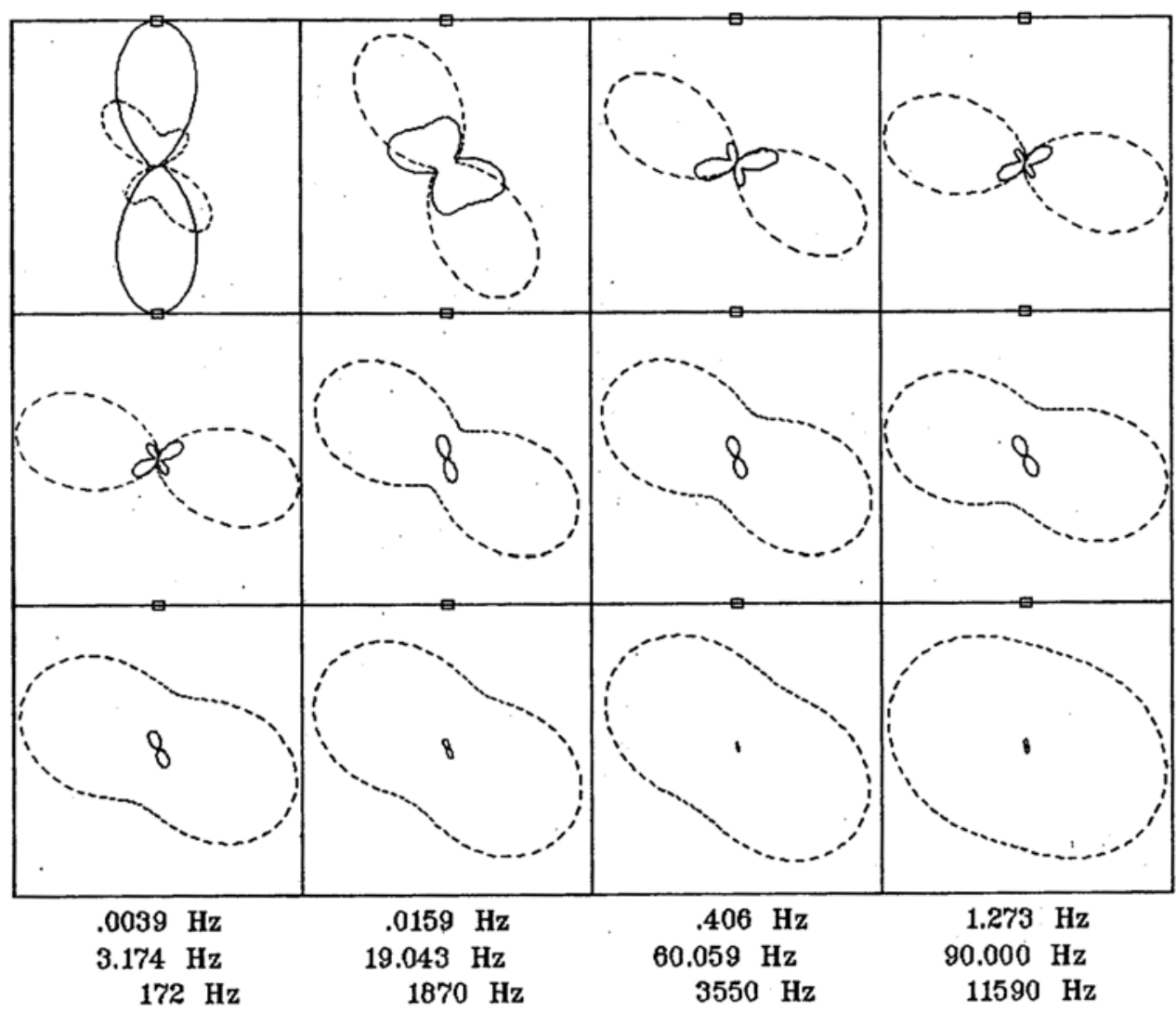

Client: DOE

Remote: none Acquired: 00:5 May 15, 2005 Survey Co:USGS
Rotation:

Filename: rm25.avg

Channels: Ch1 Ch2 Ch3 Ch4 Ch5 Ch3 Ch4

Plotted: 13:54 Jan 17, 2006

< EMI - ElectroMagnetic Instruments > 


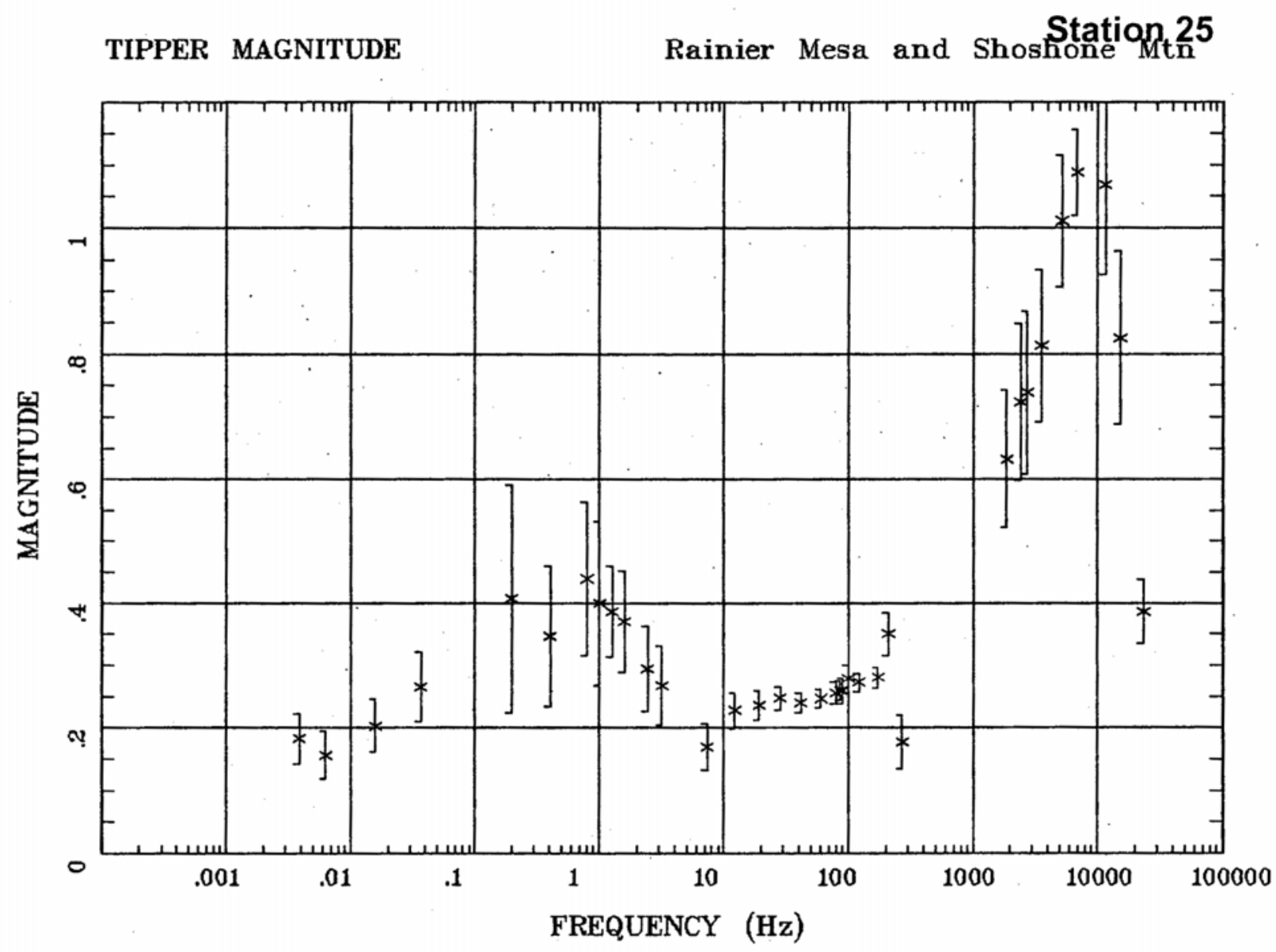

Client: DOE

Remote: none

Acquired: 00:5 May 15, 2005

Survey Co:USGS
Rotation:

Filename: rm25.avg

Channels: Ch1 Ch2 Ch3 Ch4 Ch5 Ch3 Ch4

Plotted: 13:54 Jan 17, 2006

< EMI - ElectroMagnetic Instruments > 
TIPPER STRIKE

Rainier Mesa and Shoshoneign 25

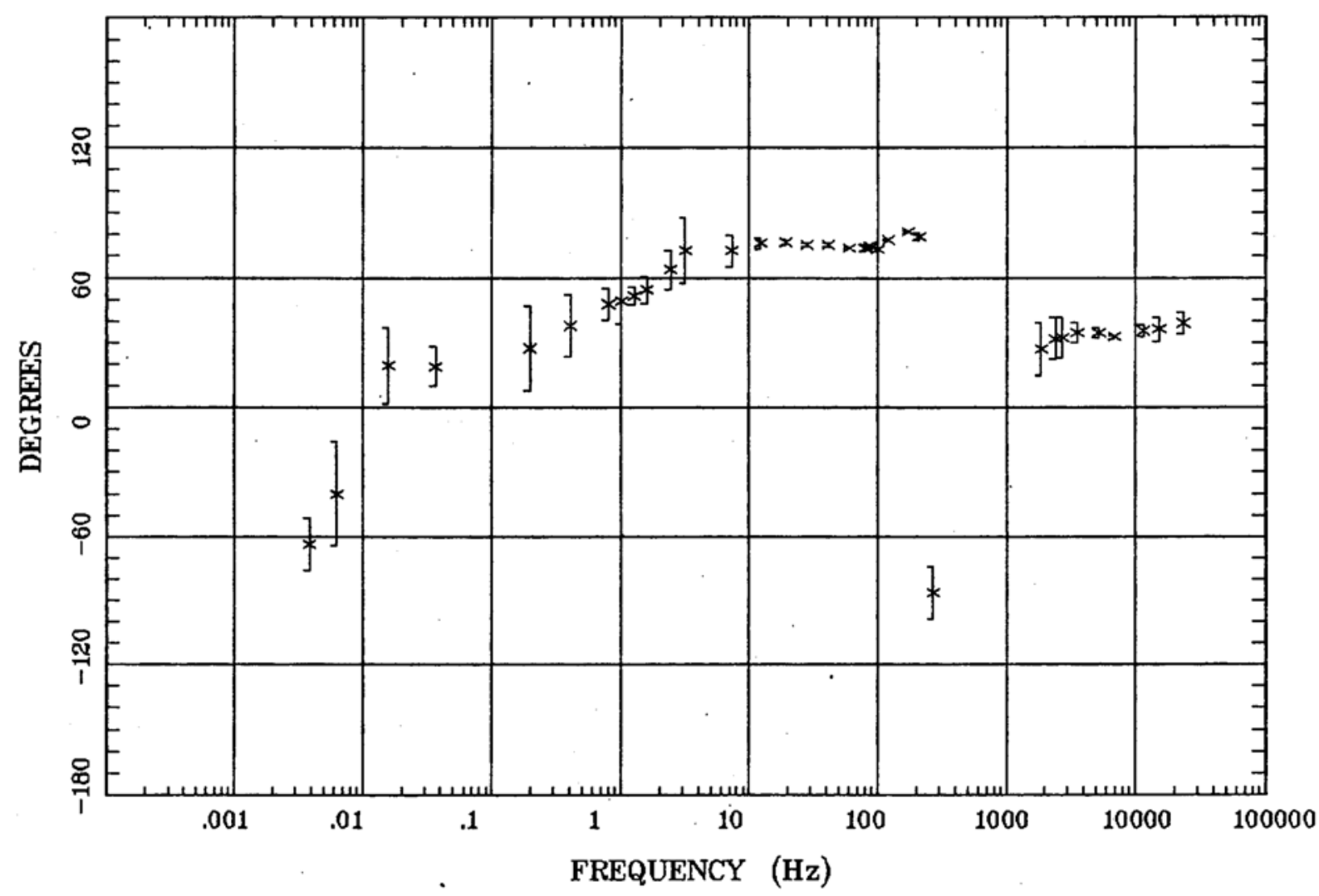

Client: $\mathrm{DOE}$

Remote: none

Acquired: 00:5 May 15, 2005

Survey Co:USGS
Rotation:

Filename: rm25.avg

Channels: Ch1 Ch2 Ch3 Ch4 Ch5 Ch3 Ch4

Plotted: 13:54 Jan 17, 2006

< EMI - ElectroMagnetic Instruments > 


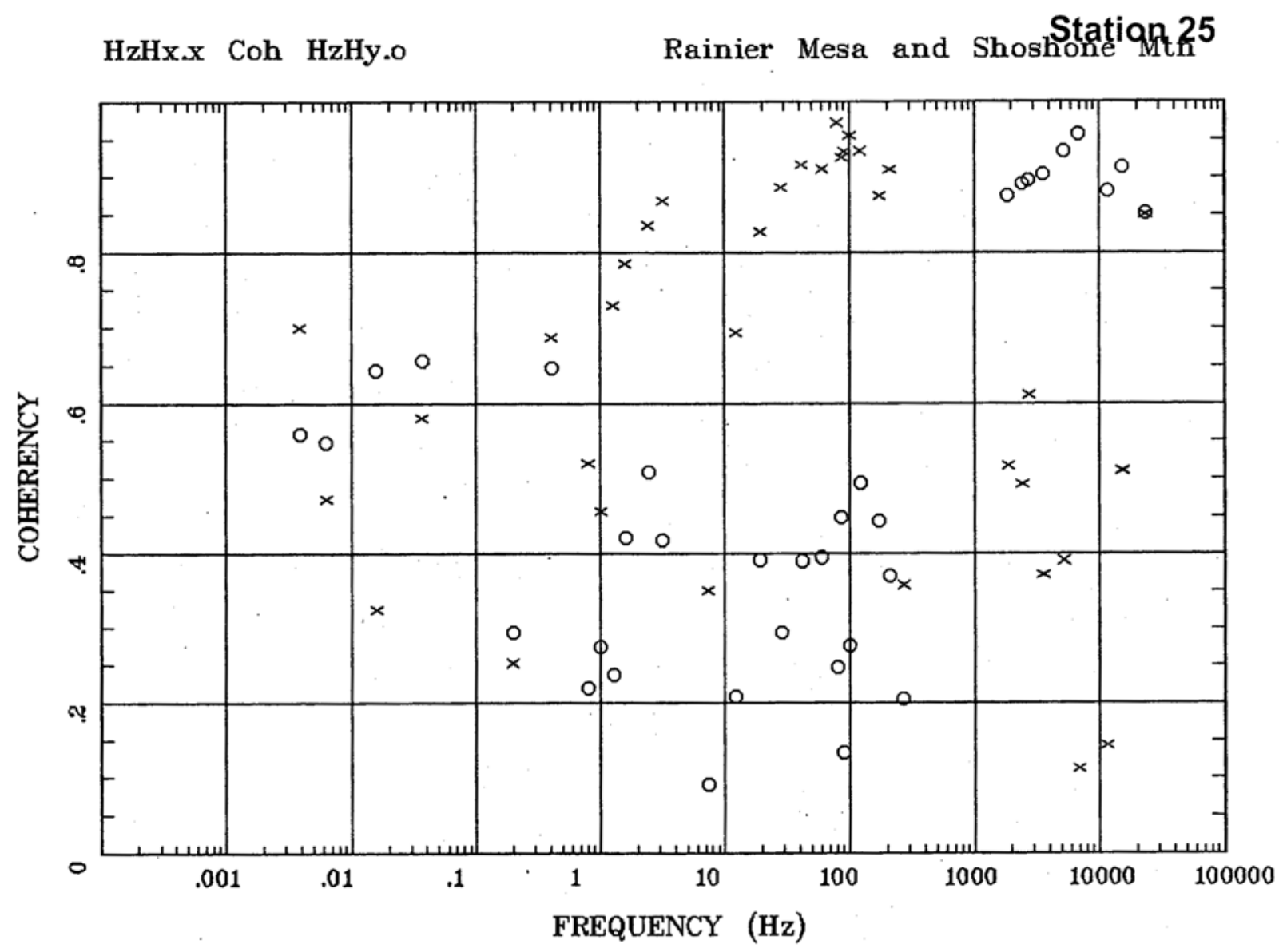

Client: DOE

Remote: none

Acquired: 00:5 May 15, 2005

Survey Co:USGS
Rotation:

Filename: rm25.avg

Channels: Ch1 Ch2 Ch3 Ch4 Ch5 Ch3 Ch4

Plotted: 13:54 Jan 17, 2006

< EMI - ElectroMagnetic Instruments > 


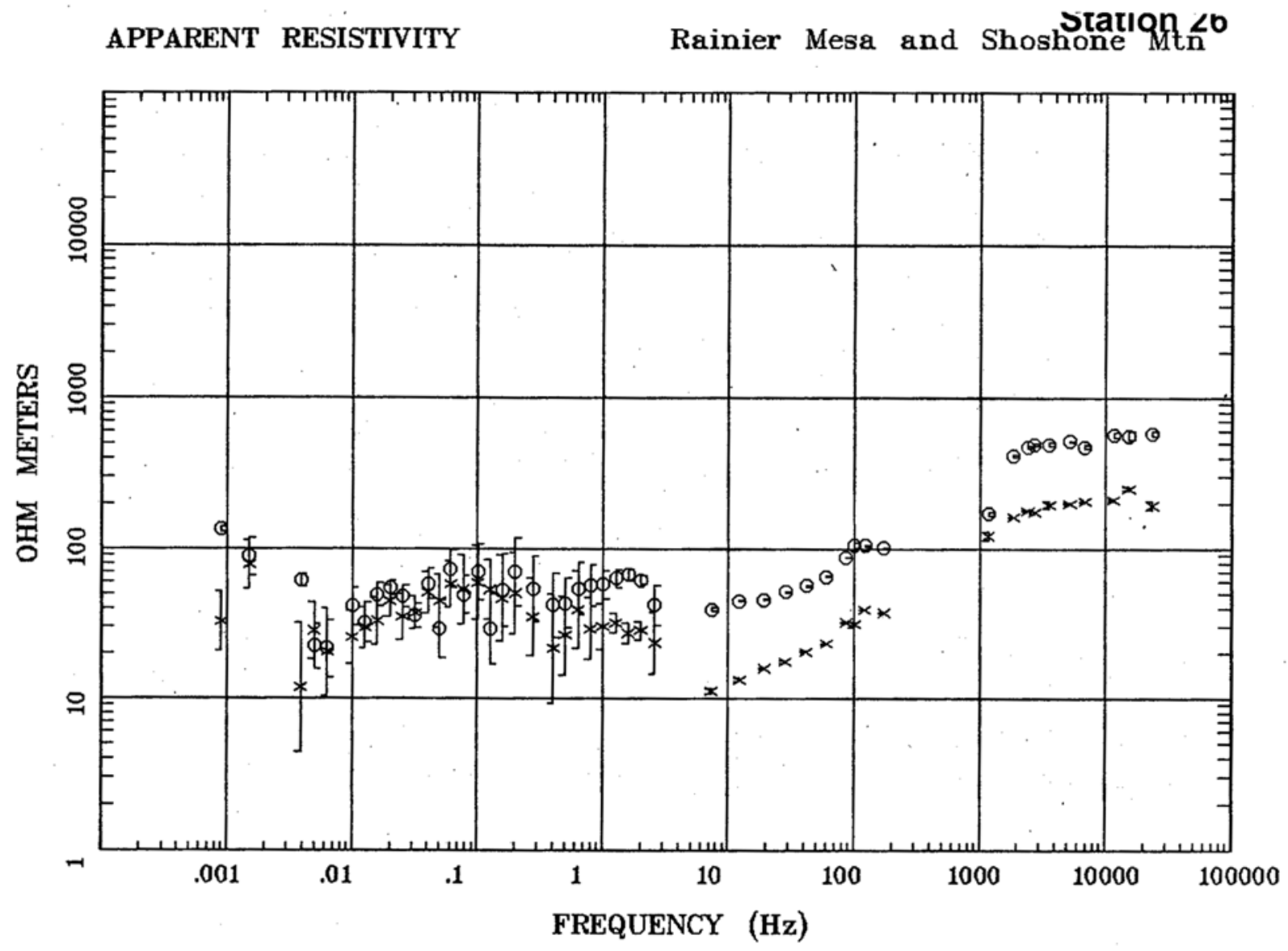

Client: DOE

Remote: none

Acquired: 22:1 May 15, 2005

Survey Co:USGS
Rotation:

Filenome: rm26.avg

Channels: Ch1 Ch2 Ch3 Ch4 Ch5 Ch3 Ch4

Plotted: 13:56 Jan 17, 2006

< EMI - ElectroMagnetic Instruments > 


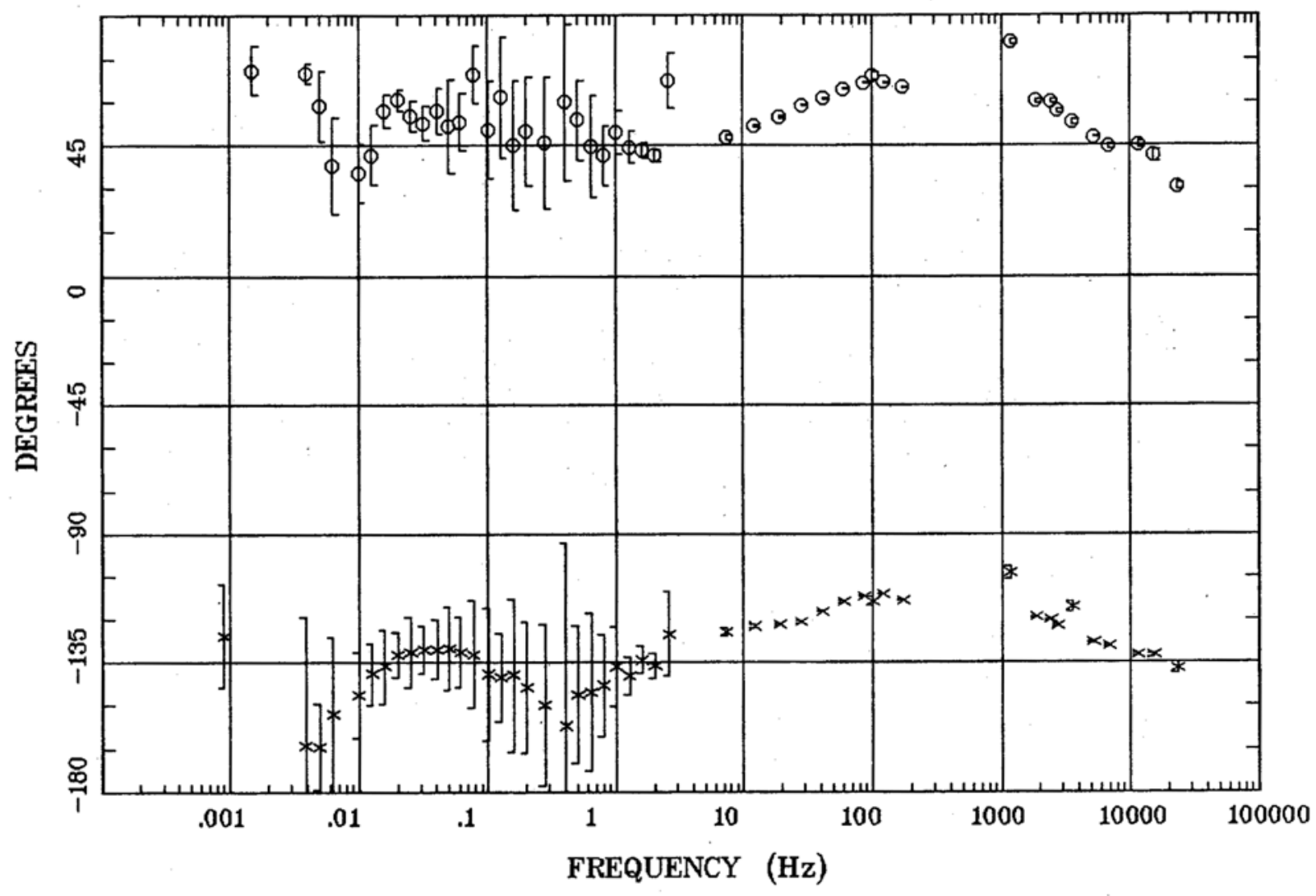

Client: DOE

Remote: none

Acquired: 22:1 May 15, 2005

Survey Co:USGS
Rainier Mesa and Shoshone Mtn 26

Rotation:

Filename: rm26.avg

Channels: Ch1 Ch2 Ch3 Ch4 Ch5 Ch3 Ch4

Plotted: 13:56 Jan 17, 2006

< EMI - ElectroMagnetic Instruments > 


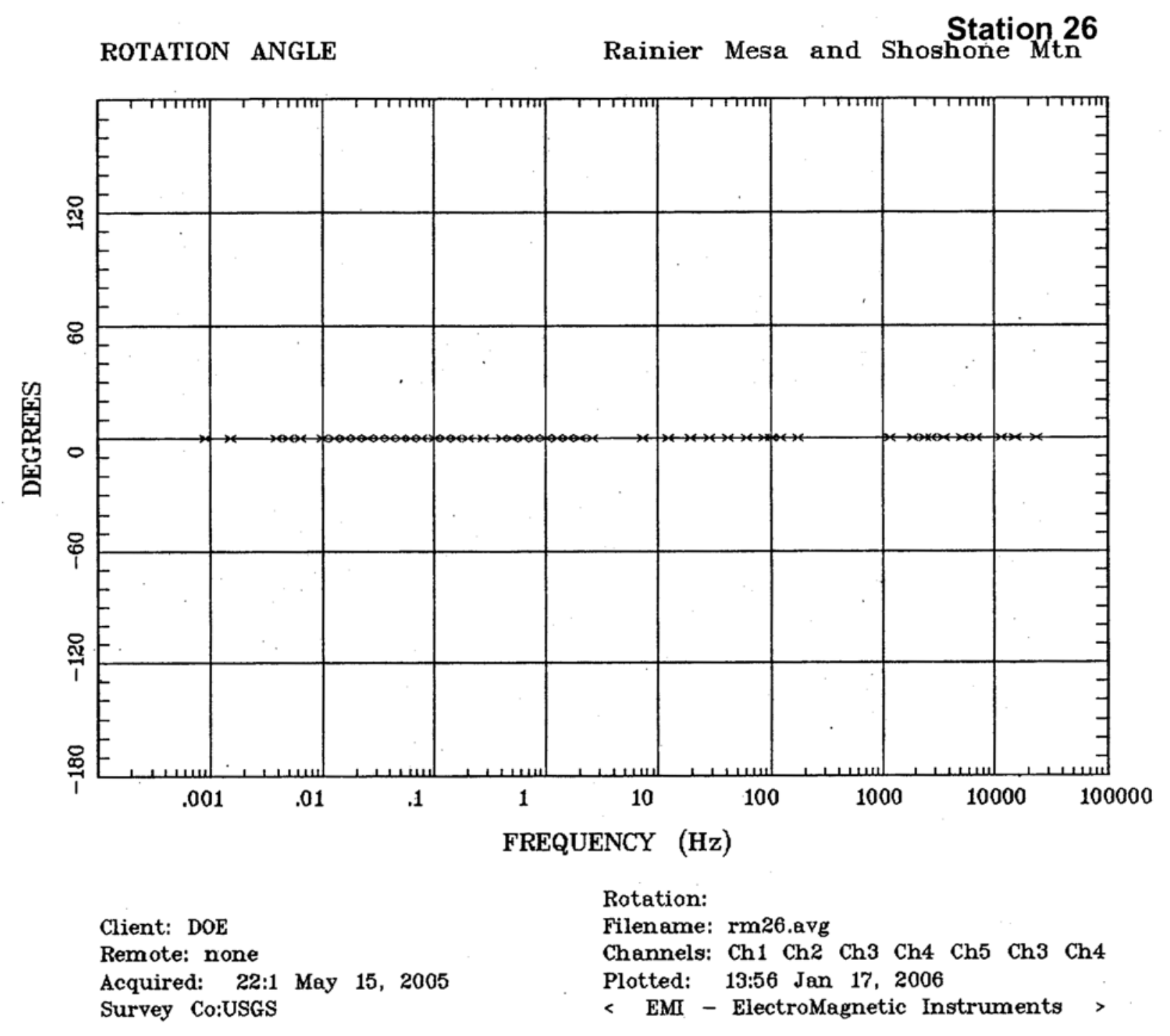




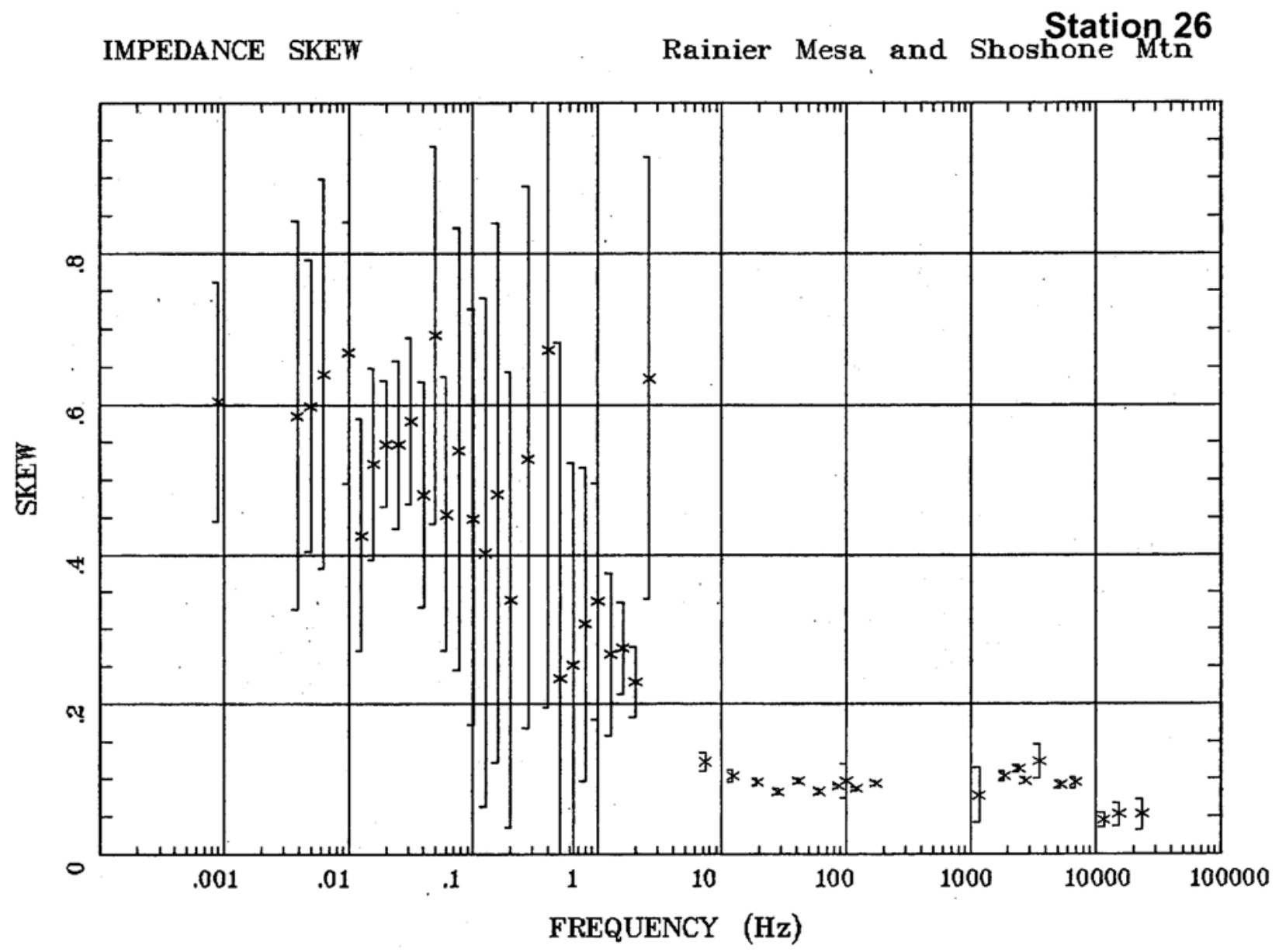

Client: DOE

Remote: none

Acquired: 22:1 May 15, 2005

Survey Co:USGS
Rotation:

Filename: rm26.avg

Channels: Ch1 Ch2 Ch3 Ch4 Ch5 Ch3 Ch4

Plotted: 13:56 Jan 17, 2006

< EMI - ElectroMagnetic Instruments > 
E MULT Coh.

Rainier Mesa and Shoshoffigh26

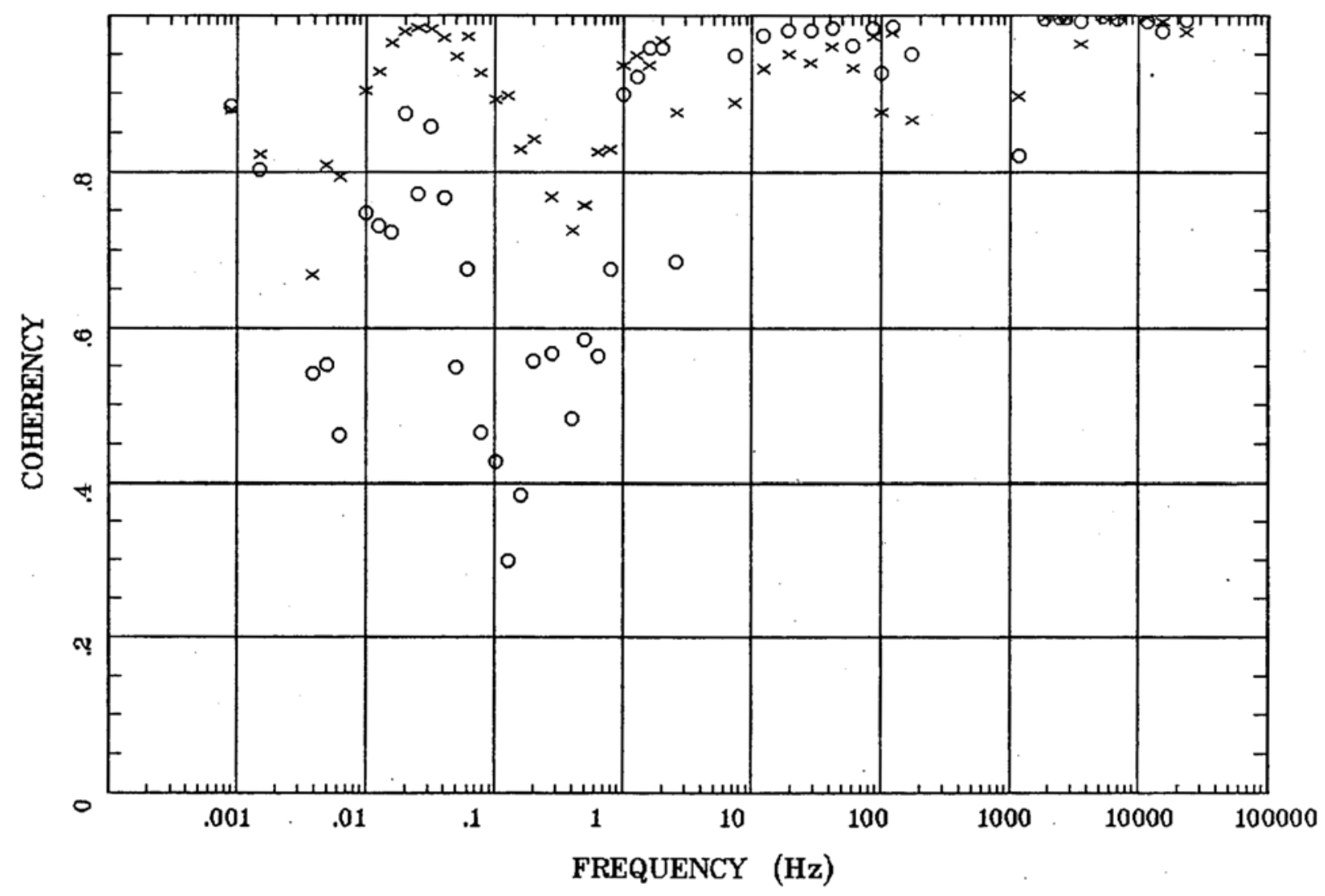

Client: DOE

Remote: none

Acquired: 22:1 May 15, 2005

Survey Co:USGS

Rotation:

Filename: rm26.avg

Channels: Ch1 Ch2 Ch3 Ch4 Ch5 Ch3 Ch4

Plotted: 13:57 Jan 17, 2006

< EMI - ElectroMagnetic Instruments > 


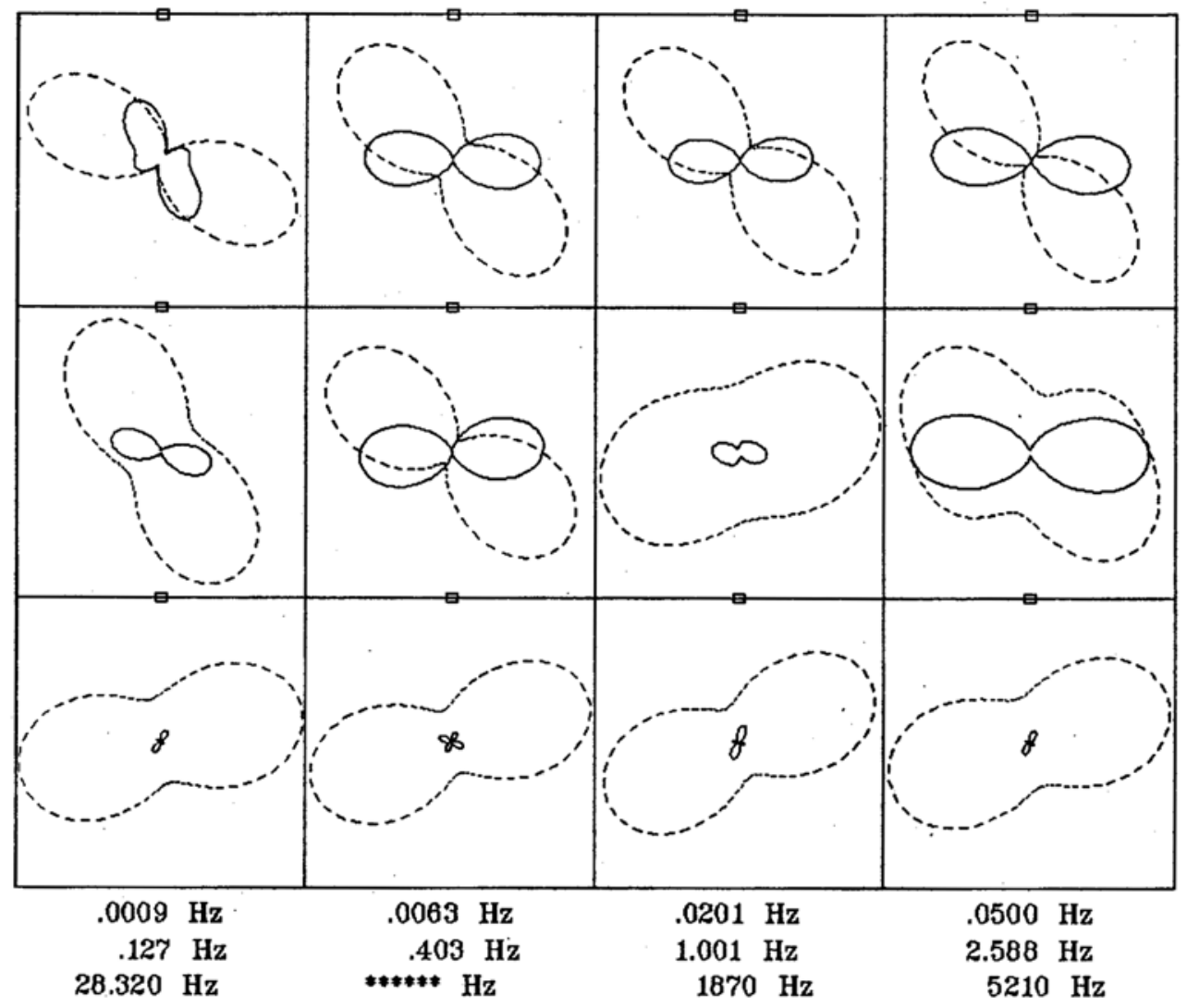

Client: DOE

Remote: none

Acquired: 22:1 May 15, 2005

Survey Co:USGS
Rotation:

Filename: rm26.avg

Channels: Ch1 Ch2 Ch3 Ch4 Ch5 Ch3 Ch4

Plotted: 13:57 Jan 17, 2006

< EMI - ElectroMagnetic Instruments > 
Rainier Mesa and Shoshoneigh 26

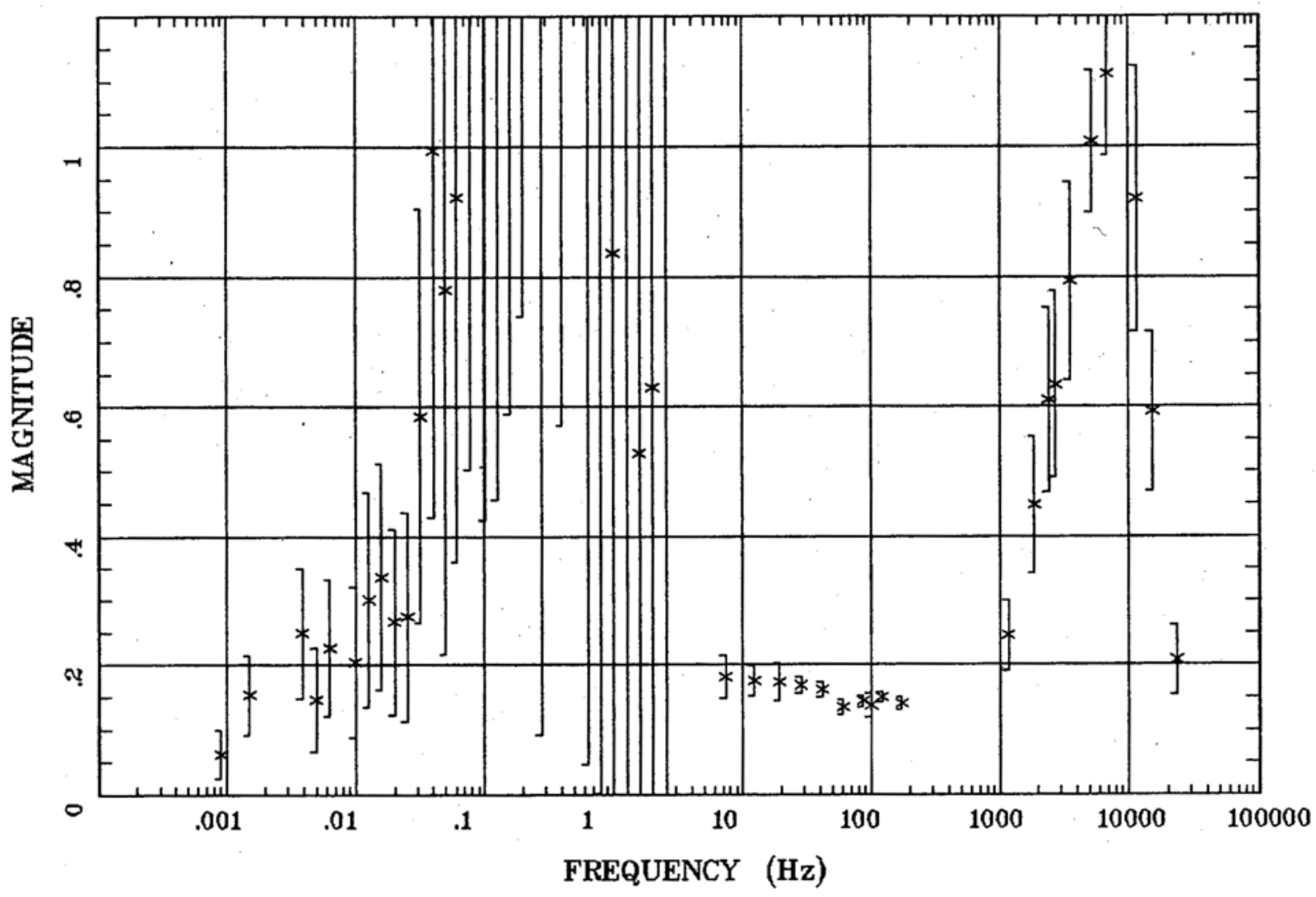

Client: $\mathrm{DOE}$

Remote: none

Acquired: 22:1 May 15, 2005

Survey Co:USGS
Rotation:

Filename: rm26.avg

Channels: Ch1 Ch2 Ch3 Ch4 Ch5 Ch3 Ch4 Plotted: 13:57 Jan 17, 2006

< EMI - ElectroMagnetic Instruments > 
TIPPER STRIKE

Station 26

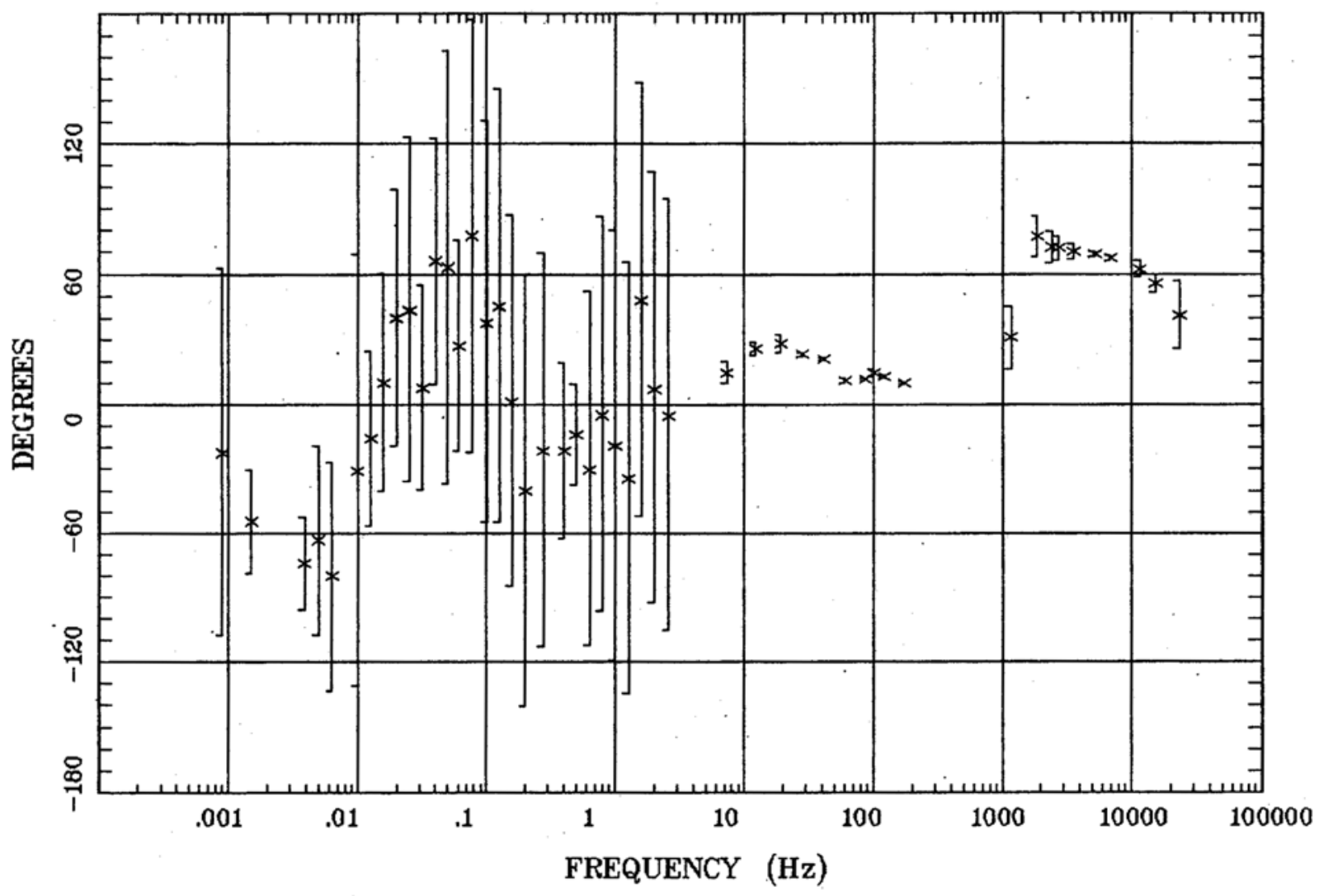

Client: $\mathrm{DOE}$

Remote: none

Acquired: 22:1 May 15, 2005

Survey Co:USGS

Rotation:

Filename: rm26.avg

Channels: Ch1 Ch2 Ch3 Ch4 Ch5 Ch3 Ch4

Plotted: 13:57 Jan 17, 2006

< EMI - ElectroMagnetic Instruments > 
HzHx.x Coh HzHy.o

Rainier Mesa and Shoshone Mtation 26

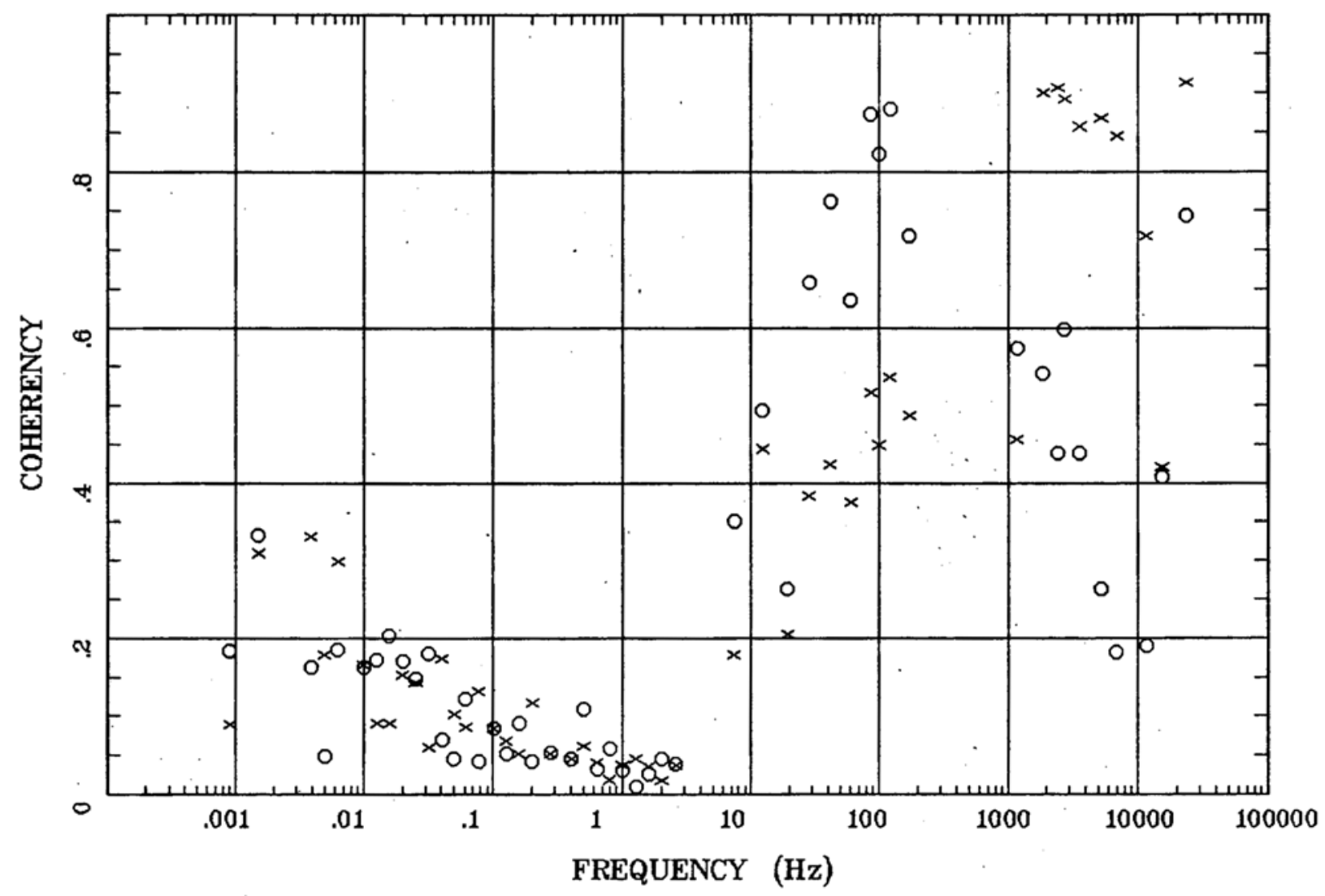

Client: DOE

Remote: none

Acquired: 22:1 May 15, 2005

Survey Co:USGS
Rotation:

Filename: rm26.avg

Channels: Ch1 Ch2 Ch3 Ch4 Ch5 Ch3 Ch4

Plotted: 13:57 Jan 17, 2006

< EMI - ElectroMagnetic Instruments > 